\title{
Manifestations of Memory in Time- and Ensemble-Average Statistical Mechanics of Low-dimensional Physical Observables
}

\author{
Dissertation \\ for the award of the degree \\ Doctor rerum naturalium
}

of the Georg-August-University Göttingen

within the doctoral program IMPRS-PBCS of the Georg-August

University School of Science (GAUSS) submitted by

Alessio Lapolla

from Italy.

Göttingen 2021 


\section{Thesis advisory committee}

Dr. Aljaž Godec, Mathematical bioPhysics group, Max Planck Institute for Biophysical Chemistry, Göttingen, Germany

Prof. Dr. Jörg Enderlein, Third Institute of Physics - Biophysics, Georg August University, Göttingen, Germany

Prof. Dr. Markus Müller, Institute for Theoretical Physics Georg August University, Göttingen, Germany

\section{Examination Board}

Dr. Aljaž Godec, Mathematical bioPhysics group, Max Planck Institute for Biophysical Chemistry, Göttingen, Germany (reviewer)

Prof. Dr. Matthias Krüger, Institute for Theoretical Physics Georg August University, Göttingen, Germany (reviewer)

Prof. Dr. Jörg Enderlein, Third Institute of Physics - Biophysics, Georg August University, Göttingen, Germany

Prof. Dr. Stephan Herminghaus, Max Planck Institute for Dynamics and Self-Organization, Göttingen, Germany

Prof. Dr. Markus Müller, Institute for Theoretical Physics Georg August University, Göttingen, Germany

Prof. Dr. Peter Sollich, Institute for Theoretical Physics Georg August University, Göttingen, Germany

Date of the oral examination: 31 May 2021. 


\begin{abstract}
The study of stochastic processes plays a very important role in our current understanding of Statistical Physics, in particular many results from this field have found successful applications in biological, socio-economics and condensed matter systems.

An important feature of stochastic process is the presence, or absence, of memory, i.e.: does the state of the system at a certain time depends on its history or not? In many physically relevant scenarios the answer is yes. Henceforth in the articles collected in this thesis we, at least partially, constructed a theory able to describe and compute relevant properties of processes with memory. This goal has been achieved in two ways: considering time-averaged observables and then obtaining explicit results for the respective expectation moments, and studying how a dimensionality reduction procedure acting on a multi-dimensional memoryless system produces a resulting process displaying memory effects. Finally the two approaches have been combined as well.

We used the theory so developed to analyze classical exactly solvable many-body systems in Statistical Mechanics: the single file and the Gaussian network models. We also applied our analysis to trajectories derived from experimental time-series and Molecular Dynamics simulations. We used the tools we developed to elucidate properties of relaxation processes towards equilibrium and to investigate the memory origin of the breaking of timetranslation invariance.
\end{abstract}




\section{Contents}

\begin{tabular}{lll}
\hline & Introduction & 7
\end{tabular}

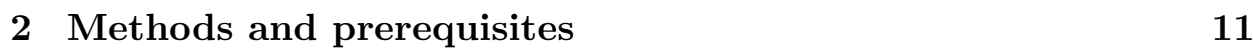

2.1 Stochastic processes . . . . . . . . . . . . . . . . . 11

$2.1 .1 \quad$ Ergodicity $\ldots \ldots \ldots \ldots \ldots$

2.2 Stochastic differential equations . . . . . . . . . . . . . . . 14

2.2 .1 The Langevin equation . . . . . . . . . . . . . . . 14

2.3 The Fokker-Planck equation . . . . . . . . . . . . . . . . . . 15

$2.3 .1 \quad$ Eigenfunction expansion . . . . . . . . . . . . . . . 16

2.4 The Feynman-Kac formula $\ldots \ldots \ldots \ldots$. . . . . . . . 17

2.5 Local time . . . . . . . . . . . . . . . . . . . . 18

3 Unfolding tagged particle histories in single-file diffusion: exact single- and two-tag local times beyond large deviation theory

4 Manifestations of Projection-Induced Memory: General Theory and the Tilted Single File

5 BetheSF: Efficient computation of the exact tagged-particle propagator in single-file systems via the Bethe eigenspectrum

6 Faster Uphill Relaxation in Thermodynamically Equidistant Temperature Quenches

7 Spectral theory of fluctuations in time-average statistical $\begin{array}{ll}\text { mechanics of reversible and driven systems } & 91\end{array}$

8 Single-file diffusion in a bi-stable potential: Signatures of memory in the barrier- crossing of a tagged-particle $\quad 109$

9 A Toolbox for Quantifying Memory in Dynamics Along Reaction Coordinates 
10 Ubiquitous Dynamical Time Asymmetry in Measurements on Materials and Biological Systems

11 Time- and ensemble-average statistical mechanics of the Gaus- 


\section{Chapter 1}

\section{Introduction}

Understanding our surrounding world has been one of the motors of civilization. Since the XVI century, with the Scientific Revolution, humanity started using a precise method to investigate Nature. Among all the Sciences, Physics aims to achieve this goal describing Nature in the language of Mathematics. This dogma is a corner stone of our modern Physics. However until the XIX century the view of the world was predominantly mechanistic and deterministic, with the XX century a more probabilistic approach took over. The paradigm shift was twofold: on one hand the quantum revolution challenged the deterministic hypothesis on a fundamental level while, on the other hand, physicists addressed more and more complicated systems, involving such a large number variables that a microscopic description of the system would be de facto impossible.

The problems related to the latter situation are traditionally grouped under the name of Statistical Mechanics: the branch of Physics that aims to describe physical systems in the language of probability and chance.

The general framework assumes that we are describing a univers ${ }^{1}$ with a huge number of degrees of freedom, these variables follow Hamilton's equation of motion. However, part of these variables may often be considered as being "fast" while the remaining are considered to be "slow". We will say that the former set belongs to a "heat bath" while the latter corresponds to the system. One physical reason why we can often divide our universe between "fast" and "slow" degrees of freedom is, for example, that we are often interested in a solute-solvent system in which the solvent particles are much smaller and lighter than the solute particles, and therefore relax towards equilibrium faster. A prime example that justifies this hypothesis can be attributed to observations of Robert Brown in 1827 [1] who noticed that tiny grains of pollen suspended in water showed an erratic motion. In

\footnotetext{
${ }^{1}$ In this context universe must not be confused with the cosmological and observable universe. We use this name just to refer to all the particles described by some particular mathematical model at hand.
} 
his experiment the pollen grain were the "system" and the much smaller water molecules were the "heat bath"; the numerous, effectively random, bumps of these molecules against the system particles cause the random motion nowadays referred to Brownian motion. In the subsequent century many notable physicists and mathematicians laid the theoretical foundations to understand phenomena akin to Brownian motion while countless experiments explored the probabilistic description of Nature. In $1905 \mathrm{Al}-$ bert Einstein gave the first theoretical explanation of Brownian motion [2]. The same year Sutherland, independently, published an article with similar equations [3]. Von Smoluchowski [4], the following year, gave an remarkably solid foundation to the atomistic theory on the basis of the Einstein's equation. In 1909 Perrin confirmed the results experimentally [5]. Further historical contributions were given by Langevin [6], Fokker [7], Planck [8, Ornstein and Uhlenbeck [9], Klein [10], and Kramers [11].

Inspired by these developments many mathematicians laid the rigorous basis to correctly describe these phenomena: Wiener [12, 13], Kolmogorov [14, 15], Feller [16], and Lévy [17, 18], to list only a few of the most prominent names.

The inexplicable power of randomness The description of natural phenomena via the physics and mathematics of Brownian motion has found application at the most disparate scales: from the incommensurately tiny quantum world [19, 20], describing the quark-gluon plasma [21, 22] and nuclear collisions [23], to the gigantic astronomical scale [24, 25] using a relativistic formulation of it [26]. Passing by all the possible intermediate sizes, Brownian motion has been used to describe systems from molecular biology [27] to flocks of birds and school of fishes [28. Moreover, ideas from Statistical Physics have been applied to the anthropic fields of Finance [29, 30], Computer Science [31], and even Social Science [32]. This ability to describe phenomena on all physical scales and beyond is quite unique. Partially this feature can be explained using powerful theorems of Probability Theory (like the Central Limit Theorem) that predict a universal behavior of random variables irrespective of any details. Nevertheless the reason why so many different phenomena satisfy, with such a remarkable agreement, the abstract hypotheses of these theorems is a mystery that we may never solve.

Ensemble and time averages In Statistical Mechanics we are, in general, only interested in the statistical property of an observable. The idea is that each realization of a given phenomenon will yield a different result for each measurement we perform on the system; in order to deal with this variability we must inspect the statistical properties of the ensemble of all possible realizations must be performed. The expected value in such an ensemble referred to as an "ensemble average", and therefore such observables 
are called "ensemble averaged" observables. According to the law of large numbers the variance of these quantities vanishes whenever we are dealing with systems that contain a large number of particles. Another way to evaluate the statistical properties is to inspect how they evolve in time along individual realizations of the experiment. The expected value over a single realization is called a "time average" and such observables are referred to as "time averaged" obervables. For random processes these time averages are stochastic quantities as well. However, under conditions typically satisfied in physical systems these time averages are guaranteed to converge to their ensemble counterparts [33].

Markovian and non-Markovian observables Most of the work of this thesis has been devoted to the study of the properties of non-Markovian processes, that is processes with memory, and their application in physical systems. The reason why these processes are so important is that they are ubiquitous in Nature. Quoting van Kampfen: "Non-Markov is the rule, Markov is the exception" [34]. Nevertheless Markov processes are much better understood for two main reasons: their mathematical treatment is often easier, and, after some reasonable approximation or on a limited time-scale, non-Markovian processes can be frequently well described by Markovian ones [35].

It is important to stress that the adjectives Markovian or non-Markovian cannot really be applied to a physical system, instead they make sense in relation to an observable used to describe the physical system.

From this point on we will understand a physical system as a set of equations of motion (in an abstract sense) describing the evolution in time, while an observable will be set of variables that we measure in the physical system. Often the observable coincides with the coordinates entering the equations of motion (positions and velocities usually) and in this sense the term "Markovian (on non-Markovian) physical system" is in fact widely used in the literature. Note, however, that it is very easy to construct a counterexample where an observable of a system described by Markovian equations of motion is in fact non-Markovian: a function that explicitly depends on the history of the process, i.e. a non-anticipating (to respect causality) and non-local function of time. Even so, the wording (non-)Markovian system is useful and descriptive. In this thesis we will discuss two common ways in which non-Markovian observables may emerge: as a result of time averaging or a projection (and combinations of these two).

Time-averaging is a common way to analyze experimental time-series and theoretical models. Here a function of the system coordinates is aver- 
aged over the entire trajectory. These observables are therefore nominally non-Markovian. Mathematically they correspond to functionals and are especially useful in the analysis of laboratory and computer experiments, since it is often challenging to obtain a sufficiently large number of statistically independent trajectories that would allow the application of ensemble Statistical Mechanics. Though under given conditions [36], we may still equate time averaged results to ensemble averaged results in the long time limit.

The other class of non-Markovian observables we studied in this thesis originates from a projection [35]. Unless the hidden, projected-out degrees of freedom are "fast", that is, they rapidly reach their equilibrium distribution, the resulting observable is in general non-Markovian [37. This situation is in fact common in experiments. Often we are interested in high-dimensional or many-body systems, like biomolecules ${ }^{2}$, where it is extremely difficult to track all degrees of freedom. Examples of experiments tracking lower dimensional coordinates (i.e. in which a projection is hardwired in the setup) are the measurements of the extension of a molecule (in optical tweezers experiments) or the distance between two fluorescent dyes attache to a molecule (in FRET experiments). The analysis and the interpretation of the results should be performed keeping in mind the non-Markovian nature of the observed process.

In summary, in this thesis we aimed at understanding the generic role of a projection in experiments on physical systems by systematically comparing Markovian and non-Markovian observables. In particular, to achieve this latter goal we developed a theory for the statistics of time-averaged functionals of the full and projected trajectories. Finally, we applied these newly developed tools to study relaxation processes and violations of the time-translational invariance in theoretical models and time-series obtained from laboratory and experiments.

\footnotetext{
${ }^{2}$ Although a molecule is "one-body" its dynamics depends on its many internal degrees of freedom.
} 


\section{Chapter 2}

\section{Methods and prerequisites}

In this chapter we present the basic background knowledge we extensively used in this thesis. Many of the topics discussed here have been developed during the XX century by some of the most prominent physicists and mathematicians in history. Nevertheless, we will summarize them here in order to provide the common base to the work presented in the following chapters. After a short, non-rigorous, and incomplete review of selected mathematical concepts from the theory of stochastic processes; we will review the main tools used in Physics to describe random systems: the Langevin equation, the Fokker-Planck equation, and the Feyman-Kac formula. For the sake of simplicity, and in order to avoid tedious technical complications and a cumbersome notation, these results will be presented for the one dimensional case; even though we will later develop the theory for systems with an arbitrary dimensionality. In most cases the extension is conceptually straightforward. In addition a selection of the most common solution methods is reported for sake of completeness.

\subsection{Stochastic processes}

Let us define the probability space [38, 39, 40] $(\Omega, \mathcal{F}, \operatorname{Pr})$ where:

- $\Omega$ is the sample space of all possible events;

- $\mathcal{F}$ is a $\sigma$-algebra, that is a collection of subsets of $\Omega$ such that: it contains $\Omega$ in itself, and it is closed under complement, countable unions, and intersections;

- Pr is the probability "law" that assigns to every $F \in \mathcal{F}$ a number between 0 and 1 ;

let us also define the mathematical space $S$ measurable under the $\sigma$-algebra

$\Sigma$. Then, given the probability space and the measurable space $(S, \Sigma)$, the 
stochastic process

$$
\left\{X_{t}, t \in T\right\}
$$

is a collection of random variables belonging to $S$ indexed by some set $T$. A follow-up important definition is the one of conditional probability that we will denote as $\operatorname{Pr}(X \mid Y)$, indicating the probability of $X$ occurring given $Y$, where $X$ and $Y$ belong to $\mathcal{F}$.

This rather abstract definition is the mathematical basis to discuss and categorize all possible process with some degree of randomness. One of the most important distinctions between different stochastic process derives from the properties of the set $T$. If said set is countable the process is referred to as a discrete time process and as a continuous time process otherwise. This thesis deals only with continuous time processes, whose basic properties are listed below.

An important class of stochastic processes are so-called Markov processes. These processes are "memoryless", that is: the next element of the process depends only on the present one. More formally the following equality must hold for $\left\{X_{n}, n \in T\right\}$ to be Markovian:

$$
\operatorname{Pr}\left(X_{n+1}=x \mid X_{1}=x_{1}, X_{2}=x_{2}, \ldots, X_{n}=x_{n}\right)=\operatorname{Pr}\left(X_{n+1}=x \mid X_{n}=x_{n}\right) .
$$

When Eq. 2.2 is not satisfied the process is said to be non-Markovian.

A continuous time stochastic process $\left\{X_{t}, t \geq 0\right\}$ is a Lévy process if the following conditions are satisfied:

- an initial state $X_{0}$ is defined;

- independence of increments: $X_{t+1}-X_{t}$ are independent for any $t$;

- stationary increments: the increment $X_{t}-X_{s}$ is equal in distribution to $X_{t-s}$;

- continuity in probability: $\lim _{h \rightarrow 0} \operatorname{Pr}\left(\left|X_{t+h}-X_{t}\right|>\epsilon\right)=0, \forall \epsilon>0$.

Lévy processes are the basis of many stochastic processes studied in Probability Theory, the most prominent one being the Wiener process $W_{t}$. It has two very important properties in addition to the normal properties of a Lévy process:

- the increments follow a normal distribution: $W_{s+t}-W_{s} \sim \mathcal{N}(0, t)$,

- $W_{t}$ is continuous in $t$, but never differentiable. 
The Wiener process is the precise mathematical definition of Brownian motion, in Physics often these two terms are used as synonyms. Another very important stochastic process often used in Physics is the continuous time Markov Chain (or Markov State Model) that is defined by:

- a countable (often, but not necessarily, finite) state space $S$;

- a transition matrix $T$ of the same dimension of $S$;

- an initial state.

Markov Chains are frequently used to describe physical process in which the dynamics can be approximated by independent jumps between discrete states.

The Wiener process and Markov Chains are the bases for many continuous time random process encountered in Physics.

\subsubsection{Ergodicity}

Many properties and results derived in Statistical Mechanics rely on the ergodic hypothesis [36]. Historically the first person to introduce this idea was Boltzmann who used it at the end of the XIX century to prove the equipartition of energy in ideal gasses. In the context of Statistical Mechanics the ergodic hypothesis assumes that after a long time a mechanical system will spend in each microstate an amount of time proportional to the Boltzmann weight. This idea has later been formalized by Poincaré in his recurrence theorem that states that an ergodic system, albeit after an astronomical time, will return infinitely close to each point along his trajectory. Subsequently, mainly Birkhoff and Khinchin formulated this idea in a more rigorous measure-theoretical and probabilistic formulation. A very important result is the Birkhoff-Khinchin theorem that makes a connection between time and ensemble averages.

A probability "law " $\operatorname{Pr}$ can be viewed as measure on the set $\Omega$ such that $\operatorname{Pr}(\Omega)=1$; then we can define:

Definition 1 The ensemble average of the integrable random variable $f$ : $\Omega \rightarrow \mathbb{R}$ is defined as:

$$
\langle f\rangle=\int f \mathrm{dPr} .
$$

While in the context of continuous time processes the time average can be defined as: 
Definition 2 The time average of the integrable random variable $f: \Omega \rightarrow \mathbb{R}$ is defined as:

$$
\bar{f}(t)=\frac{1}{t} \int_{0}^{t} f(\tau) \mathrm{d} \tau .
$$

Then, in the case of ergodic process the following holds for sufficiently long times:

$$
\lim _{t \rightarrow \infty} \bar{f}(t)=\langle f\rangle .
$$

\subsection{Stochastic differential equations}

Since Newton, differential equations have been, arguably, the most important mathematical object used in Physics. Often a differential equation is used to describe the time-evolution of a particle given some initial conditions. In the case in which, as in Statistical Physics, the differential equation contains a noisy term the mathematical theory able to correctly integrate this class of equation has been developed quite recently by Kiyosi Itô [41, 42, who founded the field of stochastic calculus, the so-called Itô calculus, in order to solve this problem.

A stochastic differential equation describing the time-evolution of the stochastic variable $X_{t}[43$ reads

$$
d X_{t}=b\left(t, X_{t}\right)+\sigma\left(t, X_{t}\right) d W_{t}
$$

where $b$ and $\sigma$ are sufficiently regular functions, and $W_{t}$ is the Wiener process. The solution to this equation, with initial condition $X_{0}$, reads

$$
X_{t}=X_{0}+\int_{0}^{t} b\left(s, X_{s}\right) d s+\int_{0}^{t} \sigma\left(s, X_{s}\right) d W_{s},
$$

where the last term is an Itô integral.

\subsubsection{The Langevin equation}

In 1908 Paul Langevin [6], a French physicist, formulated a phenomenological differential equation to describe the motion of a Brownian particle. Though a rigorous definition of his equation only came after four decades thanks to the work of Itô, it is still a useful tool in Physics since it intuitively describes the physical process. Originally Langevin described the motion of a Brownian particle considering that it is constantly accelerated by a random force and by a deterministic force, while being decelerated by the friction against the fluid surrounding the particle. Therefore Newton's equation of motion for this system read

$$
\begin{aligned}
& m \dot{v}=f(x)-\gamma v+\sqrt{2 \gamma k_{B} T} \epsilon_{t}, \\
& \dot{x}=v
\end{aligned}
$$


where $m$ is the mass of the particle, $\gamma$ is the friction coefficient, $k_{B}$ is the Boltzmann constant, $T$ is the temperature, $f$ is the external force, $x$ is the position of the particle and $v$ its velocity. The random term $\epsilon_{t}$ indicates the white noise term, that is Gaussian distributed with $\left\langle\epsilon_{t}\right\rangle=0$ and $\left\langle\epsilon_{t} \epsilon_{s}\right\rangle=$ $\delta(t-s)$. In many physical application inertial effects can be neglected, therefore it is possible to simplify Eq. 2.5), to the so-called overdamped Langevin equation that reads:

$$
\dot{x}=\frac{1}{\gamma} f(x)+\sqrt{2 D} \epsilon_{t},
$$

where we used the Einstein's relation to define the diffusion coefficient

$$
D=\frac{k_{B} T}{\gamma},
$$

a manifestation of the fluctuation-dissipation theorem.

\section{Solution of a Langevin equation}

With rare exceptions an analytic solution of the Langevin equation is unfeasible; only numerical solutions are accessible. The most basic numerical method to solve them is the Euler-Maruyama scheme. To simulate the stochastic motion (we will use the overdamped Langevin equation (2.6) as an example, further extensions are straightforward) up to time $t$, we divide this interval in $N$ equispaced subintervals of length $\Delta t$. Then, given an initial condition $x_{0}$ the successive positions are calculated according to:

$$
x_{n+1}=x_{n}+\frac{1}{\gamma} f\left(x_{n}\right) \Delta t+\sqrt{2 D \Delta t} \epsilon,
$$

where $\epsilon$ is a random number drawn from the normal distribution $\mathcal{N}(0,1)$.

\subsection{The Fokker-Planck equation}

The Fokker-Planck equation [44] (that sometimes also carries the name of two other prominent scientists: Kolmogorov and/or Smoluchowski) is a partial differential equation describing the evolution of the conditional probability density function of a stochastic process, that is, its solution describes the probability density of finding a process (e.g. the position of a particle) at time $t$ in the point $x$ that it was at $x_{0}$ at time $t_{0}$ and reads:

$$
\frac{\partial G\left(x, t \mid x_{0}, t_{0}\right)}{\partial t}=-\mu \frac{\partial G\left(x, t \mid x_{0}, t_{0}\right)}{\partial x}+D \frac{\partial^{2} G\left(x, t \mid x_{0}, t_{0}\right)}{\partial x^{2}}
$$

with initial condition $G\left(x, t_{0} \mid x_{0}, t_{0}\right)=\delta\left(x-x_{0}\right)$, where $\delta\left(x-x_{0}\right)$ is the Dirac's delta function, while $\mu$ and $D$ represent the constant drift and diffusion coefficients respectively. Eq. (2.9) must be completed by opportune 
boundary conditions imposed by the nature of the stochastic process. The solution $G\left(x, t \mid x_{0}, t_{0}\right)$ to Eq. (2.9) is often called the Green's function in agreement with the nomenclature commonly used in linear operator theory. Other common names are generator function or the particularly pictorial name of propagator since $G\left(x, t \mid x_{0}, t_{0}\right)$ "propagates" the solution from the initial to the final state.

Equation (2.9) is also referred as a forward equation since it describes the evolution from the initial condition at $t_{0}$ towards future times $t>t_{0}$. Equivalently it is possible to describe the same process backward in time, that is, we are asking for the probability of starting in a given state given that we end up in $x$ at time $t$. Hence we are propagating our solution backward in time for times $s<t$. The backward equation reads

$$
-\frac{\partial G\left(x, t \mid x_{0}, s\right)}{\partial s}=\mu \frac{\partial G\left(x, t \mid x_{0}, s\right)}{\partial x}+D \frac{\partial^{2} G\left(x, t \mid x_{0}, s\right)}{\partial x^{2}}
$$

and initial condition $G\left(x, t \mid x_{0}, t\right)=\delta\left(x-x_{0}\right)$, again with appropriate boundary conditions.

The two descriptions are equivalent; the forward description is often more useful in applications since it describes measurable experimental quantities as a function of the observed time; the backward equation has proven useful in diverse contexts, in particular in the study of first passage time problems [45].

\subsubsection{Eigenfunction expansion}

The Fokker-Planck equation belongs to the class of parabolic differential equations. These equations have a long history in mathematics and therefore several methods have been devised to solve them. One of the most powerful (with roots in the Strum-Liouville operator theory) is the eigenfunction expansion method [44, 33]. We define the Fokker-Plank operator $\hat{L}$ such that Eq. (2.9) reads:

$$
G\left(x, t \mid x_{0}, 0\right)=-\hat{L} G\left(x, t \mid x_{0}, 0\right) ;
$$

whose formal solution reads

$$
\frac{\partial G\left(x, t \mid x_{0}, 0\right)}{\partial t}=\mathrm{e}^{-\hat{L} t} G\left(x, 0 \mid x_{0}, 0\right),
$$

that can be expressed using the eigenvalues $\lambda_{k}$, the left eigenfunctions $\psi_{k}^{L}(x)$, and the right eigenfunctions $\psi_{k}^{R}(x)$ of $\hat{L}$,

$$
G\left(x, t \mid x_{0}, 0\right)=\sum_{k} \psi_{k}^{R}(x) \psi_{k}^{L}\left(x_{0}\right) \mathrm{e}^{-\lambda_{k}\left(t-t_{0}\right)} .
$$


The eigenfunctions are bi-orthonormal (introducing the Kronecker delta $\delta_{k l}$ )

$$
\int d x \psi_{k}^{R}(x) \psi_{k}^{L}(x)=\delta_{k l} .
$$

and for ergodic systems $\lambda_{0}=0$. The eigenvalues satisfy the following inequality: $\lambda_{0} \leq \Re\left(\lambda_{1}\right) \leq \cdots \leq \Re\left(\lambda_{k}\right) \leq \cdots \leq \infty$. Therefore the differential equation is reduced to and eigenvalue problem, that is often easier to solve, given by the following set of equations:

$$
\hat{L} \psi_{k}^{R}(x)=\lambda_{k} \psi_{k}^{R}(x) \quad \hat{L}^{\dagger} \psi_{k}^{L}(x)=\lambda_{k}^{\dagger} \psi_{k}^{L}(x),
$$

where $\hat{L}^{\dagger}$ denotes the adjoint backward Fokker-Planck operator (see Eq. 2.10) and $\lambda_{k}^{\dagger}$ its eigenvalues.

Other popular methods to solve the parabolic differential equation are based on the Fourier and Laplace transform or on the reflection method.

\subsection{The Feynman-Kac formula}

The forward Fokker-Planck equation (2.9) is a parabolic differential equation akin to the Schrödinger equation (up the imaginary unit factor). In the 1940s Richard Feynman [46] in his $\mathrm{PhD}$ thesis reformulated the quantum theory using the path integral: an average over all the possible paths of the particle. Subsequently Mark Kac [47] realized that a similar representation may be formulated for diffusion processes. More generally [43] for a drift-diffusion Itô equation

$$
d X_{t}=\mu\left(X_{t}\right) d t+\sqrt{2 D\left(X_{t}\right)} d W_{t}
$$

describing the evolution of our stochastic process for all the possible initial conditions $X_{0}=x_{0}$, the backward Fokker-Planck operator (in the anti-Itô sense) reads

$$
\hat{L}^{\dagger}=D(x) \frac{\partial^{2}}{\partial x^{2}}+\mu(x) \frac{\partial}{\partial x} .
$$

Let $V(x)$ be a function bounded from below. Then for sufficiently well behaved $\mu(x)$ and $D(x)$ the function $u(x, t)$ defined as

$$
u(x, t)=\left\langle\exp \left(-\int_{0}^{t} d \tau V\left(X_{\tau}\right)\right)\right\rangle_{f},
$$

satisfies the differential equation

$$
\frac{\partial u}{\partial t}=\hat{L}^{\dagger} u-V u
$$

for well behaved initial conditions $u(x, 0)=f(x)$. The brackets in Eq. (2.18) $\langle\cdot\rangle_{f}$ represent the average over all the possible realization of the process with 
the initial condition given by $f(x)$.

These last two equations represent the core of the Feynman-Kac formula and allow us to calculate the distribution of the functional

$$
\int_{0}^{t} d \tau V\left(X_{\tau}\right)
$$

of the stochastic process $X_{\tau}$ solving a "tilted " backward Fokker-Planck equation 2.10). This result connects several important aspects of stochastic processes and it proved to be an invaluable tool in Mathematics, Physics and Finance.

\subsection{Local time}

A big part of this thesis is devoted to the analysis of a specific stochastic functional: the so-called local time. This functional enjoys a long tradition in Mathematics tradition [48, 49], for a continuous time Itô process $Z_{t}$ it can be defined as

$$
L_{x}(t)=\int_{0}^{t} \delta\left(Z_{s}-x\right) d s,
$$

where we used the Dirac's delta in the definition. This functional is extremely important because for every bounded function $f$ of the stochastic process we can use the following powerful identity

$$
\int_{0}^{t} f\left(Z_{s}\right) d s=\int f(x) L_{x}(t) d x .
$$

Analogously, for a discrete space process the local time can be defined using the characteristic function:

$$
L_{x}(t)=\int_{0}^{t} \mathbb{1}_{x}\left(Z_{s}\right) d s .
$$

In this thesis the time average of the local time

$$
\theta_{x}(t)=L_{x}(t) / t
$$

has been used as a concrete example to compute the time-averaged statistic of several theoretical and experimental models. Thanks to the property defined in 2.22), these results can easily be extended to a wide class of observables. 


\section{Publications}

In the following chapters all the publications produced during the doctorate program so far are reported in their published form in chronological order. All the references and cross-references in each article only refer to the article in itself. All the references in the articles must be considered as part of the reference list of the thesis, although some of them are repeated to respect the format of the original publication. 


\section{Chapter 3}

\section{Unfolding tagged particle histories in single-file diffusion: exact single- and two-tag local times beyond large deviation theory}

This article has been published in the New Journal of Physics under Creative Commons Attribution 3.0 Licence [50]. In this work we derived explicit formulae for the mean, the variance and the covariance of the local time for stochastic processes starting from equilibrium initial conditions using the Feynman-Kac theorem and the diagonal form of the Fokker-Planck operator. We applied our results to the study of a paradigmatic stochastic many-body system: a single file in a box. Interestingly, studying the correlation of histories between different particles we found a dynamic crystallization-like transition in dense single-file systems. 
New Journal of Physics

The open access journal at the forefront of physics
Published in partnership with: Deutsche Physikalische

Gesellschaft and the Institute of Physics

PAPER

CrossMark

OPEN ACCESS

RECEIVED

13 July 2018

REVISE

4 October 2018

ACCEPTED FOR PUBLICATION

22 October 2018

PUBLISHED

14 November 2018

Original content from thi work may be used under the terms of the Creative

Commons Attribution 3.0 licence.

Any further distribution of this work must maintain

attribution to the author(s) and the title of

the work, journal citation and DOI.

\title{
Unfolding tagged particle histories in single-file diffusion: exact single- and two-tag local times beyond large deviation theory
}

\author{
Alessio Lapolla and Aljaž Godec \\ Mathematical Biophysics Group, Max-Planck-Institute for Biophysical Chemistry, Göttingen D-37077, Germany \\ E-mail: agodec@mpibpc.mpg.de
}

Keywords: stochastic processes, occupation time functional, single-file diffusion, projection-induced memory, stochastic many-body systems, generating functional, large deviations

\begin{abstract}
Strong positional correlations between particles render the diffusion of a tracer particle in a single file anomalous and non-Markovian. While ensemble average observables of tracer particles are nowadays well understood, little is known about the statistics of the corresponding functionals, i.e. the timeaverage observables. It even remains unclear how the non-Markovian nature emerges from correlations between particle trajectories at different times. Here, we first present rigorous results for fluctuations and two-tag correlations of general bounded functionals of ergodic Markov processes with a diagonalizable propagator. They relate the statistics of functionals on arbitrary time-scales to the relaxation eigenspectrum. Then we study tagged particle local times- the time a tracer particle spends at some predefined location along a single trajectory up to a time $t$. Exact results are derived for one- and two-tag local times, which reveal how the individual particles' histories become correlated at higher densities because each consecutive displacement along a trajectory requires collective rearrangements. Our results unveil the intricate meaning of projection-induced memory on a trajectory level, invisible to ensemble-average observables, and allow for a detailed analysis of singlefile experiments probing tagged particle exploration statistics.
\end{abstract}

\section{Introduction}

Single-file dynamics refers to the motion of particles in a narrow, effectively one-dimensional channel, which prevents their crossing, and is central to the transport in biological channels [1] the kinetics of transcription regulation [2], transport in zeolites [3] and in superionic conductors [4]. Recent advances in single-particle tracking and nanofluidics enabled experimental studies of single file dynamics in colloidal systems, which directly probe the fundamental physical principles of tagged particle motion to an unprecedented precision $[5,6]$.

The motion of particles in a single file is strongly correlated, which gives rise to a rich and intricate phenomenology. In a Brownian single file the non-crossing constraint leads to subdiffusion with the ensemble mean squared displacement (MSD) of a tagged particle scaling as $\left\langle[x(t)-x(0)]^{2}\right\rangle \propto \sqrt{t}$ [7]. When confined to a finite interval the subdiffusive scaling of the MSD is transient, saturating at an equilibrium variance, with the extent of the subdiffusive regime growing with the particle density (see figure 1(a) and [8]). Concurrently, an effective harmonization emerges at increasing density, with the invariant measure of a tagged particle approaching a Gaussian and a vanishing kurtosis excess $\gamma_{2}=\left\langle x^{4}\right\rangle_{\mathrm{eq}} /\left\langle x^{2}\right\rangle_{\mathrm{eq}}^{2}-3$ (see inset of figure 1(a)). More generally it holds that the MSD of a tagged particle in an unconfined single file and the absolute dispersion of a free particle in the limit $t \rightarrow \infty$ are related via $\left\langle[x(t)-x(0)]^{2}\right\rangle \propto\langle|x(t)|\rangle_{\text {free }}$ [9]. The motion of particles on a many-body level is Markovian, the resulting tagged particle dynamics is, however, highly non-Markovian [8], and displays a staggering dependence on the respective initial conditions [10].

Tremendous effort has been made to study the tagged particle dynamics theoretically [11]. In particular, the tagged particle ensemble propagator has been studied using the 'reflection principle' [12], Jepsen mapping [13], momentum Bethe ansatz [8], harmonization techniques [14], and macroscopic fluctuation [15] and large 

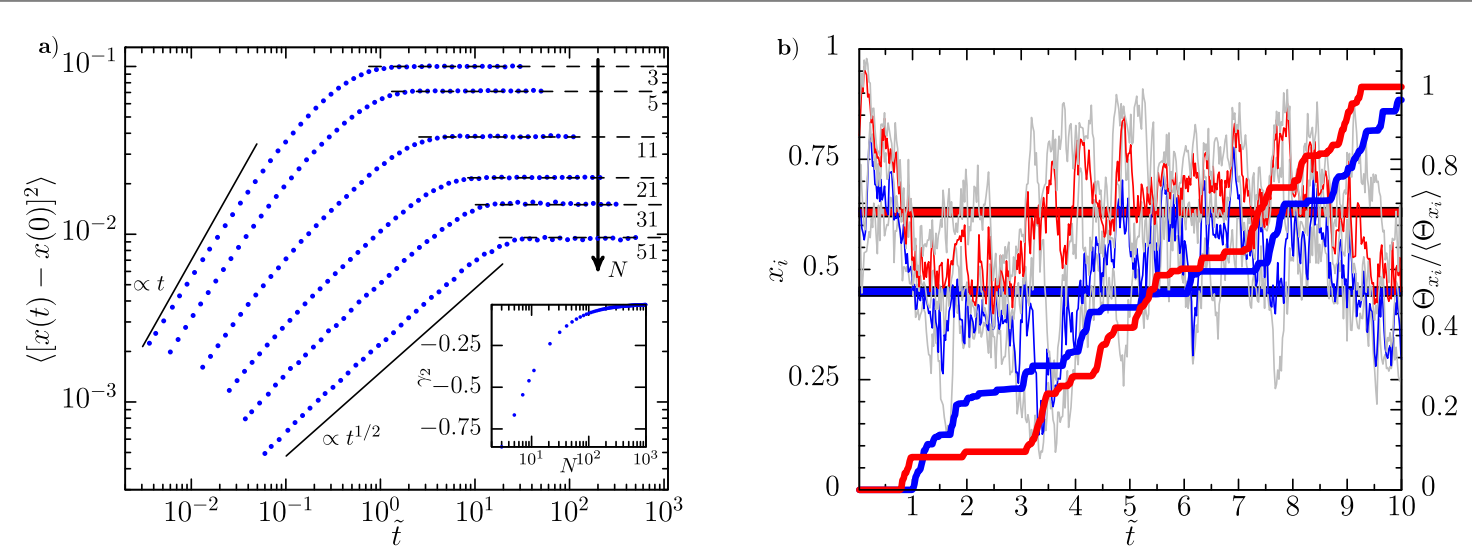

Figure 1. (a) MSD of the central particle in a single file with increasing particle number $N$ starting from equilibrium initial conditions. Time is measured in units of the mean number of collisions $\tilde{t}=D t / N^{2}$. Inset: Kurtosis excess of the invariant measure of the central particle depending on $N$; (b) trajectories of two next-nearest neighbor particles in a single file of 11 particles (red and blue curves) alongside the respective left and right nearest neighbors (gray curves). Overlaid are corresponding local time fractions up to a time $t, \theta_{t}^{i}$ in the respective red and blue shaded intervals. The remaining particle trajectories are omitted for convenience.

deviation (LD) [16] theory. Notwithstanding, these works, with isolated exceptions [17], focused on ensembleaverage properties alone. State-of-the-art experiments, however, albeit probing particle trajectories and thereby providing direct access to functionals of paths, are typically analyzed using ensemble-average concepts (see e.g. $[5,6])$. The analysis of functionals of tagged particle trajectories is thus not only feasible but also more natural than studying ensemble-average observables. Moreover, to arrive at a deeper physical understanding of projection-induced memory effects and resulting non-Markovianity, an understanding of the correlations of particle histories and their decorrelation on ergodic time-scales is required.

In particular, we here focus on the trajectory-, or time-average analogue of the tagged particle ensemble propagator [8]. Any time-average observable can be constructed from the local time fraction (see equation (A4) in appendix A), which is defined as (see figure 1(b))

$$
\theta_{t}^{j}(y)=t^{-1} \int_{0}^{t} \mathbb{1}_{y}^{j}[\mathbf{x}(\tau)] \mathrm{d} \tau
$$

where $\mathbb{1}_{y}^{j}[\mathbf{x}(\tau)]=1$ if $x_{j} \in \mathrm{d} y$ centered at $y$, and zero otherwise [18]. $\theta_{t}^{j}(y)$ in equation (1) is a random quantity denoting the fraction of the local time, $t \theta_{t}^{j}(y)$ — the time the tagged particle $j$ spends in an infinitesimal region around the point $y$ along a trajectory up until time $t . \mathbf{x}(t) \equiv\left(x_{1}(t), \ldots, x_{N}(t)\right)^{T}$ denotes the many-body trajectory written in vector form. The dynamics of a tagged particle $x_{i}(t)$ irrespective of the other $N-1$ is not Markovian, and any two tagged particle trajectories $x_{i}(t)$ and $x_{j}(t)$ are correlated on all but ergodically long times. We focus on the fluctuations and two-tag correlations of local time fractions

$$
\begin{gathered}
\sigma_{x_{i}}^{2}(t)=\left\langle\theta_{t}^{i}(x)^{2}\right\rangle-\left\langle\theta_{t}^{i}(x)\right\rangle^{2}, \\
\mathcal{C}_{x y}^{i j}(t)=\left\langle\theta_{t}^{i}(x) \theta_{t}^{j}(y)\right\rangle-\left\langle\theta_{t}^{i}(x)\right\rangle\left\langle\theta_{t}^{j}(y)\right\rangle,
\end{gathered}
$$

where $\langle\cdots\rangle$ denotes the average over all $N$-particle trajectories starting from the steady-state (in this case Boltzmann equilibrium) and propagating up to time $t$. Note that for ergodic Markov dynamics $\lim _{t \rightarrow \infty} \sigma_{x_{i}}^{2}(t)=0$ and $\lim _{t \rightarrow \infty} \mathcal{C}_{x y}^{i j}(t)=0$, reflecting the fact that on ergodically long time-scales time-average observables become deterministic and correlations between them vanish.

A general theory of local times in such correlated non-Markovian dynamics so far remained elusive. And while the statistics of functionals of the form in equation (1) in one-dimensional stochastic processes have been studied extensively in a variety of fields $[19,20]$, studies of tagged particle functionals in interacting many-body systems are sparse, and mostly limited to extreme value statistics of vicious walkers (see e.g. [21]).

Here, we present rigorous results for variances and two-tag correlations of bounded functionals ${ }^{1}$ of Markovian dynamics on arbitrary time-scales, in terms of the relaxation eigenspectrum of the corresponding propagator. The theory also covers the case, when a higher-dimensional dynamics is projected onto a smaller subspace thereby leading to non-Markovian dynamics on the reduced subspace, a hallmark example thereof being tagged-particle dynamics in a single file. The theory applies to all ergodic Markovian systems with a diagonalizable propagator. As an example we study tagged particle local times in a single file of Brownian point particles in a box. Diagonalizing the many-body propagator using the coordinate Bethe ansatz, our results uncover non-Poissonian trajectory-to-trajectory fluctuations of local times, and a cross-over from negatively to

\footnotetext{
${ }^{1}$ We consider functionals $V[\mathbf{x}(t)]$ of Markovian trajectories $\mathbf{x}(t)$, for which $V[\mathbf{x}(t)]<\infty, \forall t$ with probability 1 (see e.g. [19]).
} 
positively correlated two-tag particle histories upon increasing density, mirroring the emergence of collective fluctuations breaking Markovianity in tagged particle motion and leading to tracer subdiffusion. Clear and longlived deviations of local time statistics from shot-noise behavior demonstrate the insufficiency of harmonization concepts for describing tracer diffusion on a trajectory level. More generally, the connection to the relaxation spectrum provides an intuitive understanding of non-Poissonian statistics at sub-ergodic times in a general setting.

\section{General theory}

We consider a trajectory of a general $N$-dimensional system $\mathbf{x}(t)$ evolving according to Fokker-Planck or discrete-state Markovian dynamics. We are interested in ergodic systems with a unique steady-state $\bar{P}(\mathbf{x})$ and also assume steady state initial conditions. Due to ergodicity the mean local time fraction $\left\langle\theta_{t}^{j}(y)\right\rangle$ under these conditions is independent of $t^{2}$ and coincides with the invariant measure $\left\langle\theta_{t}^{j}(y)\right\rangle=\int \mathrm{d} \mathbf{x}^{N} \delta\left(y-x_{j}\right) \bar{P}(\mathbf{x})$, where we introduced the Dirac delta function $\delta(x)$ (for a proof see equation (A8)). In the presence of detailed balance (DB) $\bar{P}(\mathbf{x})$ is the Boltzmann-Gibbs measure $P_{\mathrm{eq}}(\mathbf{x})$.

Obtaining equations (2)-(3) essentially amounts to computing the probability generating function of the joint local time functional given by the Feynman-Kac path integral

$$
Q_{u, v}\left(x^{i}, y^{j} \mid t\right)=\hat{\mathcal{L}}_{u}^{\vartheta^{i}} \hat{\mathcal{L}}_{v}^{\vartheta^{j}}\left\langle\delta\left(\vartheta^{i}-t \theta_{t}^{i}(x)\right) \delta\left(\vartheta^{j}-t \theta_{t}^{j}(y)\right)\right\rangle,
$$

where we introduced the Laplace transform $\hat{\mathcal{L}}_{\mathfrak{s}}^{\vartheta} f(\vartheta)=\int_{0}^{\infty} \mathrm{d} \vartheta \mathrm{e}^{-s \vartheta} f(\vartheta)$. The moments in equations (2)-(3) are obtained from $\left\langle\theta_{t}^{i}(x)^{n} \theta_{t}^{j}(y)^{m}\right\rangle=\left.t^{-2} \partial_{v}^{n} \partial_{u}^{m} Q_{u, v}\left(x^{i}, y^{j} \mid t\right)\right|_{u=v=0}$ with $n+m=2$. A straightforward generalization of the trotterization in [22] shows that $Q_{u, v}\left(x^{i}, y^{j} \mid t\right)$ is the propagator of a tilted evolution operator (see appendix A)

$$
\begin{aligned}
Q_{u, v}\left(x^{i}, y^{j} \mid t\right) & =\left\langle-\left|\mathrm{e}^{-t\left(\hat{L}+u 1_{x}^{i}+v 1_{y}^{j}\right)}\right| s s\right\rangle \\
& =\left\langle s s\left|\mathrm{e}^{-t\left(\hat{L}^{\dagger}+u 1_{x}^{i}+v 1_{y}^{j}\right)}\right|-\right\rangle,
\end{aligned}
$$

where $\hat{L}$ and $\hat{L}^{\dagger}$ denote the 'bare' forward and adjoint (backward) generator of the Markov process [23], and we introduced the 'flat' $|-\rangle \equiv \int \mathrm{d} \mathbf{x}|\mathbf{x}\rangle$ and steady states $|s s\rangle=\int \mathrm{d} \mathbf{x} \bar{P}(\mathbf{x})|\mathbf{x}\rangle$ in the bra-ket notation, which are the left (right) and right (left) ground eigenstates of $\hat{L}\left(\hat{L}^{\dagger}\right)$, respectively. We obtain exact expressions for the moments in equations (2)-(3) by performing a Dyson series-expansion of equation (A3) [24], converging for any bounded functional of $\mathbf{x}(t)$ (see proof in appendix A).

Having assumed diagonalizability of $\hat{L}^{\dagger}$ (and $\left.\hat{L}\right)^{3}$, we expand the backward operator in a complete biorthogonal set of left and right eigenstates ${ }^{4}, \hat{L}^{\dagger}=\sum_{k} \lambda_{k}\left|\psi_{k}^{L}\right\rangle\left\langle\psi_{k}^{R}\right|, \lambda_{k}$ denoting the (possibly degenerate) eigenvalues and $\left\langle\psi_{k}^{L} \mid \psi_{l}^{R}\right\rangle=\delta_{k l}$. The details of the calculation of the moments are shown in appendix A. Obviously, $\left\langle\psi_{0}^{R}\left|1_{x}^{i}\right| \psi_{0}^{L}\right\rangle=\bar{P}(x)$, since the system is ergodic. The exact results for the variance and correlations are conceptually remarkably simple and read

$$
\begin{gathered}
\sigma_{x_{i}}^{2}(t)=\sum_{k \geqslant 1} 2 \frac{\Omega_{k}\left(x_{i}, x_{i}\right)}{\lambda_{k} t}\left(1-\frac{1-\mathrm{e}^{-\lambda_{k} t}}{\lambda_{k} t}\right), \\
\mathcal{C}_{x y}^{i j}(t)=\sum_{k \geqslant 1} \frac{\Omega_{k}\left(x_{i}, y_{j}\right)+\Omega_{k}\left(y_{j}, x_{i}\right)}{\lambda_{k} t}\left(1-\frac{1-\mathrm{e}^{-\lambda_{k} t}}{\lambda_{k} t}\right)
\end{gathered}
$$

where we introduced the auxiliary function $\Omega_{k}\left(x_{i}, y_{j}\right) \equiv\left\langle\psi_{0}^{R}\left|\mathbb{1}_{x}^{i}\right| \psi_{k}^{L}\right\rangle\left\langle\psi_{k}^{R}\left|\mathbb{1}_{y}^{j}\right| \psi_{0}^{L}\right\rangle$. The exact LD limits of equations (6)-(7) readily follow in the limit $t \gg \lambda_{1}^{-1}$

$$
\begin{gathered}
\sigma_{x_{i}}^{2, \mathrm{LD}}(t) \simeq 2 t^{-1} \sum_{k \geqslant 1} \lambda_{k}^{-1} \Omega_{k}\left(x_{i}, x_{i}\right), \\
\mathcal{C}_{x y}^{i j, \mathrm{LD}}(t) \simeq t^{-1} \sum_{k \geqslant 1} \lambda_{k}^{-1}\left[\Omega_{k}\left(x_{i}, y_{j}\right)+\Omega_{k}\left(y_{j}, x_{i}\right)\right],
\end{gathered}
$$

where $\simeq$ denotes asymptotic equality. Analogous formulas for LD limits of local times not connected to a spectral expansion have also been developed (see e.g. [26]). Notably, for systems obeying DB $\sigma_{x_{i}}^{2, \mathrm{LD}}(t)$ sets a universal upper bound on the variance of $\theta_{t}$ (compare equations (6) and (8)). The results in equations (6)-(9) readily extend to arbitrary functionals $t^{-1} \int_{0}^{t} \hat{V}[\mathbf{x}(\tau)] \mathrm{d} \tau$ with a bounded and local $\hat{V}$, by performing a simple

${ }^{2}$ On the level of the mean alone the time-ordering in the functional in equation (1) is not important (for a proof see equation (A8)).

${ }^{3}$ A sufficient but not necessary condition guaranteeing diagonalizability is that that the operator is normal, i.e. commutes with its adjoint, $\hat{L}^{\dagger} \hat{L}-\hat{L} \hat{L}^{\dagger}=0$.

${ }^{4}$ Note that $\hat{L}\left|\psi_{k}^{R}\right\rangle=\lambda_{k}\left|\psi_{k}^{R}\right\rangle$ and $\hat{L}^{\dagger}\left|\psi_{k}^{L}\right\rangle=\lambda_{k}\left|\psi_{k}^{L}\right\rangle[25]$. 
exchange $\mathbb{1}_{x}^{i} \rightarrow \hat{V}$, modifying only $\Omega_{k}\left(x_{i}, y_{j}\right)$ (see appendix A). Equations (6)-(7) with the aforementioned generalizations apply to all diagonalizable $\hat{L}$, thus including all systems obeying DB, and represent our first main result.

Equations (6)-(9) provide an intuitive understanding of local time statistics via a mapping onto relaxation eigenmodes, with fluctuation and correlation amplitudes proportional to the sum of transition amplitudes of excitations from the steady state to excited states and back, $\Omega_{k}\left(x_{i}, y_{j}\right)$. On ergodic time scales $\theta_{t}$ at different $t$ decorrelate, and hence display features of shot-noise, i.e. $\sigma_{x_{i}}^{2}(t)$ and $\mathcal{C}_{x y}^{i j}(t)$ decay inversely proportional to the number of independent observations of each excitation mode, $\sim \lambda_{k}^{-1} / t$. At finite times $t \lesssim \lambda_{k}^{-1}$ shot-noise statistics are altered due to a finite survival probability of the eigenmodes at a given $t,\left(1-\mathrm{e}^{-\lambda_{k} t}\right) / \lambda_{k} \forall k$, setting a hierarchy of correlation times $\lambda_{k}^{-1}$ (see correction terms in brackets of equations (6)-(7)).

\section{Local times in single-file diffusion}

Consider the dynamics of $N$ identical hard-core interacting Brownian point particles diffusing in the unit interval $[0,1]$, and set $D=1$ without loss of generality. The extension to a finite particle radius follows from a trivial change of coordinates [8]. Let $P\left(\mathbf{x}_{0}, t \mid \mathbf{x}\right) \equiv\left\langle\mathbf{x}_{0}\left|\mathrm{e}^{-t \hat{L}^{\dagger}}\right| \mathbf{x}\right\rangle$ denote the $N$-particle backward propagator of the single file with the following backward generator and $N-1$ internal non-crossing boundary conditions:

$$
\hat{L}^{\dagger}=-\sum_{i=1}^{N} \partial_{x_{i}}^{2}, \quad \lim _{x_{i+1} \rightarrow x_{i}}\left(\partial_{x_{0, i+1}}-\partial_{x_{0, i}}\right) P\left(\mathbf{x}_{0}, t \mid \mathbf{x}\right)=0 \quad \forall i .
$$

Confinement into a unit interval is imposed through external reflecting boundary conditions $\left.\partial_{x_{1}} P\left(\mathbf{x}_{0}, t \mid \mathbf{x}\right)\right|_{x_{0,1}=0}=\left.\partial_{x_{N}} P\left(\mathbf{x}, t \mid \mathbf{x}_{0}\right)\right|_{x_{0, N}=1}=0$. Under these boundary conditions we diagonalize $\hat{L^{\dagger}}$ using the coordinate Bethe ansatz [27] and obtain the Bethe eigenvalues $\lambda_{k}=\pi^{2} \sum_{i} k_{i}^{2}$ and corresponding left and right eigenvectors

$$
\psi_{k}^{L}(\mathbf{x}) \equiv\left\langle\mathbf{x} \mid \psi_{k}^{L}\right\rangle=\sum_{\left\{k_{i}\right\}}^{\prime} \prod_{i=1}^{N} 2^{\left(1-\delta_{k_{i}, 0}\right) / 2} \cos \left(k_{i} \pi x_{i}\right)
$$

and $\psi_{k}^{R}(\mathbf{x}) \equiv\left\langle\psi_{k}^{R} \mid \mathbf{x}\right\rangle=m_{k} \psi_{k}^{L}(\mathbf{x})$ with $\left\langle\psi_{k}^{R} \mid \psi_{l}^{L}\right\rangle=\delta_{k, l}$, where $m_{k}$ is the multiplicity of the Bethe eigenmode $\left|\psi_{k}^{L}\right\rangle$ (see appendix C), and $\sum_{\left\{k_{i}\right\}}{ }^{\prime}$ denotes the sum over all permutations of single-particle eigenvalues with $k_{i} \in \mathbb{N}_{0}$.

The matrix elements entering $\Omega_{k}\left(x_{i}, y_{j}\right)$ follow upon integration over the $n_{l}$ and $n_{r}$ particle coordinates to the left and right, respectively, from the tagged particle $i$ while strictly preserving the particle ordering [8], yielding (see appendix B) $\left\langle\psi_{k}^{R}\left|1_{x}^{i}\right| \psi_{0}^{L}\right\rangle=\frac{N !}{m_{k}}\left\langle\psi_{0}^{R}\left|1_{x}^{i}\right| \psi_{k}^{L}\right\rangle$ with

$$
\left\langle\psi_{0}^{R}\left|1_{x}^{i}\right| \psi_{k}^{L}\right\rangle=\frac{m_{k}}{n_{l} ! n_{r} !} \sum_{\left\{k_{i}\right\}}{ }_{x}^{1} \Lambda_{i}^{c} \prod_{j=1}^{i-1}{ }_{x}^{x} \Lambda_{j}^{s} \prod_{k=i+1}^{N}{ }_{x}^{1-x} \Lambda_{k}^{s},
$$

and $\left\langle\psi_{k}^{R}\left|\mathbb{1}_{x}^{i}\right| \psi_{0}^{L}\right\rangle=\frac{N !}{m_{k}}\left\langle\psi_{0}^{R}\left|1_{x}^{i}\right| \psi_{k}^{L}\right\rangle$. In equation (12) we have defined the auxiliary functions

$$
\begin{aligned}
& { }_{x}^{y} \Lambda_{i}^{c}=y \delta_{k_{i}, 0}-\left(1-\delta_{k_{i}, 0}\right) \sqrt{2} \cos \left(\pi k_{i} x\right) \\
& { }_{x}^{y} \Lambda_{i}^{s}=y \delta_{k_{i}, 0}-\left(1-\delta_{k_{i}, 0}\right) \sqrt{2} \sin \left(\pi k_{i} x\right) / \pi k_{i} .
\end{aligned}
$$

This delivers exact results for $\sigma_{x_{i}}^{2}(t)$ and $\mathcal{C}_{x y}^{i j}(t)$ in equations (6)-(8). An efficient numerical implementation of our analytical results can be made available upon request.

The results for $\sigma_{x_{i}}^{2}(t)$ in equation (6) for the central particle in single files with various $N$ are depicted in figure 2, and reflect large fluctuations exceeding $200 \%$ on time-scales where roughly only $50 \%$ of the particles have collided with their neighbors. The fluctuations display a non-trivial dependence on $x$, which does not follow the shape of $P_{\text {eq }}\left(x_{t}\right)=N ! x_{t}^{n_{l}}\left(1-x_{t}\right)^{n_{r}} /\left(n_{l} ! n_{r} !\right)$, and reveal striking boundary-layer effects. These deviations are clear evidence for non-Poissonian statistics and signal that harmonization concepts, which assume a locally equilibrated environment [14], break down on the more fundamental trajectory level. At longer $t$, where $\sim 50-100$ collisions / particle have occured, $\theta_{t}^{i}$ at different $t$ become uncorrelated according to the central limit theorem, with $\sigma_{x_{c}}^{2}(t)$ converging to its LD limit (8). On these time-scales the ensemble MSD has already saturated (compare figures 1 (a) and 2(c) and (d)). Notably, LD asymptotics correctly capture only small fluctuations of the order $\pm 10 \%$. As noted above and confirmed by simulations, LDs reflecting Gaussian statistics set an upper bound to the fluctuations of $\theta_{t}^{i}$ (figures 2(c) and (d)).

Single-file diffusion displays no time-scale separation in the relaxation spectrum. As a result, the projection of dynamics onto a tagged particle coordinate induces subdiffusion and strong non-Markovianity on time scales $t<\lambda_{1}^{-1}$. The respective onset of the $\sqrt{t}$ scaling of the tagged particle MSD shifts to shorter $t$ upon increasing $N$

\footnotetext{
${ }^{5}$ Note the difference with respect to the momentum-space Bethe ansatz solution [8], which does not diagonalize $\hat{L}$.
} 

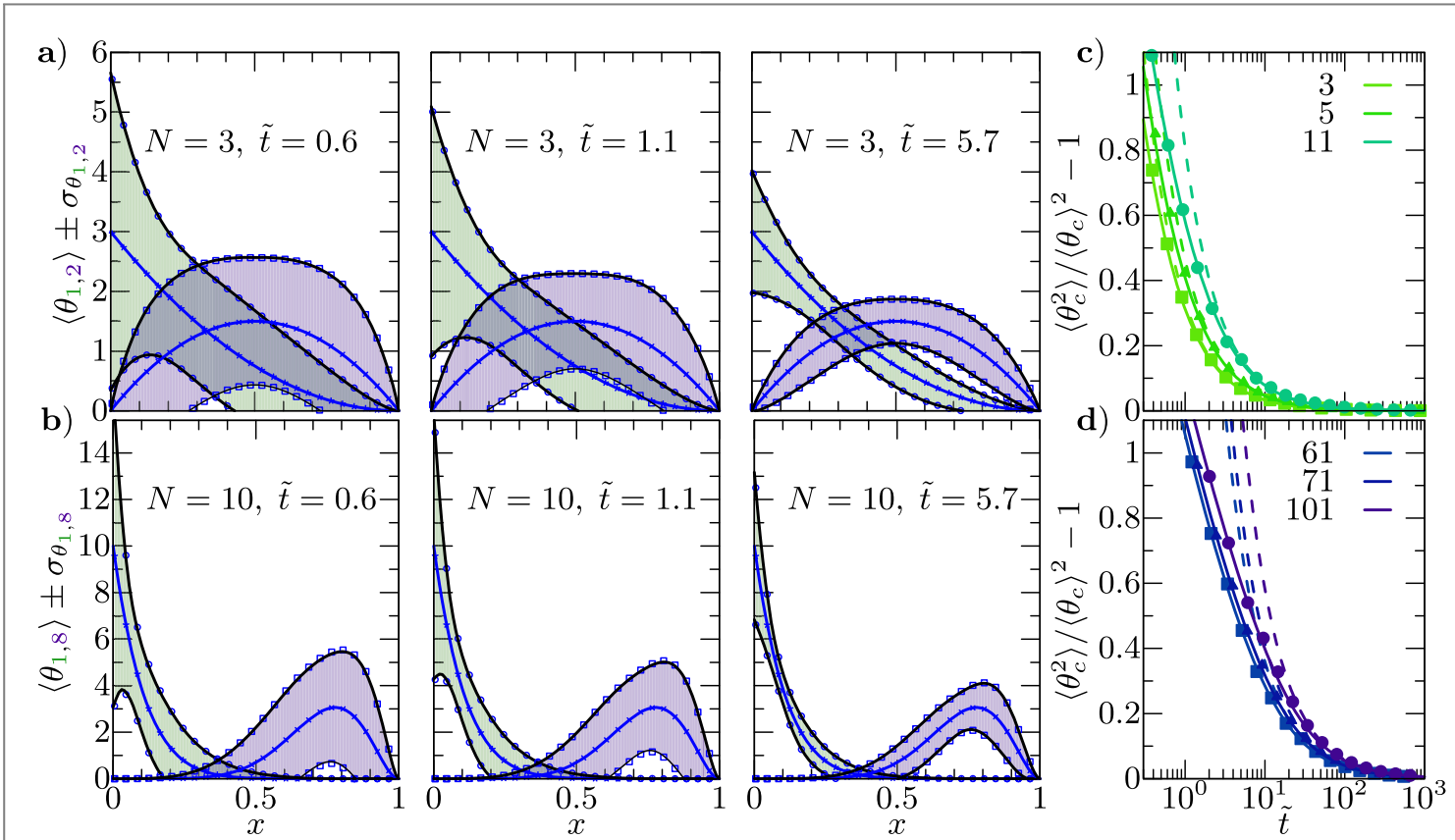

Figure 2. Statistics of local time fraction: mean, $\left\langle\theta_{t}^{i}(x)\right\rangle$, (blue lines) and fluctuations reflected by the shaded area enclosed by black lines corresponding to $\left\langle\theta_{t}^{i}(x)\right\rangle \pm \sigma_{x_{i}}(t)$ for (a) the first (green) and second (violet), and (b) first (green) and 8th (violet) tagged particle in a single file with $N=3$ and $N=10$, respectively at three different lengths of trajectories. The black lines correspond to 'error bars' on a finite-time estimate of the probability density along a single trajectory starting in the steady-state. (c) and (d): reduced variance of local time of the central particle $\sigma_{x_{c}=1 / 2}^{2}(t) /\left\langle\theta_{t}^{c}\left(x_{c}=1 / 2\right)\right\rangle^{2}$ for various odd $N$ in order to preserve the symmetry. The full lines denote exact results from equation (6) and dashed lines large deviation asymptotics equation (8). Symbols correspond to Brownian dynamics simulation of an ensemble of $10^{6}$ independent trajectories starting from equilibrium initial conditions.

(figure 1(a)). Increasing $N$ in turn leads to a high degeneracy of Bethe eigenmodes, reflecting emerging dynamical symmetries (see appendix F). As a result, fewer Bethe modes are required for a convergence of the sums in equations (6)-(7).

To gain deeper insight into the physical origin of the memory on a trajectory level we analyzed two-tag correlations between particle histories by means of the reduced covariance of local times $\tilde{\mathcal{C}}_{x y}^{i j}(t)=\mathcal{C}_{x y}^{i j}(t) /\left(\left\langle\theta_{t}^{i}(x)\right\rangle\left\langle\theta_{t}^{j}(y)\right\rangle\right)$, with $\tilde{\mathcal{C}}_{x y}^{i j}(t) \in[-1, \infty)$. Correlations between the histories of the central particle $c$ and its nearest (i.e. $c+1$ ) and next-nearest (i.e. $c+2$ ) neighbors at the midpoint between the maxima of $P_{\mathrm{eq}}\left(x_{c}\right)$ and $P_{\mathrm{eq}}\left(x_{c+1, c+2}\right)$ (see appendix E and $\mathrm{H}$ for details) are depicted in figure 3. Due to ergodicity, $\theta_{t}^{i}(x)$ become very weakly correlated at long $t$ and Gaussian statistics emerge. Consequently, $\tilde{\mathcal{C}}_{x y}^{i j}(t)$ vanishes for long times, after $\gtrsim 10^{2}$ collisions took place on average. Note that $\mathcal{C}_{x y}^{i j}(t)$ measures correlations between particle histories and not particle positions. The latter never decorrelate, i.e. two-tag position correlation functions display an algebraic decay even at equilibrium $P_{\mathrm{eq}}^{i j}\left(x_{i}, x_{j}\right)=N ! x_{i}^{n_{l}}\left(x_{j}-x_{i}\right)^{m_{l}-n_{l}}\left(1-x_{j}\right)^{m_{r}} /\left(n_{l} ! m_{r} !\left(m_{l}-n_{l}\right) !\right)-P_{\mathrm{eq}}\left(x_{i}\right) P_{\mathrm{eq}}\left(x_{j}\right) \neq 0$, where $n_{l, r}$ and $m_{l, r}$ are the number of particles to the left/right of the two tagged particles $i$ and $j$ (for details see appendix G).

Notably, we observe a transition from negatively to positively correlated tagged particle histories upon increasing density (figure 3), mirroring a change in particle dynamics from single-particle to collective fluctuations. The driving force for this transition can be found in an enhanced packing at higher densities resembling a 'crystallization' transition, where invariant tagged particle densities $P_{\mathrm{eq}}(x)$ become strongly overlapping, whereas their respective widths shrink only very slowly (see figure D1). The 'critical' density, at which the behavior shifts from negatively to positively correlated histories, depends on the topological separation between the two tagged particles and is shifted to higher values of $N$ for more distant particles (compare (a) and (b) in figure 3). In turn, this reflects a growing dynamical correlation-length with increasing $N$. As the mathematical reason for the sign-change are different signs of leading eigenvectors entering the respective elements (see equation (12)), the transition will eventually occur of any tagged pair. Moreover, upon increasing $N, \tilde{\mathcal{C}}_{x y}^{i i}(t)$ of the central particle becomes non-monotonic, with weak anti-correlations at short $t$ turning to weak correlations at large $t$, before reaching the LD limit of uncorrelated histories, where harmonization [14] ideas apply. The increasingly positive correlations with growing $N$ reflect a persistence and a finite life-time of typical collective fluctuations on a trajectory level, akin to glassy dynamics in kinetically constrained models [28]. Accordingly, positive correlations are are not observed if we tag outer particles at external boundaries (see appendix $\mathrm{H}$ ). The exact results for fluctuations and correlations of local times in single file diffusion in 

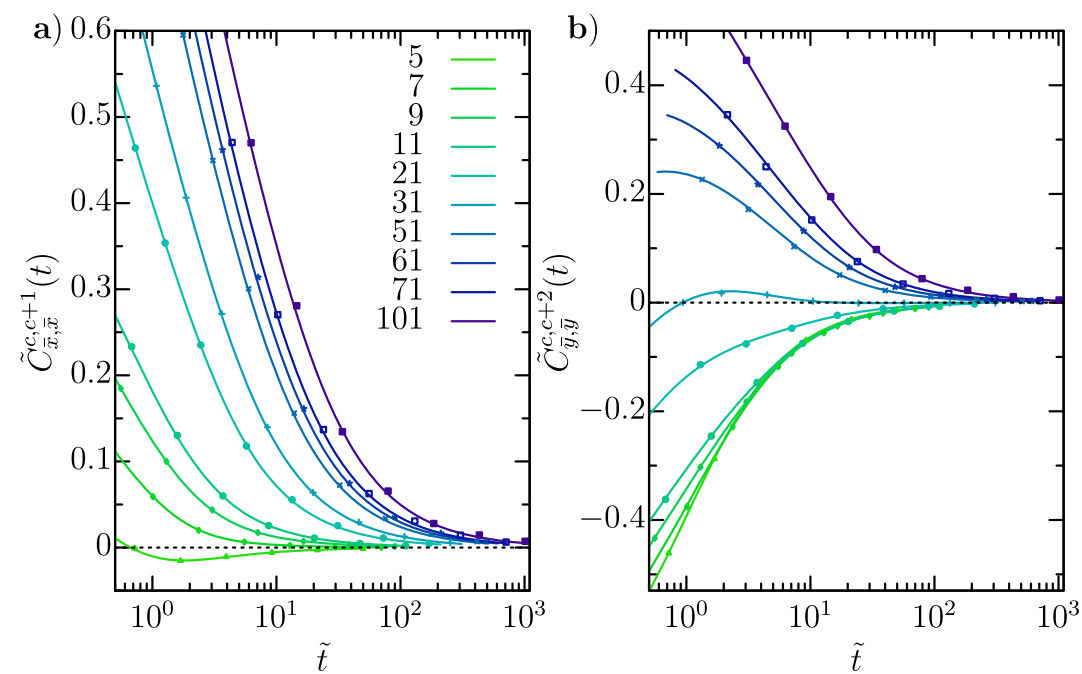

Figure 3. $\tilde{\mathcal{C}}_{x y}^{i j}(t)=\mathcal{C}_{x y}^{i j}(t) /\left(\left\langle\theta_{t}^{i}(x)\right\rangle\left\langle\theta_{t}^{j}(y)\right\rangle\right)$, reduced two-tag local time correlation functions of the central particle $c$ and its nearest (a) and next-nearest (b) neighbor for different $N$. Only odd $N$ were considered to assure the symmetry required for a meaningful comparison. Time is expressed in units of the mean collision time. Lines depict the theory in equation (7) whereas symbols correspond to Brownian dynamics simulations of $10^{6}$ independent trajectories starting from equilibrium initial conditions.

equations (11)-(13), and the explanation of the origin of broken Markovianity on a trajectory level are our second main result.

\section{Conclusions}

We established a general method for determining exactly the variance and two-tag correlations of bounded nonnegative functionals of stationary ergodic Markov processes with a diagonalizable propagator. The theory relates the statistics of functionals to the relaxation eigenspectrum, and allows for an exact treatment of non-Markovian dynamics from the corresponding higher-dimensional Markovian embedding. It also holds for diagonalizable irreversible dynamics, where a broken time-reversal symmetry can cause oscillations in higher order terms in equations (6)-(9) and/or fluctuations exceeding the LD limit in equation (8). From the spectrum of the manybody propagator obtained via the coordinate Bethe ansatz, we derived exact results for one- and two-tag local times in single file diffusion, which unveiled non-trivial correlations between tagged particle histories and the emergence of collective dynamics at increasing particle densities. Going beyond LD time-scales, our results revealed that harmonization concepts, assuming dynamics in-between local equilibria—an assumption that works well for ensemble-average observables [14]—fail on the more fundamental trajectory level. This highlights the intricate physical meaning of projection-induced memory on the level of single trajectories, which is virtually invisible to ensemble-average observables. Our results on local times can be readily tested by existing particle-tracking experiments (see e.g. [5]), and hopefully our theory will stimulate further research directed towards tagged particle functionals. Particularly interesting would be extensions to tagged particle dynamics in rugged potential landscapes [29].

\section{Acknowledgments}

We thank David Hartich for insightful discussions and critical reading of the manuscript. The financial support from the German Research Foundation (DFG) through the Emmy Noether Program 'GO 2762/1-1' (to AG), and an IMPRS fellowship of the Max Planck Society (to AL) are gratefully acknowledged.

\section{Appendix A. Proof of the main result}

Let $\mathbf{x}(t)$ be an arbitrary-dimensional ergodic Markov process on a discrete or continuous state-space. The evolution of the probability density function evolves under the corresponding diagonalizable forward generator $\hat{L}$ (e.g. Fokker-Planck- or discrete-state master equation-type) with invariant measure $\bar{P}(\mathbf{x})$ and the adjoint (i.e. backward) generator $\hat{L}^{\dagger}$. Let the respective eigenspectra be $\hat{L}=\sum_{k} \lambda_{k}\left|\psi_{k}^{R}\right\rangle\left\langle\psi_{k}^{L}\right|, \lambda_{k}$ and $\hat{L}^{\dagger}=\sum_{k} \lambda_{k}\left|\psi_{k}^{L}\right\rangle\left\langle\psi_{k}^{R}\right|, \lambda_{k}$ denoting the possibly degenerate and in general complex-valued eigenvalues. Note that $\hat{L}\left|\psi_{k}^{R}\right\rangle=\lambda_{k}\left|\psi_{k}^{R}\right\rangle$ and 
$\hat{L}^{\dagger}\left|\psi_{k}^{L}\right\rangle=\lambda_{k}\left|\psi_{k}^{L}\right\rangle$, i.e. the left and right eigenstates span a bi-orthogonal eigenspace $\left\langle\psi_{k}^{L} \mid \psi_{l}^{R}\right\rangle=\delta_{k l}$ [25]. The forward and backward propagators of the process can then be written as [25]

$$
\begin{aligned}
& P_{\mathrm{f}}\left(\mathbf{x}, t \mid \mathbf{x}_{0}\right)=\left\langle\mathbf{x}\left|\mathrm{e}^{-t \hat{L}}\right| \mathbf{x}_{0}\right\rangle=\sum_{k}\left\langle\mathbf{x} \mid \psi_{k}^{R}\right\rangle\left\langle\psi_{k}^{L} \mid \mathbf{x}_{0}\right\rangle \mathrm{e}^{-\lambda_{k} t} \\
& P_{\mathbf{b}}\left(\mathbf{x}, t \mid \mathbf{x}_{0}\right)=\left\langle\mathbf{x}_{0}\left|\mathrm{e}^{-t \hat{L}^{\dagger}}\right| \mathbf{x}\right\rangle=\sum_{k}\left\langle\mathbf{x}_{0} \mid \psi_{k}^{L}\right\rangle\left\langle\psi_{k}^{R} \mid \mathbf{x}\right\rangle \mathrm{e}^{-\lambda_{k} t} .
\end{aligned}
$$

Obviously, for $\hat{L}$ with a partially continuous spectrum ${ }^{6}$ the sum would be replaced by the corresponding integral, the probability density function of a bounded functional $\varphi_{t}=\int_{0}^{t} \hat{V}[\mathbf{x}(\tau)] \mathrm{d} \tau$ over all paths starting from a (potentially non-equlibrium) steady-state and propagating up to time $t$, is defined by the path integral

$$
\mathcal{F}(\varphi \mid t)=\iint \mathrm{d} \mathbf{x} \mathrm{d} \mathbf{x}_{0} \bar{P}\left(\mathbf{x}_{0}\right) \int_{\mathbf{x}(0)=\mathbf{x}_{0}}^{\mathrm{x}(t)=\mathbf{x}} \mathscr{D}[\mathbf{x}(t)] \mathrm{e}^{-\mathcal{S}[\mathbf{x}(t)]} \delta\left(\varphi-\int_{0}^{t} \hat{V}[\mathbf{x}(\tau)] \mathrm{d} \tau\right),
$$

with the corresponding stochastic action functional $\mathcal{S}[\mathbf{x}(t)]$ of the continuous $[22,30]$ or discrete state-space [31] Markov process $\mathbf{x}(t)$, and where we introduced the Dirac delta function $\delta(x)$. By means of a straightforward vectorial generalization of the trotterization of the the path integral (A2) in [19, 22] (for the backward and forward approach, respectively), one finds that the generating function, corresponding to the Laplace transform $\tilde{\mathcal{F}}(u \mid t)=\int_{0}^{\infty} \mathrm{d} \varphi \mathrm{e}^{-u \varphi} \mathcal{F}(\varphi \mid t)$, is the propagator of a tilted operator

$$
\tilde{\mathcal{F}}(u \mid t)=\left\langle-\left|\mathrm{e}^{-t(\hat{L}+u \hat{V})}\right| \mathrm{ss}\right\rangle=\left\langle\mathrm{ss}\left|\mathrm{e}^{-t\left(\hat{L}^{\dagger}+u \hat{V}\right)}\right|-\right\rangle,
$$

where we have introduced the 'flat' $|-\rangle \equiv \int \mathrm{d} \mathbf{x}|\mathbf{x}\rangle$ and steady states $|\mathrm{ss}\rangle=\int \mathrm{d} \mathbf{x} \bar{P}(\mathbf{x})|\mathbf{x}\rangle$, which are the left (right) and right (left) ground eigenstates of $\hat{L}\left(\hat{L}^{\dagger}\right)$, respectively. The last equality follows from $\tilde{\mathcal{F}}\left(u^{\dagger} \mid t\right)^{\dagger}=\tilde{\mathcal{F}}(u \mid t)$. In taking the Laplace transform we assumed that the functional has non-negative support (such as in the case of local times). In case the support extends to negative values one simply needs to take the Fourier transform instead.

The moments of $\mathcal{F}(\varphi \mid t)$ at any given $t$ follow immediately from $\left\langle\varphi_{t}^{n}\right\rangle=\left.(-1)^{n} \partial_{u}^{n} \tilde{\mathcal{F}}(u \mid t)\right|_{u=0}$, where $\langle\cdots\rangle$ denotes the average over all trajectories starting from a steady state and propagating up to time $t$. In case the Fourier transform is used, a corresponding change of the prefactor is required.

For bounded functionals of ergodic Markov processes all moments are finite, $\left|\left\langle\varphi_{t}^{n}\right\rangle\right| \leqslant f(t) \lim _{t \rightarrow \infty}\left|\left\langle\varphi_{t}^{n}\right\rangle\right|<\infty$ with a smooth scaling function $f(t)$, which depends on the detailed form of $\hat{V}[\mathbf{x}(t)]$. This follows from the fact that the integral is always over a finite time (see e.g. equation (1)) and hence boundedness of the integrand assures the boundedness of the time-average observable. Moreover, $\mathcal{F}(\varphi \mid t)$ obeys a LD principle [18, 32]. In the specific case of local times, $\hat{V}[\mathbf{x}(t)]=\mathbb{1}_{y}^{j}[\mathbf{x}(t)]$ and $f(t) \propto t^{n}$ for $\left\langle\varphi_{t}^{n}\right\rangle$. The finiteness of moments implies that $\tilde{\mathcal{F}}(u \mid t)$ is an analytic (i.e. holomorphic) function of $u$ at least at and near $u=0$ for any $t$.

Note that for bounded $\hat{V}[\mathrm{x}(t)]$ we can always write

$$
\begin{aligned}
& \int_{0}^{t} \mathrm{~d} t^{\prime} \hat{V}\left[\mathbf{x}\left(t^{\prime}\right)\right]=\int_{0}^{t} \mathrm{~d} t^{\prime} \int \mathrm{d} \mathbf{x} \delta\left(\mathbf{x}-\mathbf{x}\left(t^{\prime}\right)\right) V(\mathbf{x}) \\
& =\int \mathrm{d} \mathbf{x} V(x) \int_{0}^{t} \mathrm{~d} t^{\prime} \delta\left(\mathbf{x}-\mathbf{x}\left(t^{\prime}\right)\right) \equiv t \int \mathrm{d} \mathbf{x} V(\mathbf{x}) \theta_{t}(\mathbf{x}) .
\end{aligned}
$$

To obtain exact results for second moments we simply need to expand $\tilde{\mathcal{F}}(u \mid t)$ in a Dyson series to second order in $u \hat{V}$ preserving the time-ordering, and afterwards take the second derivative at $u=0$. The series is guaranteed to converge, since $\hat{V}$ is bounded. Because trivially $\left\langle-\left|\mathrm{e}^{-t \hat{L}}\right| \mathrm{ss}\right\rangle=\left\langle\mathrm{ss}\left|\mathrm{e}^{-t \hat{L}^{\dagger}}\right|-\right\rangle=1$, the Dyson expansion gives [24]

$$
\begin{aligned}
& \left\langle\mathrm{ss}\left|\mathrm{e}^{-t\left(\hat{L}^{\dagger}+u \hat{V}\right)}\right|-\right\rangle=1-u\left\langle\mathrm{ss}\left|\int_{0}^{t} \mathrm{~d} t^{\prime} \mathrm{e}^{-\hat{L}^{\dagger}\left(t-t^{\prime}\right)} \hat{V} \mathrm{e}^{-\hat{L}^{\dagger} t^{\prime}}\right|-\right\rangle \\
& +u^{2}\left\langle\mathrm{ss}\left|\int_{0}^{t} \mathrm{~d} t^{\prime} \int_{0}^{t^{\prime}} \mathrm{d} t^{\prime \prime} \mathrm{e}^{-\hat{L}^{\dagger}\left(t-t^{\prime}\right)} \hat{V} \mathrm{e}^{-\hat{L}^{\dagger}\left(t^{\prime}-t^{\prime \prime}\right)} \hat{V} \mathrm{e}^{-\hat{L}^{\dagger} t^{\prime \prime}}\right|-\right\rangle+\mathcal{O}\left(u^{3}\right),
\end{aligned}
$$

with $t \geqslant t^{\prime} \geqslant t^{\prime \prime} \geqslant 0$. An equivalent expansion can be obtained for $\hat{L}$. The Dyson series (A5) converges for $u \in \mathbb{C}<\infty$.

We first prove the convergence for any bounded linear operator $\hat{B}$. To this end we consider the operator norm. Let $\Psi$ be a complete normed linear space, and $\hat{B}: \Psi \rightarrow \Psi$. The operator norm is then defined as $\|\hat{B}\|=\sup _{\|\psi\|=1}\|\hat{B} \psi\|$ with $\psi \in \Psi$. The operator norm corresponds to the largest value $\hat{B}$ stretches an element of $\Psi$. Since $\hat{B}$ is bounded we have $\left\|\hat{B}^{N}\right\| \leqslant\|\hat{B}\|^{N}, \forall N \in \mathbb{N}$, which follows simply from $\|\hat{A} \hat{B}\| \leqslant\|\hat{A}\|\|\hat{B}\|$. The operator exponential is defined as the limit $\mathrm{e}^{\hat{B}}=\lim _{N \rightarrow \infty} \sum_{k=0}^{N} \hat{B}^{k} / k !$ and the convergence is in operator norm, since $\left\|\sum_{k=0}^{N} \hat{B}^{k} / k !\right\| \leqslant \sum_{k=0}^{N}\|\hat{B}\|^{k} / k !, \forall N \in \mathbb{N}$. The series on the right hand side converges absolutely for any number $\|\hat{B}\| \in \mathbb{C}$. Due to the completeness of the space $\Psi$, $\mathrm{e}^{\hat{B}}$ as well belongs to a complete normed linear space, and moreover $\left\|\mathrm{e}^{\hat{B}}\right\| \leqslant \mathrm{e}^{\|\hat{B}\|}$. Taking $\hat{B}=u \hat{V}$ with $u \in \mathbb{C}$ completes the proof of convergence of the series (A5).

${ }^{6}$ The ground state is always discrete as we assume the existence of an invariant measure. 
We now show that the following results also hold for bounded nonlinear functionals $\hat{V}$ such that the two-term Dyson expansion in equation (A5) is always well-behaved. Utilizing the identities in equation (A4) we find that

$$
\begin{gathered}
\langle V\rangle=t \int \mathrm{d} \mathbf{x} V(\mathbf{x})\left\langle\theta_{t}(\mathbf{x})\right\rangle, \\
\left\langle V^{2}\right\rangle=t^{2} \int \mathrm{d} \mathbf{x} \int \mathrm{d} \mathbf{x}^{\prime} V(\mathbf{x}) V\left(\mathbf{x}^{\prime}\right)\left\langle\theta_{t}(\mathbf{x}) \theta_{t}\left(\mathbf{x}^{\prime}\right)\right\rangle .
\end{gathered}
$$

Since both $\left\langle\theta_{t}(\mathbf{x})\right\rangle$ and $\left\langle\theta_{t}(\mathbf{x}) \theta_{t}\left(\mathbf{x}^{\prime}\right)\right\rangle$ are strictly bounded, $\langle V\rangle$ and $\left\langle V^{2}\right\rangle$ are also bounded, because $\hat{V}[\mathbf{x}(t)]$ is by definition bounded. For bounded $\hat{V}$ (linear or nonlinear) this proves that at least the two-term Dyson expansion is thus always finite and well behaved (in fact all orders are a.s.).

Utilizing now the spectral expansion $\hat{L}^{\dagger}=\sum_{k} \lambda_{k}\left|\psi_{k}^{L}\right\rangle\left\langle\psi_{k}^{R}\right|$ in equation (A5) we obtain for the first order term

$$
\int_{0}^{t} \mathrm{~d} t^{\prime}\left\langle\mathrm{ss}\left|\sum_{k}\right| \psi_{k}^{L}\right\rangle\left\langle\psi_{k}^{R}\left|\mathrm{e}^{-\lambda_{k}\left(t-t^{\prime}\right)} \hat{V} \sum_{l}\right| \psi_{l}^{L}\right\rangle\left\langle\psi_{l}^{R}\left|\mathrm{e}^{-\lambda_{l} t^{\prime}}\right|-\right\rangle=t V_{00}
$$

where we introduced $V_{l k}=\left\langle\psi_{l}^{R}|\hat{V}| \psi_{k}^{L}\right\rangle$ and we used the fact that $\langle\mathrm{ss}|$ and $|-\rangle$ are the left and right ground states of $\hat{L}^{\dagger}$ as well as the bi-orthogonality of the eigenbasis. The second order term follows similarly

$$
\int_{0}^{t} \mathrm{~d} t^{\prime} \int_{0}^{t^{\prime}} \mathrm{d} t^{\prime \prime} \sum_{l} V_{l 0} V_{0 l} \mathrm{e}^{-\lambda_{l}\left(t^{\prime}-t^{\prime \prime}\right)}=\frac{V_{00}^{2} t^{2}}{2}+t^{2} \sum_{l \neq 0} \frac{V_{l 0} V_{0 l}}{\lambda_{l} t}\left(1-\frac{1-\mathrm{e}^{-\lambda_{l} t}}{\lambda_{l} t}\right) .
$$

We can now trivially extend $u \hat{V} \rightarrow u \hat{A}+v \hat{B}$ for $u, v \in \mathbb{C}$ and any two bounded operators $\hat{A}$ and $\hat{B}$. In the specific case of tagged particle local times studied in the main text we have $\hat{A}=1_{y}^{i}[\mathbf{x}(t)]$ and $\hat{B}=1_{z}^{j}[\mathbf{x}(t)]$, where $\mathbb{1}_{y}^{j}[\mathbf{x}(\tau)]=1$ if $x_{j} \in \mathrm{d} y$ centered at $y$, and zero otherwise [18]. The exact second moments are now obtained from $\left.\partial_{u} \partial_{v} \tilde{\mathcal{F}}(u \mid t)\right|_{u=v=0}$ and $\left.\partial_{u}^{2} \tilde{\mathcal{F}}(u \mid t)\right|_{u=0}$ by considering the corresponding operators $\hat{A}$ and $\hat{B}$.

Finally, since we consider the local time fraction and not the total local time, we must take $\left.t^{-2} \partial_{u} \partial_{v} \tilde{\mathcal{F}}(u \mid t)\right|_{u=v=0}$ and $\left.t^{-2} \partial_{u}^{2} \tilde{\mathcal{F}}(u \mid t)\right|_{u=0}$, respectively. This completes the proof of the main general results, i.e. equations (6) and (7).

\section{Appendix B. Extended phase-space integration in single-file diffusion}

The integrals involved in the evaluation of invariant measures and matrix elements in single-file diffusion involve nesting, i.e. the ordering of particles is strictly preserved

$$
\oint_{a}^{b} f(\mathbf{x}) \mathrm{d} \mathbf{x}=\int_{a}^{b} \mathrm{~d} x_{1} \int_{x_{1}}^{b} \mathrm{~d} x_{2} \cdots \int_{x_{N-2}}^{b} \mathrm{~d} x_{N-1} \int_{x_{N-1}}^{b} \mathrm{~d} x_{N} f(\mathbf{x}) .
$$

This imposes non-trivial topology of the phase space of the system. A tremendous simplification is achieved through the so-called 'Extended Phase-Space Integration' developed by Lizana and Ambjörnsson, which exactly reduces the nested high-dimensional integrals to scaled single particle integrals, e.g. [8]:

$$
\oint_{a}^{b} f(\mathbf{x}) \delta\left(x_{\mathrm{m}}-z\right) \mathrm{d} \mathbf{x}=\left(\prod_{i=1}^{m-1} \int_{a}^{z} \mathrm{~d} x_{i}\right)\left(\prod_{j=m+1}^{N} \int_{z}^{b} \mathrm{~d} x_{j}\right) \frac{f\left(x_{m}=z, \mathbf{x}_{N-1}\right)}{n_{l} ! n_{r} !},
$$

where $n_{l}$ and $n_{r}$ are the number of particles (integrals) to the left and right of the tagged particle $m$, respectively. The extended phase-space integration in equation (B2) applies to all functions $f(\mathbf{x})$, which are invariant under the exchange $x_{i} \leftrightarrow x_{i+1}$. Throughout our work all nested integrals included in the bra-s $\left\langle\psi_{k}\right|$ (scalar products, matrix elements etc) are evaluated using the extended phase-space integration.

\section{Appendix C. Eigenmode multiplicity and eigenvalue degeneracy}

As described in the main text we diagonalize the many-body Fokker-Planck operator using the coordinate Bethe ansatz method. Each Bethe eigenstate of a Single-File of $N$ particles is uniquely defined by a tuple $k=\left(k_{1}, k_{2}, \ldots, k_{N}\right)$. To each tuple corresponds one eigenvalue through the relation:

$$
\lambda_{k}=\sum_{i=1}^{N} \pi^{2} k_{i}^{2}
$$

since more than one tuple may correspond to the same eigenvalue, these are degenerate. To each tuple $k$ it is possible to associate a set $\mathcal{K}$ containing the elements of $k$ counted once. Defining $n_{\mathcal{K}_{i}}$ as the number of times the element $\mathcal{K}_{i}$ appears in the tuple $k$, we define the multiplicity of the eigenvectors associated to $k$ as

$$
m_{k}=\prod_{i} n_{\mathcal{K}_{i}} !
$$



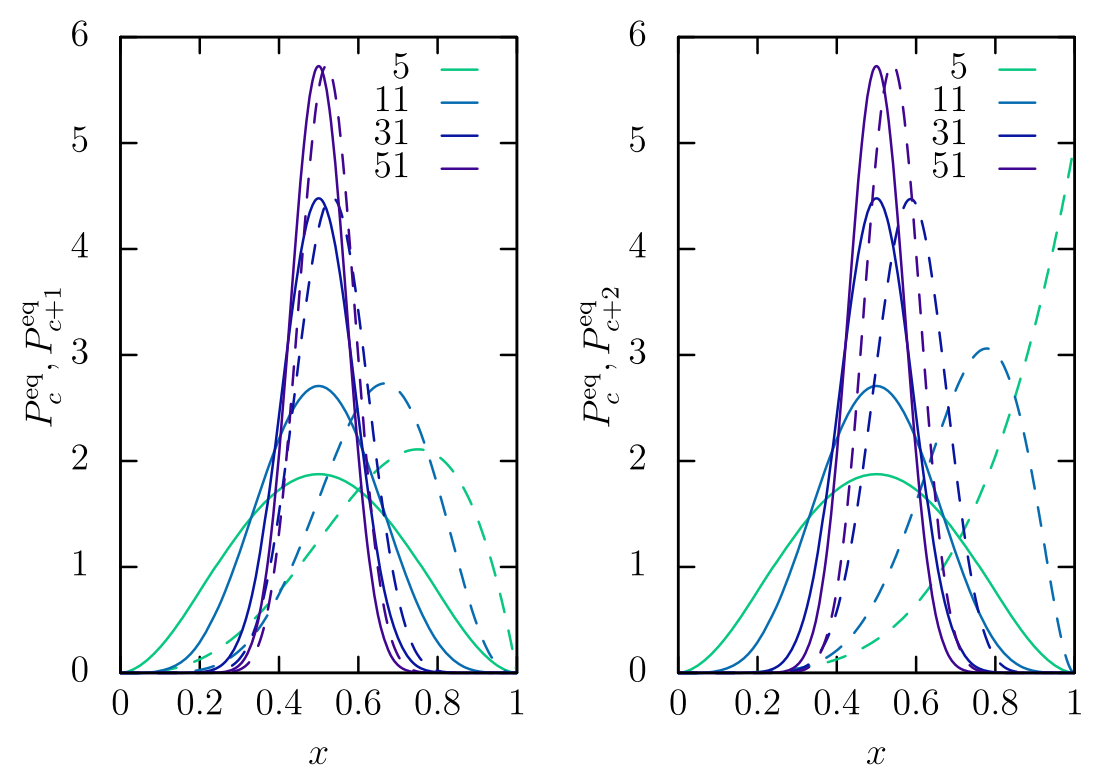

Figure D1. The solid lines represent the equilibrium probability density (D1) of the central particle and the dashed lines of the right nearest neighbor (left) and the next-nearest neighbor (right), respectively.

\section{Appendix D. Tagged particle equilibrium probability densities}

The exact tagged particle equilibrium probability density function of the tagged particle $i$ is obtained by a nested integration of all other particle positions

$$
P_{i}^{\mathrm{eq}}(x)=\oint_{0}^{1} P_{\mathrm{eq}}(\mathbf{x}) \delta\left(x_{i}-x\right) \mathrm{d} \mathbf{x}=\frac{N !}{n_{l} ! n_{r} !} x^{n_{l}}(1-x)^{n_{r}},
$$

where $n_{l}$ and $n_{r}$ are, respectively, the number of particles to the left and to the right of the tagged particle $i$. Figure D1 depicts results for $P_{i}^{\mathrm{eq}}(x)$ for the central particle $c$ and the two nearest neighbors to the right, $c+1$ and $c+2$, respectively. The probability density of the central particle approaches a Gaussian shape as the number density $N$ increases. For large enough $N$ the width of $P_{i}^{\text {eq }}(x)$ stops decreasing appreciably, while the probability densities of neighboring particles begin to overlap strongly. This has important physical consequences for correlations of particle histories, as we explained in the discussion of figure 3 in the main text.

\section{Appendix E. Reference points in the study of the density dependence of tagged particle local time statistics}

In order to allow for a meaningful comparison of results for different particle numbers $N$ we need to choose appropriate reference conditions. To do so, we focus only on odd particle numbers, for which the system is symmetric with respect to the peak of the invariant measure of the central particle $P_{\mathrm{eq}}\left(x_{c}\right)$. This way a comparison of correlations with nearest $c+1$ and next-nearest $c+2$ neighbors at different densities is indeed consistent. Moreover, in order to compare equilibrium and near-equilibrium tagged particle excursions with far-from equilibrium fluctuations we choose the following reference points with respect to $P_{\text {eq }}\left(x_{i}\right)$ : the point $x_{50}$, in which $\int_{0}^{x_{50}} P_{\text {eq }}\left(x_{i}\right) \mathrm{d} x_{i}=0.5$, point $x_{75}$, where $\int_{0}^{x_{75}} P_{\text {eq }}\left(x_{i}\right) \mathrm{d} x_{i}=0.75$, and point $x_{90}$, for which 


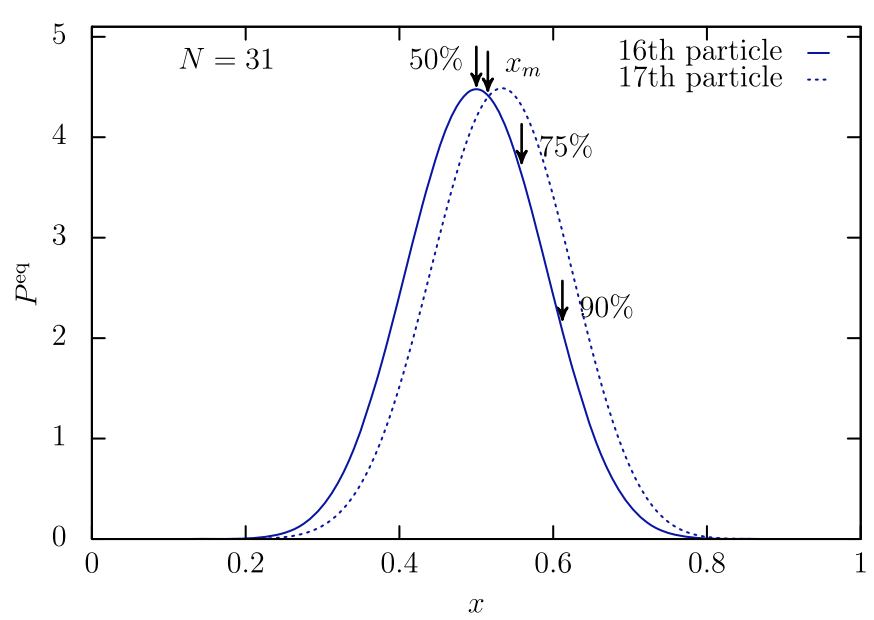

Figure E1. Invariant measures for the central particle and its nearest neighbor, denoting the different kinds of reference points.

$\int_{0}^{x_{90}} P_{\text {eq }}\left(x_{i}\right) \mathrm{d} x_{i}=0.9$ (see also figure E1). In the study of correlations of particle histories for two particles $i$ and $j$ we focus on the mid-point $x_{m}(i, j)=\left(x_{50, i}+x_{50, j}\right) / 2$.

\section{Appendix F. Convergence rates of series and eigenvalue degeneracy}

The exact expressions for variance and covariance of local time of a tagged particles in equations (6) and (7) in the main text involve an infinite series, whose rate of convergence is difficult to predict, as it strongly depends on the particular position $x$ of the tagged particle under inspection, as well as on the number of particles $N$ and $\mathcal{D}(k)$, the degeneracy of Bethe eigenvalue $\lambda_{k}$. To inspect the rate of convergence of the series we compute the relative deviation of the results for the variance of local time of the central particle truncated at the $k$ th Bethe eigenvalue, $\left|\sigma_{x_{c}}^{2}(t)-\sigma_{k}^{2}(t)\right| / \sigma^{2}(t)$ as a function of $k$ at different positions $x$ and at different lengths of trajectories $t$. Figure F1 depicts how fast the series for the variance of local time of the central particle (equation (6) in the main text) truncated at the $k$ th term converges to the exact value $k \rightarrow \infty$. n order to compare systems with different $N$ we focused on points $x_{50}$ and $x_{75}$ of the central particle, with the specific values given in table F1.

Intuitively, the convergence rate increases with increasing length of the observation $t$, since faster modes must become less and less important. The convergence rate also increases with increasing $N$, which is due to an increasing degeneracy of lower-lying eigenvalues at larger $N$. Degenerate low-lying eigenvalues allow for a mixing of different collective slow modes, which become dominant. Finally, by comparing the columns of figure F1 we notice that the rate of convergence also depends on the tagging position, which in turn depends on the curvature of the modes at different $N$. 


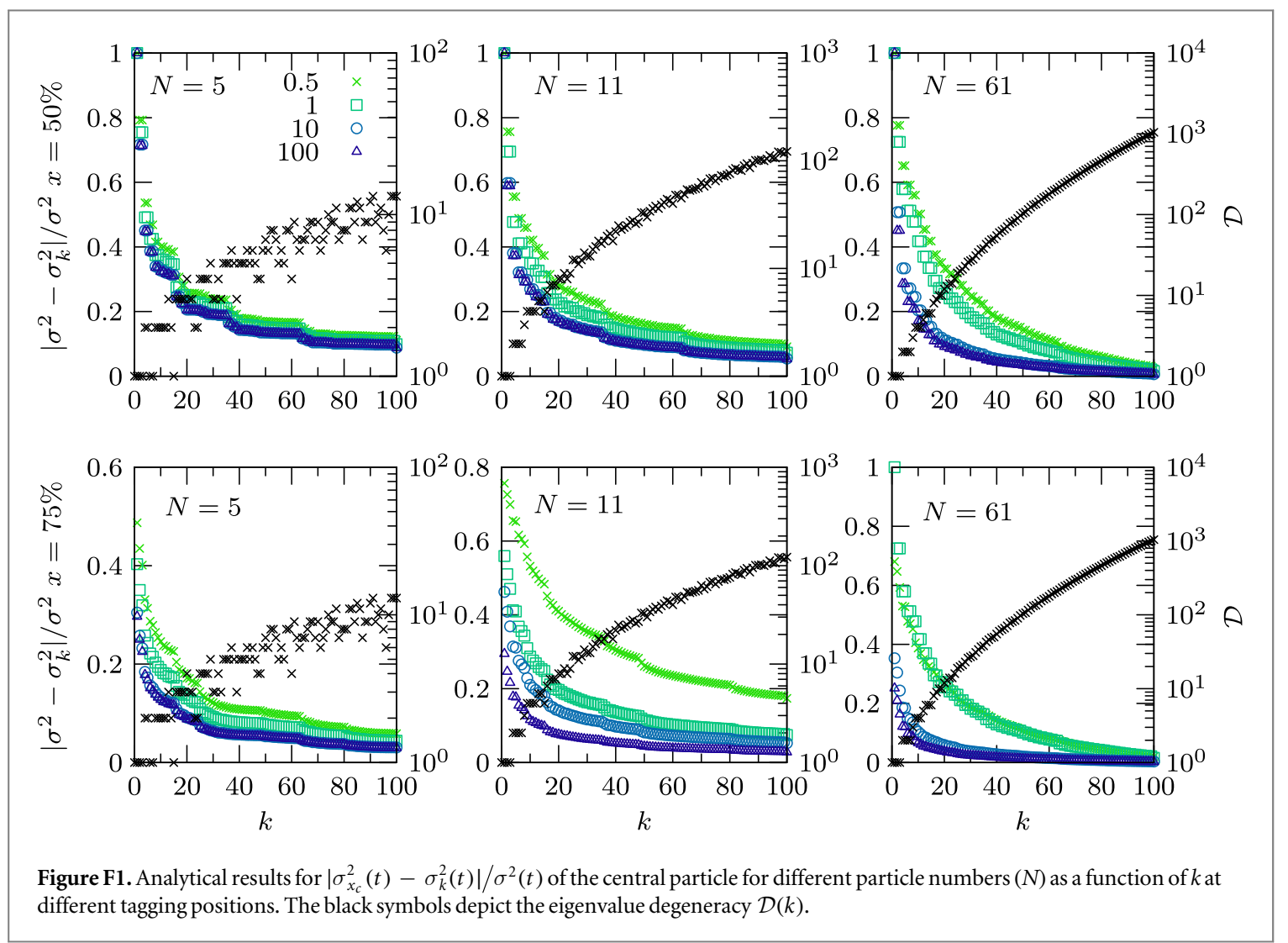

\begin{tabular}{|c|c|c|}
\hline$N$ & $x_{50}$ & $x_{75}$ \\
\hline 5 & 0.500 & 0.641 \\
\hline 11 & 0.500 & 0.598 \\
\hline 61 & 0.500 & 0.544 \\
\hline
\end{tabular}

\section{Appendix G. Equilibrium position correlation function}

In the main text we focused on the covariance of tagged particle local times $\tilde{C}_{x y}^{i j}(t)=\frac{\tilde{C}_{x y}^{i j}(t)}{\left\langle\theta_{t}^{i}(x)\right\rangle\left\langle\theta_{t}^{j}(y)\right\rangle}$ reflecting the correlations between particle histories. We found that histories decorrelate at long times as a consequence of the central limit theorem. Conversely, the particle positions on the ensemble average level do not decorrelate, not even in equilibrium. To demonstrate this we compute exactly the pair correlation function

$$
\begin{aligned}
P_{\mathrm{eq}}^{i j}\left(x_{i}, x_{j}\right) & =\oint_{0}^{1} P_{\mathrm{eq}}(\mathbf{x}) \delta\left(x_{i}-x\right) \delta\left(x_{j}-y\right) \mathrm{d} \mathbf{x}-\int_{0}^{1} P_{\mathrm{eq}}(\mathbf{x}) \delta\left(x_{i}-x\right) \mathrm{d} \mathbf{x} \int_{0}^{1} P_{\mathrm{eq}}(\mathbf{x}) \delta\left(x_{j}-y\right) \mathrm{d} \mathbf{x} \\
& =\frac{N ! x_{i}^{n_{l}}\left(x_{j}-x_{i}\right)^{m_{l}-n_{l}}\left(1-x_{j}\right)^{m_{r}}}{n_{l} ! m_{r} !\left(m_{l}-n_{l}\right) !}-\frac{(N !)^{2} x_{i}^{n_{l}}\left(1-x_{i}\right)^{n_{r}} x_{j}^{m_{l}}\left(1-x_{j}\right)^{m_{r}}}{n_{l} ! n_{r} ! m_{l} ! m_{r} !}
\end{aligned}
$$

where $n_{l, r}$ and $m_{l, r}$ are the number of particles to the left/right of the two tagged particles $i$ and $j$. Here we want to focus on the ensemble-average reduced pair correlation function $P_{\mathrm{eq}}^{i j}\left(x_{i}, x_{j}\right) /\left(P_{\mathrm{eq}}\left(x_{i}\right) P_{\mathrm{eq}}\left(x_{j}\right)\right)$ depicted in figure $\mathrm{G} 1$ and corresponding table G1. The latter is in general different from 0 , while the former goes to 0 in the limit $t \rightarrow \infty . P_{\mathrm{eq}}^{c, c+1}(x, y)$ depends on $N$ and for large $N$ changes monotonically from positive to negative correlations as a function of particle separation. At small $N$ the correlations becomes weaker with increasing separation, but remains positive. Intuitively, as one particle lies in-between, the dependence of $P_{\mathrm{eq}}^{c, c+2}$ on the interparticle separation is for large $N$ non-monotonic, going from perfectly anticorrelated to correlated and back to anti- 

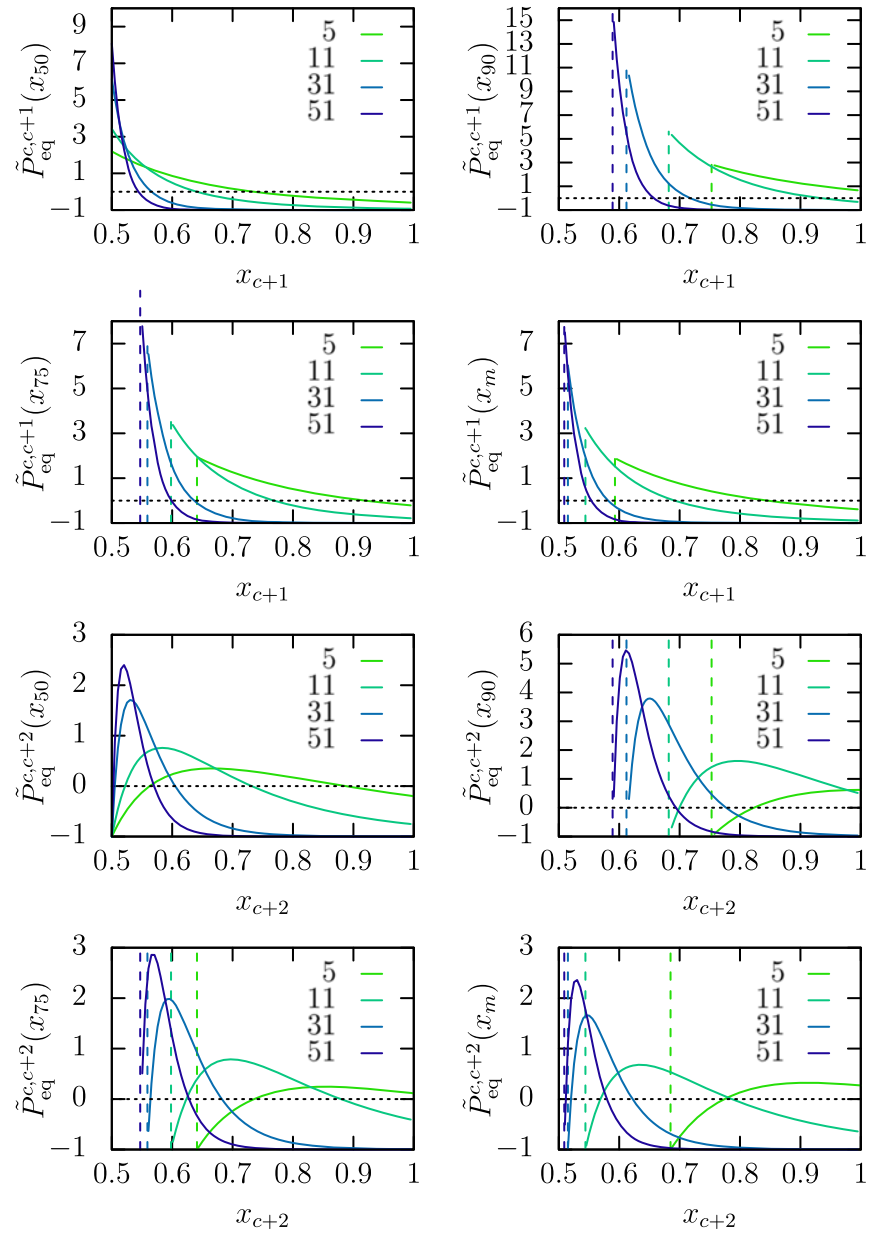

Figure G1. $P_{\mathrm{eq}}^{i j}\left(x_{i}, x_{j}\right)$ between the central particle $c$ and the right nearest and next-nearest neighbor, $c+1$ and $c+2$ respectively, when the central particle is tagged at $x_{c}=x_{50}, x_{75}, x_{90}$ and when $x_{c}=x_{m}$ (see table $\mathrm{G} 1$ for the exact positions). The vertical dashed lines are drawn at the position of the central particle (see table G1) to denote the non-crossing boundary condition.

Table G1. Reference points for the results of figure G1.

\begin{tabular}{lcccc}
\hline$N$ & $x_{50}$ & $x_{75}$ & $x_{90}$ & $x_{m}$ \\
\hline 5 & 0.500 & 0.651 & 0.753 & 0.593 \\
11 & 0.500 & 0.598 & 0.682 & 0.544 \\
31 & 0.500 & 0.559 & 0.612 & 0.515 \\
51 & 0.500 & 0.547 & 0.589 & 0.509 \\
\hline
\end{tabular}

correlation. At low $N P_{\mathrm{eq}}^{c, c+2}$ depends non-trivially on the interparticle separation, such that the aforementioned terminal anticorrelation disappears for small enough $N$. Notably, for $x_{c}=x_{90}$ the correlations are much stronger, which suggest that more extensive excursions are entropically penalized as they demand collective fluctuations.

\section{Appendix H. Two-tag correlation function of local times}

In the main text (in particular in figure 3) we analyzed one-point two-particle histories $\tilde{C}_{x_{m}, x_{m}}^{c, c+1,2}(t)$ at the respective mid-point positions $x_{m}$ listed in table $\mathrm{H} 1$.

To gain further insight we also compute the first and central-particle two-point reduced correlation functions of local times $\tilde{C}_{x y}^{1,1}(t)$ and $\tilde{C}_{x y}^{c, c}(t)$ with $x$ and $y$ given in table H2. Figure H1. In the left plot we show the self-reduced reveals weak anti-correlations at short times $t$ turning to weak correlations at longer $t$, before reaching the LD limit of uncorrelated histories. As already mentioned in the main text, the correlations for the outer particles are weak and become weaker with increasing number of particles $N$, since the outer particles are constrained between the reflecting 

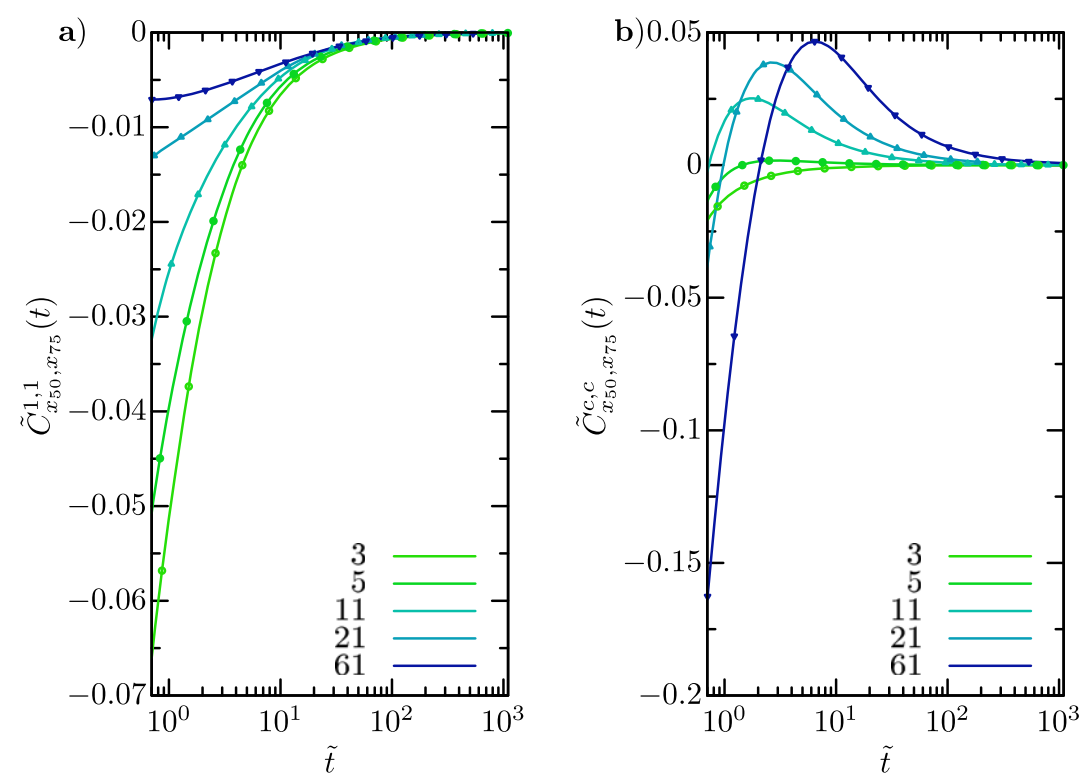

Figure H1. (a) $\tilde{C}_{x_{50} y_{75}}^{1,1}(t)$ of the first particle and (b) $\tilde{C}_{x_{50} y_{75}}^{c, c}(t)$ of the central particle.

Table H1. The location of the midpoint between the central particle and the right nearest and second nearest neighbors used in figure 3 in the main text.

\begin{tabular}{lcc}
\hline$N$ & $x_{m}$ & $y_{m}$ \\
\hline 5 & 0.593 & 0.685 \\
7 & 0.567 & 0.635 \\
9 & 0.553 & 0.606 \\
11 & 0.544 & 0.588 \\
21 & 0.523 & 0.546 \\
31 & 0.515 & 0.531 \\
51 & 0.509 & 0.519 \\
61 & 0.508 & 0.516 \\
71 & 0.507 & 0.514 \\
101 & 0.505 & 0.510 \\
\hline
\end{tabular}

Table H2. Reference points for the results of figure $\mathrm{H} 1$.

\begin{tabular}{llllll}
\hline & \multicolumn{2}{c}{ First } & & \multicolumn{2}{c}{ Central } \\
\cline { 2 - 3 } \cline { 5 - 6 }$N$ & $x_{50}$ & $x_{75}$ & & $x_{50}$ & $x_{75}$ \\
\hline 3 & 0.206 & 0.370 & & 0.500 & 0.673 \\
5 & 0.129 & 0.242 & & 0.500 & 0.641 \\
11 & 0.061 & 0.118 & & 0.500 & 0.598 \\
21 & 0.032 & 0.064 & & 0.500 & 0.572 \\
61 & 0.011 & 0.022 & & 0.500 & 0.544 \\
\hline
\end{tabular}

wall and the right nearest neighbor. In the case of the central particle we observe further evidence of the emergence of persistent collective fluctuations at higher densities, observed and described in the main text. Notably here even nearequilibrium fluctuations reveal signatures of collective behavior in the form of persistent histories (note that we are tagging at $x_{50}$ and $\left.x_{75}\right)$, i.e. $\tilde{C}_{x_{50}, x_{75}}^{c, c}(t)$ turns from purely negative to weakly positive correlations.

\section{References}

[1] Hodgkin A L and Keynes R D 1955 J. Physiol. 12861

Hummer G, Rasaiah J C and Noworyta J P 2001 Nature 414188

Beckstein O and Sansom M S P 2003 Proc. Natl Acad. Sci. USA 1007063 
[2] Lomholt M A, Ambjörnsson T and Metzler R 2005 Phys. Rev. Lett. 95260603

Li G-W, Berg O G and Elf J 2009 Nat. Phys. 5294

Mahmutovic A, Berg O G and Elf J 2015 Nucl. Acids Res. 433454

Shvets A A and Kolomeisky A B 2016 J. Phys. Chem. Lett. 72502

[3] Kärger J and Ruthven D 1992 Diffusion in Zeolites and Other Microporous Solids (New York: Wiley)

Chou T and Lohse D 1999 Phys. Rev. Lett. 823552

[4] Richards P M 1977 Phys. Rev. B 161393

[5] Lutz C, Kollmann M and Bechinger C 2004 Phys. Rev. Lett. 93026001

Chou C-Y and Eng B C 2006 J. Chem. Phys. 124044902

Lin B et al 2005 Phys. Rev. Lett. 94216001

[6] Locatelli E et al 2016 Phys. Rev. Lett. 117038001

Taloni A et al 2017 Soft Matter 131096

[7] Harris T E 1965 J. Appl. Probab. 2323

[8] Lizana L and Ambjörnsson T 2008 Phys. Rev. Lett. 100100601

Lizana L and Ambjörnsson T 2009 Phys. Rev. E 80051103

[9] Percus J K 1974 Phys. Rev. A 9557

[10] Aslangul C 1998 Europhys. Lett. 44284

Flomenbom O and Taloni A 2008 Europhys. Lett. 8320004

van Beijeren H 1991 J. Stat. Phys. 6347

Leibovich N and Barkai E 2013 Phys. Rev. E 88032107

[11] Jepsen D W 1965 J. Math. Phys. 6405

Lebowitz J L and Percus J K 1967 Phys. Rev. 155122

Lebowitz J L and Sykes J 1972 J. Stat. Phys. 6157

van Beijeren H, Kehr K W and Kutner R 1983 Phys. Rev. B 285711

Majumdar S N and Barma M 1991 Phys. Rev. B 445306

Kollmann M 2003 Phys. Rev. Lett. 90180602

Taloni A and Marchesoni F 2006 Phys. Rev. Lett. 96020601

Gupta S, Majumdar S N, Godréche C and Barma M 2007 Phys. Rev. E 76021112

Illien P et al 2013 Phys. Rev. Lett. 111038102

Bénichou O et al 2013 Phys. Rev. Lett. 111260601

Sanders L P et al 2014 New J. Phys. 1611305

[12] Rödenbeck C, Kärger J and Hahn K 1998 Phys. Rev. E 574382

[13] Barkai E and Silbey R 2009 Phys. Rev. Lett. 102050602 Barkai E and Silbey R 2010 Phys. Rev. E 81041129

[14] Lizana L et al 2010 Phys. Rev. E 81051118

[15] Krapivsky P L, Mallick K and Sadhu T 2014 Phys. Rev. Lett. 113078101 Krapivsky P L, Mallick K and Sadhu T 2015 J. Stat. Phys. 160885

[16] Hegde C, Sabhapandit S and Dhar A 2014 Phys. Rev. Lett. 113120601 Sadhu T and Derrida B 2015 J. Stat. Mech. 2015 P09008

[17] Bénichou O and Desbois J 2015 J. Stat. Mech. 2015 P03001

[18] Stroock D W and Varadhan S R S 2006 Multidimensional Diffusion Processes (Berlin: Springer)

[19] Kac M 1949 Trans. Am. Math. Soc. 651

[20] Lamperti J 1958 Trans. Am. Math. Soc. 88380

Godréche C and Luck J M 2001 J. Stat. Phys. 104489

Ehrhardt G C M A, Majumdar S N and Bray A J 2004 Phys. Rev. E 69016106

Comtet A, Desbois J and Texier C 2005 J. Phys. A 38 R341

Majumdar S N 2005 Curr. Sci. 892076

Margolin G and Barkai E 2005 Phys. Rev. Lett. 94080601

Bel G and Barkai E 2005 Phys. Rev. Lett. 94240602

Barkai E 2006 J. Stat. Phys. 123883

Bénichou O et al 2005 J. Phys. A 387205

Zatloukal V 2017 Phys. Rev. E 95052136

[21] Majumdar S N and Comtet A 2005 J. Stat. Phys. 119777

Schehr G, Majumdar S N, Comtet A and Randon-Furling J 2008 Phys. Rev. Lett. 101150601

Schehr G 2012 J. Stat. Phys. 149385

Kundu A, Majumdar S N and Schehr G 2013 Phys. Rev. Lett. 110220602

[22] Majumdar S N and Comtet A 2002 Phys. Rev. Lett. 89060601

Sabhapandit S, Majumdar S N and Comtet A 2006 Phys. Rev. E 73051102

[23] Chetrite R and Gawȩdzki K 2008 Commun. Math. Phys. 282469

[24] Dyson F J 1949 Phys. Rev. 75486

[25] Gardiner C W 1985 Handbook of Stochastic Methods for Physics, Chemistry and the Natural Sciences (Berlin: Springer)

[26] Majumdar S N and Bray A J 2002 Phys. Rev. E 6551112 Hartich D and Seifert U 2016 Phys. Rev. E 94042416

[27] Korepin V E, Bogoliubov N M and Izergin A G 1993 Quantum Inverse Scattering Method and Correlation Functions (Cambridge: Cambridge University Press)

[28] Ritort F and Sollich P 2003 Adv. Phys. 52219

[29] Hartich D and Godec A 2018 arXiv:1802.10046; arXiv:1802.10049

[30] Kleinert H 2009 Path Integrals In Quantum Mechanics, Statistics, Polymer Physics, And Financial Markets 5th edn (Singapore: World Scientific)

[31] Weber M F and Frey E 2017 Rep. Prog. Phys. 80046601

[32] Majumdar S N and Dean D S 2002 Phys. Rev. E 66041102 


\section{Chapter 4}

\section{Manifestations of Projection-Induced Memory: General Theory and the Tilted Single File}

This article has been published in Frontiers in Physics under the Creative Commons Attribution License [51. In this article we analyzed how the simple projection of several (non-fast) degrees of freedoms in a system described by a diagonalizable Fokker-Planck equation yields, almost always, to a nonMarkovian observable. Using these findings we derived a sufficient condition to find a projected renewal process. Since often projected observables are of greater importance in single-molecule experiments, their analysis greatly favors the usage of functionals of the trajectory. In particular we studied the tagged particle dynamics in a single file in a tilted potential looking at its local time statistics and comparing its dynamics to a Markovian process presenting the same equilibrium distribution. 


\title{
Manifestations of Projection-Induced Memory: General Theory and the Tilted Single File
}

\author{
Alessio Lapolla and Aljaž Godec* \\ Mathematical Biophysics Group, Max Planck Institute for Biophysical Chemistry, Göttingen, Germany
}

Over the years the field of non-Markovian stochastic processes and anomalous diffusion evolved from a specialized topic to mainstream theory, which transgressed the realms of physics to chemistry, biology and ecology. Numerous phenomenological approaches emerged, which can more or less successfully reproduce or account for experimental observations in condensed matter, biological and/or single-particle systems. However, as far as their predictions are concerned these approaches are not unique, often build on conceptually orthogonal ideas, and are typically employed on an ad-hoc basis. It therefore seems timely and desirable to establish a systematic, mathematically unifying and clean approach starting from more fine-grained principles. Here we analyze projection-induced ergodic non-Markovian dynamics, both reversible as well as irreversible, using spectral theory. We investigate dynamical correlations between histories of projected and latent observables that give rise to memory in projected dynamics, and rigorously establish conditions under which projected dynamics is Markovian or renewal. A systematic metric is proposed for quantifying the degree of non-Markovianity. As a simple, illustrative but non-trivial example we study single file diffusion in a tilted box, which, for the first time, we solve exactly using the coordinate Bethe ansatz. Our results provide a solid foundation for a deeper and more systematic analysis of projection-induced non-Markovian dynamics and anomalous diffusion.

Keywords: Fokker-Planck equation, spectral theory, projection operator method, occupation time, single file diffusion, Bethe ansatz, free energy landscape

\section{INTRODUCTION}

Over the past decades the field of anomalous diffusion and non-Markovian dynamics grew to a mainstream physical topic [1-10] backed up by a surge of experimental observations [11-16] (the list of works is anything but exhaustive). From a theoretical point of view the description of anomalous and non-Markovian phenomena is not universal [1] and can be roughly (and judiciously) classified according to the underlying phenomenology: (i) renewal continuous-time random walk and fractional Fokker-Planck approaches [1-3, 17, 18], (ii) diffusion in disordered media [19-27], (iii) generalized Langevin equation descriptions [28-36], (iv) spatially heterogeneous diffusion [37-43], and more recently also (v) the so-called diffusing diffusivity models [44-50].

From a more general first-principles perspective non-Markovian dynamics in physical systems are always a result of the projection of nominally deterministic and/or Markovian high-dimensional dynamics to a lower-dimensional subspace [51-60]. The projection in general induces a 
dependence of the dynamics on the initial conditions of the latent degrees of freedom, i.e., those being integrated out, thereby leading to memory $[51,54-56]$ and possibly (depending on the system) also to anomalous diffusion [61-68].

Hallmarks of broken Markovianity are the non-validity of the Chapman-Kolmogorov equation, and, on the level of individual trajectories, correlations between histories of projected observables and latent degrees of freedom [67]. The advantage of a first principles approach is that it allows for a deeper understanding and complete control over the origin and nature of memory effects. It might, however, be difficult to integrate out exactly degrees of freedom in a given microscopic model, and in practice this seems to be only possible for simple models, e.g., harmonic systems (e.g., [69]), comb-models (e.g., [70-72]) or simple obstruction models [61-67], to name but a few.

Here, instead of deriving effective evolution operators for projected dynamics [51, 54-56] we use a spectral-theoretic approach and focus on the consequences of the projection directly on the level of probability density functions of projected variables-both in a general setting as well as by means of a simplistic yet non-trivial model of single file diffusion in a tilted box. Using spectral theory we first present a rigorous and quite general analysis of the problem and establish conditions, under which the projection in fact leads to Markovian or renewaltype dynamics. We then apply these general results to the analysis of tagged particle diffusion in a single file confined in a tilted box. We obtain an exact solution of the full many-body and projected tagged particle propagators using the coordinate Bethe ansatz, and provide exact results for tagged particle local time statistics and correlations between tagged particle histories. Finally, to asses the degree of non-Markovianity induced by the projection, we compute the Kullback-Leibler divergence between the exact tagged particle propagator and the propagator of Markovian diffusion in the respective free energy landscape, i.e., in the so-called free energy landscape perspective. Our results provide a deeper understanding of projection-induced memory and anomalous diffusion and highlight important pitfalls in applications of free energy landscape-ideas in absence of a timescale separation.

\section{THEORY}

\subsection{Notation and Mathematical Preliminaries}

Although all presented result hold identically for discrete-state jump dynamics governed by a Markovian master equation we will here throughout be interested in projections of strongly Markovian diffusion in continuous time and in a continuous domain $\Omega \in \mathbb{R}^{d}$ in a vector field $\mathbf{F}(\mathbf{x}): \mathbb{R}^{d} \rightarrow \mathbb{R}^{d}$ (not necessarily a potential field), which is either nominally confining (in this case $\Omega$ is open) or is accompanied by corresponding reflecting boundary conditions at $\partial \Omega$ (in this case $\Omega$ is closed) thus guaranteeing the existence of an invariant measure and hence ergodicity. The dynamics are governed by the (forward) Fokker-Planck operator $\hat{\mathcal{L}}: V \rightarrow V$ or its adjoint (or backward) operator $\hat{\mathcal{L}}^{\dagger}: W \rightarrow W$, where $V$ is a complete normed linear vector space with elements $f \in C^{2}\left(\mathbb{R}^{d}\right)$, and $W$ is the space dual to $V$. In particular,

$$
\hat{\mathcal{L}}=\nabla \cdot \mathbf{D} \nabla-\nabla \cdot \mathbf{F}(\mathbf{x}), \quad \hat{\mathcal{L}}^{\dagger}=\nabla \cdot \mathbf{D} \nabla+\mathbf{F}(\mathbf{x}) \cdot \nabla,
$$

where $\mathbf{D}$ is the symmetric positive-definite diffusion matrix. $\hat{\mathcal{L}}$ propagates probability measures $\mu_{t}(\mathbf{x})$ in time, which will throughout be assumed to posses well-behaved probability density functions $P(\mathbf{x}, t)$, i.e., $d \mu_{t}(\mathbf{x})=P(\mathbf{x}, t) d \mathbf{x}$ [thereby posing some restrictions on $\mathbf{F}(\mathbf{x})$ ]. On the level of individual trajectories Equation (1) corresponds to the Itô equation $d \mathbf{x}_{t}=\mathbf{F}\left(\mathbf{x}_{t}\right) d t+$ $\sigma d \mathbf{W}_{t}$ with $\mathbf{W}_{t}$ being a $d$-dimensional vector of independent Wiener processes whose increments have a Gaussian distribution with zero mean and variance $d t$, i.e., $\left\langle d W_{t, i} d W_{t^{\prime}, j}\right\rangle=\delta_{i j} \delta(t-$ $\left.t^{\prime}\right) d t$, and where $\sigma$ is a $d \times d$ symmetric noise matrix such that $\mathbf{D}=\sigma \sigma^{T} / 2$. Moreover, we assume that $\mathbf{F}(\mathbf{x})$ admits the following decomposition into a potential (irrotational) field $-\mathbf{D} \nabla \varphi(\mathbf{x})$ and a non-conservative component $\boldsymbol{\vartheta}(\mathbf{x}), \mathbf{F}(\mathbf{x})=-\mathbf{D} \nabla \varphi(\mathbf{x})+\boldsymbol{\vartheta}(\mathbf{x})$ with the two fields being mutually orthogonal $\nabla \varphi(\mathbf{x}) \cdot \boldsymbol{\vartheta}(\mathbf{x})=0$ [73]. By insertion into Equation (1) one can now easily check that $\hat{\mathcal{L}} \mathrm{e}^{-\varphi(\mathbf{x})}=0$, such that the stationary solution of the FokkerPlanck equation (also referred to as the steady state [74, 75], which is the terminology we adopt here) by construction does not depend on the non-conservative part $\boldsymbol{\vartheta}(\mathbf{x})$. Before proceeding we first establish the decomposition of the drift field $\mathbf{F}(\mathbf{x})$ of the full dynamics, which with the knowledge of $\varphi(\mathbf{x})$ can be shown to have the form

$$
\mathbf{F}(\mathbf{x})=-\mathbf{D} \nabla \varphi(\mathbf{x})+\mathrm{e}^{\varphi(\mathbf{x})} \mathbf{j}_{\mathrm{ss}}(\mathbf{x}),
$$

$\mathbf{j}_{\mathrm{ss}}(\mathbf{x})$ denoting the steady-state probability current and $\boldsymbol{\vartheta}(\mathbf{x}) \equiv \mathrm{e}^{\varphi(\mathbf{x})} \mathbf{j}_{\mathrm{ss}}(\mathbf{x})$ being incompressible. The proof follows straightforwardly. We take $\boldsymbol{\vartheta}(\mathbf{x})=\mathbf{F}(\mathbf{x})+\mathbf{D} \nabla \varphi(\mathbf{x})$ and use $\varphi(\mathbf{x})$ to determine the steady-state current $\mathbf{j}_{\mathrm{ss}}(\mathbf{x})=(\boldsymbol{\vartheta}(\mathbf{x})-\mathbf{D} \nabla \varphi(\mathbf{x})) \mathrm{e}^{-\varphi(\mathbf{x})}+\mathbf{D} \nabla \mathrm{e}^{-\varphi(\mathbf{x})}$, such that immediately $\boldsymbol{\vartheta}(\mathbf{x})=\mathrm{e}^{\varphi(\mathbf{x})} \mathbf{j}_{\text {ss }}(\mathbf{x})$ and in turn follows $\mathbf{F}(\mathbf{x})$ in Equation (2). To check for incompressibility we note that $\mathbf{j}_{\mathrm{ss}}(\mathbf{x})$ is by definition divergence free and so $\nabla \cdot \boldsymbol{\vartheta}(\mathbf{x})=\mathrm{e}^{\varphi(\mathbf{x})}\left(\mathbf{j}_{\mathrm{ss}}(\mathbf{x}) \cdot \nabla \varphi(\mathbf{x})\right) \equiv \boldsymbol{\vartheta}(\mathbf{x}) \cdot \nabla \varphi(\mathbf{x})=0$, i.e., $\mathrm{e}^{\varphi(\mathbf{x})} \mathbf{j}_{\text {ss }}(\mathbf{x})$ is divergence-free, as claimed.

We define the forward and backward propagators by $\hat{U}(t)=$ $\mathrm{e}^{\hat{\mathcal{L}} t}$ and $\hat{U}^{\dagger}(t)=\mathrm{e}^{\hat{\mathcal{L}}^{\dagger} t}$ such that $\hat{\mathcal{L}}$ and $\hat{\mathcal{L}}^{\dagger}$ are generators of a semi-group $\hat{U}\left(t+t^{\prime}\right)=\hat{U}(t) \hat{U}\left(t^{\prime}\right)$ and $\hat{U}^{\dagger}\left(t+t^{\prime}\right)=\hat{U}^{\dagger}(t) \hat{U}^{\dagger}\left(t^{\prime}\right)$, respectively. $\hat{\mathcal{L}}$ propagates probability measures $\mu_{t}(\mathbf{x})$ in time, whereas $\hat{\mathcal{L}}^{\dagger}$ propagates observables $\mathcal{A}\left(\mathbf{x}_{t}\right)$ in time, which is best seen from the definition of the expectation

$$
\begin{array}{r}
\left\langle\mathcal{A}\left(\mathbf{x}_{t}\right)\right\rangle \equiv \int \mathcal{A}(\mathbf{x}) d \mu_{t}(\mathbf{x})=\int \mathcal{A}(\mathbf{x}) \mathrm{e}^{\hat{\mathcal{L}} t} P(\mathbf{x}, 0) d \mathbf{x}= \\
\int P(\mathbf{x}, 0) \mathrm{e}^{\hat{\mathcal{L}}^{\dagger} t} \mathcal{A}(\mathbf{x}) d \mathbf{x} \equiv \int d \mu_{0}\left(\mathbf{x}_{0}\right)\left\langle\mathcal{A}\left(\mathbf{x}_{0}, t\right)\right\rangle,
\end{array}
$$

where $\left\langle\mathcal{A}\left(\mathbf{x}_{0}, t\right)\right\rangle$ was defined to give a correct behavior after averaging over the realizations of the Itô process but before averaging over the initial conditions $P(\mathbf{x}, 0)$ for the forward in time process (or end-point conditions for the adjoint, backward 
in time process). The propagation of measures by $\hat{\mathcal{L}}$ corresponds to the "Schrödinger" picture of quantum mechanics, whereas the propagation of observables resembles the "Heisenberg" picture.

For convenience we introduce the bra-ket notation with the "ket" $|f\rangle$ representing a vector in $V$ (or $W$, respectively) written in position basis as $f(\mathbf{x}) \equiv\langle\mathbf{x} \mid f\rangle$, and the "bra" $\langle g|$ as the integral $\int d \mathbf{x} g^{\dagger}$. The scalar product is defined as $\langle g \mid f\rangle=\int d \mathbf{x} g^{\dagger}(\mathbf{x}) f(\mathbf{x})$. Therefore we have, in operator notation, the following evolution equation for the conditional probability density function starting from an initial condition $\left|p_{0}\right\rangle:\left|p_{t}\right\rangle=\mathrm{e}^{\hat{\mathcal{L}} t}\left|p_{0}\right\rangle$. Since the process is ergodic we have $\lim _{t \rightarrow \infty} \mathrm{e}^{\hat{\mathcal{L}} t}\left|p_{0}\right\rangle=|s s\rangle$, where we have defined the equilibrium or non-equilibrium steady state, $\hat{\mathcal{L}}|s s\rangle=0$ and $\langle s \mathrm{~s}| \hat{\mathcal{L}}^{\dagger}=0$, as a result of the duality. The steady state refers to a probability density function $\langle\mathbf{x}|$ ss $\rangle$ of the invarant measure, which might carry a time-independent non-vanishing probability current $\mathbf{j}_{\mathrm{ss}}(\mathbf{x})$. We also define the (typically non-normalizable) "flat" state $|-\rangle$, such that $\langle\mathbf{x} \mid-\rangle=1$ and $\left\langle-\mid p_{t}\right\rangle=1$. Hence, $\partial_{t}\left\langle-\mid p_{t}\right\rangle=0$ and $\langle-| \hat{\mathcal{L}}=0$ and $\hat{\mathcal{L}}^{\dagger}|-\rangle=0$. We define the Green's function of the process as the conditional probability density function for a localized initial condition $\left\langle\mathbf{x} \mid p_{0}\right\rangle=\delta\left(\mathbf{x}-\mathbf{x}_{0}\right)$ as

$$
G\left(\mathbf{x}, t \mid \mathbf{x}_{0}, 0\right)=\left\langle\mathbf{x}|\hat{U}(t)| \mathbf{x}_{0}\right\rangle \equiv\left\langle\mathbf{x}_{0}\left|\hat{U}^{\dagger}(t)\right| \mathbf{x}\right\rangle
$$

such that the conditional probability density starting from a general initial condition $\left|p_{0}\right\rangle$ becomes $P\left(\mathbf{x}, t \mid p_{0}, 0\right)=$ $\left\langle\mathbf{x}|\hat{U}(t)| p_{0}\right\rangle \equiv \int d \mathbf{x}_{0} p_{0}\left(\mathbf{x}_{0}\right) G\left(\mathbf{x}, t \mid \mathbf{x}_{0}, 0\right)$. Moreover, as $\mathbf{F}(\mathbf{x})$ is assumed to be sufficiently confining (i.e., $\lim _{\mathbf{x} \rightarrow \infty} P(\mathbf{x}, t)=0, \forall t$ sufficiently fast), such that $\hat{\mathcal{L}}$ corresponds to a coercive and densely defined operator on $V$ (and $\hat{\mathcal{L}}^{\dagger}$ on $W$, respectively) [7678]. Finally, $\hat{\mathcal{L}}$ is throughout assumed to be normal, i.e., $\hat{\mathcal{L}}^{\dagger} \hat{\mathcal{L}}-$ $\hat{\mathcal{L}} \hat{\mathcal{L}}^{\dagger}=0$ and thus henceforth $V=W$, where for reversible system (i.e., those obeying detailed balance) we have $\hat{\mathcal{L}} \Leftrightarrow \hat{\mathcal{L}}^{\dagger}$. Because any normal compact operator is diagonalizable [79], we can expand $\hat{\mathcal{L}}$ (and $\hat{\mathcal{L}}^{\dagger}$ ) in a complete bi-orthonormal set of left $\left\langle\psi_{k}^{L}\right|$ and right $\left|\psi_{k}^{R}\right\rangle\left(\left\langle\psi_{k}^{R}\right|\right.$ and $\left|\psi_{k}^{L}\right\rangle$, respectively) eigenstates

$$
\hat{\mathcal{L}}\left|\psi_{k}^{R}\right\rangle=-\lambda_{k}\left|\psi_{k}^{R}\right\rangle, \quad \hat{\mathcal{L}}^{\dagger}\left|\psi_{k}^{L}\right\rangle=-\alpha_{k}\left|\psi_{k}^{L}\right\rangle
$$

with $\operatorname{Re}\left(\lambda_{k}\right) \geq 0$, and according to our definition of the scalar product we have

$$
\left\langle\psi_{k}^{L}|\hat{\mathcal{L}}| \psi_{k}^{R}\right\rangle=-\lambda_{k}\left\langle\psi_{k}^{L} \mid \psi_{k}^{R}\right\rangle=\left(\left\langle\psi_{k}^{R}\left|\hat{\mathcal{L}}^{\dagger}\right| \psi_{k}^{L}\right\rangle\right)^{\dagger}=-\alpha_{k}^{\dagger}\left\langle\psi_{k}^{R} \mid \psi_{k}^{L}\right\rangle
$$

and hence the spectra of $\hat{\mathcal{L}}$ and $\hat{\mathcal{L}}^{\dagger}$ are complex conjugates, $\alpha_{k}=\lambda_{k}^{\dagger}$. Moreover, $\lambda_{0}=0,\left|\psi_{0}^{R}\right\rangle=|\mathrm{ss}\rangle,\left\langle\psi_{0}^{L}\right|=\langle-|$, and $\left\langle\psi_{k}^{L} \mid \psi_{l}^{R}\right\rangle=\delta_{k l}$. Finally, we also have the resolution of identity $\mathbf{1}=\sum_{k}\left|\psi_{k}^{R}\right\rangle\left\langle\psi_{k}^{L}\right|$ and the propagator $\hat{U}(t)=$ $\sum_{k}\left|\psi_{k}^{R}\right\rangle\left\langle\psi_{k}^{L}\right| \mathrm{e}^{-\lambda_{k} t}$. It follows that the spectral expansion of the Green's function reads

$G\left(\mathbf{x}, t \mid \mathbf{x}_{0}, 0\right)=\sum_{k} \psi_{k}^{R}(\mathbf{x}) \psi_{k}^{L \dagger}\left(\mathbf{x}_{0}\right) \mathrm{e}^{-\lambda_{k} t} \equiv \sum_{k} \psi_{k}^{L}\left(\mathbf{x}_{0}\right) \psi_{k}^{R \dagger}(\mathbf{x}) \mathrm{e}^{-\lambda_{k}^{\dagger} t}$,
We now define, $\hat{\mathcal{P}}_{\mathbf{x}}(\boldsymbol{\Gamma} ; \mathbf{q})$, a (potentially oblique) projection operator into a subspace of random variables - a mapping $\mathbf{q}=$ $\boldsymbol{\Gamma}(\mathbf{x}): \mathbb{R}^{d} \rightarrow \mathbb{R}^{a}$ to a subset of coordinates $\mathbf{q}$ lying in some orthogonal system in Euclidean space, $\mathbf{q} \in \Xi\left(\mathbb{R}^{a}\right) \subset \Omega\left(\mathbb{R}^{d}\right)$ with $a<d$. For example, the projection operator applied to some function $R(\mathbf{x}) \in V$ gives

$$
\hat{\mathcal{P}}_{\mathbf{x}}(\boldsymbol{\Gamma} ; \mathbf{q}) R(\mathbf{x})=\int_{\Omega} d \mathbf{x} \delta(\boldsymbol{\Gamma}(\mathbf{x})-\mathbf{q}) R(\mathbf{x}) .
$$

The spectral expansion of $\hat{\mathcal{L}}$ (and $\hat{\mathcal{L}}^{\dagger}$ ) in the bi-orthogonal Hilbert space alongside the projection operator $\hat{\mathcal{P}}_{\mathbf{X}}(\boldsymbol{\Gamma} ; \mathbf{q})$ will now allow us to define and analyze projection-induced nonMarkovian dynamics.

\subsection{General Results}

\subsubsection{Non-Markovian Dynamics and (Non)Existence of a Semigroup}

Using the projection operator $\hat{\mathcal{P}}_{\mathbf{X}}(\boldsymbol{\Gamma} ; \mathbf{q})$ defined in Equation (8) we can define the (in general) non-Markovian Green's function of the projected dynamics as the conditional probability density of projected dynamics starting from a localized initial condition $\mathbf{q}_{0}$

$$
\begin{array}{r}
Q_{p_{0}}\left(\mathbf{q}, t \mid \mathbf{q}_{0}, 0\right)=\frac{Q_{p_{0}}\left(\mathbf{q}, t, \mathbf{q}_{0}, 0\right)_{p_{0}}}{Q_{p_{0}}^{0}\left(\mathbf{q}_{0}\right)} \\
\equiv \frac{\hat{\mathcal{P}}_{\mathbf{x}}(\Gamma ; \mathbf{q}) \hat{\mathcal{P}}_{\mathbf{x}_{0}}\left(\Gamma ; \mathbf{q}_{0}\right) G\left(\mathbf{x}, t \mid \mathbf{x}_{0}, 0\right) p_{0}\left(\mathbf{x}_{0}\right)}{\hat{\mathcal{P}}_{\mathbf{x}_{0}}\left(\Gamma ; \mathbf{q}_{0}\right) p_{0}\left(\mathbf{x}_{0}\right)},
\end{array}
$$

which demonstrates that the time evolution of projected dynamics starting from a fixed condition $\mathbf{q}_{0}$ depends on the initial preparation of the full system $p_{0}\left(\mathbf{x}_{0}\right)$ as denoted by the subscript. This is a first signature of the non-Markovian and nonstationary nature of projected dynamics and was noted upon also in [55]. Obviously, $\int_{\Xi} d \mathbf{q} Q_{p_{0}}\left(\mathbf{q}, t \mid \mathbf{q}_{0}, 0\right)=1$ for any initial condition $\mathbf{q}_{0}$. We will refer to $\mathbf{q}$ as the projected degrees of freedom, whereas those integrated out will be called latent. For the sake of simplicity we will here mostly limit our discussion to a stationary preparation of the system, i.e., $p_{0}\left(\mathbf{x}_{0}\right)=p_{\mathrm{ss}}\left(\mathbf{x}_{0}\right)=$ $\left\langle\mathbf{x}_{0} \mid s s\right\rangle$. In order to avoid duplicating results we will explicitly carry out the calculation with the spectral expansion of $\hat{\mathcal{L}}$ but note that equivalent results are obtained using $\hat{\mathcal{L}}^{\dagger}$. Using the spectral expansion Equation (7) and introducing $\Psi_{k l}(\mathbf{q})$, the elements of an infinite-dimensional matrix

$$
\Psi_{k l}(\mathbf{q})=\left\langle\psi_{k}^{L}|\delta(\boldsymbol{\Gamma}(\mathbf{x})-\mathbf{q})| \psi_{l}^{R}\right\rangle
$$

we find from Equation (9)

$$
Q_{p_{\mathrm{ss}}}\left(\mathbf{q}, t \mid \mathbf{q}_{0}, 0\right)=\sum_{k} \Psi_{0 k}(\mathbf{q})\left(\Psi_{k 0}\left(\mathbf{q}_{0}\right) / \Psi_{00}\left(\mathbf{q}_{0}\right)\right) \mathrm{e}^{-\lambda_{k} t}
$$

with $\Psi_{00}\left(\mathbf{q}_{0}\right)=Q_{p_{\mathrm{ss}}}^{0}\left(\mathbf{q}_{0}\right)$. If one would to identify $\Psi_{0 k}(\mathbf{q})=$ $\Psi_{0 k}^{R}(\mathbf{q})$ and $\Psi_{00}\left(\mathbf{q}_{0}\right)^{-1} \Psi_{0 k}(\mathbf{q})=\Psi_{0 k}^{L}(\mathbf{q})$, Equation (11) at first sight looks deceivingly similar to the Markovian Green's function in Equation (7). Moreover, a hallmark of Markovian dynamics is that it obeys the Chapman-Kolmogorov equation and indeed, 
since $\left\langle\psi_{k}^{L} \mid \psi_{l}^{R}\right\rangle=\delta_{k l}$, we find from the spectral expansion Equation (7) directly for any $0<t^{\prime}<t$ that

$$
\begin{aligned}
& \int_{\Omega} d \mathbf{x}^{\prime} G\left(\mathbf{x}, t \mid \mathbf{x}^{\prime}, t^{\prime}\right) G\left(\mathbf{x}^{\prime}, t^{\prime} \mid \mathbf{x}_{0}, 0\right) \\
& \quad=\sum_{k, l} \psi_{k}^{R}(\mathbf{x})\left\langle\psi_{k}^{L} \mid \psi_{l}^{R}\right\rangle \psi_{l}^{L \dagger}\left(\mathbf{x}_{0}\right) \mathrm{e}^{-\lambda_{k}\left(t-t^{\prime}\right)-\lambda_{l} t^{\prime}} \\
& \quad \equiv G\left(\mathbf{x}, t \mid \mathbf{x}_{0}, 0\right) .
\end{aligned}
$$

For non-Markovian dynamics with a stationary $p_{0}(\mathbf{x})$ we here prove the following

Proposition 2.2.1.1. Let the full system be prepared in a steady state, $p_{0}(\mathbf{x})=p_{\text {ss }}(\mathbf{x})$, and let non-Markovian Green's function be defined by Equation (9). We take $\Psi_{k l}(\mathbf{q})$ as defined in Equation (10) and define a scalar product with respect to a Lebesgue measure $w$ as $\langle f \mid g\rangle_{w} \equiv \int d \mathbf{x} w(\mathbf{x}) f^{\dagger}(\mathbf{x}) g(\mathbf{x})$. Then the Green's function of the projected process will obey the ChapmanKolmogorov equation if and only if $\left\langle\Psi_{l 0} \mid \Psi_{k 0}\right\rangle_{\Psi_{00}^{-1}}=0, \forall k, l$.

We need to prove if and under which conditions

$$
\int_{\Xi} d \mathbf{q}^{\prime} Q_{p_{s s}}\left(\mathbf{q}, t \mid \mathbf{q}^{\prime}, t^{\prime}\right) Q_{p_{\mathrm{ss}}}\left(\mathbf{q}^{\prime}, t^{\prime} \mid \mathbf{q}_{0}, 0\right)
$$

can be equal to $Q_{p_{s s}}\left(\mathbf{q}, t \mid \mathbf{q}_{0}, 0\right)$. As this will generally not be the case this essentially means that the projected dynamics is in general non-Markovian. The proof is established by noticing that $\Psi_{k l}\left(\mathbf{q}^{\prime}\right)=\Psi_{l k}^{\dagger}\left(\mathbf{q}^{\prime}\right)$ such that $\left\langle\Psi_{l 0} \mid \Psi_{k 0}\right\rangle_{\Psi_{00}^{-1}} \equiv$ $\int_{\Xi} d \mathbf{q}^{\prime} \Psi_{00}\left(\mathbf{q}^{\prime}\right)^{-1} \Psi_{0 l}\left(\mathbf{q}^{\prime}\right) \Psi_{k 0}\left(\mathbf{q}^{\prime}\right)$. As a result Equation (13) can be written analogously to the first equality in Equation (12) as

$$
\sum_{k, l} \Psi_{0 k}(\mathbf{q})\left\langle\Psi_{l 0} \mid \Psi_{k 0}\right\rangle_{\Psi_{00}^{-1}}\left(\Psi_{0 l}^{\dagger}\left(\mathbf{q}_{0}\right) / \Psi_{00}\left(\mathbf{q}_{0}\right)\right) \mathrm{e}^{-\lambda_{k}\left(t-t^{\prime}\right)-\lambda_{l} t^{\prime}} .
$$

But since the projection mixes all excited eigenstates with $k>0$ (to a $k$-dependent extent) with the left and right ground states [see Equation (10)], the orthogonality between $\Psi_{00}(\mathbf{q})^{-1 / 2} \Psi_{0 l}(\mathbf{q})$ and $\Psi_{00}(\mathbf{q})^{-1 / 2} \Psi_{k 0}(\mathbf{q})$ is in general lost, and $\left\langle\Psi_{l 0} \mid \Psi_{k 0}\right\rangle_{\Psi_{00}^{-1}} \neq 0$ for $k \neq l$ as claimed above. The Chapman-Kolmogorov equation can hence be satisfied if and only if $\left\langle\Psi_{l 0} \mid \Psi_{k 0}\right\rangle_{\Psi_{00}^{-1}}=0$ for all $k \neq l$.

The possibility that the Chapman-Kolmogorov equation remains valid for non-Markovian process has been demonstrated previously on the hand of specific models (see e.g., [80, 81]). Here we establish the necessary and sufficient conditions for this to be the case in a quite general setting. In turn, even if $\left\langle\Psi_{l 0} \mid \Psi_{k 0}\right\rangle_{\Psi_{00}^{-1}}=$ $0, \forall k \neq l$ that this does not guarantee that the projected process is actually Markovian. The computation of higher-order probability densities is necessary in order to check for Markovianity.

\subsubsection{When Is the Projected Dynamics Markovian or Renewal?}

A) Projected Dynamics is Markovian

A particularly useful aspect of the present spectral-theoretic approach is its ability to establish rigorous conditions for the emergence of (exactly) Markovian and (exactly) renewal-type dynamics from a microscopic, first principles point of view. Note that in this section we assume a general, non-stationary preparation of the system [i.e., $p_{0}\left(\mathbf{x}_{0}\right) \neq p_{\mathrm{ss}}\left(\mathbf{x}_{0}\right)$ ]. By inspection of Equations (10) and (11) one can establish that:

Theorem 2.2.2.1. The necessary and sufficient condition for the projected dynamics to be Markovian if is that the projection $\hat{\mathcal{P}}_{\mathbf{x}}(\boldsymbol{\Gamma} ; \mathbf{q})$ (whatever its form) nominally projects into the nullspace of latent dynamics. In other words, the latent and projected dynamics remain decoupled and orthogonal for all times. This means that (i) there exists a bijective map $\mathbf{y}=f(\mathbf{x})$ to $a$ decomposable coordinate system $\mathbf{y}=\left(\mathbf{q}, \mathbf{q}^{\prime \prime}\right)$, in which the forward generator decomposes to $\hat{\mathcal{L}}=\hat{\mathcal{L}}_{p}+\hat{\mathcal{L}}_{l}$, where $\hat{\mathcal{L}}_{p}$ only acts and depends on the projected degrees of freedom $\mathbf{q} \in \Xi\left(\mathbb{R}^{a}\right) \subset \Omega\left(\mathbb{R}^{d}\right)$ with $a<d$ and $\hat{\mathcal{L}}_{l}$ only acts and depends on the latent coordinates $\mathbf{q}^{\prime \prime} \in \Xi^{c}\left(\mathbb{R}^{d}\right) \subset \Omega\left(\mathbb{R}^{d}\right)$ (with, $\Xi \cap \Xi^{\prime \prime}=\emptyset, \Omega=\Xi \cup \Xi^{\prime \prime}$ ), (ii) the boundary conditions on $\partial \Xi$ and $\partial \Xi^{c}$ are decoupled, and (iii) the projection operator $\hat{\mathcal{P}}_{\mathbf{y}}(\cdot ; \mathbf{q})=\int d \mathbf{q}^{\prime \prime}$ onto the subset of coordinates $\mathbf{q} \in \Xi\left(\mathbb{R}^{a}\right) \subset \Omega$ corresponds to an integral over the subset of latent coordinates $\mathbf{q}^{\prime \prime} \in \Xi^{c}\left(\mathbb{R}^{d-a}\right) \subset \Omega$, which does not mix projected and latent degrees of freedom, or alternatively $\hat{\mathcal{L}}_{l} p_{0}\left(\mathbf{q}_{0}, \mathbf{q}_{0}^{\prime \prime}\right)=0$.

The statement of the theorem is intuitive and has most likely already been presented elsewhere in the existing literature, although we were not able to find it in the present form. The proof is rather straightforward and follows from the fact that if (and only if) the projected dynamics is Markovian it must be governed as well by a formal (Markovian) Fokker-Planck generator $\hat{\mathcal{L}}_{p}$ as in Equation (1), in which the projected and latent degrees of freedom are separable $\hat{\mathcal{L}}=\hat{\mathcal{L}}_{p}+\hat{\mathcal{L}}_{l}$, and that the full Hilbert space is a direct sum of Hilbert spaces of the $V=V_{p} \oplus V_{l}$, that is $\hat{\mathcal{L}}: V \rightarrow V, \hat{\mathcal{L}}_{p}: V_{p} \rightarrow V_{p}$ and $\hat{\mathcal{L}}_{l}: V_{l} \rightarrow V_{l}$ and $V_{p} \cap V_{l}=\emptyset$. This also requires that there is no boundary condition coupling vectors from $V_{p}$ and $V_{l}$. In turn this implies assertion (i) above. If $\hat{\mathcal{P}}_{\mathbf{y}}(\cdot ; \mathbf{q})$ is such that it does not mix eigenfunctions in $V_{p}$ and $V_{l}$ (i.e., it only involves vectors from $V_{p}$ ) then ecause of biorthonormality and the fact that $\langle-| \hat{\mathcal{L}}=0$ the projected Green's function in full space $Q\left(\mathbf{q}, t \mid \mathbf{q}_{0}\right)$ for $\mathbf{q} \in \Xi\left(\mathbb{R}^{a}\right)$ will be identical to the full Green's function in the isolated domain $G\left(\mathbf{x}, t \mid \mathbf{x}_{0}\right)$ for $\mathbf{x} \in \boldsymbol{\Xi}\left(\mathbb{R}^{a}\right)$ and the non-mixing condition is satisfied. The effect is the same if the latent degrees of freedom already start in a steady state, $\hat{\mathcal{L}}_{l} p_{0}\left(\mathbf{q}_{0}, \mathbf{q}_{0}^{\prime \prime}\right)=0$. This establishes sufficiency. However, as soon as the projection mixes the two Hilbert spaces $V_{p}$ and $V_{l}$, the generator of projected dynamics will pick up contributions from $\hat{\mathcal{L}}_{l}$ and will, upon integrating out the latent degrees of freedom, not be Markovian. This completes the proof.

B) Projected Dynamics is Renewal

We can also rigorously establish sufficient conditions for the projected dynamics to poses the renewal property. Namely, the physical notion of a waiting time or a random change of timescale (see e.g., $[2,3]$ ) can as well be attributed a microscopic origin. The idea of a random waiting time (or a random change of time scale) nominally implies a period of time and thereby the existence of some subdomain, during which and within the latent degrees evolve while the projected dynamics does not change. For this to be the case the latent degrees of freedom must be perfectly orthogonal to the projected degrees of freedom, both 
in the two domains as well as on their boundaries (a prominent simple example is the so-called comb model [70-72]). Moreover, the projected degrees of freedom evolve only when the latent degrees of freedom reside in some subdomain $\Upsilon \subset \Xi^{c}\left(\mathbb{R}^{d-a}\right)$. In turn, this means that the dynamics until a time $t$ ideally partitions between projected and latent degrees of freedom, which are coupled solely by the fact that the total time spent in each must add to $t$, which effects the waiting time. In a comb-setting the motion along the backbone occurs only when the particle is in the center of the orthogonal plane. In the context of a lowdimensional projection of ergodic Markovian dynamics, we can in fact prove the following general theorem:

Theorem 2.2.2.2. Let there exists a bijective map $\mathbf{y}=f(\mathbf{x})$ to a decomposable coordinate system $\mathbf{y}=\left(\mathbf{q}, \mathbf{q}^{\prime \prime}\right)$ as in A) with the projected $\mathbf{q} \in \Xi\left(\mathbb{R}^{a}\right)$ and latent degrees of freedom $\mathbf{q}^{\prime \prime} \in$ $\Xi^{c}\left(\mathbb{R}^{d-a}\right) \equiv \Omega\left(\mathbb{R}^{d}\right) \backslash \Xi\left(\mathbb{R}^{a}\right)$. Furthermore, let $\Upsilon \subset \Xi^{c}\left(\mathbb{R}^{d-a}\right)$ and let $\mathbb{1}_{\Upsilon}\left(\mathbf{q}^{\prime \prime}\right)$ denote the indicator function of the region $\Upsilon$ (i.e., $\mathbb{1}_{\Upsilon}\left(\mathbf{q}^{\prime \prime}\right)=1$ if $\mathbf{q}^{\prime \prime} \in \Upsilon$ and zero otherwise). Moreover, let the full system be prepared in an initial condition $p_{0}\left(\mathbf{q}, \mathbf{q}^{\prime \prime}\right)$. Then a sufficient condition for renewal-type dynamics is (i) that the forward generator in $\left(\mathbf{q}, \mathbf{q}^{\prime \prime}\right)$ decomposes $\hat{\mathcal{L}}=\mathbb{1}_{\Upsilon}\left(\mathbf{q}^{\prime \prime}\right) \hat{\mathcal{L}}_{p}+\hat{\mathcal{L}}_{l}$, and where $\hat{\mathcal{L}}_{p}$ only acts and depends on $\mathbf{q}$ and $\hat{\mathcal{L}}_{l}$ only acts and depends on $\mathbf{q}^{\prime \prime}$, and (ii) the boundary conditions do not cause a coupling of latent and projected degrees of freedom (as in the Markov case above).

Theorem 2.2.2.2 and lemma 2.2.2.2.1 below appear to be new, and the proof can be established by an explicit construction of the exact evolution equation for the projected variables. Let $G_{l}\left(\mathbf{q}^{\prime \prime}, t \mid \mathbf{q}_{0}^{\prime \prime}\right)$ denote the Green's functions of the Markovian problem for the latent degrees of freedom, $G_{l}\left(\mathbf{q}^{\prime \prime}, t \mid \mathbf{q}_{0}^{\prime \prime}\right)=$ $\left\langle\mathbf{q}^{\prime \prime}\left|\mathrm{e}^{\hat{\mathcal{L}}_{l} t}\right| \mathbf{q}_{0}^{\prime \prime}\right\rangle=\sum_{k}\left\langle\mathbf{q}^{\prime \prime} \mid \psi_{k}^{l, R}\right\rangle\left\langle\psi_{k}^{l, L} \mid \mathbf{q}_{0}^{\prime \prime}\right\rangle \mathrm{e}^{-\lambda_{k}^{l} t}$ and let $\tilde{g}(s)=$ $\int_{0}^{\infty} \mathrm{e}^{-s t} g(t) d t$ denoted the Laplace transform of a function $g(t)$. The projection operator in this case corresponds to $\hat{\mathcal{P}}_{\mathbf{q}^{\prime \prime}}(\cdot ; \mathbf{q})=\int_{\Xi^{c}} d \mathbf{q}^{\prime \prime}$. We introduce the shorthand notation $\bar{p}_{0}(\mathbf{q})=\int_{\Xi^{c}} d \mathbf{q}_{0}^{\prime \prime} p_{0}\left(\mathbf{q}_{0}, \mathbf{q}_{0}^{\prime \prime}\right)$ and define the conditional initial probability density $p_{0}\left(\mathbf{q}_{0}^{\prime \prime} \mid \mathbf{q}_{0}\right)=p_{0}\left(\mathbf{q}_{0}, \mathbf{q}_{0}^{\prime \prime}\right) / \bar{p}_{0}\left(\mathbf{q}_{0}\right)$. The Green's function of projected dynamics becomes $Q_{p_{0}}\left(\mathbf{q}, t \mid \mathbf{q}_{0}\right)=$ $\int_{\Xi^{c}} d \mathbf{q}^{\prime \prime} \int_{\Xi^{c}} d \mathbf{q}_{0}^{\prime \prime} G\left(\mathbf{q}, \mathbf{q}^{\prime \prime}, t \mid \mathbf{q}_{0}, \mathbf{q}_{0}^{\prime \prime}\right) p_{0}\left(\mathbf{q}_{0}, \mathbf{q}_{0}^{\prime \prime}\right) / \bar{p}_{0}\left(\mathbf{q}_{0}\right)$. We then have the following

Lemma 2.2.2.2.1. Under the specified assumptions $Q\left(\mathbf{q}, t \mid \mathbf{q}_{0}\right)$ exactly obeys the renewal-type non-Markovian Fokker-Planck equation

$$
\partial_{t} Q_{p_{0}}\left(\mathbf{q}, t \mid \mathbf{q}_{0}\right)=\int_{0}^{t} d \tau K_{p_{0}}(t-\tau) \hat{\mathcal{L}}_{p} Q_{p_{0}}\left(\mathbf{q}, \tau \mid \mathbf{q}_{0}\right),
$$

with the memory kernel

$$
\begin{aligned}
K_{p_{0}}(t)= & \left(\delta(t)+\partial_{t}\right) \int_{\Upsilon} d \mathbf{q}^{\prime \prime} \int_{\Xi^{c}} d \mathbf{q}_{0}^{\prime \prime} p_{0}\left(\mathbf{q}_{0}^{\prime \prime} \mid \mathbf{q}_{0}\right)\left\langle\mathbf{q}^{\prime \prime}\left|\mathrm{e}^{\hat{\mathcal{L}}_{l} t}\right| \mathbf{q}_{0}^{\prime \prime}\right\rangle \\
= & \sum_{k}\left(\int_{\Xi^{c}} d \mathbf{q}_{0}^{\prime \prime} \psi_{k}^{l, L^{\dagger}}\left(\mathbf{q}_{0}^{\prime \prime}\right) p_{0}\left(\mathbf{q}_{0}^{\prime \prime} \mid \mathbf{q}_{0}\right)\right) \\
& \left(\int_{\Upsilon} d \mathbf{q}^{\prime \prime} \psi_{k}^{l, R}\left(\mathbf{q}^{\prime \prime}\right)\right)\left(\delta(t)-\lambda_{k}^{l} \mathrm{e}^{-\lambda_{k}^{l} t}\right)
\end{aligned}
$$

that is independent of $\mathbf{q}$. Moreover, $Q\left(\mathbf{q}, t \mid \mathbf{q}_{0}\right)>0$ for all $t>0$ and for all $\mathbf{q}, \mathbf{q}_{0} \in \Xi$.

To prove the lemma we Laplace transform equation $(t \rightarrow$ u) $\partial_{t} G\left(\mathbf{q}, \mathbf{q}^{\prime \prime}, t \mid \mathbf{q}_{0}, \mathbf{q}_{0}^{\prime \prime}\right)=\hat{\mathcal{L}} G\left(\mathbf{q}, \mathbf{q}^{\prime \prime}, t \mid \mathbf{q}_{0}, \mathbf{q}_{0}^{\prime \prime}\right)$ and realize that the structure of $\hat{\mathcal{L}}$ implies that its solution with initial condition $\delta\left(\mathbf{q}-\mathbf{q}_{0}\right) \delta\left(\mathbf{q}^{\prime \prime}-\mathbf{q}_{0}^{\prime \prime}\right)$ in Laplace space factorizes $\tilde{G}\left(\mathbf{q}, \mathbf{q}^{\prime \prime}, u \mid \mathbf{q}_{0}, \mathbf{q}_{0}^{\prime \prime}\right)=f_{u}\left(\mathbf{q} \mid \mathbf{q}_{0}\right) g_{u}\left(\mathbf{q}^{\prime \prime} \mid \mathbf{q}_{0}^{\prime \prime}\right)$ with $g_{u}$ and $f_{u}$ to be determined. Note that $\int_{\Xi} d \mathbf{q} \int_{\Xi^{c}} d \mathbf{q}^{\prime \prime} \tilde{G}\left(\mathbf{q}, \mathbf{q}^{\prime \prime}, u \mid \mathbf{q}_{0}, \mathbf{q}_{0}^{\prime \prime}\right)=$ $\int_{\Xi} d \mathbf{q} f_{u}\left(\mathbf{q} \mid \mathbf{q}_{0}\right) \int_{\Xi^{c}} d \mathbf{q}^{\prime \prime} g_{u}\left(\mathbf{q}^{\prime \prime} \mid \mathbf{q}_{0}^{\prime \prime}\right)=u^{-1}$ and we can chose, without any loss of generality that $\int_{\Xi} d \mathbf{q} f_{u}\left(\mathbf{q} \mid \mathbf{q}_{0}\right)=1$. Plugging in the factorized ansatz and rearranging leads to

$$
\begin{array}{r}
g_{u}\left(\mathbf{q}^{\prime \prime} \mid \mathbf{q}_{0}^{\prime \prime}\right)\left(u f_{u}\left(\mathbf{q} \mid \mathbf{q}_{0}\right)-\mathbb{1}_{\Upsilon}\left(\mathbf{q}^{\prime \prime}\right) \hat{\mathcal{L}}_{p} f_{u}\left(\mathbf{q} \mid \mathbf{q}_{0}\right)\right) \\
-f_{u}\left(\mathbf{q} \mid \mathbf{q}_{0}\right) \hat{\mathcal{L}}_{l} g_{u}\left(\mathbf{q}^{\prime \prime} \mid \mathbf{q}_{0}^{\prime \prime}\right)-\delta\left(\mathbf{q}-\mathbf{q}_{0}\right) \delta\left(\mathbf{q}^{\prime \prime}-\mathbf{q}_{0}^{\prime \prime}\right)=0 .
\end{array}
$$

Noticing that $\int_{\Xi} d \mathbf{q} \hat{\mathcal{L}}_{p} f\left(\mathbf{q} \mid \mathbf{q}_{0}\right)=0$ as a result of the divergence theorem (as we assumed that $\mathbf{F}(\mathbf{x})$ is strongly confining implying that the current vanishes at the boundaries) we obtain, upon integrating Equation (17) over $\mathbf{q}$

$$
u g_{u}\left(\mathbf{q}^{\prime \prime} \mid \mathbf{q}_{0}^{\prime \prime}\right)-\delta\left(\mathbf{q}^{\prime \prime}-\mathbf{q}_{0}^{\prime \prime}\right)-\hat{\mathcal{L}}_{l} g_{u}\left(\mathbf{q}^{\prime \prime} \mid \mathbf{q}_{0}^{\prime \prime}\right)=0,
$$

implying that $g_{u}\left(\mathbf{q}^{\prime \prime} \mid \mathbf{q}_{0}^{\prime \prime}\right)=\tilde{G}_{l}\left(\mathbf{q}^{\prime \prime}, u \mid \mathbf{q}_{0}^{\prime \prime}\right)$. As $\tilde{G}_{l}\left(\mathbf{q}^{\prime \prime}, u \mid \mathbf{q}_{0}^{\prime \prime}\right)$ is the Laplace image of a Markovian Green's function we use $\int_{\Xi^{c}} d \mathbf{q}^{\prime \prime} \tilde{G}_{l}\left(\mathbf{q}^{\prime \prime}, u \mid \mathbf{q}_{0}^{\prime \prime}\right)=u^{-1}$ in order to deduce that $\tilde{Q}_{p_{0}}\left(\mathbf{q}, u \mid \mathbf{q}_{0}\right)=f_{u}\left(\mathbf{q} \mid \mathbf{q}_{0}\right) / u$. The final step involves using the identified functions $f_{u}$ and $g_{u}$ in Equation (17), multiplying with $p_{0}\left(\mathbf{q}_{0}^{\prime \prime} \mid \mathbf{q}_{0}\right)$, integrating over $\mathbf{q}^{\prime \prime}$ and $\mathbf{q}_{0}^{\prime \prime}$ while using the divergence theorem implying $\int_{\Xi^{c}} d \mathbf{q}^{\prime \prime} \hat{\mathcal{L}}_{l} \tilde{G}_{l}\left(\mathbf{q}^{\prime \prime}, u \mid \mathbf{q}_{0}^{\prime \prime}\right.$ ) $=0$ (as before) to obtain

$$
\begin{aligned}
& u \tilde{Q}_{p_{0}}\left(\mathbf{q}, u \mid \mathbf{q}_{0}\right)-\delta\left(\mathbf{q}-\mathbf{q}_{0}\right) \\
& =\left(u \int_{\Upsilon} d \mathbf{q}^{\prime \prime} \int_{\Xi^{c}} d \mathbf{q}_{0}^{\prime \prime} \tilde{G}_{l}\left(\mathbf{q}^{\prime \prime}, u \mid \mathbf{q}_{0}^{\prime \prime}\right) p_{0}\left(\mathbf{q}_{0}^{\prime \prime} \mid \mathbf{q}_{0}\right)\right) \hat{\mathcal{L}}_{p} \tilde{Q}_{p_{0}}\left(\mathbf{q}, u \mid \mathbf{q}_{0}\right) .
\end{aligned}
$$

Finally, since the Laplace transform of $\partial_{t} g(t)+\delta(t) g(0)$ corresponds to $u \tilde{g}(u)$, taking the inverse Laplace transform of Equation (19) finally leads to Equations (15) and (16) and completes the proof of the lemma, since now we can take $Q_{p_{0}}\left(\mathbf{q}, t \mid \mathbf{q}_{0}\right)>0$ by definition because Equation (15) is an identity of Equation (1) integrated over $\mathbf{q}^{\prime \prime}$. Moreover, the rate of change of the Green's function $Q_{p_{0}}\left(\mathbf{q}, t \mid \mathbf{q}_{0}\right)$ in Equation (15) depends, at any instance $t$, position $\mathbf{q}$ and for any initial condition $\mathbf{q}_{0}$ only on the current position $\mathbf{q}$ and a waiting time (or random time-change) encoded in the memory kernel $K(t) ; Q_{p_{0}}\left(\mathbf{q}, t \mid \mathbf{q}_{0}\right)$ is the Green's function of a renewal process. This completes the proof of sufficiency.

Furthermore, for the situation where the full system is prepared in a stationary state, i.e., $p_{0}(\mathbf{x})=p_{s}(\mathbf{x})$, we have the following

Corollary 2.2.2.2.1. Let the system and projection be defined as in Theorem 2.2.2.2. If the full system is prepared such that the latent degrees of freedom are in a stationary state $p_{0}\left(\mathbf{q}_{0}, \mathbf{q}_{0}^{\prime \prime}\right)$, such that 
$\hat{\mathcal{L}}_{l} p_{0}\left(\mathbf{q}_{0}^{\prime \prime} \mid \mathbf{q}_{0}\right)=0, \forall \mathbf{q}_{0} \in \boldsymbol{\Xi}$ and hence also $\bar{p}_{0}\left(\mathbf{q}_{0}^{\prime \prime}\right)=\bar{p}_{\text {ss }}\left(\mathbf{q}_{0}^{\prime \prime}\right)$, then $p_{0}\left(\mathbf{q}_{0}^{\prime \prime} \mid \mathbf{q}_{0}\right)=\psi_{0}^{l, R}\left(\mathbf{q}_{0}^{\prime \prime}\right)$ and consequently $K_{p_{0}}=\delta(t) \int_{\Upsilon} d \mathbf{q}_{0}^{\prime \prime} \bar{p}_{s s}\left(\mathbf{q}_{0}^{\prime \prime}\right)$, and therefore the projected dynamics is Markovian. Moreover, if the system is prepared such that the latent degrees of freedom are not in a stationary state, i.e., $p_{0}\left(\mathbf{q}_{0} \mid \mathbf{q}_{0}^{\prime \prime}\right) \neq \bar{p}_{\mathrm{ss}}\left(\mathbf{q}_{0}^{\prime \prime}\right), \forall \mathbf{q}_{0}$, there exists a finite time $t_{M}>0$ after which the dynamics will be arbitrarily close to being Markovian.

The statement of this corollary is again intuitive. The proof of the first part follows from the bi-orthogonality of eigenfunctions of latent dynamics $\left\langle\psi_{k}^{l, R} \mid \psi_{0}^{l, R}\right\rangle=\delta_{k, 0}$, rendering all terms in Equation (16) in Lemma 2.2.2.2.1 identically zero except for $k=0$ with $\lambda_{k}^{l}=0$. The second part is established by the fact that for times $t_{M} \gg 1 / \lambda_{1}^{l}$, with $-\lambda_{1}^{l}$ being the largest (i.e., least negative) non-zero eigenvalue, all terms but the $k=0$ term in Equation (16) in Lemma 2.2.2.2.1 become arbitrarily small.

Having established sufficiency, we now also comment on necessity of the conditions (i) and (ii) above for renewal dynamics. It is clear that the splitting of $\hat{\mathcal{L}}$ into $\hat{\mathcal{L}}_{p}$ and $\hat{\mathcal{L}}_{l}$, where $\hat{\mathcal{L}}_{l}$ does not act nor depend on projected variables, is also necessary condition for renewal. This can be established by contradiction as loosening these assumptions leads to dynamics that is not renewal. This can be understood intuitively, because it must hold that the latent degrees of freedom remain entirely decoupled from the projected ones (but not vice versa) and that the motion along both is mutually orthogonal. To illustrate this think of the paradigmatic comb model (see schematic in Figure 1) [70-72] and realize that renewal will be violated as soon as we tilt the side-branches for some angle from being orthogonal to the backbone.

However, since it is difficult to establish the most general class of admissible functions $h\left(\mathbf{q}^{\prime \prime}\right)$ used in $\hat{\mathcal{L}}=h\left(\mathbf{q}^{\prime \prime}\right) \hat{\mathcal{L}}_{p}+$ $\hat{\mathcal{L}}_{l}$, we are not able to prove necessity. Based on the present

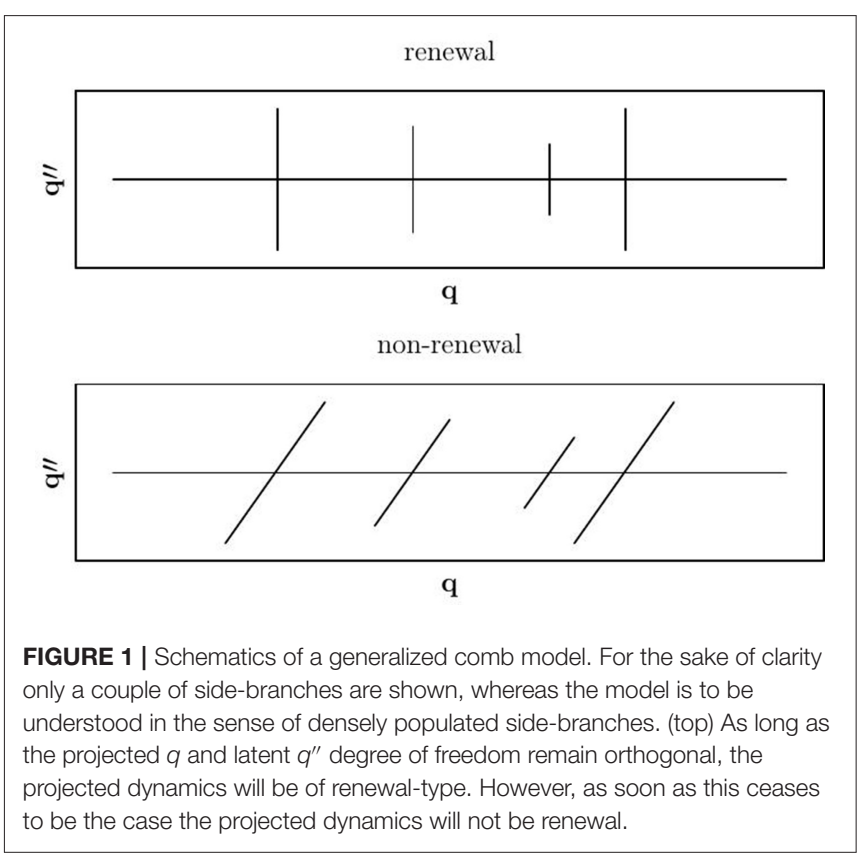

analysis it seems somewhat difficult to systematically relax the assumptions for projected dynamics to be renewal without assuming, in addition, some sort of spatial discretization. We therefore hypothesize that the sufficient conditions stated in Theorem 2.2.2.2, potentially with some additional assumptions on $h\left(\mathbf{q}^{\prime \prime}\right)$ are also necessary conditions. Notably, however, that microscopic derivations of non-Markovian master equations of the form given in Equation (15) often start in discretized space or ad-hoc introduce a random change in time scale (see e.g., $[2,17,82])$. We end this section with the following final

Remark 2.2.2.1. An arbitrary projection $\hat{\mathcal{P}}_{\mathbf{X}}(\boldsymbol{\Gamma} ; \mathbf{q})$ defined in Equation (8) will most likely lead to dynamics that is neither Markovian nor renewal.

This follows from the strong assumptions required for Markovian and renewal dynamics, respectively. The properties of the corresponding general evolution operator will be described in a separate publication.

\subsubsection{Markovian Approximation and the Degree of Non-Markovianity}

In order to quantify the degree of non-Markovianity induced by the projection we propose to compare the full non-Markovian dynamics with projected dynamics evolving under a complete time-scale separation, i.e., under the assumption of all latent degrees of freedom being in the stationary state. To do so we proceed as follows. The projected coordinates $\mathbf{q}$ are now assumed to represent a subset of another $d$-dimensional orthogonal system in Euclidean space $\mathbf{q}^{\prime} \in \mathbb{R}^{d}$, and we assume the map $\mathbf{q}^{\prime}(\mathbf{x})$ is bijective. We denote the conditional probability density in this system by $G^{\prime}\left(\mathbf{q}^{\prime}, t \mid \mathbf{q}_{0}^{\prime}, 0\right)$. The underlying physical idea is that an observer can only see the projected dynamics, which since it is non-Markovian stems from a projection but not necessarily onto Cartesian coordinates. Therefore, from a physical perspective not too much generality seems to be lost with this assumption.

As a concrete example one can consider the non-spherically symmetric Fokker-Planck process in a sphere, corresponding to the full Markovian parent system projected onto angular variables (either one or both). This way one first transforms from $\mathbf{x} \in \mathbb{R}^{3}$ to spherical coordinates $\mathbf{q}^{\prime}=(r, \phi, \theta)$ and then, e.g., projects on the the lines $\mathbf{q}=\phi \in[0,2 \pi)$.

Since the transformation of the Fokker-Planck equation under a general change of coordinates is well-known [83] the task is actually simple. Under the complete map $\mathbf{q}^{\prime}=\boldsymbol{\Gamma}(\mathbf{x})$ with $\Gamma: \mathbb{R}^{d} \rightarrow \mathbb{R}^{d}$ the forward Fokker-Planck operator in Equation (1) transforms as $\hat{\mathcal{L}}^{\prime}=\nabla_{\mathbf{q}^{\prime}} \otimes \nabla_{\mathbf{q}^{\prime}}: \tilde{D}\left(\mathbf{q}^{\prime}\right)-\nabla_{\mathbf{q}^{\prime}} \cdot \tilde{\mathbf{F}}\left(\mathbf{q}^{\prime}\right)$, where $\otimes$ and : denote, respectively, the tensor and double-dot product, and the transformed drift field and diffusion tensor can be written as

$$
\begin{array}{r}
\left(\tilde{\mathbf{F}}\left(\mathbf{q}^{\prime}\right)\right)_{k}=\sum_{i=1}^{d} \frac{\partial q_{k}^{\prime}}{\partial x_{i}} \mathbf{F}_{i}+\sum_{i, j=1}^{d} D_{i j} \frac{\partial^{2} q_{k}}{\partial x_{i} \partial x_{j}}, \\
\left(\tilde{D}\left(\mathbf{q}^{\prime}\right)\right)_{k l}=\sum_{i, j=1}^{d} D_{i j} \frac{\partial q_{k}^{\prime}}{\partial x_{i}} \frac{\partial q_{l}^{\prime}}{\partial x_{j}} .
\end{array}
$$


We note that unless the mapping is linear, the old diffusion matrix affects the new drift vector and the diffusion matrix picks up a spatial dependence. For an excellent account of the transformation properties in the more general case of a position dependent diffusion matrix [i.e., $\mathbf{D} \rightarrow \mathbf{D}(\mathbf{x})$ ] we refer the reader to [84]. We now want to marginalize over the remaining (i.e., non-projected) coordinates $\mathbf{q}^{\prime \prime} \in \Omega \backslash \Xi$ but beforehand make the Markovian approximation $G^{\prime}\left(\mathbf{q}^{\prime}, t \mid \mathbf{q}_{0}, 0\right) \approx Q_{M}\left(\mathbf{q}, t \mid \mathbf{q}_{0}\right) p_{\mathrm{ss}}\left(\mathbf{q}^{\prime \prime}\right)$. Then we have $\hat{\mathcal{L}}^{\prime} G^{\prime}\left(\mathbf{q}^{\prime}, t \mid \mathbf{q}_{0}, 0\right) \approx p_{\text {ss }}\left(\mathbf{q}^{\prime \prime}\right) \hat{\mathcal{L}}^{\prime} Q_{M}\left(\mathbf{q}, t \mid \mathbf{q}_{0}\right)$, implying that the operator $\hat{\mathcal{L}}^{\prime}$ approximately splits into one part operating on the projected coordinates alone, $\hat{\mathcal{L}}_{M}^{\prime}$, and one operating only on the latent stationary coordinates, $\hat{\mathcal{L}}^{\prime \prime}$, for which $\hat{\mathcal{L}}^{\prime \prime} p_{\mathrm{ss}}\left(\mathbf{q}^{\prime \prime}\right)=0$. The physical idea behind the Markovian approximation is that the latent degrees of freedom relax infinitely fast compared to the projected ones. Therefore, we can straightforwardly average the Fokker-Planck operator over the stationary latent coordinates $\mathbf{q}^{\prime \prime},\left\langle\hat{\mathcal{L}}_{M}^{\prime}\right\rangle_{\mathbf{q}^{\prime \prime}}$, where we have defined the latent averaging operation $\langle\cdot\rangle_{\mathbf{q}^{\prime \prime}} \equiv \int d \mathbf{q}^{\prime \prime} p_{\mathrm{ss}}\left(\mathbf{q}^{\prime \prime}\right) \cdot$. Note that the remaining dependence of $\hat{\mathcal{L}}^{\prime}$ on the latent stationary coordinates $\mathbf{q}^{\prime \prime}$ is only due to $\tilde{\mathbf{F}}\left(\mathbf{q}^{\prime}\right)$ and $\tilde{D}\left(\mathbf{q}^{\prime}\right)$. The averaged drift field and diffusion matrix now become

$$
\begin{array}{r}
\langle\tilde{\mathbf{F}}(\mathbf{q})\rangle_{k}=\sum_{i=1}^{d}\left\langle\frac{\partial q_{k}^{\prime}}{\partial x_{i}} \mathbf{F}_{i}\right\rangle_{\mathbf{q}^{\prime \prime}}+\sum_{i, j=1}^{d} D_{i j}\left\langle\frac{\partial^{2} q_{k}}{\partial x_{i} \partial x_{j}}\right\rangle_{\mathbf{q}^{\prime \prime}}, \\
\langle\tilde{D}(\mathbf{q})\rangle_{k l}=\sum_{i, j=1}^{d} D_{i j}\left\langle\frac{\partial q_{k}^{\prime}}{\partial x_{i}} \frac{\partial q_{l}^{\prime}}{\partial x_{j}}\right\rangle_{\mathbf{q}^{\prime \prime}} .
\end{array}
$$

We can further decompose the effective drift field into a conservative and a non-conservative part

$$
\left\langle\frac{\partial q_{k}^{\prime}}{\partial x_{i}} \mathbf{F}_{i}\right\rangle_{\mathbf{q}^{\prime \prime}}=-\left\langle\frac{\partial q_{k}^{\prime}}{\partial x_{i}}(\mathbf{D} \nabla \varphi)_{i}\right\rangle_{\mathbf{q}^{\prime \prime}}+\left\langle\mathrm{e}^{\varphi} \frac{\partial q_{k}^{\prime}}{\partial x_{i}}\left(\mathbf{j}_{s s}\right)_{i}\right\rangle_{\mathbf{q}^{\prime \prime}},
$$

which establishes the Markovian approximation also for a broad class of irreversible systems. The approximate effective FokkerPlanck operator for the projected dynamics in turn reads

$$
\left\langle\hat{\mathcal{L}}^{\prime}\right\rangle_{\mathbf{q}^{\prime \prime}}=\nabla_{\mathbf{q}} \otimes \nabla_{\mathbf{q}}:\langle\tilde{D}(\mathbf{q})\rangle_{\mathbf{q}^{\prime \prime}}-\nabla_{\mathbf{q}} \cdot\langle\tilde{\mathbf{F}}(\mathbf{q})\rangle_{\mathbf{q}^{\prime \prime}}
$$

By design the kernel of $\left\langle\hat{\mathcal{L}}^{\prime}\right\rangle_{\mathbf{q}^{\prime \prime}}$ is equal to $p_{\text {ss }}(\mathbf{q}) \equiv \hat{\mathcal{P}}_{\mathbf{x}}(\boldsymbol{\Gamma} ; \mathbf{q}) p_{\text {ss }}(\mathbf{x})$, hence $\left\langle\hat{\mathcal{L}}^{\prime}\right\rangle_{\mathbf{q}^{\prime \prime}}$ governs the relaxation toward the steady-state density (not necessarily equilibrium) evolving from some initial state $\mathbf{q}_{0}$ in the Markovian approximation with the corresponding Green's function $Q_{M}\left(\mathbf{q}, t \mid \mathbf{q}_{0}, 0\right) \equiv\left\langle\mathbf{q}\left|\mathrm{e}^{\left\langle\hat{\mathcal{L}}^{\prime}\right\rangle_{\mathbf{q}^{\prime \prime}}}\right| \mathbf{q}_{0}\right\rangle$.

In order to quantify the departure of the exact dynamics from the corresponding Markovian behavior we propose to evaluate the Kullback-Leibler divergence between the Green's functions of the exact and Markovian propagator as a function of time

$$
\mathcal{D}_{t}\left(Q \| Q_{M}\right)=\int_{\Xi} d \mathbf{q} Q\left(\mathbf{q}, t \mid \mathbf{q}_{0}, 0\right) \ln \left(\frac{Q\left(\mathbf{q}, t \mid \mathbf{q}_{0}, 0\right)}{Q_{M}\left(\mathbf{q}, t \mid \mathbf{q}_{0}, 0\right)}\right) .
$$

By definition $\mathcal{D}_{t}\left(Q \| Q_{M}\right) \geq 0$ and since the non-Markovian behavior of the exact projected dynamics is transient with a lifetime $\lambda_{1}^{-1}$, we have that $\lim _{t \rightarrow \infty} \mathcal{D}_{t}\left(Q \| Q_{M}\right)=0$. Our choice of quantifying the departure of the exact dynamics from the corresponding Markovian behavior is not unique. The KullbackLeibler divergence introduced here can hence be used to quantify how fast the correlation of the latent degrees of freedom with the projected degrees of freedom dies out. Notably, in a related manner the Kullback-Leibler divergence was also used in the context of stochastic thermodynamics in order to disprove the hypothesis about the monotonicity of the entropy production as a general time evolution principle [85].

\subsubsection{Functionals of Projected Dynamics}

In order to gain deeper insight into the origin and manifestation of non-Markovian behavior it is instructive to focus on the statistics of time-average observables, that is functionals of projected dynamics. As in the previous sections we assume that the full system was prepared in a (potentially non-equilibrium current-carrying) steady state. To that end we have, using Feynman-Kac theory, recently proven a theorem connecting any bounded additive functional $\Phi_{t}[\mathbf{q}(\tau)]=t^{-1} \int_{0}^{t} Z(\mathbf{q}(\tau)) d \tau$ (with a function $Z: \Xi\left(\mathbb{R}^{a}\right) \rightarrow \mathbb{R}$ locally strictly bounded in $\left.\Xi\right)$ of projected dynamics $\mathbf{q}(\tau)$ of a parent Markovian diffusion $\mathbf{x}(t)$ to the eigenspectrum of the Markov generator of the full dynamics $\hat{\mathcal{L}}$ or $\hat{\mathcal{L}}^{\dagger}$ [67]. The central quantity of the theory is $\theta_{t}(\mathbf{s})$, the so-called local time fraction spent by a trajectory $\mathbf{q}(\tau)$ in a infinitesimal volume element $d \mathbf{s}$ centered at $\mathbf{s}$ up until a time $t$ enabling

$$
\theta_{t}(\mathbf{s})=t^{-1} \int_{0}^{t} d \tau \mathbb{1}_{\mathbf{s}}(\mathbf{q}(\tau)) \rightarrow \Phi_{t}[\mathbf{q}(\tau)]=\int_{\Xi} d \mathbf{s} Z(\mathbf{s}) \theta_{t}(\mathbf{s}),
$$

where the indicator function $\mathbb{1}_{\mathbf{s}}(\mathbf{q})=1$ if $\mathbf{q}=\mathbf{s}$ and zero otherwise. We are here interested in the fluctuations of $\theta_{t}(\mathbf{s})$ and correlation functions between the local time fraction of a projected observable $\mathbf{q}(t)$ at a point $\mathbf{s}$ and $\theta^{\prime \prime}\left(\mathbf{s}^{\prime}\right)$, the local time some latent (hidden) observable $\mathbf{q}^{\prime \prime}(t)$ a the point $\mathbf{s}^{\prime}$ :

$$
\begin{array}{r}
\sigma_{t}^{2}(\mathbf{s})=\left\langle\theta_{t}^{2}(\mathbf{s})\right\rangle-\left\langle\theta_{t}(\mathbf{s})\right\rangle^{2}, \quad \mathcal{C}_{t}\left(\mathbf{s} ; \mathbf{s}^{\prime}\right) \\
=\left\langle\theta_{t}(\mathbf{s}) \theta_{t}^{\prime \prime}\left(\mathbf{s}^{\prime}\right)\right\rangle-\left\langle\theta_{t}(\mathbf{s})\right\rangle\left\langle\theta_{t}^{\prime \prime}\left(\mathbf{s}^{\prime}\right)\right\rangle
\end{array}
$$

where $\langle\cdot\rangle$ now denotes the average over all forward paths starting from the steady state $\left|\mathbf{q}_{0}\right\rangle=|s s\rangle$ (and ending anywhere, i.e., $\langle\mathbf{q}|=\langle-|$ ), or, using the backward approach, all paths starting in the flat state $|\mathbf{q}\rangle=|-\rangle$ and propagating backward in time toward the steady state $\left\langle\mathbf{q}_{0}\right|=\langle$ ss $|$. We note that any correlation function of a general additive bounded functional $\Phi_{t}^{i}[\mathbf{q}(\tau)]$ of the form $\left\langle\Phi_{t}^{i}[\mathbf{q}(\tau)] \Phi_{t}^{j}\left[\mathbf{q}^{\prime \prime}(\tau)\right]\right\rangle$ (as well as the second moment of $\left.\Phi_{t}^{i}[\mathbf{q}(\tau)]\right)$ follows directly from the local time fraction, namely, $\left\langle\Phi_{t}^{i}[\mathbf{q}(\tau)] \Phi_{t}^{j}\left[\mathbf{q}^{\prime \prime}(\tau)\right]\right\rangle=\int_{\Xi} \int_{\Xi} d \mathbf{s} d \mathbf{s}^{\prime} Z_{i}(\mathbf{s}) Z_{j}\left(\mathbf{s}^{\prime}\right)\left\langle\theta_{t}(\mathbf{s}) \theta_{t}^{\prime \prime}\left(\mathbf{s}^{\prime}\right)\right\rangle$. For details of the theory and corresponding proofs please see [67], here we will simply state the main result:

Theorem 2.2.4.1. Let the Green's function of the full parent dynamics $\mathbf{x}(t)$ be given by Equation (7) and the local time fraction $\theta_{t}(\mathbf{s})$ by Equation (25), then the variance and correlation function 
defined in Equation (26) is given exactly as

$$
\begin{aligned}
\sigma_{t}^{2}(\mathbf{s})= & 2 \sum_{k>0} \frac{\left\langle-\left|\mathbb{1}_{\mathbf{s}}\right| \psi_{k}^{R}\right\rangle\left\langle\psi_{k}^{L}\left|\mathbb{1}_{\mathbf{s}}\right| \mathrm{ss}\right\rangle}{\lambda_{k} t}\left(1-\frac{1-\mathrm{e}^{-\lambda_{k} t}}{\lambda_{k} t}\right) \\
\mathcal{C}_{t}\left(\mathbf{s} ; \mathbf{s}^{\prime}\right)= & \sum_{k>0} \frac{\left\langle-\left|\mathbb{1}_{\mathbf{s}}\right| \psi_{k}^{R}\right\rangle\left\langle\psi_{k}^{L}\left|\mathbb{1}_{\mathbf{s}^{\prime}}^{\prime \prime}\right| \mathrm{ss}\right\rangle+\left\langle-\left|\mathbb{1}_{\mathbf{s}^{\prime}}^{\prime \prime}\right| \psi_{k}^{R}\right\rangle\left\langle\psi_{k}^{L}\left|\mathbb{1}_{\mathbf{s}}\right| \mathrm{ss}\right\rangle}{\lambda_{k} t} \\
& \left(1-\frac{1-\mathrm{e}^{-\lambda_{k} t}}{\lambda_{k} t}\right)
\end{aligned}
$$

and analogous equations are obtained using the backward approach [67].

The usefulness of Equation (27) can be understood as follows. By varying $\mathbf{s}$ and $\mathbf{s}^{\prime}$ one can establish directly the regions in space responsible for the build-up (and subsequent decay) of memory in projected dynamics and simultaneously monitor the fluctuations of the time spent of a projected trajectory in said regions. Note that because the full process is assumed to be ergodic, the statistics of $\theta_{t}(\mathbf{s})$ will be asymptotically Gaussian obeying the large deviation principle. This concludes our general results. In the following section we apply the theoretical framework to the analysis of projected dynamics in a strongly-correlated stochastic many-body system, namely to tagged particle dynamics in a single file confined to a tilted box.

\section{SINGLE FILE DIFFUSION IN A TILTED BOX}

We now apply the theory developed in the previous section (here we use the backward approach) to the paradigmatic single file diffusion in a unit interval but here with a twist, namely, the diffusing particles experience a constant force. In particular, the full state-space is spanned by the positions of all $N$-particles defining the state vector $\mathbf{x}_{0}=\left(x_{0,1}, \ldots, x_{0, N}\right)^{T} \in[0,1]^{N}$ and diffusion coefficients of all particles are assumed to be equal and the thermal (white) fluctuations due to the bath are assumed to be independent, i.e., $\mathbf{D}=D \mathbf{1}$. In addition to being confined in a unit interval, all particles experience the same constant force $\mathbf{F}\left(\mathbf{x}_{0}\right)=-\beta D F$ with $\beta=\left(k_{B} T\right)^{-1}$ is the inverse thermal energy. The evolution of the Green's function is governed by the FokkerPlanck equation Equation (1) equipped with the external and internal (i.e., non-crossing) reflecting boundary conditions for the backward generator $\hat{\mathcal{L}}^{\dagger}=\sum_{i=1}^{N} D\left(\partial_{x_{0, i}}^{2}-\beta F \partial_{x_{0, i}}\right)$ :

$$
\begin{array}{r}
\left.\partial_{x_{0,1}} G\left(\mathbf{x}, t \mid \mathbf{x}_{0}\right)\right|_{x_{0,1}=0}=\left.\partial_{x_{0, N}} G\left(\mathbf{x}, t \mid \mathbf{x}_{0}\right)\right|_{x_{0, N}=1}=0, \\
\lim _{x_{0, i} \rightarrow x_{0, j}}\left(\partial_{x_{0, i+1}}-\partial_{x_{0, i}}\right) G\left(\mathbf{x}, t \mid \mathbf{x}_{0}\right)=0,
\end{array}
$$

where we adopted the notation in Equation (7). The boundary conditions in Equation (28) restrict the domain to a hypercone $\mathbf{x}_{0} \in \Xi$ such that $x_{0, i} \leq x_{0, i+1}$ for $i=1, \ldots, N-1$. The dynamics is reversible, hence the steady state current vanishes and all eigenvalues and eigenfunctions are real. Moreover, for systems obeying detailed balance $\varphi(\mathbf{x})$ corresponds to the density of the Boltzmann-Gibbs measure and it is known that $\left|\psi_{k}^{L}\right\rangle \equiv$ $\mathrm{e}^{-\varphi(\mathbf{x})}\left|\psi_{k}^{R}\right\rangle$. The single file backward generator already has a separated form $\hat{\mathcal{L}}^{\dagger}=\sum_{i=1}^{N} \mathcal{L}_{i}^{\dagger}$ and the coupling between particles enters solely through the non-crossing boundary condition Equation (28) and is hence Bethe-integrable [86]. However, because the projected and latent degrees of freedom are coupled through the boundary conditions Equation (28) the tagged particle dynamics is not of renewal type.

\subsection{Diagonalization of the Generator With the Coordinate Bethe Ansatz}

Specifically, the backward generator $\hat{\mathcal{L}}^{\dagger}$ can be diagonalized exactly using the coordinate Bethe ansatz (see e.g., [67]). To that end we first require the solution of the separated (i.e., single particle) eigenvalue problem $\mathcal{L}_{i}^{\dagger}\left|\psi_{k_{i}}^{L}\right\rangle=-\lambda_{k_{i}}\left|\psi_{k_{i}}^{L}\right\rangle$ under the imposed external boundary conditions. Since $\varphi\left(x_{0, i}\right)=F x_{0, i}+$ const we find that $p_{\mathrm{ss}}\left(x_{0, i}\right)=\beta F \mathrm{e}^{-\beta F x_{0, i}}\left(1-\mathrm{e}^{-\beta F}\right)^{-1}$ and because of the confinement we also have $\lambda_{0, i}=0$ as well as $\psi_{0_{i}}^{L}\left(x_{0, i}\right) \equiv$ $\left\langle x_{0, i} \mid \psi_{0_{i}}^{L}\right\rangle=1$ and $\psi_{0_{i}}^{R}\left(x_{0, i}\right) \equiv\left\langle\psi_{0_{i}}^{R} \mid x_{0, i}\right\rangle=p_{\mathrm{ss}}\left(x_{0, i}\right)$. We are here interested in the role of particle number $N$ and not of the magnitude of the force $F$, therefore we will henceforth set, for the sake of simplicity, $\beta F=D=1$. The excited separated eigenvalues and eigenfunctions then read

$$
\begin{aligned}
\lambda_{k_{i}}= & \pi^{2} k_{i}^{2}+\frac{1}{4}, \\
\psi_{k_{i}}^{L}\left(x_{0, i}\right)= & \frac{\mathrm{e}^{x_{0, i} / 2}}{\left(2 \pi^{2} k_{i}^{2}+1 / 2\right)^{1 / 2}}\left(\sin \left(k_{i} \pi x_{0, i}\right)-2 k_{i} \pi \cos \left(k_{i} \pi x_{0, i}\right)\right), \\
& \forall k_{i} \in \mathbb{Z}^{+},
\end{aligned}
$$

with $\psi_{k_{i}}^{R}\left(x_{0, i}\right)=\mathrm{e}^{-x_{0, i}} \psi_{k_{i}}^{L}\left(x_{0, i}\right)$. It is straightforward to check that $\left\langle\psi_{k_{i}}^{R} \mid \psi_{l_{i}}^{L}\right\rangle=\delta_{k_{i}, l_{i}}$. Denoting by $\mathbf{k}=\left(k_{i}, k_{2}, \ldots, k_{N}\right)$ the $N$-tuple of all single-state indices $k_{i}$ one can show by direct substitution that the many-body eigenvalues are given by $\lambda_{\mathbf{k}}=\sum_{i=1}^{N} \lambda_{k_{i}}$ and the corresponding orthonormal many-body eigenfunctions that obey the non-crossing internal boundary conditions Equation (28) have the form

$$
\begin{aligned}
& \boldsymbol{\psi}_{\mathbf{0}}^{L}\left(\mathbf{x}_{0}\right)=1, \quad \boldsymbol{\psi}_{\mathbf{0}}^{R}\left(\mathbf{x}_{0}\right)=N ! \prod_{i=1}^{N} \frac{\mathrm{e}^{-x_{0, i}}}{1-\mathrm{e}^{-1}} \\
& \boldsymbol{\psi}_{\mathbf{k}}^{L}\left(\mathbf{x}_{0}\right)=\sum_{\left\{k_{i}\right\}} \prod_{i=1}^{N} \psi_{k_{i}}^{L}\left(x_{0, i}\right), \boldsymbol{\psi}_{\mathbf{k}}^{R}\left(\mathbf{x}_{0}\right)=\mathbf{m}_{\mathbf{k}} ! \sum_{\left\{k_{i}\right\}} \prod_{i=1}^{N} \psi_{k_{i}}^{R}\left(x_{0, i}\right),
\end{aligned}
$$

where $\sum_{\left\{k_{i}\right\}}$ denotes the sum over all permutations of the elements of the $N$-tuple $\mathbf{k}$ and $\mathbf{m}_{\mathbf{k}}$ ! $=\prod_{i} m_{k_{i}}$ ! is the respective multiplicity of the eigenstate with $m_{k_{i}}$ corresponding to the number of times a particular value of $k_{i}$ appears in the tuple. It can be checked by explicit computation that the eigenfunctions defined in Equation (30) form a complete bi-orthonormal set, that is $\left\langle\psi_{\mathbf{k}}^{R} \mid \psi_{\mathbf{l}}^{L}\right\rangle=\delta_{\mathbf{k}, \mathbf{l}}$ and $\sum_{\mathbf{k}} \psi_{\mathbf{k}}^{L}\left(\mathbf{x}_{0}\right) \boldsymbol{\psi}_{\mathbf{k}}^{R}(\mathbf{x})=\delta\left(\mathbf{x}-\mathbf{x}_{0}\right)$.

\subsection{Projection-Induced Non-Markovian Tagged Particle Dynamics}

In the case of single file dynamics the physically motivated projection corresponds to the dynamics of a tagged particle upon integrating out the dynamics of the remaining particles. As 
before, we assume that the full system is prepared in a steady state. The projection operator for the dynamics of the $j$-th particle is therefore defined as

$$
\hat{\mathcal{P}}_{\mathbf{x}}\left(\delta ; q_{j}\right)=\int_{\Xi} d \mathbf{x} \delta\left(x_{j}-q_{j}\right)=\left[\hat{O} \prod_{i=1}^{N} \int_{0}^{1} d x_{i}\right] \delta\left(x_{j}-q_{j}\right),
$$

where the operator $\hat{O}$ orders the integration limits $\int_{0}^{1} d x_{N} \int_{0}^{x_{N}} d x_{N-1} \cdots \int_{0}^{x_{2}} d x_{1}$ since the domain $\Xi$ is a hypercone. Here, the projection is from $\mathbb{R}^{N}$ to $\mathbb{R}$. Integrals of this kind are easily solvable with the so-called 'extended phase-space integration' $[62,87]$. The non-Markovian Green's function is defined as

$$
Q\left(q_{j}, t \mid q_{0, j}\right)=\frac{\hat{\mathcal{P}}_{\mathbf{x}}\left(\delta ; q_{j}\right) \hat{\mathcal{P}}_{\mathbf{x}_{0}}\left(\delta ; q_{0, j}\right) G\left(\mathbf{x}, t \mid \mathbf{x}_{0}\right) p_{\mathrm{ss}}\left(\mathbf{x}_{0}\right)}{\hat{\mathcal{P}}_{\mathbf{x}_{0}}\left(\delta ; q_{0, j}\right) p_{\mathrm{ss}}\left(\mathbf{x}_{0}\right)}
$$

and can be computed exactly according to Equation (10) to give

$$
Q\left(q_{j}, t \mid q_{0, j}\right)=\Psi_{00}\left(q_{0, j}\right)^{-1} \sum_{\mathbf{k}} \Psi_{0 \mathbf{k}}\left(q_{j}\right) \Psi_{\mathbf{k} \mathbf{0}}\left(q_{0, j}\right) \mathrm{e}^{-\lambda_{\mathbf{k}} t},
$$

where the sum is over all Bethe eigenstates. If we denote the number of left and right neighbors by $N_{L}=(N-j+1)$ and $N_{R}=j-1$, respectively, all terms in Equation (33) read explicitly

$$
\begin{aligned}
& \Psi_{\mathbf{0 0}}\left(q_{j}\right)=\frac{N !}{N_{L} ! N_{R} !\left(1-\mathrm{e}^{-1}\right)^{N}} \mathrm{e}^{-q_{j}}\left(1-\mathrm{e}^{-q_{j}}\right)^{N_{L}}\left(\mathrm{e}^{-q_{j}}-\mathrm{e}^{-1}\right)^{N_{R}} \\
& \Psi_{\mathbf{k} \mathbf{0}}\left(q_{j}\right)=\frac{N !}{N_{L} ! N_{R} !\left(1-\mathrm{e}^{-1}\right)^{N}} \sum_{\left\{k_{i}\right\}} T\left(q_{j}\right) \prod_{i=1}^{j-1} L\left(q_{j}\right) \prod_{i=j+1}^{N} R\left(q_{j}\right)
\end{aligned}
$$

and $\Psi_{0 \mathbf{k}}\left(q_{j}\right) \equiv \Psi_{\mathbf{k} 0}^{\dagger}\left(q_{j}\right)=\frac{\mathbf{m}_{\mathbf{k}} !\left(1-\mathrm{e}^{-1}\right)^{N}}{N !} \Psi_{\mathbf{k} 0}\left(q_{j}\right)$. In Equation (34) we have introduced the auxiliary functions

$$
\begin{aligned}
T\left(q_{j}\right)= & \delta_{\lambda_{j}, 0} \mathrm{e}^{-q_{j}}+\left(1-\delta_{\lambda_{j}, 0}\right) \frac{\mathrm{e}^{-q_{j} / 2}}{\sqrt{1 / 2+2 \pi^{2} \lambda_{j}^{2}}} \\
& \left(\sin \left(\lambda_{j} \pi q_{j}\right)-2 \lambda_{j} \pi \cos \left(\lambda_{j} \pi q_{j}\right)\right) \\
L\left(q_{j}\right)= & \delta_{\lambda_{j}, 0}\left(1-\mathrm{e}^{-q_{j}}\right)-2\left(1-\delta_{\lambda_{j}, 0}\right) \frac{\mathrm{e}^{-q_{j} / 2} \sin \left(\lambda_{j} \pi q_{j}\right)}{\sqrt{1 / 2+2 \pi^{2} \lambda_{j}^{2}}} \\
R\left(q_{j}\right)= & \delta_{\lambda_{j}, 0}\left(\mathrm{e}^{-q_{j}}-\mathrm{e}^{-1}\right)+2\left(1-\delta_{\lambda_{j}, 0}\right) \frac{\mathrm{e}^{-q_{j} / 2} \sin \left(\lambda_{j} \pi q_{j}\right)}{\sqrt{1 / 2+2 \pi^{2} \lambda_{j}^{2}}}
\end{aligned}
$$

To the best of our knowledge, Equations (33) to (35) delivering the exact non-Markovian Green's function for the dynamics of the $j$-th particle in a tilted single file of $N$ particles, have not yet been derived before. Note that one can also show that $\int_{0}^{1} d q_{j} \Psi_{0 \mathbf{k}}\left(q_{j}\right) \Psi_{\mathbf{l} 0}\left(q_{j}\right) \neq 0$ and hence the Chapman-Kolmogorov equation is violated in agreement with Equation (13) confirming that the tagged particle diffusion is indeed non-Markovian on time-scales $t \lesssim \lambda_{1}^{-1}$.

\subsection{Markovian Approximation and Degree of Broken Markovianity}

Since the projection leaves the coordinates untransformed the effective Markovian approximation in Equation (23) is particularly simple and corresponds to diffusion in the presence of an effective force deriving from the free energy of the tagged particle upon integrating out all the remaining particles assumed to be in equilibrium $\left\langle F\left(q_{j}\right)\right\rangle_{\mathbf{x}^{\prime \prime}}=-\left\langle\beta D F \delta\left(x_{j}-q_{j}\right)\right\rangle_{\mathbf{x}^{\prime \prime}}$ or, since $-\beta D F p_{\mathrm{ss}}(\mathbf{x})=\partial_{x_{j}} p_{\mathrm{ss}}(\mathbf{x})$, explicitly defined as

$\left\langle F\left(q_{j}\right)\right\rangle_{\mathbf{x}^{\prime \prime}}=\frac{\int_{\Xi} d \mathbf{x} \delta\left(x_{j}-q_{j}\right) \partial_{x_{j}} p_{s s}(\mathbf{x})}{\int_{\Xi} d \mathbf{x} \delta\left(x_{j}-q_{j}\right) p_{s s}(\mathbf{x})} \equiv \frac{\partial_{q_{j}} \int_{\Xi} d \mathbf{x} \delta\left(x_{j}-q_{j}\right) p_{s s}(\mathbf{x})}{\int_{\Xi} d \mathbf{x} \delta\left(x_{j}-q_{j}\right) p_{s s}(\mathbf{x})}$.

Upon taking as before $D=\beta F=1$, and noticing that $\Psi_{00}\left(q_{j}\right)=$ $\int_{\Xi} d \mathbf{x} \delta\left(x_{j}-q_{j}\right) p_{\text {ss }}(\mathbf{x})$ we find

$$
\begin{aligned}
& \langle\hat{\mathcal{L}}\rangle_{\mathbf{q}^{\prime \prime}}=\partial_{q_{j}}^{2}+\partial_{q_{j}}\left\{\partial_{q_{j}} \ln \Psi_{\mathbf{0 0}}\left(q_{j}\right)\right\}, \\
& \left\langle\hat{\mathcal{L}}^{\dagger}\right\rangle_{\mathbf{q}^{\prime \prime}}=\partial_{q_{j}}^{2}-\left\{\partial_{q_{j}} \ln \Psi_{\mathbf{0 0}}\left(q_{j}\right)\right\} \partial_{q_{j}}
\end{aligned}
$$

where the curly bracket $\{\cdot\}$ denotes that the operator inside the bracket only acts within the bracket. The Markovian approximation of the Green's function thus becomes $Q_{M}\left(q_{j}, t \mid q_{0, j}\right)=\left\langle q_{0, j}\left|\mathrm{e}^{\left\langle\hat{\mathcal{L}}^{\dagger}\right\rangle_{\mathbf{x}^{\prime \prime}} t}\right| q_{j}\right\rangle$ and is to be compared to the exact non-Markovian Green's function (33) via the Kullback-Leibler divergence in Equation (24).

Our focus here is to asses how the "degree" of the projection, i.e., $d=N, a=1$ and thus $d-a=N-1-$ the number of latent degrees of freedom (here positions of non-tagged particles) being integrated out affects the timedependence of the Kullback-Leibler divergence. Since the Markovian generator cannot be diagonalized analytically we used a finite element numerical method cross-checked with Brownian dynamics simulations to calculate $Q_{M}\left(q_{j}, t \mid q_{0, j}\right)$. The corresponding Kullback-Leibler divergence (24) was in turn calculated by means of a numerical integration. We present results for the time dependence $\mathcal{D}_{t}\left(Q \| Q_{M}\right)$ in two different representations, the absolute (dimensionless) time $t$ and in units of the average number of collisions $\tilde{t}=t / N^{2}$, tagging the third particle $(j=3)$. The reason to adopt this second choice as the natural physical time-scale is that collisions in fact establish the effective dynamics and hence a typical collision time sets the natural time-scale.

Before going into details we comment on the following. Because we start from the same initial condition for projected coordinate (i.e., tagged particle) in both, the non-Markovian and Markovian setting, it follows trivially that $\lim _{t \rightarrow 0} \mathcal{D}_{t}\left(Q \| Q_{M}\right)=$ 0 . A zero $\mathcal{D}_{t}\left(Q \| Q_{M}\right)$ would persist until the typical time of occurrence of the first collision event. This collision time is, however, much shorter than $t / N^{2}$ because we start from equilibrium initial conditions on the full, many-body level implying a continuous (Boltzmann-weighted) distribution of initial distances of the tagged particle to its nearest neighbors. Using a spectral expansion, however, such vanishingly short time-scales are very difficult to capture, i.e., it would require an astronomically large number of eigenstates, which is 
computationally not feasible. Conversely, because the taggedparticle invariant measures are by definition the same for the single file and its Markovian approximation [i.e., $\Psi_{00}\left(q_{j}\right)$ is equal for both; the first of Equation (34) enters Equation (37)], it also follows that $\lim _{t \rightarrow \infty} \mathcal{D}_{t}\left(Q \| Q_{M}\right)=0$. The relaxation time $\lambda_{1}^{-1}$ in the many-body problem corresponds to the exploration of the entire system of length $L$ (here set to unity); for further details see [67]. For a finite single file deviations from Markovianity are therefore transient, starting at zero, passing through a maximum, and decaying back to zero at times longer than the relaxation time $\lambda_{1}^{-1}$ of the full, many-body model.

The results for $\mathcal{D}_{t}\left(Q \| Q_{M}\right)$ for intermediate and long times are shown in Figure 2. From Figure 2, we confirm that the Markovianity is broken transiently (on time-scales $t \lesssim \lambda_{1}^{-1}$, which holds for any ergodic dynamics in the sense of generating an invariant measure. Notably, the relaxation time $\lambda_{1}$ does not depend on $N$ and is hence equal for all cases considered here. Moreover, as expected, the magnitude of broken Markovianity increases with the "degree" of the projection (here with the particle number $N$ ), as is best seen on a natural time-scale (see Figure 2B). Conversely, on the absolute time-scale the relaxation rate of the Markovian approximation, describing diffusion on a free energy landscape $f\left(q_{3}\right)=-\beta \ln \Psi_{00}\left(q_{3}\right)$, which can be defined as

$$
\lambda_{1}^{M}=-\lim _{t \rightarrow \infty} t^{-1} \ln \left(Q_{M}\left(q_{j}, t \mid q_{0, j}\right)-\Psi_{00}\left(q_{j}\right)\right)
$$

increases with increasing $N$ (see inset in Figure 2B). Therefore, while both have by construction the same invariant measure, the Markovian approximation overestimates the rate of relaxation. This highlights the pitfall in using free energy landscape ideas in absence of a time-scale separation.

\subsection{Tagged Particle Local Times Probing the Origin of Broken Markovianity}

In order to gain deeper insight into the origin and physical meaning of memory emerging from integrating out latent degrees of freedom we inspect how a given tagged particle explores the configuration space starting from a stationary (equilibrium) initial condition. To that end we first compute the variance of local time of a tagged particle, $\theta_{t}\left(q_{j}\right)$ in Equation (25), given in the general form in Equation (26), which applied to tagged particle diffusion in a tilted single file reads:

$$
\sigma_{t}^{2}\left(q_{j}\right)=2 \sum_{\mathbf{k}} \frac{\Psi_{0 \mathbf{k}}\left(q_{j}\right) \Psi_{\mathbf{k} 0}\left(q_{j}\right)}{\lambda_{\mathbf{k}} t}\left(1-\frac{1-\mathrm{e}^{-\lambda_{\mathbf{k}} t}}{\lambda_{\mathbf{k}} t}\right)
$$

where $\Psi_{\mathbf{k} \mathbf{0}}\left(q_{j}\right)$ is given by Equation (34) and $\Psi_{\mathbf{0 k}}\left(q_{j}\right)=$ $\frac{\mathbf{m}_{\mathbf{k}} !}{N !} \Psi_{\mathbf{k} \mathbf{0}}\left(q_{j}\right)$. Note that since the process in ergodic we have $\left\langle\theta_{t}\left(q_{j}\right)\right\rangle=\Psi_{00}\left(q_{j}\right)$, and because the projected dynamics becomes asymptotically Gaussian (i.e., the correlations between $\theta_{t}\left(q_{j}\right)$ at different $t$ gradually decorrelate) we also have the large deviation $\lim _{t \rightarrow \infty} t \sigma_{t}^{2}\left(q_{j}\right)=2 \sum_{\mathbf{k}} \lambda_{\mathbf{k}}^{-1} \Psi_{0 \mathbf{k}}\left(q_{j}\right) \Psi_{\mathbf{k} 0}\left(q_{j}\right) \neq$ $f(t)$. Moreover, because of detailed balance the large deviation principle represents an upper bound to fluctuations of timeaverage observables $\sigma_{t}^{2}\left(q_{j}\right) \leq 2 \sum_{\mathbf{k}} \frac{\Psi_{0 \mathbf{k}}\left(q_{j}\right) \Psi_{\mathbf{k} 0}\left(q_{j}\right)}{\lambda_{\mathbf{k}} t}, \forall t$.

In order to gain more intuition we inspect the statistics of $\theta_{t}\left(q_{j}\right)$ for a single file of four particles (see Figure 3) at different lengths of trajectory $t$ (plotted here on the absolute time-scale). In Figure 3, we show $\left\langle\theta_{t}\left(q_{j}\right)\right\rangle$ with full lines, and the region bounded by the standard deviation $\pm \sigma_{t}\left(q_{j}\right)$ with the shaded area.

The scatter of $\theta_{t}\left(q_{j}\right)$ is largest near the respective free energy minima.

To understand further how this coupling to non-relaxed latent degrees of freedom arises we inspect the correlations between
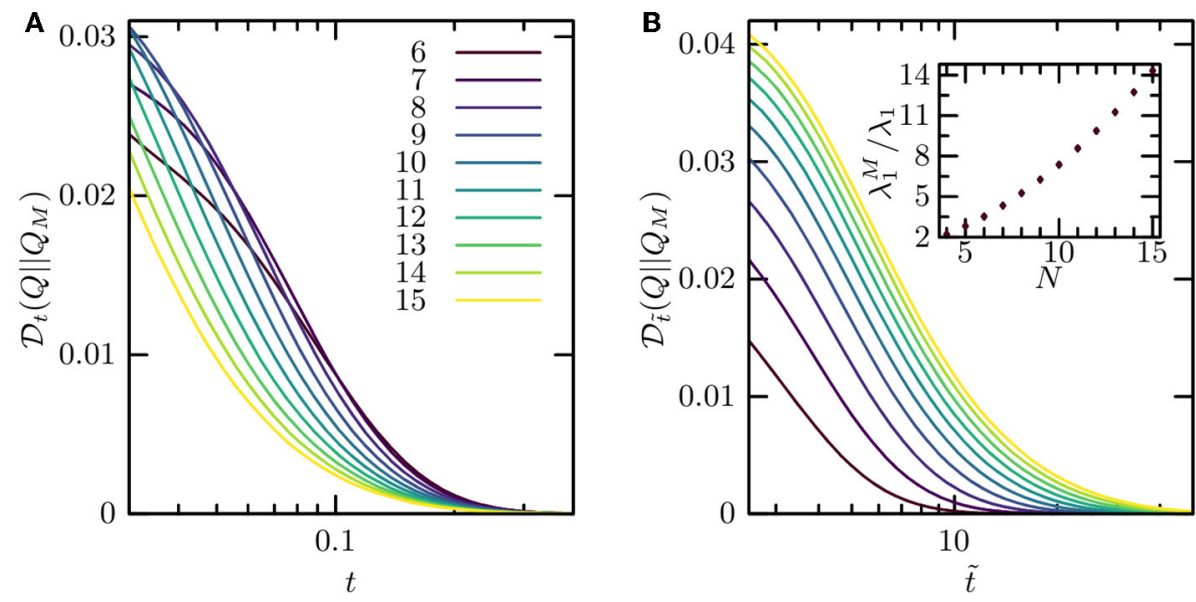

FIGURE 2 | The Kullback-Leibler divergence between the exact non-Markovian Green's function $Q\left(q_{3}, t \mid q_{0,3}\right)$ and the Markovian approximation $Q_{M}\left(q_{3}, t \mid q_{0,3}\right)$ as a function of time (measured in units of collision time) for increasing values of particle numbers $N$ : (A) results shown on the absolute (dimensionless) time-scale and (B) on the natural time-scale, that is, expressed in units of collision time $\tilde{t}$. Inset: $\lambda_{1}^{M}$, the slowest relaxation rate of $Q_{M}\left(q_{3}, t \mid q_{0,3}\right)$ compared to the corresponding eigenvalue $\lambda_{1}$ of the exact $Q\left(q_{3}, t \mid q_{0,3}\right)$ 


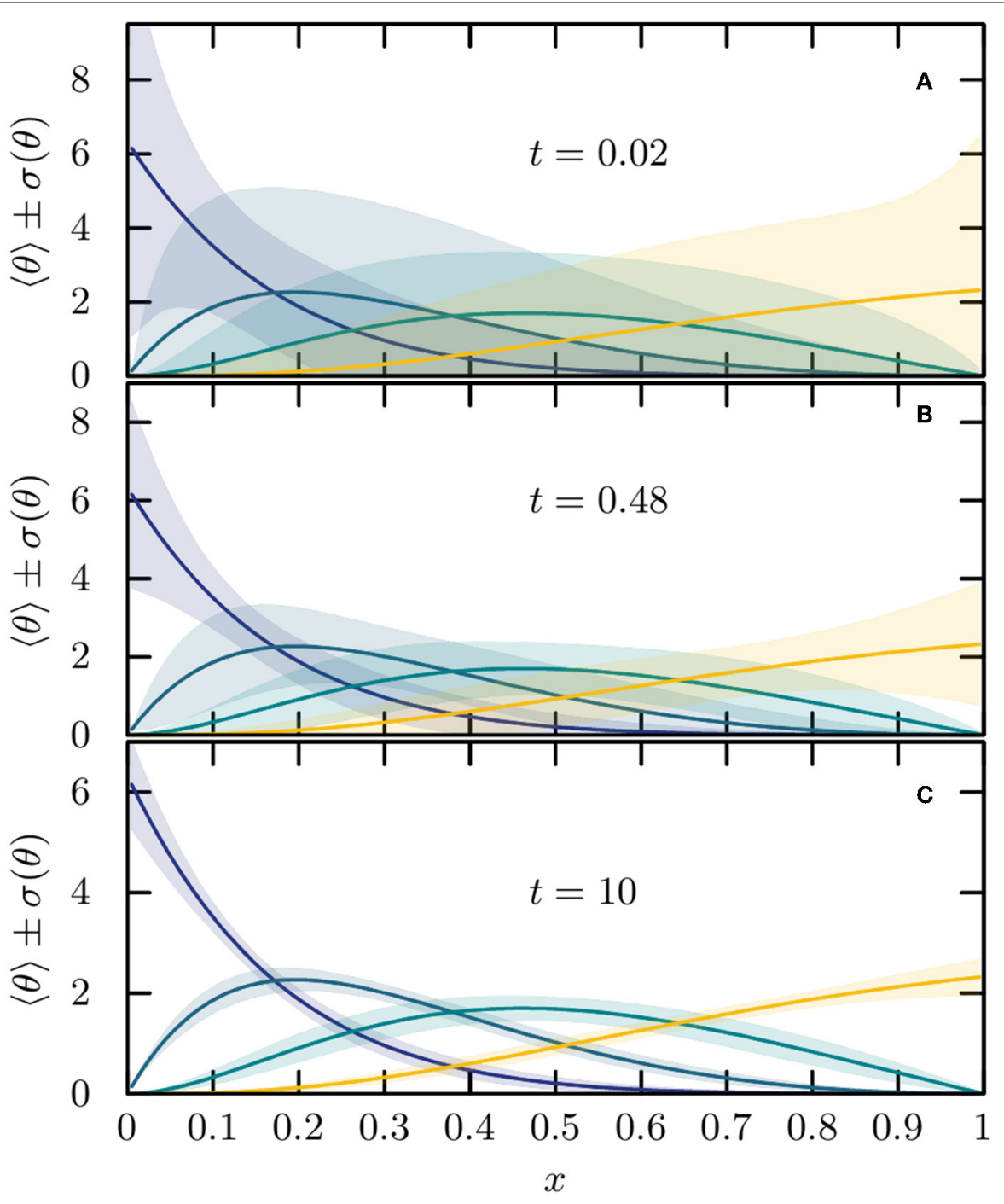

FIGURE 3 | Statistics of tagged particle local time for all members of a single file of four particles starting from stationary initial conditions; $\left\langle\theta_{t}\left(q_{j}\right)\right\rangle$ is represented by full lines and the region bounded by the standard deviation $\pm \sigma_{t}\left(q_{j}\right)$ with the corresponding shaded area. The color code is: $j=1$ violet, $j=2$ blue, $j=3$, green and $j=4$ yellow. The relaxation time corresponds to $\lambda_{1}^{-1} \simeq 0.1$. Therefore, panel $\mathbf{( A )}$ depicts fluctuations on a time scale much shorter that $\lambda_{\mathbf{1}}^{-1}$, whereas $(\mathbf{B}, \mathbf{C})$ already belong deeply into the ergodic large deviation regime.

tagged particle histories

$$
\mathcal{C}_{t}\left(q_{i} ; q_{j}\right)=\sum_{\mathbf{k}} \frac{\Psi_{0 \mathbf{k}}\left(q_{i}\right) \Psi_{\mathbf{k} 0}\left(q_{j}\right)+\Psi_{0 \mathbf{k}}\left(q_{j}\right) \Psi_{\mathbf{k} 0}\left(q_{i}\right)}{\lambda_{k} t}\left(1-\frac{1-\mathrm{e}^{-\lambda_{k} t}}{\lambda_{k} t}\right),
$$

where as before $\lim _{t \rightarrow \infty} t \mathcal{C}_{t}\left(q_{i} ; q_{j}\right) \equiv \overline{\mathcal{C}}_{t}\left(q_{i} ; q_{j}\right)=$ $\sum_{\mathbf{k}} \lambda_{\mathbf{k}}^{-1}\left(\Psi_{0 \mathbf{k}}\left(q_{i}\right) \Psi_{\mathbf{k} \mathbf{0}}\left(q_{j}\right)+\Psi_{\mathbf{0 k}}\left(q_{j}\right) \Psi_{\mathbf{k} \mathbf{0}}\left(q_{i}\right)\right) \neq f(t)$ as a manifestation of the central limit theorem, since $\theta_{t}\left(q_{i}\right)$ and $\theta_{t}\left(q_{j}\right)$ asymptotically decorrelate. In other words, taking $\mathcal{C}_{t}\left(q_{i} ; q_{i}\right) \equiv \sigma_{t}^{2}\left(q_{i}\right)$, the complete large deviation statistics of $\theta_{t}\left(q_{i}\right)$ (i.e., on ergodically long time-scales) is a $N$-dimensional Gaussian with covariance matrix $t^{-1} \overline{\mathcal{C}}_{t}\left(q_{i} ; q_{j}\right)$.
To visualize these results we present in Figures 4, 5 twotag nearest neighbor and next-nearest correlations, $\mathcal{C}_{t}\left(q_{1} ; q_{3}\right)$ and as $\mathcal{C}_{t}\left(q_{2} ; q_{3}\right)$ respectively, for a single file of $N=4$ and $N=7$ particles at two different trajectory lengths. We find that, alongside the fact that correlations intuitively increase with the $N$, both the magnitude and the sign of $\mathcal{C}_{t}$ depend on which particles we tag and even more so, where we tag these particles. Along the (upward shifted) diagonal $\mathcal{C}_{t}$ is positive, implying the two tagged particles along a stochastic many-body trajectory effectively (in the sense of the local time) move together, such that if one particle spends more time in a given region, so will the other. At fixed $F$ (here assumed to be equal to 1 ) the magnitude of the upward shift depends on which particles we tag as well as on $N$. This 

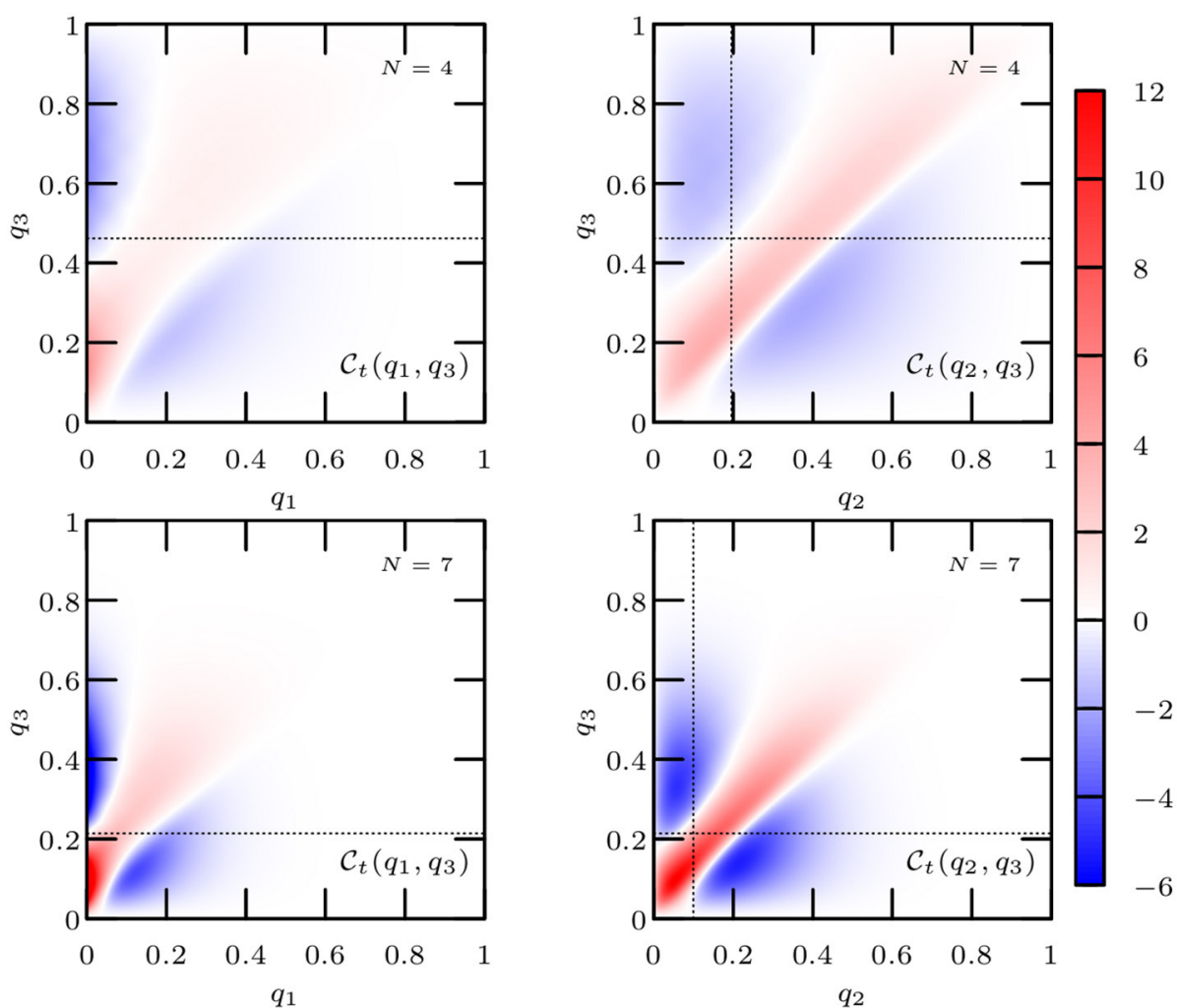

FIGURE 4 | Two-tag local time correlations $\mathcal{C}_{t}\left(q_{1} ; q_{3}\right)$ (left) and $\mathcal{C}_{t}\left(q_{2} ; q_{3}\right)$ (right) for a single file of $N=4$ (top) and $N=7$ (bottom) particles for a (very short) trajectory length $t=0.01$. The relaxation time corresponds to $\lambda_{1}^{-1} \simeq 0.1$. The dashed lines denote the positions of the two free energy minima.

intuitive idea is backed up mathematically by realizing that the lowest excited Bethe-eigenfunctions correspond to collective ("in phase") motion (see Equations 29, 30). Furthermore, defining the free energy minima of the tagged particles with $q_{i}^{\min }$ and $q_{i}^{\min }$ (see dashed lines in Figures 4, 5) we would expect, if the particles were to explore their respective free energy minima, a peak localized at $\left(q_{i}^{\min }, q_{i}^{\min }\right)$ (i.e., at the crossing of dashed line in Figures 4, 5). We find, however, that this is not the case, all together implying that the tagged particles do not, along a many-body trajectory, explore their respective free energy minima. Instead, as mentioned above, they move collectively close to each other. The collective dynamics is therefore non-trivial and the tagged particle dynamics cannot be, at least for $t \lesssim \lambda_{1}^{-1}$ coarse grained to a Markovian diffusion on $-\beta \ln \Psi_{00}\left(q_{j}\right)$, the free energy landscape of the tagged particle $j$. Conversely, the fact that all correlations (positive and negative) die our as $q_{i, j} \rightarrow 1$ is a straightforward consequence of the tilting of the confining box.

Focusing now on the dependence on the length of the trajectory we see at very short time (much shorter than the relaxation time) the correlations are stronger, and that positive correlations peak further away from the two respective tagged particle free energy minima (compare Figure 4 and Figure 5). In addition, the maximum of $\mathcal{C}_{t}\left(q_{i} ; q_{j}\right)$ appears to be somewhat more localized at longer (nearly ergodic) times (see Figure 5). In addition, the tagged particle dynamics seem to be localized more strongly near the free energy minimum if we tag the first particle and if $N$ is larger, presumably because of a faster relaxation due to the presence of the wall effecting more frequent collisions with the wall, during which the particle eventually loses memory.

\section{SUMMARY AND OUTLOOK}

Non-Markovian dynamics and anomalous diffusion are particularly ubiquitous and important in biophysical systems [1-16]. There, however, it appears that the quite many non-Markovian observations are described theoretically by phenomenological approaches with ad-hoc memory kernels, which in specific cases can lead to mathematically unsound or even unphysical behavior [82]. It therefore seems timely and useful to provide a theoretical perspective of non-Markovian dynamics starting from more fine-grained principles and considering a projection to some effective lower-dimensional configuration space.

The ideas presented here are neither new nor completely general. Projection-operator concepts date back to the original works by Zwanzig, Mori, Nakajima, van Kampen, Hänggi and other pioneers. However, these seminal contributions focused mostly on the derivation and analysis of effective non-Markovian evolution operators, whereas here we provide a thorough analysis of the manifestations of the projection on the level of Green's functions with the aim to somewhat relieve the need for choosing a particular model based solely on physical intuition. 

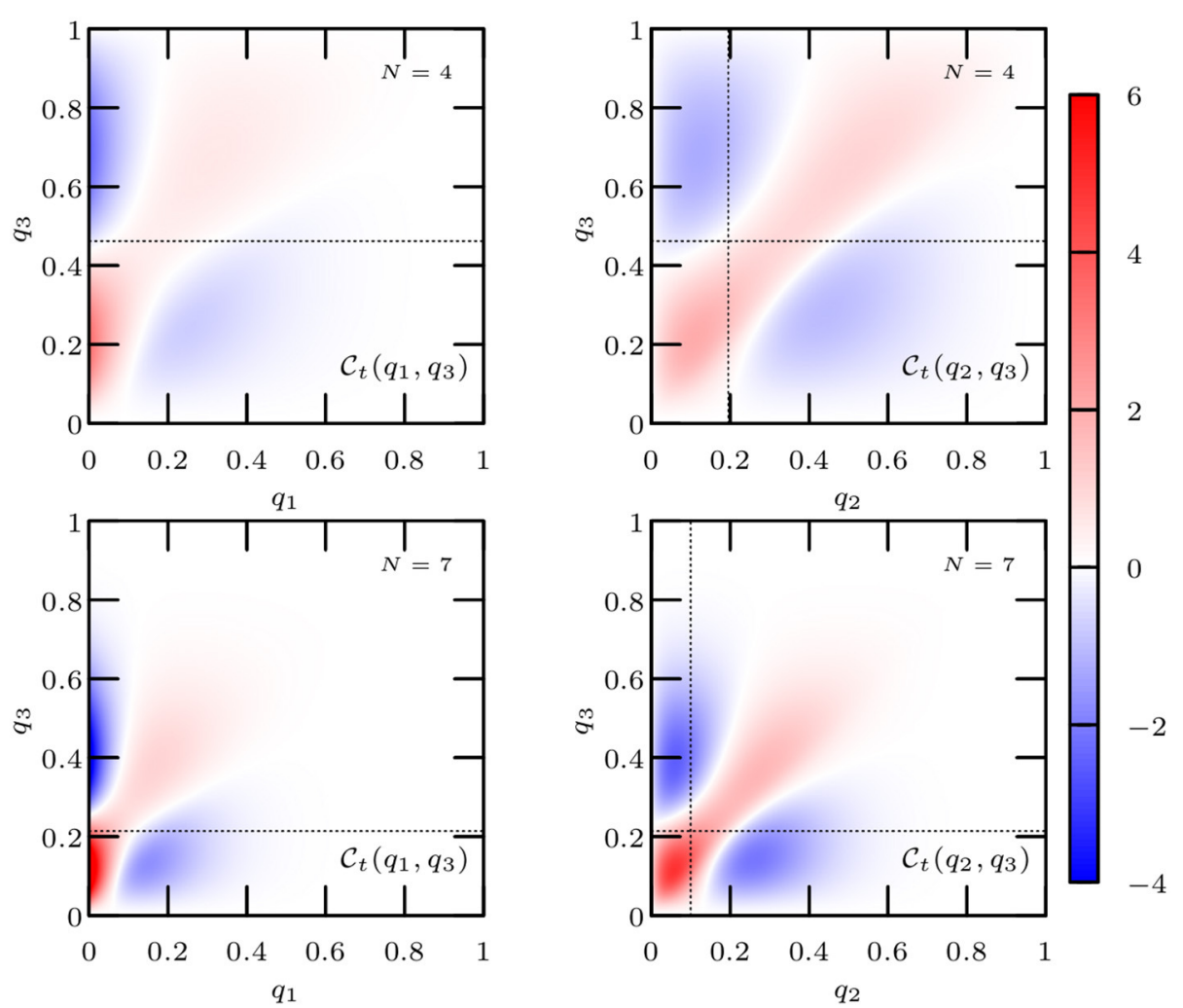

FIGURE 5 | Two-tag local time correlations $\mathcal{C}_{t}\left(q_{1} ; q_{3}\right)$ (left) and $\mathcal{C}_{t}\left(q_{2} ; q_{3}\right)$ (right) for a single file of $N=4$ (top) and $N=7$ (bottom) particles for a trajectory length comparable to the relaxation time $t=0.12 \simeq \lambda_{1}^{-1}$. The relaxation time corresponds to $\lambda_{1}^{-1} \simeq 0.1$. The dashed lines denote the positions of the two free energy minima.

Furthermore, we rigorously establish conditions under which the projected dynamics become Markovian and renewal-type, and derive Markovian approximations to projected generators. As a diagnostic tool we propose a novel framework for the assessment of the degree of broken Markovianity as well as for the elucidation of the origins of non-Markovian behavior.

An important remark concerns the transience of broken Markovianity, which is a consequence of the fact that we assumed that the complete dynamics is ergodic. First we note that (i) for any finite observation of length $t$ it is de facto not possible to discern whether the observation (and the dynamics in general) will be ergodic or not on a time scale $\tau>t$. (ii) All physical observations are (trivially) finite. (iii) In a nominally ergodic dynamics on any finite time scale $t$, where the dynamics starting from some non-stationary initial condition $\mathbf{x}_{0}$ has not yet reached the steady state (in the language of this work $t<\lambda_{1}^{-1}$ ), it is not possible to observe the effect of a sufficiently distant confining boundary $\partial \Omega(\mathbf{x})$ (potentially located at infinity if the drift field $\mathbf{F}(\mathbf{x})$ is sufficiently confining) that would assure ergodicity (in the language of this work $\forall t \ll \lambda_{1}^{-1}$ such that $G\left(\boldsymbol{l}_{\min }, t \mid \mathbf{x}_{0}, 0\right) \simeq$ 0 where $\left.\left|\boldsymbol{l}_{\min }\right| \equiv \min _{\mathbf{x}}\left|\mathbf{x}_{0}-\partial \Omega(\mathbf{x})\right|\right)$. Therefore no generality is lost in our work by assuming that the complete dynamics is nominally ergodic, even in a rigorous treatment of so-called weakly non-ergodic dynamics with diverging mean waiting times (see e.g., $[1,6]$ ) or generalized Langevin dynamics with diverging correlation times (see e.g., [29-34]) on finite time-scales. As a corollary, in the description of such dynamics on any finite timescale it is a priori by no means necessary to assume that the dynamics is non-ergodic or has a diverging correlation time. This does not imply, however, that the assumption of diverging mean waiting times or diverging correlation times cannot render the analysis of specific models simpler.

Notably, our work considers parent dynamics with a potentially broken time-reversal symmetry and hence includes the description of projection-induced non-Markovian dynamics in non-equilibrium (i.e., irreversible) systems. In the latter case the relaxation process of the parent microscopic process might not be monotonic (i.e., may oscillate), and it will be very interesting to explore the manifestations and importance of these oscillations in projected non-Markovian dynamics.

In the context of renewal dynamics our work builds on firm mathematical foundations of Markov processes and therefore provides mathematically and physically consistent explicit (but notably not necessarily the most general) memory kernels derived from microscopic (or fine-grained) principles, which can serve for the development, assessment and fine-tuning of empirical memory kernels that are used frequently in the theoretical modeling of non-Markovian phenomena (e.g., power-law, exponential, stretched exponential etc; [2, 82]). In particular, power-law kernels are expected to emerge as transients 
in cases, where the latent degrees of freedom relax over multiple time-scales with a nearly continuous and self-similar spectrum. Conversely, the quite strongly restrictive conditions imposed on the microscopic (parent) dynamics that lead to renewal dynamics, which we reveal here, suggest that renewal type transport in continuous space (e.g., continuous-time random walks [1, 2]) might not be the most abundant processes underlying projection-induced non-Markovian dynamics in physical systems, but are more likely to arise due to some disorder averaging. In general, it seems natural that coarse graining involving some degree of spatial discretization should underly renewal type ideas.

From a more general perspective beyond the theory of anomalous diffusion our results are relevant for the description and understanding of experimental observables $a(\mathbf{q})$ coupled to projected dynamics $\mathbf{q}(t)$ in presence of slow latent degrees of freedom (e.g., a FRET experiment measuring the distance within a protein or a DNA molecule [88]), as well as for exploring stochastic thermodynamic properties of projected dynamics with slow hidden degrees of freedom [89-91]. An important field of applications of the spectral-theoretic ideas developed here is the field of statistical kinetics in the context of first passage concepts (e.g., [92-94]), where general results for non-Markovian dynamics are quite sparse $[46,49,95-100]$ and will be the subject of our future studies.

\section{REFERENCES}

1. Metzler R, Klafter J. The random walk's guide to anomalous diffusion: a fractional dynamics approach. Phys Rep. (2000) 339:1-77. doi: 10.1016/S0370-1573(00)00070-3

2. Metzler R, Klafter $J$. The restaurant at the end of the random walk: recent developments in the description of anomalous transport by fractional dynamics. J Phys A Math Gen. (2004) 37:R161-208. doi: 10.1088/0305-4470/37/31/R01

3. Sokolov IM, Klafter J. From diffusion to anomalous diffusion: a century after Einstein's Brownian motion. Chaos. (2005) 15:026103. doi: 10.1063/1.1860472

4. Klages R, Radons G, Sokolov IM. Anomalous Transport: Foundations and Applications. Weinheim: Wiley-VCH Verlag GmbH and Co. (2008).

5. Godec A, Bauer M, Metzler R. Collective dynamics effect transient subdiffusion of inert tracers in flexible gel networks. N J Phys. (2014) 16:092002. doi: 10.1088/1367-2630/16/9/092002

6. Metzler R, Jepn JH, Cherstvy AG, Barkai E. Anomalous diffusion models and their properties: non-stationarity, non-ergodicity, and ageing at the centenary of single particle tracking. Phys Chem Chem Phys. (2014) 16:24128-64. doi: 10.1039/C4CP03465A

7. Höfling F, Franosch T. Anomalous transport in the crowded world of biological cells. Rep Prog Phys. (2013) 76:046602. doi: 10.1088/0034-4885/76/4/046602

8. Sokolov IM. Models of anomalous diffusion in crowded environments. Soft Matter. (2012) 8:9043-52. doi: 10.1039/c2sm25701g

9. Metzler R, Jeon JH, Cherstvy AG. Non-Brownian diffusion in lipid membranes: experiments and simulations. Biochim Biophys Acta Biomemb. (2016) 1858:2451-67. doi: 10.1016/j.bbamem.2016.01.022

10. Oliveira FA, Ferreira RMS, Lapas LC, Vainstein MH. Anomalous diffusion: a basic mechanism for the evolution of inhomogeneous systems. Front Phys. (2019) 7:18. doi: 10.3389/fphy.2019.00018

11. Woringer M, Darzacq X. Protein Motion in the Nucleus: From Anomalous Diffusion to Weak Interactions. Biochemical Society Transactions (2018).

\section{DATA AVAILABILITY STATEMENT}

All datasets generated for this study are included in the article/supplementary material.

\section{AUTHOR CONTRIBUTIONS}

$\mathrm{AL}$ and $\mathrm{AG}$ conceived the research, performed the research, and wrote and reviewed the paper.

\section{FUNDING}

The financial support from the German Research Foundation (DFG) through the Emmy Noether Program GO 2762/1-1 (to AG), and an IMPRS fellowship of the Max Planck Society (to AL) are gratefully acknowledged.

\section{ACKNOWLEDGMENTS}

We thank David Hartich for fruitful discussions and critical reading of the manuscript. AG in addition thanks Ralf Metzler for introducing him to the field on anomalous and non-Markovian dynamics and for years of inspiring and encouraging discussions.

Available online at: http://www.biochemsoctrans.org/content/early/2018/07/ 29/BST20170310

12. Dix JA, Verkman AS. Crowding effects on diffusion in solutions and cells. Annu Rev Biophys. (2008) 37:247-63. doi: 10.1146/annurev.biophys.37.032807.125824

13. Krapf D. Chapter 5: Mechanisms underlying anomalous diffusion in the plasma membrane. In: Kenworthy AK, editor. Lipid Domains. Vol. 75 of Current Topics in Membranes. Academic Press (2015). p. 167-207.

14. Golding I, Cox EC. Physical nature of bacterial cytoplasm. Phys Rev Lett. (2006) 96:098102. doi: 10.1103/PhysRevLett.96.098102

15. Jeon JH, Javanainen M, Martinez-Seara H, Metzler R, Vattulainen I. Protein crowding in lipid bilayers gives rise to non-Gaussian anomalous lateral diffusion of phospholipids and proteins. Phys Rev X. (2016) 6:021006. doi: 10.1103/PhysRevX.6.021006

16. Rienzo CD, Piazza V, Gratton E, Beltram F, Cardarelli F. Probing short-range protein Brownian motion in the cytoplasm of living cells. Nat Commun. (2014) 5:5891. doi: 10.1038/ncomms6891

17. Metzler R, Klafter J. Anomalous stochastic processes in the fractional dynamics framework: fokker:planck equation, dispersive transport, and non?exponential relaxation. In: Prigogine I and Rice SA editors. Advances in Chemical Physics, Vol 116. JohnWiley and Sons, Ltd (2007). doi: 10.1002/9780470141762.ch3

18. Lomholt MA, Lizana L, Metzler R, Ambjörnsson T. Microscopic origin of the logarithmic time evolution of aging processes in complex systems. Phys Rev Lett. (2013) 110:208301. doi: 10.1103/PhysRevLett.110. 208301

19. Bouchaud JP, Georges A. Anomalous diffusion in disordered media: statistical mechanisms, models and physical applications. Phys Rep. (1990) 195:127-93.

20. Bouchaud JP, Comtet A, Georges A, Doussal PL. Classical diffusion of a particle in a one-dimensional random force field. Ann Phys. (1990) 201:285341.

21. Sinai YG. The limiting behavior of a one-dimensional random walk in a random medium. Theory Prob Appl. (1982) 27:285-341. 
22. Oshanin G, Rosso A, Schehr G. Anomalous fluctuations of currents in sinaitype random chains with strongly correlated disorder. Phys Rev Lett. (2013) 110:100602. doi: 10.1103/PhysRevLett.110.100602

23. Dean DS, Gupta S, Oshanin G, Rosso A, Schehr G. Diffusion in periodic, correlated random forcing landscapes. J Phys A Math Theor. (2014) 47:372001. doi: 10.1088/1751-8113/47/37/372001

24. Radons G. Anomalous transport in disordered dynamical systems. Phys $D$ Nonlinear Phenomena. (2004) 187:3-19. doi: 10.1016/j.physd.2003.09.001

25. Godec A, Chechkin AV, Barkai E, Kantz H, Metzler R. Localisation and universal fluctuations in ultraslow diffusion processes. J Phys A Math Theor. (2014) 47:492002. doi: 10.1088/1751-8113/47/49/492002

26. Krüsemann H, Godec A, Metzler R. First-passage statistics for aging diffusion in systems with annealed and quenched disorder. Phys Rev E. (2014) 89:040101. doi: 10.1103/PhysRevE.89.040101

27. Krsemann H, Godec A, Metzler R. Ageing first passage time density in continuous time random walks and quenched energy landscapes. J Phys $A$ Math Theor. (2015) 48:285001. doi: 10.1088/1751-8113/48/28/285001

28. Coffey WT, Kalmykov YP, Waldron JT. The Langevin Equation. World Scientific (1996). Available online at: https://www.worldscientific.com/doi/ abs/10.1142/2256

29. Jeon JH, Metzler R. Fractional Brownian motion and motion governed by the fractional Langevin equation in confined geometries. Phys Rev E. (2010) 81:021103. doi: 10.1103/PhysRevE.81.021103

30. Lutz E. Fractional Langevin equation. Phys Rev E. (2001) 64:051106. doi: 10.1103/PhysRevE.64.051106

31. Deng W, Barkai E. Ergodic properties of fractional Brownian-Langevin motion. Phys Rev E. (2009) 79:011112. doi: 10.1103/PhysRevE.79.011112

32. Metzler R, Klafter J. Subdiffusive transport close to thermal equilibrium From the Langevin equation to fractional diffusion. Phys Rev E. (2000) 61:6308-11. doi: 10.1103/PhysRevE.61.6308

33. Kou SC, Xie XS. Generalized Langevin equation with fractional Gaussian noise: subdiffusion within a single protein molecule. Phys Rev Lett. (2004) 93:180603. doi: 10.1103/PhysRevLett.93.180603

34. Burov S, Barkai E. Critical exponent of the fractional Langevin equation. Phys Rev Lett. (2008) 100:070601. doi: 10.1103/PhysRevLett.100.070601

35. Goychuk I. Viscoelastic subdiffusion: generalized langevin equation approach. In: Rice SA, Dinner AR editors. Advances in Chemical Physics, Vol 150. JohnWiley and Sons, Ltd (2012). doi: 10.1002/9781118197 714.ch5

36. Dubkov AA, Hänggi P, Goychuk I. Non-linear Brownian motion the problem of obtaining the thermal Langevin equation for a nonGaussian bath. J Stat Mech Theory Exp. (2009) (2009) 2009:P01034. doi: 10.1088/1742-5468/2009/01/P01034

37. Cherstvy AG, Chechkin AV, Metzler R. Anomalous diffusion and ergodicity breaking in heterogeneous diffusion processes. N J Phys. (2013) 15:083039. doi: 10.1088/1367-2630/15/8/083039

38. Cherstvy AG, Metzler R. Nonergodicity, fluctuations, and criticality in heterogeneous diffusion processes. Phys Rev E. (2014) 90:012134. doi: 10.1103/PhysRevE.90.012134

39. Massignan P, Manzo C, Torreno-Pina JA, García-Parajo MF, Lewenstein M, Lapeyre GJ. Nonergodic subdiffusion from Brownian motion in an inhomogeneous medium. Phys Rev Lett. (2014) 112:150603. doi: 10.1103/PhysRevLett.112.150603

40. Guérin T, Dean DS. Force-induced dispersion in heterogeneous media. Phys Rev Lett. (2015) 115:020601. doi: 10.1103/PhysRevLett.115.020601

41. Godec A, Metzler R. Optimization and universality of Brownian search in a basic model of quenched heterogeneous media. Phys Rev E. (2015) 91:052134. doi: 10.1103/PhysRevE.91.052134

42. Vaccario G, Antoine C, Talbot J. First-passage times in $d$ dimensional heterogeneous media. Phys Rev Lett. (2015) 115:240601. doi: 10.1103/PhysRevLett.115.240601

43. Godec A, Metzler R. First passage time distribution in heterogeneity controlled kinetics: going beyond the mean first passage time. Sci Rep. (2016) 6:20349. doi: 10.1038/srep20349

44. Chubynsky MV, Slater GW. Diffusing diffusivity: a model for anomalous, yet Brownian, diffusion. Phys Rev Lett. (2014) 113:098302. doi: 10.1103/PhysRevLett.113.098302
45. Chechkin AV, Seno F, Metzler R, Sokolov IM. Brownian yet non-Gaussian diffusion: from superstatistics to subordination of diffusing diffusivities. Phys Rev X. (2017) 7:021002. doi: 10.1103/PhysRevX.7.021002

46. Godec A, Metzler R. First passage time statistics for two-channel diffusion. J Phys A Math Theor. (2017) 50:084001. doi: 10.1088/1751-8121/aa5204

47. Sposini V, Chechkin AV, Seno F, Pagnini G, Metzler R. Random diffusivity from stochastic equations: comparison of two models for Brownian yet non-Gaussian diffusion. N J Physics. (2018) 20:043044. doi: 10.1088/1367-2630/aab696

48. Lanoiselée Y, Grebenkov DS. A model of non-Gaussian diffusion in heterogeneous media. J Phys A Math Theor. (2018) 51:145602. doi: 10.1088/1751-8121/aab15f

49. Grebenkov DS. A unifying approach to first-passage time distributions in diffusing diffusivity and switching diffusion models. J Phys A Math Theor. (2019) 52:174001. doi: 10.1088/1751-8121/ab0dae

50. Lanoiselee Y, Moutal N, Grebenkov DS. Diffusion-limited reactions in dynamic heterogeneous media. Nat Commun. (2018) 9:4398. doi: 10.1038/s41467-018-06610-6

51. Zwanzig R. Ensemble method in the theory of irreversibility. J Chem Phys. (1960) 33:1338-41. doi: 10.1063/1.1731409

52. Nordholm S, Zwanzig R. A systematic derivation of exact generalized Brownian motion theory. J Stat Phys. (1975) 13:347-71. doi: $10.1007 / \mathrm{BF} 01012013$

53. Mori H. Transport, collective motion, and Brownian motion*). Prog Theor Phys. (1965) 33:423-55. doi: 10.1143/PTP.33.423

54. Grabert H, Talkner P, Hänggi P. Microdynamics and time-evolution of macroscopic non-Markovian systems. Z B Condensed Matter. (1977) 26:38995. doi: 10.1007/BF01570749

55. Hänggi $\mathrm{P}$, Thomas $\mathrm{H}$. Time evolution, correlations, and linear response of non-Markov processes. Z Condensed Matter. (1977) 26:85-92. doi: 10.1007/BF01313376

56. Grabert $\mathrm{H}$, Talkner $\mathrm{P}$, Hänggi $\mathrm{P}$, Thomas $\mathrm{H}$. Microdynamics and timeevolution of macroscopic non-Markovian systems. II. Z B Condensed Matter. (1978) 29:273-80. doi: 10.1007/BF01321192

57. Hynes JT, Kapral R, Weinberg M. Microscopic theory of Brownian motion: mori friction kernel and Langevin-equation derivation. Phys A Stat Mech Appl. (1975) 80:105-27.

58. Haken H. Cooperative phenomena in systems far from thermal equilibrium and in nonphysical systems. Rev Mod Phys. (1975) 47:67-121. doi: 10.1103/RevModPhys.47.67

59. Ferrario $M$, Grigolini $P$. The non-Markovian relaxation process as "contraction" of a multidimensional one of Markovian type. J Math Phys. (1979) 20:2567-72. doi: 10.1063/1.524019

60. Grigolini P. A Fokker-Planck equation for canonical non-Markovian systems: a local linearization approach. J Chem Phys. (1988) 89:4300-8. doi: $10.1063 / 1.454812$

61. Harris TE. Diffusion with "collisions" between particles. J Appl Probab. (1965) 2:323-38.

62. Lizana L, Ambjörnsson T. Single-file diffusion in a box. Phys Rev Lett. (2008) 100:200601. doi: 10.1103/PhysRevLett.100.200601

63. Barkai E, Silbey R. Theory of single file diffusion in a force field. Phys Rev Lett. (2009) 102:050602. doi: 10.1103/PhysRevLett.102.050602

64. Sanders LP, Lomholt MA, Lizana L, Fogelmark K, Metzler R, Ambjrnsson T. Severe slowing-down and universality of the dynamics in disordered interacting many-body systems: ageing and ultraslow diffusion. N J Phys. (2014) 16:113050. doi: 10.1088/1367-2630/16/11/113050

65. Illien P, Bénichou O, Mejía-Monasterio C, Oshanin G, Voituriez R. Active transport in dense diffusive single-file systems. Phys Rev Lett. (2013) 111:038102. doi: 10.1103/PhysRevLett.111.038102

66. Bertrand T, Illien P, Bénichou O, Voituriez R. Dynamics of run-andtumble particles in dense single-file systems. N J Phys. (2018) 20:113045. doi: 10.1088/1367-2630/aaef6f

67. Lapolla A, Godec A. Unfolding tagged particle histories in single-file diffusion: exact single- and two-tag local times beyond large deviation theory. N J Phys. (2018) 20:113021. doi: 10.1088/1367-2630/aaea1b

68. Godec A, Metzler R. Signal focusing through active transport. Phys Rev E. (2015) 92:010701. doi: 10.1103/PhysRevE.92.010701 
69. Deutch JM, Silbey R. Exact generalized Langevin equation for a particle in a harmonic lattice. Phys Rev A. (1971) 3:2049-52. doi: 10.1103/PhysRevA.3.2049

70. Havlin S, Kiefer JE, Weiss GH. Anomalous diffusion on a random comblike structure. Phys Rev A. (1987) 36:1403-8. doi: 10.1103/PhysRevA.36.1403

71. Pottier N. Analytic study of a model of diffusion on random comb-like structures. Il Nuovo Cimento D. (1994) 16:1223-30. doi: 10.1007/BF02458804

72. Berezhkovskii AM, Dagdug L, Bezrukov SM. From normal to anomalous diffusion in comb-like structures in three dimensions. J Chem Phys. (2014) 141:054907. doi: 10.1063/1.4891566

73. Qian H. A decomposition of irreversible diffusion processes without detailed balance. J Math Phys. (2013) 54:053302. doi: 10.1063/1.4803847

74. Gray AH. Uniqueness of steady-state solutions to the Fokker-Planck equation. J Math Phys. (1965) 6:644-7. doi: 10.1063/1.1704316

75. Huang W, Ji M, Liu Z, Yi Y. Steady states of Fokker-Planck equations: I existence. J Dyn Diff Equ. (2015) 27:721-42. doi: 10.1007/s10884-015-9454-x

76. Helffer B, Nier F. Hypoelliptic Estimates and Spectral Theory for Fokker Planck Operators and Witten Laplacians. Berlin; Heidelberg: Springer (2005).

77. Chupin L. Fokker-Planck equation in bounded domain. Ann Inst Fourier. (2010) 60:217. doi: 10.5802/aif.2521

78. Reed M, Simon B. Methods of Modern Mathematical Physics I: Functional Analysis. New York, NY: Academic Press (1972).

79. Conway JB. A Course in Functional Analysis. New York, NY: Springer-Verlag (1985).

80. Feller W. Non-Markovian processes with the semigroup property. Ann Math Statist. (1959) 30:1252-3. doi: 10.1214/aoms/1177706110

81. Hanggi P, Thomas H, Grabert H, Talkner P. Note on time evolution of nonMarkov processes. J Stat Phys. (1978) 18:155-9. doi: 10.1007/BF01014306

82. Sokolov IM. Solutions of a class of non-Markovian Fokker-Planck equations. Phys Rev E. (2002) 66:041101. doi: 10.1103/PhysRevE.66.041101

83. Risken H, Haken H. The Fokker-Planck Equation: Methods of Solution and Applications. 2nd ed. Springer (1989).

84. Polettini M. Generally covariant state-dependent diffusion. J Stat Mech Theory Exp. (2013) 2013:P07005. doi: 10.1088/1742-5468/2013/07/P07005

85. Polettini M, Esposito M. Nonconvexity of the relative entropy for Markov dynamics: a Fisher information approach. Phys Rev E. (2013) 88:012112. doi: 10.1103/PhysRevE.88.012112

86. Korepin VE, Bogoliubov NM, Izergin AG. Quantum Inverse Scattering Method and Correlation Functions. Cambridge University Press (1993) doi: $10.1017 / \mathrm{CBO} 9780511628832$

87. Lizana L, Ambjörnsson T. Diffusion of finite-sized hard-core interacting particles in a one-dimensional box: tagged particle dynamics. Phys Rev E. (2009) 80:051103. doi: 10.1103/PhysRevE.80.051103

88. Gopich IV, Szabo A. Theory of the energy transfer efficiency and fluorescence lifetime distribution in single-molecule FRET. Proc Natl Acad Sci USA. (2012) 109:7747-52. doi: 10.1073/pnas.120512 0109
89. Seifert U. Stochastic thermodynamics, fluctuation theorems and molecular machines. Rep Prog Phys. (2012) 75:126001. doi: 10.1088/0034-4885/75/12/126001

90. Mehl J, Lander B, Bechinger C, Blickle V, Seifert U. Role of hidden slow degrees of freedom in the fluctuation theorem. Phys Rev Lett. (2012) 108:220601. doi: 10.1103/PhysRevLett.108.220601

91. Uhl M, Pietzonka P, Seifert U. Fluctuations of apparent entropy production in networks with hidden slow degrees of freedom. $J$ Stat Mech Theory Exp. (2018) 2018:023203. doi: 10.1088/1742-5468/ aaa78b

92. Hartich D, Godec A. Duality between relaxation and first passage in reversible Markov dynamics: rugged energy landscapes disentangled. $\mathrm{N} \mathrm{J}$ Phys. (2018) 20:112002. doi: 10.1088/1367-2630/aaf038

93. Hartich D, Godec A. Interlacing relaxation and first-passage phenomena in reversible discrete and continuous space Markovian dynamics. J Stat Mech Theory Exp. (2019) 2019:024002. doi: 10.1088/1742-5468/ab00df

94. Hartich D, Godec A. Extreme value statistics of ergodic Markov processes from first passage times in the large deviation limit. J Phys A Math Theor. (2019) 52:244001. doi: 10.1088/1751-8121/ableca

95. Hänggi P, Talkner P. Non-Markov processes: the problem of the mean first passage time. Z B Condensed Matter. (1981) 45:79-83. doi: 10.1007/BF01294279

96. Balakrishnan V, Van den Broeck C, Hänggi P. First-passage times of nonMarkovian processes: the case of a reflecting boundary. Phys Rev A. (1988) 38:4213-22. doi: 10.1103/PhysRevA.38.4213

97. McKane AJ, Luckock HC, Bray AJ. Path integrals and non-Markov processes. I. General formalism. Phys Rev A. (1990) 41:644-56. doi: 10.1103/PhysRevA.41.644

98. Reimann P, Schmid GJ, Hänggi P. Universal equivalence of mean first-passage time and Kramers rate. Phys Rev E. (1999) 60:R1-4. doi: 10.1103/PhysRevE.60.R1

99. Bray AJ, Majumdar SN, Schehr G. Persistence and first-passage properties in nonequilibrium systems. Adv Phys. (2013) 62:225-361. doi: 10.1080/00018732.2013.803819

100. Guerin T, Levernier N, Benichou O, Voituriez R. Mean first-passage times of non-Markovian random walkers in confinement. Nature. (2016) 534:356-9. doi: 10.1038 /nature18272

Conflict of Interest: The authors declare that the research was conducted in the absence of any commercial or financial relationships that could be construed as a potential conflict of interest.

Copyright (c) 2019 Lapolla and Godec. This is an open-access article distributed under the terms of the Creative Commons Attribution License (CC BY). The use, distribution or reproduction in other forums is permitted, provided the original author(s) and the copyright owner(s) are credited and that the original publication in this journal is cited, in accordance with accepted academic practice. No use, distribution or reproduction is permitted which does not comply with these terms. 


\section{Chapter 5}

\section{BetheSF: Efficient computation of the exact tagged-particle propagator in single-file systems via the Bethe eigenspectrum}

This article has been published in Computer Physics Communications under the $C C-B Y$-NC-ND License [52]. The study of tagged-particle dynamics in a single-file is one of the main examples of non-Markovian dynamics and sub-diffusive behavior. In this article we explicitly show how to diagonalize the propagator of the tagged-particle using the coordinate Bethe-Ansatz. Unfortunately the direct evaluation of such a solution poses a serious computational challenge since it relies on the generation of all possible permutation with repetition of the multisets labeling the eigenfunctions, a classic non-polynomial problem. In this paper we explain an ingenious algorithm able to reduce the generation of the permutations to the fastest generation of combinations of these multisets thanks to the exchange symmetry characterizing single file systems. Moreover, the article is accompanied by an efficient (and extendable) open-source $\mathrm{C}++$ implementation of the algorithm able to compute the propagator of the tagged-particle for several external potentials. 


\title{
BetheSF: Efficient computation of the exact tagged-particle propagator in single-file systems via the Bethe eigenspectrum
}

\author{
Alessio Lapolla*, Aljaž Godec \\ Mathematical bioPhysics Group, Max Planck Institute for Biophysical Chemistry, Am Fassberg 11, 37077 Göttingen, Germany
}

\section{A R T I C L E I N F O}

\section{Article history:}

Received 5 March 2020

Received in revised form 3 July 2020

Accepted 10 August 2020

Available online 27 August 2020

\section{Keywords:}

Single-file diffusion

Stochastic many-body system

Tagged-particle dynamics

Spectral expansion

Coordinate Bethe ansatz

Non-Markovian dynamics

\begin{abstract}
A B S T R A C T
Single-file diffusion is a paradigm for strongly correlated classical stochastic many-body dynamics and has widespread applications in soft condensed matter and biophysics. However, exact results for singlefile systems are sparse and limited to the simplest scenarios. We present an algorithm for computing the non-Markovian time-dependent conditional probability density function of a tagged-particle in a single-file of $N$ particles diffusing in a confining external potential. The algorithm implements an eigenexpansion of the full interacting many-body problem obtained by means of the coordinate Bethe ansatz. While formally exact, the Bethe eigenspectrum involves the generation and evaluation of permutations, which becomes unfeasible for single-files with an increasing number of particles $N$. Here we exploit the underlying exchange symmetries between the particles to the left and to the right of the tagged-particle and show that it is possible to reduce the complexity of the algorithm from the worst case scenario $\mathcal{O}(N$ !) down to $\mathcal{O}(N)$. A C++ code to calculate the non-Markovian probability density function using this algorithm is provided. Solutions for simple model potentials are readily implemented including single-file diffusion in a flat and a 'tilted' box, as well as in a parabolic potential. Notably, the program allows for implementations of solutions in arbitrary external potentials under the condition that the user can supply solutions to the respective single-particle eigenspectra.

Program summary

Program Title: BetheSF

CPC Library link to program files: http://dx.doi.org/10.17632/3bs74vf72n.1

Licensing provisions: MIT

Programming language: $\mathrm{C}++(\mathrm{C}++17$ support required $)$

Supplementary material: makefile, README, SingleFileBluePrint.hpp

Nature of problem: Diffusive single-files are mathematical models of effectively one-dimensional strongly correlated many-body systems. While the dynamics of the full system is Markovian, the diffusion of a tracer-particle in a single-file is an example of non-Markovian and anomalous diffusion. The many-body Fokker-Planck equation governing the system's dynamics can be solved using the coordinate Bethe ansatz. A naïve implementation of such a solution runs in non-polynomial time since it requires the generation of permutations of the elements of a multiset.

Solution method: In this paper we show how, exploiting the exchange symmetries of the system, it is possible to reduce the complexity of the algorithm to evaluate the solution, using a permutationgeneration algorithm, from $\mathcal{O}(N$ !) in the worst case scenario to $\mathcal{O}(N)$ in the best case scenario, which corresponds to tagging the first or the last particle, where $N$ stands for the number of particles in the single-file.

Additional comments including restrictions and unusual features: The code may overflow for large singlefiles $N \geq 170$. All the benchmarks ran on the following CPU: Intel Xeon E3-1270 v2 $3.50 \mathrm{GHz} 4$ cores. The compiler used is g++ 7.3.1 (SUSE Linux) with the optimization-O3 turned on. The code to produce all the data in the figures is included in the files: figure2.cpp, figure3.cpp, figure4.cpp, figure5a.cpp and figure5b.cpp
\end{abstract}

(c) 2020 Elsevier B.V. All rights reserved.

\footnotetext{
The review of this paper was arranged by Prof. Hazel Andrew.

this paper and its associated computer program are available via the Computer Physics Communication homepage on ScienceDirect (http://www.sciencedirect. com/science/journal/00104655).

* Corresponding author.

E-mail address: alessio.lapolla@mpibpc.mpg.de (A. Lapolla).
} 


\section{Introduction}

Single-file diffusion refers to the dynamics of one-dimensional systems composed of identical hard-core particles, that is, to manyparticle diffusion subject to non-crossing boundary conditions. Diffusive single-file models are a paradigm for the stochastic dynamics of classical strongly correlated many-body systems. As such they have been studied extensively both theoretically (see e.g. [1-10]) as well as experimentally [11-13]. Single-file diffusion underlies the dynamics in biological channels [14], molecular search processes of transcription factors in gene regulation [15], transport in zeolites [16,17] and superionic conductors [18], and diverse phenomena in soft matter systems [19].

Whereas the dynamics of the entire $N$-particle single-file is Markovian, the typically observed "tagged-particle" diffusion - the projection of the many-body dynamics onto the motion of a single tracer particle - is strongly non-Markovian [10]. Namely, by focusing on a tagged-particle alone, the $N-1$ remaining so-called latent degrees of freedom (i.e. the coordinates of the remaining particles) that become coarse-grained out, relax on exactly the same time scale as the tagged particle [10]. This renders single-file diffusion somewhat special as compared to other physical examples probing low-dimensional projections, such as for example the dynamics of individual protein molecules [20] involving degrees freedom with relaxation times that span several orders of magnitude in time [21]. As there are no "fast" degrees of freedom in a single-file, low-dimensional projections give rise to strong memory effects, i.e. the Markov property is said to be strongly broken. In other words, the dynamics of a tagged-particle is fundamentally different (by extent as well as duration) from the adiabatic, Markovian approximation of the dynamics of a single particle diffusing in a potential of mean force created if the remaining particles were to relax to equilibrium instantaneously [10].

Tagged-particle diffusion in a single-file is also a representative toy model for diffusion in so-called crowded systems, in particular when the dynamics is effectively one-dimensional and anomalous [22], i.e. when the mean squared displacement of a particle $\left\langle(x(t)-x(0))^{2}\right\rangle \propto t^{\alpha}$ (where $\langle\cdot\rangle$ denotes the average over an ensemble of trajectories) is not linear in time as in the case of (normal) Brownian motion (i.e. $\alpha_{\text {Brown }}=1$ ) but scales sub-linearly with $\alpha=1 / 2$, which is referred to as subdiffusion [23]. The theoretical analysis of tagged-particle dynamics has been carried out by several different techniques: the so-called "reflection principle" applicable to single-files with both finite and infinite number of elements [4], Jepsen mapping for the central particle in a finite [5] or infinite single-file [24], the so-called momentum Bethe ansatz for a finite single-file [7], harmonization techniques for infinite single-files [8], etc.

Here, we focus on the propagator (or the "non-Markovian Green's function") of a tagged-particle in a finite single-file of $N$ particles diffusing in an arbitrary confining potential, that is, the conditional probability density function to find the tagged-particle at position $x$ at a time $\tau$ assuming that at $\tau=0$ it was at $x_{0}$, while the positions of the remaining $N-1$ particles were drawn from the equilibrium distribution compatible with the initial position of the tagged-particle. In the past few years a number of detailed analyses of ensemble$[7,10]$ and time- $[9,10]$ averaged physical observables have been carried out focusing on the motion of a tagged-particle in a single-file, which provided a generic, conceptual insight into the emergence of memory in projection-induced non-Markovian dynamics.

In our previous work $[9,10]$ we determined the propagator exactly by means of the coordinate Bethe ansatz (CBA) [25]. The power of the CBA lies in the fact that it diagonalizes the many-body Fokker-Planck operator that governs the dynamics of the single-file. In other words, it expresses the dynamics of the full $\mathrm{N}$-body system in a given potential in terms of a complete set of eigenfunctions and corresponding eigenvalues, which describe exactly how the system relaxes to equilibrium in terms of irreducible collective relaxation modes on different time-scales. By projecting these collective modes onto the motion of a tagged-particle we were able to disentangle the microscopic, collective origin of subdiffusion and memory in tagged-particle dynamics in simple confining potentials $[9,10,26]$.

However, the implementation of the analytical results obtained by the CBA poses a computational challenge since it involves an algorithm whose complexity is non-polynomial in $N$. Here we present an efficient algorithm (that in some cases runs in polynomial time) for evaluating the tagged-particle propagator that exploits the exchange-symmetry of the problem. We also present a $\mathrm{C}++$ code to perform such a computation for selected examples. The code is easily extendable to other potentials.

Notably, a common alternative method to analyze tagged-particle dynamics in finite single-files is to perform Brownian Dynamics computer simulations. To do so efficient algorithms have been designed based on the Gillespie algorithm [6], on the Ermak algorithm [27] or on the Verlet algorithm [28]. Nevertheless, these algorithms may still suffer from time- and space-discretization artifacts since they only provide an approximate solution to the problem. Moreover, they neither readily reveal the collective relaxation eigenmodes, nor do they establish how these affect tagged-particle motion. In addition, the computational cost of such Brownian Dynamics simulations is much larger than the one of the present algorithm (for a comparison see Section 5).

\section{Problem and solution by means of the coordinate Bethe ansatz}

The evolution of the (Markovian) probability density function of a diffusive single-file of $N$ particles in the over-damped regime under the influence of an external force $F(x)=-\partial_{x} U(x), G\left(\mathbf{x}, \tau \mid \mathbf{x}_{0}\right)$, evolving from an initial condition $G\left(\mathbf{x}, 0 \mid \mathbf{x}_{0}\right)=\delta\left(\mathbf{x}-\mathbf{x}_{0}\right)$ is described by the Fokker-Planck equation

$$
\begin{aligned}
{\left[\partial_{\tau}-\sum_{i=1}^{N}\left(D \partial_{x_{i}}^{2}-\mu \partial_{x_{i}} F\left(x_{i}\right)\right)\right] G\left(\mathbf{x}, \tau \mid \mathbf{x}_{0}\right) } & =0, \\
\left.\left(\partial_{x_{i+1}}-\partial_{x_{i}}\right) G\left(\mathbf{x}, \tau, \mathbf{x}_{0}\right)\right|_{x_{i+1}=x_{i}} & =0, \quad \forall i,
\end{aligned}
$$

where $D$ is the diffusion coefficient, $\mu=D / k_{\mathrm{B}} T$ is the mobility given by the fluctuation-dissipation theorem, and $\delta\left(\mathbf{x}-\mathbf{x}_{0}\right)=$ $\prod_{i=1}^{N} \delta\left(x_{i}-x_{0 i}\right)$. Eq. (1) is accompanied by appropriate external boundary conditions for the first and last particles of the single-file. Here we will only consider so-called natural ('zero probability at infinity', i.e. $\lim _{|\mathbf{x}| \rightarrow \infty} G\left(\mathbf{x}, \tau \mid \mathbf{x}_{0}\right)=0$ ) or reflecting ('zero flux') boundary conditions, which are selected according to the specific nature of the external potential $U(x)$. We will assume that $U(x)$ is sufficiently confining to assure that the eigenspectrum of the generator $\hat{L}_{N} \equiv \sum_{i=1}^{N}\left[D \partial_{x_{i}}^{2}-\mu \partial_{x_{i}} F\left(x_{i}\right)\right]$ is discrete [29]. In Eq. (1) we assumed that 
each particle experiences the same external force $F(x)$ and throughout we will assume that $D$ is equal for all particles. Note that the corresponding over-damped (Itô) Langevin equation that describes individual trajectories of the single-file and would be integrated numerically in a Brownian Dynamics simulation reads

$$
d x_{i}(t)=\mu F\left(x_{i}(t)\right) d t+\sqrt{2 D} d W_{t}^{i},\left\langle d W_{t}\right\rangle=0,\left\langle d W_{t}^{i} d W_{t^{\prime}}^{j}\right\rangle=\delta_{i j} \delta\left(t-t^{\prime}\right) d t, \forall i,
$$

where $d W_{t}$ is an increment of the Wiener process (Gaussian white noise), whereby we must enforce that particles remain ordered at all times, i.e. $x_{i}(t) \leq x_{i+1}(t), \forall i, t$.

The boundary value problem in Eq. (1) can be solved exactly by means of the coordinate Bethe ansatz [25], which requires that we (only) know the eigenexpansion of the single-particle Green's function. That is, we are required to solve the following single-particle Fokker-Planck equation with the same external boundary conditions

$$
\left(\partial_{\tau}-\hat{L}_{1}\right) \Gamma\left(x_{i}, \tau \mid x_{0 i}\right)=0
$$

with initial condition $\Gamma\left(x_{i}, 0 \mid x_{0 i}\right)=\delta\left(x_{i}-x_{0 i}\right)$, which can be conveniently expressed by means of a (bi)spectral expansion

$$
\Gamma\left(x_{i}, \tau \mid x_{0 i}\right)=\sum_{k=0}^{\infty} \psi_{k_{i}}^{R}\left(x_{i}\right) \psi_{k_{i}}^{L}\left(x_{0 i}\right) \mathrm{e}^{-\lambda_{k_{i}} \tau},
$$

where $-\lambda_{k_{i}}<0, \forall i>0$ and $\lambda_{0}=0$ are the eigenvalues, and $\psi_{k_{i}}^{L / R}(x)$ are respectively the $k_{i}$ th left and the right eigenfunction of the operator $\hat{L}_{1}$, which form a complete bi-orthonormal basis. Here we assume detailed balance to be obeyed and hence $\psi_{l}^{R}(x) \propto$ $\mathrm{e}^{-\beta U(x)} \psi_{l}^{L}(x)$ [30], where $\beta=1 /\left(k_{B} T\right)$ is the inverse of the thermal energy. The solution to the many-body Fokker-Planck equation can be written as

$$
G\left(\mathbf{x}, \tau \mid \mathbf{x}_{0}\right)=\sum_{\mathbf{k}} \Psi_{\mathbf{k}}^{R}(\mathbf{x}) \Psi_{\mathbf{k}}^{L}\left(\mathbf{x}_{0}\right) \mathrm{e}^{-\Lambda_{\mathbf{k}} \tau} .
$$

The many-body eigenvalue $\mathbf{k}$ corresponds to a multiset containing the $N$ natural numbers $\left\{k_{1}, k_{2}, \ldots, k_{N}\right\}$ and $\mathbf{0}$ denotes the unique ground state of the many-body system in which each single-particle eigenvalue is equal to zero. Each pair of many-body eigenvalues and eigenfunctions satisfies the eigenvalue problem

$$
\hat{L}_{N} \Psi_{\mathbf{k}}^{R}=\Lambda_{\mathbf{k}} \Psi_{\mathbf{k}}^{R} .
$$

The Bethe ansatz solution postulates that the right eigenfunction has the following form

$$
\Psi_{\mathbf{k}}^{R}=\hat{O}_{\mathbf{x}} \sum_{\{\mathbf{k}\}} \prod_{i=1}^{N} c_{i} \psi_{k_{i}}^{R}\left(x_{i}\right) ;
$$

where $\sum_{\{\mathbf{k}\}}$ denotes the sum over all the possible permutations of the multiset $\mathbf{k}$ (see Appendix A) and $\hat{O}_{\mathbf{x}}$ denotes the particle-ordering operator defined as

$$
\hat{O}_{\mathbf{x}} \equiv \prod_{i=2}^{N} \Theta\left(x_{i}-x_{i-1}\right),
$$

where $\Theta(x)$ denotes the Heaviside step function.

The $N$ constants $\left\{c_{i}\right\}$ and the many-body eigenvalue are fixed imposing the $N-1$ internal boundary conditions in Eq. (1) alongside the pair of external boundary conditions. This leads to the many-body eigenvalue

$$
\Lambda_{\mathbf{k}}=\sum_{i=1}^{N} \lambda_{k_{i}},
$$

and in the case of zero-flux boundary conditions all $c_{i}$ turn out to be equal to one. Finally, a proper orthonormalization between left and right many-body eigenfunctions must be assured, for example

$$
\Psi_{\mathbf{k}}^{L / R}(\mathbf{x})=\mathcal{N}^{-1 / 2} \hat{O}_{\mathbf{x}} \sum_{\{\mathbf{k}\}} \prod_{i=1}^{N} \psi_{k_{i}}^{L / R}\left(x_{i}\right),
$$

where the normalization factor $\mathcal{N}$ is equal to the number of permutations of the multiset $\mathbf{k}$ (see Appendix A)

Here we are interested in the non-Markovian Green's function referring to the propagation of a tagged-particle starting from a fixed initial condition $x_{0 i}$ while the remaining particles are drawn from those equilibrium configurations that are compatible with the initial condition of the tagged-particle [10]

$$
\mathcal{G}\left(x_{i}, \tau \mid x_{0 i}\right)=V_{\mathbf{0 0}}^{-1}\left(x_{0 i}\right) \sum_{\mathbf{k}} V_{\mathbf{0 k}}\left(x_{i}\right) V_{\mathbf{k} \mathbf{0}}\left(x_{0 i}\right) \mathrm{e}^{-\Lambda_{\mathbf{k}} \tau},
$$

where the 'overlap elements' are defined as

$$
V_{\mathbf{k l}}(z)=\int d \mathbf{x} \delta\left(z-x_{i}\right) \Psi_{\mathbf{k}}^{L}(\mathbf{x}) \Psi_{\mathbf{1}}^{R}(\mathbf{x})
$$


and $\delta(x)$ is Dirac's delta. In the specific case of equilibrated initial conditions for background particles only the special cases

$$
\begin{aligned}
V_{\mathbf{k} \mathbf{0}}(z) & =\int d \mathbf{x} \delta\left(z-x_{i}\right) \Psi_{\mathbf{k}}^{L}(\mathbf{x}) \Psi_{\mathbf{0}}^{R}(\mathbf{x}), \\
V_{\mathbf{0 k}}(z) & =\int d \mathbf{x} \delta\left(z-x_{i}\right) \Psi_{\mathbf{0}}^{L}(\mathbf{x}) \Psi_{\mathbf{k}}^{R}(\mathbf{x})
\end{aligned}
$$

are important. Note that any numerical implementation of Eq. (11) involves a truncation at some maximal eigenvalue $\Lambda_{\mathbf{M}}$. The ordering operator allows us to evaluate the integrals (13) as nested integrals, i.e.

$$
\int_{a}^{b} f(\mathbf{x}) d \mathbf{x}=\int_{a}^{b} d x_{1} \int_{x_{1}}^{b} d x_{2} \ldots \int_{x_{N-2}}^{b} d x_{N-1} \int_{x_{N-1}}^{b} d x_{N} f(\mathbf{x}) .
$$

Since by construction the integrand is invariant under exchange of the $\left\{x_{i}\right\}$ coordinates we can take advantage of the so-called extended phase-space integration [31] and greatly simplify the multi-dimensional nested integral to a product of one-dimensional integrals

$$
\oint_{a}^{b} f(\mathbf{x}) \delta\left(z-x_{i}\right) d \mathbf{x}=\left(\prod_{j=1}^{i-1} \int_{a}^{z} d x_{j}\right)\left(\prod_{j=i+1}^{N} \int_{z}^{b} d x_{j}\right) \frac{f\left(x_{i}=z,\left\{x_{j}, j \neq i\right\}\right)}{N_{L} ! N_{R} !},
$$

where $a$ and $b$ are the lower and upper boundaries of the domain, respectively, and $N_{L}\left(N_{R}\right)$ is the number of particles to the left (right) of the tagged one. These last two equations allow us to write Eq. (12) as

$$
V_{\mathbf{k} \mathbf{l}}(z)=\frac{m_{\mathbf{l}}}{N_{L} ! N_{R} !} \sum_{\{\mathbf{k}\}} \sum_{\{\mathbf{l}\}} T_{i}(z) \prod_{j=1}^{i-1} L_{j}(z) \prod_{j=i+1}^{N} R_{j}(z),
$$

where $m_{\mathbf{l}}$ is the multiplicity of the multiset $\mathbf{l}$ defined in Appendix A and we have introduced the auxiliary functions

$$
\begin{aligned}
& T_{i}(z)=\psi_{k_{i}}^{L}(z) \psi_{l_{i}}^{R}(z), \\
& L_{j}(z)=\int_{a}^{z} d x \psi_{k_{j}}^{L}(x) \psi_{l_{j}}^{R}(x), \\
& R_{j}(z)=\int_{z}^{b} d x \psi_{k_{j}}^{L}(x) \psi_{l_{j}}^{R}(x) .
\end{aligned}
$$

Once substituted into Eq. (11) Eqs. (16)-(17) deliver the tagged particle propagator sought for.

\section{Avoiding permutations}

Although the extended phase-space integration (cf. Eqs. (12) and (16)) substantially simplifies the integrals involved in the computation of the tagged particle propagator we still need to sum over all the permutations of $\mathbf{l}$ and $\mathbf{k}$ in Eq. (16). A brute force (or naïve) approach is thus not feasible, not even for rather small single-files since we need to evaluate the products $V_{\mathbf{0 k}}\left(x_{i}\right) V_{\mathbf{k} \mathbf{0}}\left(x_{0 i}\right)$ in Eq. (11) up to $2 \times N$ ! times in the worst case scenario for a calculation involving only the Green's function; and for a general element $V_{\mathbf{l k}}$ up to $(N !)^{2}$.

The main contribution of this paper is Algorithm 1 that reduces the number of terms in the Bethe ansatz solution entering Eq. (16) that need to be computed explicitly. Namely, since the full single-file diffusion model is symmetric with respect to the exchange of particles many terms arising from the permutations of the eigennumbers of the multisets in Eq. (16) happen to be identical. Algorithm 1 counts how many terms are equal and computes only those that are unique, and does so only once. These unique terms are then multiplied by their respective multiplicity and summed up to yield the result equation (11). Algorithm 1 thereby avoids going through the large number of equivalent permutations of the multisets in the sum with the larger number of terms between $\sum_{\{\mathbf{k}\}}$ and $\sum_{\{\mathbf{1}\}}$ in Eq. (16). In the specific case of the tagged-particle Green's function defined in Eq. (11), where one of the two multisets $\{\mathbf{k}\},\{\mathbf{l}\}$ corresponds to the ground state (having only one permutation), the algorithm in fact avoids permutations entirely.

More precisely (i.e. for a general $V_{\mathbf{k} \mathbf{l}}\left(x_{i}\right)$ ), the algorithm first generates all permutations of the multiset having the smallest number of permutations $P(\mathbf{l})$ (for sake of simplicity let us assume that this is the multiset $\mathbf{l}$ with $\mathcal{N}_{\mathbf{l}}$ distinct permutations). Then, for each of these permutations a multiset of pairs is created: $\mathbf{p}=\left\{\left\{k_{1}, l_{1}^{*}\right\}, \ldots,\left\{k_{N}, l_{N}^{*}\right\}\right\}$. The function $S(\mathbf{p})$ selects the largest possible set from $\mathbf{p}$ and generates for each element $u$ of the resulting set the 'difference multiset': $\mathbf{r}=\mathbf{p} \backslash u$. In the following it determines $t=\min \left(N_{L}, N_{R}\right)$ and all the $t$-combinations of $\mathbf{r}$ are generated via $C(\mathbf{r}, t)$ (note that $t$ here does not refer to time). For each of these combinations $\mathbf{s}$ the complementary multiset $\mathbf{d}=\mathbf{r} \backslash \mathbf{s}$ is created and the number of permutations of $\mathbf{s}$ and $\mathbf{d}$ is computed. Finally, the products in Eq. (16) are calculated (where $u$ is the pair of eigennumbers belonging to the tagged-particle) and accounted for their multiplicity.

In summary, our algorithm exploits the fact that the extended phase-space integration allows us to ignore the ordering of the particles to the left and to the right of the tagged-particle, respectively. A consequence of this symmetry is that several terms that appear in Eq. (16) are identical. Therefore, we can substitute the permutations of one multiset in Eq. (16) with all its combinations that are not tied to any ordering by definition. This makes the algorithm more efficient. A pseudocode-implementation is presented in Algorithm 1 and an explicit flowchart is depicted in Fig. 1. The reduction of the computational time achieved by our algorithm compared to a naïve implementation ispresented in Fig. 2. 


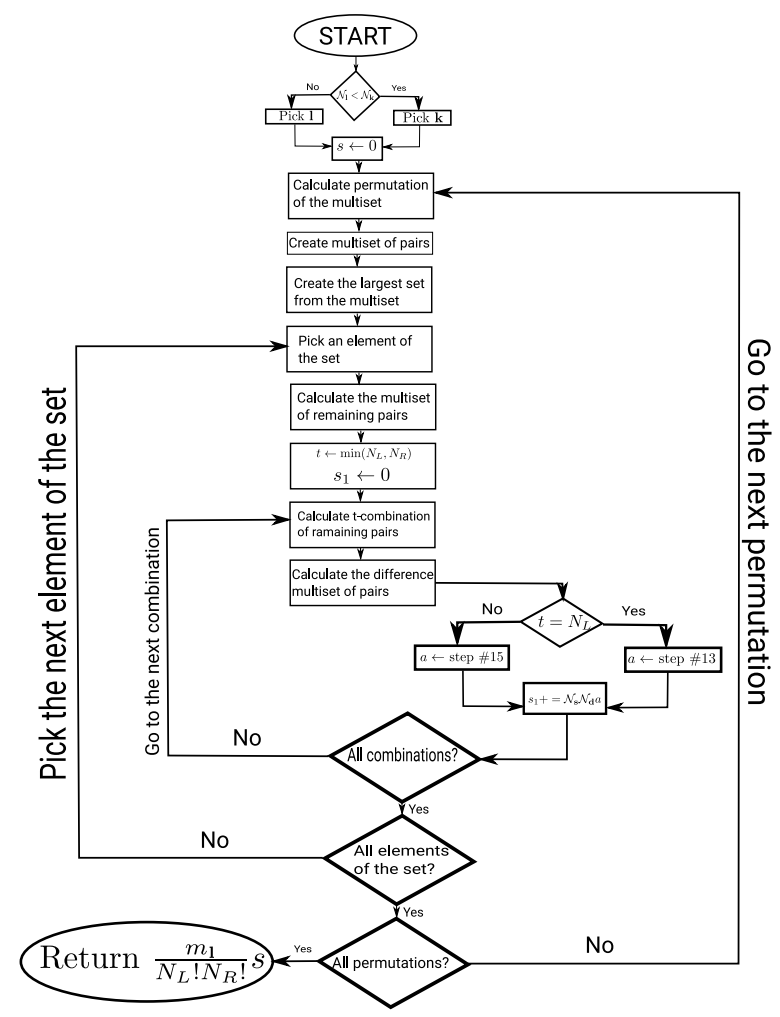

Fig. 1. The flowchart of Algorithm 1. The steps \#13 and \#15 are not reported for spatial constraints and can be found in the explanation of the algorithm.

Algorithm 1 Calculate $V_{\mathbf{k l}}(z)$

Require:

- $\mathbf{k}, 1$ multisets;

- $z \in \mathbb{R}, a \leq z \leq b$;

- functions: $T_{i}(\bar{z}), L_{j}(z), R_{j}(z)$;

- a function to generate all the permutation of multiset $P(\mathbf{k})$;

- a function to calculate the number of permutation of a multiset: $\mathcal{N}_{\mathbf{k}}$;

- a function to generate all the $t$-combinations of a multiset $C(\mathbf{k}, t)$;

- a function to compute the multiset difference $\mathbf{k} \backslash \mathbf{1}$;

- a function to create the largest set from a multiset $\tilde{k}=S(\mathbf{k})$;

1: calculate $\mathcal{N}_{\mathbf{k}}$ and $\mathcal{N}_{\mathbf{l}}$ and pick the multiset with the smallest number of permutations (let us assume it is $\mathbf{1}$ );

2: initialize $s \leftarrow 0$;

3: for all $\mathbf{l}^{*} \in P(\mathbf{l})$ do

create the multiset of pairs $\mathbf{p}=\left\{\left\{k_{1}, l_{1}^{*}\right\}, \cdots,\left\{k_{N}, l_{N}^{*}\right\}\right\}$;

$\tilde{u} \leftarrow S(\mathbf{p})$;

for $u \in \tilde{u}$ do

$\mathbf{r} \leftarrow \mathbf{p} \backslash u$

$t \leftarrow \min \left(N_{L}, N_{R}\right)$

initialize $s_{1} \leftarrow 0$;

for all $\mathbf{s} \in C(\mathbf{r}, t)$ do

$\mathbf{d} \leftarrow \mathbf{r} \backslash \mathbf{s}$

if $t=N_{L}$ then

else

$a \leftarrow T_{i}(z) \prod_{j \in \mathbf{s}} L_{j}(z) \prod_{j \in \mathbf{d}} R_{j}(z) ;$

$a \leftarrow T_{i}(z) \prod_{j \in \mathbf{d}} L_{j}(z) \prod_{j \in \mathbf{s}} R_{j}(z)$

end if

$s_{1}+=\mathcal{N}_{\mathbf{s}} \mathcal{N}_{\mathbf{d}} a ;$

end for

$s+=s_{1}$;

end for

end for

return $\frac{m_{1}}{N_{L} ! N_{R} !} S$. 


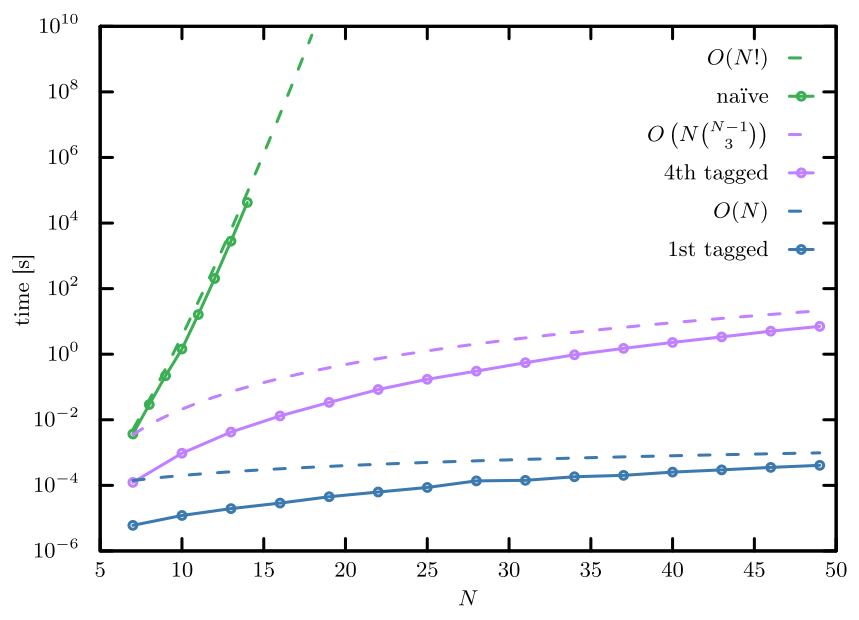

Fig. 2. Computational time (in seconds) required to calculate $V_{\mathbf{k} \mathbf{0}}$ for a single-file confined to a flat box depending on the number of particles in the single-file $N$; the $\mathbf{k}$-multiset has been chosen to represent the worst case scenario, i.e. $\mathbf{k}=\{1,2, \ldots, N\}$. The green line corresponds to the running time of the naïve implementation for comparison (which does not depend on which particle is tagged), while the blue and purple lines depict the running time of our program tagging the first and the fourth particle, respectively. Dashed lines depict the computational complexity for the various cases. (For interpretation of the references to color in this figure legend, the reader is referred to the web version of this article.)

Computational complexity of Algorithm 1. The computational complexity of the algorithm can be derived by following its flow (see Fig. 1). For the sake of simplicity we will (only initially) assume that the multiset $\mathbf{l}$ has only one possible permutation. Let $\mathcal{U}$ be the number of unique elements belonging to the multiset $\mathbf{k}$. Then for each unique element $u \in \mathbf{k}$ we need to iterate over all the $t$-combinations of the multiset $\mathbf{k} \backslash u$, where $t=\min \left(N_{L}, N_{R}\right)$. The number of these combinations is given by the function $\mathcal{M}(N-1, t)-$ an algorithm describing and computing this function is presented in Appendix A. Hence, the complexity of the algorithm is given by $\mathcal{O}\left(\mathcal{U} \cdot \mathcal{M}\left(N-1, \min \left(N_{L}, N_{R}\right)\right)\right)$. In the worst-case scenario, in which all the elements of $\mathbf{k}$ are different, the complexity is $\mathcal{O}\left(N \cdot\left(\begin{array}{c}N-1 \\ \min \left(N_{L}, N_{R}\right)\end{array}\right)\right)$. However, even in this worst case scenario the algorithm scales linearly $\mathcal{O}(N)$ in the number of particles if we tag the first or the last particle (see Fig. 2). In the general case when $\mathbf{l} \neq \mathbf{0}$, i.e. the one in which $\mathbf{l}$ admits more permutations (which, however, is not required for evaluating Eq. (11)), the complexity deteriorates fast since the evaluation of all permutations of $\mathbf{1}$ must be considered; the computational complexity in this case is $\mathcal{O}\left(\mathcal{N}_{\mathbf{l}} \cdot \mathcal{U} \cdot \mathcal{M}\left(N-1, \min \left(N_{L}, N_{R}\right)\right)\right)$, where $\mathcal{N}_{\mathbf{l}}$ is the number of permutations with repetitions of $\mathbf{l}$ and we assume that $\mathcal{N}_{\mathbf{1}} \leq \mathcal{N}_{\mathbf{k}}$.

\section{Implementation}

The main goal of the code attached to this article is to compute the Green's function of any tagged-particle in a single-file of $N$ elements given a potential $U(x)$. For this reason we opt for an object-oriented approach that allows the user to easily extend the code to incorporate any potential satisfying the constraints on $\hat{L}_{N}$. The code defines the abstract base class: class SingleFile (in SingleFile.hpp) responsible for the interface and for the functions that are responsible for the computation of the overlap elements (Eq. (16)). Conversely, all functions directly related to some specific potential $U(x)$ are private pure abstract base functions and must be implemented by the user in a derived class.

In our codebase we provide three different derived classes:

class SingleFileFlat : public SingleFile; ,

class SingleFileOnSlope : public SingleFile; ,

class SingleFileHarmonic : public SingleFile; in the header file SingleFileDerived.hpp, covering several different 'canonical' cases of single-file systems.

The base class. The base class class SingleFile provides a common interface to all single-file systems. It contains the following functions: the equilibrium probability density function virtual double eq_prob(const double $\mathrm{x}$ ) const; , the two-point joint density

double joint2dens(const double $\mathrm{x}$, const double $\mathrm{t}$, const double $\mathrm{x} 0$ ); and the Green's function

double green_function(const double $\mathrm{x}$, const double $\mathrm{t}$, const double $\mathrm{x} 0$ ); for a specific tagged-particle implementing the analytical solution in Eq. (11). The function evaluating the equilibrium probability density function of a tagged-particle, i.e. $\mathcal{G}_{\text {eq }}\left(x_{i}\right)=\lim _{t \rightarrow \infty} \mathcal{G}\left(x_{i}, \tau \mid x_{0 i}\right)$, is virtual since for a given potential $U(x)$ it often has a relatively simple form. In addition, naïve implementations directly computing all permutations have also been defined in the interface: double joint2dens_naive(const double $\mathrm{x}$, const double $\mathrm{t}$, const double $\mathrm{x} 0$ ); and double green_function_naive(const double $\mathrm{x}$, const double $\mathrm{t}$, const double $\mathrm{x} 0$ ); . These two functions call the function

double Vkl_element_naive(std::vector<int>\& k_vec, std::vector<int $>\&$ I_vec, const double $x)$ const; that implements slavishly Eq. (12). Finally, the interface of the class is completed by several tiny functions that allow changing the parameters of an instance of the class, like the tagged-particle or the diffusion coefficient $D$.

The base class is also responsible for the internal machinery to use our fast algorithm implementing (among its private members): double Vkl_element(std::vector<int $>\&$ k_vec, std::vector<int $>\&$ I_vec, const double $\mathrm{x}$ ) const; ; and its specialized versions, defined by default in its terms: virtual double V0k_element(std::vector<int $>\& \mathrm{k}_{-}$vec, const double $\mathrm{x}$ ) const; and

virtual double Vk0_element(std::vector<int>\& k_vec, const double $\mathrm{x}$ ) const; . 
These specialized versions are made virtual to allow a derived class to override them if they require special settings (one such example is the single-file in a linear potential). The declarations and definitions of these functions can be found in the files SingleFile.hpp and SingleFile.cpp. Our algorithm computes the $t$-combinations of a multiset and this feature is provided by the friend class template<typename $\mathrm{T}>$ class UCombinations; that implements (in the file combinations.hpp) a classical algorithm given in [32]. We use std::next_permutation for the computation of permutations. Finally, this base abstract class defines the private members responsible for the calculation of the single-particle eigenvalues and for the evaluation of Eqs. (17). These are pure virtual functions since they depend on the specific external potential, and hence they must be implemented by the derived class. Finally, the pure virtual function virtual int eigenfunction_condition(const int i) const=0; defines the rule to initialize the private member std::vector<std::vector<int>> eigenfunction_store; that contains (row-wise) all the multisets considered in the evaluation of Eq. (5) for a given specific potential $U(x)$. For this reason the derived class is responsible for initializing this last member (in its constructor, for example). We provide the protected function void eigenfunction_store_init (); to initialize this data structure (details are given below). However, the user may implement a different way to initialize the container as well, for example by importing it from an existing file.

The derived classes. Three types of analytically solvable potentials: $U(x)=0, U(x)=g x$ and $U(x)=\gamma x^{2} / 2$, are implemented $(g, \gamma$ being real and positive). These implementations assume that the positions of non-tagged-particles are drawn from their respective equilibrium distributions conditioned on the initial position of the tagged-particle. The many-body Bethe eigenvalues for these models are given by

$$
\begin{aligned}
& \Lambda_{\mathbf{k}}=\sum_{i=1}^{N} D \pi^{2} k_{i}^{2}, \\
& \Lambda_{\mathbf{k}}=\sum_{i=1}^{N}\left(1-\delta_{k_{i}, 0}\right)\left(D \pi^{2} k_{i}^{2}+\frac{g^{2}}{4 D}\right), \\
& \Lambda_{\mathbf{k}}=\sum_{i=1}^{N} \gamma k_{i},
\end{aligned}
$$

for $U(x)=0, U(x)=g x$ and $U(x)=\gamma x^{2} / 2$ respectively. In the files SingleFileDerived.hpp and SingleFileDerived.cpp the functions related to single-particle solutions (further details are given in Appendix B) that enter the Bethe-ansatz solution in Eq. (16) are implemented as overridden private member functions of the derived classes. The function double lambda_single(const int $n)$ const; calculates the single-particle eigenvalue while the functions double tagged(const int lambda_k, const int lambda_l, const double $\mathrm{x}$ ) const;

double lefttagged(const int lambda_k, const int lambda_l, const double $\mathrm{x}$ ) const override;

double righttagged(const int lambda_k, const int lambda_l, const double x) const override; implement respectively $T_{i}, L_{i}$ and $R_{i}$ defined in Eq. (17). These last four functions must be implemented following the template in SingleFileBluePrint.hpp if the user wishes to implement a solution for a different potential $U(x)$. In our implementation the constructor of a derived class takes a parameter int max_many_eig $\equiv M$. This positive parameter is proportional to the maximum eigenvalues we want to consider in the implementation of Eq. (11).

Note that by fixing the largest eigenvalue $\Lambda_{\mathbf{M}}$ we consider in the computation of the Green's function in Eq. (11) we implicitly determine the shortest time-scale for which the solution is reliable, i.e. the solution is exact for times $\tau \gtrsim 1 / \Lambda_{\mathbf{M}}[33$,34]. Since the eigenspectra of single-file systems are always degenerate, once fixed $M$ is used to select the multisets $\mathbf{k}$ (each of them uniquely identifies an eigenfunction) that must be considered in Eq. (11). However, the rule for selecting allowed multisets is system dependent; in the case of the flat and linear potentials (cf. Eqs. (18) and (19)) we can only accept multisets satisfying $\sum_{i=1}^{N} k_{i}^{2} \leq M$, and for the harmonic potential (Eq. (20)) only those satisfying $\sum_{i=1}^{N} k_{i} \leq M$ are allowed. These constraints must be implemented in the pure abstract function virtual int eigenfunction_condition(const int i) const $=0$; .

According to this function the constructors of our derived classes fill std::vector<std::vector<int>> eigenfunction_store; using a slightly modified implementation of a classical algorithm for computing integer partitions found in [32], which takes into account the possibility that one (or more) of the $k_{i}$ can be equal to 0 . This implementation is provided in the friend class class IntegerPartitions; in the file IntegerPartitions.hpp. The number of integer partitions (see Appendix A for an example) generated by this algorithm is the sum of all the possible bounded compositions of $N$ numbers such that their sum is between 0 and $M$. The number of bounded composition of $N$ elements summing to $M$ (see e.g. Eq. (9) alongside the specific values of $\lambda_{k_{i}}$ given in Appendix B) is equivalent to the $N$-combinations of multiset in which all the numbers between 0 and $M$ appear at most $N$ times [32]. The function virtual int eigenfunction_condition(const int i) const $=0$; then selects from those only the allowed ones. All these steps are wrapped in the aforementioned void eigenfunction_store_init (); function. The function virtual int eigenfunction_condition(const int $\mathrm{i}$ ) const=0; must be implemented by the user in a new derived class implementing a different potential.

In Fig. 3 we show how many multisets must be considered for the convergence of the sum on a time-scale $\tau \gtrsim 1 / \Lambda_{\mathbf{M}}$. Since these multisets are saved in std::vector<std::vector<int >> eigenfunction_store; , the size of this data structure prescribes the memory requirements of our program. The program saves these values to allow for a flexible way to compute the non-Markovian Green's function (11) for the same system when tagging a different particle without the need to re-compute the necessary multisets. Though this number can become huge for some systems, for example in the case of the harmonic potential, it has been proved that for regular Sturm-Liouville problems the eigenvalues scale quadratically for large $k_{i}$ [35]. Since often also non-regular Sturm-Liouville problems on a infinite domain are treated numerically using truncation methods [36] our choice to save these numbers to enhance the flexibility and readability of the code is justified. Nevertheless, it would be equally possible not to save the necessary multisets and instead do all calculations on-the-fly.

Moreover, according to the specific properties of the Fokker-Planck operator $\hat{L}_{N}$ we sometimes find that $V_{\mathbf{k} \mathbf{0}}(x)=V_{\mathbf{0 k}}(x)$. A result of both functions can be implemented in terms of a single function responsible for $V_{\mathbf{k l}}(x)$ without the necessity of code duplication. However, for the single-file in a linear potential this is not the case [10]. For this reason the functions for calculating the 'overlaps' 


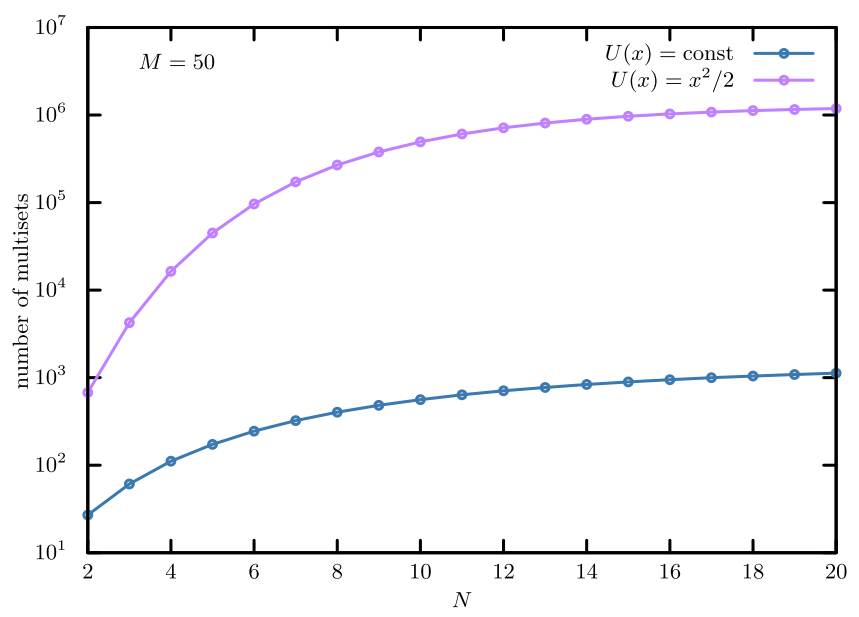

Fig. 3. The number of multisets that must be considered assuming max_many_eig $\equiv M=50$ for the single-file in a flat (blue line) or in a harmonic (purple line) potential. (For interpretation of the references to color in this figure legend, the reader is referred to the web version of this article.)

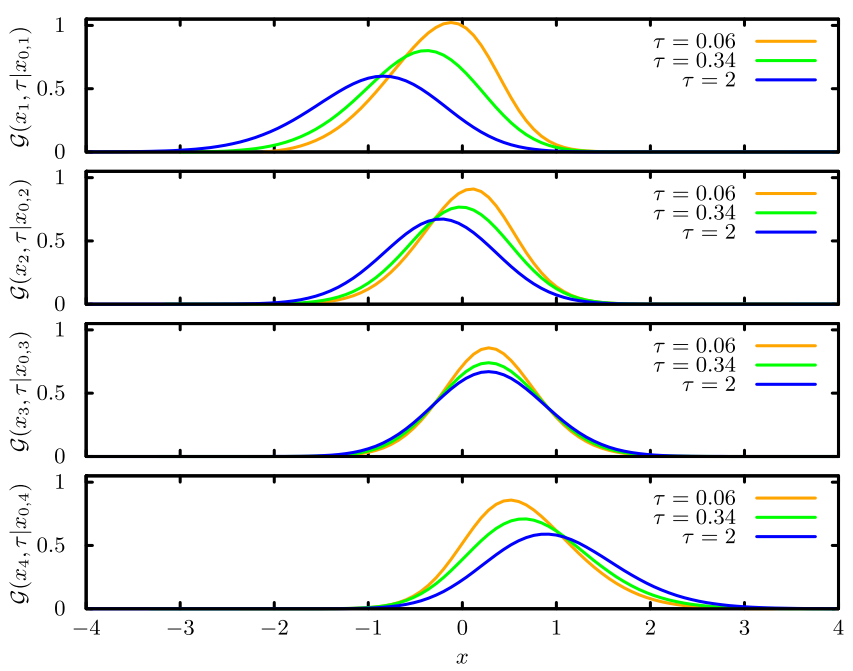

Fig. 4. The tagged-particle Green's function for different particles of a single-file of 4 particles in a harmonic potential $U(x)=x^{2} / 2$ at different times (the relaxation time is $\sim \lambda_{1}^{-1}=1$ ). Each particle's initial position is $x_{0}=0.305$ assuming that all the other particles are in their respective equilibrium conditioned on the position of the tagged-particle.

(i.e. Eqs. (12)) with the ground state are virtual, such that they can be implemented without re-factoring the code. In our implementation of class SingleFileOnSlope; the function Vk0_element is overridden with a marginally faster version to take into account the asymmetry of the single-file in a linear potential.

In order to illustrate our final result we depict in Fig. 4 the computed Green's function for a single-file of 4 particles in a harmonic potential $U(x)=x^{2} / 2$.

Exceptions. Two classes for managing exceptions:

class NotImplementedException : public std::logic_error; and

class NotAllowedParameters : public std::logic_error; are included in the code base. The former allows to write a partially implemented derived class, while the latter just throws in the case that an ill-posed parameter is provided. All exceptions throw without any attempt to catch them.

Parallelization. By construction the evaluation of Eq. (5) for different $x, \tau$ and $x_{0}$ is parallelizable. A non-trivial parallelization may be achieved implementing a reduction for Eq. (5). However, for many systems (see Fig. 3) the number of terms in the sum is relatively small and a parallel approach is unnecessary unless the single-file is very big and/or we are interested in very small time-scales. The present code does not support parallelization and thread-safety is not guaranteed.

\section{Comparison with Brownian dynamics simulations}

A fair comparison between our algorithm implementing a solution based on Eq. (11) and a Brownian Dynamics simulation integrating the Langevin equation (2) numerically is somewhat tricky. The reason is that the computational effort of a Brownian Dynamics simulation grows "forward" in time, while the eigenexpansion solution becomes challenging "backward" in time. In the former case the 

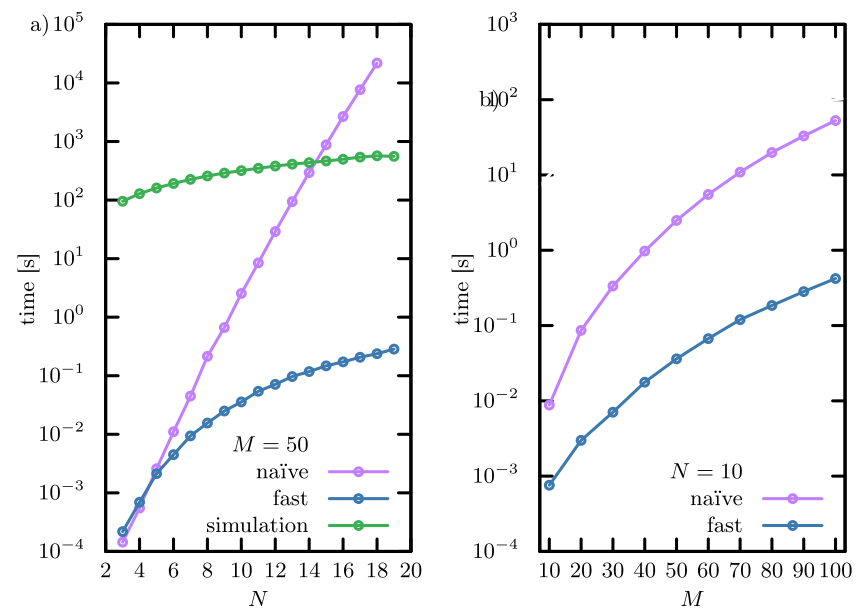

Fig. 5. Computational time for calculating the tagged-particle Green's function, in one specific space-time point a single-file in a flat potential. We compare our algorithm (blue lines) with the naïve implementation (purple lines) to a Brownian Dynamics simulation of $10^{4}$ trajectories with $2 \times 10^{4}$ integration-steps of length $10^{-3}$ using Algorithm 2 (green line). In (a) we fix the max many-body eigenvalue and tag the central particle while in (b) we fix the total number of particles of the single-file and tag the fifth particle. For both plots the time to calculate all the available multisets in Eq. (5) has not been taken into account since it is the same the naïve and efficient implementations of the CBA solution. (For interpretation of the references to color in this figure legend, the reader is referred to the web version of this article.)

longer the time-scale we are interested in the more integration steps we must perform, while if we are interested in shorter time-scales a smaller integration time-step must be used. In the implementation of the Bethe ansatz solution in Eq. (11) we need to consider more and more terms in the sum over Bethe eigenvalues $\Lambda_{\mathbf{k}}$ in order to obtain reliable results for shorter time-scales. In contrast, essentially only two terms (i.e. the ground state $\Lambda_{\mathbf{0}}$ and the first excited state $\Lambda_{\mathbf{1}}$ ) are required if we are interested only in the long-time dynamics, i.e. $t>1 / \Lambda_{1}$.

In Algorithm 2 we present a convenient method to simulate single-file diffusion based on the Jepsen mapping [2]. The key step is the sorting of the particles' positions (step 6) that allows avoiding a costly chain of if statements required to implement non-crossing conditions. To perform this step we use the sorting routine std::sort included in the C++ standard library. A comparison of this algorithm with the analytical solution can be found in [9].

Algorithm 2 Brownian Dynamics simulation of a single-file

\section{Require:}

- number of particles $N$;

- time-step $\Delta t$, final time $t_{f}$, list of sampling times $t_{s}$;

- number of trajectories $N_{t}$;

- the initial position of the tagged-particle $x_{0}$;

- a function to update a histogram.

1: for $i=1: N_{t}$ do

2: $\quad t \leftarrow 0$;

3: Generate the initial position for the $N-1$ particle from their equilibrium distribution conditioned on the position of the tagged-particle $x_{0}$;

4: $\quad$ while $t \leq t_{f}$ do

5: Integrate the Langevin equation (using a Euler-Maruyama scheme for example) of $N$ independent particles using the time-step $\Delta t$;

6: $\quad$ Sort in ascending order the particles' positions to satisfy the non-crossing condition;

7: $\quad$ if $t \in t_{s}$ then

8: $\quad$ Update the histogram containing the Green's function of the tagged-particle;

9: $\quad$ end if

10: $\quad t+=\Delta t$

11: end while

12: end for

13: return the histogram;

In Fig. 5 we present the computational time required to evaluate the Green's function in a single point in space and time fixing either the maximum eigenvalue (panel a) or the number of particles (panel b). In the left panel we also plot the time required to compute the tagged-particle Green's function of the single-file in a flat potential for different number of particles $N$ by means of a Brownian Dynamics simulation. We simulate $10^{4}$ trajectories with a time-step of $10^{-3}$ until time $t_{f}=20 \approx 2 / \Lambda_{1}$ to ensure that the final equilibrium distribution is reached. Using these parameters the statistical error of the simulation is $\sim 5 \%-10 \%$ using $10^{4}$ trajectories and $1 \%-2 \%$ if instead we generate $10^{5}$ trajectories, in agreement with the Gaussian central limit theorem. Note that since we are considering enough terms in the series expansion (11) the analytic solution may be reliably considered to be exact on the time-scale of 
interest. Because the error of the Brownian Dynamics simulation can be reduced by increasing the number of independent trajectories, $N_{\text {traj }}$, and since Algorithm 2 scales linearly with $N_{\text {traj }}$, it is easy to extrapolate from Fig. 5 the computational effort required to obtain more accurate results.

Conversely, if we are interested only in short time-scales we must carry out the numerical integration of Eq. (2) for a small number (say 100) of steps and thereby obtain better results (and with less computational effort) than the analytic solution. This is so because the analytical solution suffers from the Runge-phenomenon for short times, since we are approaching a delta-function distribution. However, such very short time-scales are less interesting since the tagged-particle behaves like a free-particle for times shorter the average collision-time with neighboring particles [7]. For the same reason a smaller integration time-step must also be taken to capture all the non-trivial physics in a Brownian Dynamics simulation if we consider a single-file with a large number of particles $N$. In addition, if the Green's function of the tagged-particle is peaked, the binning of the histogram in the analysis of simulations must be made sufficiently small, which imposes additional constraints on the integration time-step in order to obtain reliable results.

\section{Conclusions}

We presented an efficient numerical implementation of the exact coordinate Bethe ansatz solution of the non-Markovian taggedparticle propagator in a single-file in a general confining potential. Motivated by the fact that the Bethe eigenspectrum solution nominally carries a large computational cost when the number of particles is large we developed an efficient algorithm, which enables investigations of tagged-particle diffusion on a broad span of time-scales and for various numbers of particles. Our code exploits exchange symmetries in order to reduce the number of combinatorial operations. One of the main advantages of the Bethe ansatz solution, aside from the fact that it provides an exact solution of the problem and that it ties the tagged-particle dynamics to manybody relaxation eigenmodes, is that it is easy to generalize to take into account for any confining external potential or initial condition. For this reason we provide a header file SingleFileBluePrint.hpp that allows an easy extension of our codebase. With this goal in mind the expressiveness and the tools of modern $\mathrm{C}++$ were used to achieve modularity and simplicity of use. The code can be easily extended in order to allow for a calculation of other key quantities related to the non-Markovian dynamics of a tagged-particle, e.g. the mean square displacement [31] as well as local-time statistics and other local additive functionals of tagged-particle trajectories [9,10].

\section{Declaration of competing interest}

The authors declare that they have no known competing financial interests or personal relationships that could have appeared to influence the work reported in this paper.

\section{Acknowledgments}

The financial support from the German Research Foundation (DFG) through the Emmy Noether Program GO 2762/1-1 (to AG), and an IMPRS fellowship of the Max Planck Society, Germany (to AL) are gratefully acknowledged.

\section{Appendix A. Combinatorics}

Permutations. Let $\mathbf{k}$ be a multiset of $N$ elements. Let $r_{i}$ denote the multiplicity of each of the $m$ distinct elements of $\mathbf{k}$ such that $\sum_{i=1}^{m} r_{i}=N$. Then the number of distinct permutations of this multiset is

$$
\left(\begin{array}{c}
N \\
r_{1}, r_{2}, \ldots, r_{m}
\end{array}\right)=\frac{N !}{\prod_{i=1}^{m} r_{i} !} .
$$

The denominator of Eq. (A.1) is what we call the multiplicity $m_{\mathbf{k}}$ of the multiset $\mathbf{k}$. For example, the distinct permutations of $\{1,1,2,3\}$ are: $\{\{1,1,2,3\},\{1,1,3,2\},\{1,2,1,3\},\{1,2,3,1\},\{1,3,1,2\},\{1,3,2,1\},\{2,1,1,3\},\{2,1,3,1\},\{2,3,1,1\},\{3,1,1,2\},\{3,1,2,1\}$, $\{3,2,1,1\}\}$.

$t$-combinations. The problems of enumerating and computing the combinations of a multiset can be mapped to the equivalent bounded composition problems [32]. James Bernoulli in 1713 enumerated them for the first time, observing that the number of the $t$-combinations of a multiset $\mathbf{k}$ with $m$ distinct elements, where each of them is contained $r_{m}$ times, is equal to the $t$ th coefficient of the polynomial $P_{\mathbf{k}}(z)$ :

$$
\begin{gathered}
P_{\mathbf{k}}(z)=\prod_{i=1}^{m} p_{i}(z) \\
p_{i}(z)=\sum_{j=0}^{r_{i}} z^{j}
\end{gathered}
$$

For example the 2-combinations of $\{1,1,1,2,3\}$ are: $\{\{1,1\},\{1,2\},\{1,3\},\{2,3\}\}$.

Integer partitions. We refer to the integer partition of a number $N$ in $m$ parts as the number of ways in which $N$ can be expressed as a sum of all the numbers smaller than or equal to itself. For example for $N=4$ and $m=4:\{0,0,0,4\},\{0,0,1,3\},\{0,0,2,2\},\{0,1,1,2\}$, $\{1,1,1,1\}$. 


\section{Appendix B. Single-particle eigenspectra}

The dynamics of a single Brownian particle in a unit box in a constant potential with reflecting external boundary conditions is governed by the Sturm-Liouville problem

$$
\begin{gathered}
\left(\partial_{\tau}-D \partial_{x}^{2}\right) \Gamma\left(x, \tau \mid x_{0}\right)=0, \\
\left.\partial_{x} \Gamma\right|_{x=0}=\left.\partial_{x} \Gamma\right|_{x=1}=0
\end{gathered}
$$

with the initial condition $\Gamma\left(x, 0 \mid x_{0}\right)=\delta\left(x-x_{0}\right)$. The corresponding Green's function can be expressed in terms of a spectral expansion

$$
\Gamma\left(x, \tau \mid x_{0}\right)=\sum_{k} \psi_{k}^{R}(x) \psi_{k}^{L}\left(x_{0}\right) \mathrm{e}^{-\lambda_{k} \tau}
$$

where

$$
\begin{gathered}
\psi_{0}^{L}(x)=\psi_{0}^{R}(x)=1, \\
\psi_{k}^{L}(x)=\psi_{k}^{R}(x)=\sqrt{2} \cos (k \pi x), \\
\lambda_{k}=k^{2} \pi^{2} .
\end{gathered}
$$

On the other hand, if we add a linear potential the corresponding Fokker-Planck equation for the Green's function becomes

$$
\left(\partial_{\tau}-D \partial_{x}^{2}-g \partial_{x}\right) \Gamma\left(x, \tau \mid x_{0}\right)=0,
$$

with initial condition $\Gamma\left(x, 0 \mid x_{0}\right)=\delta\left(x-x_{0}\right)$, and the eigenexpansion is given by

$$
\begin{gathered}
\lambda_{0}=0, \\
\lambda_{k}=D k^{2} \pi^{2}+\frac{g^{2}}{4 D}, \\
\psi_{0}^{L}(x)=1, \\
\psi_{k}^{L}(x)=\frac{\mathrm{e}^{\frac{g x}{2 D}}}{\sqrt{1 / 2+2 D k^{2} \pi^{2} / g^{2}}}(\sin (k \pi x)-2 D k \pi \cos (k \pi x) / g), \\
\psi_{0}^{R}(x)=\frac{g}{D} \frac{\mathrm{e}^{-\frac{g x}{D}}}{1-\mathrm{e}^{-\frac{g}{D}}}, \\
\mathrm{e}^{-\frac{g x}{2 D}}(x)=\frac{}{\sqrt{1 / 2+2 D k^{2} \pi^{2} / g^{2}}}(\sin (k \pi x)-2 D k \pi \cos (k \pi x) / g) .
\end{gathered}
$$

Finally for an Ornstein-Uhlenbeck process with natural boundary conditions (i.e. $\lim _{|x| \rightarrow \infty} \Gamma\left(x, \tau \mid x_{0}\right)=0$ ) the Green's function is given by

$$
\left(\partial_{\tau}-D \partial_{x}^{2}-\gamma \partial_{x} x\right) \Gamma\left(x, \tau \mid x_{0}\right)=0
$$

with initial condition $\Gamma\left(x, 0 \mid x_{0}\right)=\delta\left(x-x_{0}\right)$ and eigenexpansion

$$
\begin{aligned}
\psi_{k}^{L}(x) & =\frac{1}{\sqrt{2^{k} k !}} H_{k}(x \sqrt{\gamma / 2 D}) \\
\psi_{k}^{R}(x) & =\sqrt{\frac{\gamma}{2 \pi D}} \mathrm{e}^{-\frac{\gamma x^{2}}{2 D}} \psi_{k}^{L}(x) \\
\lambda_{k} & =\gamma k,
\end{aligned}
$$

where $H_{k}(x)$ denotes the $k$ th "physicist's" Hermite polynomial [37].

\section{References}

[1] T.E. Harris, J. Appl. Probab. 2 (2) (1965) 323-338, http://dx.doi.org/10.2307/3212197.

[2] D.W. Jepsen, J. Math. Phys. 6 (3) (1965) 405-413, http://dx.doi.org/10.1063/1.1704288, URL http://aip.scitation.org/doi/10.1063/1.1704288,

[3] H. van Beijeren, J. Stat. Phys. 63 (1-2) (1991) 47-58, http://dx.doi.org/10.1007/BF01026591, URL http://link.springer.com/10.1007/BF01026591.

[4] C. Rödenbeck, J. Kärger, K. Hahn, Phys. Rev. E 57 (4) (1998) 4382-4397, http://dx.doi.org/10.1103/PhysRevE.57.4382, URL https://link.aps.org/doi/10.1103/ PhysRevE.57.4382.

[5] E. Barkai, R. Silbey, Phys. Rev. Lett. 102 (5) (2009) 050602, http://dx.doi.org/10.1103/PhysRevLett.102.050602, URL https://link.aps.org/doi/10.1103/PhysRevLett. 102.050602.

[6] T. Ambjörnsson, L. Lizana, M.A. Lomholt, R.J. Silbey, J. Chem. Phys. 129 (18) (2008) 185106, http://dx.doi.org/10.1063/1.3009853, URL https://aip.scitation.org/ doi/10.1063/1.3009853.

[7] L. Lizana, T. Ambjörnsson, Phys. Rev. Lett. 100 (20) (2008) 200601, http://dx.doi.org/10.1103/PhysRevLett.100.200601, URL https://link.aps.org/doi/10.1103/ PhysRevLett.100.200601.

[8] L. Lizana, T. Ambjörnsson, A. Taloni, E. Barkai, M.A. Lomholt, Phys. Rev. E 81 (5) (2010) 051118, http://dx.doi.org/10.1103/PhysRevE.81.051118, URL https: //link.aps.org/doi/10.1103/PhysRevE.81.051118.

[9] A. Lapolla, A. Godec, New J. Phys. 20 (11) (2018) 113021, https://iopscience.iop.org/article/10.1088/1367-2630/aaea1b.

[10] A. Lapolla, A. Godec, Front. Phys. 7 (2019) http://dx.doi.org/10.3389/fphy.2019.00182, URL https://www.frontiersin.org/articles/10.3389/fphy.2019.00182/full.

[11] C. Lutz, M. Kollmann, C. Bechinger, Phys. Rev. Lett. 93 (2) (2004) 026001, http://dx.doi.org/10.1103/PhysRevLett.93.026001, URL https://link.aps.org/doi/10.1103/ PhysRevLett.93.026001. 
[12] B. Lin, M. Meron, B. Cui, S.A. Rice, H. Diamant, Phys. Rev. Lett. 94 (21) (2005) 216001, http://dx.doi.org/10.1103/PhysRevLett.94.216001, URL https://link.aps.org/ doi/10.1103/PhysRevLett.94.216001.

[13] E. Locatelli, M. Pierno, F. Baldovin, E. Orlandini, Y. Tan, S. Pagliara, Phys. Rev. Lett. 117 (3) (2016) 038001, http://dx.doi.org/10.1103/PhysRevLett.117.038001, URL https://link.aps.org/doi/10.1103/PhysRevLett.117.038001.

[14] G. Hummer, J.C. Rasaiah, J.P. Noworyta, Nature 414 (6860) (2001) 188-190, http://dx.doi.org/10.1038/35102535, URL http://www.nature.com/articles/35102535.

[15] S. Ahlberg, T. Ambjörnsson, L. Lizana, New J. Phys. 17 (4) (2015) 043036, http://dx.doi.org/10.1088/1367-2630/17/4/043036.

[16] T. Chou, D. Lohse, Phys. Rev. Lett. 82 (17) (1999) 3552-3555, http://dx.doi.org/10.1103/PhysRevLett.82.3552, URL https://link.aps.org/doi/10.1103/PhysRevLett. 82.3552.

[17] J. Kärger, D.M. Ruthven, Diffusion in Zeolites and Other Microporous Solids, in: A Wiley Interscience publication, Wiley, New York, 1992, OCLC: 22451684.

[18] P.M. Richards, Phys. Rev. B 16 (4) (1977) 1393-1409, http://dx.doi.org/10.1103/PhysRevB.16.1393, URL https://link.aps.org/doi/10.1103/PhysRevB.16.1393.

[19] A. Taloni, O. Flomenbom, R. Castaeda-Priego, F. Marchesoni, Soft Matter 13 (6) (2017) 1096-1106, http://dx.doi.org/10.1039/C6SM02570F, URL http://xlink.rsc. org/?DOI=C6SM02570F.

[20] X. Hu, L. Hong, M. Dean Smith, T. Neusius, X. Cheng, J. Smith, Nat. Phys. 12 (2) (2015) 171-174, http://dx.doi.org/10.1038/nphys3553, URL http://dx.doi.org/10. 1038/nphys3553.

[21] K. Henzler-Wildman, D. Kern, Nature 450 (7172) (2007) 964-972, http://dx.doi.org/10.1038/nature06522, URL http://www.nature.com/articles/nature06522.

[22] G.-W. Li, O.G. Berg, J. Elf, Nat. Phys. 5 (4) (2009) 294-297, http://dx.doi.org/10.1038/nphys1222, URL http://www.nature.com/articles/nphys1222.

[23] E. Barkai, R. Silbey, Phys. Rev. E 81 (4) (2010) 041129, http://dx.doi.org/10.1103/PhysRevE.81.041129, URL https://link.aps.org/doi/10.1103/PhysRevE.81.041129.

[24] N. Leibovich, E. Barkai, Phys. Rev. E 88 (3) (2013) 032107, http://dx.doi.org/10.1103/PhysRevE.88.032107, URL https://link.aps.org/doi/10.1103/PhysRevE.88. 032107.

[25] V.E. Korepin, N.M. Bogoliubov, A.G. Izergin, Quantum Inverse Scattering Method and Correlation Functions, in: Cambridge Monographs on Mathematical Physics, Cambridge University Press, 1997.

[26] A. Lapolla, A. Godec, Phys. Rev. Lett. 125 (2020) 110602, http://dx.doi.org/10.1103/PhysRevLett.125.110602.

[27] S. Herrera-Velarde, R. Castaeda-Priego, Phys. Rev. E 77 (4) (2008) 041407, http://dx.doi.org/10.1103/PhysRevE.77.041407, URL https://link.aps.org/doi/10.1103/ PhysRevE.77.041407.

[28] S. Herrera-Velarde, G. Pérez-Angel, R. Castaeda-Priego, Soft Matter 12 (44) (2016) 9047-9057, http://dx.doi.org/10.1039/C6SM01558A, URL http://xlink.rsc.org/ ?DOI=C6SM01558A.

[29] L. Chupin, Ann. Inst. Fourier 60 (1) (2010) 217-255, http://dx.doi.org/10.5802/aif.2521.

[30] J. Kurchan, arXiv:0901.1271 [cond-mat] arXiv:0901.1271. URL http://arxiv.org/abs/0901.1271.

[31] L. Lizana, T. Ambjörnsson, Phys. Rev. E 80 (5) (2009) 051103, http://dx.doi.org/10.1103/PhysRevE.80.051103, URL https://link.aps.org/doi/10.1103/PhysRevE.80. 051103.

[32] D.E. Knuth, The Art of Computer Programming, Vol. 4a, fourth ed., Addison-Wesley, 2013.

[33] C.W. Gardiner, Handbook of Stochastic Methods for Physics, Chemistry and Natural Sciences, second ed., Springer-Verlag, 1985.

[34] H. Risken, T. Frank, The Fokker-Planck Equation: Methods of Solution and Applications, second ed., in: Springer Series in Synergetics, Springer-Verlag, Berlin Heidelberg, 1996, http://dx.doi.org/10.1007/978-3-642-61544-3, URL https://www.springer.com/gp/book/9783540615309.

[35] H. Hochstadt, Commun. Pure Appl. Math. 14 (4) (1961) 749-764, http://dx.doi.org/10.1002/cpa.3160140408, URL https://onlinelibrary.wiley.com/doi/abs/10.1002/ cpa.3160140408.

[36] J.P. Boyd, Chebyshev and Fourier Spectral Methods, Dover, New York, 2001.

[37] M. Abramowitz, I.A. Stegun, Handbook of Mathematical Functions with Formulas, Graphs, and Mathematical Tables, Ninth Dover printing, tenth GPO printing ed., Dover, New York, 1964 


\section{Chapter 6}

\section{Faster Uphill Relaxation in Thermodynamically Equidistant Temperature Quenches}

This article has been published in Physical Review Letters under the Creative Commons Attribution 4.0 International license [53]. Thermal relaxation processes have been one of the main topics of Thermodynamics since its conception. Looking at systems in which fluctuations dominate the dynamics, e.g. nanoscale systems, we discovered that cooling and heating process show a fundamental asymmetry: in the case in which the underlying potential is composed by a single well the relaxation process starting form a lower temperature is always faster compared to its counterpart starting from the higher one. We analyzed relaxation dynamics for Markovian and non-Markovian observables, motivating this asymmetry via the instantaneous energetic and entropic contributions of the systems' dynamic. The article it is followed by its supplementary material. 


\title{
Faster Uphill Relaxation in Thermodynamically Equidistant Temperature Quenches
}

\author{
Alessio Lapolla@ and Aljaž Godec $\oplus^{*}$ \\ Mathematical bioPhysics group, Max Planck Institute for Biophysical Chemistry, Göttingen 37077, Germany
}

(Received 6 February 2020; revised 13 June 2020; accepted 10 August 2020; published 11 September 2020)

We uncover an unforeseen asymmetry in relaxation: for a pair of thermodynamically equidistant temperature quenches, one from a lower and the other from a higher temperature, the relaxation at the ambient temperature is faster in the case of the former. We demonstrate this finding on hand of two exactly solvable many-body systems relevant in the context of single-molecule and tracer-particle dynamics. We prove that near stable minima and for all quadratic energy landscapes it is a general phenomenon that also exists in a class of non-Markovian observables probed in single-molecule and particle-tracking experiments. The asymmetry is a general feature of reversible overdamped diffusive systems with smooth singlewell potentials and occurs in multiwell landscapes when quenches disturb predominantly intrawell equilibria. Our findings may be relevant for the optimization of stochastic heat engines.

DOI: 10.1103/PhysRevLett.125.110602

Relaxation processes are a paradigm for condensed matter [1,2], single-molecule experiments [3] and tracer-particle transport in complex media [4-8]. Relaxation close to equilibrium was described by the mechanical Onsager-Casimir $[9,10]$ and thermal Kubo-Yokota-Nakajima [11] linear laws. These pioneering ideas were consistently generalized in numerous ways, most notably, to thermodynamics along individual stochastic trajectories driven far from equilibrium at weak [12,13] and strong [14-18] coupling with the bath, anomalous diffusion phenomena [19-22], and the so-called "frenesis" focusing on the dynamical activity-a dynamic counterpart to changes in entropy $[23,24]$. Many of these new concepts have been verified by and/or successfully applied in experiments in colloidal systems [25-27] and single-molecule experiments on nucleic acids [28-30] and larger biomolecular machines [31].

Not as much is known about transients, in particular those evolving from nonstationary initial conditions. Our present understanding of thermodynamics and in particular the kinetics in transient systems, reversible as well as irreversible, is mostly limited to small deviations from equilibrium $[9,10]$, nonequilibrium steady states [23,32-35], and statistics of the "housekeeping" heat [36,37] and entropy production [38]. The role of initial conditions in relaxation was recently studied in the context of the "Mpemba effect"- the phenomenon where a hot system can cool down faster than the same system initiated at

Published by the American Physical Society under the terms of the Creative Commons Attribution 4.0 International license. Further distribution of this work must maintain attribution to the author(s) and the published article's title, journal citation, and DOI. Open access publication funded by the Max Planck Society. a lower temperature [39,40]. Notable recent advances include an information-theoretic bound on the entropy production during relaxation far from equilibrium [41] and a spectral duality between relaxation and first passage processes $[42,43]$.

It is meanwhile possible to probe the transient, nonequilibrium dynamics of colloids and single molecules, e.g., by temperature-modulated particle tracking [4] and timemodulated [44], temperature-modulated [45], temperaturejump [46], and holographic [47] optical tweezers, as well as optical pushing [48]. These experiments allow for systematic investigations of the dependence of relaxation on the direction of the displacement from equilibrium, which is the central question of the present Letter.

Notwithstanding all progress in the field, the dependence of relaxation on the direction of the displacement from
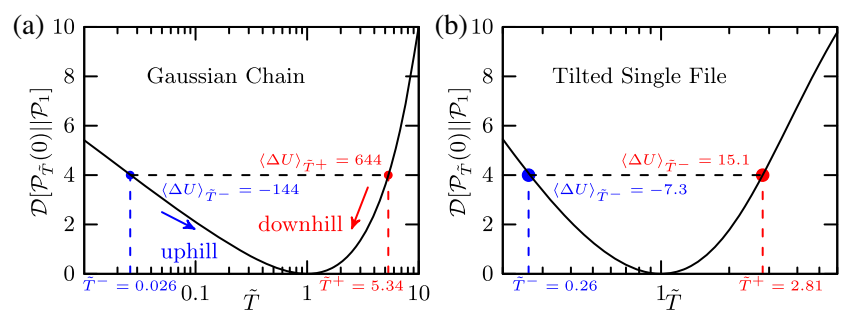

FIG. 1. Nonequilibrium free energy after a temperature quench $T \rightarrow T_{\text {eq }}$ at time $t=0$ in units of $k_{B} T_{\text {eq }}, \Delta F_{\tilde{T}}=\mathcal{D}\left[\mathcal{P}_{\tilde{T}}(t=\right.$ $\left.\left.0^{+}\right) \| \mathcal{P}_{1}^{\mathrm{eq}}\right]$ [see Eq. (3)], as a function of the relative prequench temperature $\tilde{T}=T / T_{\text {eq }}$ (note the logarithmic scale); (a) refers to the end-to-end distance of a Gaussian chain with 100 beads and (b) to the 7 th in a single file of 10 particles in a linear potential with slope 10 confined to a unit box. The blue and red points depict a pair of thermodynamically equidistant temperature quenches, $\tilde{T}^{-}$and $\tilde{T}^{+}$, with corresponding excess potential energies $\langle\Delta U\rangle_{\tilde{T}^{ \pm}} \equiv\left\langle U\left(0^{+}\right)\right\rangle_{\tilde{T}^{ \pm}}-\langle U\rangle_{1}$. 
equilibrium (see Fig. 1) remains elusive. Moreover, as a result of the projection to a lower-dimensional subspace, it is expected that observables in many experiments, in particular those tracking individual particles [4] and single molecules [46,47], relax in a manner that is not Markovian [8].

Here, we address relaxation from an instantaneous temperature quench $T \rightarrow T_{\text {eq }}$ at time $t=0$ with respect to its directionality, that is, $T^{-\uparrow} T_{\text {eq }}$ versus $T^{+} \downarrow T_{\text {eq }}$. We uncover an unforeseen dependence on the direction of the quench: For a given pair of temperatures $T^{-}<T_{\text {eq }}<T^{+}$at which the thermodynamic displacement from equilibrium at $t=0^{+}$in the sense of $\mathcal{D}_{T^{ \pm}}\left(0^{+}\right)$, the nonequilibrium free energy difference or "lag" [49-55], is equal, i.e., $\mathcal{D}_{T^{+}}\left(0^{+}\right)=\mathcal{D}_{T^{-}}\left(0^{+}\right)$(see Fig. 1), relaxation evolves, contrary to intuition, faster "uphill" $\left(\langle\Delta U\rangle_{T^{-}}<0\right)$ than "downhill" $\left(\langle\Delta U\rangle_{T^{+}}>0\right)$ in the energy landscape. This always holds for single-well potentials and occurs in near degenerate multiwell potentials with high energy barriers under Markovian dynamics, as well as for a class of non-Markovian observables probed by single-molecule and particle-tracking experiments. We demonstrate the asymmetry on the hand of the Gaussian polymer chain [56], single-file diffusion in a tilted box [8], and for diffusion in nearly degenerate multiwell potentials. For relaxation near a stable minimum and thus for all reversible OrnsteinUhlenbeck processes, we prove that the asymmetry, albeit counterintuitive, is general.

Theory.-We consider $d$-dimensional Markovian diffusion with a $d \times d$ symmetric positive-definite diffusion matrix $\mathbf{D}$ and mobility tensor $\mathbf{M}_{T}=\mathbf{D} / k_{B} T$ in a drift field $\mathbf{F}(\mathbf{x})$ such that $\mathbf{M}_{T}^{-1} \mathbf{F}(\mathbf{x})=-\nabla U(\mathbf{x})$ is a gradient flow. The evolution of the probability density at temperature $T$ is governed by the Fokker-Planck operator $\hat{\mathcal{L}}_{T} \equiv \nabla \cdot \mathbf{D} \nabla-\nabla \cdot \mathbf{M}_{T} \mathbf{F}(\mathbf{x})$. We let $G_{T}\left(\mathbf{x}, t \mid \mathbf{x}_{0}\right)$ be the Green's function of the initial value problem $\left(\partial_{t}-\right.$ $\left.\hat{\mathcal{L}}_{T}\right) G_{T}\left(\mathbf{x}, t \mid \mathbf{x}_{0}\right)=0$ and assume that the potential $U(\mathbf{x})$ is confining (i.e., $\lim _{|\mathbf{x}| \rightarrow \infty} U(\mathbf{x})=\infty$ ). This assures the existence of an invariant Maxwell-Boltzmann measure with density $\lim _{t \rightarrow \infty} G_{T}\left(\mathbf{x}, t \mid \mathbf{x}_{0}\right) \equiv P_{T}^{\mathrm{eq}}(\mathbf{x})=$ $Q_{T}^{-1} \mathrm{e}^{-U(\mathbf{x}) / k_{B} T}, \quad \forall \mathbf{x}_{0}$ with partition function $Q_{T}=$ $\int \mathrm{e}^{-U(\mathbf{x}) / k_{B} T} d \mathbf{x}$.

The system is prepared at equilibrium with a temperature $T, P_{T}^{\text {inv }}(\mathbf{x})$, whereupon an instantaneous temperature quench is performed to the ambient temperature $T_{\text {eq }}$ at $t=0$. The relaxation evolves at $T_{\text {eq }}$ according to $\hat{\mathcal{L}}_{T_{\text {eq }}}$ and for a given system it is uniquely characterized by $T$. For convenience we define $\tilde{T} \equiv T / T_{\text {eq }}[57]$ such that

$$
P_{\tilde{T}}(\mathbf{x}, t)=\int d \mathbf{x}_{0} G_{1}\left(\mathbf{x}, t \mid \mathbf{x}_{0}\right) P_{\tilde{T}}^{\mathrm{eq}}\left(\mathbf{x}_{0}\right) \underset{t \rightarrow \infty}{\rightarrow} P_{1}^{\mathrm{eq}}(\mathbf{x})
$$

The instantaneous entropy and mean energy are given by $S_{\tilde{T}}(t) \equiv-k_{B} \int d \mathbf{x} P_{\tilde{T}}(\mathbf{x}, t) \ln P_{\tilde{T}}(\mathbf{x}, t) \quad$ and $\langle U(t)\rangle_{\tilde{T}}=\int d \mathbf{x} P_{\tilde{T}}(\mathbf{x}, t) U(\mathbf{x})$, respectively, where $\langle\cdot\rangle_{\tilde{T}}$ denotes an average over all paths $\mathbf{x}(t)$ starting from $P_{\tilde{T}}^{\text {inv }}\left(\mathbf{x}_{0}\right)$.

Let the measured physical observable be $\mathbf{q}=\boldsymbol{\Gamma}(\mathbf{x})$. Its probability density function corresponds to [8]

$\mathcal{P}_{\tilde{T}}(\mathbf{q}, t)=\hat{\Pi}_{\mathbf{x}}(\mathbf{q}) P_{\tilde{T}}(\mathbf{x}, t) \equiv \int d \mathbf{x} \delta[\boldsymbol{\Gamma}(\mathbf{x})-\mathbf{q}] P_{\tilde{T}}(\mathbf{x}, t),(2)$

which in general displays non-Markovian dynamics as soon as $\mathbf{q}$ corresponds to a low-dimensional projection [8]. Once equilibrium is reached, we have $\lim _{t \rightarrow \infty} \mathcal{P}_{\tilde{T}}(\mathbf{q}, t)=\mathcal{P}_{1}^{\mathrm{eq}}(\mathbf{q})$, or, expressed via the so-called potential of mean force $\mathcal{U}(\mathbf{q})[58], \mathcal{P}_{1}^{\mathrm{eq}}(\mathbf{q})=\mathrm{e}^{-\beta_{\mathrm{eq}} \mathcal{U}(\mathbf{q})}$ [14,17,59]. Obviously, when $\boldsymbol{\Gamma}(\mathbf{x})=\mathbf{x}$, we have $\mathcal{P}_{\tilde{T}}(\mathbf{q}, t)=P_{\tilde{T}}(\mathbf{x}, t)$.

We quantify the instantaneous displacement from equilibrium with the Kullback-Leibler divergence [49-55]:

$\mathcal{D}\left[\mathcal{P}_{\tilde{T}}(t) \| \mathcal{P}_{1}^{\mathrm{eq}}\right]=\int d \mathbf{q} \mathcal{P}_{\tilde{T}}(\mathbf{q}, t) \ln \left[\mathcal{P}_{\tilde{T}}(\mathbf{q}, t) / \mathcal{P}_{1}^{\mathrm{eq}}(\mathbf{q})\right]$

Writing this out for the Markovian case, we find, upon identifying $S_{\tilde{T}}(t)$ and $\left\langle U_{\tilde{T}}(t)\right\rangle$,

$$
\mathcal{D}\left[P_{\tilde{T}}(t) \| P_{1}^{\mathrm{eq}}\right]=-S_{\tilde{T}}(t) / k_{B}+\beta_{\mathrm{eq}}\langle U(t)\rangle_{\tilde{T}}+\ln Q_{T_{\mathrm{eq}}} .
$$

Recalling the definition of free energy $F=-\beta_{\mathrm{eq}}^{-1} \ln Q_{T_{\mathrm{eq}}}$ and defining the instantaneous generalized free energy (GFE) [52] or "lag" [55] as $F_{\tilde{T}}(t)=\langle U(t)\rangle_{\tilde{T}}-T_{\text {eq }} S_{\tilde{T}}(t)$, we see, upon multiplying through by $\beta_{\text {eq }}^{-1}=k_{B} T_{\text {eq }}$, that in the Markovian case Eq. (3) is the excess GFE in units of $k_{B} T_{\text {eq }}$, i.e., $\mathcal{D}_{\tilde{T}}^{M}(t) \equiv \mathcal{D}\left[P_{\tilde{T}}(t) \| P_{1}^{\mathrm{eq}}\right]=\beta_{\text {eq }}\left[F_{\tilde{T}}(t)-F\right]$ [51,52]. Writing out Eq. (3) for the non-Markovian case and identifying $\mathcal{S}_{\tilde{T}}(t)$ and $\mathcal{U}(\mathbf{q})$ (calligraphic letters denote potentials of projected observables), we find

$$
\mathcal{D}_{\tilde{T}}^{n M}(t) \equiv \mathcal{D}\left[\mathcal{P}_{\tilde{T}}(t) \| \mathcal{P}_{1}^{\mathrm{eq}}\right]=-\mathcal{S}_{\tilde{T}}(t) / k_{B}+\beta_{\mathrm{eq}}\langle\mathcal{U}(t)\rangle_{\tilde{T}},
$$

which is the non-Markovian GFE $\mathcal{D}_{\tilde{T}}^{n M}(t)=\beta_{\text {eq }} \mathcal{F}_{\tilde{T}}(t)$. Note that $\mathcal{U}(\mathbf{q})$ itself is an effective free energy, i.e., $\quad \beta_{\text {eq }} \mathcal{U}(\mathbf{q}) \equiv-\ln \langle\delta[\boldsymbol{\Gamma}(\mathbf{x})-\mathbf{q}]\rangle_{1}=-\ln \int d \mathbf{x} \delta[\boldsymbol{\Gamma}(\mathbf{x})-$ $\mathbf{q}] \mathrm{e}^{-\beta_{\mathrm{eq}} U(\mathbf{x})}+\ln Q_{T_{\mathrm{eq}}}$ and $\mathcal{S}_{1}=-\langle\mathcal{U}\rangle_{1}$. We henceforth express energies in units of $k_{B} T_{\mathrm{eq}}$. If (and only if) latent degrees of freedom (i.e., those integrated out) relax much faster than $\mathbf{q}(t)$, Eqs. (4) and (5) are equivalent and $\mathbf{q}(t)$ is a Markovian diffusion in the free energy landscape $\mathcal{U}(\mathbf{q})$ [8]. In the absence of a time-scale separation, however, both $\mathcal{S}_{\tilde{T}}(t)$ and $\langle\mathcal{U}(t)\rangle_{\tilde{T}}$ contain contributions from the (hidden) relaxation of the latent degrees of freedom.

Consider now a pair of temperatures, $\tilde{T}^{+}>1$ and $\tilde{T}^{-}<1$, corresponding to equal displacements immediately after the quench $\mathcal{D}_{\tilde{T}^{-}}^{M, n M}\left(0^{+}\right)=\mathcal{D}_{\tilde{T}^{+}}^{M, n M}\left(0^{+}\right)$. The existence of (at least) two such temperatures is guaranteed within an interval $\tilde{T} \in\left(\tilde{T}_{\min }, \tilde{T}_{\max }\right)$ where $\mathcal{D}_{\tilde{T}}^{M, n M}\left(0^{-}\right)=f(\tilde{T})$ has 

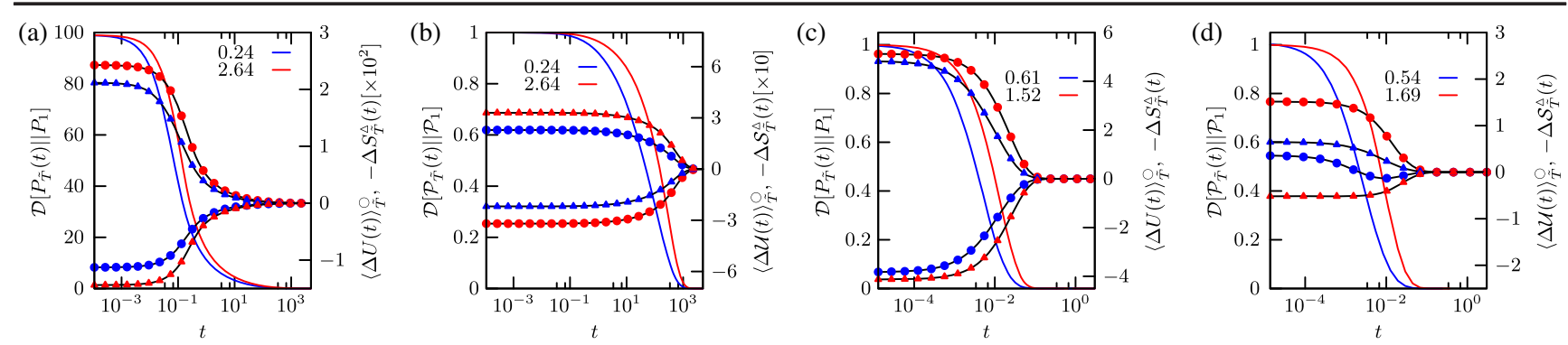

FIG. 2. $\mathcal{D}\left[\mathcal{P}_{\tilde{T}}(t) \| \mathcal{P}_{1}^{\mathrm{eq}}\right]$ (full lines) for the Gaussian chain $[(\mathrm{a}),(\mathrm{b})]$ and single file with 10 particles in a linear potential with slope $g=10$ [(s),(d)]. (a) refers to the entire chain of 100 beads [Eq. (4)] and (b) to the end-to-end distance [Eq. (5)] for equidistant quenches from $\tilde{T}^{-}=0.24$ (blue) and $\tilde{T}^{+}=2.64$ (red); (c) stands for the full single file for equidistant quenches from $\tilde{T}^{-}=0.61$ (blue) and $\tilde{T}^{+}=1.52$ (red); (d) the 7th particle for equidistant quenches from $\tilde{T}^{-}=0.54$ (blue) and $\tilde{T}^{+}=1.69$. The circles refer to $\langle\Delta U(t)\rangle_{\tilde{T}^{ \pm}}$and $\langle\Delta \mathcal{U}(t)\rangle_{\tilde{T}^{ \pm}}$ in (a) and (c), and (b) and (d), respectively, and triangles denote $\Delta S_{\tilde{T}^{ \pm}}(t)$ and $\Delta \mathcal{S}_{\tilde{T}}(t)$. Note the second axes for $\langle\Delta U(t)\rangle_{\tilde{T}^{ \pm}},\langle\Delta \mathcal{U}(t)\rangle_{\tilde{T}^{ \pm}}$ and $\Delta S_{\tilde{T}^{ \pm}}(t), \Delta \mathcal{S}_{\tilde{T}}(t)$. Note that $\mathcal{S}_{\tilde{T}}(\infty)=\mathcal{S}_{1}=-\langle\mathcal{U}\rangle_{1}$.

no local maximum. The central question of this Letter addresses the rate of the "uphill" ( $\left.\tilde{T}^{-}<1\right)$ versus "downhill" $\left(\tilde{T}^{+}>1\right)$ relaxation.

Gaussian Chain.-In the context of single-molecule experiments, we consider the overdamped dynamics of a chain of $N+1$ beads with coordinates $\left\{\mathbf{r}_{i}\right\}$ connected by harmonic springs with potential $U\left(\left\{\mathbf{r}_{i}\right\}\right)=$ $\sum_{i=1}^{N}\left(\mathbf{r}_{i+1}-\mathbf{r}_{i}\right)^{2}$ (general harmonic networks are treated in [60]). In the Markovian setting, we consider all monomers, $P_{\tilde{T}}\left(\left\{\mathbf{r}_{i}\right\}, t\right)$ in Eq. (1), while single-molecule experiments (e.g., Förster resonance energy transfer [73,74] or optical tweezers $[46,47])$ typically track a single (e.g., end to end) distance within the macromolecule $\mathbf{q} \equiv d=$ $\left|\mathbf{r}_{1}-\mathbf{r}_{N}\right|$ with $\mathcal{P}_{\tilde{T}}(d, t)$ from Eq. (2), evolving according to non-Markovian dynamics.

The excess GFE is given by (see derivation in [60])

$$
\begin{gathered}
\mathcal{D}_{\tilde{T}}^{M}(t)=\frac{3}{2} \sum_{k=1}^{N}\left[\Lambda_{k}^{\tilde{T}}(t)-1-\ln \Lambda_{k}^{\tilde{T}}(t)\right] \\
\mathcal{D}_{\tilde{T}}^{n M}(t)=\frac{3}{2}\left[\frac{\mathcal{A}_{\tilde{T}}^{1 N}(t)}{\mathcal{A}_{1}^{1 N}(0)}-1-\ln \frac{\mathcal{A}_{\tilde{T}}^{1 N}(t)}{\mathcal{A}_{1}^{1 N}(0)}\right],
\end{gathered}
$$

where $\quad \Lambda_{k}^{\tilde{T}}(t) \equiv 1+(\tilde{T}-1) \mathrm{e}^{-2 \mu_{k} t} \quad$ with $\quad \mu_{k}=$ $4 \sin ^{2}\{k \pi /[2(N+1)]\}$, and we introduced $\mathcal{A}_{\tilde{T}}^{i j}(t) \equiv$ $\sum_{k=1}^{N} \Lambda_{k}^{\tilde{T}}(t) \mathcal{C}_{k}^{i j} / 2 \mu_{k}$ with $\mathcal{C}_{k}^{i j} \geq 0$ given explicitly in [60]. The initial excess free energies are both convex in $\tilde{T}$ and read

$$
\mathcal{D}_{\tilde{T}}^{M}\left(0^{+}\right)=3 N(\tilde{T}-1-\ln \tilde{T}) / 2=N \mathcal{D}_{\tilde{T}}^{n M}\left(0^{+}\right) .
$$

The instantaneous potential energy of the full system and the potential of mean force in turn read $\langle U(t)\rangle_{\tilde{T}}=$ $\frac{3}{2} \sum_{k=1}^{N} \Lambda_{k}^{\tilde{T}}(t) \quad$ and $\quad \mathcal{U}(d)=-\ln \mathcal{P}_{1}^{\mathrm{eq}}(d), \quad$ respectively. Aside from specific values of $\mu_{k}$ and $\mathcal{C}_{k}^{i j}$, Eqs. (6)-(8) hold for any reversible Ornstein-Uhlenbeck" process (OUp), that is, for any $\tilde{T}$, connectivity or stability matrix, and tagged distance.

The results for $\mathcal{D}_{\tilde{T}}^{M, n M}(t)$ and their decomposition into $\langle U\rangle_{\tilde{T}},\langle\mathcal{U}(t)\rangle_{\tilde{T}}, S_{\tilde{T}}(t)$, and $\mathcal{S}_{\tilde{T}}(t)$ for a pair of equidistant temperature quenches are shown in Fig. 2 and demonstrate that the uphill relaxation is always faster than the downhill relaxation. As we prove below, this is true for any reversible OUp quenched arbitrarily far from equilibrium.

The energy and entropy differences relative to their equilibrium values (i.e., at $t=\infty$ ) in Fig. 2(a) suggest that the Markovian uphill and downhill relaxation are dominated by $\langle\Delta U(t)\rangle_{\tilde{T}^{+}}$and $\Delta S_{\tilde{T}^{-}}$, respectively. Surprisingly, entropy pushing the system uphill against the deterministic force is more efficient. Notably, the magnitude of individual contributions is smaller for uphill relaxation, i.e., $\langle\Delta U\rangle_{\tilde{T}^{+}}>-\langle\Delta U\rangle_{\tilde{T}^{-}}$and $\Delta S_{\tilde{T}^{+}}>-\Delta S_{\tilde{T}^{-}}$. Thus, a larger energy excess and entropy deficit are dissipated during downhill relaxation. Conversely, the partitioning into $\mathcal{S}_{\tilde{T}}(t)$ and $\langle\mathcal{U}(t)\rangle_{\tilde{T}}$ of the non-Markovian relaxation depends on the details of the projection and is less intuitive [in our example in Fig. 2(b) it is, in fact, reversed].

To explain why uphill relaxation is faster, we inspect in Fig. 3 local contributions to $\mathcal{D}_{\tilde{T}}^{M}(t)$ for a one-dimensional OUp. An uphill quench localizes $P_{\tilde{T}^{-}}\left(x, 0^{+}\right)$near the origin, whereas a downhill quench broadens $P_{\tilde{T}^{+}}\left(x, 0^{+}\right)$, rendering the integrand of Eq. (4) nonzero over a larger domain [Fig. 3(a), red line]. The evolution of $P_{\tilde{T}}(x, t)$ is driven by diffusion $\propto \partial_{x}^{2} P_{\tilde{T}}$ and advection $\propto \partial_{x} x P_{\tilde{T}}$. By forcing probability mass toward the origin, advection seems to oppose uphill relaxation [triangles in Fig. 3(b)] but thereby actually sustains an even faster diffusion rate compared to free diffusion [compare circles and dashed line in Fig. 3(b)]. The net effect is an overall relaxation nearly as fast as free diffusion [compare full and dashed line in Fig. 3(b)]. Downhill relaxation is advection-dominated and weakly opposed by diffusion, which is almost unaffected by the potential [Fig. 3(c)]. The overall dynamics is much slower [compare full lines in Fig. 3(b), (c)]. Faster diffusion from a localized initial distribution thereby renders uphill 


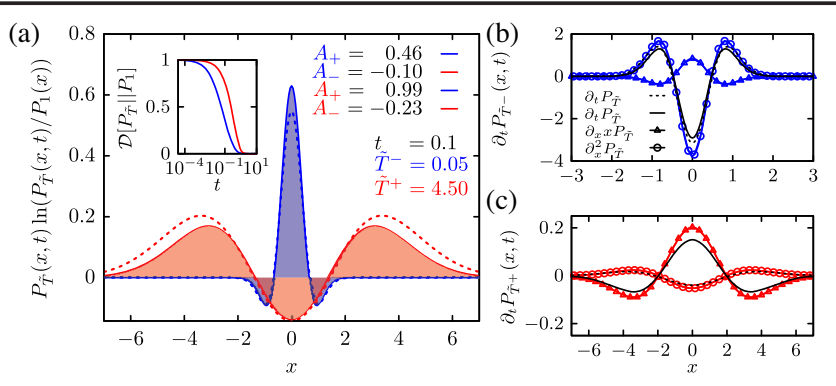

FIG. 3. (a) Integrand of Eq. (3) at $t=0.1$ for a one-dimensional OUp (full line) for uphill (blue) and downhill (red) relaxation with the positive $A_{+}$and negative $A_{-}$area under the curve. Inset: The corresponding $\mathcal{D}_{\tilde{T}}^{M}(t)$. (b), (c) Decomposition of $\partial_{t} P_{\tilde{T}}(x, t)$ into diffusive $\partial_{x}^{2} P_{\tilde{T}}$ (circles) and advective $\partial_{x} x P_{\tilde{T}}$ (triangles) contribution for uphill (b) and downhill (c) relaxation. Dashed lines correspond to free diffusion evolving from the same initial condition.

relaxation faster - an effect that will exist in any confining potential well with ruggedness $\ll k_{B} T_{\text {eq }} . \hat{\mathcal{L}}_{T}$ of any reversible OUp is diagonalizable and thus uniquely decomposable into one-dimensional OUps, extending our explanation to arbitrary dimensions.

Non-Markovian relaxation displays the same asymmetry, but the dominant driving forces, here $\mathcal{S}_{\tilde{T}}$ and $\left\langle\mathcal{U}_{\tilde{T}}\right\rangle$, may become reversed [see Fig. 2(b)]. Since $\mathcal{U}_{\tilde{T}}$ contains entropic effects of latent degrees of freedom, the partitioning between $\mathcal{S}_{\tilde{T}}$ and $\left\langle\mathcal{U}_{\tilde{T}}\right\rangle$ is in general projectiondependent.

Tilted single file.-In the context of tracer-particle dynamics, we consider $N$ hard-core Brownian point particles with positions $\left\{x_{i}(t)\right\}$ (the extension to a finite diameter is straightforward [5,6]) diffusing in a box of unit length in the presence of a linear potential (e.g., the gravitational field), $U\left(\left\{x_{i}\right\}\right)=\sum_{i=1}^{N} g x_{i}$. The probability density of $\left\{x_{i}(t)\right\}$ upon a quench from $\tilde{T}, P_{\tilde{T}}\left(\left\{x_{i}(t)\right\}, t\right)$, evolves according to $\hat{\mathcal{L}}_{1}=\sum_{i=1}^{N}\left(\partial_{x_{i}}^{2}+g \partial_{x_{i}}\right)$ under noncrossing conditions $[7,8]$. In [60] we solve the problem exactly via the coordinate Bethe ansatz [7,8], both for the Markovian complete single file and the nonMarkovian probability density of a tagged particle $\mathcal{P}_{\tilde{T}}(z, t)$ (i.e., $\mathbf{q} \equiv x_{\mathcal{T}}=z$ ).

$\mathcal{D}_{\tilde{T}}^{M}(t)$ with corresponding $\langle\Delta U(t)\rangle_{\tilde{T}},\langle\mathcal{U}(t)\rangle_{\tilde{T}}, \Delta S_{\tilde{T}}(t)$, and $\mathcal{S}_{\tilde{T}}(t)$ for the complete and tagged particle dynamics are shown in Fig. 2(c), (d). As for the Gaussian chain uphill relaxation in the tilted single file, both full as well as for a tagged particle seems to always be faster irrespective of which particle we tag, and for any $\tilde{T}, N$ and tilting strength $g>0$ (see also [60]). The Markovian uphill relaxation is dominated by $\Delta S_{\tilde{T}^{-}}(t)$ and downhill by $\langle\Delta U(t)\rangle_{\tilde{T}^{+}}$, and a larger energy and entropy difference must be dissipated during downhill relaxation [see Fig. 2(c)]. For a tagged particle, the partitioning between $\langle\mathcal{U}(t)\rangle_{\tilde{T}^{-}}$and $\mathcal{S}(t)_{\tilde{T}^{-}}$ varies depending on which particle we tag as a result of the shape of $\mathcal{U}(z)$ and the dependence of $\mathcal{P}_{\tilde{T}}\left(z, 0^{-}\right)$on $\tilde{T}$, which in turn both depend on the tagged particle as well as $T_{\text {eq }}, N$, and $g$.

Is the asymmetry universal?-We first focus on dynamics near a stable minimum at $\mathbf{R}_{0}, \delta \mathbf{R}(t)=\mathbf{R}(t)-\mathbf{R}_{0}$, which is well described by an OUp, i.e., $d \delta \mathbf{R}(t)=$ $\mathbf{H} \delta \mathbf{R}(t) d t+\sqrt{2} d \mathbf{W}_{t}$, where $(\mathbf{H})_{i j}=\left.\sum_{i j} \partial_{R_{i}} \partial_{R_{j}} U(\mathbf{R})\right|_{\mathbf{R}_{0}}$ is the Hessian.

Theorem 1.-For a general diffusion sufficiently close to a stable minimum and for any stable reversible OUp, the relaxation from a pair of equidistant quenches of arbitrary magnitude (as defined above) is always faster uphill.

Proof of Theorem 1.-Any pair $0<\tilde{T}^{-} \leq 1$ and $1 \leq$ $\tilde{T}^{+}<\infty$ with $\mathcal{D}_{\tilde{T}^{+}}\left(0^{+}\right)=\mathcal{D}_{\tilde{T}^{-}}\left(0^{+}\right)$satisfies by construction $\tilde{T}^{+}-\tilde{T}^{-}=\ln \left(\tilde{T}^{+} / \tilde{T}^{-}\right)$. We first prove the claim for the Markovian setting, where Eq. (6) has the structure $\mathcal{D}_{\tilde{T}}^{M}(t)=\sum_{k=1}^{N} \mathcal{D}_{k}^{ \pm}(t)$. We set $\varphi \equiv \tilde{T}^{+} / \tilde{T}^{-}>1$, $\delta_{ \pm} \equiv \tilde{T}^{ \pm}-1$, and write $\Delta \mathcal{D}_{k}(t) \equiv \mathcal{D}_{k}^{+}(t)-\mathcal{D}_{k}^{-}(t)=$ $\ln Z_{\varphi}\left(\mu_{k} t\right)$, such that

$$
\begin{aligned}
Z_{\varphi}(\tau) & =\varphi^{\mathrm{e}^{-\tau}}\left(1+\delta_{-} \mathrm{e}^{-\tau}\right) /\left(1+\delta_{+} \mathrm{e}^{-\tau}\right) \\
& =\left[\varphi\left(1+\delta_{-} \mathrm{e}^{-\tau}\right) \mathrm{e}^{\tau^{\tau}}\right]^{-\tau} /\left(1+\delta_{+} \mathrm{e}^{-\tau}\right) \\
& \geq\left[\varphi\left(1+\delta_{-}\right) /\left(1+\delta_{+}\right)\right]^{\mathrm{e}^{-\tau}} \geq 1,
\end{aligned}
$$

where we have used both generalized Bernoulli inequalities, i.e., for any real $0 \leq y_{-} \leq 1, y_{+} \geq 1$ and $x \geq-1$ we have $(1+x)^{y_{+}} \geq 1+y_{+} x$ and $(1+x)^{y_{-}} \leq 1+y_{-} x$. Recalling the definition of $\Delta \mathcal{D}_{k}(t)$ completes the proof.

To prove the claim in the non-Markovian setting for projections of type $q=\left|\delta \mathbf{R}_{i}-\delta \mathbf{R}_{j}\right|$, we first realize that $\dot{\mathcal{A}}_{\tilde{T}^{+}}^{i j}(t) \leq 0$ and $\dot{\mathcal{A}}_{\tilde{T}^{-}}{ }^{j}(t) \geq 0$, where $\dot{f}(t) \equiv(d / d t) f(t)$. Setting $\Delta \mathcal{D}(t) \equiv \mathcal{D}_{\tilde{T}^{+}}^{n M}(t)-\mathcal{D}_{\tilde{T}^{-}}^{n M}(t)$ and using Eq. (7) we find, upon taking the derivative,

$$
\Delta \dot{\mathcal{D}}(t)=\frac{\dot{\mathcal{A}}_{\tilde{T}^{+}}^{i j}(t)}{\mathcal{A}_{\tilde{T}^{+}}^{i j}(0)}-\frac{\dot{\mathcal{A}}_{\tilde{T}^{+}}^{i j}(t)}{\mathcal{A}_{\tilde{T}^{+}}^{i j}(t)}-\frac{\dot{\mathcal{A}}_{\tilde{T}^{-}}^{i j}(t)}{\mathcal{A}_{\tilde{T}^{-}}^{i j}(0)}+\frac{\dot{\mathcal{A}}_{\tilde{T}^{-}}^{i j}(t)}{\mathcal{A}_{\tilde{T}^{-}}^{i j}(t)}
$$

Eq. (10) implies $\Delta \dot{\mathcal{D}}(t) \geq 0$ because $\mathcal{A}_{\tilde{T}^{+}}^{i j}(t) \geq \mathcal{A}_{\tilde{T}^{+}}^{i j}(0)$ while $\mathcal{A}_{\tilde{T}^{-}}^{i j}(t) \leq \mathcal{A}_{\tilde{T}^{+}}^{i j}(0)$, which completes the proof.

The fact that tilted single file diffusion, being anharmonic and asymmetric with nonperturbative interactions, displays the asymmetry for quenches of arbitrary magnitude and for any steepness of the potential hints that the asymmetry might be more general. Note that tagging different particles in different slopes $g>0$, we can construct $\mathcal{U}(z)$ with arbitrary asymmetry. Alongside the physical principle underlying the asymmetry established for the OUp and Theorem 1, this strongly suggests that uphill relaxation in smooth single-well potentials could be universally faster (see [60]). Since the projection (2) is independent of $\tilde{T}$, these statements should extend also to non-Markovian observables, in particular those probed in many single-molecule and particle-tracking experiments. 
As a corollary, uphill relaxation is faster also in multiwell potentials for equidistant quenches that predominantly disturb only the intrawell equilibria, in particular for nearly degenerate basins separated by sufficiently high barriers [75] (for reasoning and examples, see [60]). This is violated in asymmetric multiwells, and examples with faster downhill relaxation are constructed in [60].

Conclusion.-We uncovered an unforeseen asymmetry in the relaxation to equilibrium in equidistant temperature quenches. Uphill relaxation was found to be faster-a phenomenon we proved to be universal for quenches of dynamics near stable minima. We hypothesize that it is a general phenomenon in reversible overdamped diffusion in single-well potentials extending to degenerate multiwell potentials for quenches leaving interwell equilibria virtually intact. The dependence on the direction of the quench, which so far seems to have been overlooked, implies a systematic asymmetry in the dissipation of the system's entropy $\dot{S}_{\tilde{T}}(t)$ versus heat $\langle\dot{U}(t)\rangle_{\tilde{T}}[12]$ and, for specific projections, the modified entropy $\dot{\mathcal{S}}_{\tilde{T}}(t)$ versus "strong coupling heat" $\langle\dot{\mathcal{U}}(t)\rangle_{\tilde{T}}[14,17]$, which seems to be relevant for the efficiency of stochastic heat engines [44,76,77]. Implying that the hot isothermal step can be shorter than the cold one, which reduces cycle times, the asymmetry may also be relevant for the optimization of the engine's output power $[44,76,77]$. Our results can readily be tested by single-molecule and particle-tracking experiments [4,44-48]. To understand the asymmetry on the level of individual trajectories, it would be interesting to analyze relaxation from equidistant quenches in terms of occupation measures $[7,8]$ and from the perspective of stochastic thermodynamics $[12,14]$.

We thank David Hartich for fruitful discussions. The financial support from the German Research Foundation (DFG) through the Emmy Noether Program GO 2762/1-1 to A. G. is gratefully acknowledged.

* Corresponding author. agodec@mpibpc.mpg.de

[1] A. Farhan, P. M. Derlet, A. Kleibert, A. Balan, R. V. Chopdekar, M. Wyss, J. Perron, A. Scholl, F. Nolting, and L. J. Heyderman, Direct Observation of Thermal Relaxation in Artificial Spin Ice, Phys. Rev. Lett. 111, 057204 (2013).

[2] S. Dattagupta, Relaxation Phenomena in Condensed Matter Physics (Elsevier, New York, 2012).

[3] H. Chen, E. Rhoades, J. S. Butler, S. N. Loh, and W. W. Webb, Dynamics of equilibrium structural fluctuations of apomyoglobin measured by fluorescence correlation spectroscopy, Proc. Natl. Acad. Sci. U.S. A. 104, 10459 (2007).

[4] P. Wang, C. Song, and H. A. Makse, Dynamic particle tracking reveals the ageing temperature of a colloidal glass, Nat. Phys. 2, 526 (2006).

[5] L. Lizana and T. Ambjörnsson, Single-File Diffusion in a Box, Phys. Rev. Lett. 100, 200601 (2008).
[6] L. Lizana and T. Ambjörnsson, Diffusion of finite-sized hard-core interacting particles in a one-dimensional box: \{Tagged\} particle dynamics, Phys. Rev. E 80, 051103 (2009).

[7] A. Lapolla and A. Godec, Unfolding tagged particle histories in single-file diffusion: exact single- and two-tag local times beyond large deviation theory, New J. Phys. 20, 113021 (2018).

[8] A. Lapolla and A. Godec, Manifestations of projectioninduced memory, Front. Phys. 7, 182 (2019).

[9] L. Onsager, Reciprocal relations in irreversible processes I, Phys. Rev. 37, 405 (1931).

[10] L. Onsager, Reciprocal relations in irreversible processes II, Phys. Rev. 38, 2265 (1931).

[11] R. Kubo, M. Yokota, and S. Nakajima, Statistical-Mechanical Theory of Irreversible Processes. II. Response to Thermal Disturbance, J. Phys. Soc. Jpn. 12, 1203 (1957).

[12] U. Seifert, Stochastic thermodynamics, fluctuation theorems and molecular machines, Rep. Prog. Phys. 75, 126001 (2012).

[13] C. Jarzynski, Equalities and inequalities: Irreversibility and the second law of thermodynamics at the nanoscale, Annu. Rev. Condens. Matter Phys. 2, 329 (2011).

[14] U. Seifert, First and Second Law of Thermodynamics at Strong Coupling, Phys. Rev. Lett. 116, 020601 (2016).

[15] P. Strasberg, G. Schaller, N. Lambert, and T. Brandes, Nonequilibrium thermodynamics in the strong coupling and non-Markovian regime based on a reaction coordinate mapping, New J. Phys. 18, 073007 (2016).

[16] P. Strasberg and M. Esposito, Stochastic thermodynamics in the strong coupling regime: An unambiguous approach based on coarse graining, Phys. Rev. E 95, 062101 (2017).

[17] C. Jarzynski, Stochastic and Macroscopic Thermodynamics of Strongly Coupled Systems, Phys. Rev. X 7, 011008 (2017).

[18] P. Talkner and P. Hänggi, Colloquium: Statistical mechanics and thermodynamics at strong coupling: quantum and classical, arXiv:1911.11660 [Rev. Mod. Phys. (to be published).]

[19] R. Metzler, E. Barkai, and J. Klafter, Anomalous Diffusion and Relaxation Close to Thermal Equilibrium: A Fractional Fokker-Planck Equation Approach, Phys. Rev. Lett. 82, 3563 (1999).

[20] R. Metzler and J. Klafter, The random walk's guide to anomalous diffusion: a fractional dynamics approach, Phys. Rep. 339, 1 (2000).

[21] J.-H. Jeon and R. Metzler, Inequivalence of time and ensemble averages in ergodic systems: Exponential versus power-law relaxation in confinement, Phys. Rev. E 85, 021147 (2012).

[22] I. M. Sokolov and J. Klafter, From diffusion to anomalous diffusion: A century after Einstein's Brownian motion, Chaos 15, 026103 (2005).

[23] M. Baiesi and C. Maes, An update on the nonequilibrium linear response, New J. Phys. 15, 013004 (2013).

[24] C. Maes, Frenetic Bounds on the Entropy Production, Phys. Rev. Lett. 119, 160601 (2017).

[25] V. Blickle, T. Speck, L. Helden, U. Seifert, and C. Bechinger, Thermodynamics of a Colloidal Particle in a 
Time-Dependent Nonharmonic Potential, Phys. Rev. Lett. 96, 070603 (2006).

[26] T. M. Hoang, R. Pan, J. Ahn, J. Bang, H. Quan, and T. Li, Experimental Test of the Differential Fluctuation Theorem and a Generalized Jarzynski Equality for Arbitrary Initial States, Phys. Rev. Lett. 120, 080602 (2018).

[27] J.-H. Jeon, N. Leijnse, L. B. Oddershede, and R. Metzler, Anomalous diffusion and power-law relaxation of the time averaged mean squared displacement in worm-like micellar solutions, New J. Phys. 15, 045011 (2013).

[28] D. Collin, F. Ritort, C. Jarzynski, S. B. Smith, I. Tinoco, and C. Bustamante, Verification of the Crooks fluctuation theorem and recovery of RNA folding free energies, Nature (London) 437, 231 (2005).

[29] E. Dieterich, J. Camunas-Soler, M. Ribezzi-Crivellari, U. Seifert, and F. Ritort, Single-molecule measurement of the effective temperature in non-equilibrium steady states, Nat. Phys. 11, 971 (2015).

[30] J. Camunas-Soler, A. Alemany, and F. Ritort, Experimental measurement of binding energy, selectivity, and allostery using fluctuation theorems, Science 355, 412 (2017).

[31] K. Hayashi, H. Ueno, R. Iino, and H. Noji, Fluctuation Theorem Applied toF1-ATPase, Phys. Rev. Lett. 104, 218103 (2010).

[32] T. Hatano and S.-i. Sasa, Steady-State Thermodynamics of Langevin Systems, Phys. Rev. Lett. 86, 3463 (2001).

[33] C. Maes, K. Netočný, and B. Wynants, Monotonic Return to Steady Nonequilibrium, Phys. Rev. Lett. 107, 010601 (2011).

[34] M. Polettini and M. Esposito, Nonconvexity of the relative entropy for Markov dynamics, Phys. Rev. E 88, 012112 (2013).

[35] C. Maes, Frenesy: Time-symmetric dynamical activity in nonequilibria, Phys. Rep. 850, 1 (2020).

[36] T. Speck and U. Seifert, Integral fluctuation theorem for the housekeeping heat, J. Phys. A 38, L581 (2005).

[37] R. Chétrite, S. Gupta, I. Neri, and É. Roldán, Martingale theory for housekeeping heat, Europhys. Lett. 124, 60006 (2019).

[38] H.-M. Chun and J.D. Noh, Universal property of the housekeeping entropy production, Phys. Rev. E 99, 012136 (2019).

[39] Z. Lu and O. Raz, Nonequilibrium thermodynamics of the Markovian Mpemba effect and its inverse, Proc. Natl. Acad. Sci. U.S.A. 114, 5083 (2017).

[40] I. Klich, O. Raz, O. Hirschberg, and M. Vucelja, Mpemba Index and Anomalous Relaxation, Phys. Rev. X 9, 021060 (2019).

[41] N. Shiraishi and K. Saito, Information-Theoretical Bound of the Irreversibility in Thermal Relaxation Processes, Phys. Rev. Lett. 123, 110603 (2019).

[42] D. Hartich and A. Godec, Duality between relaxation and first passage in reversible Markov dynamics: rugged energy landscapes disentangled, New J. Phys. 20, 112002 (2018).

[43] D. Hartich and A. Godec, Interlacing relaxation and firstpassage phenomena in reversible discrete and continuous space Markovian dynamics, J. Stat. Mech. (2019) 024002.

[44] I. A. Martínez, E. Roldán, L. Dinis, D. Petrov, J. M. R. Parrondo, and R.A. Rica, Brownian Carnot engine, Nat. Phys. 12, 67 (2016).
[45] I. A. Martínez, E. Roldán, J. M. R. Parrondo, and D. Petrov, Effective heating to several thousand kelvins of an optically trapped sphere in a liquid, Phys. Rev. E 87, 032159 (2013).

[46] S. de Lorenzo, M. Ribezzi-Crivellari, J. Arias-Gonzalez, S. Smith, and F. Ritort, A temperature-jump optical trap for single-molecule manipulation, Biophys. J. 108, 2854 (2015).

[47] J. Gladrow, M. Ribezzi-Crivellari, F. Ritort, and U. F. Keyser, Experimental evidence of symmetry breaking of transition-path times, Nat. Commun. 10, 55 (2019).

[48] G. Sitters, N. Laurens, E. de Rijk, H. Kress, E. Peterman, and G. Wuite, Optical pushing: A tool for parallelized biomolecule manipulation, Biophys. J. 110, 44 (2016).

[49] S. Kullback and R. Leibler, On information and sufficiency, Ann. Math. Stat. 22, 79 (1951).

[50] J. L. Lebowitz and P. G. Bergmann, Irreversible gibbsian ensembles, Ann. Phys. (N.Y.) 1, 1 (1957).

[51] M. C. Mackey, The dynamic origin of increasing entropy, Rev. Mod. Phys. 61, 981 (1989).

[52] H. Qian, A decomposition of irreversible diffusion processes without detailed balance, J. Math. Phys. 54, 053302 (2013).

[53] C. Van den Broeck and M. Esposito, Three faces of the second law. II. Fokker-Planck formulation, Phys. Rev. E 82, 011144 (2010).

[54] M. Esposito and C. Van den Broeck, Three Detailed Fluctuation Theorems, Phys. Rev. Lett. 104, 090601 (2010).

[55] S. Vaikuntanathan and C. Jarzynski, Dissipation and lag in irreversible processes, Europhys. Lett. 87, 60005 (2009).

[56] M. Doi and S.F. Edwards, The Theory of Polymer Dynamics (Clarendon Press, Oxford, 1988).

[57] $T>T_{\text {eq }}$ implies $\tilde{T}>1$ and $T<T_{\text {eq }}$ implies $0<\tilde{T}<1$.

[58] The name comes from the fact that $\mathcal{U}(\mathbf{q})$ delivers the mean force, i.e., $-\nabla_{\mathbf{q}} \mathcal{U}(\mathbf{q})=-\left\langle\nabla_{\mathbf{x}} U(\mathbf{x}) \delta[\mathbf{\Gamma}(\mathbf{x})-\mathbf{q}]\right\rangle$.

[59] J. G. Kirkwood, Statistical mechanics of fluid mixtures, J. Chem. Phys. 3, 300 (1935).

[60] See Supplemental Material at http://link.aps.org/ supplemental/10.1103/PhysRevLett.125.110602 for details of calculations, proofs, and supplementary examples, as well as Refs. [61-72].

[61] I. Chatzigeorgiou, Bounds on the lambert function and their application to the outage analysis of user cooperation, IEEE Commun. Lett. 17, 1505 (2013).

[62] A. Godec, T. Ukmar, M. Gaberšček, and F. Merzel, Inversion of pore size dependence of solute transport kinetics from increasingly attractive ordered porous matrix, Europhys. Lett. 92, 60011 (2010).

[63] E. B. Mpemba and D. G. Osborne, Cool?, Phys. Educ. 14, 410 (1979)

[64] M. Jeng, The Mpemba effect: When can hot water freeze faster than cold?, Am. J. Phys. 74, 514 (2006).

[65] J. I. Katz, When hot water freezes before cold, Am. J. Phys. 77, 27 (2009).

[66] P. Chaddah, S. Dash, K. Kumar, and A. Banerjee, Overtaking while approaching equilibrium, arXiv:1011.3598.

[67] P. A. Greaney, G. Lani, G. Cicero, and J. C. Grossman, Mpemba-like behavior in carbon nanotube resonators, Metall. Mater Trans. A 42, 3907 (2011). 
[68] C. Hu, J. Li, S. Huang, H. Li, C. Luo, J. Chen, S. Jiang, and L. An, Conformation directed mpemba effect on polylactide crystallization, Cryst. Growth Des. 18, 5757 (2018).

[69] Y.-H. Ahn, H. Kang, D.-Y. Koh, and H. Lee, Experimental verifications of $\{$ Mpemba\}-like behaviors of clathrate hydrates, Korean J. Chem. Eng. 33, 1903 (2016).

[70] A. Gijón, A. Lasanta, and E. R. Hernández, Paths towards equilibrium in molecular systems: The case of water, Phys. Rev. E 100, 032103 (2019).

[71] M. Baity-Jesi et al. (J. Collaboration), The Mpemba effect in spin glasses is a persistent memory effect, Proc. Natl. Acad. Sci. U.S.A. 116, 15350 (2019).

[72] I. Klich and M. Vucelja, Solution of the Metropolis dynamics on a complete graph with application to the Markov chain Mpemba effect, arXiv:1812.11962.
[73] S. Yang, J. B. Witkoskie, and J. Cao, Single-molecule dynamics of semiflexible Gaussian chains, J. Chem. Phys. 117, 11010 (2002).

[74] C. Joo, H. Balci, Y. Ishitsuka, C. Buranachai, and T. Ha, Advances in single-molecule fluorescence methods for molecular biology, Annu. Rev. Biochem. 77, 51 (2008).

[75] G. J. Moro, Kinetic equations for site populations from the Fokker-Planck equation, J. Chem. Phys. 103, 7514 (1995).

[76] T. Schmiedl and U. Seifert, Efficiency at maximum power: An analytically solvable model for stochastic heat engines, Europhys. Lett. 81, 20003 (2008).

[77] H. Ouerdane, Y. Apertet, C. Goupil, and P. Lecoeur, Continuity and boundary conditions in thermodynamics: From Carnot's efficiency to efficiencies at maximum power, Eur. Phys. J. Special Topics 224, 839 (2015). 


\title{
Supplementary Material for: \\ Faster uphill relaxation in thermodynamically equidistant temperature quenches
}

\author{
Alessio Lapolla and Aljaž Godec \\ Mathematical bioPhysics Group, Max Planck Institute for Biophysical Chemistry, 3707r Göttingen, Germany
}

\begin{abstract}
In this Supplementary Material (SM) we present detailed derivations of the main results for the Gaussian-Chain and tilted single-file diffusion model presented in the main Letter, as well as several supplementary examples with figures. We also present counterexamples demonstrating that the uphilldownhill asymmetry is not universal as it vanishes in sufficiently asymmetric multi-well potentials. However, we establish generic conditions under which the asymmetry is obeyed. Finally, we also discuss the non-Markovian Mpemba effect.
\end{abstract}

\section{GAUSSIAN CHAIN AND ORNSTEIN-UHLENBECK PROCESS}

We consider a Gaussian Chain with $\mathrm{N}+1$ beads with coordinates $\mathbf{R}=\left\{\mathbf{r}_{i}\right\}$ connected by harmonic springs with potential energy $U(\mathbf{R})=\frac{1}{2} \sum_{i=1}^{N}\left|\mathbf{r}_{i}-\mathbf{r}_{i+1}\right|^{2}$. The overdamped Langevin equation governing the dynamics of a Gaussian Chain with $\mathrm{N}+1$ beads connected by ideal springs with zero rest-length and diffusion coefficient $D$ is given by the set of coupled Itô equations

$$
\begin{aligned}
d \mathbf{r}_{1}(t) & =\left[-\mathbf{r}_{1}(t)+\mathbf{r}_{2}(t)\right] d t+\sqrt{2 D} \boldsymbol{\xi}_{1}(t) \\
d \mathbf{r}_{i}(t) & =\left[\mathbf{r}_{i-1}(t)-2 \mathbf{r}_{i}(t)+\mathbf{r}_{i+1}(t)\right] d t+\sqrt{2 D} \boldsymbol{\xi}_{i}(t) \\
d \mathbf{r}_{N+1}(t) & =\left[-\mathbf{r}_{N+1}(t)+\mathbf{r}_{N}(t)\right] d t+\sqrt{2 D} \boldsymbol{\xi}_{N+1}(t),
\end{aligned}
$$

where $\boldsymbol{\xi}_{i}(t)$ stands for zero mean Gaussian white noise, i.e.

$$
\left\langle\boldsymbol{\xi}_{i}(t)\right\rangle=0, \quad\left\langle\xi_{i, k}(t) \xi_{i, l}\left(t^{\prime}\right)\right\rangle=\delta_{k l} \delta\left(t-t^{\prime}\right) .
$$

It is straightforward to generalize these formulas to any reversible $M$-dimensional Ornstein-Uhlenbeck process $\mathbf{R}(t) \equiv$ $\left\{\mathbf{r}_{i}(t)\right\}$ with some $\mathbb{R}^{M} \times \mathbb{R}^{M}$ symmetric force matrix $\boldsymbol{\Xi}$ and potential energy function $U(\mathbf{R})=\frac{1}{2} \mathbf{R}^{T} \boldsymbol{\Xi} \mathbf{R}$

$$
d \mathbf{R}(t)=\boldsymbol{\Xi R}(t) d t+\sqrt{2} d \mathbf{W}_{t}
$$

where $d \mathbf{W}_{t}$ is the $M$-dimensional super-vector of independent Wiener increments with zero mean and unit variance, $\mathbb{E}\left[d W_{i, t} d W_{j, t^{\prime}}\right]=\delta_{i, j} \delta\left(t-t^{\prime}\right)$. In this super-vector/super-matrix notation the Gaussian chain is recovered by introducing $\mathbb{R}^{3(N+1)} \times \mathbb{R}^{3(N+1)}$ tridiagonal super-matrix $\boldsymbol{\Xi}$ with elements

$$
\boldsymbol{\Xi}_{i i}=\mathbb{1}, \quad \boldsymbol{\Xi}_{i i+1}=\boldsymbol{\Xi}_{i i-1}=\left(-1+(-1)^{\delta_{i, 1}+\delta_{i, N+1}}\right) \mathbb{1},
$$

where $\mathbb{1}$ is the $3 \times 3$ identity matrix. This leads to the equations of motion presented in the Letter. Since $\boldsymbol{\Xi}$ is supposed to be symmetric these equations can be decoupled by diagonalizing $\boldsymbol{\Xi}$ i.e. by passing to normal coordinates $\mathbf{R} \rightarrow \mathbf{X} \equiv\left\{\mathbf{x}_{i}\right\}:$

$$
\mathbf{A}^{T} \mathbf{\Xi A}=\operatorname{diag}(\boldsymbol{\mu})
$$

where the diagonal matrix has elements $\operatorname{diag}(\boldsymbol{\mu})_{k k}=\mu_{k}$. This yields eigenvalues $\mu_{i}$ and orthogonal super-matrices $(\mathbf{A})_{i j}$, where the $i$ th column $A_{j i}^{\mathbf{k}}, j=1, N+1$ with $\mathbf{k}=1,2,3$ corresponds to a "vector" of eigenvectors $x_{i}$, i.e. $\mathbf{x}_{i}=\left\{x_{i+1}, x_{i+2}, x_{i+3}\right\}$ with eigenvalues $\mu_{i+k}$ with $k=1,2,3$. In the specific case of the Gaussian chain $(\mathbf{A})_{i j}$ refer to super-matrices $(\mathbf{A})_{i j} \equiv A_{i j} \mathbb{1}$, where the $i$ th column $A_{j i}, j=1, M$ corresponds to an eigenvector of the 1-dimensional contraction of $\boldsymbol{\Xi}$ (see e.g. Eq. (S4) for the Gaussian chain, i.e. $\boldsymbol{\Xi}_{i i} \rightarrow 1$ and $\boldsymbol{\Xi}_{i i-1} \rightarrow\left(-1+(-1)^{\delta_{i, 1}+\delta_{i, N+1}}\right)$ ).

In the particular case of the Gaussian chain with $M=3(N+1)$ the eigenvalues and eigenvectors read

$$
\mu_{k}=4 \sin ^{2}\left(\frac{k \pi}{2(N+1)}\right), \quad A_{i j}=\sqrt{\frac{2^{1-\delta_{j, 0}}}{N+1}} \cos \left[\frac{(2 i-1) j \pi}{2(N+1)}\right] .
$$


The back-transformation in general corresponds to $\left(\mathbf{r}_{i}\right)_{k}=\sum_{j=1}^{M} A_{i j}^{k}\left(\mathbf{x}_{j}\right)_{k}$. In normal coordinates the the potential energy reads $U(\mathbf{X})=\frac{1}{2} \sum_{k} \mu_{k} x_{k}^{2}$ while the corresponding Fokker-Planck equation for the evolution of the Green's function of internal degrees of freedom (i.e. excluding center of mass motion) at a temperature $T, G_{T}\left(\mathbf{x}, t \mid \mathbf{x}_{0}\right)$, reads

$$
\left[\partial_{t}-D \sum_{k}^{\prime}\left(\partial_{\mathbf{x}_{k}}^{2}+\beta \mu_{k} \partial_{\mathbf{x}_{k}} \mathbf{x}_{k}\right)\right] G_{T}\left(\mathbf{x}, t \mid \mathbf{x}_{0}\right)=\delta\left(\mathbf{x}-\mathbf{x}_{0}\right)
$$

where $\beta=1 / k_{\mathrm{B}} T$ and the primed sum runs over all non-zero eigenvalues $\mu_{k}$, i.e. $\sum_{k}^{\prime}=\sum_{k ; \mu_{k} \neq 0}$. Note that we are interested only in internal dynamics and not on the center-of-mass dynamics, therefore we ignore in Eq. (S7) and what follows all contributions with $\mu_{k}=0$, as these pertain to (ideal) rigid-body motions motion (i.e. center of mass translation and rotation). Alternatively, we consider expansions around stable minima, such that $\boldsymbol{\Xi}$ is positive definite. Without any loss of generality we henceforth set $D=1$ and measure energies in units of $k_{\mathrm{B}} T_{\text {eq }}$, where $T_{\text {eq }}$ is the equilibrium (post-quench) temperature as defined in the manuscript. Moreover, since we are only interested in the evolution at temperature $T_{\text {eq }}$, we further express temperature relative to $T_{\text {eq }}$, i.e. $\tilde{T} \equiv T / T_{\text {eq }}$, such that $\tilde{T}=1$ corresponds to $T_{\text {eq }}$. The stationary solution of Eq. (S7) corresponds to the Boltzmann-Gibbs density

$$
P_{\tilde{T}}^{\mathrm{eq}}(\mathbf{X})=\prod_{k}^{\prime}\left(\frac{\mu_{k}}{2 \pi}\right)^{3 / 2} \exp \left(-\frac{\mu_{k} \mathbf{x}_{k}^{2}}{2 \tilde{T}}\right)
$$

where $\prod_{k}{ }^{\prime}=\prod_{k ; \mu_{k} \neq 0}$. The probability density of $\mathbf{X}$ starting from an initial probability density function $P_{\tilde{T}}^{\mathrm{eq}}(\mathbf{X})$ is obtained from the Green's function via

$$
P_{\tilde{T}}(\mathbf{X}, t)=\int d \mathbf{X}_{0} G_{1}\left(\mathbf{X}, t \mid \mathbf{X}_{0}\right) P_{\tilde{T}}^{\mathrm{eq}}\left(\mathbf{X}_{0}\right)
$$

where

$$
G_{1}\left(\mathbf{X}, t \mid \mathbf{X}_{0}, 0\right)=\prod_{k}^{\prime}\left(\frac{\mu_{k}}{2 \pi\left(1-\mathrm{e}^{-2 \mu_{k} t}\right)}\right)^{3 / 2} \exp \left[-\frac{\mu_{k}}{2\left(1-\mathrm{e}^{-2 \mu_{k} t}\right)}\left(\mathbf{x}_{k}^{2}-2 \mathbf{x}_{k} \cdot \mathbf{x}_{0 k} \mathrm{e}^{-\mu_{k} t}+\mathbf{x}_{0 k}^{2} \mathrm{e}^{-2 \mu_{k} t}\right)\right]
$$

is the well-known Green's function of an Ornstein-Uhlenbeck process. Note that $\lim _{t \rightarrow \infty} G_{\tilde{T}}\left(\mathbf{X}, t \mid \mathbf{X}_{0}, 0\right)=P_{\tilde{T}}^{\mathrm{eq}}(\mathbf{X})$. The intergal Eq. (S9) can easily be performed analytically and yields

$$
P_{\tilde{T}}(\mathbf{X}, t)=\prod_{k}^{\prime}\left(\frac{\mu_{k}}{2 \pi\left[1+(\tilde{T}-1) \mathrm{e}^{-2 \mu_{k} t}\right]}\right) \exp \left(-\frac{\mu_{k} \mathbf{x}_{k}^{2}}{2\left[1+(\tilde{T}-1) \mathrm{e}^{-2 \mu_{k} t}\right]}\right) .
$$

Eq. (S11) can now be used to calculate the Kullback-Leibler divergence (Eq. (3) in the Letter) to yield the first of Eqs. (8) in the Letter. Furthermore, the average potential energy and the system's entropy are defined as

$$
\langle U(t)\rangle_{\tilde{T}} \equiv \int d \mathbf{x} P_{\tilde{T}}(\mathbf{x}, t) U(\mathbf{x}), \quad S_{\tilde{T}}(t)=-\int d \mathbf{x} P_{\tilde{T}}(\mathbf{x}, t) \ln P_{\tilde{T}}(\mathbf{x}, t)
$$

and read, upon performing the integration and introducing $\Lambda_{k}^{\tilde{T}}(t) \equiv 1+(\tilde{T}-1) \mathrm{e}^{-2 \mu_{k} t}$,

$$
\langle U(t)\rangle_{\tilde{T}}=\frac{3}{2} \sum_{k}^{\prime} \Lambda_{k}^{\tilde{T}}(t), \quad S_{\tilde{T}}(t)=\frac{3}{2} \sum_{k}^{\prime}\left[1-\ln \left(\frac{\mu_{k}}{2 \pi \Lambda_{k}^{\tilde{T}}(t)}\right)\right] .
$$

In the projected, non-Markovian setting we are interested in the dynamics of an internal distance $d_{i j}(t) \equiv \mid \mathbf{r}_{i}(t)-$ $\mathbf{r}_{j}(t) \mid$. In normal coordinates this corresponds to

$$
d_{i j} \equiv\left|\mathbf{r}_{i}-\mathbf{r}_{j}\right|=\sum_{k}^{\prime}\left|\left(A_{i k}-A_{j k}\right) \mathbf{x}_{k}\right|
$$

By doing so we project out $3(N-1)$ latent degrees of freedom and track only $d_{i j}$. The 'non-Markovian Green's function', that is, the probability density of $d_{i j}$ and time $t$ given that the full system evolves from $P_{\tilde{T}}^{\text {eq }}\left(\mathbf{X}_{0}\right)$ is defined 
as

$$
\begin{aligned}
\mathcal{P}_{\tilde{T}}(d, t) & =\int d \Omega \int d \mathbf{X}_{0} \delta\left(\sum_{k}^{\prime}\left[A_{i k}-A_{j k}\right] \mathbf{x}_{k}-\mathbf{d}\right) G_{1}\left(\mathbf{X}, t \mid \mathbf{X}_{0}, 0\right) P_{\tilde{T}}^{\mathrm{eq}}\left(\mathbf{X}_{0}\right) \\
& =d^{2} \int_{0}^{\infty} d l_{0} l_{0}^{2} \int d \Omega \int d \Omega_{0} \delta\left(\sum_{k}^{\prime}\left[A_{i k}-A_{j k}\right] \mathbf{x}_{k}-\mathbf{d}\right) \delta\left(\sum_{k}^{\prime}\left[A_{i k}-A_{j k}\right] \mathbf{x}_{k, 0}-\mathbf{l}_{0}\right) G_{1}\left(\mathbf{X}, t \mid \mathbf{X}_{0}, 0\right) P_{\tilde{T}}^{\mathrm{eq}}\left(\mathbf{X}_{0}\right) \\
& \equiv \int_{0}^{\infty} d l_{0} \mathcal{P}_{\tilde{T}}\left(d, t, l_{0} ; P_{\tilde{T}}^{\mathrm{eq}}\right)
\end{aligned}
$$

where we first project onto the vectors $\mathbf{d}$ and $\mathbf{d}_{0}$ and afterwards marginalize over all respective angles $\Omega$ and $\Omega_{0}$. Note that the stept in line 2 of Eq. (S15) is actually not necessary but is preferable if one also wants to access the general non-Markovian two-point joint density $\mathcal{P}_{\tilde{T}}\left(d, t, d_{0} ; P_{\tilde{T}}^{\text {eq }}\right)$. The calculation proceeds as follows.

We first preform two 3-dimensional Fourier transforms $\mathbf{d}_{0} \rightarrow \mathbf{u}$ and $\mathbf{d} \rightarrow \mathbf{v}$ :

$$
\begin{aligned}
\hat{\mathcal{P}}_{\tilde{T}}\left(\mathbf{u}, t, \mathbf{v}_{0} ; P_{\tilde{T}}^{\mathrm{eq}}\right) & \equiv \frac{1}{(2 \pi)^{6}} \int d \mathbf{d} \mathrm{e}^{-i \mathbf{v} \cdot \mathbf{d}} \int d \mathbf{d}_{0} \mathrm{e}^{-i \mathbf{u} \cdot \mathbf{d}_{0}} \mathcal{P}_{\tilde{T}}\left(\mathbf{d}, t, \mathbf{d}_{0} ; P_{\tilde{T}}^{\mathrm{eq}}\right) \\
& =\frac{1}{(2 \pi)^{6}} \prod_{k}^{\prime} \exp \left[-\frac{\mathcal{C}_{k}^{i j}}{2 \mu_{k}}\left(1+(\tilde{T}-1) \mathrm{e}^{-2 \mu_{k} t}\right) \mathbf{v}^{2}-\frac{\mathcal{C}_{k}^{i j}}{2 \mu_{k}} \mathbf{u}^{2}-2 \frac{\mathcal{C}_{k}^{i j}}{2 \mu_{k}} \mathrm{e}^{-\mu_{k} t} \mathbf{v} \cdot \mathbf{u}\right],
\end{aligned}
$$

where we have introduced the short-hand notation

$$
\mathcal{C}_{k}^{i j} \equiv\left(A_{i k}-A_{j k}\right)^{2} .
$$

Now we define, as in the main text, $\Lambda_{k}^{\tilde{T}}(t) \equiv 1+(\tilde{T}-1) \mathrm{e}^{-2 \mu_{k} t}$ as well as

$$
\mathcal{A}_{\tilde{T}}^{i j}(t) \equiv \sum_{k}^{\prime} \Lambda_{k}^{\tilde{T}}(t) \mathcal{C}_{k}^{i j} / 2 \mu_{k}, \quad \mathcal{B}_{\tilde{T}}^{i j}(t) \equiv \tilde{T} \sum_{k}^{\prime} \mathcal{C}_{k}^{i j} \mathrm{e}^{-\mu_{k} t} / 2 \mu_{k}
$$

and rewrite Eq. (S16) as

$$
\hat{\mathcal{P}}_{\tilde{T}}\left(\mathbf{u}, t, \mathbf{v}_{0} ; P_{\tilde{T}}^{\mathrm{eq}}\right)=\frac{1}{(2 \pi)^{6}} \exp \left(-\mathcal{A}_{\tilde{T}}^{i j}(t) \mathbf{v}^{2}-\mathcal{A}_{\tilde{T}}^{i j}(0) \mathbf{u}^{2}-2 \mathcal{B}_{\tilde{T}}^{i j}(t) \mathbf{v} \cdot \mathbf{u}\right),
$$

which can be easily inverted back to give

$$
\mathcal{P}_{\tilde{T}}\left(\mathbf{d}, t, \mathbf{d}_{0} ; P_{\tilde{T}}^{\mathrm{eq}}\right)=(4 \pi)^{-3}\left[\mathcal{A}_{\tilde{T}}^{i j}(t) \mathcal{A}_{\tilde{T}}^{i j}(0)-\mathcal{B}_{\tilde{T}}^{i j}(t)^{2}\right]^{-3 / 2} \exp \left(-\frac{1}{4} \frac{\mathcal{A}_{\tilde{T}}^{i j}(0) \mathbf{d}^{2}-2 \mathcal{B}_{\tilde{T}}^{i j}(t) \mathbf{d} \cdot \mathbf{d}_{0}+\mathcal{A}_{\tilde{T}}^{i j}(t) \mathbf{d}_{0}^{2}}{\mathcal{A}_{\tilde{T}}^{i j}(t) \mathcal{A}_{\tilde{T}}^{i j}(0)-\mathcal{B}_{\tilde{T}}^{i j}(t)^{2}}\right) .
$$

The marginalization is henceforth straightforward and yields

$$
\begin{aligned}
\mathcal{P}_{\tilde{T}}\left(d, t, d_{0} ; P_{\tilde{T}}^{\mathrm{eq}}\right) & =\frac{\left(d d_{0}\right)^{2} \exp \left(-\frac{1}{4} \frac{\mathcal{A}_{\tilde{T}}^{i j}(0) \mathbf{d}^{2}+\mathcal{A}_{\tilde{T}}^{i j}(t) \mathbf{d}_{0}^{2}}{\mathcal{A}_{\tilde{T}}^{i j}(t) \mathcal{A}_{\tilde{T}}^{i j}(0)-\mathcal{B}_{\tilde{T}}^{i j}(t)^{2}}\right)}{2 \pi\left[\mathcal{A}_{\tilde{T}}^{i j}(t) \mathcal{A}_{\tilde{T}}^{i j}(0)-\mathcal{B}_{\tilde{T}}^{i j}(t)^{2}\right]^{3 / 2}} \int_{0}^{\pi} d \cos \theta \exp \left(\frac{1}{2} \frac{d d_{0} \mathcal{B}_{\tilde{T}}^{i j}(t) \cos \theta}{\mathcal{A}_{\tilde{T}}^{i j}(t) \mathcal{A}_{\tilde{T}}^{i j}(0)-\mathcal{B}_{\tilde{T}}^{i j}(t)^{2}}\right) \\
& =\frac{d d_{0}}{2 \pi \mathcal{B}_{\tilde{T}}^{i j}(t)} \frac{\exp \left(-\frac{1}{4} \frac{\mathcal{A}_{\tilde{T}}^{i j}(0) \mathbf{d}^{2}+\mathcal{A}_{\tilde{T}}^{i j}(t) \mathbf{d}_{0}^{2}}{\mathcal{A}_{\tilde{T}}^{i j}(t) \mathcal{A}_{\tilde{T}}^{i j}(0)-\mathcal{B}_{\tilde{T}}^{i j}(t)^{2}}\right)}{\left[\mathcal{A}_{\tilde{T}}^{i j}(t) \mathcal{A}_{\tilde{T}}^{i j}(0)-\mathcal{B}_{\tilde{T}}^{i j}(t)^{2}\right]^{1 / 2}} \sinh \left(\frac{1}{2} \frac{\mathcal{B}_{\tilde{T}}^{i j}(t) d d_{0}}{\mathcal{A}_{\tilde{T}}^{i j}(t) \mathcal{A}_{\tilde{T}}^{i j}(0)-\mathcal{B}_{\tilde{T}}^{i j}(t)^{2}}\right) .
\end{aligned}
$$

The probability density of $d$ at time $t$ after having started from an initial density $P_{\tilde{T}}^{\text {eq }}\left(\mathbf{X}_{0}\right)$ (i.e. the pre-quench equilibrium) follows by simple integration and finally reads

$$
\mathcal{P}_{\tilde{T}}(d, t)=\int_{0}^{\infty} d l_{0} \mathcal{P}_{\tilde{T}}\left(d, t, l_{0} ; P_{\tilde{T}}^{\mathrm{eq}}\right) \equiv \frac{d^{2}}{2 \sqrt{\pi}} \mathcal{A}_{\tilde{T}}^{i j}(t)^{-3 / 2} \mathrm{e}^{-d^{2} / 4 \mathcal{A}_{\tilde{T}}^{i j}(t)},
$$

which is precisely Eq. (7) in the manuscript. The average potential of mean force, $\langle\mathcal{U}(t)\rangle_{\tilde{T}} \equiv-\left\langle\ln \mathcal{P}_{1}^{\mathrm{eq}}(d)\right\rangle_{\tilde{T}}$ and entropy, $\mathcal{S}_{\tilde{T}}(t) \equiv-\left\langle\ln \mathcal{P}_{\tilde{T}}(d, t)\right\rangle_{\tilde{T}}$ (in units of $\left.k_{\mathrm{B}} T\right)$, where $\langle f(d)\rangle_{\tilde{T}} \equiv \int d l \mathcal{P}_{\tilde{T}}(l, t) f(l)$, in turn read

$$
\begin{gathered}
\langle\mathcal{U}(t)\rangle_{\tilde{T}}=\ln \left(2 \sqrt{\pi} \mathcal{A}_{\tilde{T}}^{i j}(0)^{3 / 2}\right)-\mathcal{A}_{\tilde{T}}^{i j}(t)^{1 / 2}\left(2-\gamma_{\mathrm{e}}+\ln \mathcal{A}_{\tilde{T}}^{i j}(t)\right)+\frac{3}{2} \frac{\mathcal{A}_{\tilde{T}}^{i j}(t)}{\mathcal{A}_{\tilde{T}}^{i j}(0)} \\
\mathcal{S}_{\tilde{T}}(t)=\ln \left(2 \sqrt{\pi} \mathcal{A}_{\tilde{T}}^{i j}(t)^{3 / 2}\right)-\mathcal{A}_{\tilde{T}}^{i j}(t)^{1 / 2}\left(2-\gamma_{\mathrm{e}}+\ln \mathcal{A}_{\tilde{T}}^{i j}(t)\right)+\frac{3}{2}
\end{gathered}
$$


where $\gamma_{\mathrm{e}}$ denotes Euler's gamma. Using the results in Eq. (S23) as well as the definition of the equilibrium free energy, $F=-\ln Q_{1} \equiv-\ln \int d \mathbf{X} \mathrm{e}^{-U(\mathbf{X})}$, (where all potentials are in units of $k_{\mathrm{B}} T_{\text {eq }}$ ) we arrive at

$$
\mathcal{D}\left[P_{\tilde{T}}(t) \| P_{1}\right]=\left\langle U_{\tilde{T}}(t)\right\rangle-S_{\tilde{T}}(t)-F, \quad \mathcal{D}\left[\mathcal{P}_{\tilde{T}}(t) \| \mathcal{P}_{1}\right]=\left\langle\mathcal{U}_{\tilde{T}}^{\mathrm{eff}}(t)\right\rangle-\mathcal{S}_{\tilde{T}}(t),
$$

which are exactly Eqs. (4) and (5) in the Letter. For any stable symmetric matrix $\boldsymbol{\Xi}$ the condition of equidistant quenches $\mathcal{D}\left[P_{\tilde{T}^{+}}\left(0^{+}\right) \| P_{1}\right]=\mathcal{D}\left[P_{\tilde{T}^{-}}\left(0^{+}\right) \| P_{1}\right]$ is satisfied by

$$
\tilde{T}^{+}-\tilde{T}^{-}=\ln \left(\tilde{T}^{+} / \tilde{T}^{-}\right) \quad \rightarrow \quad \tilde{T}^{+}\left(\tilde{T}^{-}\right)=-W_{-1}\left(-\tilde{T}^{-} \mathrm{e}^{-\tilde{T}^{-}}\right)
$$

where $W_{-1}(x)$ defined for $x \in\left[-\mathrm{e}^{-1}, 0\right)$ denotes the second real branch of the Lambert-W function, which in turn satisfies the following sharp two-sided bound [1]

$$
\frac{2}{3}\left[1+\sqrt{2\left(\tilde{T}^{-}-1-\ln \tilde{T}^{-}\right)}+\tilde{T}^{-}-1-\ln \tilde{T}^{-}\right] \leq \tilde{T}^{+}\left(\tilde{T}^{-}\right) \leq 1+\sqrt{2\left(\tilde{T}^{-}-1-\ln \tilde{T}^{-}\right)}+\tilde{T}^{-}-1-\ln \tilde{T}^{-} .
$$

\section{Kullback-Leibler divergence and uphill/downhill asymmetry in relaxation of a random Gaussian network}

In the Letter we prove that for any reversible ergodic Ornstein-Uhlenbeck process uphill relaxation (i.e. for a quench from $\tilde{T}^{-} \uparrow 1$ for which $\left.\left\langle U\left(0^{+}\right)\right\rangle_{\tilde{T}^{-}}-\langle U\rangle_{1}<0\right)$ is always faster that downhill relaxation (i.e. for a quench from $\tilde{T}^{+} \downarrow 1$ for which $\left.\left\langle U\left(0^{+}\right)\right\rangle_{\tilde{T}^{-}}-\langle U\rangle_{1}>0\right)$, where the pair of equidistant quenches $\tilde{T}^{+}$and $\tilde{T}^{-}$is defined in the Letter. To visualize this on hand of an additional instructive example, we generated a random Gaussian network with 10 beads by filling elements of the upper-triangular part of the connectivity matrix with a -1 according to a Bernulli distribution with $p=0.7$. The resulting matrix was then symmetrized and the diagonal elements chosen to assure sure mechanical stability (i.e. 'connectedness'). The resulting connectivity matrix $\boldsymbol{\Gamma}$ is related to the general Ornstein-Uhlenbeck matrix in Eq. (S3) via $\boldsymbol{\Xi}=\boldsymbol{\Gamma} \otimes \mathbb{1}$, where

$$
\boldsymbol{\Gamma}=\left(\begin{array}{cccccccccc}
5 & -1 & -1 & 0 & -1 & 0 & 0 & 0 & -1 & -1 \\
-1 & 5 & -1 & 0 & -1 & -1 & 0 & 0 & 0 & -1 \\
-1 & -1 & 8 & -1 & -1 & 0 & -1 & -1 & -1 & -1 \\
0 & 0 & -1 & 7 & -1 & -1 & -1 & -1 & -1 & -1 \\
-1 & -1 & -1 & -1 & 9 & -1 & -1 & -1 & -1 & -1 \\
0 & -1 & 0 & -1 & -1 & 7 & -1 & -1 & -1 & -1 \\
0 & 0 & -1 & -1 & -1 & -1 & 7 & -1 & -1 & -1 \\
0 & 0 & -1 & -1 & -1 & -1 & -1 & 7 & -1 & -1 \\
-1 & 0 & -1 & -1 & -1 & -1 & -1 & -1 & 8 & -1 \\
-1 & -1 & -1 & -1 & -1 & -1 & -1 & -1 & -1 & 9
\end{array}\right) .
$$

The corresponding results for $\mathcal{D}\left[\mathcal{P}_{\tilde{T}}(t) \| \mathcal{P}_{1}\right]$, whereby we tagged the distance between the 1 st and 10 th bead, i.e. $d=\left|\mathbf{r}_{1}-\mathbf{r}_{10}\right|$ are shown in Fig. S4.

\section{TILTED SINGLE FILE}

We consider a system of $N$ hard-core point-particles (the extension to a finite diameter is straightforward [2, 3]) diffusing in a box of unit length with a diffusion coefficient $D$, which we set equal to 1 and express energies in units of $k_{\mathrm{B}} T_{\text {eq }}$ without any loss of generality. The particles with positions $\mathbf{x}=\left\{x_{i}\right\}$ feel the presence of a linear potential $U\left(\left\{x_{i}\right\}\right)=\sum_{i=1}^{N} g x_{i}$. The Green's function of the system obeys the many-body Fokker-Planck equation

$$
\left(\partial_{t}-\hat{\mathcal{L}}_{\tilde{T}}\right) G_{\tilde{T}}\left(\mathbf{x}, t \mid \mathbf{x}_{0}\right) \equiv\left(\partial_{t}-\sum_{i=1}^{N}\left(\partial_{x_{i}}^{2}+g \tilde{T}^{-1} \partial_{x_{i}}\right)\right) G_{\tilde{T}}\left(\mathbf{x}, t \mid \mathbf{x}_{0}\right)=\delta\left(\mathbf{x}-\mathbf{x}_{0}\right)
$$

The confining walls are assumed to be perfectly reflecting, i.e $\left.J\left(x_{i}\right)\right|_{x_{i}=0}=\left.J\left(x_{i}\right)\right|_{x_{i}=1}=-D\left(g / \tilde{T}-\partial_{x_{i}}\right) G_{\tilde{T}}\left(\mathbf{x}, t \mid \mathbf{x}_{0}\right)=$ $0, \forall i$. Moreover, particles are not allowed to cross, which introduces the following set of internal boundary conditions

$$
\left.\left(\partial_{x_{i+1}}-\partial_{x_{i}}\right) G_{\tilde{T}}\left(\mathbf{x}, t \mid \mathbf{x}_{0}\right)\right|_{x_{i+1}-x_{i}=0}=0, \forall i .
$$




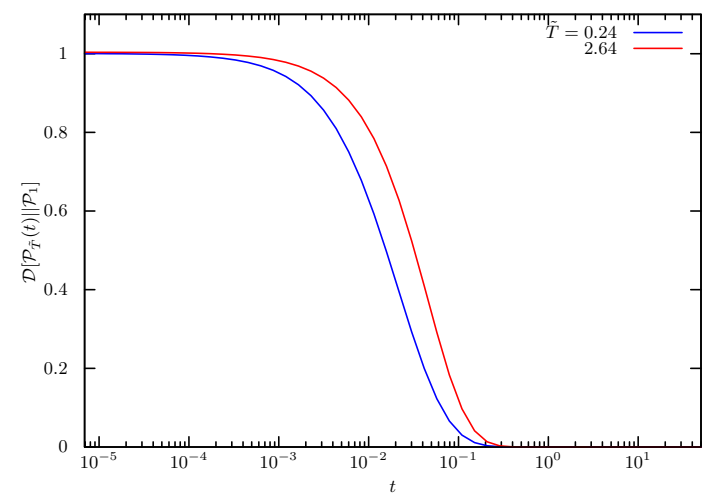

Figure S4. $\mathcal{D}\left[\mathcal{P}_{\tilde{T}^{ \pm}}(t) \| \mathcal{P}_{1}\right]$ as a function of time for a pair of equidistant quenches with $\tilde{T}^{+}=2.64$ and $\tilde{T}^{-}=0.24$, which illustrates the asymmetry in the thermal relaxation holds for any Gaussian Network (according to our proof).

Eq. (S28) with reflecting external boundary conditions $\left.J\left(x_{i}\right)\right|_{x_{i}=0}=\left.J\left(x_{i}\right)\right|_{x_{i}=1}=0, \forall i$ and internal boundary conditions in Eq. (S29) is solved exactly using the coordinate Bethe ansatz (we do not repeat the results here as they can be found in [4]). It is convenient to introduce the particle-ordering operator

$$
\hat{\mathcal{O}}_{\mathbf{x}} \equiv \prod_{i=2}^{N} \theta\left(x_{i}-x_{i-1}\right)
$$

where $\theta(x)$ is the Heaviside step-function. Let $\zeta_{\tilde{T}}\left(x_{i}, t \mid x_{0 i}\right)$ be the Green's function of the corresponding single-particle problem and $P_{\tilde{T}}^{\mathrm{eq}}\left(x_{i}\right)=\lim _{t \rightarrow \infty} \zeta_{\tilde{T}}\left(x_{i}, t \mid x_{0 i}\right)$ the density of the equilibrium measure at temperature $\tilde{T}$, then the Green's function can be written directly as

$$
G_{1}\left(\mathbf{x}, t \mid \mathbf{x}_{0}\right)=N ! \hat{O}_{\mathbf{x}} \prod_{i=1}^{N} \zeta_{1}\left(x_{i}, t \mid x_{0 i}\right) \rightarrow P_{\tilde{T}}(\mathbf{x}, t)=N ! \hat{O}_{\mathbf{x}} \prod_{i=1}^{N} \int_{0}^{1} d x_{i 0} \zeta_{1}\left(x_{i}, t \mid x_{i 0}\right) P_{\tilde{T}}^{\mathrm{eq}}\left(x_{i 0}\right),
$$

where the normalization factor $N$ ! assures a correct re-weighing of non-crossing trajectories [4]. We expand the Green's function for a single particle at $\tilde{T}=1$ in a bi-orthonormal eigenbasis, $\zeta\left(x, t \mid x_{0}\right)=\sum_{k} \phi_{k}^{R}(x) \phi_{k}^{L}\left(x_{0}\right) \mathrm{e}^{-\lambda_{k} t}$, where $\lambda_{0}=0, \lambda_{k}=\pi^{2} k^{2}+g^{2} / 4$ and

$$
\phi_{k}^{L}(x)=\frac{\mathrm{e}^{g x / 2}}{\sqrt{2 \lambda_{k}}}(g \sin (k \pi x)-2 k \pi \cos (k \pi x)), k>0
$$

and $\phi_{k}^{R}(x)=\mathrm{e}^{-g x} \phi_{k}^{L}(x)$, whereas for $k=0$ we have $\phi_{0}^{L}(x)=1, \phi_{0}^{R}(x)=P_{1}^{\mathrm{eq}}(x)$.

A key simplification in the calculation of order-preserving integrals as well as all projected, tagged-particle observables (incl. functionals; see e.g. [4]) is the so-called 'extended phase space integration' introduced by Lizana and Ambjörnsson $[2,3]$, according to which for any $1 \leq M \leq N$ and some function $f(\mathbf{x})$ that is symmetric with respect to permutation of coordinates $x_{i}$

$$
\hat{O}_{\mathbf{x}} \prod_{i=1}^{N} \int_{0}^{1} d x_{i 0} f(\mathbf{x}) \delta\left(z-x_{M}\right)=\prod_{i=1}^{M-1} \int_{0}^{z} d x_{i 0} \prod_{j=M+1}^{N} \int_{z}^{1} d x_{j 0} \frac{f\left(x_{M}=z,\left\{x_{i \neq M}\right\}\right)}{(M-1) !(N-M) !} .
$$

With the aid of Eq. (S33) it is possible to calculate the Kullback-Leibler divergence as

$$
\mathcal{D}\left[P_{\tilde{T}} \| P_{1}\right]=\int d \mathbf{x} P_{\tilde{T}}(\mathbf{x}, t) \ln \left(P_{\tilde{T}}(\mathbf{x}, t) / P_{1}(\mathbf{x})\right) \equiv\left[\int_{0}^{1} d x P_{\tilde{T}}^{1}(x, t) \ln \left(P_{\tilde{T}}^{1}(x, t) / P_{1}^{1}(x)\right)\right]^{N},
$$

where $P_{\tilde{T}}^{1}(x, t)=\int_{0}^{1} d x_{0} \zeta_{1}\left(x, t \mid x_{0}\right) P_{\tilde{T}}^{\mathrm{eq}}\left(x_{0}\right)$, and the second equality is a result of applying Eq. (S33). The result in Eq. (S34) for a single file of 10 particles is depicted in Fig. (3a) in the Letter. For the sake of completeness, we also present the exact explicit result for $\langle U(t)\rangle_{\tilde{T}} \equiv g N\langle x(t)\rangle_{\tilde{T}}$, which reads

$$
\langle U(t)\rangle_{\tilde{T}}=g N\left(\frac{1-\mathrm{e}^{g}+g}{g\left(1-\mathrm{e}^{g}\right)}+8 \sum_{k=1}^{\infty}\left(\frac{g k \pi}{\lambda_{k}}\right)^{2} \frac{(\tilde{T}-1)\left(\mathrm{e}^{g / 2}-(-1)^{k}\right)\left(\mathrm{e}^{g / \tilde{T}}-(-1)^{k} \mathrm{e}^{g / 2}\right)}{\left(\mathrm{e}^{g / \tilde{T}}-1\right)\left[(\tilde{T}-2)^{2} g^{2}+(2 \pi k \tilde{T})^{2}\right]} \mathrm{e}^{-\lambda_{k} t}\right) .
$$


The results for the non-Markovian tagged-particle dynamics can be derived analogously. The probability density function for tagging the $M$ th particle is defined as

$$
\mathcal{P}_{\tilde{T}}(z, t)=\hat{\Pi}_{\mathbf{x}}(z) P_{\tilde{T}}(\mathbf{x}, t) \equiv \hat{O}_{\mathbf{x}} \prod_{i=1}^{N} \int_{0}^{1} d x_{i 0} \delta\left(z-x_{\mathcal{T}}\right) P_{\tilde{T}}(\mathbf{x}, t)
$$

and since $P_{\tilde{T}}(\mathbf{x}, t)$ is symmetric to permutation of particle indices Eq. (S33) can be applied. The exact result has the form of a spectral expansion and reads

$$
\mathcal{P}_{\tilde{T}}(z, t)=\sum_{\mathbf{k}} V_{0 \mathbf{k}}(z) \mathcal{V}_{\mathbf{k} 0}^{\tilde{T}} \mathrm{e}^{-\lambda_{\mathbf{k}} t}
$$

where $\mathbf{k}=\left\{k_{i}\right\}$ is a $N$-tuple of non-negative integers and $\lambda_{\mathbf{k}}=\sum_{n=1}^{N} \lambda_{k_{n}}$ are Bethe eigenvalues of the operator $\hat{\mathcal{L}_{1}}=\sum_{i=1}^{N}\left(\partial_{x_{i}}^{2}+g \partial_{x_{i}}\right)$ in a unit box under non-crossing conditions with $\lambda_{0}=0$ and $\lambda_{k_{i}}=\pi^{2} k_{i}^{2}+g^{2} / 4, \forall k>0$. Let $N_{L}=\mathcal{T}-1$ and $N_{R}=N-\mathcal{T}$ be the total number of particles to the left and to the right of the tagged particle, respectively. Then $V_{0 \mathbf{k}}(z)$ and $\mathcal{V}_{\mathbf{k} 0}^{\tilde{T}}$ in Eq. (S37) are defined as

$$
\begin{aligned}
& V_{0 k}(z)=\frac{m_{\mathbf{k}}}{N_{L} ! N_{R} !} \frac{2 g \alpha}{\tilde{T} \Omega_{\tilde{T}}^{g}(0,1)} \sum_{\left\{k_{i}\right\}} T_{\mathcal{T}}^{1}(z) \prod_{i=1}^{N_{L}} L_{i}^{1}(z) \prod_{i=N_{L}+2}^{N} R_{i}^{1}(z) \\
& \mathcal{V}_{\mathbf{k} 0}^{\tilde{T}}=\frac{N !}{N_{L} ! N_{R} !} \frac{2 g \alpha}{\tilde{T} \Omega_{\tilde{T}}^{g}(0,1)} \int_{0}^{1} d z \sum_{\left\{k_{i}\right\}} T_{\mathcal{T}}^{\tilde{T}}(z) \prod_{i=1}^{N_{L}} L_{i}^{\tilde{T}}(z) \prod_{i=N_{L}+2}^{N} R_{i}^{\tilde{T}}(z)
\end{aligned}
$$

where $\alpha=\tilde{T} /(2-\tilde{T}), \Omega_{\tilde{T}}^{g}(x, y) \equiv \mathrm{e}^{-g x / \tilde{T}}-\mathrm{e}^{-g y / \tilde{T}}$, and $m_{\mathbf{k}}=\prod_{i} n_{k_{i}} !$ is the multiplicity of the Bethe eigenstate corresponding to the $N$-tuple $\mathbf{k}$, and the number $n_{k_{i}}$ counts how many times the eigenindex $k_{i}$ appears in the Bethe eigenstate [4]. In Eq. (S39) we have introduced the auxiliary functions

$$
T_{\mathcal{T}}^{\tilde{T}}(z)=P_{\tilde{T}}^{\mathrm{eq}}(z) \frac{\mathrm{e}^{g x / 2}\left(g \sin \left(k_{\mathcal{T}} \pi z\right)-2 k_{\mathcal{T}} \pi \cos \left(k_{\mathcal{T}} \pi z\right)\right)}{\sqrt{2 \lambda_{k_{\mathcal{T}}}}}, \forall k_{\mathcal{T}}>0
$$

and $T_{\mathcal{T}}^{\tilde{T}}(z)=P_{\tilde{T}}^{\mathrm{eq}}(z)$ for $k_{\mathcal{T}}>0$ where $P_{\tilde{T}}^{\mathrm{eq}}(z)$ is defined as

$$
\mathcal{P}_{\tilde{T}}^{\mathrm{eq}}(z)=\frac{g N !}{N_{L} ! N_{R} !} \frac{\Omega_{\tilde{T}}^{g}(0, z)^{N_{L}} \Omega_{\tilde{T}}^{g}(z, 1)^{N_{R}}}{\tilde{T} \Omega_{\tilde{T}}^{g}(0,1)} \mathrm{e}^{-g z / \tilde{T}},
$$

as well as

$$
\begin{aligned}
L_{i}^{\tilde{T}}(z) & =\left\{\begin{array}{l}
\Omega_{\tilde{T}}^{g}(0, z) / \Omega_{\tilde{T}}^{g}(0,1), \quad k_{i}=0 \\
\lambda_{\sqrt{\alpha} k_{i}} \Phi_{k_{i}}^{g, \alpha}(0, z)+k_{i} \pi g \Psi_{k_{i}}^{g, \alpha}(0, z)(\tilde{T}-1) /(2-\tilde{T}), \quad k_{i}>0
\end{array}\right. \\
R_{i}^{\tilde{T}}(z) & =\left\{\begin{array}{l}
\Omega_{\tilde{T}}^{g}(z, 1) / \Omega_{\tilde{T}}^{g}(0,1), \quad k_{i}=0 \\
\lambda_{\sqrt{\alpha} k} \Phi_{k_{i}}^{g, \alpha}(z, 1)+k_{i} \pi g \Psi_{k_{i}}^{g, \alpha}(z, 1)(\tilde{T}-1) /(2-\tilde{T}), \quad k_{i}>0,
\end{array}\right.
\end{aligned}
$$

Note that $\lambda_{x k} \equiv \pi^{2}\left(x k_{i}\right)^{2}+g^{2} / 4, \forall k>0$, and $\sum_{\left\{k_{i}\right\}}$ denotes the sum over all possible permutations of $\mathbf{k}$ and the functions $\Phi_{k}^{g, \alpha}(x, y)$ and $\Psi_{k}^{g, \alpha}(x, y)$ are defined as

$$
\begin{aligned}
\Phi_{k}^{g, \alpha}(x, y) & =\frac{\mathrm{e}^{-g x / 2 \alpha} \sin (k \pi x)-\mathrm{e}^{-g y / 2 \alpha} \sin (k \pi y)}{\lambda_{\alpha k} \sqrt{2 \lambda_{k}}} \\
\Psi_{k}^{g, \alpha}(x, y) & =\frac{\mathrm{e}^{-g x / 2 \alpha} \cos (k \pi x)-\mathrm{e}^{-g y / 2 \alpha} \cos (k \pi y)}{\lambda_{\alpha k} \sqrt{2 \lambda_{k}}} .
\end{aligned}
$$

Details of the calculations can be found in [4]. The evaluation of Kullback-Leibler divergence, $S_{\tilde{T}}(t), \mathcal{S}_{\tilde{T}}(t)$ as well as $\langle\mathcal{U}(t)\rangle_{\tilde{T}}$ cannot be carried out analytically and we therefore resort to efficient and accurate numerical quadratures. The results are presented in Fig. (3) in the Letter.

We performed extensive systematic calculations for different values of $g$ and $N$, various combinations of $\tilde{T}^{ \pm}$as well as for different choices for tagged particles. All these calculations gave the same qualitative picture - without any exceptions 'uphill' relaxation was always faster. However, we are not able to prove rigorously that this is indeed always the case. Therefore, for the single file the universally faster uphill relaxation is only a conjecture. 


\section{NON-EXISTENCE OF A UNIQUE RELAXATION ASYMMETRY IN MULTI-WELL POTENTIALS AND GENERIC CONDITIONS WHEN THE ASYMMETRY IS OBEYED}

In the letter we demonstrated that the relaxation in single-well potentials is faster uphill than downhill. We have proven that this is always the case near stable minima and for any reversible Ornstein-Uhlenbeck process. Based on additional physical arguments we hypothesized that the asymmetry is a general feature of diffusion in single-well potentials. However, as we remarked in the Letter, it is not difficult to construct counterexamples proving that the asymmetry is not a general phenomenon in all reversible ergodic diffusion processes.

To that end we condider Markovian diffusion in rugged, multi-well potentials parametrized by

$$
U(x)=e\left(a x^{6}+b x^{4}+c x^{3}+d x^{2}\right)
$$

with some appropriately chosen constants $a, b, c, d$ and $e$. Let the dynamics evolve according to $\hat{L}_{\tilde{T}}=\partial_{x}^{2}-\tilde{T}^{-1} \partial_{x} F(x)$, where $F(x)=-6 e\left(a x^{5}+4 b x^{3}+3 c x^{2}+2 d x\right)$ in a finite domain $a \leq x \leq b$ with reflecting boundaries, and let the corresponding Green's function be the solution of the following initial-boundary value problem

$$
\left(\partial_{t}-\hat{L}_{\tilde{T}}\right) G_{\tilde{T}}\left(x, t \mid x_{0}\right)=\delta\left(x-x_{0}\right), \quad-\left.\left(\partial_{x}-\tilde{T}^{-1} F(x)\right) G_{\tilde{T}}\left(x, t \mid x_{0}\right)\right|_{x=a}=-\left.\left(\partial_{x}-\tilde{T}^{-1} F(x)\right) G_{\tilde{T}}\left(x, t \mid x_{0}\right)\right|_{x=b}=0 .
$$

We solve the Fokker-Plank equation so defined via the Method of Lines. The results for three distinct parameter sets is shown in Fig. (S5). We did not perform a systematic analysis of all the possible potentials. However, based
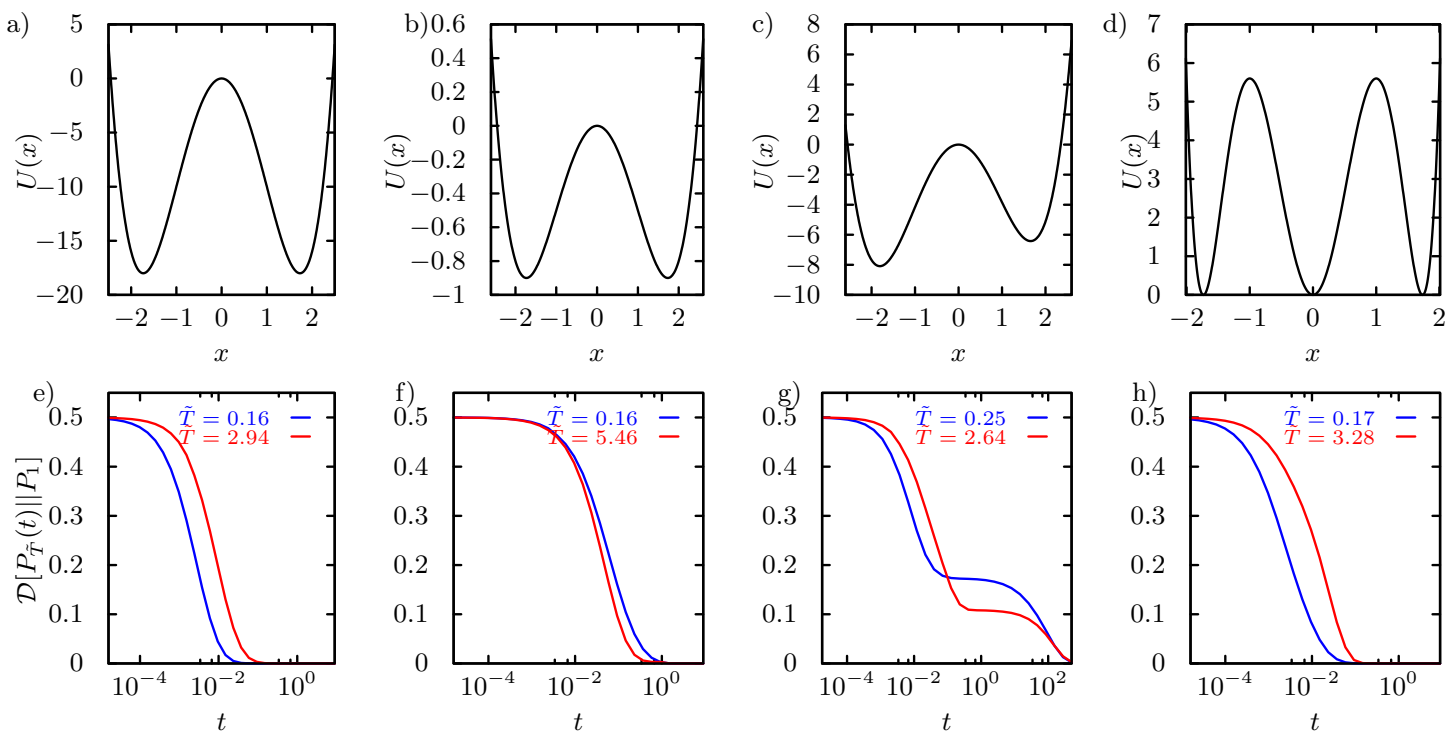

Figure S5. In panels a,b) and e,f) the potential is a quartic with parameter $a=0, b=1, c=0, d=-6, e=2$ in panels $\mathrm{a}$ and $\mathrm{f}$ and $a=0, b=1, c=0, d=-6, e=0.1$ in panels $\mathrm{b}$ and $\mathrm{f}$. In the asymmetric potential in panels $\mathrm{c}$ and $\mathrm{g}$ with $a=0, b=1, c=0.2, d=-6, e=0.8$ and panels $\mathrm{c}$ and $\mathrm{f}$ with $a=1, b=-6, c=0, d=9, e=1.4$, respectively, the single-well asymmetry-pattern in fact becomes reversed. In a tripple-well with equally deep wells the asymmetry is again obeyed despite the middle well being wider.

on our observations it seems that the different uphill/downhill relaxation patterns depend on how different entropic contributions (i.e. intra-well entropy versus inter-well configuration entropy) change qualitatively with temperature for potentials with several minima.

If we focus on the asymmetric case (Fig. S5c) we find that uphill relaxation is initially always faster, which is a direct result of the physical mechanism at play that we present in the Letter. At longer time the asymmetry gets inverted by the slow inter-well partitioning of probability mass. It is now not diffcult to understand that by making the asymmetry smaller we will move the crossing point, where the corves intersect, closer to $\mathcal{D}\left[P_{\tilde{T}^{ \pm}}(t) \| P_{1}^{\text {eq }}\right]=0$, such that for a sufficiently small asymmetry - which in the letter we refer to near degeneracy - uphill relaxation will eventually be faster for all times, for which $\mathcal{D}\left[P_{\tilde{T}^{ \pm}}(t) \| P_{1}^{\text {eq }}\right]$ differs from zero by an amount that is not neglgible/detectable. For a formal discussion of this situation see below. 
It is interesting and important to note that the asymmetry is also obeyed if the barrier is moderately high, i.e. such that a small but non-neglible probability mass is located at the barrier (see Fig. S6). However, the quench must then not be too strong. That is, an 'infinitely' high barrier effecting a strict time-scale separation betwenn intra-well and inter-well relaxation is not a neccessarry condition for the asymmetry to occur. To demonstrate this we inspect overdamped relaxation according to Eq. (S44) in the following double well potential $U(x)=\Delta\left(x^{2}-1\right)^{2}$, where we choose (in units of $\left.k_{\mathrm{B}} T_{\text {eq }}\right) \Delta=3$ and $F(x)=-U(x)^{\prime}$.
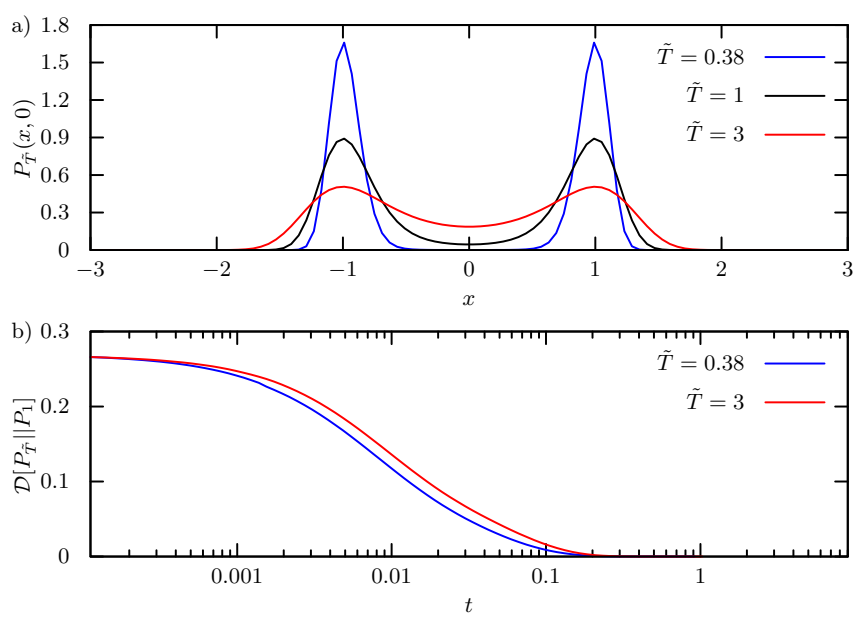

Figure S6. a) Density of invariant measure at $\tilde{T}=1$ (i.e. equilibrium probability density), and the equidistant post-quench probability densities at $\tilde{T}^{+}=3$ and $\tilde{T}^{-}=0.38$; b) Corresponding time evolution of the Kullback-Leibler divergence depicting that the asymmetry is obeyed.

In order to check that the observed effect in multi-well potentials is not an artifact of one-dimensional systems now also inspect 2-dimensional multi-well potentials. To that end we consider 4 -well potentials parametrized by

$$
U(x, y)=\Delta_{x}\left(x^{2}-x_{0}^{2}\right)^{2}+\Delta_{y}\left(y^{2}-y_{0}^{2}\right),
$$

where energy is measured in units of $k_{\mathrm{B}} T_{\text {eq. }}$. We solve the problem by the Alternating Direction Implicit method (ADI) developed in [5] with 4-step operator splitting. We first focus on the limit of high barriers and quenches leaving the inter-well partitioning of probability mass unaffected (see Fig. S7). According to the proposed principle and prediction the symmetry is obeyes and uphill relaxation is always faster.

In Fig. S8 now inspect the case of a moderately high barriers (where the probability density on top of the barriers does not vanishes). As expected the asymmetry is obeyed only for sufficiently small quenches, whereas it becomes violated for stronger quenches (compare full and dashed lines). The reason for the violation is the fact that the inter-well redistribution becomes the dominant step for strong quenches.

It seems that the asymmetry observed in single-well potentials persists in nearly degenerate potentials and ceases to exists as soon as the potential becomes sufficiently asymmetric with sufficiently deep wells, where entropy attains an additional inter-well configurational component, such that during relaxation the probability mass becomes redistributed between the wells in an asymmetric manner.

\section{The asymmetry is obeyed in degenerate potentials in the presence of a time-scale separation}

We now provide also formal arguments confirming that the symmetry must be obeyed in degenerate potentials in the presence of a time-scale separation. We follow the work of Moro [6]. Since we are dealing with systems obeying detailed balance the generator of the relaxation dynamics $\hat{\mathcal{L}}$ is always diagonalizable, i.e.

$$
\hat{\mathcal{L}}_{T}=\sum_{k \geq 0}-\lambda_{k} \psi_{k}^{R}(\mathbf{x}) \psi_{k}^{L}\left(\mathbf{x}_{0}\right)
$$

where $\psi_{k}^{R}(\mathbf{x})$ and $\psi_{k}^{L}(\mathbf{x})$ are the orthonormal right and left eigenfunctions, respectively, (i.e. $\left.\int \psi_{k}^{L}(\mathbf{x}) \psi_{l}^{R}(\mathbf{x}) d \mathbf{x}=\delta_{k l}\right)$ and $-\lambda_{k}$ are real eigenvalues ( $\lambda_{0}=0$ as we have assumed that the potential is confining and the dynamics is ergodic). 

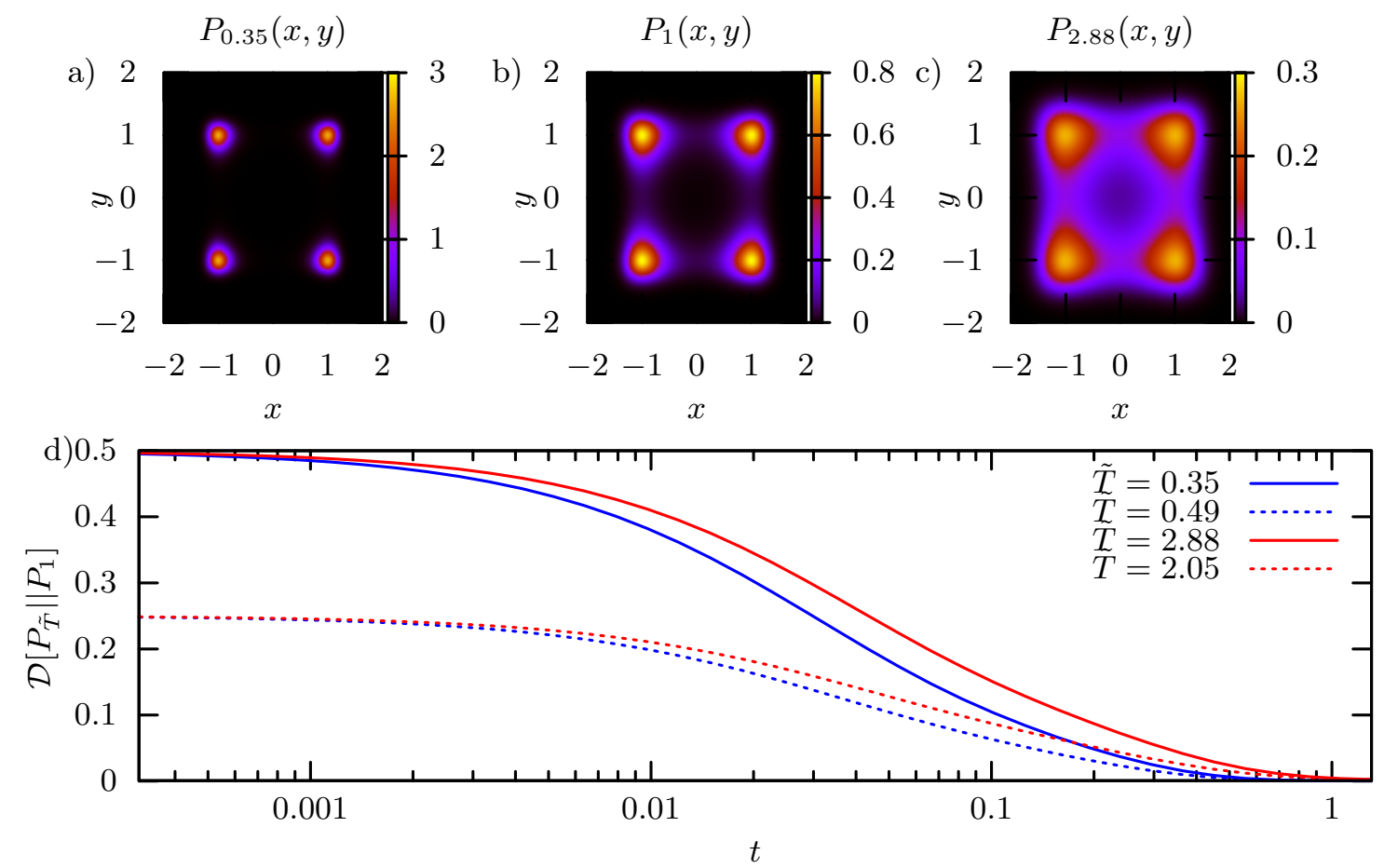

Figure S7. Density of invariant measure at $\tilde{T}=1$ (b) (i.e. equilibrium probability density), and the equidistant post-quench probability densities at (c) $\tilde{T}^{+}=2.88$ and (a) $\tilde{T}^{-}=0.35$ for the 4 -well potential in Eq. (S45) with parameters $\Delta_{x}=\Delta_{y}=3$ and $x_{0}=y_{0}=1 . ;$ d) Corresponding time evolution of the Kullback-Leibler divergence depicting that the asymmetry is obeyed for two pairs of equidistant temperatures.

The eigenfunctions constitute a complete bi-orthonormal basis, $\sum_{k} \psi_{k}^{L}(\mathbf{x}) \psi_{k}^{R}\left(\mathbf{x}^{\prime}\right)=\delta\left(\mathbf{x}-\mathbf{x}^{\prime}\right)$. As a result of detailed balance we have $\psi_{k}^{R}(\mathbf{x})=\mathrm{e}^{-U(\mathbf{x}) / k_{\mathrm{B}} T} \psi_{k}^{L}(\mathbf{x})$ and $\psi_{0}^{R}(\mathbf{x})=P_{T}^{\mathrm{eq}} \equiv \mathrm{e}^{-U(\mathbf{x}) / k_{\mathrm{B}} T} / \int \mathrm{e}^{-U(\mathbf{x}) / k_{\mathrm{B}} T} d \mathbf{x}$ and $\psi_{0}^{L}(\mathbf{x})=1$. Let $\hat{\mathcal{L}}_{T}^{\dagger}$ be the adjoint (or 'backward') generator, then we have the pair of eigenproblems $\hat{\mathcal{L}}_{T} \psi_{k}^{R}(\mathbf{x})=-\lambda_{k} \psi_{k}^{R}(\mathbf{x})$ and $\hat{\mathcal{L}}_{T}^{\dagger} \psi_{k}^{L}(\mathbf{x})=-\lambda_{k} \psi_{k}^{L}(\mathbf{x})$.

The Green's function of the relaxation problem, $\left(\partial_{t}-\hat{\mathcal{L}}_{T}\right) G_{T}\left(\mathbf{x}, t \mid \mathbf{x}_{0}\right)=0$ with $G_{T}\left(\mathbf{x}, 0 \mid \mathbf{x}_{0}\right)=\delta\left(\mathbf{x}-\mathbf{x}_{0}\right)$, decomposes to

$$
G_{T}\left(\mathbf{x}, t \mid \mathbf{x}_{0}\right)=\sum_{k \geq 0} \psi_{k}^{R}(\mathbf{x}) \psi_{k}^{L}\left(\mathbf{x}_{0}\right) \mathrm{e}^{-\lambda_{k} t} \rightarrow \quad P_{\tilde{T}}(\mathbf{x}, t)=\int G_{1}\left(\mathbf{x}, t \mid \mathbf{x}_{0}\right) P_{\tilde{T}}^{\mathrm{eq}}\left(\mathbf{x}_{0}\right) d \mathbf{x}_{0}
$$

In presence of a time-scale separation (as a result of the existence of one or more high energy barriers) the eigenvalue spectrum of $\hat{\mathcal{L}}$ has a gap, i.e. $\exists k_{\min }$ such that $\lambda_{k_{\min }+l} \gg k_{\min } \forall l \geq 1$.

Assume now a set of $M$ well-defined deep minima at $\hat{\mathbf{x}}_{i}, i=1, \ldots, M$. This implies $k_{\min }=M-1$. Let us define localizing functions $g_{i}(\mathbf{x}), i \in[1, M]$ such that

$$
c_{i}^{\mathrm{eq}} \equiv \int g_{i}(\mathbf{x}) P_{1}^{\mathrm{eq}}(\mathbf{x}) d \mathbf{x} \quad \rightarrow \quad \int g_{i}(\mathbf{x}) \psi_{k}^{R}(\mathbf{x})=0, \forall k \geq M
$$

$c_{i}^{\text {eq }}$ are the equilibrium site populations. The localizing functions therefore by definition separate the intra-well relaxation from the inter-well 'hopping' of probability mass. In turn this implies that $g_{i}(\mathbf{x})$ belong to the subspace $\left\{\psi_{k}^{L}(\mathbf{x})\right\}, k<M$, i.e.

$$
g_{i}(\mathbf{x})=\sum_{k=0}^{M-1} B_{i k} \psi_{k}^{L}(\mathbf{x}), \forall i \in[1, M]
$$

and are thus by construction linearly independent but are so far only defined up to the expansion matrix $\mathbf{B}$. We determine $\mathbf{B}$ by imposing that the localizing functions should be localized near only one minimum $\hat{\mathbf{x}}_{i}$ and vanish at 

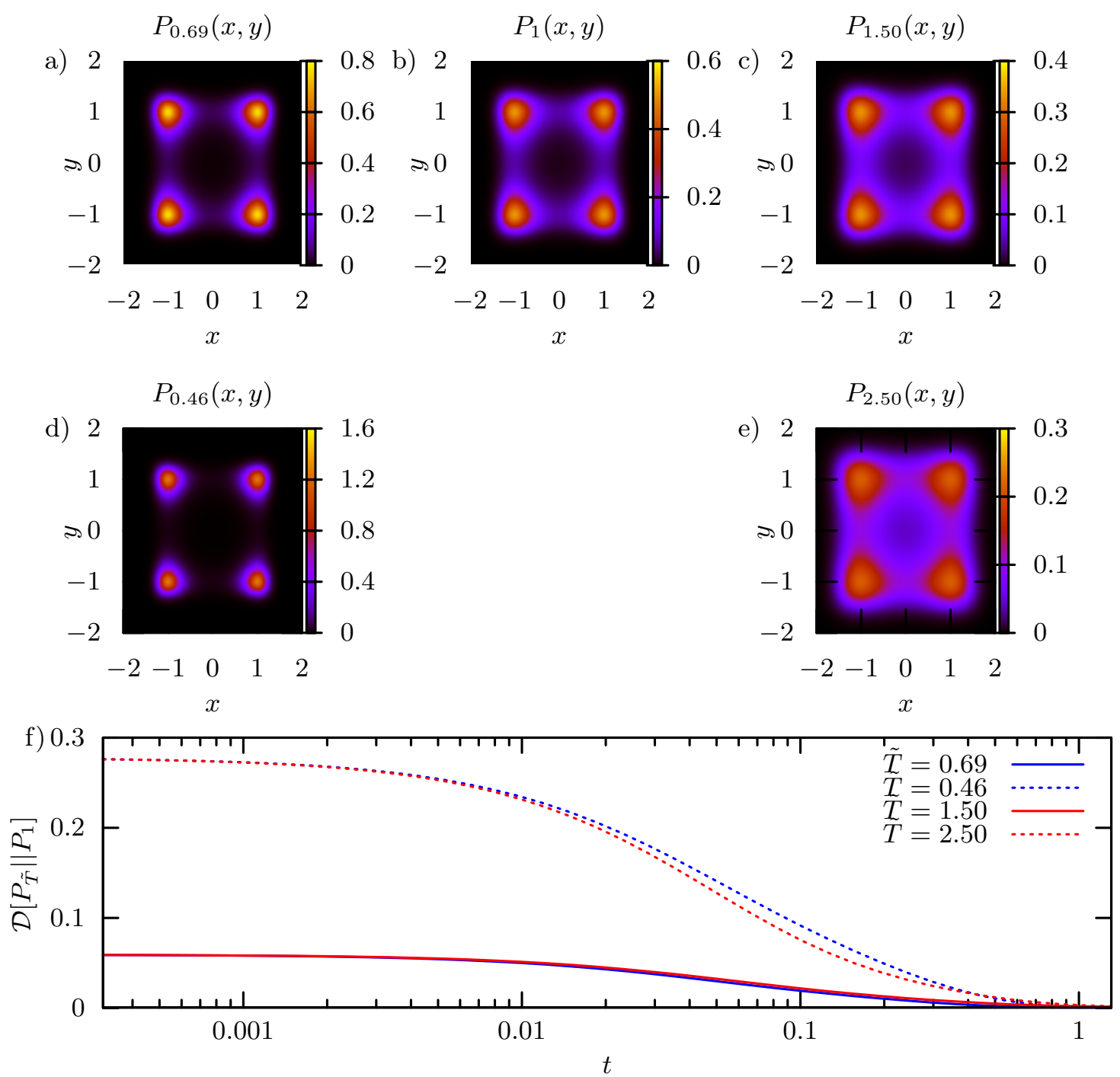

Figure S8. Density of invariant measure at (a) $\tilde{T}^{-}=0.69$ (b) at $\tilde{T}=1$, and at (c) $\tilde{T}^{+}=1.5$, (d) $\tilde{T}^{-}=0.46$ and (e) $\tilde{T}^{+}=2.5$ corresponding to the 4 -well potential in Eq. (S45) with parameters $\Delta_{x}=\Delta_{y}=2$ and $x_{0}^{2}=y_{0}^{2}=1$; b) Corresponding time evolution of the Kullback-Leibler divergence depicting that the asymmetry is obeyed for small quenches (a and c) and violated for strong quenches (d and e).

all remaining minima, i.e. $g_{i}\left(\hat{\mathbf{x}}_{j}\right) \simeq \delta_{i, j}$. Let the inverse of $\mathbf{B}$ be $\mathbf{B}^{-1}, \mathbf{B}^{-1} \mathbf{B}=\mathbb{1}$. We finally fix $g_{i}(\mathbf{x})$ by imposing the following resolution of identity $\sum_{I=1}^{M} g_{i}(\mathbf{x})=1$, which allows us to write

$$
\psi_{i}^{L}(\mathbf{x})=\sum_{k=1}^{M} B_{i k}^{-1} g_{k}(\mathbf{x}), \forall i \in[0, M-1],
$$

We now define the time-dependent population of the localizing sites (i.e. basins)

$$
c_{i}(t) \equiv \int g_{i}(\mathbf{x}) G\left(\mathbf{x}, t \mid \mathbf{x}_{0}\right) d \mathbf{x}
$$

The fact that $g_{j}(\mathbf{x})$ decompose unity implies that the total site population is conserved in time, i.e.

$$
\sum_{i=1}^{M} c_{i}(t) \equiv \sum_{i=1}^{M} \int g_{i}(\mathbf{x}) G\left(\mathbf{x}, t \mid \mathbf{x}_{0}\right) d \mathbf{x}=\int \sum_{i=1}^{M} g_{i}(\mathbf{x}) G\left(\mathbf{x}, t \mid \mathbf{x}_{0}\right) d \mathbf{x}=1,
$$


where we have used the fact that the integral and sum commute by Fubini's theorem (note that we can write the sum as an integral with respect to a counting measure). The localizing functions are linearly independent but not orthonormal. For this purpose we define the $M \times M$ superposition matrix $\mathbf{S}$ with elements $S_{i j} \equiv \int g_{i}(\mathbf{x}) P_{1}^{\mathrm{eq}}(\mathbf{x}) g_{j}(\mathbf{x}) d \mathbf{x}$ such that we can re-write the equilibrium site population as

$$
c_{i}^{\mathrm{eq}}=\int g_{i}(\mathbf{x}) P_{1}^{\mathrm{eq}}(\mathbf{x}) \sum_{j=1}^{M} g_{j}(\mathbf{x}) d \mathbf{x}=\sum_{j=1}^{M} S_{i j} .
$$

We now define a projection operator projecting onto the space of localizing functions

$$
\hat{\mathrm{P}} q(\mathbf{x}) \equiv \sum_{i=1}^{M} q_{i} g_{i}(\mathbf{x}), \quad q_{i} \equiv \sum_{k=1}^{M} S_{i, k}^{-1} \int g_{i}(\mathbf{x}) P_{1}^{\mathrm{eq}}(\mathbf{x}) q(\mathbf{x}) d \mathbf{x} .
$$

The time evolution of site populations then follows

$$
\begin{aligned}
\frac{d c_{j}(t)}{d t} & =\int g_{j}(\mathbf{x}) \partial_{t} G_{\tilde{T}}\left(\mathbf{x}, t \mid \mathbf{x}_{0}\right) d \mathbf{x}=\int g_{j}(\mathbf{x}) \hat{\mathcal{L}}_{1} G_{\tilde{T}}\left(\mathbf{x}, t \mid \mathbf{x}_{0}\right) d \mathbf{x}=\int G_{\tilde{T}}\left(\mathbf{x}, t \mid \mathbf{x}_{0}\right) \hat{\mathcal{L}}_{1}^{\dagger} g_{j}(\mathbf{x}) d \mathbf{x} \\
& \equiv \int G_{\tilde{T}}\left(\mathbf{x}, t \mid \mathbf{x}_{0}\right) \hat{\mathrm{P}} \hat{\mathcal{L}}_{1}^{\dagger} g_{j}(\mathbf{x}) d \mathbf{x}=\sum_{k, i} c_{k}(t) S_{k, i}^{-1} \int g_{i}(\mathbf{y}) \hat{\mathcal{L}}_{1}^{\dagger} P_{1}^{\mathrm{eq}}(\mathbf{y}) g_{j}(\mathbf{y}) d \mathbf{y} \equiv \sum_{k, i} c_{k}(t) S_{k, i}^{-1} \Gamma_{i j}
\end{aligned}
$$

where in the second line we used the fact that $\hat{\mathcal{L}}_{1}^{\dagger} g_{j}(\mathbf{x})$ already lies in the subspace of localizing functions (because $\hat{\mathcal{L}}_{T}^{\dagger} \psi_{k}^{L}(\mathbf{x})=-\lambda_{k} \psi_{k}^{L}(\mathbf{x})$ and Eq. (S49)) and the projection operator projects back onto said subspace. By defining $\mathbf{c}(t)=\left(c_{i}(t), \ldots, c_{M}(t)\right)^{T}$ we recognize from Eq. (S56) that the site populations obey the Markovian master equation

$$
\frac{d}{d t} \mathbf{c}(t)=\mathbf{M c}(t), \quad M_{j k} \equiv \sum_{i} S_{k, i}^{-1} \Gamma_{i j}
$$

where it can be shown that the transition rates entering $\mathbf{M}$ obey detailed balance [6]. It is obvious that $\mathbf{M c} \mathbf{c}^{e q}=$ 0 and therefore an equilibrated site-population does not lead to any inter-well dynamics. The evolution upon a temperature quench from $\tilde{T}$ follows from the evolution of the Green's function, i.e. $P_{\tilde{T}}(\mathbf{x}, t)=\int G_{1}\left(\mathbf{x}, t \mid \mathbf{x}_{0}\right) P_{\tilde{T}}^{\text {eq }}\left(\mathbf{x}_{0}\right) d \mathbf{x}_{0}$. Therefore, any quench that will leave the site populations given the potential $U(\mathbf{x})$ and Fokker-Planck operator $\hat{\mathcal{L}}_{1}$ $\left(\hat{\mathcal{L}}_{1}^{\dagger}\right.$ respectively) almost unaffected, i.e.

$$
\mathbf{M c}(0) \simeq 0, \quad \text { where } \quad c_{i}(0)=\int g_{i}(\mathbf{x}) P_{\tilde{T}}\left(\mathbf{x}, t=0 \mid \mathbf{x}_{0}\right) d \mathbf{x} \equiv \int g_{i}\left(\mathbf{x}_{0}\right) P_{\tilde{T}}^{\mathrm{eq}}\left(\mathbf{x}_{0}\right) d \mathbf{x}_{0},
$$

will lead to a faster uphill relaxation as a direct consequence of the fact that the intra-well (i.e. in each individual well) relaxation is faster uphill. The above arguments can be arranged in a form that is fully rigorous, but since the argumentation is essentially straightforward, we do not find it necessary to do so.

\section{Small local modulations do not spoil the asymmetry}

As stated in the Letter, small local modulations of the potential ( $\left.\ll k_{\mathrm{B}} T_{\text {eq }}\right)$ do not affect the asymmetry as longs as the uphill quench is sufficiently small to assure that the modulation is $\lesssim k_{\mathrm{B}} T^{-}$. Then the system relaxes similarly as in a perfectly smooth single well. To demonstrate that this is indeed the case we inspect the relaxation from equidistant quenches in the potential in Eq. (S45) with $\Delta_{x}=\Delta_{y}=2$ and $x_{0}^{2}=y^{2}=0.4$ depicted in Fig. S9. If, however, we make the quench too severe, such that the local modulations of the potential effectively reach $|\Delta U(\mathbf{x})| \gtrsim k_{\mathrm{B}} T^{-}$the asymmetry would become violated and the curves will eventually cross, rendering downhill relaxation faster.

A a final example we focus on an asymmetric quadruple-well with a pair of high barriers and a pair of low barriers (the latter creating a small local modulation of the potential). In particular, we consider the relaxation in the potential given in Eq. (S45) with parameters $\Delta_{x}=3, \Delta_{y}=2$ and $x_{0}=0.5, y_{0}=1$ and inspect in Fig. S10 the following pairs of thermodynamically equidistant temperatures, $\tilde{T}^{-}=0.8, \tilde{T}^{+}=1.25$ and $\tilde{T}^{-}=0.5, \tilde{T}^{+}=2$.

As anticipated, the uphill relaxation is faster for sufficiently small quenches (see Fig. S10f) and becomes violated for stronger quenches (see Fig. S10g), where the Kullback-Leibler divergences also display an Mpemba-like effect (see also next section). 

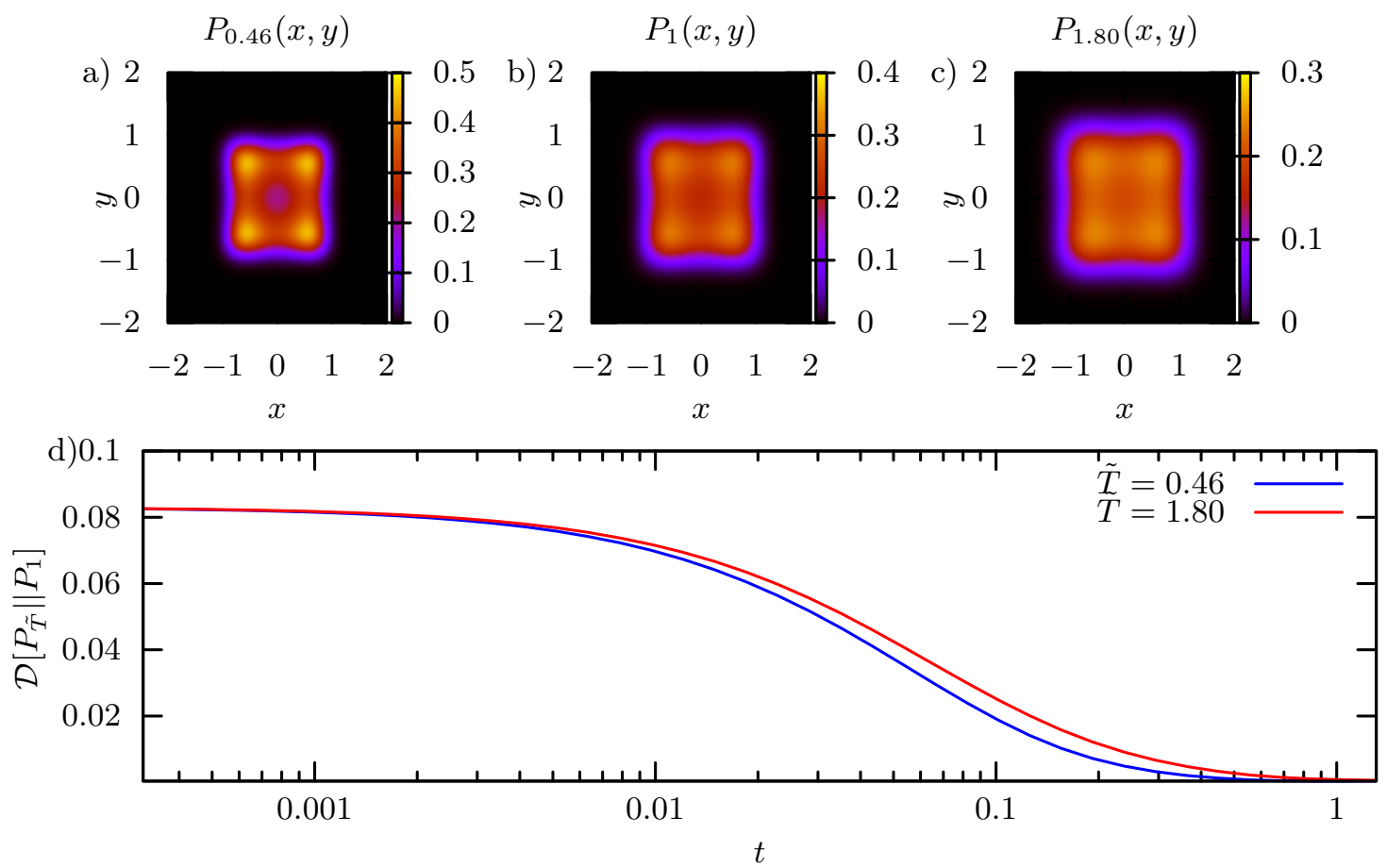

Figure S9. a) Density of invariant measure at $\tilde{T}=1$ (i.e. equilibrium probability density), and the equidistant post-quench probability densities at $\tilde{T}^{+}=1.8$ and $\tilde{T}^{-}=0.46$ for the 4 -well potential in Eq. (S45) with parameters $\Delta_{x}=\Delta_{y}=2$ and $x_{0}^{2}=y_{0}^{2}=0.4$; b) Corresponding time evolution of the Kullback-Leibler divergence depicting that the asymmetry is obeyed.

\section{GENERALIZED MPEMBA EFFECT FOR NON-MARKOVIAN DYNAMICS}

A phenomenon closely linked to relaxation from a quench is the so-called Mpemba effect [7-9], according to which a liquid upon cooling can freeze faster if its initial temperature is higher. Meanwhile the phenomenon has been extended to cover relaxation processes in different systems: magneto-resistors [10], carbon-nanotubes [11], polymers crystallization [12], clathrate hydrates [13], granular systems [14] and spin glasses [15]. Recently theoretical generalizations of it for Markovian observables have been published [16-18]. Not long ago the phenomenon was also adressed in more detail in the context of Markovian stochastic dynamics [16, 18].

Here we further extend the concept of the Mpemba effect to projected, non-Markovian observables. As before we focus on the distance of two different generic configurations displaced from equilibrium at $t=0$, such that one is displaced further away than the other, whereas the time-evolution of the entire system is governed by the same FokkerPlanck operator. In this setting, there are cases, where the more distant initial configuration reaches equilibrium faster that the closer one. One can observe this effect in the two systems analyzed in the Letter (see Fig. S11). It is worth to stress that the presence of the generalized Mpemba effect not only depends on the system and the initial condition (like in the Markovian case) but also on the particular type of projection In Fig. S12 we demonstrate, on hand of the same system (a tilted single file of 5 particles) from the same pair of pre-quench temperatures, that we can switch the generalized Mpemba effect on and off by simply changing the particle we are tagging. 

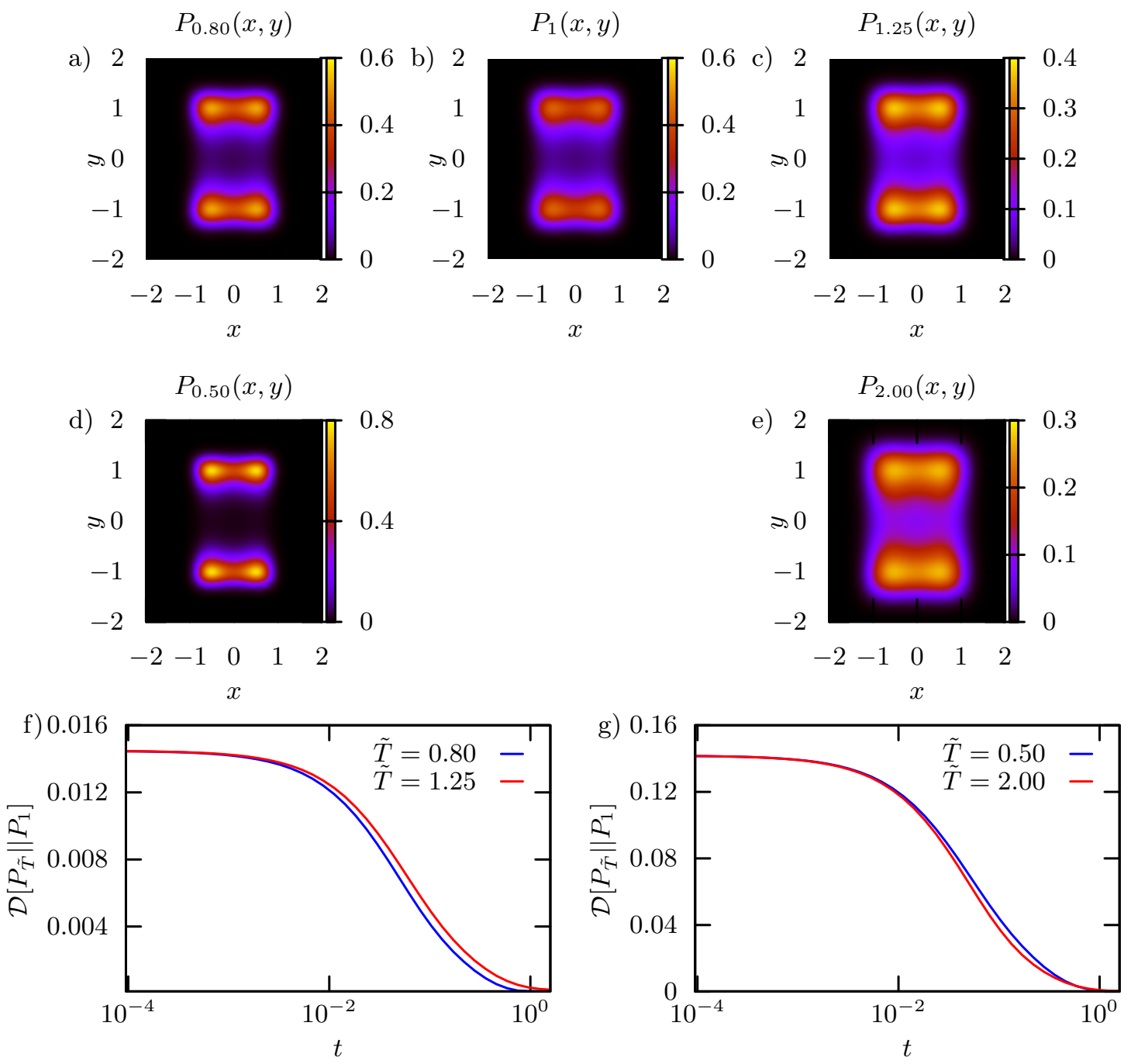

Figure S10. b) Density of invariant measure at $\tilde{T}=1$ (i.e. equilibrium probability density), and two pairs of equidistant postquench probability densities at $\tilde{T}^{+}=1.25$ (c) and 2 (e) and corresponding equidistant $\tilde{T}^{-}=0.8$ (a) and 0.5 (d), respectively, for the 4-well potential in Eq. (S45) with parameters $\Delta_{x}=3, \Delta_{y}=2$ and $x_{0}=0.5, y_{0}=1$; f $\mathrm{f}$ ) ) Corresponding time evolution of the Kullback-Leibler divergence depicting that the asymmetry is obeyed for small enough quenches but becomes violated (in the form of an Mpemba-like effect) for stronger quenches.

* agodec@mpibpc.mpg.de

[1] I. Chatzigeorgiou, IEEE Communications Letters 17, 1505-1508 (2013).

[2] L. Lizana and T. Ambjörnsson, Phys. Rev. E 80, 051103 (2009).

[3] L. Lizana and T. Ambjörnsson, Phys. Rev. Lett. 100, 200601 (2008).

[4] A. Lapolla and A. Godec, Front. Phys. 7 (2019), 10.3389/fphy.2019.00182.

[5] A. Godec, T. Ukmar, M. Gaberšček, and F. Merzel, EPL (Europhysics Letters) 92, 60011 (2010).

[6] G. J. Moro, J. Chem. Phys. 103, 7514-7531 (1995).

[7] E.B. Mpemba D.G. Osborne, Physics Education 14, 410 (1979).

[8] M. Jeng, American Journal of Physics 74, 514 (2006).

[9] J. I. Katz, American Journal of Physics 77, 27 (2009).

[10] P. Chaddah, S. Dash, K. Kumar, and A. Banerjee, arXiv:1011.3598 [cond-mat, physics:physics] (2010), arXiv: 1011.3598.

[11] P. A. Greaney, G. Lani, G. Cicero, and J. C. Grossman, Metall and Mat Trans A 42, 3907 (2011).

[12] C. Hu, J. Li, S. Huang, H. Li, C. Luo, J. Chen, S. Jiang, and L. An, Crystal Growth \& Design 18, 5757 (2018). 

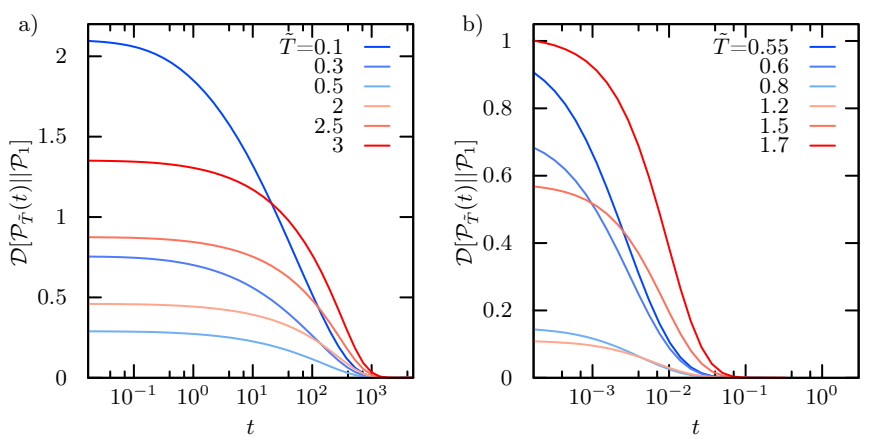

Figure S11. In the left panel we show time dependence of the Kullback-Leibler divergence for a Gaussian Chain of 100 beads, while the right panel depicts a Single File of 10 particles $(g=5)$. In both cases we focus on non-Markovian observables, the end-to-end distance for the Gaussian chain and on the 7th particle of the single file, respectively. For some pairs of initial temperatures we notice the generalized Mpemba effect: systems that start further away from the equilibrium approach the equilibrium configuration faster.

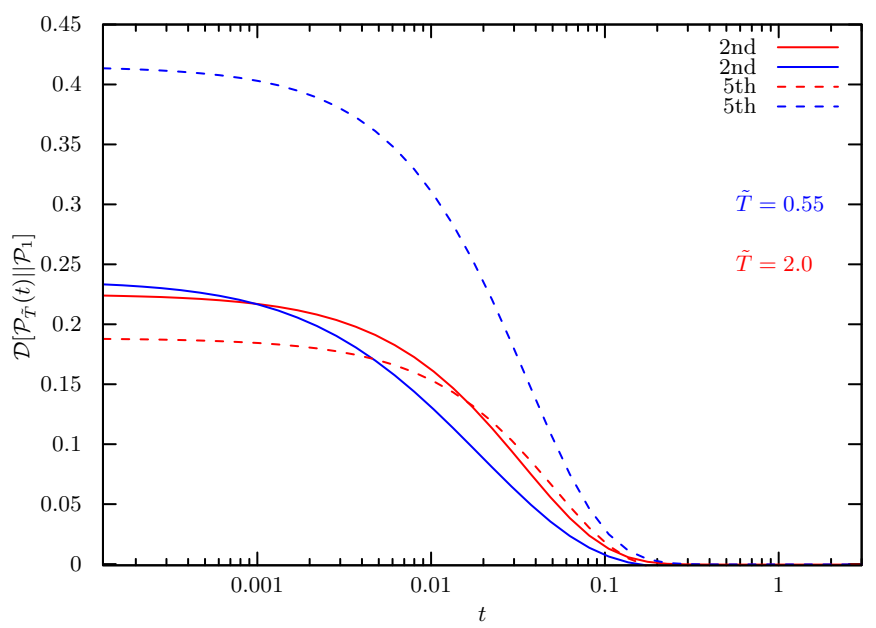

Figure S12. Kullback-Leibler divergences for a single file of 5 particles with $g=1$. If we tag the 2nd particle (solid lines) or the 5 th (dashed lines) for the same pair of pre-quench temperatures one projection displays the generalized Mpemba effect while the other one does not.

[13] Y.-H. Ahn, H. Kang, D.-Y. Koh, and H. Lee, Korean J. Chem. Eng. 33, 1903 (2016).

[14] A. Lasanta, F. Vega Reyes, A. Prados, and A. Santos, Phys. Rev. Lett. 119, 148001 (2017).

[15] J. collaboration, M. Baity-Jesi, E. Calore, A. Cruz, L. A. Fernandez, J. M. Gil-Narvion, A. Gordillo-Guerrero, D. Iñiguez, A. Lasanta, A. Maiorano, E. Marinari, V. Martin-Mayor, J. Moreno-Gordo, A. Muñoz-Sudupe, D. Navarro, G. Parisi, S. Perez-Gaviro, F. Ricci-Tersenghi, J. J. Ruiz-Lorenzo, S. F. Schifano, B. Seoane, A. Tarancon, R. Tripiccione, and D. Yllanes, Proc Natl Acad Sci USA 116, 15350 (2019), arXiv: 1804.07569.

[16] Z. Lu and O. Raz, Proc Natl Acad Sci USA 114, 5083 (2017).

[17] I. Klich and M. Vucelja, arXiv:1812.11962 [cond-mat, physics:math-ph] (2019), arXiv: 1812.11962.

[18] I. Klich, O. Raz, O. Hirschberg, and M. Vucelja, Phys. Rev. X 9, 021060 (2019). 


\section{Chapter 7}

\section{Spectral theory of fluctuations in time-average statistical mechanics of reversible and driven systems}

This article has been published in Physical Review Research under the Creative Commons Attribution 4.0 International license [54]. The timeaveraged statistics of a well-behaved functional of a stochastic process is investigated. Explicit expressions for the linear and quadratic moments of such functional are derived using two perturbative approaches and the Feynman-Kac formula. These results hold for any well-posed initial condition and any ergodic system, including systems out of detailed balance. Moreover, we explicitly show the emergence of the Central Limit Theorem for such variables in the long time limit, a generic upper bound for the fluctuations of the local time fraction in the case of reversible dynamics, and apply our results to bound the precision of receptor signaling in biological settings. 


\title{
Spectral theory of fluctuations in time-average statistical mechanics of reversible and driven systems
}

\author{
Alessio Lapolla @, David Hartich, and Aljaž Godec ๑ * \\ Mathematical bioPhysics group, Max Planck Institute for Biophysical Chemistry, Göttingen 37077, Germany
}

(Received 20 April 2020; accepted 25 August 2020; published 15 October 2020)

\begin{abstract}
We present a spectral-theoretic approach to time-average statistical mechanics for general, nonequilibrium initial conditions. We consider the statistics of bounded, local additive functionals of reversible as well as irreversible ergodic stochastic dynamics with continuous or discrete state-space. We derive exact results for the mean, fluctuations, and correlations of time-average observables from the eigenspectrum of the underlying generator of Fokker-Planck or master equation dynamics, and we discuss the results from a physical perspective. Feynman-Kac formulas are rederived using Itô calculus and combined with non-Hermitian perturbation theory. The emergence of the universal central limit law in a spectral representation is shown explicitly on largedeviation timescales. For reversible dynamics with equilibrated initial conditions, we derive a general upper bound to fluctuations of occupation measures in terms of an integral of the return probability. Simple, exactly solvable examples are analyzed to demonstrate how to apply the theory. As a biophysical example, we revisit the Berg-Purcell problem on the precision of concentration measurements by a single receptor. Our results are directly applicable to a diverse range of phenomena underpinned by time-average observables and additive functionals in physical, chemical, biological, and economical systems.
\end{abstract}

DOI: 10.1103/PhysRevResearch.2.043084

\section{INTRODUCTION}

Many experiments on soft and biological matter, such as single-particle tracking [1-4] and single-molecule spectroscopy [5-13], probe individual trajectories. It is typically not feasible to repeat these experiments enough times to allow for ensemble-averaging. It is, however, straightforward to analyze such data by means of time-averaging along individual realizations. However, except for (ergodically) long observations, time-averages inferred from individual trajectories are random with nontrivial statistics. This naturally leads to the study of statistical properties of time-averages which formally represent functionals of stochastic processes.

The study of functionals of stochastic processes has a long tradition in mathematics (see, e.g., [14-21]) and finance $[22,23]$. In physics, they were found to be relevant in the context of diffusion-controlled chemical reactions (e.g., [24-26]), transport in porous media [27], chemical inference [28-37], astrophysical observations [38], medical diagnostics [39], optical imaging [40], the study of growing surfaces [41], blinking of colloidal quantum dots [42,43], mesoscopic physics [44], climate [45] and computer science [46], and most recently in single-molecule spectroscopy [47-50] and diffusion studies [51], to name a few.

\footnotetext{
*agodec@mpibpc.mpg.de
}

Published by the American Physical Society under the terms of the Creative Commons Attribution 4.0 International license. Further distribution of this work must maintain attribution to the author(s) and the published article's title, journal citation, and DOI.
From a theoretical point of view, analytical results were obtained for the occupation time statistics for discrete-state Markov switching [47-50], for the local time at zero and occupation time above zero of a Brownian particle diffusing in a simple one-dimensional potential [46,52,53], the occupation time inside a spherical domain of a Brownian particle moving in free space [51], and for a free, uniformly biased and harmonically bound particle undergoing subdiffusion $[54,55]$. Exact results were also obtained for occupation time statistics for a general class of Markov processes [56] and a discrete stationary non-Markovian sequence [57]. Large deviation functions for various nonlinear functionals of a class of Gaussian stationary Markov processes were studied in [45]. Numerous important results on functionals have also been obtained in the context of persistence in spatially extended nonequilibrium systems [58]. Exact results were recently obtained on local times for projected observables in stochastic many-body systems $[59,60]$, which provided insight into the emergence of memory on the level of individual non-Markovian trajectories. Notwithstanding, a general approach to fluctuations in time-average statistical mechanics for arbitrary initial conditions remains elusive.

Here, we present a spectral-theoretic approach to finite time-average statistical mechanics of ergodic systems. In mathematical terms, we focus on the statistics of bounded, local, additive functionals of normal ergodic Markovian stochastic processes with continuous and discrete statespaces, including functionals of their (non-Markovian) lowerdimensional projections. The paper is organized as follows. We first provide in Sec. II a brief introduction into timeaverage statistical mechanics. In Sec. III we rederive the well-known Feynman-Kac formulas for Markovian diffusion using Itô calculus. In Sec. IV A spectral theory combined 


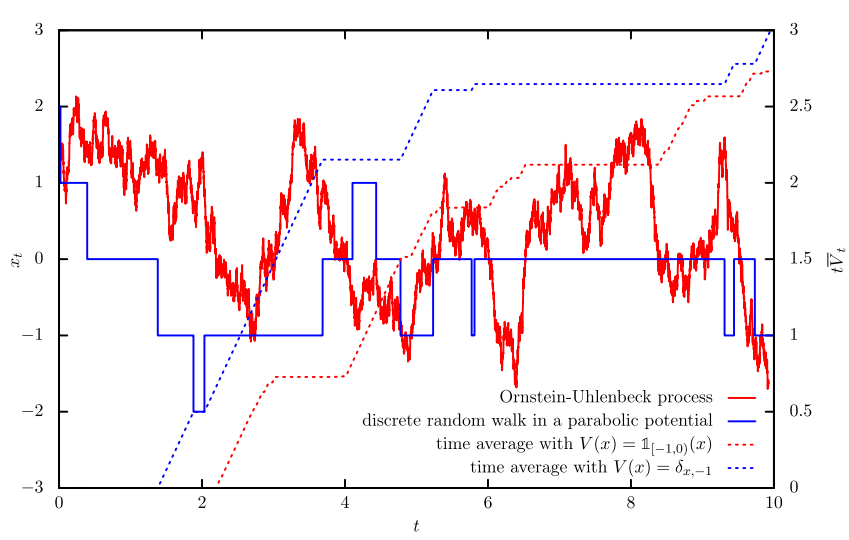

FIG. 1. Realization of a trajectory $\mathbf{x}_{t}$ (solid lines) of a continuous Ornstein-Uhlenbeck diffusion (red) and a Markovian discretespace continuous-time random walk in a quadratic potential (blue). The dotted lines refer to the time average, Eq. (3), with $t \bar{V}_{t}=$ $\int_{0}^{t} V\left(x_{\tau}\right) d \tau$, where we chose $V(x)=\mathbb{1}_{[0,1)}(x)$ for the OrnsteinUhlenbeck and $V(x)=\delta_{-1, x}$ for the discrete random walk.

with non-Hermitian perturbation theory is applied to obtain our main result-exact expressions for the mean, variance, and correlations of time-average observables for any nonstationary preparation of the system, expressed explicitly in terms of the eigenspectrum of the underlying generator of the dynamics, which may correspond to Fokker-Planck diffusion or Markovian dynamics governed by a master equation. We demonstrate explicitly the emergence of a central limit law in a spectral representation on large-deviation timescales. In Sec. IV C we derive our second main result - a general upper bound on fluctuations of occupation measures in terms of an integral of the, generally non-Markovian, return probability that is valid for generators of overdamped dynamics obeying detailed balance. Finally, simple analytically solvable examples are provided in Sec. V to demonstrate how to apply the theory. We conclude in Sec. VI.

\section{TIME-AVERAGE STATISTICAL MECHANICS}

\section{A. Ensemble- versus time-average observables}

Traditional (classical) ensemble statistical mechanics describes physical observations as averages over individual realizations of the dynamics at single (or multiple) predetermined times. For example, the ensemble average of an observable $V\left(\mathbf{x}_{t}\right)$ at a time $t$ for an ergodic stochastic process $\mathbf{x}_{\tau}(0 \leqslant \tau \leqslant t)$ starting from some nonstationary initial condition $p_{0}\left(\mathbf{x}_{0}\right)$ is defined by

$$
\left\langle V\left(\mathbf{x}_{t}\right)\right\rangle_{p_{0}} \equiv \begin{cases}\int_{\Omega} d \mathbf{x} \int_{\Omega} d \mathbf{x}_{0} V(\mathbf{x}) P_{t}\left(\mathbf{x} \mid \mathbf{x}_{0}\right) p_{0}\left(\mathbf{x}_{0}\right), & \Omega \subset \mathbb{R}^{d}, \\ \sum_{\mathbf{x} \in \Omega} \sum_{\mathbf{x}_{0} \in \Omega} V(\mathbf{x}) P_{t}\left(\mathbf{x} \mid \mathbf{x}_{0}\right) p_{0}\left(\mathbf{x}_{0}\right), & \Omega \text { discrete },\end{cases}
$$

where $\Omega$ is the state space of the process and $P_{t}\left(\mathbf{x} \mid \mathbf{x}_{0}\right)$ is the so-called propagator, i.e., $P_{t}\left(\mathbf{x} \mid \mathbf{x}_{0}\right) d \mathbf{x}$ (upper line) is the probability that the process is found in $\mathbf{x} \in \Omega$ within the increment $d \mathbf{x}$ at time $t$ given that it started at $t=0$ at $\mathbf{x}_{0}$. Note that if $\mathbf{x}$ is continuously valued ( $\mathbf{x} \in \Omega \subset \mathbb{R}^{d}$; see Fig. 1, solid red line), then $P_{t}\left(\mathbf{x} \mid \mathbf{x}_{0}\right)$ is a probability density, whereas if $\mathbf{x}$ is from a discrete state space $\Omega$ (Fig. 1, solid blue line), the integral in Eq. (1) (upper line) becomes a sum, $\int_{\Omega} d \mathbf{x} \rightarrow \sum_{\mathbf{x} \in \Omega}$, and $P_{t}\left(\mathbf{x} \mid \mathbf{x}_{0}\right)$ becomes a plain probability as shown in the lower line of Eq. (1). If $\mathbf{x}_{0}$ is sampled and averaged over a stationary (invariant) measure, $p\left(\mathbf{x}_{0}\right)=P_{\text {inv }}\left(\mathbf{x}_{0}\right)$, or if $t$ becomes sufficiently (i.e., ergodically) large, $P_{\infty}\left(\mathbf{x} \mid \mathbf{x}_{0}\right) \equiv P_{\text {inv }}(\mathbf{x})$, the ensemble average becomes time-independent,

$$
\langle V\rangle_{\mathrm{inv}} \equiv \int d \mathbf{x} V(\mathbf{x}) P_{\mathrm{inv}}(\mathbf{x}) .
$$

Conversely, in single-molecule dynamics, single-particle tracking, and other related experiments, one probes individual realizations of $\mathbf{x}_{\tau}$ within the interval $0 \leqslant \tau \leqslant t$ and instead analyzes the observation by taking a time-average. Such time-average observables are in general random, fluctuating quantities with nontrivial statistics. For example, for a physical observable $V\left(\mathbf{x}_{t}\right)$, which may correspond to the squared displacement $[61,62]$ or local time $[51,59,60,63]$ in single-particle tracking or the FRET efficiency [5-8] or distance between two optical traps [9-13] in single-molecule fluorescence and force spectroscopy, respectively, the (local) time-average is defined as

$$
\bar{V}_{t} \equiv t^{-1} \int_{0}^{t} V\left(\mathbf{x}_{\tau}\right) d \tau
$$

and it depends on the entire history of $\mathbf{x}_{\tau}$ until time $t$ (see also the dotted lines in Fig. 1). The statistical evolution of $\bar{V}_{t}$ is therefore a non-Markovian process characterized by the probability density that the random observable $\bar{V}_{t}$ attains, in a given realization of $\mathbf{x}_{\tau}$, the value $v[46,52,53,59,60]$, which is defined as

$$
\mathcal{P}_{t}^{\bar{V}}\left(v \mid \mathbf{x}_{0}\right) \equiv\left\langle\delta\left(v-\bar{V}_{t}\right)\right\rangle_{\mathbf{x}_{0}}
$$

where $\delta(z)$ is the Dirac delta function and $\langle\cdot\rangle_{\mathbf{x}_{0}}$ denotes the average over all paths starting at $\mathbf{x}_{0}$, i.e., $p_{0}(\mathbf{x})=\delta\left(\mathbf{x}-\mathbf{x}_{0}\right)$, and propagating until time $t$. The corresponding result for arbitrary initial conditions $p_{0}\left(\mathbf{x}_{0}\right)$ follows by superposition, i.e., $\mathcal{P}_{t}^{\bar{V}}\left(\nu \mid p_{0}\right) \equiv \int_{\Omega} \mathcal{P}_{t}^{\bar{V}}\left(\nu \mid \mathbf{x}_{0}\right) p_{0}\left(\mathbf{x}_{0}\right) d \mathbf{x}_{0}$ (see also Sec. III B).

The random "empirical density" [64] $\theta_{\mathbf{x}}(t)$ determined from a single trajectory in time-average statistical mechanics is the so-called local-time fraction defined as $[21,59,60]$

$$
\theta_{\mathbf{x}}(t) \equiv t^{-1} \int_{0}^{t} d \tau \delta\left(\mathbf{x}-\mathbf{x}_{\tau}\right)
$$

which allows us to rewrite the time average (3) in the form

$$
\bar{V}_{t}=t^{-1} \int_{0}^{t} d \tau \int_{\Omega} d \mathbf{x} \delta\left(\mathbf{x}-\mathbf{x}_{\tau}\right) V(\mathbf{x}) \equiv \int_{\Omega} d \mathbf{x} V(\mathbf{x}) \theta_{\mathbf{x}}(t),
$$

where $\delta\left(\mathbf{x}-\mathbf{x}_{\tau}\right)$ denotes the Dirac delta function if $\mathbf{x} \in \Omega$ is continuous, whereas $\delta\left(\mathbf{x}-\mathbf{x}_{\tau}\right)$ denotes the Kronecker delta if $\mathbf{x} \in \Omega$ is integer-valued. Note that it is often useful to generalize the local-time fraction in a point $\mathbf{x}$ in Eq. (5) to the notion of occupation time within the hypersurface $V(\mathbf{x})=\mathcal{V}$ defined as

$$
\theta_{\mathcal{V}}(t) \equiv t^{-1} \int_{0}^{t} \delta\left(\mathcal{V}-V\left(\mathbf{x}_{\tau}\right)\right) d \tau
$$


Accordingly, we can rewrite Eq. (6) equivalently in terms of $\theta_{\mathcal{V}}(t)$ as

$$
\bar{V}_{t}=t^{-1} \int_{0}^{t} d \tau \int d \mathcal{V} \delta\left(\mathcal{V}-V\left(\mathbf{x}_{\tau}\right)\right) \mathcal{V} \equiv \int d \mathcal{V} \mathcal{V} \theta_{\mathcal{V}}(t)
$$

Because the dynamics $\mathbf{x}_{t}$ is assumed to be ergodic, we have $\lim _{t \rightarrow \infty} \theta_{\mathbf{x}}(t)=P_{\text {inv }}(\mathbf{x})$ and $\lim _{t \rightarrow \infty} \theta_{\mathcal{V}}(t)=P_{\text {inv }}(\mathcal{V})$ $[52,59,60]$, and

$$
\begin{aligned}
& \lim _{t \rightarrow \infty} \bar{V}_{t}=\int d \mathbf{x} V(\mathbf{x}) P_{\text {inv }}(\mathbf{x})=\int d \mathcal{V} \mathcal{V} P_{\text {inv }}(\mathcal{V}) \equiv\langle V\rangle_{\text {inv }}, \\
& \lim _{t \rightarrow \infty} \mathcal{P}_{t}^{\bar{V}}\left(\nu \mid \mathbf{x}_{0}\right)=\delta\left(v-\langle V\rangle_{\text {inv }}\right),
\end{aligned}
$$

where we have defined the stationary (or invariant) measure of $\bar{V}\left(\mathbf{x}_{t}\right)$, i.e., $P_{\text {inv }}(\mathcal{V}) \equiv \int d \mathbf{x} \delta(\mathcal{V}-V(\mathbf{x})) P_{\text {inv }}(\mathbf{x})$.

Equations (9) reflect the strong law of large numbers on timescales where $\bar{V}_{t}$ for different values of $t$ decorrelate. Moreover, on the so-called large-deviation timescale, i.e., on the timescale that is finite but longer that the longest relaxation time of $\mathbf{x}_{t}$, we find convergence in the mean, $\left\langle\bar{V}_{t_{\mathrm{LD}}}\right\rangle=$ $\langle V\rangle_{\text {inv }}$, and Gaussian fluctuations around the mean value $[45,59,60,65,66]$. For finite, and in particular subergodic (i.e., supralarge-deviation) times, the statistics of $\bar{V}_{t}$ is, however, nontrivial. Below we provide intuition about the local-time fraction from a practical perspective.

\section{B. Local-time fraction as a histogram inferred from a single trajectory}

To gain more intuition about the local-time fraction (or "empirical density"), we consider, as an example, a Brownian particle diffusing in a harmonic potential. A single trajectory starting from $x_{0}=1.2$ is recorded as a function of time (see the full red line in Fig. 1). We are interested in the distribution (i.e., a histogram) of the particle's position $x_{t}$ inferred from a single trajectory of length $t$ (see Fig. 2). Note that in Fig. 2 we consider a histogram with a finite bin-size $\Delta$, which we denote explicitly as $\theta_{x}^{\Delta}(t)$. In this sense, the local-time fraction (5) is simply a mathematical idealization of a histogram, i.e., $\theta_{x}(t)=\theta_{x}^{\Delta \rightarrow 0}(t)$.

If the trajectory is sufficiently long (i.e., $t \rightarrow \infty), \theta_{x}^{\Delta}(t)$ converges, up to small fluctuations of order $1 / \sqrt{t}$, to a Gaussian stationary (invariant) measure $P_{\text {inv }}(x) \propto e^{-x^{2} / 2}$. This convergence is depicted explicitly in Fig. 2(b). According to Eq. (9), this result depends neither on the initial condition $x_{0}$ nor on the particular realization of the trajectory.

We may also infer a histogram of the particle's position from a short trajectory. The resulting histogram $\theta_{x}^{\Delta}(t)$ appears "rough" and far from Gaussian [see the histogram in Fig. 2(a)]. If we were to repeat the analysis for many trajectories and infer an averaged histogram $\left\langle\theta_{x}^{\Delta}(t)\right\rangle$, we would find as well that it deviates strongly from a Gaussian [see the line in Fig. 2(a)] with large fluctuations around the mean, $\delta \theta_{x}^{\Delta}(t) \equiv\left|\theta_{x}^{\Delta}(t)-\left\langle\theta_{x}^{\Delta}(t)\right\rangle\right| \sim\left\langle\theta_{x}^{\Delta}(t)\right\rangle$ [see the histogram in Fig. 2(a)]. Moreover, both the mean histogram $\left\langle\theta_{x}^{\Delta}(t)\right\rangle$ and the fluctuations around the mean, $\delta \theta_{x}^{\Delta}(t)$, depend not only on $t$ but also on the initial position $x_{0}$, where we observe a persistent cusp [Fig. 2(a)].
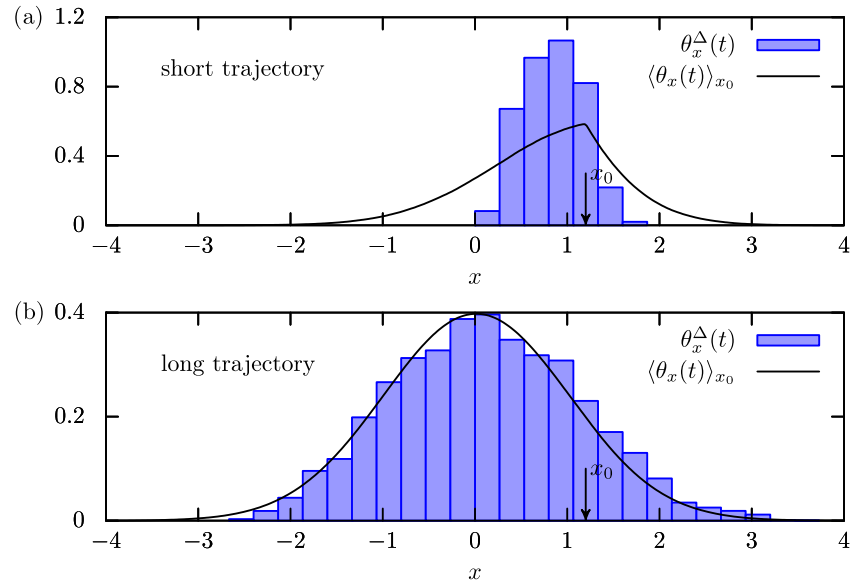

FIG. 2. Local time fraction (or "empirical density") for a Brownian particle in a harmonic potential. The histogram $\theta_{x}^{\Delta}(t)$ (blue) inferred from a single trajectory starting from $x_{0}=1.2$ compared the mean local-time fraction $\left\langle\theta_{y}(t)\right\rangle_{x_{0}}=\lim _{\Delta \rightarrow 0}\left\langle\theta_{y}^{\Delta}(t)\right\rangle_{x_{0}}$. The histogram with bin-size $\Delta$ (here we assume $\Delta=0.3$ ) is defined by $\theta_{x}^{\Delta}(t)=\Delta^{-1} \int_{x-\Delta / 2}^{x+\Delta / 2} \theta_{y}(t) d y$, that is, $\theta_{x}^{\Delta}(t)=\bar{V}_{t}$ with $V(x) \equiv$ $\Delta^{-1} \mathbb{1}_{[x-\Delta / 2, x+\Delta / 2]}(x)$, where " $\mathbb{1}$ " denotes the indicator function being 1 if $x \in[x-\Delta / 2, x+\Delta / 2]$ and 0 otherwise. Parameters: $x_{0}=1.2$; (a) $t=1$, (b) $t=100$ (the trajectory is ten times as long as in Fig. 1).

In the reminder of this work, we will focus on the mean values and fluctuations of entries, as well as linear correlations between entries of such (random, realization-dependent) histograms inferred from finite, individual trajectories starting from general initial conditions.

\section{Fluctuations of time averages}

To exploit the role of the local-time fraction $\theta_{\mathbf{x}}(t)$ as a "propagator" in time-average statistical mechanics [via $\bar{V}_{t}=$ $\left.\int_{\Omega} d \mathbf{x} V(\mathbf{x}) \theta_{\mathbf{x}}(t)\right]$, we now relate the statistics of $\theta_{\mathbf{x}}(t)$ to the probability density of a general time-averaged physical observable $\bar{V}_{t}$ defined in Eq. (3). In the example in Fig. 2, $\bar{V}_{t}=\theta_{x}^{\Delta}$ accounts for the value of the histogram at position $x$. The characteristic function of $\bar{V}_{t}$, i.e., the Laplace transform $\tilde{\mathcal{P}}_{t}^{\bar{V}}\left(u \mid \mathbf{x}_{0}\right) \equiv \int_{0}^{\infty} e^{-u v} \mathcal{P}_{t}^{\bar{V}}\left(v \mid \mathbf{x}_{0}\right) d v$ if $v \geqslant 0$, reads

$$
\tilde{\mathcal{P}}_{t}^{\bar{V}}\left(u \mid \mathbf{x}_{0}\right)=\left\langle e^{-u \bar{V}_{t}}\right\rangle_{\mathbf{x}_{0}}=\left\langle e^{-u \int_{\Omega} V(\mathbf{x}) \theta_{\mathbf{x}}(t) d \mathbf{x}}\right\rangle_{\mathbf{x}_{0}},
$$

where we have used Eq. (6) to arrive at the second equality. Equation (10) relates the statistics of the time average $\bar{V}_{t}$ to the statistics of all local-time fractions $\theta_{\mathbf{x}}(t)(\mathbf{x} \in \Omega)$. We note that Eq. (10) must be modified if $V(\mathbf{x})$ can also become negative such that a Fourier transform is required instead, which amounts to replacing $u \rightarrow i \omega$ with $\omega \in \mathbb{R}$ in Eq. (10). The probability density is obtained from Eq. (10) by Laplace inversion,

$$
\mathcal{P}_{t}^{\bar{V}}\left(v \mid \mathbf{x}_{0}\right)=\frac{1}{2 \pi \mathrm{i}} \int_{c-i \infty}^{c+i \infty} e^{u v} \tilde{\mathcal{P}}_{t}^{\bar{V}}\left(u \mid \mathbf{x}_{0}\right) d u
$$

where $c \in \mathbb{R}$ lies to the right of all singularities of $\tilde{\mathcal{P}}_{t}^{\bar{V}}\left(u \mid \mathbf{x}_{0}\right)$ and we have assumed that $\mathcal{P}_{t}^{\bar{V}}\left(\nu \mid \mathbf{x}_{0}\right)$ is of exponential order for sufficiently large $v$ [in the following section, the conditions on $V\left(\mathbf{x}_{\tau}\right)$ will be made more precise]. If the support extends to 
negative values, Eq. (11) becomes the inverse Fourier transform. Here we are particularly interested in fluctuations of time averages $\bar{V}_{t}$ and $\bar{W}_{t}$ of different physical observables $V\left(\mathbf{x}_{\tau}\right)$ and $W\left(\mathbf{x}_{\tau}\right)$, which are quantified by $[59,60]$

$$
\begin{aligned}
\sigma_{\bar{V}}^{2}(t) & \equiv\left\langle\bar{V}_{t}^{2}\right\rangle_{\mathbf{x}_{0}}-\left\langle\bar{V}_{t}\right\rangle_{\mathbf{x}_{0}}^{2}, \\
C_{\overline{V W}}(t) & \equiv\left\langle\bar{V}_{t} \bar{W}_{t}\right\rangle_{\mathbf{x}_{0}}-\left\langle\bar{V}_{t}\right\rangle_{\mathbf{x}_{0}}\left\langle\bar{W}_{t}\right\rangle_{\mathbf{x}_{0}},
\end{aligned}
$$

where $\sigma_{V}^{2}(t)=C_{\overline{V V}}(t)$ denotes the variance of $\bar{V}_{t}$, and $C_{\overline{V W}}(t)$ denotes the covariance between $\bar{V}_{t}$ and $\bar{W}_{t}$.

In the example in Fig. 2, where $\bar{V}_{t}=\theta_{x}^{\Delta}(t)$ corresponds to the entry $x$ in the histogram, $\sigma_{\bar{V}}^{2}(t) \equiv\left\langle\delta \theta_{x}^{\Delta}(t)^{2}\right\rangle$ refers to the variance of said entries between different realizations of the histogram [i.e., the scatter of $\theta_{x}^{\Delta}(t)$ around $\left.\left\langle\theta_{x}(t)\right\rangle\right]$. Analogously, $C_{\overline{V W}}(t)$ accounts for linear correlations between pairs of entries at $x$ and $y, \theta_{x}^{\Delta}(t)$ and $\theta_{y}^{\Delta}(t)$, in a histogram inferred from a single trajectory of length $t$.

Using Eq. (10), we obtain more generally

$$
\begin{aligned}
\left\langle\bar{V}_{t}^{n} \bar{W}_{t}^{m}\right\rangle_{\mathbf{x}_{0}}= & \prod_{i=1}^{n} \int_{\Omega} d \mathbf{x}_{i} V\left(\mathbf{x}_{i}\right) \prod_{j=1}^{m} \int_{\Omega} d \mathbf{y}_{j} W\left(\mathbf{y}_{j}\right) \\
& \times\left\langle\theta_{\mathbf{x}_{1}}(t) \cdots \theta_{\mathbf{x}_{n}}(t) \theta_{\mathbf{y}_{1}}(t) \cdots \theta_{\mathbf{y}_{m}}(t)\right\rangle_{\mathbf{x}_{0}},
\end{aligned}
$$

where $\mathbf{x}_{i}, \mathbf{y}_{j} \in \Omega$, respectively. Thereby, the ensemble average corresponding to the last term in Eq. (13) is obtained by differentiating the characteristic function with respect to the Laplace (or Fourier) variable.

It therefore follows that the fluctuations and (linear) correlations of general time-average observables are fully specified by multipoint correlation functions of the local-time fraction. These are derived on the basis of the Feynman-Kac formalism, which is presented below for continuous diffusion processes. The extension to discrete state dynamics is discussed afterward.

\section{FLUCTUATIONS OF ADDITIVE FUNCTIONALS}

\section{A. Itô approach to Feynman-Kac theory of additive functionals}

It seems to be customary in the physics literature to start from a path-integral approach to Feynman-Kac theory [46] and then to derive a backward Fokker-Planck equation for the characteristic function $[52,53]$. Here we provide a simple derivation of the "forward" Feynman-Kac theory based on Itô calculus.

We consider a $d$-dimensional Markovian diffusion $\mathbf{x}_{t} \in$ $\Omega \subset \mathbb{R}^{d}$ process in the presence of a drift $\mathbf{F}(\mathbf{x})$ driven by $d$-dimensional Gaussian white noise governed by the Itô equation

$$
d \mathbf{x}_{t}=\mathbf{F}\left(\mathbf{x}_{t}\right) d t+\sigma d \mathbf{W}_{t},
$$

where $\sigma$ is a $d \times d$ noise matrix such that $\mathbf{D}=\sigma \sigma^{T} / 2$ becomes a symmetric positive-(semi)definite diffusion matrix, and $d \mathbf{W}_{t}$ is an increment of a $d$-dimensional Wiener process, such that $\left\langle W_{t}\right\rangle=\mathbf{0}$ and $\left\langle d W_{t, i} d W_{t^{\prime}, j}\right\rangle=\delta\left(t-t^{\prime}\right) \delta_{i j} d t$. We assume throughout that $\mathbf{F}(\mathbf{x})$ is sufficiently confining to ensure that the process $\mathbf{x}_{t}$ is ergodic with a steady-state probability density $P_{\text {inv }}(\mathbf{x})$. Multiplying the time average in Eq. (3) by the trajectory length $t$, we transform the time average to the additive functional

$$
\psi_{t} \equiv t \bar{V}_{t}=\int_{0}^{t} V\left(\mathbf{x}_{\tau}\right) d \tau .
$$

We now consider the joint process of $\mathbf{x}_{t}$ and $\psi_{t}$. According to Itô's lemma, any twice differentiable function $f(\mathbf{x}, \psi)$ with $\mathbf{x}_{t}$ and $\psi_{t}$ defined by Eqs. (14) and (15), respectively, satisfies

$$
\begin{aligned}
d f\left(\mathbf{x}_{t}, \psi_{t}\right)= & {\left[\mathbf{F}\left(\mathbf{x}_{t}\right) \cdot \nabla_{\mathbf{x}} f\left(\mathbf{x}_{t}, \psi_{t}\right)+\nabla_{\mathbf{x}} \cdot \mathbf{D} \nabla_{\mathbf{x}} f\left(\mathbf{x}_{t}, \psi_{t}\right)\right] d t } \\
& +\nabla_{\mathbf{x}} f\left(\mathbf{x}_{t}, \psi_{t}\right) \cdot \boldsymbol{\sigma} d \mathbf{W}_{t}+V\left(\mathbf{x}_{t}\right) \partial_{\psi} f\left(\mathbf{x}_{t}, \psi_{t}\right) d t
\end{aligned}
$$

where we inserted the diffusion matrix $\mathbf{D}=\sigma \boldsymbol{\sigma}^{T} / 2$, and for the last term we used $d \psi_{t}=V\left(\mathbf{x}_{t}\right) d t$, which follows from Eq. (15).

Using Itô's lemma (16), we derive in the following the time evolution of the joint probability density $Q_{t}\left(\mathbf{x}, \psi \mid \mathbf{x}_{0}\right)$ to find the system in state $\mathbf{x}$ and the functional $\psi_{t}$ in Eq. (15) to attain the value $\psi$ at time $t$ given that the system started from $\mathbf{x}_{0}$. For convenience, we first focus on positive functionals $(\psi \geqslant 0)$. Using a test function that vanishes at the boundary $f(\mathbf{x}, 0)=$ 0 , we obtain after some calculations [67]

$$
\begin{aligned}
\frac{d}{d t}\left\langle f\left(x_{t}, \psi_{t}\right)\right\rangle_{\mathbf{x}_{0}} & =\int_{0}^{\infty} d \psi \int_{\Omega} d \mathbf{x} f(\mathbf{x}, \psi) \partial_{t} Q_{t}\left(\mathbf{x}, \psi \mid \mathbf{x}_{0}\right) \\
& =\int_{0}^{\infty} d \psi \int_{\Omega} d \mathbf{x} Q_{t}\left(\mathbf{x}, \psi \mid \mathbf{x}_{0}\right)\left[\mathbf{F}(\mathbf{x}) \cdot \nabla_{\mathbf{x}} f(\mathbf{x}, \psi)+\nabla_{\mathbf{x}} \cdot \mathbf{D} \nabla_{\mathbf{x}} f(\mathbf{x}, \psi)+V(\mathbf{x}) \partial_{\psi} f(\mathbf{x}, \psi)\right] \\
& =\int_{0}^{\infty} d \psi \int_{\Omega} d \mathbf{x} f(\mathbf{x}, \psi)\left[-\nabla_{\mathbf{x}} \cdot \mathbf{F}(\mathbf{x})+\nabla_{\mathbf{x}} \cdot \mathbf{D} \nabla_{\mathbf{x}}-V(\mathbf{x}) \partial_{\psi}\right] Q_{t}\left(\mathbf{x}, \psi \mid \mathbf{x}_{0}\right)
\end{aligned}
$$

which is obtained as follows. In the first line of Eq. (17), we differentiate both sides of the identity $\int_{0}^{\infty} d \mathbf{x} \int d \psi f(\mathbf{x}, \psi) Q_{t}\left(\mathbf{x}, \psi \mid \mathbf{x}_{0}\right)=\left\langle f\left(\mathbf{x}_{t}, \psi_{t}\right)\right\rangle_{\mathbf{x}_{0}}$ with respect to time $t$. To obtain the second line, we inserted Itô's lemma (16) and finally performed an integration by parts. Since Eq. (17) holds for any function $f$ that vanishes at the boundary

$$
\psi=0, \text { we obtain }
$$

$$
\partial_{t} Q_{t}\left(\mathbf{x}, \psi \mid \mathbf{x}_{0}\right)=\left[\hat{L}-V(\mathbf{x}) \partial_{\psi}-V(\mathbf{x}) \delta(\psi)\right] Q_{t}\left(\mathbf{x}, \psi \mid \mathbf{x}_{0}\right),
$$

where we have defined the forward generator $\hat{L}=\nabla_{\mathbf{x}}$. $\mathbf{D} \nabla_{\mathbf{x}}-\nabla_{\mathbf{x}} \cdot \mathbf{F}(\mathbf{x})$ and further introduced a boundary term 
$V(\mathbf{x}) \delta(\psi) Q_{t}\left(\mathbf{x}, 0 \mid \mathbf{x}_{0}\right)$ that vanishes for $\psi>0$ and is derived in the following two steps. First, Eq. (17) holds for all functions $f(\mathbf{x}, \psi)$ with $f(\mathbf{x}, 0)=0$, which immediately gives Eq. (18) without the last term for $\psi>0$ (see also [67]). Second, the last term in Eq. (18) is required to guarantee the conservation of probability $\int_{0}^{\infty} d \psi \int_{\Omega} d \mathbf{x} \partial_{t} Q_{t}\left(\mathbf{x}, \psi \mid \mathbf{x}_{0}\right)=0$, i.e., to correct for the fact that there is a nonzero probability that the functional has a vanishing value $\psi=0$. Finally, performing a Laplace transform of Eq. (18), $\tilde{Q}_{t}\left(\mathbf{x}, u \mid \mathbf{x}_{0}\right) \equiv$ $\int_{0}^{\infty} e^{-u \psi} Q_{t}\left(\mathbf{x}, \psi \mid \mathbf{x}_{0}\right) d \psi=\left\langle\delta\left(\mathbf{x}-\mathbf{x}_{t}\right) e^{-u \psi_{t}}\right\rangle_{\mathbf{x}_{0}}$, we obtain the forward Feynman-Kac partial differential equation for the characteristic function of the joint density of position and $\psi$,

$$
\partial_{t} \tilde{Q}_{t}\left(\mathbf{x}, u \mid \mathbf{x}_{0}\right)=[\hat{L}-u V(\mathbf{x})] \tilde{Q}_{t}\left(\mathbf{x}, u \mid \mathbf{x}_{0}\right),
$$

where $Q_{t}\left(\mathbf{x}, \psi \mid \mathbf{x}_{0}\right)$ is the central object of the "forward" Feynman-Kac approach $[15,46]$.

We now relax the assumption by allowing for a negative support of $\psi_{t}$, which we denote explicitly by $\psi_{t} \rightarrow$ $\Psi_{t}$. In this case, we need not make any additional assumptions on $f\left(\mathbf{x}_{t}, \Psi_{t}\right)$ for $\Psi_{t}=0$ because naturally $\lim _{|\Psi| \rightarrow \infty} Q_{t}\left(\mathbf{x}, \Psi \mid \mathbf{x}_{0}\right)=0$. The lower boundary of integration over $\Psi$ in Eq. (17) is extended to $-\infty$, and the boundary terms resulting from the partial integration vanish as a result of the boundary conditions. The resulting Eq. (17) implies

$$
\partial_{t} Q_{t}\left(\mathbf{x}, \Psi \mid \mathbf{x}_{0}\right)=\left(\hat{L}-V(\mathbf{x}) \partial_{\Psi}\right) Q_{t}\left(\mathbf{x}, \Psi \mid \mathbf{x}_{0}\right),
$$

which upon taking a Fourier transform $\tilde{Q}_{t}\left(\mathbf{x}, \omega \mid \mathbf{x}_{0}\right) \equiv$ $\int_{-\infty}^{\infty} e^{-i \omega \Psi} Q_{t}\left(\mathbf{x}, \Psi \mid \mathbf{x}_{0}\right) d \Psi$ leads to the forward Feynman-Kac partial differential equation,

$$
\partial_{t} \tilde{Q}_{t}\left(\mathbf{x}, \omega \mid \mathbf{x}_{0}\right)=[\hat{L}-i \omega V(\mathbf{x})] \tilde{Q}_{t}\left(\mathbf{x}, \omega \mid \mathbf{x}_{0}\right) .
$$

Note that the generalization to the case of a joint problem for multiple functionals $\psi_{t}^{i}$ with $i=1, \ldots, p$ is straightforward. Introducing the vectorial notation $\psi_{t} \equiv \int_{0}^{t} \mathbf{V}\left(\mathbf{x}_{\tau}\right) d \tau$ with the corresponding Laplace images $\mathbf{u}$, the resulting equation reads

$$
\partial_{t} \tilde{Q}_{t}\left(\mathbf{x}, \mathbf{u} \mid \mathbf{x}_{0}\right)=[\hat{L}-\mathbf{u} \cdot \mathbf{V}(\mathbf{x})] \tilde{Q}_{t}\left(\mathbf{x}, \mathbf{u} \mid \mathbf{x}_{0}\right),
$$

which allows for the computation of higher-order correlation functions (13). What we actually seek is the marginal probability density of $\boldsymbol{\psi}$ given $\mathbf{x}_{0}$ defined by

$$
\mathcal{P}_{t}^{\psi}\left(\boldsymbol{\psi} \mid \mathbf{x}_{0}\right) \equiv \int_{\Omega} Q_{t}\left(\mathbf{x}, \boldsymbol{\psi} \mid \mathbf{x}_{0}\right) d \mathbf{x},
$$

which is also the statement of the Feynman-Kac theorem [15]. Note that the corresponding characteristic function $\tilde{\mathcal{P}}_{t}^{\psi}\left(\mathbf{u} \mid \mathbf{x}_{0}\right)=\int e^{-\mathbf{u} \cdot \psi} \mathcal{P}_{t}^{\psi}\left(\boldsymbol{\psi} \mid \mathbf{x}_{0}\right) d \boldsymbol{\psi} \equiv\left\langle e^{-\mathbf{u} \cdot \psi_{t}}\right\rangle_{\mathbf{x}_{0}}$ is the solution of the "backward" Feynman-Kac problem [52,53]. Moreover, the marginal probability density of $\mathbf{x}$ given $\mathbf{x}_{0}$ corresponds to the plain propagator $P_{t}\left(\mathbf{x} \mid \mathbf{x}_{0}\right) \equiv \int Q_{t}\left(\mathbf{x}, \boldsymbol{\psi} \mid \mathbf{x}_{0}\right) d \boldsymbol{\psi}$, which solves the (forward) Fokker-Planck equation $\left(\partial_{t}-\right.$ $\hat{L}) P_{t}\left(\mathbf{x} \mid \mathbf{x}_{0}\right)=0$ with initial data $P_{0}\left(\mathbf{x} \mid \mathbf{x}_{0}\right)=\delta\left(\mathbf{x}-\mathbf{x}_{0}\right)$.

Note that the characteristic functions $\tilde{\mathcal{P}}_{t}^{\overline{\mathbf{V}}}$ and $\tilde{\mathcal{P}}_{t}^{\psi}$ are equivalent up to a trivial rescaling of the independent variable, i.e.,

$$
\tilde{\mathcal{P}}_{t}^{\overline{\mathbf{V}}}\left(\mathbf{u} \mid \mathbf{x}_{0}\right)=\left\langle e^{-\mathbf{u} \cdot \overline{\mathbf{V}}_{t}}\right\rangle=\left\langle e^{-\mathbf{u} / t \cdot \psi_{t}}\right\rangle=\tilde{\mathcal{P}}_{t}^{\psi}\left(\mathbf{u} / t \mid \mathbf{x}_{0}\right) .
$$

Therefore, once $\tilde{\mathcal{P}}_{t}^{\psi}$ is determined according to the FeynmanKac program, a simple change of scale of the Laplace image $\mathbf{u} \rightarrow \mathbf{u} / t$ delivers $\tilde{\mathcal{P}}_{t}^{\overline{\mathbf{V}}}$.

\section{B. From the forward to the backward Feynman-Kac equation}

For convenience, we henceforth adopt the bra-ket notation, where the "ket" $|h\rangle$ denotes a vector, the "bra" the integral operator $\langle g| \equiv \int_{\Omega} d \mathbf{x} g^{\dagger}(\mathbf{x})$, and the scalar product is defined as $\langle g \mid h\rangle \equiv \int_{\Omega} d \mathbf{x} g^{\dagger}(\mathbf{x}) h(\mathbf{x})$. Introducing, moreover, the "flat" state $|-\rangle \equiv \int_{\Omega} d \mathbf{x}|\mathbf{x}\rangle$ and $\langle-| \equiv \int_{\Omega} d \mathbf{x}\langle\mathbf{x}|$, Eqs. (21) and (22) for a general initial condition $p_{0}(\mathbf{x})$, i.e., $\left|p_{0}\right\rangle=$ $\int_{\Omega} d \mathbf{x}_{0} p_{0}\left(\mathbf{x}_{0}\right)\left|\mathbf{x}_{0}\right\rangle$ and $\left\langle p_{0}\right|=\int_{\Omega} d \mathbf{x}_{0} p_{0}\left(\mathbf{x}_{0}\right)\left\langle\mathbf{x}_{0}\right|$, have the solution [59]

$$
\tilde{\mathcal{P}}_{t}^{\psi}\left(\mathbf{u} \mid p_{0}\right) \equiv\left\langle-\left|e^{t[\hat{L}-\mathbf{u} \cdot \mathbf{V}(\mathbf{x})]}\right| p_{0}\right\rangle=\left\langle p_{0}\left|e^{t\left[\hat{L}^{\dagger}-\mathbf{u} \cdot \mathbf{V}(\mathbf{x})\right]}\right|-\right\rangle .
$$

To arrive at the second equality we have used Green's identity, introduced the adjoint (or backward) Fokker-Planck operator $\hat{L}^{\dagger}=\nabla_{\mathbf{x}} \cdot \mathbf{D} \nabla_{\mathbf{x}}+\mathbf{F}(\mathbf{x}) \cdot \nabla_{\mathbf{x}}$, and used that the Laplace transform of a real function $f(t)$ transforms as $\tilde{f}\left(s^{\dagger}\right)=\tilde{f}^{\dagger}(s)$ under complex conjugation. In the following subsection, we show that for Markov-jump processes (25), the theory can be adopted one-to-one.

\section{Markov-jump dynamics and additive functionals}

Markov-jump processes correspond to a discrete statespace $\Omega$ in which the system jumps with a constant rate $w_{\mathbf{x y}}$ from state $\mathbf{x} \in \Omega$ to another state $\mathbf{y} \in \Omega$, such that the propagator $P_{t}\left(\mathbf{x} \mid \mathbf{x}_{0}\right)$ satisfies the master equation

$$
\partial_{t} P_{t}\left(\mathbf{x} \mid \mathbf{x}_{0}\right)=\sum_{\mathbf{y}}\langle\mathbf{x}|\hat{L}| \mathbf{y}\rangle P_{t}\left(\mathbf{y} \mid \mathbf{x}_{0}\right)
$$

where $\langle\mathbf{x}|\hat{L}| \mathbf{y}\rangle=w_{\mathbf{y x}}$ if $\mathbf{x} \neq \mathbf{y}$ and $\langle\mathbf{x}|\hat{L}| \mathbf{x}\rangle=-\sum_{\mathbf{y} \neq \mathbf{x}}\langle\mathbf{y}|\hat{L}| \mathbf{x}\rangle$ such that $-\langle\mathbf{x}|\hat{L}| \mathbf{x}\rangle>0$ is the rate of leaving state $\mathbf{x}$. According to the celebrated Gillespie algorithm [68], a single trajectory $\mathbf{x}_{\tau}(0 \leqslant \tau \leqslant t)$ consists of a sequence of exponentially distributed local waiting times $\tau_{i}$ in state $\mathbf{x}_{i} \in \Omega$ followed by an instantaneous transition to another state $\mathbf{x}_{i+1} \neq$ $\mathbf{x}_{i}$, with the total time being the sum of waiting times $\sum_{i} \tau_{i}=$ $t-\tau_{R}$, where $\tau_{R}$ is the duration of the final epoch that contains no jump. More precisely, whenever the system is in a state $\mathbf{x}_{i}$ at time $t_{i}$, the probability density to leave said state $\mathbf{x}_{i}$ exactly at time $t_{i+1}=t_{i}+\tau_{i}$ is exponentially distributed with a waiting time density $-\left\langle\mathbf{x}_{i}|\hat{L}| \mathbf{x}_{i}\right\rangle e^{\left\langle\mathbf{x}_{i}|\hat{L}| \mathbf{x}_{i}\right\rangle \tau_{i}}$. After the waiting time, a new (accessible) state, $\mathbf{x}_{i+1}$, is randomly chosen with probability $-\left\langle\mathbf{x}_{i+1}|\hat{L}| \mathbf{x}_{i}\right\rangle /\left\langle\mathbf{x}_{i}|\hat{L}| \mathbf{x}_{i}\right\rangle$. Therefore, the joint probability density that the system, starting from state $\mathbf{x}_{i}$, jumps after time $\tau_{i}$ and the following state is $\mathbf{x}_{i+1}$ becomes $\left\langle\mathbf{x}_{i+1}|\hat{L}| \mathbf{x}_{i}\right\rangle e^{\left\langle\mathbf{x}_{i}|\hat{L}| \mathbf{x}_{i}\right\rangle \tau_{i}}$.

Denoting the number of transitions from state $\mathbf{x}$ to state $\mathbf{y}$ until a time $t$ by $n_{\mathbf{x y}}(t)=\sum_{i} \delta_{\mathbf{x}_{i}, \mathbf{x}} \delta_{\mathbf{x}_{i+1}, \mathbf{y}}$ and identifying the sum of all local waiting times in state $\mathbf{x}$ up to time $t$ by $t \theta_{\mathbf{x}}(t)=\sum_{i} \delta_{\mathbf{x}_{i}, \mathbf{x}} \tau_{i}$, the path probability (or path weight) of $\mathbf{x}_{\tau}(0 \leqslant \tau \leqslant t)$ starting from $\mathbf{x}_{0}$ generated by the Markov dynamics (26) can be written as [37]

$$
P\left(\left\{\mathbf{x}_{\tau}\right\} \mid \mathbf{x}_{0}\right)=\prod_{\mathbf{x} \neq \mathbf{y}}[\langle\mathbf{x}|\hat{L}| \mathbf{y}\rangle]^{n_{\mathbf{y x}}(t)} e^{\sum_{\mathbf{x}} t \theta_{\mathbf{x}}(t)\langle\mathbf{x}|\hat{L}| \mathbf{x}\rangle} .
$$

Replacing the integral in Eq. (6) by a sum, $\bar{V}_{t}=$ $\sum_{\mathbf{x}} V(\mathbf{x}) \theta_{\mathbf{x}}(t)$, allows us to identify the characteristic function 


$$
\begin{aligned}
& \text { of } \psi_{t}=t \bar{V}_{t} \text { by [37] } \\
& \qquad \begin{aligned}
\tilde{\mathcal{P}}_{t}^{\psi}\left(u \mid \mathbf{x}_{0}\right) & \equiv\left\langle e^{-u t \bar{V}_{t}}\right\rangle_{\mathbf{x}_{0}} \\
& =\int d\left\{\mathbf{x}_{\tau}\right\} P\left(\left\{\mathbf{x}_{\tau}\right\} \mid \mathbf{x}_{0}\right) e^{-u \sum_{\mathbf{x}} V(\mathbf{x}) t \theta_{\mathbf{x}}(t)} \\
& =\left\langle-\left|e^{\hat{L}(u) t}\right| \mathbf{x}_{0}\right\rangle,
\end{aligned}
\end{aligned}
$$

where in the second line we inserted the path weight Eq. (27). While passing from the second to the third line, we tilted the diagonal of the generator in the path weight (27) according to $\langle\mathbf{x}|\hat{L}(u)| \mathbf{x}\rangle \equiv\langle\mathbf{x}|\hat{L}| \mathbf{x}\rangle-u V(\mathbf{x})$, which effectively moves $e^{-u t \bar{V}_{t}}$ into the tilted path weight. In other words, identifying $\hat{L}(u)$ in the second line of Eq. (28) yields the third line. Note that the off-diagonal elements of the tilted generator remain unchanged, that is, $\langle\mathbf{x}|\hat{L}(u)| \mathbf{y}\rangle \equiv\langle\mathbf{x}|\hat{L}| \mathbf{y}\rangle$ if $\mathbf{x} \neq \mathbf{y}$. Since all elements of $\hat{L}$ and $V(\mathbf{x})$ are real, Eq. (25) holds also for Markov-jump processes. As shown in Eq. (24), the characteristic function of $\bar{V}_{t}=\psi_{t} / t$ follows from a trivial change of scale, $\tilde{\mathcal{P}}_{t}^{\bar{V}}\left(u \mid \mathbf{x}_{0}\right)=\tilde{\mathcal{P}}_{t}^{\psi}\left(u / t \mid \mathbf{x}_{0}\right)$. In the following, we develop a spectral theory, which unifies diffusion processes and Markov-jump processes.

\section{Spectral theory of non-Hermitian generators}

We henceforth employ a spectral-theoretic approach and are thus required to make some more specific assumptions about the underlying dynamics in order to assure that the generator $\hat{L}$ is diagonalizable. An excellent account of the theory for Markov-jump dynamics governed by a discrete-state master equation can be found in [69]. In the case of FokkerPlanck dynamics, we consider that $\mathbf{x}_{t}$ is an ergodic Markovian diffusion evolving according to Eq. (14) with the drift field $\mathbf{F}(\mathbf{x})$ not necessarily corresponding to a potential field (which thus includes systems with a broken detailed balance) but at the same time requiring that it is sufficiently confining, that is, it grows sufficiently fast as $|\mathbf{x}| \rightarrow \infty$ to assure that $\hat{L}$ has a pure point-spectrum. Moreover, we require that $\hat{L}$ is diagonalizable, and it can be shown that any normal operator $\hat{L}$, satisfying $\hat{L} \hat{L}^{\dagger}=\hat{L}^{\dagger} \hat{L}$, is in fact diagonalizable [70]. A more detailed mathematical exposé of the requirements for, and properties of, $\hat{L}$ can be found in [60]. In all practical examples presented below, we will in fact assume that the dynamics is overdamped. Moreover, except for the example presented in Sec. V C where detailed balance is violated, $\hat{L}$ will be assumed to obey detailed balance [67,71], implying that it is orthogonally equivalent to a self-adjoint operator and hence automatically diagonalizable.

Let $-\lambda_{k}\left[\operatorname{Re}\left(\lambda_{k}\right) \geqslant 0\right],\left\langle L_{k}\right|$, and $\left|R_{k}\right\rangle$ denote the eigenvalue and orthonormal left and right eigenstates of $\hat{L}$, and let $-\lambda_{k}^{\dagger},\left\langle R_{k}\right|$, and $\left|L_{k}\right\rangle$ denote the corresponding orthonormal eigenstates of $\hat{L}^{\dagger}$ [60], i.e., $\left\langle L_{k} \mid R_{l}\right\rangle=\left\langle R_{k} \mid L_{l}\right\rangle=\delta_{k l}$, with the resolution of identity $\sum_{k}\left|R_{k}\right\rangle\left\langle L_{k}\left|\equiv \sum_{k}\right| L_{k}\right\rangle\left\langle R_{k}\right|=\mathbf{1}$. Then written in the respective eigenbases, $\hat{L}$ and $\hat{L}^{\dagger}$ read

$$
\hat{L}=-\sum_{k} \lambda_{k}\left|R_{k}\right\rangle\left\langle L_{k}\left|, \quad \hat{L}^{\dagger}=-\sum_{k} \lambda_{k}^{\dagger}\right| L_{k}\right\rangle\left\langle R_{k}\right|,
$$

with the ground-state eigenvalue $\lambda_{0}=0$ and the corresponding null-space $\left|R_{0}\right\rangle \equiv\left|P_{\text {inv }}\right\rangle$ and $\left\langle L_{0}\right| \equiv\langle-|$. In the respective dual eigenbasis the propagator $P_{t}\left(\mathbf{x} \mid \mathbf{x}_{0}\right) \equiv\left\langle\mathbf{x}\left|e^{\hat{L} t}\right| \mathbf{x}_{0}\right\rangle=$
$\left\langle\mathbf{x}_{0}\left|e^{\hat{L}^{\dagger} t}\right| \mathbf{x}\right\rangle$ reads

$$
\begin{aligned}
P_{t}\left(\mathbf{x} \mid \mathbf{x}_{0}\right) & =\sum_{k}\left\langle\mathbf{x} \mid R_{k}\right\rangle\left\langle L_{k} \mid \mathbf{x}_{0}\right\rangle e^{-\lambda_{k} t} \\
& \equiv \sum_{k}\left\langle\mathbf{x}_{0} \mid L_{k}\right\rangle\left\langle R_{k} \mid \mathbf{x}\right\rangle e^{-\lambda_{k}^{\dagger} t},
\end{aligned}
$$

where $\left\langle\mathbf{x} \mid R_{k}\right\rangle \equiv R_{k}(\mathbf{x})$ and $\left\langle L_{k}\right| \mathbf{x}_{0} \equiv L_{k}^{\dagger}\left(\mathbf{x}_{0}\right)$, while $\left\langle\mathbf{x}_{0} \mid L_{k}\right\rangle=$ $L_{k}\left(\mathbf{x}_{0}\right)$ and $\left\langle R_{k} \mid \mathbf{x}\right\rangle=R_{k}^{\dagger}(\mathbf{x})$. For overdamped systems with an invertible diffusion matrix $\mathbf{D}$ that obey detailed balance, i.e., $\mathbf{D}^{-1} \mathbf{F}(\mathbf{x})=-\beta \nabla_{\mathbf{x}} U(\mathbf{x})$ with inverse thermal energy $\beta=1 / k_{\mathrm{B}} T$, all $\lambda_{k}$ are real, $\left|P_{\text {inv }}\right\rangle=\left|P_{\mathrm{eq}}\right\rangle$ is the BoltzmannGibbs equilibrium $P_{\mathrm{eq}}(\mathbf{x})=e^{-\beta U(\mathbf{x})} / \int_{\Omega} e^{-\beta U(\mathbf{x})} d \mathbf{x}$, and $\left|L_{k}\right\rangle=e^{\beta U(\mathbf{x})}\left|R_{k}\right\rangle[67,72]$.

\section{CHARACTERISTIC FUNCTION NEAR ZERO VIA NON-HERMITIAN PERTURBATION THEORY}

Based on Eqs. (10) and (13), we only require the momentgenerating function (25) in the limit $|\mathbf{u}| \rightarrow \mathbf{0}$ to calculate moments of arbitrary order. Moreover, recall that $\tilde{\mathcal{P}}_{t} \overline{\mathbf{v}}(\mathbf{u})=$ $\tilde{\mathcal{P}}_{t}^{\psi}(\mathbf{u} / t)$ [see Eq. (24)]. To keep the treatment general, we utilize the spectral expansion of $\hat{L}$ ( $\hat{L}^{\dagger}$, respectively). We employ perturbation theory to derive the moment-generating function (25) in the limit $|\mathbf{u}| \rightarrow \mathbf{0}$. There are (at least) two possible ways to arrive at the result: a Dyson series approach, which is presented in Appendix B, and by means of secondorder non-Hermitian perturbation theory, which is detailed below. While both yield equivalent results, the perturbationtheoretic approach is more general as it provides a (bi)spectral expansion of the perturbed generator $\hat{L}-\mathbf{u} \cdot \mathbf{V}(\mathbf{x})\left[\hat{L}^{\dagger}-\mathbf{u}\right.$. $\mathbf{V}(\mathbf{x})$, respectively] to second order in $|\mathbf{u}|$. These perturbationtheoretic results, which in the physics literature appear to be new, are applicable beyond time-average statistical mechanics in diverse problems involving perturbations of non-Hermitian and/or non-self-adjoint eigenvalue problems.

Our aim is to diagonalize the "tilted" propagator in Eq. (25) in the limit when $\mathbf{u}$ vanishes. Because $\hat{L}$ is in general not self-adjoint, we need to separately perturb left and right eigenstates. First we must confirm that the tilted propagator $\hat{L}(\mathbf{u}) \equiv$ $\hat{L}-\mathbf{u} \cdot \mathbf{V}(\mathbf{x})$ [and $\hat{L}^{\dagger}(\mathbf{u}) \equiv \hat{L}^{\dagger}-\mathbf{u} \cdot \mathbf{V}(\mathbf{x})$, respectively] is actually diagonalizable in an arbitrarily small neighborhood of $\mathbf{u}=\mathbf{0}$. We focus first on the case in which $V(\mathbf{x}) \geqslant 0$. We Laplace-transform Eq. (25), $t \rightarrow s$, yielding

$$
\tilde{\mathcal{P}}_{s}^{\psi}\left(\mathbf{u} \mid p_{0}\right) \equiv\left\langle-\left|[s-\hat{L}(\mathbf{u})]^{-1}\right| p_{0}\right\rangle=\left\langle p_{0}\left|\left[s-\hat{L}^{\dagger}(\mathbf{u})\right]^{-1}\right|-\right\rangle .
$$

The singularities of Eq. (31) correspond to the perturbed eigenvalue spectrum $\left\{-\lambda_{k}(\mathbf{u})\right\}$ of $\hat{L}(\mathbf{u})$, and diagonalizability is broken whenever one or more singularities are not simple poles (see, e.g., [73]). Equation (25) shows that $|\mathbf{u}|=\mathbf{0}$ is not an accumulation point. Moreover, $\hat{L}$ has a pure point spectrum, therefore an arbitrarily small $|\mathbf{u}|$ cannot cause the emergence of poles of second order in Eq. (31) that would break diagonalizability, akin to the "avoided crossing theorem." Therefore, in the limit $|\mathbf{u}| \rightarrow \mathbf{0}$ the tilted generator $\hat{L}(\mathbf{u})$ is diagonalizable, $\mathbf{u}$ can be taken as real [74], and the eigenspectrum of $\hat{L}(\mathbf{u})$ corresponds to a regular perturbation of the original eigenvalue problem $\hat{L}\left|R_{k}\right\rangle=-\lambda_{k}\left|R_{k}\right\rangle\left(\hat{L}^{\dagger}\left|L_{k}\right\rangle=-\lambda_{k}^{\dagger}\left|L_{k}\right\rangle\right)$, and we seek a perturbative expansion of the tilted eigenspectrum, e.g., of 
$\hat{L}^{\dagger}(\mathbf{u})$ :

$$
\begin{aligned}
-\lambda_{k}^{\dagger^{\prime}}(\mathbf{u}) & =-\lambda_{k}^{\dagger}-\sum_{i>0} \mathbf{u}^{i} \cdot \lambda_{k}^{(i)}, \\
\left|L_{k}(\mathbf{u})\right\rangle & =\left|L_{k}\right\rangle+\sum_{i>0} \mathbf{u}^{i} \cdot\left|\mathbf{L}_{\mathbf{k}}^{\mathbf{i}}\right\rangle, \\
\left\langle R_{k}(\mathbf{u})\right| & =\left\langle R_{k}\left|+\sum_{i>0} \mathbf{u}^{i} \cdot\right| \mathbf{R}_{\mathbf{k}}^{\mathbf{i}}\right\rangle .
\end{aligned}
$$

Without loss of generality, we will henceforth assume that $\mathbf{u}$ is real. Note that while the spectra of $\hat{L}$ and $\hat{L}^{\dagger}$ are complex conjugates [see Eq. (29)], the perturbation is in fact symmetric [see Eq. (31)]. Therefore, the spectra of $\hat{L}(u)$ and $\hat{L}^{\dagger}(u)$ are not complex conjugates except for the unperturbed part, which we denoted in Eq. (32) by quotation marks $\lambda^{\prime \iota^{\prime}}$. According to Eq. (32), multiple functionals yield additive perturbations. It thus suffices to carry out the calculations for $\mathbf{u} \rightarrow u$ and write the corresponding general result by inspection. We are interested in up to second-order moments (13), and therefore we need to evaluate the perturbation up to second order in $u$ :

$$
\begin{aligned}
& \left(-\hat{L}^{\dagger}+u V\right) \sum_{n=0}^{2} u^{n}\left|L_{k}^{n}\right\rangle=\sum_{n=0}^{2} u^{n} \lambda_{k}^{(n)} \sum_{m=0}^{2} u^{m}\left|L_{k}^{m}\right\rangle, \\
& \sum_{n=0}^{2} u^{n}\left\langle R_{k}^{n}\left|\left(-\hat{L}^{\dagger}+u V\right)=\sum_{n=0}^{2} u^{n} \lambda_{k}^{(n)} \sum_{m=0}^{2} u^{m}\right| R_{k}^{m}\right|,
\end{aligned}
$$

where we have adopted the convention $\lambda_{k}^{(0)} \equiv \lambda_{k}^{\dagger},\left\langle R_{k}^{0}\right| \equiv\left\langle R_{k}\right|$, and $\left|L_{k}^{0}\right\rangle \equiv\left|L_{k}\right\rangle$. In Eqs. (34) we only need to keep terms up to $u^{2}$ and equate terms of matching order in $u$. First we impose the preliminary normalization $\left\langle R_{k}(u) \mid L_{k}\right\rangle=\left\langle R_{k} \mid L_{k}(u)\right\rangle=1$, i.e.,

$$
\begin{aligned}
& 1=\left\langle R_{k} \mid L_{k}\right\rangle+u\left\langle R_{k}^{1} \mid L_{k}\right\rangle+u^{2}\left\langle R_{k}^{2} \mid L_{k}\right\rangle+O\left(u^{3}\right), \\
& 1=\left\langle R_{k} \mid L_{k}\right\rangle+u\left\langle R_{k} \mid L_{k}^{1}\right\rangle+u^{2}\left\langle R_{k} \mid L_{k}^{2}\right\rangle+O\left(u^{3}\right),
\end{aligned}
$$

which implies

$$
\left\langle R_{k}^{n} \mid L_{k}\right\rangle=\left\langle R_{k} \mid L_{k}^{n}\right\rangle=0 \quad \text { for } n>0 .
$$

The zeroth order of the expansion gives the solution of the unperturbed system. For the higher orders we need to solve Eqs. (34) matching terms of equal order. Introducing the coupling elements $V_{l k} \equiv\left\langle R_{l}|V| L_{k}\right\rangle$, we obtain (details of the calculation are shown in Appendix A)

$$
\begin{aligned}
& \lambda_{k}^{(1)}=V_{k k}, \quad \lambda_{k}^{(2)}=\sum_{l \neq k} \frac{V_{k l} V_{l k}}{\lambda_{k}^{\dagger}-\lambda_{l}^{\dagger}}, \\
& \left|L_{k}^{1}\right\rangle=\sum_{l \neq k} \frac{V_{l k}}{\lambda_{k}^{\dagger}-\lambda_{l}^{\dagger}}\left|L_{l}\right\rangle, \quad\left\langle R_{k}^{1}\right|=\sum_{l \neq k} \frac{V_{k l}}{\lambda_{k}^{\dagger}-\lambda_{l}^{\dagger}}\left\langle R_{l}\right|, \\
& \left|L_{k}^{2}\right\rangle=\sum_{l \neq k}\left[\sum_{i \neq k} \frac{V_{i k} V_{l i}}{\left(\lambda_{k}^{\dagger}-\lambda_{i}^{\dagger}\right)\left(\lambda_{k}^{\dagger}-\lambda_{l}^{\dagger}\right)}-\frac{V_{k k} V_{l k}}{\left(\lambda_{k}^{\dagger}-\lambda_{l}^{\dagger}\right)^{2}}\right]\left|L_{l}\right\rangle, \\
& \left\langle R_{k}^{2}\right|=\sum_{l \neq k}\left[\sum_{i \neq k} \frac{V_{k i} V_{i l}}{\left(\lambda_{k}^{\dagger}-\lambda_{i}^{\dagger}\right)\left(\lambda_{k}^{\dagger}-\lambda_{l}^{\dagger}\right)}-\frac{V_{k k} V_{k l}}{\left(\lambda_{k}^{\dagger}-\lambda_{l}^{\dagger}\right)^{2}}\right]\left\langle R_{l}\right| .
\end{aligned}
$$

However, while they are orthogonal by construction, the resulting perturbed eigenstates are not normalized anymore, i.e., $\left\langle R_{k}(u) \mid L_{k}(u)\right\rangle \neq 1$. Hence, we need to postnormalize them such that

$$
\mathcal{N}_{k}(u)\left\langle R_{k}(u) \mid L_{k}(u)\right\rangle=1,
$$

where from it follows that

$$
\begin{aligned}
\mathcal{N}_{k}(u) & =\left[\left(\left\langle R_{k}\left|+\sum_{i=1}^{2} u^{i}\left\langle R_{k}^{i}\right|\right)\left(\left|L_{k}\right\rangle+\sum_{i=1}^{2} u^{i}\left|L_{k}^{i}\right\rangle\right)\right]^{-1}\right.\right. \\
& =\frac{1}{1+u^{2}\left\langle R_{k}^{1} \mid L_{k}^{1}\right\rangle+O\left(u^{3}\right)} \\
& =1-u^{2} \sum_{i \neq k} \frac{V_{k i} V_{i k}}{\left(\lambda_{k}^{\dagger}-\lambda_{i}^{\dagger}\right)^{2}}+O\left(u^{3}\right) \\
& \equiv 1-u^{2} \mathcal{M}_{k}+O\left(u^{3}\right),
\end{aligned}
$$

where in the last line we have defined $\mathcal{M}_{k}$. We now use the second-order perturbed eigenspectrum to diagonalize the moment-generating function in Eq. (25),

$$
\tilde{\mathcal{P}}_{t}^{\psi}\left(u \mid p_{0}\right)=\sum_{k} \mathcal{N}_{k}(u)\left\langle p_{0} \mid L_{k}(u)\right\rangle\left\langle R_{k}(u) \mid-\right\rangle e^{-\lambda_{k}^{\mu^{\prime \prime}}(u) t},
$$

where moreover

$$
e^{-\lambda_{k}^{\dagger}(u) t}=e^{-\lambda_{k}^{\dagger} t}\left[1-u \lambda_{k}^{(1)} t+u^{2}\left(\lambda_{k}^{(1) 2} t^{2} / 2-\lambda_{k}^{(2)} t\right)\right]+O\left(u^{3}\right)
$$

and $\left\langle R_{k}(u) \mid-\right\rangle=\left\langle R_{k} \mid-\right\rangle+u\left\langle R_{k}^{1} \mid-\right\rangle+u^{2}\left\langle R_{k}^{2} \mid-\right\rangle+O\left(u^{3}\right)$ with the coefficients given by

$$
\begin{aligned}
\left\langle R_{k} \mid-\right\rangle & =\delta_{k 0}, \quad\left\langle R_{k}^{1} \mid-\right\rangle=\frac{V_{k 0}}{\lambda_{k}^{\dagger}}\left(1-\delta_{k 0}\right), \\
\left\langle R_{k}^{2} \mid-\right\rangle & =-\frac{V_{k k} V_{k 0}}{\lambda_{k}^{\dagger 2}}+\sum_{i \neq k} \frac{V_{k i} V_{i 0}}{\lambda_{k}^{\dagger}\left(\lambda_{k}^{\dagger}-\lambda_{i}^{\dagger}\right)}\left(1-\delta_{k 0}\right)
\end{aligned}
$$

and $\left\langle p_{0} \mid L_{k}(u)\right\rangle=\left\langle p_{0} \mid L_{k}\right\rangle+u\left\langle p_{0} \mid L_{k}^{1}\right\rangle+u^{2}\left\langle p_{0} \mid L_{k}^{2}\right\rangle+O\left(u^{3}\right)$, where $\left|L_{k}^{1}\right\rangle$ and $\left|L_{k}^{2}\right\rangle$ are given by Eq. (37). Using Eqs. (37), (39), as well as (41) and (42), the tilted propagator in Eq. (40) to second order in $u$ reads

$$
\tilde{\mathcal{P}}_{t}^{\psi}\left(u \mid p_{0}\right)=1+\sum_{k>0}\left(u C_{k}^{(1)}+u^{2} C_{k}^{(2)}\right) e^{-\lambda_{k}^{\ddagger} t}+O\left(u^{3}\right),
$$

where we have introduced the coefficients

$$
\begin{aligned}
C_{k}^{(1)}= & \delta_{k 0}\left(-V_{00} t+\left\langle p_{0} \mid L_{0}^{1}\right\rangle\right)+\left\langle p_{0} \mid L_{k}\right\rangle\left\langle R_{k}^{1} \mid-\right\rangle \\
C_{k}^{(2)}= & \delta_{k 0}\left[\left\langle p_{0} \mid L_{0}\right\rangle\left(\mathcal{M}_{0}+V_{00}^{2} t^{2} / 2-\lambda_{0}^{(2)} t\right)+\left\langle p_{0} \mid L_{0}^{2}\right\rangle\right] \\
& -t\left(\left\langlep_{0}\left|L_{0}^{1}\right| V_{00} \delta_{k 0}-\left\langle p_{0} \mid L_{k}\right\rangle\left\langle R_{k}^{1}|-| V_{k k}\right)\right.\right. \\
& +\left\langle p_{0} \mid L_{k}^{1}\right\rangle\left\langle R_{k}^{1} \mid-\right\rangle+\left\langle p_{0} \mid L_{k}\right\rangle\left\langle R_{k}^{2} \mid-\right\rangle
\end{aligned}
$$

with $\mathcal{M}_{0}$ defined in Eq. (39). In the special case when initial conditions $\mathbf{x}_{0}$ are drawn from the steady-state probability density, i.e., $p_{0}(\mathbf{x})=P_{\text {inv }}(\mathbf{x})$, these equations simplify to

$$
\begin{aligned}
& \left\langle P_{\text {inv }} \mid L_{k}^{2}\right\rangle=\delta_{k 0}, \quad\left\langle P_{\text {inv }} \mid L_{k}^{1}\right\rangle=\frac{V_{0 k}}{\lambda_{k}^{\dagger}}\left(1-\delta_{k 0}\right), \\
& \left\langle P_{\text {inv }} \mid L_{k}^{2}\right\rangle=-\frac{V_{k k} V_{0 k}}{\lambda_{k}^{\dagger 2}}+\sum_{i \neq k} \frac{V_{i k} V_{0 i}}{\lambda_{k}^{\dagger}\left(\lambda_{k}^{\dagger}-\lambda_{i}^{\dagger}\right)}\left(1-\delta_{k 0}\right) .
\end{aligned}
$$

Turning now to the case in which $V(\mathbf{x})$ extends negative values, we must simply replace $u$ by $i \omega$. To obtain moments up to 
second order [i.e., Eq. (12)], we are now left with evaluating derivatives of Eq. (43) with respect to $u$ at $u=0$.

\section{A. Mean value, fluctuations, and correlation functions for general initial conditions}

We can now derive the mean, variance, and covariance of time-average observables. We first focus on the case of a single time-average observable $\bar{V}_{t}=\psi_{t} / t$ [see Eqs. (3) and (15)]. According to Eq. (24), we must make the replacement $u \rightarrow u / t$ in Eq. (43). We will only present the result in terms of the spectrum of the backward generator $\hat{L}^{\dagger}$ since the results corresponding to $\hat{L}$ follow trivially from Eq. (25). Note that $\left\langle p_{0} \mid-\right\rangle=1$ for any normalized initial condition.

To order $u^{0}$, Eq. (43) simply reflects the normalization of $\mathcal{P}_{t}^{\bar{V}}\left(v \mid p_{0}\right)$, i.e., $\tilde{\mathcal{P}}_{t}^{\bar{V}}\left(0 \mid p_{0}\right)=\int_{0}^{\infty} \mathcal{P}_{t}^{\bar{V}}\left(v \mid p_{0}\right) d v=1$ (and equivalently in the case in which the support of $\bar{V}_{t}$ extends to the entire real axis). To order $u^{1}$, Eq. (43) encodes the mean value $\left\langle\bar{V}_{t}\right\rangle_{p_{0}}$ since it follows from Eq. (10) that $-\left.\partial_{u} \tilde{\mathcal{P}}_{t}^{\bar{V}}\left(u \mid p_{0}\right)\right|_{u=0}=$ $\int_{0}^{\infty} \nu \mathcal{P}_{t}^{\bar{V}}\left(\nu \mid p_{0}\right) d \nu \equiv\left\langle\bar{V}_{t}\right\rangle_{p_{0}}$. Thus the mean value of a timeaverage observable $\left\langle\bar{V}_{t}\right\rangle_{p_{0}}$ evolving from an arbitrary initial condition $p_{0}(\mathbf{x})$ is given by

$$
\left\langle\bar{V}_{t}\right\rangle_{p_{0}}=V_{00}+\frac{1}{t} \sum_{k>0} \frac{V_{k 0}}{\lambda_{k}^{\dagger}}\left\langle p_{0} \mid L_{k}\right\rangle\left(1-e^{-\lambda_{k}^{\dagger} t}\right),
$$

with the anticipated ergodic limit $V_{00}=\left\langle\bar{V}_{t \rightarrow \infty}\right\rangle_{p_{0}}=$ $\left\langle P_{\text {inv }}|V|-\right\rangle \equiv \int_{\Omega} V(\mathbf{x}) P_{\text {inv }}(\mathbf{x}) d \mathbf{x}$. The result (46) is equally valid in cases in which $V(\mathbf{x})$ can become negative (as long as it is bounded). Moreover, from Eq. (46) it is easy to discern the large-deviation asymptotic

$$
\lim _{t \gg 1 / \operatorname{Re} \lambda_{1}^{\dagger}}\left\langle\bar{V}_{t}\right\rangle_{p_{0}} \simeq V_{00}+\frac{1}{t} \sum_{k>0} \frac{V_{k 0}}{\lambda_{k}^{\dagger}}\left\langle p_{0} \mid L_{k}\right\rangle,
$$

and in the special case of steady-state initial conditions, $p_{0}\left(\mathbf{x}_{0}\right)=P_{\text {inv }}\left(\mathbf{x}_{0}\right)$, Eq. (46) reduces to the time-independent ergodic result $\left\langle\bar{V}_{t}\right\rangle_{\text {inv }}=V_{00}$. To order $u^{2}$, Eq. (43) encodes the second moment via $\left.\partial_{u}^{2} \tilde{\mathcal{P}}_{t}^{\bar{V}}\left(u \mid p_{0}\right)\right|_{u=0}=\int_{0}^{\infty} v^{2} \mathcal{P}_{t}^{\bar{V}}\left(v \mid \mathbf{x}_{0}\right) d v \equiv$ $\left\langle\bar{V}_{t}^{2}\right\rangle_{p_{0}}$, which reads

$$
\begin{aligned}
\left\langle\bar{V}_{t}^{2}\right\rangle_{p_{0}}= & V_{00}^{2}+\frac{2}{t} \sum_{k>0} \frac{V_{k 0}}{\lambda_{k}^{\dagger}}\left[V_{0 k}+\left\langle p_{0} \mid L_{k}\right\rangle\left(V_{00}+V_{k k} e^{-\lambda_{k}^{\dagger} t}\right)\right] \\
& +\frac{2}{t^{2}} \sum_{k>0} V_{k 0}\left\{\left[\left\langle p_{0} \mid L_{k}\right\rangle\left(V_{k k}-V_{00}\right)-V_{k 0}\right] \frac{\left(1-e^{-\lambda_{k}^{\dagger} t}\right)}{\lambda_{k}^{\dagger}}\right. \\
& \left.+\sum_{l>0, l \neq k} \frac{V_{l k}\left\langle p_{0} \mid L_{l}\right\rangle}{\lambda_{k}^{\dagger}-\lambda_{l}^{\dagger}}\left(\frac{1-e^{-\lambda_{l}^{\dagger} t}}{\lambda_{l}^{\dagger}}-\frac{1-e^{-\lambda_{k}^{\dagger} t}}{\lambda_{k}^{\dagger}}\right)\right\},
\end{aligned}
$$

which together with Eq. (46) yields the variance

$$
\sigma_{\bar{V}, p_{0}}^{2}(t) \equiv\left\langle\bar{V}_{t}^{2}\right\rangle_{p_{0}}-\left\langle\bar{V}_{t}\right\rangle_{p_{0}}^{2} .
$$

We further introduce the following notational convention for localized initial conditions $p_{0}(\mathbf{x})=\delta\left(\mathbf{x}-\mathbf{x}_{0}\right), \sigma_{\bar{V}, p_{0}}^{2}(t) \rightarrow$ $\sigma_{\bar{V}, \mathbf{x}_{0}}^{2}(t)$. From Eqs. (46), (48), and (49) follows the anticipated ergodic result $\left\langle\bar{V}_{t \rightarrow \infty}^{2}\right\rangle_{p_{0}}=V_{00}^{2}$, proving that in the ergodic limit $\bar{V}_{t}$ becomes deterministic [i.e., the variance vanishes, $\left.\sigma_{\bar{V}, p_{0}}^{2}(t \rightarrow \infty)=\left\langle\bar{V}_{t \rightarrow \infty}^{2}\right\rangle_{p_{0}}-\left\langle\bar{V}_{t \rightarrow \infty}\right\rangle_{p_{0}}^{2}=0\right]$. Conversely the large-deviation asymptotic reads for any initial condition $p_{0}(\mathbf{x})$,

$$
\lim _{\operatorname{Re} \lambda_{1}^{\dagger} t \gg 1}\left\langle\bar{V}_{t}^{2}\right\rangle_{p_{0}}=V_{00}^{2}+\frac{2}{t} \sum_{k>0} \frac{V_{k 0}}{\lambda_{k}^{\dagger}}\left(V_{0 k}+V_{00}\left\langle p_{0} \mid L_{k}\right\rangle\right),
$$

yielding a large-deviation variance

$$
\lim _{\operatorname{Re} \lambda_{1} t \gg 1} \sigma_{\bar{V}}^{2}(t)=\frac{2}{t} \sum_{k>0} \frac{V_{0 k} V_{k 0}}{\lambda_{k}^{\dagger}}+O\left(t^{-2}\right),
$$

which embodies the emergence of the central-limit theorem. One can further show that all higher cumulants decay to zero faster than $1 / t$. Since the only distribution with a finite number of nonzero cumulants is the Gaussian distribution [75], the large-deviation mean value (47) and variance (51) specify the entire asymptotic probability density for time-average observables along trajectories of length $t \gg 1 / \operatorname{Re} \lambda_{1}^{\dagger}$.

In the special case of steady-state initial conditions, $p_{0}(\mathbf{x})=P_{\text {inv }}(\mathbf{x})$, we find the variance satisfies (see also [59])

$$
\sigma_{\bar{V}, \text { inv }}^{2}(t)=\frac{2}{t} \sum_{k>0} \frac{V_{0 k} V_{k 0}}{\lambda_{k}^{\dagger}}\left(1-\frac{1-e^{-\lambda_{k}^{\dagger} t}}{\lambda_{k}^{\dagger} t}\right) .
$$

Note that for overdamped systems in detailed balance, we have $\lambda_{k}^{\dagger} \in \mathbb{R}$ and $\left|L_{k}\right\rangle=e^{\beta U(\mathbf{x})}\left|R_{k}\right\rangle \in \mathbb{R}$. Therefore, $V_{0 k} V_{k 0} \geqslant$ 0 , which implies [compare Eqs. (51) and (52)] that the fluctuations for stationary initial conditions are bounded from above by $\sigma_{\bar{V}, \text { inv }}^{2}(t) \leqslant \lim _{\operatorname{Re} \lambda_{1} t \gg 1} \sigma_{\bar{V}}^{2}(t)$.

We now inspect the correlation between two functionals $\bar{V}_{1, t}$ and $\bar{V}_{2, t}$ [see Eq. (12)] defined as $C_{\bar{V}_{1} \bar{V}_{2}}(t)=$ $\left\langle\bar{V}_{1, t} \bar{V}_{2, t}\right\rangle_{p_{0}}-\left\langle\bar{V}_{1, t}\right\rangle_{p_{0}}\left\langle\bar{V}_{2, t}\right\rangle_{p_{0}}$. The mean values were derived in Eq. (46), so we only require the mixed second moment $\left\langle\bar{V}_{1, t} \bar{V}_{2, t}\right\rangle_{p_{0}}$, which is obtained from the joint momentgenerating function [i.e., generalization of Eq. (40) to two variables] as $\left\langle\bar{V}_{1, t} \bar{V}_{2, t}\right\rangle_{p_{0}}=\left.\partial_{u_{1} u_{2}}^{2} \tilde{\mathcal{P}}_{t} \overline{\mathbf{V}}\left(\mathbf{u} \mid p_{0}\right)\right|_{\mathbf{u}=\mathbf{0}}$. A lengthy calculation leads, upon introducing the coupling elements $U_{k l}^{i} \equiv$ $\left\langle R_{k}\left|V_{i}(\mathbf{x})\right| L_{l}\right\rangle$ and the shorthand notation $W_{k l m n}=U_{k l}^{1} U_{m n}^{2}+$ $U_{k l}^{2} U_{m n}^{1}$, to the exact result

$$
\begin{aligned}
& \left\langle\bar{V}_{1, t} \bar{V}_{2, t}\right\rangle_{p_{0}} \\
& =\frac{W_{0000}}{2}+\frac{1}{t} \sum_{k>0} \frac{1}{\lambda_{k}^{\dagger}}\left(W_{k 00 k}+\left\langle p_{0} \mid L_{k}\right\rangle\left[W_{00 k 0}-W_{k 0 k k} e^{-\lambda_{k}^{\dagger} t}\right]\right) \\
& \quad+\frac{1}{t^{2}} \sum_{k>0}\left\{\left[\left\langle p_{0} \mid L_{k}\right\rangle\left(W_{k 0 k k}-W_{00 k 0}\right)-W_{k 00 k}\right] \frac{\left(1-e^{-\lambda_{k}^{\dagger} t}\right)}{\lambda_{k}^{\dagger 2}}\right. \\
& \left.\quad+\sum_{l>0, l \neq k} \frac{W_{k 0 l k}\left\langle p_{0} \mid L_{l}\right\rangle}{\lambda_{k}^{\dagger}-\lambda_{l}^{\dagger}}\left(\frac{1-e^{-\lambda_{l}^{\dagger} t}}{\lambda_{l}^{\dagger}}-\frac{1-e^{-\lambda_{k}^{\dagger} t}}{\lambda_{k}^{\dagger}}\right)\right\},
\end{aligned}
$$

and we note that $\left\langle\bar{V}_{1, t}\right\rangle_{\text {inv }}\left\langle\bar{V}_{2, t}\right\rangle_{\text {inv }}=W_{0000} / 2$, implying that for an ergodic system, any two functionals asymptotically decorrelate, $\lim _{t \rightarrow \infty} C_{\bar{V}_{1} \bar{V}_{2}}(t)=0$. Equations (46), (48), and (53) expressing the mean value and second moments (and together the variance and covariance) of the time average of a general physical observable $V\left(\mathbf{x}_{\tau}\right)$ of type Eq. (3) solely in 
terms of the eigenspectrum of the underlying generator are the main theoretical result of this work.

In the case of stationary initial conditions $p_{0}(\mathbf{x})=P_{\text {inv }}(\mathbf{x})$, we have that $\left\langle P_{\text {inv }} \mid L_{k}\right\rangle=\delta_{k 0}$, and as a result of Eq. (53) the covariance reduces to (note that $W_{k 00 k}=W_{0 k k 0}$ and $W_{0000} / 2=$ $\left.\left\langle\bar{V}_{1, t}\right\rangle_{\text {inv }}\left\langle\bar{V}_{2, t}\right\rangle_{\text {inv }}\right)$

$$
C_{\bar{V}_{1} \bar{V}_{2}}^{\text {inv }}(t)=\frac{1}{t} \sum_{k>0} \frac{W_{0 k k 0}}{\lambda_{k}^{\dagger}}\left(1-\frac{1-e^{-\lambda_{k}^{\dagger} t}}{\lambda_{k}^{\dagger} t}\right) .
$$

Finally, in the large-deviation regime, we recover

$$
\lim _{t \gg 1 / \lambda_{1}} C_{\bar{V}_{1} \bar{V}_{2}}(t)=\frac{1}{t} \sum_{k>0} \frac{W_{0 k k 0}}{\lambda_{k}^{\dagger}}+O\left(t^{-2}\right),
$$

the $1 / t$ scaling reflecting the emergence of the centrallimit theorem. Therefore, it follows that an arbitrary set of $m$ time-average observables $\overline{\mathbf{V}}_{t}=\left\{\bar{V}_{i, t}\right\}$ in the largedeviation limit exhibits Gaussian statistics. If we denote the vector of mean values as $\langle\overline{\mathbf{V}}\rangle_{\text {inv }}$ and introduce the symmetric covariance matrix $\mathbf{C}$ with diagonal elements $\mathbf{C}_{i i}=$ $t \lim _{t \gg 1 / \operatorname{Re} \lambda_{1}} \sigma_{\bar{V}_{i}}^{2}(t)$ [see Eq. (51)] and off-diagonal elements $\mathbf{C}_{i j}=t \lim _{t \gg 1 / \operatorname{Re} \lambda_{1}} C_{\bar{V}_{i} \bar{V}_{j}}(t)$ [see Eq. (55)], then the probability density that $\overline{\mathbf{V}}_{t}$ attains a value $\boldsymbol{v}$ obeys the asymptotic Gaussian limit law $\lim _{t \gg 1 / \operatorname{Re} \lambda_{1}} \mathcal{P}_{t}^{\overline{\mathbf{V}}}\left(\boldsymbol{v} \mid p_{0}\right) \equiv \mathcal{P}_{t}^{\mathrm{LD}}(\boldsymbol{v})$, where

$$
\mathcal{P}_{t}^{\mathrm{LD}}(\boldsymbol{v}) \simeq \frac{e^{-\frac{1}{2}\left(\boldsymbol{v}-\langle\overline{\mathbf{V}}\rangle_{\text {inv }}\right)^{T} \mathbf{C}^{-1}\left(\boldsymbol{v}-\langle\overline{\mathbf{V}}\rangle_{\text {inv }}\right) t}}{\sqrt{(2 \pi)^{m} \operatorname{det} \mathbf{C} / t}}
$$

We now introduce the rescaled variables $\hat{\boldsymbol{v}} \equiv \boldsymbol{v} \sqrt{t}$ and scaled mean $\boldsymbol{\mu} \equiv\left\langle\overline{\mathbf{V}}_{t} \sqrt{t}\right\rangle_{\text {inv }}$, which upon renormalization lead to a time-independent density. Moreover, we define

$$
\Xi=\left(\hat{v}_{i}-\mu_{i}\right) / \sqrt{\mathbf{C}_{i i}}, \quad \Xi_{i \mid j}=\left(\hat{v}_{i}-\tilde{\mu}_{i \mid j}\right) / \tilde{\sigma}_{i \mid j}
$$

with the shifted mean and stretched variance

$$
\begin{aligned}
\tilde{\mu}_{i \mid j} & =\mu_{i}+\left(\hat{v}_{j}-\mu_{j}\right) \mathbf{C}_{i j} / \mathbf{C}_{j j}, \\
\tilde{\sigma}_{i \mid j}^{2} & =\left(\mathbf{C}_{i i} \mathbf{C}_{j j}-\mathbf{C}_{i j}^{2}\right) / \mathbf{C}_{j j} .
\end{aligned}
$$

Then the limit law (56) implies that the univariate large deviations $\mathcal{P}_{t}^{\mathrm{LD}}(\hat{v})$ and conditional bivariate large deviations $\mathcal{P}_{t}^{\mathrm{LD}}\left(\hat{v}_{1} \mid \hat{v}_{2}\right) \equiv \mathcal{P}_{t}^{\mathrm{LD}}\left(\hat{v}_{1}, \hat{v}_{2}\right) / \mathcal{P}_{t}^{\mathrm{LD}}\left(\hat{v}_{2}\right)$ collapse, upon rescaling, onto a universal Gaussian master curve

$$
\begin{gathered}
\sqrt{\frac{\mathbf{C}_{i i}}{t^{1 / 2}}} \mathcal{P}_{t}^{\mathrm{LD}}\left(\hat{v}_{i}\right) \rightarrow \mathcal{N}_{\Xi}(0,1), \\
\frac{\tilde{\sigma}_{i \mid j}}{\sqrt{t}} \mathcal{P}_{t}^{\mathrm{LD}}\left(\hat{v}_{i} \mid \hat{v}_{j}\right) \rightarrow \mathcal{N}_{\Xi_{i \mid j}}(0,1),
\end{gathered}
$$

where $\mathcal{N}_{x}(0,1)$ denotes the Gaussian probability density with zero mean and unit variance. The explicit rescaling [i.e., Eq. (59) combined with Eq. (51) and Eq. (55)] leading to the collapse onto a master unit normal density in the largedeviation limit is the main practical consequence of our large-deviation result.

\section{B. Degenerate eigenspectra}

Note that if the spectrum of $\hat{L}$ has degenerate eigenstates (such as, e.g., in single-file diffusion $[59,60]$ ) special care is required for initial conditions that do not correspond to the steady state, i.e., $p_{0}(\mathbf{x}) \neq P_{\text {inv }}(\mathbf{x})$, as a result of the singularities the degeneracy causes in Eqs. (48) and (53). As is customary in regular perturbation theory (see, e.g., [76]), one must first postdiagonalize all the respective degenerate subspaces prior to using Eqs. (48) and (53). Once this has been taken care of (using any of the many possible methods [77]) and the degenerate eigenstates are replaced by their appropriate linear combinations, Eqs. (48)-(53) can be used as they stand.

\section{A general upper bound for occupation measures for overdamped reversible dynamics}

When $\hat{L}$ corresponds to reversible overdamped dynamics [i.e., $\mathbf{D}^{-1} \mathbf{F}(\mathbf{x})=-\beta \nabla_{\mathbf{x}} U(\mathbf{x})$ is a gradient field], or to a reversible Markov-jump process [i.e., the transition matrix elements in Eq. (26) satisfy the symmetry $\langle\mathbf{y}|\hat{L}| \mathbf{x}\rangle /\langle\mathbf{x}|\hat{L}| \mathbf{y}\rangle=$ $\left.e^{\beta U(\mathbf{x})-\beta U(\mathbf{y})}\right]$, the large-deviation asymptotic (51) provides an upper bound for fluctuations of the occupation time fraction in any subdomain $\mathcal{V} \subseteq \Omega, V(\mathbf{x})=\mathcal{V}$, for any duration of the trajectory [which naturally includes the local-time fraction when $V(\mathbf{x})=\mathbf{x}]$.

Let us define the projection operator $\hat{\Gamma}_{\mathbf{x}}(\mathcal{V} ; V) \equiv$ $\int_{\Omega} d \mathbf{x} \delta(\mathcal{V}-V(\mathbf{x}))$ [60], which projects the full dynamics $\mathbf{x} \subset \mathbb{R}^{d}$ onto the hypersurface compatible with a given value of the observable $V(\mathbf{x})=\mathcal{V}$. Then the (generally non-Markovian) joint probability density that the observable $V(\mathbf{x})$ starts from $\mathcal{V}$ and returns to the initial value $\mathcal{V}$ at time $t$ in an ensemble of trajectories $\mathbf{x}_{t}$ starting from the equilibrium probability density $P_{\text {inv }}\left(\mathbf{x}_{0}\right)=P_{\text {eq }}\left(\mathbf{x}_{0}\right)$ is defined as [60]

$$
G_{t}^{\mathrm{eq}}(\mathcal{V}, \mathcal{V}) \equiv \hat{\Gamma}_{\mathbf{x}}(\mathcal{V} ; V) \hat{\Gamma}_{\mathbf{x}_{0}}(\mathcal{V} ; V) P_{t}\left(\mathbf{x} \mid \mathbf{x}_{0}\right) P_{\mathrm{eq}}\left(\mathbf{x}_{0}\right)
$$

We now recall the definition of the occupation time fraction of $\mathbf{x}_{\tau}$ within the hypersurface $V(\mathbf{x})=\mathcal{V}$ in Eq. (8). Then Eq. (51) and the spectral decomposition of $P_{t}\left(\mathbf{x} \mid \mathbf{x}_{0}\right)$ in Eq. (30) imply the general upper bound on $\theta_{\mathcal{V}}(t)$,

$$
t \sigma_{\theta_{\mathcal{V}}, \text { inv }}^{2}(t) \leqslant 2 \int_{0}^{\infty}\left[G_{t}^{\mathrm{eq}}(\mathcal{V}, \mathcal{V})-G_{\infty}^{\mathrm{eq}}(\mathcal{V}, \mathcal{V})\right] d t
$$

where equality holds in the limit $t \rightarrow \infty$. Note that in the special case when $V(\mathbf{x})=\mathbf{x}$, Eq. (61) bounds the local-time fraction defined in Eq. (5).

To prove the bound (61), let us express Eq. (60) using the spectral expansion of $\hat{L}^{\dagger}$ (or equivalently $\hat{L}$ ). Since we are considering systems in detailed balance, the eigenspectrum is real. Introducing the elements $V_{k l}(\mathcal{V}) \equiv\left\langle R_{l}|\delta(\mathcal{V}-V(\mathbf{x}))| L_{k}\right\rangle$, the spectral representation of Eq. (60) reads (see also [60])

$$
G_{t}^{\mathrm{eq}}(\mathcal{V}, \mathcal{V})=\sum_{k} V_{0 k}(\mathcal{V}) V_{k 0}(\mathcal{V}) e^{-\lambda_{k} t}
$$

such that $\lim _{t \rightarrow \infty} G_{t}^{\mathrm{eq}} \equiv G_{\infty}^{\mathrm{eq}}(\mathcal{V}, \mathcal{V})=V_{00}(\mathcal{V})^{2}$. Therefore,

$$
\int_{0}^{\infty}\left[G_{t}^{\mathrm{eq}}(\mathcal{V}, \mathcal{V})-V_{00}(\mathcal{V})^{2}\right] d t=\sum_{k>0} V_{0 k}(\mathcal{V}) V_{k 0}(\mathcal{V}) / \lambda_{k}
$$

Multiplying Eq. (63) by 2 and dividing by $t$ we obtain Eq. (51) for the case when $\bar{V}_{t}=\theta_{\mathcal{V}}(t)$ defined in Eq. (8), which in turn proves asymptotic equality as $t \rightarrow \infty$. Because for systems obeying detailed balance we further have $\left|L_{k}\right\rangle=e^{\beta U(\mathbf{x})}\left|R_{k}\right\rangle$, 
each coefficient is positive, $V_{0 k}(\mathcal{V}) V_{k 0}(\mathcal{V}) \geqslant 0$, because it corresponds to $e^{-\beta U(\mathbf{x})}>0$ multiplied by the square of a real number. Together with Eq. (52) this proves that the inequality holds for any $t$ and completes the proof of the existence and tightness of the bound (61).

Equation (61) enables us to obtain an upper bound on fluctuations of $\theta_{\mathcal{V}}(t)$ - the (generally non-Markovian) occupation measure that the full dynamics $\mathbf{x}_{\tau}$ along a single trajectory is found within the hypersurface $V(\mathbf{x})=\mathcal{V}$-from the integral over the return probability (60). It thereby also bounds the fluctuations of random time-average "empirical densities," that is, local-time fractions [see Eq. (5)], by means of the corresponding deterministic (ensemble) joint return probability density (60). Equation (61) is the main practical result of this work. Interestingly, a similar bound involving the integral of the return probability has been found in Ref. [78] in the study of large-deviation asymptotics of the first passage times.

\section{Physical interpretation of the results}

We now provide some intuition about the developed theory. As time evolves, the value of $\bar{V}_{t}$ for an ergodic process eventually becomes only weakly correlated. The statistics of $\bar{V}_{t}$ passes first through the large-deviation regime (56), where the central-limit theorem kicks in with Gaussian statistics, and finally ends up in Khinchin's law of large numbers, where it becomes deterministic and equal to $\left\langle\bar{V}_{t}\right\rangle_{\text {inv }}$ [79]. For simplicity, we start in the large-deviation regime (51).

By using spectral theory, we map fluctuations of $\bar{V}_{t}$ onto the eigenmodes of $\hat{L}$ (and/or $\hat{L}^{\dagger}$, respectively), with the "similarity" to a given eigenmode reflected by the overlaps $V_{0 k}, V_{k 0}$. Since on these timescales all memory of the initial condition is lost, which is equivalent to imposing stationary initial conditions, only overlaps from and to the ground state are relevant. Moreover, due to the orthogonality of eigenmodes, these projections are statistically independent. Each eigenmode has a finite lifetime or correlation time $1 / \lambda_{k}$. Therefore, in a time $t \gg \lambda_{k}^{-1}$ any $k$ th projection acts as shot-noise, and there will be $t \lambda_{k}$ independent realizations of such a projection reducing the (co)variance by a factor $1 / t \lambda_{k}$ [see Eqs. (51) and (55)]. In the limit $t \rightarrow \infty$, the Gaussian converges to a Dirac delta, i.e., $\lim _{t \rightarrow \infty} \mathcal{P}_{t}^{\mathrm{LD}}(\boldsymbol{v})=\delta\left(\boldsymbol{v}-\langle\overline{\mathbf{V}}\rangle_{\text {inv }}\right)$.

At shorter times, nontrivial corrections to these largedeviation results arise due to strong correlations between the values of $\bar{V}_{t}$ at different times $t$. As a result of these correlations, the "completely decorrelated" large-deviation results in Eqs. (51) and (55) become reduced by a term that seems to reflect the "effective probability of mode $k$ to persist until $t$," $t^{-1} \int_{0}^{t} e^{-\lambda_{k} \tau} d \tau=\left(1-e^{-\lambda_{k} t}\right) / \lambda_{k} t$ [see Eqs. (52) and (54) as well as Eqs. (B3) and (B4)]. In the case of general initial conditions, $p_{0}(\mathbf{x})$ additional terms arise [see Eqs. (50) and (53)] that reflect the memory of the initial condition. These terms, however, are difficult to interpret beyond the point that they reflect projections that couple different excited eigenstates and thus describe fluctuation modes that are more complicated than simple excursions starting and ending in the steady state.

\section{APPLICATIONS OF THE THEORY}

We now apply the theory to a collection of simple illustrative examples. Due to the fundamental role played by the local-time fraction $\theta_{\mathbf{x}}(t)$ and because it determines the dynamics of other time-average observables [see Eq. (6)], we focus on $\theta_{\mathbf{x}}(t)$ alone. The coupling elements are therefore simply given by $V_{l k}=\int_{\Omega} d \mathbf{y} R_{l}(\mathbf{y}) \delta(\mathbf{x}-\mathbf{y}) L_{k}(\mathbf{y}) \equiv R_{l}(\mathbf{x}) L_{k}(\mathbf{x})$. We first present explicit results for local times for continuous space-time Markovian diffusion processes and an irreversible (i.e., driven) three-state unicyclic network. Next, we apply the theory to a simple two-state Markov model of the celebrated Berg-Purcell problem [28-30,36], i.e., the physical limit to the precision of receptor-mediated measurement of the concentration of ligand molecules.

As minimal, exactly solvable models of continuous-space Markovian diffusion, we consider a Wiener process confined to a unit interval with reflective boundaries and the Ornstein-Uhlenbeck process. To demonstrate the theory for Markov-jump dynamics, we consider a random walk in a finite harmonic potential and a simple three-state unicyclic network.

\section{A. Local time fraction of the Wiener process in the unit interval}

The propagator of the Wiener process confined to a unit interval (i.e., $L=1$ ) is the solution of

$$
\left(\partial_{t}-\partial_{x}^{2}\right) P_{t}^{\mathrm{W}}\left(x \mid x_{0}\right)=0,\left.\partial_{x} P_{t}^{\mathrm{W}}\right|_{x=0}=\left.\partial_{x} P_{t}^{\mathrm{W}}\right|_{x=1}=0,
$$

with initial condition $P_{0}^{\mathrm{W}}\left(x \mid x_{0}\right)=\delta\left(x-x_{0}\right)$. The eigenvalues of $\partial_{x}^{2}$ in a unit interval are given by $\lambda_{k}^{\mathrm{W}}=k^{2} \pi^{2}$ (time is expressed in units of $\tau=L^{2} / D$ ), and the eigenvectors read $[67,72]$

$$
L_{k}^{\mathrm{W}}(x)=R_{k}^{\mathrm{W}}(x)=\delta_{k 0}+\left(1-\delta_{k 0}\right) \sqrt{2} \cos (k \pi x),
$$

since $\partial_{x}^{2}$ is self-adjoint. The mean local-time fraction, $\left\langle\theta_{x}(t)\right\rangle$, the variance $\sigma_{\theta}^{2}(t)$, and the covariance $C_{\theta_{1} \theta_{2}}(t)$ for the confined Wiener process are shown in Fig. 3. In the case of equilibrium initial conditions, $\left\langle\theta_{x}(t)\right\rangle_{\text {inv }}$ is constant and equal to $P_{\text {inv }}(x)$, and the fluctuations of $\theta_{x}(t)$ are largest at the boundaries as a result of repeated collisions with the walls. Notably, starting from localized conditions, $\left\langle\theta_{x}(t)\right\rangle_{x_{0}}$ as a function of $x$, in contrast to the ensemble propagator $P_{t}^{\mathrm{W}}\left(x \mid x_{0}\right)$, displays a persistent cusp located at the initial condition $x_{0}$ [see Fig. 3(c)]. The fluctuations of $\theta_{x}(t)$ are larger near the initial condition and at the boundaries.

\section{B. Local time fraction of the Ornstein-Uhlenbeck process}

Trajectories of the one-dimensional Ornstein-Uhlenbeck process are solutions of the Itô equation

$$
d x_{t}=-\gamma x_{t}+\sqrt{2 D} d W_{t}
$$

and on the level or probability density they correspond to the Fokker-Planck equation $\left(\partial_{t}-D\left[\partial_{x}^{2}+\gamma \partial_{x} x\right]\right) P_{t}^{\mathrm{OU}}\left(x \mid x_{0}\right)=$ 0 with initial condition $P_{0}^{\mathrm{OU}}\left(x \mid x_{0}\right)=\delta\left(x-x_{0}\right)$ and natural boundary conditions $\lim _{|x| \rightarrow \infty} P_{t}^{\mathrm{OU}}\left(x \mid x_{0}\right)=0$. To connect continuous processes to discrete ones, we translate the Fokker-Planck equation of the Ornstein-Uhlenbeck process to a random walk on a lattice with spacing $\Delta x$ and the harmonic potential $\gamma x^{2}$ entering transition rates according 

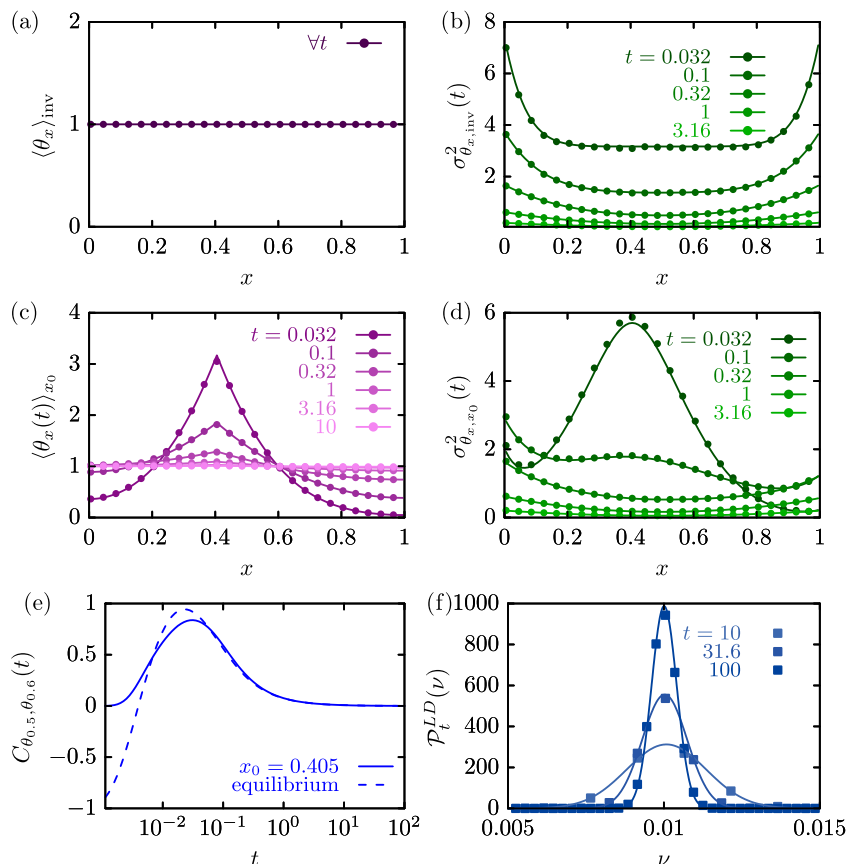

FIG. 3. Statistics of the fraction of local-time $\theta_{x}(t)$ as a function of $x$ at different times $t$ for equilibrium initial conditions, $p_{0}\left(x_{0}\right)=P_{\text {inv }}\left(x_{0}\right)[(\mathrm{a})$ and (b)] and localized initial condition at $x_{0}=0.405$ [(c) and (d)]; (a) $\left\langle\theta_{x}(t)\right\rangle_{\text {inv }}$ is constant for all times; (b) $\sigma_{\theta_{x}}^{2}$,inv $(t)$ as a function of $x$ for equilibrium initial conditions; (c) $\left\langle\theta_{x}(t)\right\rangle_{x_{0}}$ and (d) $\sigma_{\theta_{x}}^{2}(t)$; (e) covariance starting from equilibrated initial conditions, $C_{\theta_{x} \theta_{y}}^{\text {inv }}(t)$ (dashed line) and localized initial conditions, $C_{\theta_{x} \theta_{y}}(t)$ (solid line). (f) Probability density of occupation time fraction $\theta_{\mathcal{V}}(t)=t^{-1} \int_{0}^{t} \mathbb{1}_{[0.45,0.55]}\left(x_{\tau}\right) d \tau$ [see also Eq. (7)] on large-deviation timescales (symbols) and corresponding theoretical result (56) (lines). Symbols were obtained from Brownian dynamics simulations of $10^{5}$ trajectories simulated with a time-step $d t=10^{-4}$.

to $[80]$

$$
\begin{aligned}
\langle x+\Delta x|\hat{L}| x\rangle & =\frac{D}{\Delta x^{2}} e^{\frac{1}{4} \gamma\left[x^{2}-(x+\Delta x)^{2}\right]}, \\
\langle x|\hat{L}| x+\Delta x\rangle & =\frac{D}{\Delta x^{2}} e^{\frac{1}{4} \gamma\left[(x+\Delta x)^{2}-x^{2}\right]}
\end{aligned}
$$

in a confined domain $\Omega_{\text {conf }}=\{-l,-l+\Delta x, \ldots, l-$ $\Delta x, l\} \subset \Omega$. The matrix $\hat{L}$ is tridiagonal and satisfies $\sum_{y}\langle y|\hat{L}| x\rangle=0$ for all $\mathbf{x} \in \Omega$ and $x \in \mathbb{Z} \Delta x$. We diagonalized $\hat{L}$ numerically using the library from Ref. [81]. The mean, variance, and correlation function for the continuous-space Ornstein-Uhlenbeck process (66) obtained from Brownian dynamics simulations are depicted in Fig. 4 (symbols) and are in excellent agreement with the spectral-theoretic results for the corresponding lattice random-walk approximation (67) (lines). In Fig. 4(f) we also investigate the full probability density function of the fraction of occupation time in the interval $x \in[0,0.01]$, i.e., $\theta_{\mathcal{V}}(t)=t^{-1} \int_{0}^{t} \mathbb{1}_{[0,0.01]}[x(\tau)] d \tau$ [see Eq. (7)] on large-deviation timescales, and we compare it to the theoretical Gaussian prediction Eq. (51). Note that while the eigenspectrum of the generator of continuous Ornstein-Uhlenbeck dynamics is unbounded, implying that the spectral-theoretic result would require the summation
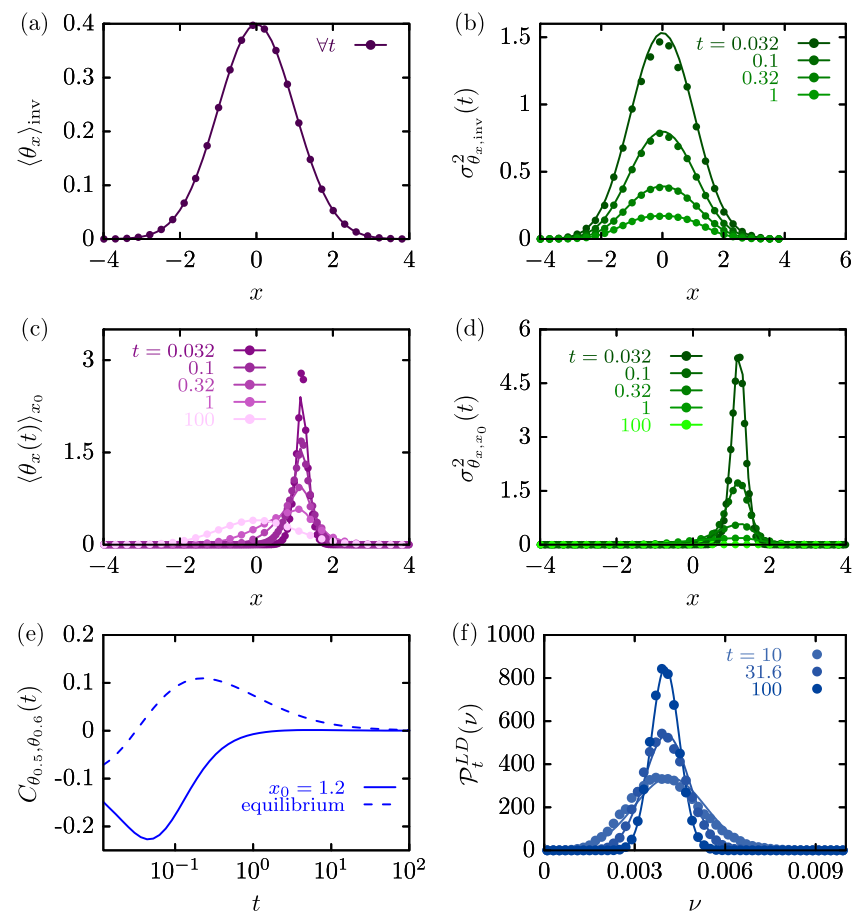

FIG. 4. Statistics of the fraction of local-time $\theta_{x}(t)$ as a function of $x$ for the Ornstein-Uhlenbeck process (symbols) and corresponding lattice random walk (67) with $10^{3}$ states in the interval $x \in$ $[-5,5]$ as a function of $x$ at different times $t$ for equilibrium initial conditions, i.e., $p_{0}\left(x_{0}\right)=P_{\text {inv }}\left(x_{0}\right)[(\mathrm{a})$ and $(\mathrm{b})]$, and initial conditions localized at $x_{0}=1.2$, i.e., $p_{0}\left(x_{0}\right)=\delta\left(x_{0}-1.2\right)$ [(c) and (d)]. (a) $\left\langle\theta_{x}(t)\right\rangle_{\text {inv }}=P_{\text {inv }}(x)$ is a time-independent Gaussian; (b) $\sigma_{\theta_{x} \text {,inv }}^{2}(t)$ at various times as a function of $x$; (c) $\left\langle\theta_{x}(t)\right\rangle_{x_{0}}$ and (d) $\sigma_{\theta_{x}, x_{0}}^{2}(t)$ at various times as a function of $x$; (e) $C_{\theta_{0.5} \theta_{0.6}}(t)$ for equilibrium (dashed line) and localized (full lines) initial conditions; (f) occupation time fraction $\theta_{\mathcal{V}}(t)=t^{-1} \int_{0}^{t} \mathbb{1}_{[0,0.01]}[x(\tau)] d \tau$ [see also Eq. (7)] with symbols derived from simulations and the solid line representing the theoretical result (56) for the lattice random walk (67). To obtain each simulation point, we generated $10^{5}$ Brownian dynamics trajectories using $D=\gamma=1$ with a time-step $d t=10^{-4}$.

of a large number of terms, the summation in the lattice approximation is limited by the number of lattice points. Therefore, except for very short times, where the lattice approximation naturally breaks down, this example demonstrates that our formalism applies equally well to Markov-jump processes and diffusion dynamics. Note that the results in Figs. 4(c) and 4(d) for times $t=1$ and 100 correspond to the "short" and "long" trajectory in Fig. 2, respectively.

\section{Local-time fraction in a driven unicyclic network}

Let us address in the following a simple three-state model with broken detailed balance to also address driven systems. The model corresponds to a simple cycle with states 1,2, and 3 , where all rates in a given direction are equal but each of them has the same forward/backward asymmetry. The model may represent, for example, a molecular motor such as the F1-ATPase driven by ATP hydrolysis [82]. The corresponding 

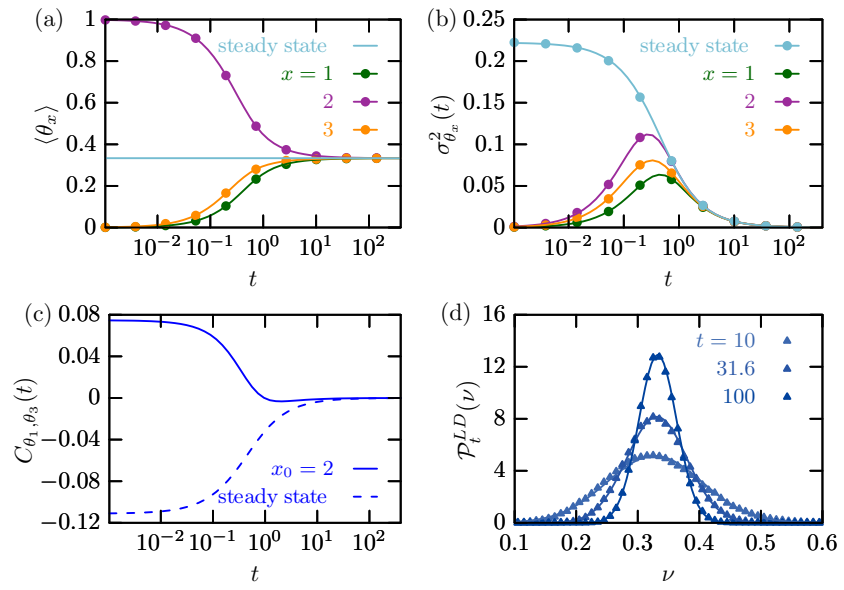

FIG. 5. (a) Mean local-time fraction $\left\langle\theta_{x}(t)\right\rangle$ in states $x=1,2,3$, respectively, for a driven unicyclic network starting from steady-state initial conditions $\left[p_{0}\left(x_{0}\right)=P_{\text {inv }}\left(x_{0}\right)\right.$, light blue line; result identical for any state] and an initial condition localized at $x_{0}=2$ (lines are theory and points simulations); (b) variance of the local-time fraction $\sigma_{\theta \text { inv }}^{2}(t)$ starting from steady-state initial conditions (light blue line; result identical for any state) and $\sigma_{\theta, 2}^{2}(t)$ in states $x=1,2,3$ starting from $x_{0}=2$; (c) covariance of local-time fraction between states $x=1$ and 2, $C_{\theta_{1} \theta_{2}}(t)$, as a function of time for stationary (dashed line) and localized (full line) initial conditions; (d) probability density function of the local-time fraction in state $x=1, \bar{V}_{t} \equiv$ $\theta_{1}(t)$, on large-deviation timescales alongside theoretical prediction of Eq. (56).

transition matrix of the model reads

$$
\hat{L}=\left(\begin{array}{rrr}
-3 & 1 & 2 \\
2 & -3 & 1 \\
1 & 2 & -3
\end{array}\right)
$$

and it has eigenvalues $\lambda_{0}=0, \lambda_{1,2}=-9 / 2 \pm i \sqrt{3} / 2$, and eigenvectors $\left|R_{0}\right\rangle=3^{-1}(1,1,1)^{T}$ and

$$
\left|R_{1,2}\right\rangle=\frac{1}{3}\left(\frac{-3 \sqrt{3} \pm i}{\sqrt{3} \mp 5 i}, \frac{2 \sqrt{3} \pm 2 i}{\sqrt{3} \mp 5 i}, 1\right)^{T} .
$$

As a result of broken detailed balance, the eigenspectrum is complex. In Fig. 5 we analyze the mean [panel (a)], fluctuations [panel (b)], and correlation function [panel (c)] of the local-time fraction $\theta_{x}(t)$ in the various states for nonequilibrium steady-state initial conditions (light blue lines) and conditions initially localized in state $x_{0}=2$, i.e., $\left|p_{0}\right\rangle=$ $(0,1,0)^{T}$. The theoretical results (lines) show an excellent agreement with simulations (symbols) carried out using the Gillespie algorithm [68]. We also confirm the Gaussian statistics of the local-time fraction $\theta_{x}(t)$ from Eq. (56) in Fig. 5(d).

\section{Generic behavior of the local-time fraction in ergodic systems}

Note that an exhaustive study of the statistics of the localtime fraction is beyond the scope of this work. Nevertheless, we discuss here some general features of $\theta_{x}(t)$. The manner in which $\left\langle\theta_{x}(t)\right\rangle$, starting from some nonequilibrium initial condition, approaches the ergodic invariant measure $P_{\text {inv }}(x)$ can be highly nontrivial and even nonmonotonic [see, e.g., Figs. 6(a)]. Even when the ergodic limit is reached, where
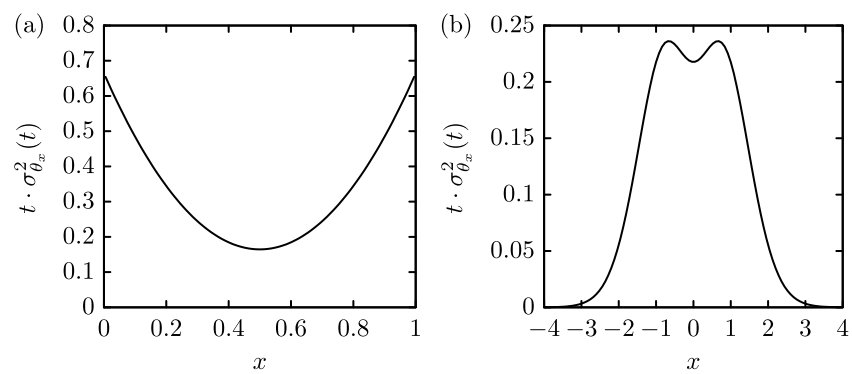

FIG. 6. Long-time behavior (i.e., $t \gg \lambda_{1}^{-1}$ ) of the scaled variance of the local-time, $t \sigma_{\theta_{x}}^{2}(t)$, that is time-independent; (a) results for the Wiener process and $(b)$ the Ornstein-Uhlenbeck processes.

the variance ceases to depend on time, i.e., $t \sigma_{\theta_{x}}^{2}(t) \neq f(t)$, the fluctuations display a nontrivial behavior (see Fig. 6). For example, in the case of the Wiener process, fluctuations are enhanced close to the boundaries, while for the OrnsteinUhlenbeck process they become depressed near the minimum. Both results may be interpreted in terms of random "oscillations" around a typical position and confined by a boundary that amplifies fluctuations.

Moreover, the time dependence of $\left\langle\theta_{x}(t)\right\rangle$ for nonstationary initial conditions is often nonmonotonic or has a nonmonotonic derivative [see Figs. 7(a) and 7(c)]. A comparison between $\left\langle\theta_{x}(t)\right\rangle$ starting from stationary (dashed lines) and localized (full lines) initial conditions illustrates the two coexisting decorrelation mechanisms of $\theta_{x}(t)$ at different times, one corresponding to self-averaging and the emergence of the central-limit theorem (compare dashed and dotted lines), the other additionally reflecting the loss of memory of the initial condition (full lines). Stationary initial conditions often give rise to larger fluctuations than nonstationary initial conditions
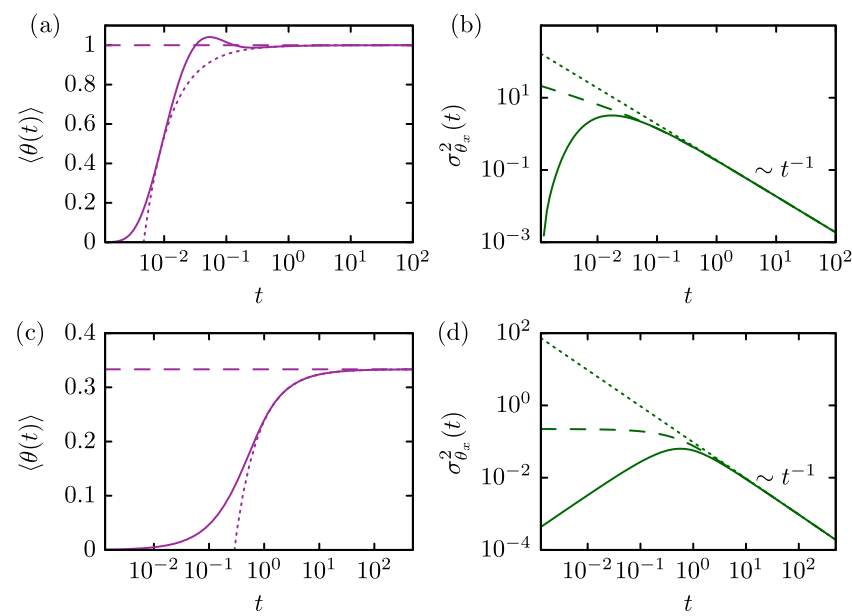

FIG. 7. (a) Mean local-time and (b) variance at $x=0.6$ for a Wiener process starting from equilibrium (dashed line) and from a localized initial condition $p_{0}(x)=\delta(x-0.405)$ (solid line). (a) Mean local-time $\left\langle\theta_{x}(t)\right\rangle$ and (b) variance $\sigma_{\theta_{x}}^{2}(t)$ at $x=0.6$ for the driven three-state cycle Eq. (68) starting from stationary (dashed line) and from a localized initial condition $p_{0}(x)=\delta(x-1)$ (solid line); the dotted lines correspond to the large-deviation limit (51) and depict the validity and long-time saturation of the upper bound Eq. (61). 
[compare the dashed and full lines in Figs. 7(b) and 7(d)], and in the particular case of equilibrium initial conditions for systems obeying detailed balance, $\sigma_{\theta}^{2}(t)$ is a monotonically decaying function of time $t$ [see Eq. (52) and Figs. 7(b) and $7(\mathrm{~d})]$ with an upper bound given by the large-deviation asymptotic [see Eq. (61)] with a $\propto 1 / t$ scaling dictated by the central-limit theorem [dotted lines in Figs. 7(b) and 7(d)].

The covariance of the local-time fraction between a pair of points $x_{1}$ and $x_{2}$ in the continuous setting [Figs. 3(e) and 4(e)] or $x=1$ and 2 in the discrete setting [Fig. 5(c)], $C_{\theta_{1} \theta_{2}}(t)$, displays a similarly nontrivial and nonmonotonic dependence on time and initial conditions $p_{0}\left(x_{0}\right)$ as shown in Figs. 3(e), 4(e), and 5(c). The striking dependence on the tagged points reflects a directional persistence of individual trajectories in-between said points and can therefore be used as a robust indicator of directional persistence and thus "temporally correlated exploration" on the level of a single trajectory.

\section{Universal asymptotic Gaussian limit law for time-average physical observables}

Finally, we comment on the universal asymptotic Gaussian limit law Eq. (56) for Markovian as well as non-Markovian time-average physical observables of type (3) of ergodic stochastic dynamics of the form given in Eqs. (14) and (26). Namely, using the asymptotic results (47), (51), and (55) in the large-deviation probability density function (56), and rescaling to the centered and time-independent variables $\Xi$ and $\Xi_{i \mid j}$ defined in Eqs. (57) and (58), we can rescale the probability density of any time-average physical observable $\mathcal{P}_{t}^{\mathrm{LD}}(\hat{v})$, and the conditional probability density of a time-average physical observable given another time-average physical observable $\mathcal{P}_{t}^{\mathrm{LD}}\left(\hat{v}_{i} \mid \hat{v}_{j}\right)$, to collapse at long times onto a unit normal probability density (59). For the three models studied here, Figs. 3(f), 4(f), and 5(d), and additionally for the conditional probability density function of the occupation time fraction in $x \in[0.1,0.4]$ given the occupation time fraction in $y \in$ $[0.6,0.9]$ for the Wiener process, we demonstrate this collapse explicitly in Fig. 8.

\section{Precision limit of concentration measurement by a single receptor}

Let us now investigate the physical limit to the precision of concentration measurements by means of the simplest twostate Markov-jump process with states $\Omega=\{0,1\}$ [28]. The receptor can either be occupied by a ligand $(x=1)$ or be empty $(x=0)$. Let the background ligand concentration be $c$ and assume that the ligand binds with a rate $k c$ and unbinds with rate $k$, ignoring for simplicity any spatial variations of concentration. The generator and its eigenvectors are given by

$\hat{L}=\left(\begin{array}{cc}-k c & k \\ k c & -k\end{array}\right),\left|R_{0}\right\rangle=\frac{1}{1+c}\left(\begin{array}{l}1 \\ c\end{array}\right),\left|R_{1}\right\rangle=\frac{1}{1+c}\left(\begin{array}{c}1 \\ -1\end{array}\right)$

with $\lambda_{1}=-k(1+c)$ being the only nonzero eigenvalue. The left eigenvectors corresponding to Eq. (70) are $\left\langle L_{0}\right|=\langle-|=$ $(1,1)$ and $\left\langle L_{1}\right|=(c,-1)$. Moreover, since the entire state space has only two states, we have $\theta_{0}(t)=1-\theta_{1}(t)$. Assuming that the system was initially in equilibrium, $\left|p_{0}\right\rangle=\left|R_{0}\right\rangle$,

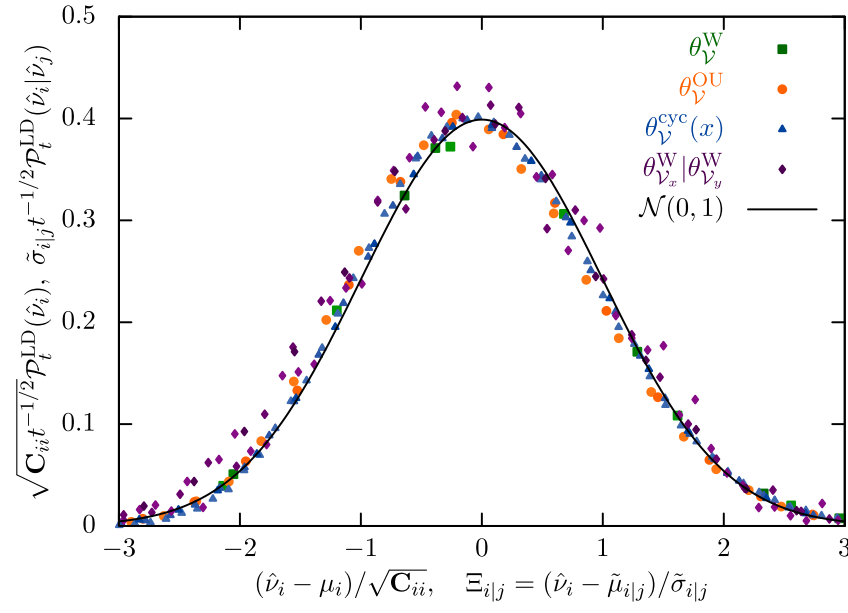

FIG. 8. Collapse of probability density functions of all studied models at long time onto a unit normal probability density. The symbols correspond to rescaled probability density of occupation time fraction $\theta_{\mathcal{V}}(t)^{\mathrm{W}, \mathrm{OU}, \mathrm{cyc}}$ [see also Eq. (7)] of the Wiener process (64), the Ornstein-Uhlenbeck process (66), the driven three-state cycle (68), respectively, and the conditional probability density function of occupation time fraction $\theta_{\mathcal{V}_{x}}(t)^{\mathrm{W}} \mid \theta_{\mathcal{V}_{y}}(t)^{\mathrm{W}}$ of the Wiener process for $\mathcal{V}_{x}=x \in[0.1,0.4]$ and $\mathcal{V}_{y}=y \in[0.6,0.9]$. The line is a zero mean unit normal probability density function $\mathcal{N}(0,1)$.

this implies that the mean values of the respective localtime fractions are given by $\left\langle\theta_{1}(t)\right\rangle=(1+c)^{-1} c$ and $\left\langle\theta_{0}(t)\right\rangle=$ $(1+c)^{-1}$.

If the receptor estimates the concentration $c$ by reading out and averaging the fraction of time the ligand is bound, $\theta_{1}$, over an interval of duration $t$, the precision of the estimate is bounded from above by the variance of the local-time fraction given by Eq. (52) and reads explicitly

$$
\sigma_{\theta_{1}}^{2}(t)=\frac{2 c}{(1+c)^{3} k t}\left[1-\frac{1-e^{-k(1+c) t}}{k(1+c) t}\right] .
$$

Typically one assumes that the measurement $t$ is longer than any correlation time $[28,30,83,84]$, which in the present setting implies $t \gg 1 /[k(1+c)]$, i.e., much longer than the correlation time of two-state Markov switching noise, $\tau_{c} \equiv$ $\lambda_{1}^{-1}=1 /(k+k c)$ [30]. In this regime, the averaging noise corresponds to shot noise such that the variance decreases with the number of statistically independent receptor measurements $\#_{t}[28,30,83,84]$, where $\#_{t} \sim t / \tau_{c}$ is the number of statistically independent realizations of the two-state process. Therefore, $\sigma_{\theta_{1}}^{2}(t) \propto 1 / \#_{t}=\tau_{c} / t$, according to the centrallimit theorem.

Based on the bound derived in Eq. (61), the shot-noise limit is in fact an upper bound to fluctuations of receptor occupancy at any duration of measurement, and it saturates only in the limit $t \gg \tau_{c}$. Namely, a direct application of the bound (61) indeed yields, using $G_{t}^{\mathrm{eq}}(1,1)=P_{t}(1 \mid 1) P_{\infty}(1 \mid 1)$,

$$
\begin{aligned}
B & \equiv 2 \int_{0}^{\infty}\left[G_{t}^{\mathrm{eq}}(1,1)-G_{\infty}^{\mathrm{eq}}(1,1)\right] d t \\
& =2 \int_{0}^{\infty} \frac{c}{(1+c)^{2}} e^{-k(1+c) t} d t=\frac{2 c}{(1+c)^{3} k},
\end{aligned}
$$


implying, according to Eq. (61),

$$
\sigma_{\theta_{1}}^{2}(t) \leqslant \frac{B}{t}=\frac{2 c}{(1+c)^{2}} \frac{1}{\#_{t}} \equiv \lim _{t \gg \tau_{c}} \sigma_{\theta_{1}}^{2}(t)
$$

Therefore, for short and particularly finite measurements, the shot-noise limit of fluctuations for long receptor read-out $[28,30,83,84]$ gives only an upper bound to the uncertainty of the estimate, whereas the inequality becomes sharp at long times.

Fundamental bounds on the precision of inferring $c$ from $\theta_{1}(t)$ can be found in [28]. Using the entire time trace of the receptor occupancy $x_{\tau}(0 \leqslant \tau \leqslant t)$ instead of the occupation time $\theta_{1}(t)$ alone, and employing a maximum-likelihood estimate of the concentration $c$, the error of the resulting estimate (i.e., its variance) is found to be reduced further by a factor of $1 / 2$ [31,32]. A detailed discussion of the precision of inferring kinetic parameters by means of nonlocal functionals can be found in Ref. [37].

\section{CONCLUDING PERSPECTIVE}

We developed a general spectral-theoretic approach to time-average statistical mechanics, i.e., to the statistics of bounded, local additive functionals of (normal) ergodic stochastic processes with continuous and discrete statespaces. In particular, we have shown how to obtain exactly the mean, variance, and correlations of time-average observables from the eigenspectrum of the underlying forward or backward generator. We rederived the famous Feynman-Kac formulas using Itô calculus and included a brief derivation for Markov-jump processes. We combined Feynman-Kac formulas with non-Hermitian perturbation theory to derive an exact spectral representation of the results. We demonstrated explicitly, and quantitatively, the emergence of the universal central-limit law in a spectral representation on large-deviation timescales. For the special case of equilibrated initial conditions and dynamics obeying detailed balance, we derived a general upper bound on fluctuations of occupation measures inferred from individual trajectories. We discussed our theoretical results from a physical perspective and provided simple but instructive practical examples to demonstrate how the theory is to be applied. Our work is applicable to continuous as well as discrete state-space processes, reversible as well as irreversible, encompassing a wide and diverse range of phenomena involving time-average observables and additive functionals in physical, chemical, and biological systems as well as financial mathematics and econophysics.

\section{ACKNOWLEDGMENTS}

The financial support from the German Research Foundation (DFG) through the Emmy Noether Program GO 2762/1-1 to A.G. is gratefully acknowledged.

\section{APPENDIX A: THE PERTURBATIVE CALCULATION}

We carry out all calculations with the spectrum of the backward generator $\hat{L}^{\dagger}$. Equivalent results can be derived using the forward generator instead. We carry out perturbative calculations (34) up to second order to derive the results in Eq. (37).

\section{Terms of first order in $u$}

Starting with a perturbation of the backward "kets" and collecting terms of first order in Eq. (34), we find

$$
-\hat{L}^{\dagger}\left|L_{k}^{1}\right\rangle+V\left|L_{k}\right\rangle=\lambda_{k}^{(1)}\left|L_{k}\right\rangle+\lambda_{k}^{\dagger}\left|L_{k}^{1}\right\rangle
$$

and multiply Eq. (A1) by $\left\langle R_{l}\right|$ from the left to obtain

$$
\left.-\left\langle R_{l}\left|\hat{L}^{\dagger}\right| L_{k}^{1}\right\rangle+\left\langle R_{l}|V| L_{k}\right\rangle=\lambda_{k}^{(1)} \delta_{k l}+\lambda_{k}^{\dagger}\left|R_{l}\right| L_{k}^{1}\right\rangle .
$$

Therefore, if $k=l$ we find

$$
\lambda_{k}^{(1)}=\left\langle R_{k}|V| L_{k}\right\rangle \equiv V_{k k}
$$

while for $k \neq l$ we obtain

$$
\left\langle R_{l} \mid L_{k}^{1}\right\rangle=\frac{V_{l k}}{\lambda_{k}^{\dagger}-\lambda_{l}^{\dagger}}
$$

and therefore

$$
\left|L_{k}^{1}\right\rangle=\sum_{l \neq k} \frac{V_{l k}}{\lambda_{k}^{\dagger}-\lambda_{l}^{\dagger}}\left|L_{l}\right\rangle .
$$

We now turn to the perturbation of $\hat{L}^{\dagger}$ acting on the bras from the right and multiply the resulting first-order equation by $\left|L_{l}\right\rangle$ from the right to obtain

$$
\left.-\left\langle R_{k}^{1}\left|\hat{L}^{\dagger}\right| L_{l}\right\rangle+\left\langle R_{k}|V| L_{l}\right\rangle=\lambda_{k}^{\dagger}\left|R_{k}^{1}\right| L_{l}\right\rangle+\lambda_{k}^{(1)} \delta_{k l} .
$$

For $k=l$ we obtain the eigenvalue-corrections (A3) while for $k \neq l$ we have

$$
\left\langle R_{k}^{1}\right|=\sum_{l \neq k} \frac{V_{k l}}{\lambda_{k}^{\dagger}-\lambda_{l}^{\dagger}}\left\langle R_{l}\right| .
$$

\section{Terms of second order in $u$}

Collecting in Eq. (34) corrections of second order to the kets we find, upon multiplying by $\left\langle R_{l}\right|$ from the left,

$$
\begin{aligned}
& -\left\langle R_{l}\left|\hat{L}^{\dagger}\right| L_{k}^{2}\right\rangle+\left\langle R_{l}|V| L_{k}^{1}\right\rangle \\
& =\lambda_{k}^{(2)} \delta_{l k}+\lambda_{k}^{(1)}\left\langle R_{l} \mid L_{k}^{1}\right\rangle+\lambda_{k}^{\dagger}\left\langle R_{l} \mid L_{k}^{2}\right\rangle,
\end{aligned}
$$

yielding, for $k=l$,

$$
\lambda_{k}^{(2)}=\left\langle R_{k}^{0}|\hat{V}| L_{k}^{1}\right\rangle,
$$

because $\left\langle R_{k} \mid L_{k}^{1}\right\rangle=0$ due to the Eq. (36) and thus

$$
\lambda_{k}^{(2)}=\sum_{l \neq k} \frac{V_{k l} V_{l k}}{\lambda_{k}^{\dagger}-\lambda_{l}^{\dagger}}\left\langle R_{l}|V| L_{k}\right\rangle .
$$

Conversely, if $k \neq l$ we obtain the second-order correction

$$
\left|L_{k}^{2}\right\rangle=\sum_{l \neq k}\left[\sum_{i \neq k} \frac{V_{i k} V_{l i}}{\left(\lambda_{i}^{\dagger}-\lambda_{k}^{\dagger}\right)\left(\lambda_{l}^{\dagger}-\lambda_{k}^{\dagger}\right)}-\frac{V_{k k} V_{l k}}{\left(\lambda_{l}^{\dagger}-\lambda_{k}^{\dagger}\right)^{2}}\right]\left|L_{l}\right\rangle .
$$

Collecting in Eq. (34) corrections of second order to the bras we find, upon multiplying from the right by $\left|L_{l}\right\rangle$,

$$
\begin{aligned}
& -\left\langle R_{k}^{2}\left|\hat{L}^{\dagger}\right| L_{l}\right\rangle+\left\langle R_{k}^{1}|V| L_{l}\right\rangle \\
& \left.=\lambda_{k}^{(2)} \delta_{k l}+\lambda_{k}^{(1)}\left\langle R_{k}^{1} \mid L_{l}\right\rangle+\lambda_{k}^{\dagger}\left|R_{k}^{2}\right| L_{l}\right\rangle .
\end{aligned}
$$


When $k=l$ we obtain Eq. (A10) while in the case when $k \neq l$ we find the second correction to the bra,

$$
\left\langle R_{k}^{2}\right|=\sum_{l \neq k}\left[\sum_{i \neq k} \frac{V_{k i} V_{i l}}{\left(\lambda_{i}^{\dagger}-\lambda_{k}^{\dagger}\right)\left(\lambda_{l}^{\dagger}-\lambda_{k}^{\dagger}\right)}-\frac{V_{k k} V_{k l}}{\left(\lambda_{l}^{\dagger}-\lambda_{k}^{\dagger}\right)^{2}}\right]\left\langle R_{l}\right|
$$

which completes the derivation of Eq. (37).

\section{APPENDIX B: DERIVATION VIA THE DYSON SERIES}

In a previous publication [59], we showed how to derive equations for the moments of $\psi_{t}$ for stationary initial conditions, $p_{0}(\mathbf{x})=P_{\text {inv }}(\mathbf{x})$ [Eqs. (52) and (54)], using a Dyson series approach. Here we sketch how to obtain the moments of $\psi_{t}$ for generic initial conditions $p_{0}(\mathbf{x})$. In contrast to Ref. [59], we use the forward approach here and expand the tilted propagator up to the second order in $u$,

$$
\left\langle-\left|e^{(\hat{L}-u V) t}\right| p_{0}\right\rangle=1-u\left\langle-\left|\int_{0}^{t} d t^{\prime} e^{\hat{L}\left(t-t^{\prime}\right)} V e^{\hat{L} t^{\prime}}\right| p_{0}\right\rangle+u^{2}\left\langle-\left|\int_{0}^{t} d t^{\prime} \int_{0}^{t^{\prime}} d t^{\prime \prime} e^{\hat{L}\left(t-t^{\prime}\right)} V e^{\hat{L}\left(t^{\prime}-t^{\prime \prime}\right)} V e^{\hat{L} t^{\prime \prime}}\right| p_{0}\right\rangle+O\left(u^{3}\right),
$$

assuming $t>t^{\prime}>t^{\prime \prime}>0$. Here we confirm that the Dyson series gives results identical to the perturbation calculation.

\section{Mean, fluctuations, and correlations}

We now derive $\left\langle\bar{V}_{t}\right\rangle, \sigma_{\bar{V}}^{2}(t)$, and $C_{\bar{V}_{1} \bar{V}_{2}}(t)$ presented in Eqs. (46)-(54) using the Dyson series. Note that this calculation does not diagonalize the tilted generator $\hat{L}^{\dagger}-u V(\mathbf{x})\left[\hat{L}^{\dagger}-u V(\mathbf{x})\right.$, respectively]. Starting from Eq. (B1), we can carry out all integrations analytically for arbitrary initial conditions $p_{0}(\mathbf{x})$. To first order in $u$, we obtain

$$
\begin{aligned}
t^{-1}\left\langle-\left|\int_{0}^{t} d t^{\prime} e^{\hat{L}\left(t-t^{\prime}\right)} V e^{\hat{L} t^{\prime}}\right| p_{0}\right\rangle & =t^{-1} \int_{0}^{t} d t^{\prime} \sum_{k} \sum_{l}\left\langle-\mid R_{l}\right\rangle\left\langle L_{l}|V| R_{k}\right\rangle\left\langle L_{k} \mid p_{0}\right\rangle e^{-\lambda_{l}\left(t-t^{\prime}\right)-\lambda_{k} t^{\prime}} \\
& =t^{-1} \int_{0}^{t} d t^{\prime} \sum_{k}\left\langle-|V| R_{k}\right\rangle\left\langle L_{k} \mid p_{0}\right\rangle e^{-\lambda_{k} t^{\prime}}=V_{00}+\frac{1}{t} \sum_{k} \frac{V_{k 0}}{\lambda_{k}}\left\langle L_{k} \mid p_{0}\right\rangle\left(1-e^{-\lambda_{k} t}\right)
\end{aligned}
$$

where for $p_{0}(\mathbf{x})=P_{\text {inv }}(\mathbf{x})$ we have $\left\langle L_{k} \mid p_{0}\right\rangle=\delta_{k 0}$ leading to $\langle\bar{V}\rangle_{\text {inv }}=V_{00}=P_{\text {inv }}(\mathbf{x})$.

To second order in $u$ we find for an arbitrary $p_{0}(\mathbf{x})$

$$
\begin{aligned}
& t^{-2}\left\langle-\left|\int_{0}^{t} d t^{\prime} \int_{0}^{t^{\prime}} d t^{\prime \prime} e^{\hat{L}\left(t-t^{\prime}\right)} V e^{\hat{L}\left(t^{\prime}-t^{\prime \prime}\right)} V e^{\hat{L} t^{\prime \prime}}\right| p_{0}\right\rangle \\
& =t^{-2} \int_{0}^{t} d t^{\prime} \int_{0}^{t^{\prime}} d t^{\prime \prime}\left\langle-\left|\sum_{m}\right| R_{m}\right\rangle\left\langle L_{m}\left|e^{-\lambda_{m}\left(t-t^{\prime}\right)} V \sum_{k}\right| R_{k}\right\rangle\left\langle L_{k}\left|e^{-\lambda_{k}\left(t^{\prime}-t^{\prime \prime}\right)} V \sum_{l}\right| R_{i}\right\rangle\left\langle L_{l}\left|e^{-\lambda_{l} t^{\prime \prime}}\right| p_{0}\right\rangle \\
& =t^{-2} \sum_{k} \sum_{l} V_{0 k} V_{k l}\left\langle L_{l} \mid p_{0}\right\rangle \int_{0}^{t} d t^{\prime} \int_{0}^{t^{\prime}} d t^{\prime \prime} e^{-\lambda_{k}\left(t^{\prime}-t^{\prime \prime}\right)-\lambda_{l} t^{\prime \prime}} ;
\end{aligned}
$$

when $k=l=0$ only $V_{00}^{2} / 2$ survives, while for $k \neq 0$ and $l=$ 0 we find

$$
\frac{1}{t} \sum_{k>0} \frac{V_{k 0} V_{0 k}}{\lambda_{k}}\left(1-\frac{1-e^{-\lambda_{k} t}}{t \lambda_{k}}\right)
$$

Conversely, when $k=0$ and $l \neq 0$ we end up with

$$
\frac{1}{t} \sum_{l>0} \frac{V_{00} V_{l 0}}{\lambda_{l}}\left\langle L_{l} \mid p_{0}\right\rangle\left(1-\frac{1-e^{-\lambda_{l} t}}{t \lambda_{l}}\right)
$$

while for $k=l$ and $k \neq 0$ and $l \neq 0$ we have

$$
\frac{1}{t} \sum_{k>0} \frac{V_{k 0} V_{k k}}{\lambda_{k}}\left\langle L_{k} \mid p_{0}\right\rangle\left(e^{-\lambda_{k} t}-\frac{1-e^{-\lambda_{k} t}}{t \lambda_{k}}\right) .
$$

Finally, when $k \neq l \neq 0$ we obtain

$$
\frac{1}{t^{2}} \sum_{k>0} \sum_{l>0, l \neq k} \frac{V_{k 0} V_{l k}}{\lambda_{k}-\lambda_{l}}\left\langle L_{l} \mid p_{0}\right\rangle\left(\frac{1-e^{-\lambda_{l} t}}{\lambda_{l}}-\frac{1-e^{-\lambda_{k} t}}{\lambda_{k}}\right) .
$$

The sum of these terms yields the sought-after result. The corresponding result for stationary initial conditions, $p_{0}(\mathbf{x})=$ $P_{\text {inv }}(\mathbf{x})$, is obtained using $\left\langle L_{l} \mid P_{\text {inv }}\right\rangle=\delta_{l 0}$, which leads to Eqs. (52) and (54). When considering correlations, we make the replacement $u V \rightarrow u_{1} V_{1}+u_{2} V_{2}$ and replace $\partial_{u_{1}}^{2} \rightarrow \partial_{u_{1} u_{2}}^{2}$ to compute the covariance. The formulas above thereby generalize in a straightforward manner.
[1] M. J. Saxton, Single-particle tracking: Connecting the dots, Nat. Methods 5, 671 (2008).
[2] R. Metzler, J.-H. Jeon, A. G. Cherstvy, and E. Barkai, Anomalous diffusion models and their properties: Non-stationarity, 
non-ergodicity, and ageing at the centenary of single particle tracking, Phys. Chem. Chem. Phys. 16, 24128 (2014).

[3] D. Ernst, J. Köhler, and M. Weiss, Probing the type of anomalous diffusion with single-particle tracking, Phys. Chem. Chem. Phys. 16, 7686 (2014).

[4] H. Shen, L. J. Tauzin, R. Baiyasi, W. Wang, N. Moringo, B. Shuang, and C. F. Landes, Single particle tracking: From theory to biophysical applications, Chem. Rev. 117, 7331 (2017).

[5] M. L. Hughes and L. Dougan, The physics of pulling polyproteins: a review of single molecule force spectroscopy using the AFM to study protein unfolding, Rep. Prog. Phys. 79, 076601 (2016).

[6] X. Sunney Xie, Single-molecule spectroscopy and dynamics at room temperature, Acc. Chem. Res. 29, 598 (1996).

[7] W. P. Ambrose, P. M. Goodwin, J. H. Jett, A. Van Orden, J. H. Werner, and R. A. Keller, Single molecule fluorescence spectroscopy at ambient temperature, Chem. Rev. 99, 2929 (1999).

[8] T. Plakhotnik, E. A. Donley, and U. P. Wild, Single-molecule spectroscopy, Annu. Rev. Phys. Chem. 48, 181 (1997).

[9] K. C. Neuman and A. Nagy, Single-molecule force spectroscopy: Optical tweezers, magnetic tweezers and atomic force microscopy, Nat. Methods 5, 491 (2008).

[10] M. Rief and H. Grubmüller, Force spectroscopy of single biomolecules, ChemPhysChem 3, 255 (2002).

[11] M. T. Woodside and S. M. Block, Reconstructing folding energy landscapes by single-molecule force spectroscopy, Annu. Rev. Biophys. 43, 19 (2014).

[12] F. Ritort, Single-molecule experiments in biological physics: methods and applications, J. Phys.: Condens. Matter 18, R531 (2006).

[13] J. Camunas-Soler, M. Ribezzi-Crivellari, and F. Ritort, Elastic properties of nucleic acids by single-molecule force spectroscopy, Annu. Rev. Biophys. 45, 65 (2016).

[14] P. Lévy, Sur certains processus stochastiques homogènes, Compositio Mathematica 7, 283 (1940).

[15] M. Kac, On distributions of certain Wiener functionals, Trans. Am. Math. Soc. 65, 1 (1949).

[16] D. A. Darling and M. Kac, On occupation times for Markoff processes, Trans. Am. Math. Soc. 84, 444 (1957).

[17] J. Lamperti, An occupation time theorem for a class of stochastic processes, Trans. Am. Math. Soc. 88, 380 (1958).

[18] W. Feller, Fluctuation theory of recurrent events, Trans. Am. Math. Soc. 67, 98 (1949).

[19] N. H. Bingham, Fluctuation theory in continuous time, Adv. Appl. Probab. 7, 705 (1975).

[20] A N Borodin, Brownian local time, Russ. Math. Surv. 44, 1 (1989).

[21] J.-Y. Yen and M. Yor, Local Times and Excursion Theory for Brownian Motion (Springer, Cham, 2013).

[22] M. Yor, Exponential Functionals of Brownian Motion and Related Processes, Springer Finance Lecture Notes (SpringerVerlag, Berlin, 2001).

[23] H. Geman and M. Yor, Bessel processes, Asian options, and perpetuities, Math. Fin. 3, 349 (1993).

[24] G. Wilemski and M. Fixman, General theory of diffusioncontrolled reactions, J. Chem. Phys. 58, 4009 (1973).

[25] A. Szabo, Theory of diffusion-influenced fluorescence quenching, J. Phys. Chem. 93, 6929 (1989).
[26] O. Bénichou, M. Coppey, M. Moreau, and G. Oshanin, Kinetics of diffusion-limited catalytically activated reactions: An extension of the Wilemski-Fixman approach, J. Chem. Phys. 123, 194506 (2005).

[27] D. S. Grebenkov, Residence times and other functionals of reflected Brownian motion, Phys. Rev. E 76, 041139 (2007).

[28] H. C. Berg and E. M. Purcell, Physics of chemoreception, Biophys. J. 20, 193 (1977).

[29] F. W. Wiegel, Diffusion and the physics of chemoreception, Phys. Rep. 95, 283 (1983).

[30] W. Bialek and S. Setayeshgar, Physical limits to biochemical signaling, Proc. Natl. Acad. Sci. (USA) 102, 10040 (2005).

[31] R. G. Endres and N. S. Wingreen, Maximum Likelihood and the Single Receptor, Phys. Rev. Lett. 103, 158101 (2009).

[32] T. Mora and N. S. Wingreen, Limits of Sensing Temporal Concentration Changes by Single Cells, Phys. Rev. Lett. 104, 248101 (2010).

[33] A. H. Lang, C. K. Fisher, T. Mora, and P. Mehta, Thermodynamics of Statistical Inference by Cells, Phys. Rev. Lett. 113, 148103 (2014).

[34] T. Mora, Physical Limit to Concentration Sensing Amid Spurious Ligands, Phys. Rev. Lett. 115, 038102 (2015).

[35] A. C. Barato and U. Seifert, Dispersion for two classes of random variables: General theory and application to inference of an external ligand concentration by a cell, Phys. Rev. E 92, 032127 (2015).

[36] G. Aquino, N. S. Wingreen, and R. G. Endres, Know the singlereceptor sensing limit? Think again, J. Stat. Phys. 162, 1353 (2015).

[37] D. Hartich and U. Seifert, Optimal inference strategies and their implications for the linear noise approximation, Phys. Rev. E 94, 042416 (2016).

[38] M. Ferraro and L. Zaninetti, Statistics of visits to sites in random walks, Physica A 338, 307 (2004).

[39] A. H. Gandjbakhche and G. H. Weiss, Descriptive parameter for photon trajectories in a turbid medium, Phys. Rev. E 61, 6958 (2000).

[40] G. H. Weiss and P. P. Calabrese, Occupation times of a CTRW on a lattice with anomalous sites, Physica A 234, 443 (1996).

[41] Z. Toroczkai, T. J. Newman, and S. Das Sarma, Sign-time distributions for interface growth, Phys. Rev. E 60, R1115 (1999).

[42] X. Brokmann, J.-P. Hermier, G. Messin, P. Desbiolles, J.-P. Bouchaud, and M. Dahan, Statistical Aging and Nonergodicity in the Fluorescence of Single Nanocrystals, Phys. Rev. Lett. 90, 120601 (2003).

[43] F. D. Stefani, J. P. Hoogenboom, and E. Barkai, Beyond quantum jumps: Blinking nanoscale light emitters, Phys. Today 62(2), 34 (2009).

[44] A. Comtet, J. Desbois, and C. Texier, Functionals of Brownian motion, localization and metric graphs, J. Phys. A 38, R341 (2005).

[45] S. N. Majumdar and A. J. Bray, Large-deviation functions for nonlinear functionals of a Gaussian stationary Markov process, Phys. Rev. E 65, 051112 (2002).

[46] S. N. Majumdar, Brownian functionals in physics and computer science, Curr. Sci. 89, 2075 (2005).

[47] S. Wennmalm, L. Edman, and R. Rigler, Conformational fluctuations in single DNA molecules, Proc. Natl. Acad. Sci. (USA) 94, 10641 (1997). 
[48] I. V. Gopich and A. Szabo, Theory of the energy transfer efficiency and fluorescence lifetime distribution in single-molecule FRET, Proc. Natl. Acad. Sci. (USA) 109, 7747 (2012).

[49] L. Fleury, J.-M. Segura, G. Zumofen, B. Hecht, and U. P. Wild, Nonclassical Photon Statistics in Single-Molecule Fluorescence at Room Temperature, Phys. Rev. Lett. 84, 1148 (2000).

[50] E. Barkai, Y. J. Jung, and R. Silbey, Theory of single-molecule spectroscopy: Beyond the ensemble average, Annu. Rev. Phys. Chem. 55, 457 (2004).

[51] N. Agmon, The residence time equation, Chem. Phys. Lett. 497, 184 (2010)

[52] S. Sabhapandit, S. N. Majumdar, and A. Comtet, Statistical properties of functionals of the paths of a particle diffusing in a one-dimensional random potential, Phys. Rev. E 73, 051102 (2006).

[53] S. N. Majumdar and A. Comtet, Local and Occupation Time of a Particle Diffusing in a Random Medium, Phys. Rev. Lett. 89, 060601 (2002).

[54] G. Bel and E. Barkai, Weak Ergodicity Breaking in the Continuous-Time Random Walk, Phys. Rev. Lett. 94, 240602 (2005).

[55] S. Carmi and E. Barkai, Fractional Feynman-Kac equation for weak ergodicity breaking, Phys. Rev. E 84, 061104 (2011).

[56] A. Dhar and S. N. Majumdar, Residence time distribution for a class of Gaussian Markov processes, Phys. Rev. E 59, 6413 (1999).

[57] S. N. Majumdar and D. S. Dean, Exact occupation time distribution in a non-Markovian sequence and its relation to spin glass models, Phys. Rev. E 66, 041102 (2002).

[58] A. J. Bray, S. N. Majumdar, and G. Schehr, Persistence and first-passage properties in nonequilibrium systems, Adv. Phys. 62, 225 (2013).

[59] A. Lapolla and A. Godec, Unfolding tagged particle histories in single-file diffusion: exact single- and two-tag local times beyond large deviation theory, New J. Phys. 20, 113021 (2018).

[60] A. Lapolla and A. Godec, Manifestations of projection-induced memory: General theory and the tilted single file, Front. Phys. 7, 182 (2019).

[61] D. Boyer, D. S. Dean, C. Mejía-Monasterio, and G. Oshanin, Optimal fits of diffusion constants from single-time data points of Brownian trajectories, Phys. Rev. E 86, 060101(R) (2012).

[62] D. Boyer, D. S. Dean, C. Mejía-Monasterio, and G. Oshanin, Optimal estimates of the diffusion coefficient of a single Brownian trajectory, Phys. Rev. E 85, 031136 (2012).

[63] J. B. Jørgensen, J. Mann, S. Ott, H. L. Pócseli, and J. Trulsen, Experimental studies of occupation and transit times in turbulent flows, Phys. Fluids 17, 035111 (2005).

[64] A. C. Barato and R. Chetrite, A formal view on level 2.5 large deviations and fluctuation relations, J. Stat. Phys. 160, 1154 (2015).
[65] H. Touchette, The large deviation approach to statistical mechanics, Phys. Rep. 478, 1 (2009).

[66] H. Touchette, Introduction to dynamical large deviations of markov processes, Physica A 504, 5 (2018).

[67] C. W. Gardiner, Handbook of Stochastic Methods, 3rd ed. (Springer, Berlin, 2004).

[68] D. T. Gillespie, Exact stochastic simulation of coupled chemical reactions, J. Phys. Chem. 81, 2340 (1977).

[69] J. Schnakenberg, Network theory of microscopic and macroscopic behavior of master equation systems, Rev. Mod. Phys. 48, 571 (1976).

[70] J. B. Conway, A Course in Functional Analysis, Graduate Texts in Mathematics (Springer-Verlag, New York, 1985).

[71] N. G. van Kampen, Stochastic Processes in Physics and Chemistry, 3rd ed., North-Holland Personal Library (Elsevier, Amsterdam, 2007).

[72] H. Risken, The Fokker-Planck Equation: Methods of Solution and Applications, 2nd ed., Springer Series in Synergetics (Springer-Verlag, Berlin, 1996).

[73] S. D. Stoller, W. Happer, and F. J. Dyson, Transverse spin relaxation in inhomogeneous magnetic fields, Phys. Rev. A 44, 7459 (1991).

[74] Note that 0 is an eigenvalue of $\hat{L}$ and $\tilde{\mathcal{P}}_{s}^{\psi}\left(\mathbf{u} \mid p_{0}\right)$ is meromorphic for small $|\mathbf{u}|$, therefore we can assume, without loss of generality, that $\mathbf{u}$ is real.

[75] J. Marcinkiewicz, Sur une propriété de la loi de Gauß, Math. Z. 44, 612 (1939).

[76] J. J. Sakurai and J. Napolitano, Modern Quantum Mechanics (Cambridge University Press, Cambridge, 2017).

[77] D. J. Klein, Degenerate perturbation theory, J. Chem. Phys. 61, 786 (1974).

[78] D. Hartich and A. Godec, Extreme value statistics of ergodic Markov processes from first passage times in the large deviation limit, J. Phys. A 52, 244001 (2019).

[79] A. I. Khinchin, Sur la loi des grands nombres, C. R. Acad. Sci. 188, 477 (1929).

[80] V. Holubec, K. Kroy, and S. Steffenoni, Physically consistent numerical solver for time-dependent Fokker-Planck equations, Phys. Rev. E 99, 032117 (2019).

[81] G. Guennebaud, B. Jacob et al., Eigen v3, http://eigen. tuxfamily.org (2010).

[82] S. Toyabe, T. Watanabe-Nakayama, T. Okamoto, S. Kudo, and E. Muneyuki, Thermodynamic efficiency and mechanochemical coupling of $F_{1}$-ATPase, Proc. Natl. Acad. Sci. (USA) 108, 17951 (2011).

[83] A. Godec and R. Metzler, Signal focusing through active transport, Phys. Rev. E 92, 010701(R) (2015).

[84] A. Godec and R. Metzler, Active transport improves the precision of linear long distance molecular signalling, J. Phys. A 49, 364001 (2016). 


\section{Chapter 8}

\section{Single-file diffusion in a bi-stable potential: Signatures of memory in the barrier- crossing of a tagged-particle}

This article has been published in The Journal of Chemical Physics under the Creative Commons Attribution 4.0 International license [55]. Barriercrossing dynamics is important in a wide variety of processes, in particular chemical reactions. Often these analyses focus on the first passage time statistics. In this article we, instead, focused on how the relaxation of a non-Markovian observable, the tagged-particle in a single-file, is affected by the memory generated by the other hidden (integrated out) degrees of freedom and a barrier in the potential. We considered the memory kernel of the correlation function obtained by the Zwanzig theory and the difference between the Markovian and the non-Markovian relaxation towards equilibrium. 


\title{
Single-file diffusion in a bi-stable potential: Signatures of memory in the barrier-crossing of a tagged-particle
}

\author{
Cite as: J. Chem. Phys. 1 53, 194104 (2020); doi: 10.1 063/5.0025785 \\ Submitted: 18 August 2020 - Accepted: 30 October $2020 \cdot$ \\ Published Online: 16 November 2020
}

\author{
Alessio Lapolla (D) and Aljaž Godec ${ }^{\text {a) }}$ (D)
}

\section{AFFILIATIONS}

Mathematical bioPhysics Group, Max Planck Institute for Biophysical Chemistry, Am Fassberg 11, 37077 Göttingen, Germany

${ }^{\text {a) }}$ Author to whom correspondence should be addressed: agodec@mpibpc.mpg.de

\begin{abstract}
We investigate memory effects in barrier-crossing in the overdamped setting. We focus on the scenario where the hidden degrees of freedom relax on exactly the same time scale as the observable. As a prototypical model, we analyze tagged-particle diffusion in a single file confined to a bi-stable potential. We identify the signatures of memory and explain their origin. The emerging memory is a result of the projection of collective many-body eigenmodes onto the motion of a tagged-particle. We are interested in the "confining" (all background particles in front of the tagged-particle) and "pushing" (all background particles behind the tagged-particle) scenarios for which we find non-trivial and qualitatively different relaxation behaviors. Notably and somewhat unexpectedly, at a fixed particle number, we find that the higher the barrier, the stronger the memory effects are. The fact that the external potential alters the memory is important more generally and should be taken into account in applications of generalized Langevin equations. Our results can readily be tested experimentally and may be relevant for understanding transport in biological ion-channels.
\end{abstract}

(c) 2020 Author(s). All article content, except where otherwise noted, is licensed under a Creative Commons Attribution (CC BY) license (http://creativecommons.org/licenses/by/4.0/). https://doi.org/10.1063/5.0025785

\section{INTRODUCTION}

Non-linear stochastic flows are at the heart of thermally driven processes in systems whose potential energy surfaces are characterized by multiple local energy minima. Pioneered by the seminal work of Kramers, ${ }^{1}$ the concept of thermally activated barrier-crossing has ever since been applied to diverse phenomena, including chemical reactions, ${ }^{2-4}$ tunnel diodes, ${ }^{5}$ laser-pumping, ${ }^{6}$ magnetic resonance, ${ }^{7}$ conformational dynamics and folding of proteins ${ }^{8-16}$ and nucleic acids, ${ }^{17,18}$ and receptor-ligand binding ${ }^{19}$ to name but a few.

From a theoretical point of view, the most detailed and precise results were obtained in the context of relaxation phenomena ${ }^{20-26}$ and first passage time statistics ${ }^{27-34}$ in Markovian (i.e., memoryless) systems. However, physical observables typically correspond to lower-dimensional projections, and the observed dynamics is Markovian only under quite restrictive conditions on the nature of the projection. ${ }^{35}$ Quoting van Kampen: "Non-Markov is the rule, Markov is the exception."
Over the years, the non-Markovian barrier-crossing has therefore received special attention. Most approaches considered a generalized Langevin equation in the underdamped regime with diverse phenomenological memory kernels for the velocity in the high ${ }^{37-39}$ and low ${ }^{40,41}$ viscosity limits. In the case of diffusion in double-well potentials, unified solutions have been obtained. ${ }^{42}$ Seminal results on non-Markovian effects in the crossing of high energy barriers have been obtained by Mel'nikov and Meshkov ${ }^{43}$ and were later extended to low barriers by Kalmykov, Coffey, and Titov. ${ }^{44}$ Important results on the non-Markovian barrier-crossing have been obtained in the context of condensed-phase dynamics. ${ }^{4-47}$ More recent studies of memory effects in bi-stable potentials have been carried out in the context of conformational dynamics of macromolecules ${ }^{16,48-50}$ and the role of hydrodynamic memory in surmounting energy barriers, ${ }^{51}$ while recent applications involve the interpretation of experiments on the folding of a DNA hairpin. ${ }^{52}$

Quite detailed analytical results have also been obtained for overdamped non-Markovian stochastic flows in bi-stable potentials, 
in particular for exponentially correlated noise. ${ }^{53-57}$ Characteristic of these studies is that the memory is introduced phenomenologically and/or the systems typically possess slow and fast degrees of freedom. Thereby, integrating out of fast degrees of freedom leads to memory, and timescales similar to, or longer than, the correlation time are of interest.

Here, we are interested in the scenario where the background degrees of freedom (i.e., those that become integrated out) relax on exactly the same time scale as the observable. In particular, we are interested in the relaxation dynamics of a tagged-particle in a single file of Brownian particles confined to a bi-stable potential and investigate the role of the height of the potential barrier. Projecting out particles' positions introduces memory and strongly breaks Markovianity. ${ }^{35}$ The more particles' coordinates become integrated out, the stronger Markovianity is broken. ${ }^{35}$ A distinguishing characteristic of our approach with respect to the existing literature is, therefore, that we do not introduce memory phenomenologically via a generalized Langevin equation. Instead, the memory arises explicitly as a result of projecting out degrees of freedom in an exactly solvable Markovian many-body system. This is important because any external potential, in general, also affects the memory in the tagged-particle's dynamics. ${ }^{35,58,59}$ One, therefore, may not employ ad hoc memory kernels that are independent of the external potential, except when the potential does not act as background degrees of freedom and the interaction between the background degrees of freedom and the tagged-particle is harmonic or negligibly weak.

Single-file models are generically used to describe strongly correlated, effectively one-dimensional, systems and processes, e.g., biological channels, ${ }^{60}$ transport in zeolites, ${ }^{61}$ crowding effects in gene regulation, ${ }^{62,63}$ superionic conductors, ${ }^{64}$ and strongly correlated one-dimensional soft matter systems in general. ${ }^{65-68}$ Over the past few years, diverse theoretical studies yielded deep insight about the anomalous tagged-particle diffusion ${ }^{69-77}$ and the emergence and meaning of memory. ${ }^{35,78,79}$ Single-file diffusion in potential landscapes has been studied by computer simulations.

It is well known that a tagged-particle's diffusion in any homogeneous overdamped system of identically interacting particles with an excluded mutual passage is asymptotically subdiffusive, i.e., the tagged-particle's mean squared displacement asymptotically scales as $\left\langle x^{2}\right\rangle \sim t^{1 / 2}$ (see, e.g., Ref. 81). However, the manner in which crowding/steric obstruction and particle correlations affect memory in barrier-crossing, and in particular in the relaxation toward equilibrium and how such memory can be inferred and quantified from measurable physical observables, has so far remained elusive. Our results are relevant in the context of search processes of proteins on DNA in the presence of macromolecular crowding involved in transcription regulation and on a conceptual level for transport in ion-channels. More generally, the methodological framework presented here does not require an analytical solution of the problem to be known and can thus also be applied in the analysis of experiments or computer simulations.

In this work, we provide in Sec. II an analytical solution to the problem using the coordinate Bethe ansatz. In Sec. III, we analyze the equilibrium correlation functions and underlying linear memory kernel as a function of the barrier height and number of particles in the single file. Section IV addresses the relaxation to equilibrium from a fixed, non-equilibrium initial condition of the tagged-particle in the "confining" and "pushing" scenario, respectively. We conclude with a brief discussion including potential applications and extensions of our results.

\section{THEORY}

We consider a single file of $N$ point-particles confined to a box of length of $L=2 \pi$. In the center of the box, there is a squaretop energy barrier of width $\pi$ and height $U_{b}$ [see Fig. 1(a)]. More precisely, each particle experiences the potential ${ }^{82,83}$

$$
U(x)= \begin{cases}0, & \pi>|x|>\pi / 2 \\ U_{b}, & |x| \leq \pi / 2 \\ \infty, & \text { otherwise. }\end{cases}
$$

The particles move according to overdamped Brownian dynamics but are not allowed to cross. For simplicity and without loss of generality, we set $D=1$, which is equivalent to expressing time in units of $4 \pi^{2} / D$, and express $U$ in units of thermal energy $k_{\mathrm{B}} T$, i.e., $U \rightarrow U / k_{\mathrm{B}} T$. The probability density of the set of positions $\left\{x_{i}\right\}=\mathbf{x}$ of the $N$ particles evolves according to the many-body Fokker-Planck equation,

$$
\left(\partial_{t}-\sum_{i=1}^{N}\left[\partial_{x_{i}}^{2}+\partial_{x_{i}}\left\{\partial_{x_{i}} U\left(x_{i}\right)\right\}\right]\right) G\left(\mathbf{x}, t \mid \mathbf{x}_{0}\right)=0
$$

with the initial condition $G\left(\mathbf{x}, 0 \mid \mathbf{x}_{0}\right)=\prod_{i=1}^{N} \delta\left(x_{i}-x_{0 i}\right)$, where the operator in curly brackets \{\} acts only within the bracket. Equation (2) is equipped with the set of external and internal boundary conditions,

$$
\begin{gathered}
\left.\partial_{x_{1}} G\left(\mathbf{x}, t \mid \mathbf{x}_{0}\right)\right|_{x_{1}=-\pi}=\left.\partial_{x_{N}} G\left(\mathbf{x}, t \mid \mathbf{x}_{0}\right)\right|_{x_{N}=\pi}=0, \\
\left.\left(\partial_{x_{i+1}}-\partial_{x_{i}}\right) G\left(\mathbf{x}, t \mid \mathbf{x}_{0}\right)\right|_{x_{i+1}=x_{i}}=0,
\end{gathered}
$$

and is solved exactly using the coordinate Bethe ansatz (for technical details, refer to Refs. 35, 79, and 84). In a nutshell, the Bethe ansatz solution exploits the intuitive fact that a trajectory of $N$ identical non-crossing Brownian particles is identical to that of an ideal Brownian gas if we re-label the particle indices such that that they are ordered at all times. As a result, one can construct the probability density of the set of particles' positions $\mathbf{x}$ by a suitable permutation of the products of probability densities of individual, non-interacting particles. In turn, an eigenfunction expansion of the many-body Fokker-Planck operator can be obtained by permuting products of single-particle eigenspectra, which is what we exploit in the present article. The resulting Bethe many-body Green's function reads

$$
G\left(\mathbf{x}, t \mid \mathbf{x}_{0}\right)=\sum_{\mathbf{k}} \Psi_{\mathbf{k}}^{R}(\mathbf{x}) \Psi_{\mathbf{k}}^{L}\left(\mathbf{x}_{0}\right) \mathrm{e}^{-\Lambda_{\mathbf{k}} \tau},
$$

where $\Psi_{\mathbf{k}}^{L}(\mathbf{x})$ and $\Psi_{\mathbf{k}}^{R}(\mathbf{x})$ are the so-called left and right Bethe eigenfunctions, respectively, defined as

$$
\Psi_{\mathbf{k}}^{L, R}(\mathbf{x}) \equiv \mathscr{N}^{1 / 2} \hat{O}_{\mathbf{x}} \sum_{\{\mathbf{k}\}} \prod_{i=1}^{N} \psi_{k_{i}}^{L, R}\left(x_{i}\right)
$$



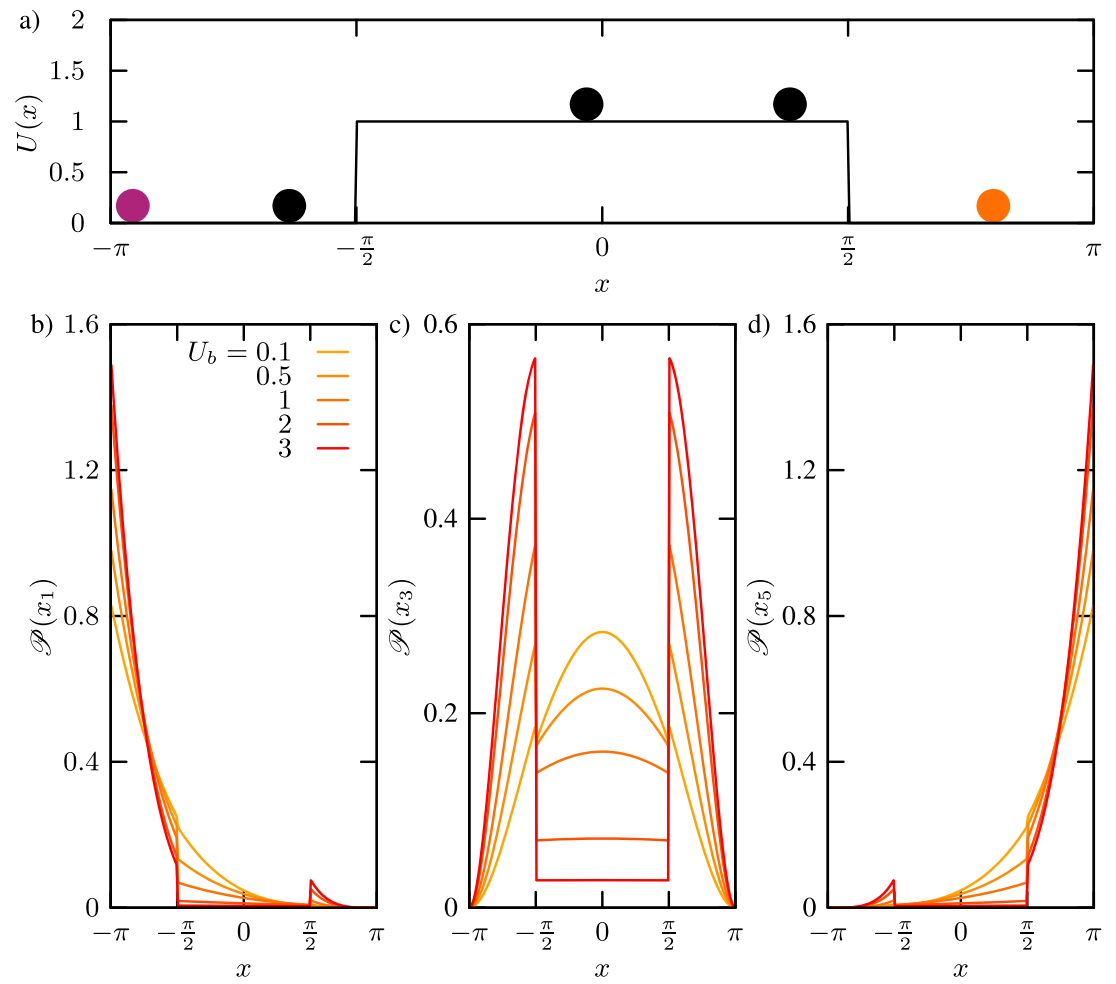

FIG. 1. (a) Schematic of the potentia $U(x)$ defined in Eq. (1); single file with $N=5$ particles. Throughout this work, we either tag the first (magenta stands for $i$ $=1$ ) or the last (orange stands for $i=N$ ) particle. (b)-(d) depict the equilibrium probability distribution $\mathscr{P}_{\text {eq }}\left(x_{i}\right)$ for $i=1$ (b), $i=3$ (c), and $i=5$ (d) in a single file with $N=5$ for different barrier-heights $U_{b}$ respectively.

where $\psi_{n}^{L, R}(x)$ are the orthonormal eigenfunctions of the singleparticle problem (given in the Appendix), the sum over $\{\mathbf{k}\}$ refers to the sum over all permutations of the multiset $\mathbf{k}$, and $\mathscr{N}$ is the number of these permutations $\mathbf{k} . \Lambda_{\mathbf{k}}=\sum_{i=1}^{N} \lambda_{k_{i}}$ refers to the Bethe eigenvalue with multi-index $\mathbf{k}=\left\{k_{i}\right\}, i \in[1, N]$, and $\hat{O}_{\mathbf{x}}$ is the particleordering operator, which ensures that $x_{1} \leq \cdots \leq x_{i} \leq \cdots \leq x_{N}$. Moreover, $\lambda_{n}$ refer to the eigenvalues of the respective one-body problem, given by ${ }^{82,83}$

$$
\lambda_{n}= \begin{cases}\frac{n^{2}}{4}, & \bmod (n, 4)=0 \\ \left(\frac{n-1}{2}+v\right)^{2}, & \bmod (n, 4)=1, \\ \frac{n^{2}}{4}, & \bmod (n, 4)=2, \\ \left(\frac{n+1}{2}-v\right)^{2}, & \bmod (n, 4)=3\end{cases}
$$

where $v=2 \arctan \left(\mathrm{e}^{-U_{b} / 2}\right) / \pi$ and $\bmod (k, l)$ stands for the remainder of the division $k / l$.

We are interested in the non-Markovian probability density of $x_{i}$ and the position of the $i$ th tagged-particle under the condition that the initial positions of the remaining particles are drawn from those equilibrium configurations that contain particle $i$ at $x_{0}$, which reads (for a derivation, see Refs. 35, 79, and 84)

$$
\mathscr{G}\left(x_{i}, t \mid x_{0 i}\right)=V_{\mathbf{0 0}}^{-1}\left(x_{0 i}\right) \sum_{\mathbf{k}} V_{\mathbf{0 k}}\left(x_{i}\right) V_{\mathbf{k} \mathbf{0}}\left(x_{0 i}\right) \mathrm{e}^{-\Lambda_{\mathbf{k}} t}
$$

where the "overlap-elements" $V_{\mathbf{k l}}\left(x_{i}\right)$ are defined as ${ }^{84}$

$$
V_{\mathbf{k} \mathbf{l}}\left(x_{i}\right)=\frac{m_{\mathbf{l}}}{N_{L} ! N_{R} !} \sum_{\{\mathbf{k}\}} \sum_{\{\mathbf{1}\}} \psi_{k_{i}}^{R}\left(x_{i}\right) \psi_{l_{i}}^{L}\left(x_{i}\right) \prod_{n=1}^{i-1} L_{n}\left(x_{i}\right) \prod_{m=i+1}^{N} R_{m}\left(x_{i}\right),
$$

with $m_{\mathbf{l}}$ being the multiplicity of the multiset $\mathbf{l}$, and $N_{L}=i-1$ and $N_{R}=N-i$ are the number of particles to the left and right of the tagged-particle, respectively. In Eq. (8), we introduced the auxiliary functions

$$
L_{n}(x)=\int_{-\pi}^{x} d z \psi_{l_{n}}^{L}(z) \psi_{k_{n}}^{R}(z), \quad R_{n}(x)=\int_{x}^{\pi} d z \psi_{l_{n}}^{L}(z) \psi_{k_{n}}^{R}(z)
$$

Note that the equilibrium probability density of the tagged-particle's position is given by [see Eq. (7)] $\mathscr{P}_{\text {eq }}\left(x_{i}\right) \equiv \lim _{t \rightarrow \infty} \mathscr{G}\left(x_{i}, t \mid x_{0 i}\right)$ $=V_{00}\left(x_{i}\right)$ and is depicted for various values of $U_{b}$ in Figs. 1(b)1 (d). Intuitively, as $U_{b}$ increases, particles become expelled from the barrier.

In Ref. 84, we have developed an algorithm designed to efficiently cope with the combinatorial complexity of the implementation of the analytical solution in Eq. (7). Due to the piece-wise constant nature of the potential $U(x)$ in Eq. (1), all integrals (9) can be computed analytically. As the resulting expressions are lengthy, we do not show them here. Instead, they are readily implemented in an extension of the code published in Ref. 84 (see the supplementary material). 


\section{LINEAR CORRELATIONS AT EQUILIBRIUM}

First, we consider linear correlations at equilibrium and limit the discussion in the reminder of this paper to tagging the first or last particle (i.e., throughout, we set $i=1$ or $i=N$ ). That is, we are interested in the normalized positional autocorrelation function of a tagged-particle, defined as

$$
C_{i}(t)=\frac{\left\langle x_{i}(t) x_{i}(0)\right\rangle-\left\langle x_{i}\right\rangle^{2}}{\left\langle x_{i}^{2}\right\rangle-\left\langle x_{i}\right\rangle^{2}},
$$

where the covariance of the position is defined as

$$
\left\langle x_{i}(t) x_{i}(0)\right\rangle \equiv \int_{-\pi}^{\pi} d x_{i} \int_{-\pi}^{\pi} d x_{0 i} x_{i} x_{0 i} \mathscr{G}\left(x_{i}, t \mid x_{0 i}\right) \mathscr{P}_{\mathrm{eq}}\left(x_{0 i}\right)
$$

and $\left\langle x_{i}^{n}\right\rangle=\int_{-\pi}^{\pi} d x_{i} x_{i}^{n} \mathscr{P}_{\text {eq }}\left(x_{i}\right)$. The above integrals have been performed numerically by means of Gauss-Kronrod quadrature. ${ }^{85}$ Note that Eq. (10) alongside Eqs. (5)-(9) necessarily implies the structure $C_{i}(t)=\sum_{\mathbf{k} \neq \mathbf{0}} a_{\mathbf{k}} \mathrm{e}^{-\Lambda_{\mathbf{k}} t}$ with $\sum_{\mathbf{k} \neq \mathbf{0}} a_{\mathbf{k}}=1$, where all $a_{\mathbf{k}} \geq 0 .{ }^{86}$ The results for $C_{1}(t)$ as a function of the barrier height $U_{b}$ are depicted in Fig. 2. Since $U(x)$ is symmetric, the autocorrelation functions of the first and last particles coincide, i.e., $C_{1}(t)=C_{N}(t)$.

The autocorrelation of an isolated particle (i.e., $N=1$ ) in Fig. 2(a) displays for a given value of $U_{b}$ to a good approximation an exponential decay with rate $\Lambda_{1}=\lambda_{1}$ given by Eq. (6). This reflects that positional correlations decay predominantly due to barrier-crossing. Conversely, as the number of particles increases, $C_{1}(t)$ decays on multiple timescales [see Fig. 2(b)] and develops an "anomalous" shoulder on shorter timescales ${ }^{78}$ whose span increases with the barrier height $U_{b}$. A comparison of $C_{1}\left(\Lambda_{1}^{-1}\right)$ reveals that the relative decay of correlations from the relaxation time $\tau_{\text {rel }} \equiv \Lambda_{1}^{-1}$ onward is substantially reduced for about a factor of 2 compared to the isolated particle case. $\tau_{\text {rel }}$ denotes the timescale on which the system reaches equilibrium from any initial condition. Note that (i) $C_{1}(t)$ measures relative correlations, and (ii) according to Eq. (7) (terminal), relaxation roughly corresponds to the particles individually crossing the barrier several times. It is also important to note that the natural timescale of a tagged-particle is set by the average collision time ${ }^{35,79} \tau_{\text {col }}=1 / N^{2}$, which decreases with an increase in $N$. That is, in units of the average number of collisions, $t \rightarrow t / \tau_{\text {col }}$ correlations decay more slowly for larger $N$.

A common means to quantify the extent of correlations found in the literature is the so-called correlation time $T_{c},{ }^{25,26,44,87}$ and it should be compared with the actual relaxation time $\tau_{\text {rel }}{ }^{8}$

$$
T_{c}=\int_{0}^{\infty} d t C_{i}(t), \tau_{\text {rel }} \equiv \Lambda_{1}^{-1}=\left(\frac{2}{\pi} \arctan \left(\mathrm{e}^{-U_{b} / 2}\right)\right)^{-2},
$$

where we note that for high barriers, i.e., $U_{B} \gg 1$, the relaxation time follows the expected Arrhenius scaling $\tau_{\text {rel }} \simeq 4 \mathrm{e}^{U_{b}} / \pi^{2}$. In Fig. 3(a), we depict the correlation time for the leftmost particle in units of $\tau_{\mathrm{col}}$ as a function of the barrier height $U_{b}$ for different $N$. For an isolated particle, $T_{c}=T_{c}^{\text {isolated }}$ agrees very well with $\tau_{\text {rel }}$ for all values of $U_{b}$, confirming the idea that $C(t)$ decays to a very good approximation as a single exponential. Note that for systems obeying detailed balance, the mathematical structure of $C_{i}(t)$ trivially implies a shorter correlation time as soon as $C_{i}(t)$ decays on multiple timescales if the longest timescale $\Lambda_{1}^{-1}$ is the same. This is particularly true when comparing $C_{i}(t)$ of a tagged-particle in a single file with an isolated particle. Namely,

$$
T_{c}=\sum_{\mathbf{k} \neq \mathbf{0}} a_{\mathbf{k}} / \Lambda_{\mathbf{k}} \leq \sum_{\mathbf{k} \neq \mathbf{0}} a_{\mathbf{k}} / \Lambda_{\mathbf{1}}=\Lambda_{\mathbf{1}}^{-1} \approx T_{c}^{\text {isolated }} .
$$

Therefore, the interpretation of $T_{c}$ should always be made cautiously and in the particular case of tagged-particle diffusion in a single file is not meaningful if we consider $T_{c}$ on an absolute scale. However, it becomes somewhat more meaningful on the natural timescale, i.e., when time is expressed in terms of the average number of interparticle collisions (see also Ref. 35). Inspecting $C_{1}(t)$ on this natural time scale, we find in Fig. 3(a) that the tagged-particle on average undergoes more collisions before it decorrelates for larger values of $N$, and this number increases with an increase in $U_{b}$.

Moreover, as $N$ increases, the space explored by a taggedparticle becomes progressively more confined ${ }^{35}$ rendering the correlation time $T_{c}$ on an absolute timescale also intuitively shorter. Indeed, in Fig. 3(b), we depict the ratio $T_{c} / \Lambda_{1}^{-1}$, which decreases with an increase in $N$ for any barrier height $U_{b}$. Note that $\Lambda_{1}^{-1}$ is independent of $N$ and the breaking of Markovianity (reflected, e.g., in the violation of the Chapman-Kolmogorov semi-group property ${ }^{35}$ ) is encoded entirely in the overlap elements $V_{\mathbf{0 k}}, V_{\mathbf{k} \mathbf{0}}$. For systems with microscopically reversible dynamics, $T_{c} / \Lambda_{1}^{-1}<1$ quite generally implies that relaxation evolves on multiple timescales. Thus, the results in Fig. 3(b) suggest, in agreement with intuition, that more and more timescales are involved in the relaxation of a taggedparticle's position in equilibrium as we increase $N$. In other words, on the level of linear correlations' signatures of memory of the initial conditions of "latent"/background, particles are reflected in the multi-scale relaxation of $C_{1}(t)$.
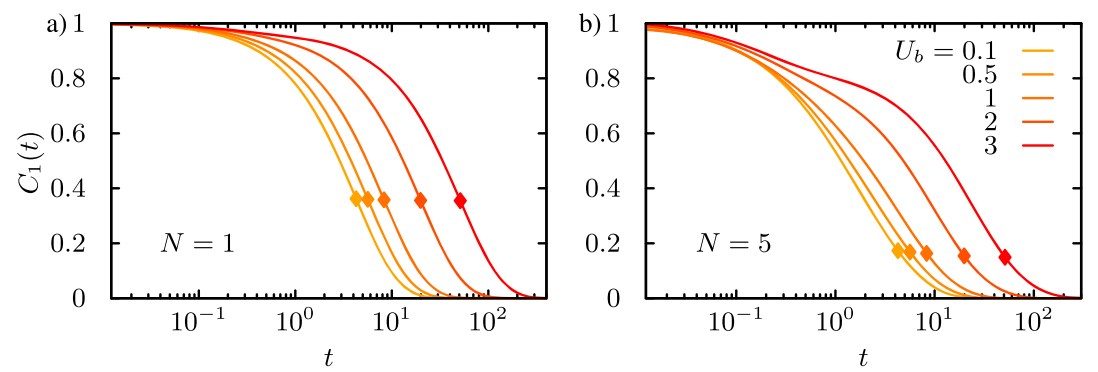

FIG. 2. Position autocorrelation function $\mathrm{C}_{1}(t)$ of an isolated particle (a) and the leftmost tagged-particle in a single file with five particles (b) as a function of the barrier height $U_{b}$. Symbols denote $C_{1}\left(\Lambda_{1}^{-1}\right)$. 

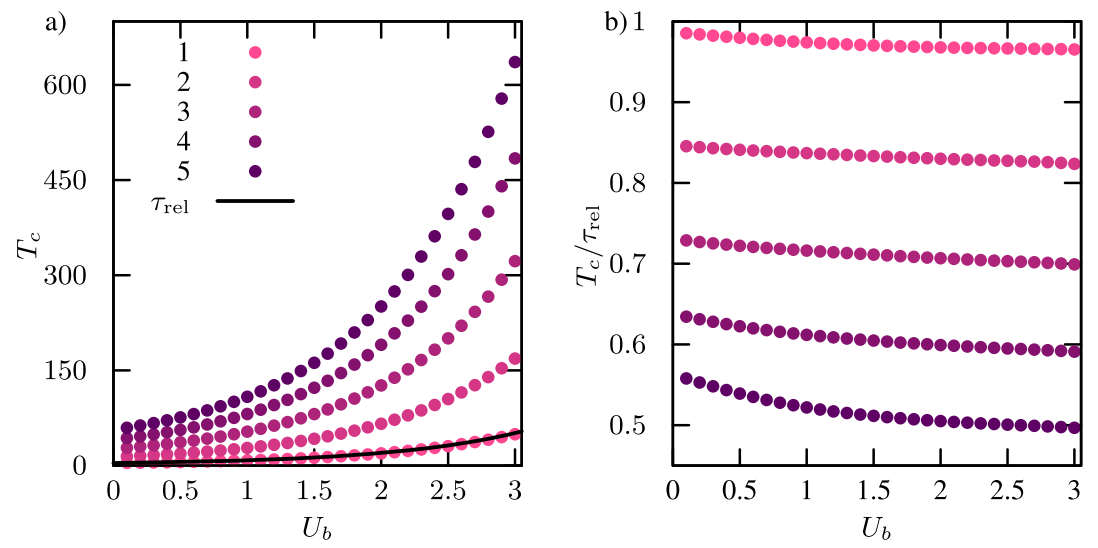

FIG. 3. (a) $T_{c}$ (in units of collision time) for the first particle (i.e., $i=1$ ) as a function of the barrier height $U_{b}$ for various values of $N$. The full line depicts $\tau_{\text {rel }} \equiv \Lambda_{1}^{-1}$ (in absolute time units). (b) Ratio $T_{c} / \tau_{\text {rel }}$ as a function of the barrier height $U_{b}$ for various values of $N$; both $T_{c}$ and $\tau_{\text {rel }}$ are measured in units of collision time.

As shown by Zwanzig from first principles, ${ }^{58,59}$ one can also analyze the memory encoded in $C_{i}(t)$ defined in Eq. (10) in terms of a memory function $K_{U}(t)$ defined through

$$
\frac{d}{d t} C_{i}(t)=-\int_{0}^{t} K_{U}(s) C_{i}(t-s) d s,
$$

where the subscript $U$ is included to stress that the memory kernel depends on the external potential. The kinetic equation (14) obeyed exactly. ${ }^{58,59}$

Note that the memory kernel $K_{U}(t)$ in the linear kinetic equation (14) is not equivalent to the memory kernel entering a nonlinear generalized Langevin equation for a tagged-particle motion in a potential of mean force. ${ }^{48,49,59}$ If, however, one were to compute $C_{i}(t)$ from such a non-linear generalized Langevin equation, this would yield Eq. (14). Here, we aim to connect quantitatively the different signatures of memory encoded in $C_{i}(t)$ and the correlation time $T_{c}$ solely by means of the information encoded in $C_{i}(t)$. Note that this approach is simple and model-free and can therefore directly be used in the analysis of experimental and simulation data. Alternatively, one may equally well use a non-linear framework (for an excellent recent example, see Ref. 89) that, however, requires more effort and a more detailed input.

We determine $K_{U}(s)$ from the Laplace transform of Eq. (14),

$$
\tilde{K}_{U}(u)=\frac{1}{\sum_{\mathbf{k} \neq \mathbf{0}} a_{\mathbf{k}} /\left(\Lambda_{\mathbf{k}}+u\right)}-u,
$$

where $\tilde{f}(u) \equiv \int_{0}^{\infty} \mathrm{e}^{-u t} f(t) d t$. The inverse Laplace transform is, in turn, determined by numerically inverting Eq. (15) using the fixed Talbot method. ${ }^{90}$

By construction, $\int_{0}^{t} K_{U}(s) C_{i}(t-s) d s \geq 0$, and therefore, $K_{U}(t)$ must have a positive contribution at least for $t \rightarrow 0$. For example, if $C_{i}(t)$ decays exactly as a single exponential with rate $\Lambda$, we have $\tilde{K}_{U}(u)=\Lambda$, and hence, $K_{U}(t)=\Lambda \delta(t)$. In fact, one can show by means of Mori's projection-operator formalism that $K_{U}(t)$ has the generic structure $K_{U}(t)=a \delta(t)+\zeta(t)$, where $a>0$ and $\zeta(t)$ is a smooth function of $t$ [see, e.g., Eq. (8.61) in Ref. 59].

More generally, when memory is short-lived, that is, when $K(t)$ decays rapidly compared to the timescale on which $C_{i}(t)$ changes appreciably, we may approximate Eq. (14) as $\frac{d}{d t} C_{i}(t)$ $\approx-\left(\int_{0}^{\infty} K(s) d s\right) C_{i}(t) \equiv-\Lambda C_{i}(t)$, and hence, the autocorrelation decays approximately as a single exponential $C_{i}(t) \approx \mathrm{e}^{-\Lambda t}-$ the system is therefore said to be effectively memoryless (Markovian). ${ }^{5}$

The memory kernel of an isolated particle and that of the leftmost tagged-particle in a single file are depicted in Fig. 4 [note that we depict $-K_{U}(t)>0$, implying an anti-persistent motion]. The unavoidable truncation of the spectral solution (7) does not allow us to determine $K_{U}(t)$ in the limit of $t \rightarrow 0$. In the case of an isolated particle [Fig. 4(a)], the memory is short-lived and decays on a timescale $t \ll \Lambda_{1}^{-1}$ much shorter than the relaxation time and decreases with the barrier height $U_{b}$. This agrees with the essentially single exponential decay of $C_{1}(t)$ found in Fig. 2 and implies that the dynamics is essentially memoryless. ${ }^{58,59}$

Conversely, in the case of a tagged-particle [see Fig. 4(b)], $K_{U}(t)$ displays a strikingly different behavior. First, the range where $K_{U}(t)$
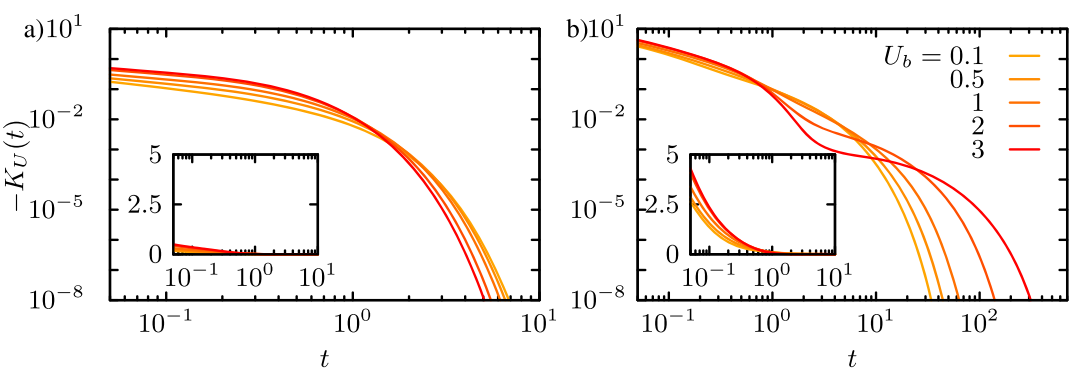

FIG. 4. Memory kernel $-K_{U}(t)$ of an isolated particle (a) and the leftmost taggedparticle in a single file with five particles (b) as a function of the barrier height $U_{b}$ shown on a double logarithmic scale. The inset provides the same results shown on a linear-logarithmic scale. We used 900 pairs $a_{k}, \Lambda_{k}$ to determine $\tilde{K}_{U}(u)$ in Eq. (15). Note that the range of $t$ in (a) and (b) is different. 
considerably differs from zero is orders of magnitude longer and increases with an increase in the barrier height $U_{b}$. Second, the antipersistence of $K_{U}(t)$ is much larger than for an isolated particle, and third, for high barriers, $K_{U}(t)$ develops a shoulder, indicating a transiently stalled decay of memory, presumably due to "jamming" in front of the barrier prior to crossing. The latter acts as a transient entropic trap.

Importantly, $U_{b}$ strongly and non-trivially affects the memory in the tagged-particle's motion [compare with the trivial effect of $U_{b}$ on $K(t)$ for an isolated particle in Fig. 4(a)]. As a result, any microscopically consistent memory kernel $K_{U}(t)$ must depend on the external potential $U(x)$. The reason is twofold: (i) the external potential also acts on the background degrees of freedom and (ii) the coupling of the background degrees of freedom and the taggedparticle's motion is strong. In fact, whenever (i) and/or (ii) hold, the external potential generally alters the memory. Conclusive evidence that the potential affects the memory function has been found, e.g., by atomistic computer simulations of molecular solutes in water.

In contrast to $C_{1}(t)$ depicted in Fig. 2, which on an absolute timescale decays to zero faster for larger $N$ as a result of being normalized and having the same relaxation time $\Lambda_{1}^{-1}$, the memory kernel $K_{U}(t)$ clearly displays long-time memory effects that become more pronounced as $N$ increases. This can be understood by noting that $K_{U}(t)$ in Eq. (14) is unaffected by the normalization. The memory kernel is thus more informative than $C_{1}(t)$ and less ambiguous than correlation times $T_{c}$. Moreover, it is not required that $C_{i}(t)$ is known analytically in order to apply the analysis.

\section{RELAXATION FROM A PINNED CONFIGURATION}

We now focus on the "complete" (i.e., including correlations to all orders) relaxation to equilibrium from a pinned configuration. That is, we are interested in those initial configurations where either the first $(i=1)$ or the last $(i=N)$ particle is pinned at $x_{0}$, while the initial conditions of the remaining particles are drawn from the corresponding pinned equilibria (i.e., those equilibrium many-body configurations where the first/last particle is located at $x_{0}$ ). In this non-stationary setting, the analysis of memory kernels seems less sensible since these would depend explicitly on time and $x_{0}$.

We quantify the relaxation dynamics by means of $\mathscr{D}\left(t, x_{0 i}\right)$, the Kullback-Leibler divergence ${ }^{92}$ between the non-Markovian probability density of the tagged-particle's position at time $t$, $\mathscr{G}\left(x_{i}, t \mid x_{0 i}\right)$ in Eq. $(7)$, and the respective equilibrium density $\mathscr{P}_{\text {eq }}\left(x_{i}\right)$ $\equiv \lim _{t \rightarrow \infty} \mathscr{G}\left(x_{i}, t \mid x_{0 i}\right)$,

$$
\mathscr{D}\left(t, x_{0 i}\right) \equiv \int_{-\pi}^{\pi} d x \mathscr{G}\left(x, t \mid x_{0 i}\right) \ln \left(\frac{\mathscr{G}\left(x, t \mid x_{0 i}\right)}{\mathscr{P}_{\mathrm{eq}}(x)}\right)
$$

In physical terms, $\mathscr{D}\left(t, x_{0 i}\right)$ represents the displacement from equilibrium in the sense of an excess instantaneous free energy, i.e., $k_{\mathrm{B}} T \mathscr{D}\left(t, x_{0 i}\right)=F(t)-F .^{93-95}$ Since the integral in Eq. (16) cannot be performed analytically, we evaluate it numerically. We always pin the initial position of the tagged-particle at $x_{0}=-2$. According to the effect of the pinning on the relaxation of the taggedparticle, the scenario in which we tag the first particle is referred to as "confining" (since background particles obstruct the relaxation of the tagged-particle) and the one in which we tag the first particle as "pushing" (since background particles exert an entropic force pushing the tagged-particle over the barrier). $\mathscr{D}\left(t, x_{0 i}\right)$ as a function of the barrier height $U_{b}$ for $N=5$ and $N=9$ is shown in Fig. 5 .

Note that $\lim _{t \rightarrow 0} \mathscr{D}\left(t, x_{0 i}\right)=\infty$ irrespective of $N$ and $U_{b}$ since we are comparing a delta distribution with a smooth probability density. Conversely, in an arbitrarily small time interval $\tau_{\varepsilon}>0$, the nonMarkovian tagged-particle density $\mathscr{G}\left(x, t \mid x_{0 i}\right)$ evolves to a smooth, well-behaved probability density such that $\mathscr{D}\left(t>0, x_{0 i}\right)$ is always finite and the "pathology" at $t=0$ is mathematical and not physical.
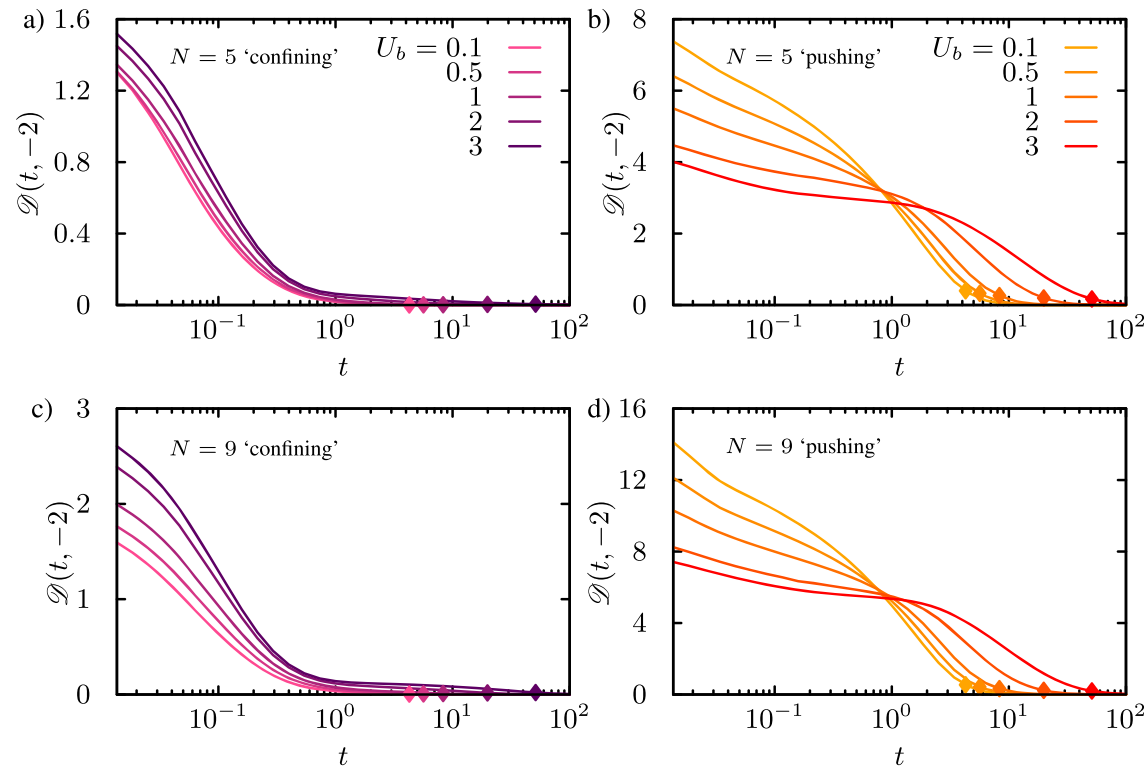

FIG. 5. Time evolution of $\mathscr{D}\left(t, x_{0 i}\right)$ for various barrier heights $U_{b}$ for $N=5[(a)$ : confining and (b): pushing] and $N=9$

[(c): confining and (d): pushing], respectively. The symbols denote $\mathscr{D}\left(\Lambda_{1}^{-1}, x_{0 i}\right)$. 
With this in mind, we observe in Fig. 5 a striking difference between the "confining" and "pushing" scenarios. In the "confining" setting, $\mathscr{D}\left(t, x_{01}\right)$ at a fixed time $t$ is a monotonically increasing function of $U_{b}$ and as a function of time decays on a timescale that seems to be rather independent of $U_{b}$. In the "confining" scenario, an increase in $U_{b}$ displaces the system at $t=0^{+}$further from equilibrium. This is intuitive because $\mathscr{P}_{\text {eq }}\left(x_{1}\right)$ becomes more strongly confined to the boundary and hence away from $x_{0}$. To a dominant extent, relaxation occurs already on timescales $t \gtrsim 1 \ll \Lambda_{1}^{-1}$. The reason may be found in the fact that $\Lambda_{1}^{-1}$ corresponds to the mixing/ergodic timescale on which the full single file (and thus the tagged-particle) explores the entire system. In the "confining" scenario, the background particles are drawn from a distribution that resembles closely the unconstrained equilibrium, and in addition, the tagged-particle is nominally unlikely to be found in the right well in equilibrium. Therefore, the fraction of paths that cross the barrier in the ensemble of relaxation paths is small, rendering $V_{\mathbf{0 k}} V_{\mathbf{k} 0}$ for low-lying $\mathbf{k}$ essentially negligible [see Eq. (7)]. Nevertheless, a second, slower relaxation stage is still discernible at $t \gtrsim 1$.

Conversely, in the "pushing" scenario depicted in Figs. 5(b) and 5(d), we find (i) the dependence of $\mathscr{D}\left(0^{+}, x_{01}\right)$ on $U_{b}$ to be inverted, and (ii) for given $N$ and $U_{b}$, relaxation extends to much longer timescales compared to the "confining" scenario. In order to rationalize (i), we consider a pair of barriers $U_{b_{1}}, U_{b_{2}}$ and take the limit

$$
\lim _{t \rightarrow 0}\left(\mathscr{D}^{b_{1}}\left(t, x_{0}\right)-\mathscr{D}^{b_{2}}\left(t, x_{0}\right)\right)=\ln \left(\mathscr{P}_{\mathrm{eq}}^{b_{2}}\left(x_{0}\right) / \mathscr{P}_{\mathrm{eq}}^{b_{1}}\left(x_{0}\right)\right)
$$

which is finite and well defined despite the fact that $\lim _{t \rightarrow 0} \mathscr{D}^{b_{1}, b_{2}}$ $\left(t, x_{0}\right)$ are infinite. Equation (17) explains that the dependence of $\mathscr{D}\left(0^{+}, x_{0}\right)$ on $U_{b}$ is not unique and depends on the pinning point $x_{0}$, which determines whether or not $\mathscr{P}_{\text {eq }}^{b_{2}}\left(x_{0}\right) / \mathscr{P}_{\text {eq }}^{b_{1}}\left(x_{0}\right)$ is greater or smaller than 1 [see Figs. 1(b) and 1(d)]. (ii) can be understood by an extension of the argument put forward in the discussion of the "confining" scenario, i.e., as a result of the pinning, the initial configurations of the background particles are displaced much further away from equilibrium, rendering $V_{\mathbf{0 k}} V_{\mathbf{k} \mathbf{0}}$ for low-lying $\mathbf{k}$ substantial [see Eq. (7)]. Therefore, a pronounced second relaxation stage is visible at longer times $t \gtrsim 1$.

Based on Fig. 5 alone we are not able to deduce whether these observations are a trivial consequence of the pinning in the sense that they have nothing to do with memory (note that a Markov process "remembers" the initial condition up to $\sim \tau_{\text {rel }}$ ) or whether they are, in fact, a signature of memory in the dynamics. Additional insight is gained by inspecting the relaxation of the full, Markovian single file evolving from the same initial condition, i.e.,

$$
\mathscr{D}_{M}\left(t, x_{0}\right) \equiv\left[\prod_{i=1}^{N} \int_{-\pi}^{\pi} d x_{i}\right] G\left(\mathbf{x}, t, P_{0}\right) \ln \left(\frac{G\left(\mathbf{x}, t, P_{0}\right)}{P_{\mathrm{eq}}(\mathbf{x})}\right)
$$

where we have introduced the joint Markovian two-point probability density $G\left(\mathbf{x}, t, P_{0}\right) \equiv \int d \mathbf{y}_{0} G\left(\mathbf{x}, t \mid \mathbf{y}_{0}\right) P_{0}\left(\mathbf{y}_{0}\right)$, and whereby, for $-\pi<x_{0}<-\pi / 2, P_{0}(y)$ is defined as

$$
P_{0}(\mathbf{x})=N !\left(\pi+x_{0}\right)^{-N_{L}} \hat{O}_{\mathbf{x}} \delta\left(x_{i}-x_{0}\right) \frac{\mathrm{e}^{-U_{b} \sum_{j=i+1}^{N} \theta\left(\pi / 2-\left|x_{j}\right|\right)}}{\left(\pi \mathrm{e}^{-U_{b}}-x_{0}\right)^{N_{R}}},
$$

where $\theta(x)$ is the Heaviside step function and $P_{\text {eq }}(\mathbf{x})$ $=\lim _{t \rightarrow \infty} G\left(\mathbf{x}, t \mid \mathbf{x}_{0}\right)$. The integration in Eq. (18) can be performed analytically (for details, see Ref. 95). Introducing the two-point joint density of the single-particle problem $\Gamma_{t}(x, a, b)$ $\equiv \sum_{k} \psi_{k}^{R}(x)\left[\int_{a}^{b} d y \psi_{k}^{L}(y) P_{0}(y)\right] \mathrm{e}^{-\lambda_{k} t}$, with $P_{0}(y) \equiv \theta(-\pi / 2-y) /$ $\left(\pi+x_{0}\right)+\theta(y+\pi / 2) \mathrm{e}^{-U(y)} /\left(\pi \mathrm{e}^{-U_{b}}-x_{0}\right)$ and the auxiliary function

$$
\Xi_{t}(a, b)=\int_{a}^{b} d x \Gamma_{t}(x, a, b) \ln \left(\Gamma_{t}(x, a, b) / P_{\mathrm{eq}}(x)\right),
$$

where $P_{\mathrm{eq}}(x)=\mathrm{e}^{-U(x)} / \pi\left(1+\mathrm{e}^{-U_{b}}\right)$, the result reads

$$
\mathscr{D}_{M}\left(t, x_{0}\right)=\Xi_{t}(-\pi, \pi) \Xi_{t}\left(-\pi, x_{0}\right)^{N_{L}} \Xi_{t}\left(x_{0}, \pi\right)^{N_{R}} .
$$

An explicit solution is obtained with the aid of Mathematica. ${ }^{96}$ As it is bulky, but straightforward, we do not show it here. The Markovian result in Eq. (21) for the same set of parameters, as in Figs. 5(a) and 5(b), is depicted in Fig. 6. A comparison of Figs. 5 and 6 reveals that the second, long-time relaxation stage observed in the "pushing" scenario of Fig. 5 is absent in the Markovian setting (compare Figs. 5 and 6 , and note that the relaxation time $\Lambda_{1}^{-1}$ is identical in both settings). This, in turn, implies that the pronounced second relaxation stage in the non-Markovian, tagged-particle scenario at times $t \gtrsim 1$ is, indeed, a signature of memory.
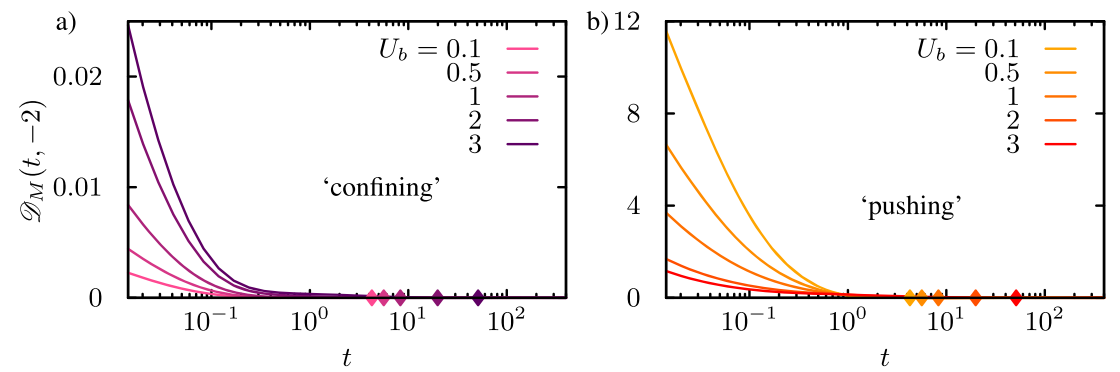

FIG. 6. Time evolution of $\mathscr{D}_{M}\left(t, x_{0 i}\right)$ for various barrier-heights $U_{b}$ for $N=5[(a)$ : confining, $i=1$ and (b): pushing, $i=N]$ The symbols denote $\mathscr{D}_{M}\left(\Lambda_{1}^{-1}, x_{0 i}\right)$. 


\section{DISCUSSION}

We identified pronounced signatures of memory in the overdamped relaxation of a tagged-particle in a single file confined to a bi-stable potential. On the level of linear correlations in equilibrium, memory is visible in the form of a multi-scale relaxation of the autocorrelation function (see Fig. 2), a substantial and long-ranged (linear) memory kernel [see Fig. 4(b)], and a seemingly paradoxical shortening of the so-called correlation time $T_{c}$ (see Fig. 3). The latter was shown to be an artifact of the definition of $T_{c}$. When including the complete correlation-structure as encoded in the so-called excess instantaneous free energy [see Eq. (16)], distinctive signatures of memory emerge in the form of a second, late-time relaxation regime.

The memory originates from the fact that the entire single file relaxes to equilibrium in the form of linearly independent manybody eigenmodes, which become projected onto the motion of a tagged-particle. ${ }^{35,79}$ The projection couples distinct modes, thus breaking Markovianity and giving rise to memory. ${ }^{35}$ It turns out to be very important which particle is tagged. Here, we were only interested in the "confining" (all background particles in front of the tagged-particle) and "pushing" (all background particles behind the tagged-particle) scenarios and found qualitatively different relaxation behaviors. A systematic analysis would be required to understand the intricate details on how the number of particles on each side affects relaxation dynamics, which is beyond the scope of the present work.

We have shown that the memory non-trivially depends on the external potential. That is, a tagged-particle was shown to experience the external potential directly (i.e., time-locally) and indirectly through the effect it exerts on the memory kernel. This effect is general-it occurs whenever the potential is sufficiently strong and also acts on either the latent degrees of freedom (i.e., those that are integrated out) or the interaction between the tagged-particle and the latent degrees of freedom that is not harmonic or, more generally, non-negligible. Direct evidence for the effect has been found in all-atom computer simulations of the hydration of molecular solutes. ${ }^{91}$ It is important to keep this in mind when applying generalized Langevin equations (GLEs) with phenomenological memory kernels or microscopically consistent GLEs "decorated" with an external potential as these may lead to erroneous conclusions or misinterpretations.

The application of the methodology for extracting and analyzing memory in tagged-particle dynamics put forward in Eqs. (10), (14), and (16) requires neither $C_{i}(t)$ nor $\mathscr{G}\left(x, t \mid x_{0 i}\right)$ to be known analytically. The quantities can be equally well determined from experiments or computer simulations. The analysis is expected to provide insight into memory effects as long as either the "crowding" (i.e., the concentration of background particles) and/or the barrier-height can be controlled. The qualitative features of the signatures of memory are expected to be preserved in most systems of effectively one-dimensional systems with obstructed tagged-particle dynamics. The proposed analysis of memory effects can be viewed as complementary to the analysis of anomalies in tagged-particle diffusion. ${ }^{97-99}$

Our results can readily be tested by existing experiments probing colloidal particle systems (see, e.g., Refs. 100-102) and may, furthermore, be relevant for a theoretical description of transport in ion-channels. ${ }^{103-106}$ Our results can be extended in diverse ways, most immediately by including other types of inter-particle interactions $^{81}$ and time-dependent energy barriers. ${ }^{107}$

\section{SUPPLEMENTARY MATERIAL}

See the supplementary material for the extension of the code published in Ref. 84 that implements the analytical results presented this article.

\section{ACKNOWLEDGMENTS}

The financial support from the German Research Foundation (DFG) through the Emmy Noether Program [Grant No. GO 2762/1-1 (to A.G.)] is gratefully acknowledged. The authors thank Kristian Blom for fruitful discussions.

\section{APPENDIX: SINGLE PARTICLE EIGENSPECTRUM}

In this appendix, we give explicit expressions for the singleparticle eigenfunctions that are required in the diagonalization of the many-body Fokker-Planck operator using the so-called coordinate Bethe ansatz. For details of the solution method, see Refs. 35, 79 , and 84 . The eigenfunctions of the corresponding single-particle eigenvalue problem are

$$
\begin{aligned}
& \left(\partial_{x}^{2}+\partial_{x}\left\{\partial_{x} U(x)\right\}\right) \psi_{k}^{R}(x)=-\lambda_{k} \psi_{k}^{R}(x), \\
& \left(\partial_{x}^{2}-\left\{\partial_{x} U(x)\right\} \partial_{x}\right) \psi_{k}^{L}(x)=-\lambda_{k} \psi_{k}^{L}(x),
\end{aligned}
$$

where $\psi_{k}^{L, R}(x):[-\pi, \pi] \rightarrow \mathbb{R}$ allow for a spectral decomposition of the single-particle Green's function,

$$
\Gamma\left(x, t \mid x_{0}\right)=\sum_{k} \psi_{k}^{R}(x) \psi_{k}^{L}\left(x_{0}\right) \mathrm{e}^{-\lambda_{k} t}
$$

$\psi_{k}^{L, R}(x)$ enter Eq. (5) and are here defined via their "Hermitianized" counterpart $\psi_{k}(x):[-\pi, \pi] \rightarrow \mathbb{R}$ as $\psi_{k}^{R}(x)=\mathrm{e}^{-U(x) / 2} \psi_{k}(x)$ and $\psi_{k}^{L}(x)=\mathrm{e}^{U(x) / 2} \psi_{k}(x)$, where ${ }^{108}$

$$
\psi_{k}(x)=\sqrt{\frac{2}{1+\delta_{k, 0}}} \frac{\mathrm{e}^{-U(x) / 2}}{\sqrt{\pi\left(1+\mathrm{e}^{-f 0}\right)}} \cos \left(\sqrt{\lambda_{k}} x\right), \quad \bmod (k, 4)=0,
$$

$$
\psi_{k}(x)= \begin{cases}-\frac{\cos \left(\sqrt{\lambda_{k}}(x+\pi)\right)}{\sqrt{\pi}}, & x<-\pi / 2 \\ \frac{\sin \left(\sqrt{\lambda_{k}} x\right)}{\sqrt{\pi}}, & |x| \leq \pi / 2, \quad \bmod (k, 4)=1, \\ \frac{\cos \left(\sqrt{\lambda_{k}}(x-\pi)\right)}{\sqrt{\pi}}, & x>\pi / 2\end{cases}
$$

$$
\psi_{k}(x)=\sqrt{2} \frac{\mathrm{e}^{U(x) / 2}}{\sqrt{\pi\left(1+\mathrm{e}^{f 0}\right)}} \cos \left(\sqrt{\lambda_{k}} x\right), \quad \bmod (k, 4)=2,
$$




$$
\psi_{k}(x)= \begin{cases}\frac{\cos \left(\sqrt{\lambda_{k}}(x+\pi)\right)}{\sqrt{\pi}}, & x<-\pi / 2 \\ \frac{\sin \left(\sqrt{\lambda_{k}} x\right)}{\sqrt{\pi}}, & |x| \leq \pi / 2, \quad \bmod (k, 4)=3, \\ -\frac{\cos \left(\sqrt{\lambda_{k}}(x-\pi)\right)}{\sqrt{\pi}}, & x>\pi / 2\end{cases}
$$

where $\delta_{k, 0}$ is the Kronecker delta.

\section{DATA AVAILABILITY}

The data that support the findings of this study are available from the corresponding author upon reasonable request.

\section{REFERENCES}

${ }^{1}$ H. A. Kramers, "Brownian motion in a field of force and the diffusion model of chemical reactions," Physica 7(4), 284-304 (1940).

${ }^{2}$ A. Nitzan, P. Ortoleva, J. Deutch, and J. Ross, "Fluctuations and transitions at chemical instabilities: The analogy to phase transitions," J. Chem. Phys. 61(3), 1056-1074 (1974)

${ }^{3}$ F. Schlögl, "Chemical reaction models for non-equilibrium phase transitions," Z. Phys. 253(2), 147-161 (1972).

${ }^{4}$ I. Matheson, D. F. Walls, and C. W. Gardiner, "Stochastic models of firstorder nonequilibrium phase transitions in chemical reactions," J. Stat. Phys. 12(1), 21 34 (1975)

${ }^{5} \mathrm{R}$. Landauer, "The role noise in negative resistance circuits," J. Phys. Soc. Jpn. 41(2), 695-696 (1976).

${ }^{6}$ H. Risken, "Distribution- and correlation-functions for a laser amplitude," Z. Phys. 186(1), 85-98 (1965).

${ }^{7}$ R. Kubo, K. Matsuo, and K. Kitahara, "Fluctuation and relaxation of macrovariables,” J. Stat. Phys. 9(1), 51-96 (1973).

${ }^{8}$ H. Lannon, J. S. Haghpanah, J. K. Montclare, E. Vanden-Eijnden, and J. Brujic, "Force-clamp experiments reveal the free-energy profile and diffusion coefficient of the collapse of protein molecules," Phys. Rev. Lett. 110(12), 128301 (2013).

${ }^{9}$ H. S. Chung and W. A. Eaton, "Protein folding transition path times from single molecule FRET," Curr. Opin. Struct. Biol. 48, 30-39 (2018).

${ }^{10}$ H. Yu, A. N. Gupta, X. Liu, K. Neupane, A. M. Brigley, I. Sosova, and M. T. Woodside, "Energy landscape analysis of native folding of the prion protein yields the diffusion constant, transition path time, and rates," Proc. Natl. Acad. Sci. U. S. A. 109(36), 14452-14457 (2012).

${ }^{11}$ A. P. Manuel, J. Lambert, and M. T. Woodside, "Reconstructing folding energy landscapes from splitting probability analysis of single-molecule trajectories," Proc. Natl. Acad. Sci. U. S. A. 112(23), 7183-7188 (2015).

${ }^{12}$ K. Truex, H. S. Chung, J. M. Louis, and W. A. Eaton, "Testing landscape theory for biomolecular processes with single molecule fluorescence spectroscopy," Phys. Rev. Lett. 115(1), 018101 (2015).

${ }^{13}$ H. S. Chung, K. McHale, J. M. Louis, and W. A. Eaton, "Single-molecule fluorescence experiments determine protein folding transition path times," Science 335(6071), 981-984 (2012).

${ }^{14}$ D. de Sancho, A. Sirur, and R. B. Best, "Molecular origins of internal friction effects on protein-folding rates," Nat. Commun. 5(1), 4307 (2014).

${ }^{15} \mathrm{R}$. Best and G. Hummer, "Diffusive model of protein folding dynamics with Kramers turnover in rate," Phys. Rev. Lett. 96(22), 228104 (2006).

${ }^{16}$ J. Kappler, J. O. Daldrop, F. N. Brünig, M. D. Boehle, and R. R. Netz, "Memoryinduced acceleration and slowdown of barrier crossing," J. Chem. Phys. 148(1), 014903 (2018).

${ }^{17}$ K. Neupane, A. P. Manuel, J. Lambert, and M. T. Woodside, "Transition-path probability as a test of reaction-coordinate quality reveals DNA hairpin folding is a one-dimensional diffusive process," J. Phys. Chem. Lett. 6(6), 1005-1010 (2015).

${ }^{18}$ K. Neupane, D. A. N. Foster, D. R. Dee, H. Yu, F. Wang, and M. T. Woodside, "Direct observation of transition paths during the folding of proteins and nucleic acids,” Science 352(6282), 239-242 (2016).

${ }^{19}$ F. Rico, A. Russek, L. González, H. Grubmüller, and S. Scheuring, "Heterogeneous and rate-dependent streptavidin-biotin unbinding revealed by high-speed force spectroscopy and atomistic simulations," Proc. Natl. Acad. Sci. U. S. A. 116(14), 6594-6601 (2019).

${ }^{20}$ N. G. van Kampen, “A soluble model for diffusion in a bistable potential," J. Stat. Phys. 17(2), 71-88 (1977).

${ }^{21}$ B. Caroli, C. Caroli, and B. Roulet, "Diffusion in a bistable potential: A systematic WKB treatment," J. Stat. Phys. 21(4), 415-437 (1979).

${ }^{22}$ Y. Saito, "Relaxation in a bistable system," J. Phys. Soc. Jpn. 41(2), 388-393 (1976).

${ }^{23}$ A. Bunde and J.-F. Gouyet, "Brownian motion in the bistable potential at intermediate and high friction: Relaxation from the instability point," Physica A 132(2), 357-374 (1985).

${ }^{24}$ P. Hanggi, H. Grabert, P. Talkner, and H. Thomas, "Bistable systems: Master equation versus Fokker-Planck modeling,” Phys. Rev. A 29, 371-378 (1984).

${ }^{25}$ A. Perico, R. Pratolongo, K. F. Freed, R. W. Pastor, and A. Szabo, "Positional time correlation function for one-dimensional systems with barrier crossing: Memory function corrections to the optimized Rouse-Zimm approximation," J. Chem. Phys. 98(1), 564-573 (1993).

${ }^{26}$ A. Perico, R. Pratolongo, K. F. Freed, and A. Szabo, "Torsional time correlation function for one-dimensional systems with barrier crossing: Periodic potential," J. Chem. Phys. 101(3), 2554-2561 (1994).

${ }^{27}$ P. Hänggi, P. Talkner, and M. Borkovec, "Reaction-rate theory: Fifty years after Kramers,” Rev. Mod. Phys. 62(2), 251-341 (1990).

${ }^{28}$ B. U. Felderhof, "Escape by diffusion from a square well across a square barrier," Physica A 387(1), 39-56 (2008).

${ }^{29} \mathrm{~V}$. Berdichevsky and M. Gitterman, "One-dimensional diffusion through single- and double-square barriers,” J. Phys. A: Math. Gen. 29(8), 1567-1580 (1996).

${ }^{30} \mathrm{~B}$. Jun and D. L. Weaver, "One-dimensional potential barrier model of protein folding with intermediates," J. Chem. Phys. 116(1), 418 (2002).

${ }^{31}$ G. R. Fleming, S. H. Courtney, and M. W. Balk, "Activated barrier crossing: Comparison of experiment and theory," J. Stat. Phys. 42(1-2), 83-104 (1986).

${ }^{32}$ A. M. Berezhkovskii and D. E. Makarov, "Communication: Transition-path velocity as an experimental measure of barrier crossing dynamics," J. Chem. Phys. 148(20), 201102 (2018).

${ }^{33}$ D. Hartich and A. Godec, "Duality between relaxation and first passage in reversible Markov dynamics: Rugged energy landscapes disentangled," New J. Phys. 20(11), 112002 (2018).

${ }^{34}$ D. Hartich and A. Godec, "Interlacing relaxation and first-passage phenomena in reversible discrete and continuous space Markovian dynamics," J. Stat. Mech. Theory Exp. 2019(2), 024002.

${ }^{35}$ A. Lapolla and A. Godec, "Manifestations of projection-induced memory: General theory and the tilted single file," Front. Phys. 7, 182 (2019).

${ }^{36}$ N. G. van Kampen, "Remarks on non-Markov processes," Braz. J. Phys. 28(2), 90-96 (1998)

${ }^{37}$ H. Peter and F. Mojtabai, "Thermally activated escape rate in presence of longtime memory," Phys. Rev. A 26(2), 1168-1170 (1982)

${ }^{38}$ S. Okuyama and D. W. Oxtoby, "The generalized Smoluchowski equation and non-Markovian dynamics," J. Chem. Phys. 84(10), 5824-5829 (1986).

${ }^{39}$ S. Okuyama and D. W. Oxtoby, "Non-Markovian dynamics and barrier crossing rates at high viscosity," J. Chem. Phys. 84(10), 5830-5835 (1986).

${ }^{40}$ B. Carmeli and A. Nitzan, "Non-Markoffian theory of activated rate processes," Phys. Rev. Lett. 49(7), 423-426 (1982).

${ }^{41}$ B. Carmeli and A. Nitzan, "Non-Markovian theory of activated rate processes. I. Formalism," J. Chem. Phys. 79(1), 393-404 (1983).

${ }^{42}$ B. Carmeli and A. Nitzan, "Non-Markovian theory of activated rate processes. IV. The double well model,” J. Chem. Phys. 80(8), 3596-3605 (1984). 
${ }^{43}$ V. I. Mel'nikov and S. V. Meshkov, "Theory of activated rate processes: Exact solution of the Kramers problem," J. Chem. Phys. 85(2), 1018-1027 (1986).

${ }^{44}$ Y. P. Kalmykov, W. T. Coffey, and S. V. Titov, "Thermally activated escape rate for a Brownian particle in a double-well potential for all values of the dissipation," J. Chem. Phys. 124(2), 024107 (2006).

${ }^{45}$ R. F. Grote and J. T. Hynes, "The stable states picture of chemical reactions. II. Rate constants for condensed and gas phase reaction models," J. Chem. Phys, 73(6), 2715-2732 (1980)

${ }^{46} \mathrm{D}$. Chandler, "Statistical mechanics of isomerization dynamics in liquids and the transition state approximation," J. Chem. Phys. 68(6), 2959 (1978).

${ }^{47}$ R. Chakrabarti, "Exact analytical evaluation of time dependent transmission coefficient from the method of reactive flux for an inverted parabolic barrier," J. Chem. Phys. 126(13), 134106 (2007).

${ }^{48}$ R. Satija and D. E. Makarov, "Generalized Langevin equation as a model for barrier crossing dynamics in biomolecular folding," J. Phys. Chem. B 123(4), 802-810 (2019).

${ }^{49}$ D. E. Makarov, "Interplay of non-Markov and internal friction effects in the barrier crossing kinetics of biopolymers: Insights from an analytically solvable model," J. Chem. Phys. 138(1), 014102 (2013).

${ }^{50}$ J. Kappler, V. B. Hinrichsen, and R. R. Netz, "Non-Markovian barrier crossing with two-time-scale memory is dominated by the faster memory component," Eur. Phys. J. E 42(9), 119 (2019).

${ }^{51}$ S. L. Seyler and S. Pressé, "Surmounting potential barriers: Hydrodynamic memory hedges against thermal fluctuations in particle transport," J. Chem. Phys. 153(4), 041102 (2020)

${ }^{52}$ A. G. T. Pyo and M. T. Woodside, "Memory effects in single-molecule force spectroscopy measurements of biomolecular folding," Phys. Chem. Chem. Phys. 21(44), 24527-24534 (2019).

${ }^{53}$ P. Hänggi, F. Marchesoni, and P. Grigolini, "Bistable flow driven by coloured Gaussian noise: A critical study," Z. Phys. B: Condens. Matter 56(4), 333-339 (1984).

${ }^{54} \mathrm{P}$. Hänggi, "Path integral solutions for non-Markovian processes," Z. Phys. B: Condens. Matter 75(2), 275-281 (1989).

${ }^{55}$ R. F. Fox, "Functional-calculus approach to stochastic differential equations," Phys. Rev. A 33, 467-476 (1986).

${ }^{56}$ C. R. Doering, P. S. Hagan, and C. D. Levermore, "Bistability driven by weakly colored Gaussian noise: The Fokker-Planck boundary layer and mean first-passage times," Phys. Rev. Lett. 59, 2129-2132 (1987).

${ }^{57}$ J. Casademunt, R. Mannella, P. V. E. McClintock, F. E. Moss, and J. M. Sancho, "Relaxation times of non-Markovian processes," Phys. Rev. A 35, 51835190 (1987).

${ }^{58}$ R. Zwanzig, "Statistical mechanics of irreversibility," in Lectures in Theoretical Physiscs (Wiley, New York, 1961), p. 106.

${ }^{59}$ R. Zwanzig, Nonequilibrium Statistical Mechanics (Oxford University Press, 2004).

${ }^{60}$ G. Hummer, J. C. Rasaiah, and J. P. Noworyta, "Water conduction through the hydrophobic channel of a carbon nanotube," Nature 414(6860), 188-190 (2001).

${ }^{61}$ T. Chou and D. Lohse, "Entropy-driven pumping in zeolites and biological channels," Phys. Rev. Lett. 82(17), 3552-3555 (1999).

${ }^{62}$ S. Ahlberg, T. Ambjörnsson, and L. Lizana, "Many-body effects on tracer particle diffusion with applications for single-protein dynamics on DNA," New J. Phys. 17(4), 043036 (2015).

${ }^{63}$ G.-W. Li, O. G. Berg, and J. Elf, "Effects of macromolecular crowding and DNA looping on gene regulation kinetics," Nat. Phys. 5(4), 294-297 (2009).

${ }^{64}$ P. M. Richards, "Theory of one-dimensional hopping conductivity and diffusion,” Phys. Rev. B 16(4), 1393-1409 (1977).

${ }^{65}$ A. Taloni, O. Flomenbom, R. Castañeda-Priego, and F. Marchesoni, "Single file dynamics in soft materials," Soft Matter 13(6), 1096-1106 (2017).

${ }^{66}$ C. Lutz, M. Kollmann, and C. Bechinger, "Single-file diffusion of colloids in one-dimensional channels,” Phys. Rev. Lett. 93, 026001 (2004).

${ }^{67}$ B. Lin, M. Meron, B. Cui, S. A. Rice, and H. Diamant, "From random walk to single-file diffusion,” Phys. Rev. Lett. 94, 216001 (2005).
${ }^{68}$ E. Locatelli, M. Pierno, F. Baldovin, E. Orlandini, Y. Tan, and S. Pagliara, "Single-file escape of colloidal particles from microfluidic channels," Phys. Rev. Lett. 117, 038001 (2016).

${ }^{69}$ T. E. Harris, “Diffusion with 'collisions' between particles," J. Appl. Probab. 2(2), 323-338 (1965).

${ }^{70} \mathrm{D}$. W. Jepsen, “Dynamics of a simple many-body system of hard rods," J. Math. Phys. 6(3), 405-413 (1965).

${ }^{71}$ L. Lizana and T. Ambjörnsson, "Single-file diffusion in a box," Phys. Rev. Lett. 100(20), 200601 (2008).

${ }^{72}$ L. Lizana and T. Ambjörnsson, "Diffusion of finite-sized hard-core interacting particles in a one-dimensional box: Tagged particle dynamics," Phys. Rev. E 80(5), 051103 (2009).

${ }^{73}$ E. Barkai and R. Silbey, "Theory of single file diffusion in a force field," Phys. Rev. Lett. 102(5), 050602 (2009).

${ }^{74}$ E. Barkai and R. Silbey, "Diffusion of tagged particle in an exclusion process," Phys. Rev. E 81(4), 041129 (2010).

${ }^{75} \mathrm{~N}$. Leibovich and E. Barkai, "Everlasting effect of initial conditions on single-file diffusion,” Phys. Rev. E 88(3), 032107 (2013).

${ }^{76} \mathrm{O}$. Flomenbom and A. Taloni, "On single-file and less dense processes," Europhys. Lett. 83(2), 20004 (2008).

${ }^{77}$ R. Metzler, L. Sanders, M. A. Lomholt, L. Lizana, K. Fogelmark, and T. Ambjörnsson, “Ageing single file motion,” Eur. Phys. J.: Spec. Top. 223(14), 3287-3293 (2014).

${ }^{78}$ L. Lizana, T. Ambjörnsson, A. Taloni, E. Barkai, and M. A. Lomholt, "Foundation of fractional Langevin equation: Harmonization of a many-body problem," Phys. Rev. E 81(5), 051118 (2010).

${ }^{79}$ A. Lapolla and A. Godec, "Unfolding tagged particle histories in single-file diffusion: Exact single- and two-tag local times beyond large deviation theory," New J. Phys. 20(11), 113021 (2018).

${ }^{80}$ S. D. Goldt and E. M. Terentjev, "Role of the potential landscape on the single-file diffusion through channels," J. Chem. Phys. 141(22), 224901 (2014).

${ }^{81}$ M. Kollmann, "Single-file diffusion of atomic and colloidal systems: Asymptotic laws," Phys. Rev. Lett. 90, 180602 (2003).

${ }^{82} \mathrm{M}$. Morsch, H. Risken, and H. D. Vollmer, "One-dimensional diffusion in soluble model potentials,” Z. Phys. B: Condens. Matter Quanta 32(2), 245-252 (1979).

${ }^{83}$ H. Risken and T. Frank, The Fokker-Planck Equation: Methods of Solution and Applications, Springer Series in Synergetics, 2nd ed. (Springer-Verlag, Berlin, Heidelberg, 1996).

${ }^{84}$ A. Lapolla and A. Godec, "BetheSF: Efficient computation of the exact taggedparticle propagator in single-file systems via the Bethe eigenspectrum," Comput. Phys. Commun. 258, 107569 (2021).

${ }^{85}$ See https://www.boost.org/doc/libs/1_71_0/libs/math/doc/html/quadrature. html for details about the quadrature library used; accessed 20 Septemer 2019.

${ }^{86}$ Note that Eqs. (5)-(9) imply that $a_{\mathrm{k}}$ is a positive constant times the square of a real number.

${ }^{87}$ G. Lipari and A. Szabo, "Model-free approach to the interpretation of nuclear magnetic resonance relaxation in macromolecules. 1 . Theory and range of validity," J. Am. Chem. Soc. 104(17), 4546-4559 (1982).

${ }^{88}$ Note that $\tau_{\text {rel }}$ does not depend on $N$.

${ }^{89}$ E. Herrera-Delgado, J. Briscoe, and P. Sollich, "Tractable nonlinear memory functions as a tool to capture and explain dynamical behaviors,” Phys. Rev. Res. 2, 043069 (2020).

${ }^{90} \mathrm{~J}$. Abate and P. P. Valkó, "Multi-precision Laplace transform inversion," Int. J. Numer. Methods Eng. 60(5), 979-993 (2004).

${ }^{91}$ J. O. Daldrop, B. G. Kowalik, and R. R. Netz, "External potential modifies friction of molecular solutes in water," Phys. Rev. X 7, 041065 (2017).

${ }^{92}$ S. Kullback and R. A. Leibler, "On information and sufficiency," Ann. Math. Stat. 22(1), 79-86 (1951).

${ }^{93}$ M. C. Mackey, "The dynamic origin of increasing entropy," Rev. Mod. Phys. 61(4), 981-1015 (1989).

${ }^{94} \mathrm{H}$. Qian, “A decomposition of irreversible diffusion processes without detailed balance,” J. Math. Phys. 54(5), 053302 (2013). 
${ }^{95}$ A. Lapolla and A. Godec, "Faster uphill relaxation in thermodynamically equidistant temperature quenches," Phys. Rev. Lett. 125, 110602 (2020).

${ }^{96}$ Wolfram Research, Inc. Mathematica, Version 12.0, 2019.

${ }^{97}$ Y. Meroz, I. M. Sokolov, and J. Klafter, "Test for determining a subdiffusive model in ergodic systems from single trajectories," Phys. Rev. Lett. 110, 090601 (2013).

${ }^{98}$ M. Schwarzl, A. Godec, and R. Metzler, "Quantifying non-ergodicity of anomalous diffusion with higher order moments," Sci. Rep. 7(1), 3878 (2017).

${ }^{99}$ R. Metzler, J.-H. Jeon, A. G. Cherstvy, and E. Barkai, "Anomalous diffusion models and their properties: Non-stationarity, non-ergodicity, and ageing at the centenary of single particle tracking," Phys. Chem. Chem. Phys. 16(44), 24128-24164 (2014).

${ }^{100}$ Q.-H. Wei, C. Bechinger, and P. Leiderer, "Single-file diffusion of colloids in one-dimensional channels," Science 287(5453), 625-627 (2000).

${ }^{101}$ R. D. L. Hanes, C. Dalle-Ferrier, M. Schmiedeberg, M. C. Jenkins, and S. U. Egelhaaf, "Colloids in one dimensional random energy landscapes," Soft Matter 8(9), 2714 (2012).
${ }^{102}$ A. L. Thorneywork, J. Gladrow, Y. Qing, M. Rico-Pasto, F. Ritort, H. Bayley, A. B. Kolomeisky, and U. F. Keyser, "Direct detection of molecular intermediates from first-passage times," Sci. Adv. 6(18), eaaz4642 (2020).

${ }^{103}$ B. Roux, T. Allen, S. Bernèche, and W. Im, "Theoretical and computational models of biological ion channels," Q. Rev. Biophys. 37(1), 15-103 (2004).

${ }^{104}$ A. Pohorille, M. A. Wilson, and C. Wei, "Validity of the electrodiffusion model for calculating conductance of simple ion channels," J. Phys. Chem. B 121(15), 3607-3619 (2017).

${ }^{105}$ W. Kopec, D. A. Köpfer, O. N. Vickery, A. S. Bondarenko, T. L. C. Jansen, B. L. de Groot, and U. Zachariae, "Direct knock-on of desolvated ions governs strict ion selectivity in K+ channels," Nat. Chem. 10(8), 813-820 (2018).

${ }^{106}$ R. Epsztein, R. M. DuChanois, C. L. Ritt, A. Noy, and M. Elimelech, "Towards single-species selectivity of membranes with subnanometre pores," Nat. Nanotechnol. 15(6), 426-436 (2020).

${ }^{107}$ E. Subrt and P. Chvosta, "Diffusion in the time-dependent double-well potential," Czech J. Phys. 56(2), 125-139 (2006).

${ }^{108} \mathrm{We}$ here correct typos found in the original publications. 


\section{Chapter 9}

\section{A Toolbox for Quantifying Memory in Dynamics Along Reaction Coordinates}

This article is currently under review. The pre-print is freely available from the ArXiv repository [56]. Often experimental time-series represent a projected observable and the presence and extent of memory in these traces is not easy to quantify. In this work we provide two practical tools to measure memory effects: the first relies on the comparison with a related Markovian (by definition) process displaying the same equilibrium probability density function, while the second checks if the process satisfies the Markovian semi-group property. We test both methods against a theoretical model for flexible polymers (the Rouse chain) and against an experimental series describing the end-to-end distance of a DNA hairpin kindly provided by the Woodside lab from the University of Alberta (Canada). 


\title{
A Toolbox for Quantifying Memory in Dynamics Along Reaction Coordinates
}

\author{
Alessio Lapolla and Aljaž Godec* \\ Mathematical bioPhysics Group, Max Planck Institute for Biophysical Chemistry, Göttingen 37077, Germany
}

(Dated: January 14, 2021)

\begin{abstract}
Memory effects in time-series of experimental observables are ubiquitous, have important consequences for the interpretation of kinetic data, and may even affect the function of biomolecular nanomachines such as enzymes. Here we propose a set of complementary methods for quantifying conclusively the magnitude and duration of memory in a time series of a reaction coordinate. The toolbox is general, robust, easy to use, and does not rely on any underlying microscopic model. As a proof of concept we apply it to the analysis of memory in the dynamics of the end-to-end distance of the analytically solvable Rouse-polymer model and an experimental time-series of extensions of a single DNA hairpin measured in an optical tweezers experiment.
\end{abstract}

The dynamics of complex, high-dimensional physical systems such as complex biomolecules is frequently described by means of memory-less, Markovian diffusion along a low-dimensional reaction coordinate [1-9]. Such simplified models often accurately describe selected observations in experiments [10-14] and computer simulations $[1,6,7]$. However, as soon as latent, hidden degrees of freedom that become projected out do not relax instantaneously on the time scale we observe the reaction coordinate [15], or the reaction coordinate does not locally equilibrate in long-lived meta-stable meso-states [16], almost any projection of high-dimensional dynamics onto a lower dimensional coordinate introduces memory [15-24].

Memory effects can have intriguing manifestations in the evolution of both, ensemble- $[15,25-28]$ and timeaverage observables [15, 29], and are often particularly well-pronounced in observations that reflect, or couple to, intra-molecular distances in conformationally flexible biomolecules [16, 18, 20-22, 25, 30-38]. Moreover, if the dynamics is ergodic in the sense that the system relaxes to a unique equilibrium probability density function from any initial condition (i.e. the reaction coordinate has a unique free energy landscape) then the memory is necessarily transient [15]. Whether or not memory is in fact relevant depends on how its extent compares to the relaxation time and whether or not the latter is reached in an experiment. If the extent of memory is comparable to, or longer than, the time-scale on which biomolecules operate, such e.g. enzymes catalyzing chemical reactions $[39,40]$, non-Markovian effects shape biological function.

It is therefore important to asses the presence and duration of memory effects in the dynamics along reaction coordinates. An elegant "test of Markovianity" of a reaction coordinate has recently been proposed by Berezhkovskii and Makarov, who considered the behavior of transition paths [41]. The authors provide a pair of inequalities whose violation conclusively reflects that the dynamics is non-Markovian. However, memory-effects are typically transient [15] although their extent may exceed the duration of experimental observations [33]. There is thus a need to determine not only the presence of memory in a time-series of a reaction coordinate but also its extent and attenuation at different time-scales.

Here, we fill this gap by providing a toolbox for quantifying memory in a time-series of a low-dimensional reaction coordinate. We propose a set of model-free complementary methods that are easy to use and are suited to treat reaction coordinates with arbitrary dimensionality. As a proof of concept we apply these methods to the analysis of an experimental time-series of the extension of a DNA-hairpin measured in an optical tweezers experiment and the exactly-solvable Rouse model of polymer chain.

Theory. - Our approach rests on a comparison of the true time-evolution with a pair reference processes: one constructed as a fictitious, Markovian time-series and the other testing for the semi-group property. Let $q_{t}$ with $0 \leq t \leq T$ denote the time-series of the reaction coordinate and $q_{t}^{\mathrm{M}}$ the fictitious Markovian series. Without any loss of generality we assume that the reaction coordinate is one-dimensional - the generalization to multiple dimensions is straightforward. We assume $q_{t}$ and $q_{t}^{\mathrm{M}}$ to be ergodic with an equilibrium probability density $p_{\text {eq }}(q)$ that is by construction identical for both processes. Let $G\left(q, t \mid q_{0}\right)=\left\langle\delta\left(q_{t}-q\right)\right\rangle_{q_{0}}$ denote the probability density that the reaction coordinate evolving from $q_{t=0}=q_{0}$ is found at time $t$ to have a value in an infinitesimal neighborhood of $q$ and $G^{\mathrm{M}}\left(q, t \mid q_{0}\right)=\left\langle\delta\left(q_{t}^{\mathrm{M}}-q\right)\right\rangle_{q_{0}}$ the corresponding Markovian counterpart, where $\delta(x)$ denotes Dirac's delta function and the angular brackets $\langle\cdot\rangle_{q_{0}}$ the average over all realizations of $q_{t}$ evolving from $q_{0}$. We then have $\lim _{t \rightarrow \infty} G\left(q, t \mid q_{0}\right)=\lim _{t \rightarrow \infty} G_{M}\left(q, t \mid q_{0}\right)=$ $p_{\text {eq }}(q)$ as a result of ergodicity. In practice the limits are achieved as soon as $t$ becomes sufficiently larger than the relaxation time $t_{\text {rel }}$, i.e. $t \gtrsim t_{\text {rel }}$, which may or may not be reached in an experiment. Note that the relaxation times of the true and Markovian reference process are typically not equal.

We use two descriptors, the Kullback-Leibler divergence between the transition probabilities of the true and 
a fictitious reference process defined as [42]

$$
\mathcal{D}_{q_{0}}^{\mathrm{a}}(t) \equiv \int d q G\left(q, t \mid q_{0}\right) \ln \left[G\left(q, t \mid q_{0}\right) / G^{\mathrm{a}}\left(q, t \mid q_{0}\right)\right],
$$

where $a=$ M,CK denotes the particular kind of reference process that we detail below. By construction $\mathcal{D}_{q_{0}}^{\mathrm{a}}(t) \neq 0$ if and only if $G\left(q, t \mid q_{0}\right) \neq G^{\mathrm{a}}\left(q, t \mid q_{0}\right)$ and thus non-zero values of $\mathcal{D}_{q_{0}}^{\mathrm{a}}(t)$ reflect memory in the dynamics of $q_{t}$.

When $q_{t}$ reaches equilibrium in the coarse of the experiment we may also consider the normalized equilibrium autocorrelation function defied as

$$
C_{, M}(t) \equiv \frac{\left\langle q_{t} q_{0}\right\rangle_{, M}-\langle q\rangle_{\mathrm{eq}}^{2}}{\left\langle q^{2}\right\rangle_{\mathrm{eq}}-\langle q\rangle_{\mathrm{eq}}^{2}}
$$

where we have introduced

$$
\begin{aligned}
\left\langle q_{t} q_{0}\right\rangle_{, M} & \equiv \iint q q_{0} G^{, M}\left(q, t \mid q_{0}\right) p_{\text {eq }}\left(q_{0}\right) d q d q_{0} \\
& \stackrel{T \gg t_{\text {rel }}}{=}(T-t)^{-1} \int_{0}^{T-t} q_{\tau+t} q_{\tau} d \tau, t \ll T, \\
\left\langle q^{n}\right\rangle_{\mathrm{eq}} & \equiv \int q^{n} p_{\mathrm{eq}}(q) d q, \text { for } n=1,2 \\
& \stackrel{T \gg t_{\mathrm{rel}}}{=} T^{-1} \int_{0}^{T} q_{\tau}^{n} d \tau
\end{aligned}
$$

where the respective definitions in terms of time-averages hold if the trajectory is much longer than the relaxation time, i.e. $T \gg t_{\text {rel }}$. The absence of an index refers to the true process and the index $M$ to the fictitious Markovian counterpart.

In the comparison we consider two distinct reference processes. The first one builds on the semi-group property of Markov processes, i.e. the so-called ChapmanKolmogorov equation that we may write as

$$
G_{\tau}^{\mathrm{CK}}\left(q, t \mid q_{0}\right) \equiv \int G\left(q, t-\tau \mid q^{\prime}\right) G\left(q^{\prime}, \tau \mid q_{0}\right) d q^{\prime}
$$

because the Green's function of a time-homogeneous Markov process is time-translation invariant, $G(q, t-$ $\left.\tau \mid q^{\prime}\right)=G\left(q, t \mid q^{\prime}, \tau\right)[43]$. The physical interpretation of the construction is that we observe the true dynamics $q_{t}$ until time $\tau$ and then instantaneously reset the memory (if any) to zero.

If $q_{t}$ is indeed memoryless we have identically $G_{\tau}^{\mathrm{CK}}\left(q, t \mid q_{0}\right)=G\left(q, t \mid q_{0}\right)$ for any $\tau$ and thus $\mathcal{D}_{\tau, q_{0}}^{\mathrm{CK}}(t)=$ 0 for any $t$ and $\tau$. If $G_{\tau}^{\mathrm{CK}}\left(q, t \mid q_{0}\right) \neq G\left(q, t \mid q_{0}\right)$ for some $t$ and $\tau$ then $q_{t}$ is conclusively non-Markovian and $\mathcal{D}_{\tau, q_{0}}^{\text {CK }}(t)>0$ but the converse is not true. Namely, there exist non-Markovian processes that satisfy the ChapmanKolmogorov equation [15, 44]. Note that this method does not require $q_{t}$ to reach equilibrium during an experiment and requires only $G\left(q, t \mid q_{0}\right)$ that is straightforward to determine from a time series $q_{t}$. However, if equilibrium is reached then $\lim _{t \gg t_{\text {rel }}} \mathcal{D}_{\tau, q_{0}}^{C K}(t)=0$ for any $q_{0}$. By analyzing $\mathcal{D}_{\tau, q_{0}}^{\mathrm{CK}}(t)$ we can quantify the degree of memory and its range as a function of $\tau$ and $q_{0}$ which we demonstrate below.

In the second method we construct from $q_{t}$ a corresponding Markovian time-series $q_{t}^{\mathrm{M}}$ evolving under the influence of the potential of mean force $w(q) \equiv$ $-k_{\mathrm{B}} T \ln p_{\text {eq }}(q)$ according to the Itô Langevin equation

$$
\frac{d}{d t} q_{t}^{\mathrm{M}}=D f\left(q_{t}^{\mathrm{M}}\right) / k_{\mathrm{B}} T+\xi_{t}
$$

where $f\left(q_{t}^{\mathrm{M}}\right) \equiv-\left.k_{\mathrm{B}} T \partial_{q} \ln p_{\text {eq }}(q)\right|_{q=q_{t}^{\mathrm{M}}}$ and $\xi_{t}$ denotes zero mean Gaussian white noise with variance $\left\langle\xi_{t} \xi_{t^{\prime}}\right\rangle=$ $2 D \delta\left(t-t^{\prime}\right)$ and $D$ is the diffusion coefficient. This method assumes that we are able to determine the equilibrium probability density $p_{\text {eq }}(q)$ and thus requires $q_{t}$ to reach equilibrium. In the simplest model, which we adopt here, it is assumed that the diffusion coefficient does not depend on $q$. In this case inferring $D$ is straightforward. However, we note that the best possible Markovian approximation would allow that the diffusion coefficient depends on $q$, i.e. $D \rightarrow D(q)$ [45] and efficient methods have been developed to treat this case as well [46, 47]. In this case Eq. (5) is to be interpreted in the thermodynamically consistent anti-Itô or post-point convention [16].

On the level of the probability density function Eq. (5) corresponds to the Fokker-Planck equation

$$
\partial_{t} G^{\mathrm{M}}\left(q, t \mid q_{0}\right)=\partial_{q} D\left[\partial_{q}-\left(k_{\mathrm{B}} T\right)^{-1} f(q)\right] G^{\mathrm{M}}\left(q, t \mid q_{0}\right)
$$

with initial condition $G^{\mathrm{M}}\left(q, t \mid q_{0}\right)=\delta\left(q-q_{0}\right)$ and natural boundary conditions imposed by the underlying physics. Depending on the specific problem $G^{\mathrm{M}}\left(q, t \mid q_{0}\right)$ can be found by a numerical integration of the Langevin equation and subsequent histogram analysis, i.e. $G^{\mathrm{M}}\left(q, t \mid q_{0}\right)=\left\langle\delta\left(q_{t}^{\mathrm{M}}-q\right)\right\rangle_{q_{0}}$, or directly by solving Eq. (6) as e.g. [15, 48]. In the following we illustrate both approaches.

End-to-end distance of a Rouse polymer. - As a first example we consider a Rouse polymer chain with $N+1$ beads ( $N$-bonds) in absence of hydrodynamic interactions $[49,50]$ and focus on the end-to-end distance as the reaction coordinate, i.e. $q_{t} \equiv\left|\mathbf{r}_{1}-\mathbf{r}_{N+1}\right|$. An important feature of this model is that it is exactly solvable. We express time in units of $t_{\mathrm{Kuhn}}$, the characteristic diffusion time of a Kuhn-segment, i.e. $t_{\mathrm{Kuhn}}=b^{2} / D$, where $b$ is the Kuhn-length and $D$ the diffusion coefficient of a bead. The probability density function for the positions of all beads $\left\{\mathbf{r}_{i}\right\}$ is well-known $[49,50]$ and allows us to determine exactly the probability density of the end-to-end distance. Introducing $\nu_{k} \equiv k \pi / 2(N+1), \alpha_{k}=4 \sin ^{2}\left(\nu_{k}\right)$ as well as $Q_{i k} \equiv \sqrt{2 /(N+1)} \cos \left(\nu_{k}[2 i-1]\right)$ and $\eta_{t} \equiv$ $\sum_{k=1}^{N}\left(Q_{1 k}-Q_{1 k}\right)^{2} \mathrm{e}^{-\alpha_{k} t} / 2 \alpha_{k}$ the equilibrium probability density of $q$ is given by $p_{\text {eq }}(q)=q^{2} \mathrm{e}^{-q^{2} / 4 \eta_{0}} / 2 \sqrt{\pi} \eta_{0}^{3 / 2}$ for $q \in[0, \infty)$ with the mean extension $\langle d\rangle=4 \sqrt{\eta_{0} / \pi}$ 
and mean square extension $\left\langle d^{2}\right\rangle=6 \eta_{0}$. The probability density function of $q$ reads exactly (for a derivation see Ref. [26])

$G_{R}\left(q, t \mid q_{0}\right)=\frac{q q_{0} \mathrm{e}^{-\eta_{0}\left(q^{2}+q_{0}^{2}\right) / 2\left(\eta_{0}^{2}-\eta_{t}^{2}\right)}}{p_{\text {eq }}(q) 2 \pi \eta_{t} \sqrt{\eta_{0}^{2}-\eta_{t}^{2}}} \sinh \left(\frac{\eta_{t} q q_{0}}{2\left(\eta_{0}^{2}-\eta_{t}^{2}\right)}\right)$.

The exact autocorrelation function in Eq. (2) is in turn obtained in the form

$$
C(t)=\frac{6 \sqrt{\eta_{0}^{2}-\eta_{t}^{2}}}{(3 \pi-8) \eta_{0}}+\frac{4\left(\eta_{0}^{2}+\eta_{t}^{2}\right) \arctan \left(\eta_{t} / \sqrt{\eta_{0}^{2}-\eta_{t}^{2}}\right)}{(3 \pi-8) \eta_{0} \eta_{t}}
$$

The Fokker-Planck equation in the Markovian approximation to the evolution of $q$ for the Rouse polymer (i.e. Eq. (6)) can be obtained exactly in the form of a spectral expansion [48] and reads $G_{R}^{\mathrm{M}}\left(q, t \mid q_{0}\right)=$ $\sum_{k=0}^{\infty} \psi_{k}^{R}(q) \psi_{k}^{L}\left(q_{0}\right) \mathrm{e}^{-k t / \eta_{0}}$ where

$$
\psi_{k}^{L}(x) \equiv \sqrt{\frac{k ! \sqrt{\pi}}{2 \Gamma(3 / 2+k)}} L_{k}^{1 / 2}\left(\frac{x^{2}}{4 \eta_{0}}\right)
$$

where $\Gamma(x)$ denotes the Gamma-function and $L_{k}^{1 / 2}(x)$ the generalized Laguerre polynomial of degree $k$ with parameter $1 / 2$ (see [51]) that we compute using the Arblibrary [52] and $\psi_{k}^{R}(x)=p_{\mathrm{eq}}(x) \psi_{k}^{L}(x)$. Here from it is straightforward to obtain the autocorrelation function in the Markovian approximation that reads

$C_{M}(t)=\frac{\sqrt{\pi}}{3-8 / \pi} \sum_{k=1}^{\infty} \mathrm{e}^{-k t / \eta_{0}}\left[k ! \Gamma(3 / 2-k)^{2} \Gamma(3 / 2+k)\right]^{-1}$.

A comparison of the true autocorrelation function $C(t)$ given by Eq. (8) and the Markovian approximation $C_{M}(t)$ given by Eq. (10) is shown in Fig. 1a, with the inset depicting the corresponding equilibrium probability density function $p_{\text {eq }}(q)$. Note that when the free energy landscape $w(q)$ overestimates the confining effect of hidden degrees of freedom on $q_{t}$ the approximate Markovian evolution always overestimates the relaxation rate (e.g. [15]; see also SM). Namely, the Markovian evolution assumes the hidden degrees of freedom to remain at equilibrium at all times whereas the instantaneous fluctuating restoring force on $q_{t}$ is in this case smaller than the force arising from the free energy landscape that corresponds to the average of the fluctuating force.

The Chapman-Kolmogorov-construct for the Rouse polymer, $G_{\tau}^{\mathrm{CK}}\left(q, t \mid q_{0}\right)$, is somewhat lengthy and is thefore given explicitly in the Supplementary Material, SM. $G_{\tau}^{\mathrm{CK}}\left(q, t \mid q_{0}\right)$ differs from Eq. (7) for all expect large values of $t-\tau$. A quantification of the discrepancy between the true and "Chapman-Kolmogorov" evolution of the end-to-end distance of the Rouse-polymer in terms of the Kullback-Leibler divergence (1) is shown in Fig. 2a.

A typical time evolution of $\mathcal{D}_{\tau, q_{0}}^{\mathrm{CK}}(t)$ gradually increases
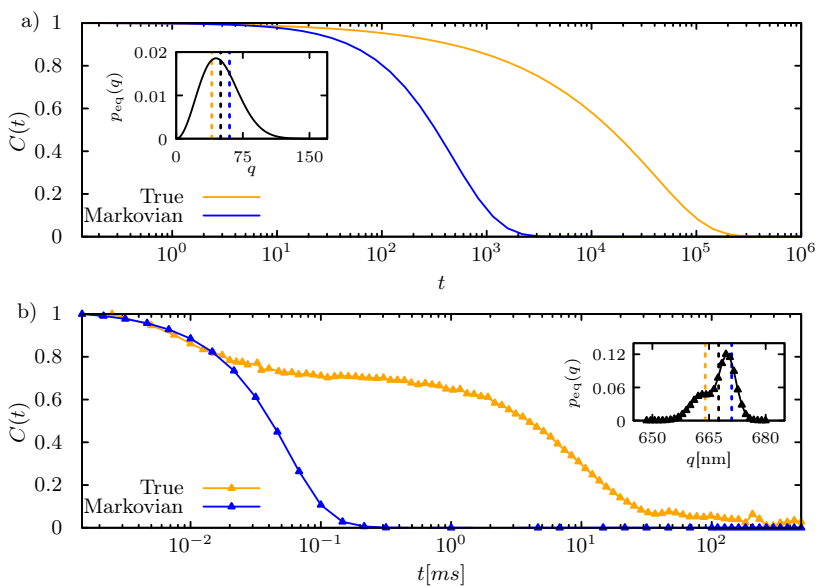

Figure 1. Comparison of the true autocorrelation function $C(t)$ (orange) with the Markovian approximation $C_{M}(t)$ (blue) for a) a Rouse-polymer with 1000 monomers with time expressed in units of the diffusion time of a Kuhn-segment $t_{\mathrm{Kuhn}}$; b) the extension of a DNA-hairpin. The insets depict the respective equilibrium probability density function $p_{\text {eq }}(q)$. The dashed lines depict the initial conditions we consider in Fig. 3.
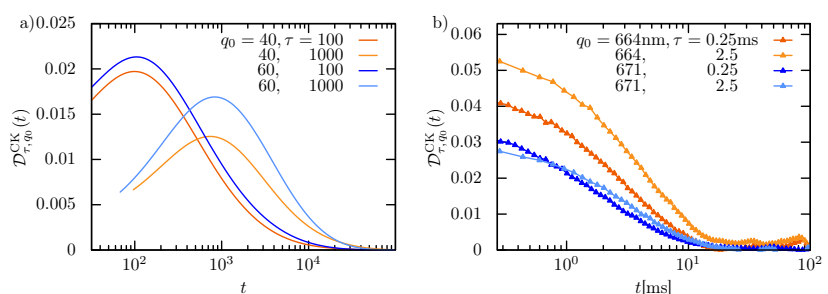

Figure 2. Kullback-Leibler divergence defined in Eq. (1) between the true Green's function $G\left(q, t \mid q_{0}\right)$ and the approximate Green's function constructed from the ChapmanKolmogorov equation (4) as a function of time $t$ for a) the Rouse-polymer with 1000 beads and b) the extension of a DNA-hairpin. Several values of $\tau$ are considered in the construction of $G_{\tau}^{\mathrm{CK}}\left(q, t \mid q_{0}\right)$ as well as a pair of different initial conditions $q_{0}$. Note that due to the particular construction of Eq. (4) times shorter than depicted are not accessible due to numerical instability and poor statistics.

from zero, reaches a maximum and afterwards returns back to 0 , which reflects the gradual build-up and attenuation of memory because $q_{t}$ "remembers" the initial condition of the hidden degrees of freedom [15]. As a result, the Chapman-Kolmogorov Green's function $G_{\tau}^{\mathrm{CK}}\left(q, t \mid q_{0}\right)$ fails to predict the true evolution of $q_{t}$, and $\mathcal{D}_{\tau, q_{0}}^{\mathrm{CK}}(t)$ constructed this way depends on both, $\tau$ and initial condition $q_{0}$. For the Rouse-polymer with 1000 beads $\mathcal{D}_{\tau, q_{0}}^{\text {CK }}(t) \neq 0$ at least up to $t \sim 10^{4} \times t_{\text {Kuhn }}$.

Next we examine $\mathcal{D}_{q_{0}}^{\mathrm{M}}(t)$, the Kullback-Leibler divergence (1) between the true Green's function $G\left(q, t \mid q_{0}\right)$ and the Markovian approximation corresponding to the white-noise Markovian diffusion in the exact free energy 

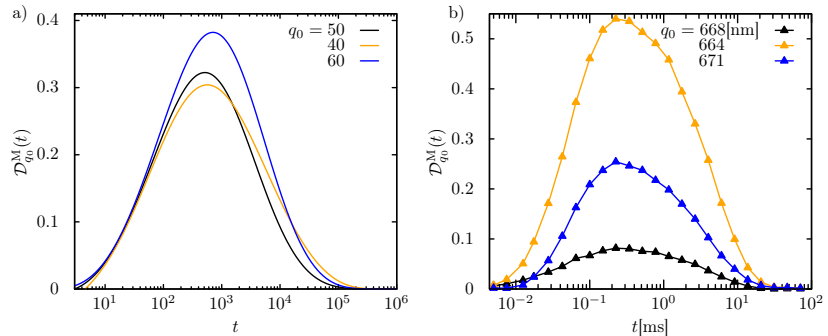

Figure 3. Kullback-Leibler divergence $\mathcal{D}_{t}^{\mathrm{M}}$ defined in Eq. (1) between the true Green's function $G\left(q, t \mid q_{0}\right)$ and the Markovian approximation to the Green's function $G^{\mathrm{M}}\left(q, t \mid q_{0}\right)$ corresponding to the evolution equation (5) as a function of time $t$ for a) the Rouse-polymer with 1000 beads evolving from $q_{0}=40$ (orange), $q_{0}=50$ (black), $q_{0}=60$ (blue) and b) the extension of a DNA-hairpin evolving from a distance within a bin of thickness $1 \mathrm{~nm}$ centered at $q_{0}=664 \mathrm{~nm}$ (orange), $q_{0}=668$ (black) and $q_{0}=671 \mathrm{~nm}$ (blue).

landscape (i.e. Eq. (5)). The results are shown in Fig. 3a.

The qualitative features of the time-dependence of $\mathcal{D}_{q_{0}}^{\mathrm{M}}(t)$ are similar to those observed in Fig. 2 - memory builds up in a finite interval and smoothly returns back to zero from the attained maximum. The intuition behind this result is that it takes a finite time to allow for distinct evolutions of hidden degrees of freedom that introduce memory in the dynamics of the reaction coordinate $q_{t}$. At long times memory is progressively lost as a result of the gradual relaxation of the hidden degrees of freedom to their respective equilibrium that in turn renders the dynamics of the reaction coordinate effectively memory-less and correspondingly $\mathcal{D}_{q_{0}}^{\mathrm{M}}(t)$ vanishes.

Single-molecule experiments on a DNA hairpin.-As a second example we consider an experimental time-series of the end-to-end distance of a single-strand DNA hairpin measured in an optical tweezers experiment performed by the Woodside group [53]. The data-set contains 11 million measurements of the extension of the DNA hairpin 30R50T4 held in a pair of optical traps with stiffness $0.63 \mathrm{pN} / \mathrm{nm}$ and $1.1 \mathrm{pN} / \mathrm{nm}$, respectively, sampled with a $2.5 \mu \mathrm{s}$ temporal resolution. It has been shown that this time-series is non-Markovian [38]. We find the length of the time-series to be much larger that the relaxation time (see Fig. 1b) and therefore slice it into several pieces that are statistically independent. More precisely, we use a conservative estimate, namely the time-scale $t_{\text {cut }}$ where the autocorrelation function of the extension, $C(t)$, falls to below $\simeq 0.05$. Note that this enssures $t_{\text {cut }} \gg t_{\text {rel }}$ and yields an ensemble of 50 statistically independent trajectories.

We determine the equilibrium probability density $p_{\text {eq }}(q)$ (see inset of Fig. 1b) and two-point joint probability density $p\left(q, t, q_{0}, 0\right)=p\left(q, t_{0}+t, q_{0}, t_{0}\right)$ by performing a standard histogram analysis with a bin-size of $l_{\text {bin }}=0.35 \mathrm{~nm}$, such that $q$ refers to a bin of width $l_{\text {bin }}$ centered at $q$. The Greens function is thereupon obtained by the law of conditional probability, $G\left(q, t \mid q_{0}\right)=$ $p\left(q, t, q_{0}, 0\right) / p_{\text {eq }}(q)$ while the autocorrelation function in Eq. (2) is determined directly from the respective second lines of Eq. (3).

The Chapman-Kolmogorov construct is determined from $G\left(q, t \mid q_{0}\right)$ by direct integration of Eq. (4) and is used to determine $\mathcal{D}_{\tau, q_{0}}^{\mathrm{CK}}(t)$, while the corresponding fictitious Markovian process evolves as Markovian diffusion in a free energy landscape $w(q) \equiv-k_{\mathrm{B}} T \ln p_{\text {eq }}(q)$ with a diffusion coefficient $D$ that we parameterize as follows. We first determine the first and second moment of the displacement from each bin-point $q_{l}$ after a single time-step $\delta t=2.5 \mu$ s, i.e. $\left\langle\delta q_{\delta t}^{2}(l)\right\rangle$ and $\left\langle\delta q_{\delta t}(l)\right\rangle$ where $\delta q_{\delta t}(l)=q_{t+\delta t}-\left.q_{t}\right|_{q_{t}=q_{l}}$. We consider two bin-sizes, $l_{D}=0.01 \mathrm{~nm}$ and $l_{D}=0.001 \mathrm{~nm}$, and find the result to be essentially independent on the precise value of $l_{D}$ we choose (see SM). Moreover, the results are also rather independent of the location of the bin $q_{l}$ (see SM), implying that to a good approximation $D$ may indeed be taken as being constant. It is of course possible to extend the analysis to include a coordinate dependent diffusion coefficient $D(q)$ (see e.g. [47]), which, however, is beyond the scope of this proof-of-concept paper.

From the first and second local moments of $q$ the diffusivity $D$ is in turn determined according to

$$
D=\sum_{l=1}^{N_{b}} \frac{\left\langle\delta q_{\delta t}^{2}(l)\right\rangle-\left\langle\delta q_{\delta t}(l)\right\rangle^{2}}{2 N_{b} \delta t}
$$

where the brackets $\langle\cdot\rangle$ here denote the average over all displacements from this bin observed during the entire time-series. The analysis yields $D=447 \pm 9 \mathrm{~nm}^{2} / \mathrm{ms}$ for $l_{D}=0.001 \mathrm{~nm}$ and $D=448 \pm 9 \mathrm{~nm}^{2} / \mathrm{ms}$ for $l_{D}=0.01$ $\mathrm{nm}$, respectively. Using the values for and $D$ obtained in this way we generate the Markovian time-series $q_{t}^{\mathrm{M}}$ by integrating the Itô Langevin equation (5) using the EulerMayurama scheme, and determine $\mathcal{D}_{t}^{\mathrm{M}}\left(q_{0}\right)$ in Eq. (1) and $C_{M}(t)$ in Eq. (2), respectively.

In contrast to the Rouse-polymer the DNA hairpin exists in two characteristic conformational states - folded and unfolded. As a result, the equilibrium probability density function $p_{\text {eq }}(q)$ is bimodal and the dynamics of $q_{t}$ displays signatures of metastability [53]. However, since the two peaks corresponding to the two sub-populations are not separated (see inset of Fig.1b) the potential of mean force $w(q)$ is expected to underestimate the free energy barrier and therefore the Markovian evolution is likely to overestimate the relaxation rate. In complete agreement Fig.1b displays an overestimation of the rate of decay of autocorrelations in the Markovian approximation by two orders of magnitude in time. Moreover, a long-lived plateau is observed in the true $C(t)$ spanning more than an order of magnitude in time. 
In order to assess whether the mismatch between true and Markovian time evolution is predominantly due to an underestimation of the free energy barrier between folded and unfolded states of the hairpin we inspect he Kullback-Leibler divergence (1) between the true and "Chapman-Kolmogorov evolution" of the extension of the hairpin is shown in Fig. 2b. The result clearly shows pronounced signatures of memory in the evolution of the end-to-end distance of the hairpin that extend over more than $\sim 10 \mathrm{~ms}$. Note that the "Chapman-Kolmogorov evolution" describes the correct evolution until time $t=\tau$ whereupon memory is reset to zero. Therefore a nonzero $\mathcal{D}_{\tau, q_{0}}^{\mathrm{Ck}}(t)$ is a clear signature of memory arising from the dynamical coupling of $q_{t}$ to hidden degrees of freedom. Similar to the Rouse-polymer $\mathcal{D}_{\tau, q_{0}}^{\mathrm{CK}}(t)$ depends on the initial condition $q_{0}$

A build-up and decay of memory similar to the Rousepolymer is also observed in the time evolution of $\mathcal{D}_{q_{0}}^{\mathrm{M}}(t)$, the Kullback-Leibler divergence between the Green's function of the true evolution and the white-noise Markovian diffusion in the exact free energy landscape shown in Fig. 3b. Notably, Fig. 2b and Fig. 3b display essentially the same extent of memory (though the peak is attained sooner in the white-noise Markovian diffusion), demonstrating that metastability does not necessarily destroy nor dominate memory in the evolution of reaction coordinates. Note that the presence of memory in metastable systems is not unusual (see e.g. [20, 21] and [28]). In total, the analysis conclusively identifies the presence of extended memory effects in the dynamics of the extension of the hairpin.

It is important to note that the extent of memory (of the order of $\sim 10 \mathrm{~ms}$ ) is clearly shorter than the relaxation time (compare Figs. 1b and 3b), and therefore the decay of memory does not coincide with the relaxation time and the corresponding "forgetting" of initial conditions of the coordinate itself. Instead the memory reflects the correlations between $q_{t}$ and the initial conditions of the hidden degrees of freedom [15]. The information encoded in $C(t)$ and $\mathcal{D}^{\mathrm{M}, \mathrm{CK}}(t)$ is therefore different $-\mathcal{D}^{\mathrm{M}, \mathrm{CK}}(t)$ is a genuine measure of the extent and duration of memory.

Conclusion. - We presented a set of complementary methods to quantify conclusively the degree and duration of memory in a time series of a reaction coordinate $q_{t}$. The proposed toolbox does not assume any particular physical model. Instead it exploits the semi-group property of Markov processes and constructs a fictitious Markovian diffusion process in the free energy landscape of $q_{t}$, and compares the artificially constructed transition probability density with the observed probability density. The analysis not only determines whether the dynamics of $q_{t}$ has memory but also quantifies the magnitude and duration of memory and thus complements the recently proposed "test for Markovianity" based on transition paths [41]. Whereas in our examples we considered only one-dimensional coordinates and constant diffusion coefficients the toolbox generalizes straightforwardly to higher-dimensional reaction coordinates and diffusion landscapes $D(q)$. The method is general, robust, and easy to use. We therefore hope that it will find numerous applications involving time-series derived from experiments and computer simulations.

Acknowledgments We thank Krishna Neupane and Michael T. Woodside for providing unlimited access to their DNA-hairpin data. The financial support from the German Research Foundation (DFG) through the Emmy Noether Program GO 2762/1-1 to AG is gratefully acknowledged.

agodec@mpibpc.mpg.de

[1] R. B. Best and G. Hummer, Reaction coordinates and rates from transition paths, Proc. Natl. Acad. Sci. 102, 6732-6737 (2005).

[2] J. J. Portman, S. Takada, and P. G. Wolynes, Microscopic theory of protein folding rates. ii. local reaction coordinates and chain dynamics, J. Chem. Phys. 114, 5082-5096 (2001).

[3] B. Peters, P. G. Bolhuis, R. G. Mullen, and J.-E. Shea, Reaction coordinates, one-dimensional smoluchowski equations, and a test for dynamical self-consistency, J. Chem. Phys. 138, 054106 (2013).

[4] A. K. Faradjian and R. Elber, Computing time scales from reaction coordinates by milestoning, J. Chem. Phys. $\mathbf{1 2 0}$, 10880-10889 (2004).

[5] A. Berezhkovskii and A. Szabo, One-dimensional reaction coordinates for diffusive activated rate processes in many dimensions, J. Chem. Phys. 122, 014503 (2005).

[6] G. Hummer, Position-dependent diffusion coefficients and free energies from bayesian analysis of equilibrium and replica molecular dynamics simulations, New J. Phys. 7, 34-34 (2005).

[7] R. B. Best and G. Hummer, Coordinate-dependent diffusion in protein folding, Proc. Natl. Acad. Sci. 107, 1088-1093 (2009).

[8] W. Zhang, C. Hartmann, and C. Schütte, Effective dynamics along given reaction coordinates, and reaction rate theory, Faraday Discussions 195, 365-394 (2016).

[9] A. M. Berezhkovskii and D. E. Makarov, Communication: Coordinate-dependent diffusivity from single molecule trajectories, J. Chem. Phys. 147, 201102 (2017).

[10] O. K. Dudko, G. Hummer, and A. Szabo, Theory, analysis, and interpretation of single-molecule force spectroscopy experiments, Proceedings of the National Academy of Sciences 105, 15755-15760 (2008).

[11] K. Neupane, A. P. Manuel, and M. T. Woodside, Protein folding trajectories can be described quantitatively by one-dimensional diffusion over measured energy landscapes, Nat. Phys. 12, 700 (2016).

[12] K. Neupane, D. A. N. Foster, D. R. Dee, H. Yu, F. Wang, and M. T. Woodside, Direct observation of transition paths during the folding of proteins and nucleic acids, Science 352, 239 (2016).

[13] J. Gladrow, M. Ribezzi-Crivellari, F. Ritort, and U. F. Keyser, Experimental evidence of symmetry breaking of transition-path times, Nat. Commun. 10, 10.1038/s41467- 
018-07873-9 (2019).

[14] A. L. Thorneywork, J. Gladrow, Y. Qing, M. Rico-Pasto, F. Ritort, H. Bayley, A. B. Kolomeisky, and U. F. Keyser, Direct detection of molecular intermediates from firstpassage times, Sci. Adv. 6, 10.1126/sciadv.aaz4642 (2020).

[15] A. Lapolla and A. Godec, Manifestations of ProjectionInduced Memory: General Theory and the Tilted Single File, Front. Phys. 7, 10.3389/fphy.2019.00182 (2019).

[16] D. Hartich and A. Godec, Emergent memory and kinetic hysteresis in strongly driven networks, arXiv:2011.04628 (2020), arXiv:2011.04628 [cond-mat.stat-mech].

[17] N. van Kampen, Remarks on Non-Markov Processes, Brazilian J. Phys. 28, 10.1590/S0103-97331998000200003 (1998).

[18] S. S. Plotkin and P. G. Wolynes, Non-markovian configurational diffusion and reaction coordinates for protein folding, Phys. Rev. Lett. 80, 5015 (1998).

[19] R. Zwanzig, Nonequilibrium statistical mechanics (Oxford Univ. Press, 2010).

[20] D. E. Makarov, Interplay of non-markov and internal friction effects in the barrier crossing kinetics of biopolymers: Insights from an analytically solvable model, J. Chem. Phys. 138, 014102 (2013).

[21] M. Ozmaian and D. E. Makarov, Transition path dynamics in the binding of intrinsically disordered proteins: A simulation study, J. Chem. Phys. 151, 235101 (2019).

[22] H. Meyer, P. Pelagejcev, and T. Schilling, Non-markovian out-of-equilibrium dynamics: A general numerical procedure to construct time-dependent memory kernels for coarse-grained observables, EPL (Europhys. Lett.) 128, 40001 (2020)

[23] E. Herrera-Delgado, J. Briscoe, and P. Sollich, Tractable nonlinear memory functions as a tool to capture and explain dynamical behaviors, Phys. Rev. Research 2, 043069 (2020).

[24] F. Müller, U. Basu, P. Sollich, and M. Krüger, Coarsegrained second-order response theory, Phys. Rev. Research 2, 043123 (2020).

[25] W. Min, G. Luo, B. J. Cherayil, S. C. Kou, and X. S. Xie, Observation of a power-law memory kernel for fluctuations within a single protein molecule, Phys. Rev. Lett. 94, 198302 (2005).

[26] A. Lapolla and A. Godec, Faster uphill relaxation in thermodynamically equidistant temperature quenches, Phys. Rev. Lett. 125, 10.1103/physrevlett.125.110602 (2020).

[27] A. Lapolla and A. Godec, Bethesf: Efficient computation of the exact tagged-particle propagator in single-file systems via the bethe eigenspectrum, Comput. Phys. Commun. 258, 107569 (2021).

[28] A. Lapolla and A. Godec, Single-file diffusion in a bistable potential: Signatures of memory in the barriercrossing of a tagged-particle, J. Chem. Phys. 153, 194104 (2020).

[29] A. Lapolla and A. Godec, Unfolding tagged particle histories in single-file diffusion: exact single- and two-tag local times beyond large deviation theory, New J. Phys. 20, 113021 (2018).

[30] S. C. Kou and X. S. Xie, Generalized langevin equation with fractional gaussian noise: Subdiffusion within a single protein molecule, Phys. Rev. Lett. 93, 180603 (2004).

[31] T. Neusius, I. Daidone, I. M. Sokolov, and J. C. Smith, Subdiffusion in peptides originates from the fractal-like structure of configuration space, Phys. Rev. Lett. 100, 188103 (2008).
[32] S. Pressé, J. Peterson, J. Lee, P. Elms, J. L. MacCallum, S. Marqusee, C. Bustamante, and K. Dill, Single molecule conformational memory extraction: P5ab rna hairpin, J. Phys. Chem. B 118, 6597-6603 (2014).

[33] X. Hu, L. Hong, M. Dean Smith, T. Neusius, X. Cheng, and J. Smith, The dynamics of single protein molecules is non-equilibrium and self-similar over thirteen decades in time, Nature Physics 12, 171-174 (2015).

[34] A. K. Sangha and T. Keyes, Proteins Fold by Subdiffusion of the Order Parameter, J. Phys. Chem. B 113, 15886 (2009).

[35] S. M. Avdoshenko, A. Das, R. Satija, G. A. Papoian, and D. E. Makarov, Theoretical and computational validation of the Kuhn barrier friction mechanism in unfolded proteins, Sci. Rep. 7, 269 (2017).

[36] Y. Cote, P. Senet, P. Delarue, G. G. Maisuradze, and H. A. Scheraga, Anomalous diffusion and dynamical correlation between the side chains and the main chain of proteins in their native state, Proc. Natl. Acad. Sci. 109, 10346 (2012).

[37] I. Grossman-Haham, G. Rosenblum, T. Namani, and H. Hofmann, Slow domain reconfiguration causes powerlaw kinetics in a two-state enzyme, Proc. Natl. Acad. Sci. 115, 513 (2018).

[38] A. G. T. Pyo and M. T. Woodside, Memory effects in single-molecule force spectroscopy measurements of biomolecular folding, Phys. Chem. Chem. Phys. 21, 24527 (2019).

[39] H. P. Lu, Single-molecule enzymatic dynamics, Science 282, 1877-1882 (1998).

[40] B. P. English, W. Min, A. M. van Oijen, K. T. Lee, G. Luo, H. Sun, B. J. Cherayil, S. C. Kou, and X. S. Xie, Ever-fluctuating single enzyme molecules: Michaelismenten equation revisited, Nat. Chem. Biol. 2, 87-94 (2005).

[41] A. M. Berezhkovskii and D. E. Makarov, Single-Molecule Test for Markovianity of the Dynamics along a Reaction Coordinate, J. Phys. Chem. Lett. 9, 2190 (2018).

[42] S. Kullback and R. Leibler, On information and sufficiency, Ann. Math. Statist 22, 79 (1951).

[43] Gardiner, C.W., Handbook of Stochastic Methods for Physics, Chemistry and Natural Sciences, 2nd ed. (Springer-Verlag, 1985).

[44] W. Feller, Non-Markovian Processes with the Semigroup Property, The Annals of Mathematical Statistics 30, 1252 (1959).

[45] A. Berezhkovskii and A. Szabo, Time scale separation leads to position-dependent diffusion along a slow coordinate, J. Chem. Phys. 135, 074108 (2011).

[46] B. J. Berne, M. Borkovec, and J. E. Straub, Classical and modern methods in reaction rate theory, J. Phys. Chem. 92, 3711-3725 (1988).

[47] G. Hummer, Position-dependent diffusion coefficients and free energies from bayesian analysis of equilibrium and replica molecular dynamics simulations, New J. Phys. 7, 34 (2005).

[48] S. Sunagawa and M. Doi, Theory of Diffusion-Controlled Intrachain Reactions of Polymers, Polymer J. 7, 604 (1975).

[49] P. E. Rouse, A Theory of the Linear Viscoelastic Properties of Dilute Solutions of Coiling Polymers, J. Chem. Phys. 21, 1272 (1953).

[50] K. H. Ahn, J. L. Schrag, and S. J. Lee, Bead-spring chain model for the dynamics of dilute polymer solutions, J. 
Non-Newton Fluid Mech. 50, 349 (1993).

[51] Abramowitz, Milton and Stegun, Irene A., Handbook of Mathematical Functions with Formulas, Graphs, and Mathematical Tables, in Handbook of Mathematical Functions with Formulas, Graphs, and Mathematical Tables (Dover, New York, 1964) ninth dover printing, tenth gpo printing ed.

[52] F. Johansson, Arb: Efficient Arbitrary-Precision Midpoint-Radius Interval Arithmetic, IEEE Transactions on Computers 66, 1281 (2017).

[53] K. Neupane, A. P. Manuel, J. Lambert, and M. T. Woodside, Transition-Path Probability as a Test of ReactionCoordinate Quality Reveals DNA Hairpin Folding Is a One-Dimensional Diffusive Process, J. Phys. Chem. Lett. 6, 1005 (2015). 


\title{
Supplementary Material for: \\ A Toolbox for Quantifying Memory in Dynamics Along Reaction Coordinates
}

\author{
Alessio Lapolla and Aljaž Godec \\ Mathematical bioPhysics Group, Max Planck Institute for Biophysical Chemistry, 37077 Göttingen, Germany
}

\begin{abstract}
In this Supplementary Material (SM) we present the exact result for the "Champman-Kolmogorov" construction of the Green's function of the end-to-end distance and other internal coordinates of the Rouse polymer defined in Eq. (4) in the manuscript as well as a local error analysis of the inferred diffusion coefficient $D$ entering the Markovian approximation of the end-to-end diffusion of the DNA hairpin. In addition, supplementary figures are included showing the various Green's functions for the Rouse polymer and DNA hairpin.
\end{abstract}

\section{DETAILS OF THE PROJECTION AFFECT THE RELAXATION TIME AND EXTENT OF MEMORY}

In the main text we consider Rouse polymer chain composed of 1000 beads and we focus on the autocorrelation function of its end-to-end distance as the reaction coordinate $q_{t}$. We find that the fictitious Markovian reference process describing Brownian diffusion in the free energy landscape overestimates the relaxation rate; a similar observation is also made in the case of the experimental hairpin data. However this difference in the rate of relaxation is non-unique and in fact depends on the observable, i.e. on details of the projection.

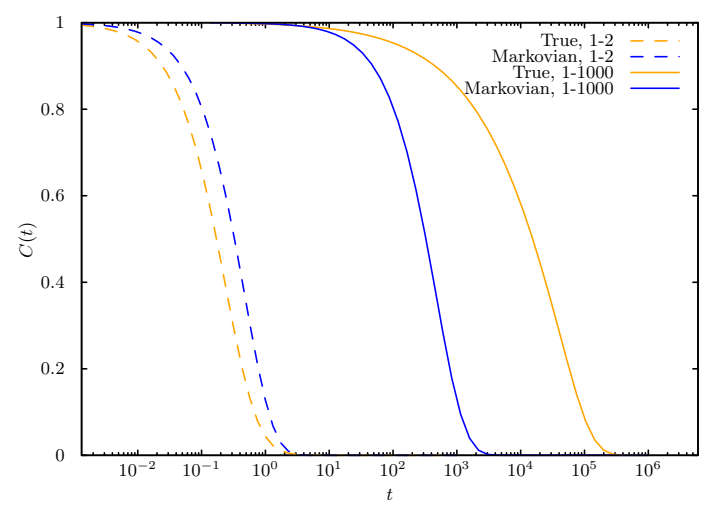

Figure S4. The autocorrelation function of the distance between the first and the second bead (dashed lines) and first and last bead (full lines) of a Rouse chain composed by 1000 according to the true (orange) and fictitious Markovian evolution (blue). Note the the free energy landscape for both orange-blue pairs is by construction identical. The continuous lines are those shown in the main text.

For example we demonstrate in Fig. S4 the opposite trend that arises when we observe the autocorrelation function of the distance between the first and the second bead of the same Rouse Chain (see dashed lines).

In addition, is worth to note that if the Green's function $\mathcal{G}$ describing the full many-dimensional system is diagonalizable (like in the Rouse chain case [1] or any Markovian dynamics obeying detailed balance), it can be written as

$$
\mathcal{G}\left(\mathbf{x}, t \mid \mathbf{x}_{0}\right)=\sum_{k} \psi_{k}^{R}(\mathbf{x}) \psi_{k}^{L}\left(\mathbf{x}_{0}\right) \mathrm{e}^{-\lambda_{k} t}
$$

where $\psi_{k}^{R}$ and $\psi_{k}^{L}$ are respectively the right and left eigenfunctions of the underlying Fokker-Planck-Smoluchowski operator, while $\lambda_{k}$ denotes the eigenvalues. Then the Green's function of the projected observable - the reaction coordinate $q=\Gamma(\mathbf{x})$ - can be written in full generality [2] as

$$
G\left(q, t \mid q_{0}\right)=\sum_{k} V_{k}^{R}(q ; \Gamma) V_{k}^{L}\left(q_{0} ; \Gamma\right) \mathrm{e}^{-\lambda_{k} t}
$$


where the elements $V_{k}^{R}$ and $V_{k}^{L}$ depend both on $\psi_{k}^{R}$ and $\psi_{k}^{L}$, and on the projection $\Gamma(\mathbf{x})$. In turn the autocorrelation function can be easily computed as:

$$
C(t)=\sum_{k}\left(\int q V_{k}^{R}(q ; \Gamma) d q\right)\left(\int q_{0} V_{k}^{L}\left(q_{0} ; \Gamma\right) d q_{0}\right) \mathrm{e}^{-\lambda_{k} t} \equiv \sum_{k} a_{k}^{R, \Gamma} b_{k}^{L, \Gamma} \mathrm{e}^{-\lambda_{k} t},
$$

and one can show that for systems obeying detailed balance $a_{k}^{R, \Pi} b_{k}^{L, \Pi} \geq 0[2]$. The analysis shows that the projection only affects the weights whereas the exponentiated eigenvalues (and thus time-scales) are those of the full system's dynamics.

Nevertheless, the autocorrelation function of different observables of the same system may decay on widely disparate time-scales; compare the dashed and continuous lines in Fig. S4 where in the end-to-end distance the relaxation time is $\sim 10^{6}$ while in the first-to-second distance is $\sim 10^{1}$. This disparity is simply a result of the projection that determines the relative contribution of different eigenfunctions.

\section{THE CHAPMAN-KOLMOGOROV CONSTRUCTION}

In the case of the Rouse chain the integral defined in Eq. (4) in the main text can be solved analytically via a straightforward but tedious calculation using Eq. (7) in the main text. The result of the integral reads exactly

$$
\begin{aligned}
G_{t_{1}}^{C K}\left(q, t \mid q_{0}\right)= & \frac{\eta_{0}^{7 / 2} q}{q_{0} \eta_{t-\tau} \eta_{\tau} \sqrt{\pi \Xi_{\tau, t-\tau}}} \sinh \left(\frac{q q_{0} \eta_{0} \eta_{\tau} \eta_{t-\tau}}{2 \Xi_{\tau, t-\tau}}\right) \times \\
& \exp \left[-\frac{q^{2} \eta_{0}}{2 \Omega_{t-\tau}^{-}}\left(1-\frac{\eta_{t-\tau}^{2} \Omega_{\tau}^{-}}{2 \Xi_{\tau, t-\tau}}\right)-\frac{q_{0}^{2}}{4 \Omega_{\tau}^{-}}\left(\frac{\eta_{0}^{2} \Omega_{\tau}^{-}-\eta_{t-\tau}^{2} \Omega_{\tau}^{+}}{\Omega_{t-\tau}^{-}}-\frac{\eta_{0} \eta_{\tau}^{2} \Omega_{t-\tau}^{-}}{\Xi_{\tau, t-\tau}}\right)\right]
\end{aligned}
$$

having defined

$$
\Omega_{t}^{ \pm}=\eta_{0}^{2} \pm \eta_{t}^{2}, \quad \Xi_{\tau, t-\tau}=4 \eta_{0}^{4}-\Omega_{\tau}^{+} \Omega_{t-\tau}^{+} .
$$

Notably, the structure of Eq. (S4) is identical to the structure of the plain Green's function (Eq. (7) in the main text) but here the temporal dependence is obviously different.

Note that in when the observation time is much larger than the relaxation time of the observable $t_{\text {rel }}$, we find

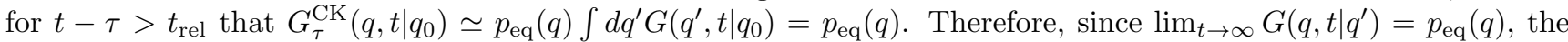
definition of $G_{\tau}^{\mathrm{CK}}\left(q, t \mid q_{0}\right)$ (Eq. (4) in the main text) by construction ensures $\lim _{t \rightarrow \infty} \mathcal{D}_{\tau, q_{0}}^{\mathrm{CK}}(t)=0$. In Fig. S5 we explicitly show the Green's function that is required for the computation of the Kullback-Liebler divergence.

\section{ESTIMATION OF THE DIFFUSION COEFFICIENT}

Eq. (12) in the main text demonstrates how one can estimate the ( $q$-independent) diffusion coefficient $D$ from a time-series using the first two moments of the local displacement.

The moments are in turn determined as follows: if $q_{t}$ is found during an epoch in the bin centered around $q$ and of size $l_{D}$ its displacement from the previous epoch is determined and its square and both are averaged over all epochs in the time-series yielding $\left\langle\delta q_{\delta t}(l)\right\rangle$ and $\left\langle\delta q_{\delta t}^{2}(l)\right\rangle$ respectively.

This procedure is repeated for different positions $q$ on the support of the equilibrium distribution. We find that the resulting diffusion coefficient changes little over the entire support even for different bin-sizes $l_{D}$ (see Fig.S6). Therefore we treat it, as a first approximation, as being independent of $q$ and average over all bins to obtain $D \simeq \bar{D}=448$ $\mathrm{nm}^{2} / \mathrm{ms}$. Using this value of $D$ we perform Brownian Dynamics simulations of the fictitious Markovian process according to the Euler-Mayurama scheme. 

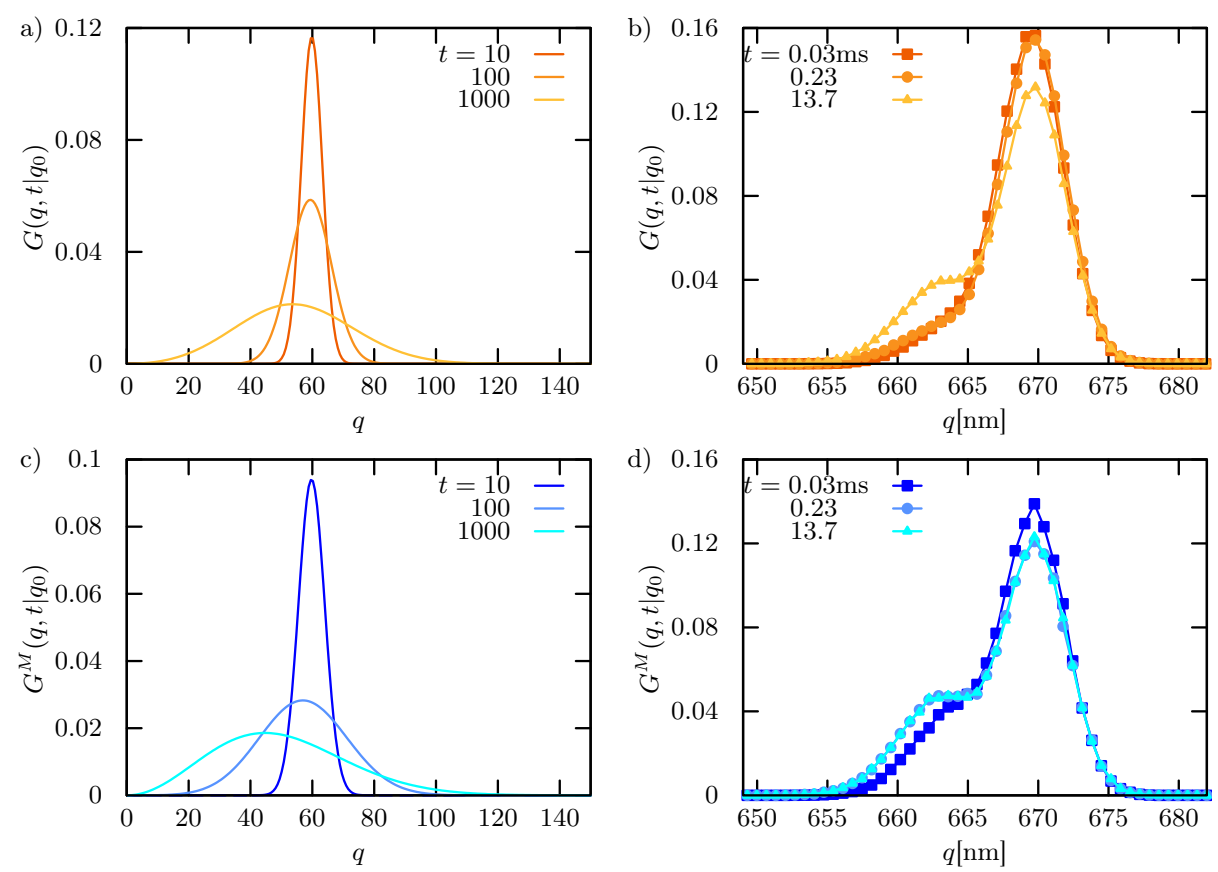

Figure S5. Green's function at different times for both considered systems. a) and b) depict the true Green's function for the end-to-end distance of the Rouse chain and of the DNA hairpin respectively. Panels c) and d) show the Green's function of their respective fictitious Markovian processes at the same times. The initial conditions are $q_{0}=60$ for the Rouse chain and $q_{0}=671 \mathrm{~nm}$ for the hairpin.

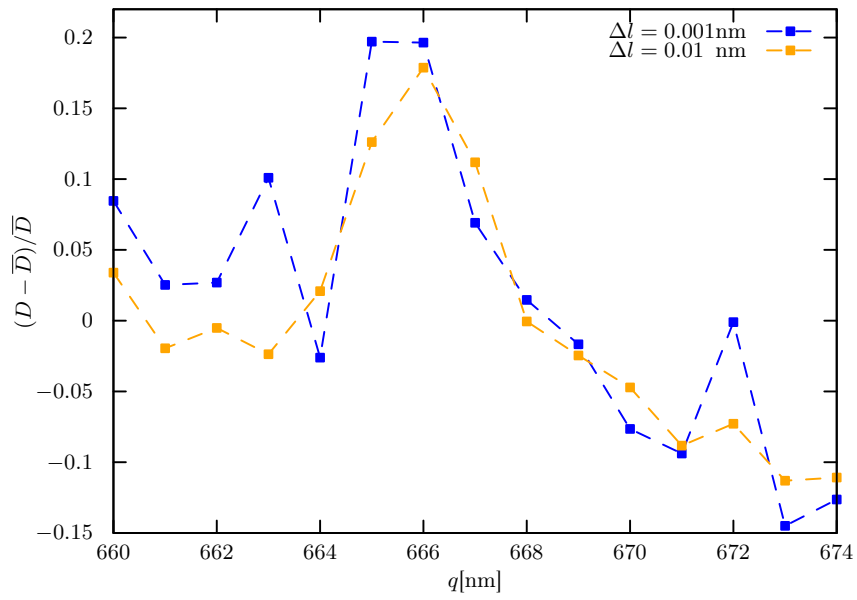

Figure S6. Relative deviation of the local diffusion coefficient in a given bin $D_{l}$ from the average value $\bar{D}=N^{-1} \sum_{l=1}^{N} D_{l}$ as a function of the position of the bin. In the case of $\Delta l=0.001 \mathrm{~nm}$ we find $\bar{D}=447 \mathrm{~nm}^{2} / \mathrm{ms}$ with a deviation $\pm 9 \mathrm{~nm}{ }^{2} / \mathrm{ms}$ and for $\Delta l=0.01 \mathrm{~nm}$ we find $\bar{D}=448 \mathrm{~nm}^{2} / \mathrm{ms}$ with a deviation $\pm 9 \mathrm{~nm}^{2} / \mathrm{ms}$. In a first approximation the values of $D_{l}$ are independent of $l$ and we thus set $D \approx \bar{D} \simeq 448 \mathrm{~nm}^{2} / \mathrm{ms}$. 
* agodec@mpibpc.mpg.de

[1] G. Wilemski and M. Fixman, Diffusion-controlled intrachain reactions of polymers. I Theory, J. Chem. Phys. 60, 866 (1974).

[2] A. Lapolla and A. Godec, Manifestations of Projection-Induced Memory: General Theory and the Tilted Single File, Front. Phys. 7, 10.3389/fphy.2019.00182 (2019). 


\section{Chapter 10}

\section{Ubiquitous Dynamical Time Asymmetry in Measurements on Materials and Biological Systems}

This article is currently under review. The pre-print is freely available on the ArXiv repository [57]. The article investigates a particularly striking effect of the memory generated by a projection. Specifically, we are interested in the breaking of the time-translation invariance, in other words: given a starting time $t_{0}$ and a waiting time $t_{1}, t_{1}>t_{0}$, we ask if and to which extent the evolution between times $t_{1}$ and $t_{1}+t_{0}$ depends on $t_{0}$. We find that (unless the probing times are very different or very close to each others) this effect naturally arises because of the memory generated by the projection. We prove that even if the full Markovian dynamics of the entire system evolves from equilibrated initial condition the breaking of the invariance is always present, as long as the projected observable does not evolve from its equilibrium distribution (which includes the case in which the equilibrium distribution is undersampled). We explicitely show our findings via theoretical models, experimental time-series, and time-series generated by computer experiments. 


\title{
Ubiquitous Dynamical Time Asymmetry in Measurements on Materials and Biological Systems
}

\author{
Alessio Lapolla, ${ }^{1}$ Jeremy C. Smith, ${ }^{2,3}$ and Aljaž Godec ${ }^{1, *}$ \\ ${ }^{1}$ Mathematical bioPhysics Group, Max Planck Institute for Biophysical Chemistry, Göttingen 37077, Germany \\ ${ }^{2}$ Center for Molecular Biophysics, Oak Ridge National Laboratory, Oak Ridge, Tennessee 37830, USA \\ ${ }^{3}$ Department of Biochemistry and Cellular and Molecular Biology, \\ University of Tennessee, Knoxville, Tennessee 37996, USA
}

\begin{abstract}
Many measurements on soft condensed matter (e.g., biological and materials) systems track lowdimensional observables projected from the full system phase space as a function of time. Examples are dynamic structure factors, spectroscopic and rheological response functions, and time series of distances derived from optical tweezers, single-molecule spectroscopy and molecular dynamics simulations. In many such systems the projection renders the reduced dynamics non-Markovian and the observable is not prepared in, or initially sampled from and averaged over, a stationary distribution. We prove that such systems always exhibit non-equilibrium, time asymmetric dynamics. That is, they evolve in time with a broken time-translation invariance in a manner closely resembling aging dynamics. We identify the entropy associated with the breaking of time-translation symmetry that is a measure of the instantaneous thermodynamic displacement of latent, hidden degrees of freedom from their stationary state. Dynamical time asymmetry is a general phenomenon, independent of the underlying energy surface, and is frequently even visible in measurements on systems that have fully reached equilibrium. This finding has fundamental implications for the interpretation of many experiments on, and simulations of, biological and materials systems.
\end{abstract}

\section{INTRODUCTION}

Relaxation refers to the dynamics of approaching a stationary state (e.g. thermodynamic equilibrium) and is a hallmark of non-equilibrium physics, from condensed matter [1-3] to single-molecule systems [4] initially perturbed near [3, 5-13] or far [14-19] from equilibrium. In extreme cases the non-stationary behavior of a system extends over all experimentally accessible time-scales a phenomenon often referred to as "aging" [20-24]. Aging is typically assumed to occur in systems whose energy landscapes contain a large number (scaling exponentially with the system size) of meta-stable states [20-25]. It has been observed in polymeric [26, 27], spin [28, 29] and colloidal glasses [30, 31], supercooled liquids [32-35] and recently in protein internal dynamics [36-39], where it may also affect biological function [40-43].

Typical manifestations of aging are a complex, nonexponential relaxation spectrum and non-stationary correlation and response functions $[26-34,36-39,44,45]$ that depend strongly and systematically on the time elapsed since the system was prepared [15, 26, 46-48] or, when derived from time-series measurements, on the duration of the observation $[39,45]$. The temporal extent of apparent aging dynamics in experimental systems (e.g. spin glass materials), although very long, may be finite [29]. Throughout we will refer to aging systems with experimentally observable equilibration as "transiently aging" irrespective of the precise manner in which the relaxation time depends on the system size.

Theoretical studies on aging have focused mainly

\footnotetext{
*agodec@mpibpc.mpg.de
}

on non-stationary correlations and responses $[24,45-$ $52]$ as well as generalizations to aging systems of the fluctuation-dissipation relation [14, 15, 53-55]. Aging dynamics has frequently been associated with the existence of deep traps with unbounded depth in the potential energy function $[21,23]$, fractal properties of the underlying free energy landscape $[36,37,56]$, the presence of disorder $[48,53]$, and other effects $[25,46,47,57,58]$.

Recent efforts in understanding relaxation dynamics that are not limited to systems with unobservable stationary states focus on diverse aspects of the thermodynamics of relaxation, e.g. the rôle of initial conditions in the context of the so-called "Mpemba effect" (i.e. the phenomenon where a system can cool down faster when initiated at a higher temperature) [16, 17], asymmetries in the kinetics of relaxation from thermodynamically equidistant temperature quenches [19], a spectral duality between relaxation and first-passage processes $[59,60]$, so-called "frenetic" concepts $[12,13]$, and the statistics of the 'house-keeping' heat $[61,62]$ and entropy production [63]. Important advances in understanding transients of relaxation also include information-theoretic bounds on the entropy production during relaxation far from equilibrium [18] and the so-called "thermodynamic uncertainty relation" for non-stationary initial conditions that bounds transient currents by means of the total entropy production [64].

Here, we look at non-stationary physical observables from a more general, "first principles" perspective. By directly analyzing the mathematical structure of the underlying multi-point probability density functions we reveal the universality of a broken time-translation invariance that we coin as dynamical time asymmetry (DTA). We prove the established linear aging correlation functions to be ambiguous indicators of broken time-translation in- 
variance. DTA has many of the properties commonly associated with aging but, unlike theoretical models of aging $[20-25,65]$, does not require any particular functional form of the dependence on the aging time nor that the relaxation time increases exponentially with system size and is therefore experimentally unobservable. Moreover, we here show that specific properties, such as deep traps in the potential energy function $[21,23]$, fractal properties of the underlying free energy landscape [36, 37, 56], or the presence of disorder $[48,53]$ that are often required for aging to occur, are not required for DTA dynamics, although they can amplify the breaking of time-translation invariance. In fact, DTA typically implies (transient) aging but the converse is not true. Instead, we prove DTA to emerge whenever (i) a physical observable corresponds to a lower-dimensional projection in configuration space that renders the reduced dynamics non-Markovian, and (ii) the projected physical observable is not prepared in, or initially sampled from and averaged over, a stationary distribution i.e., a distribution that does not change in time.

Most measurements on condensed matter correspond to projections of type (i), examples being structure factors in scattering experiments $[30,31,33,34,56]$, spectroscopic response functions (e.g. magnetization [28, 29, 53] and dielectric responses $[27,32,49])$, the rheology of soft materials [66,67], diverse empirical order parameters [45-47] and measurements of mechanical responses [26]. These projections also inevitably arise in single-particle tracking [34, 45, 56] and measurements of various reaction coordinates in all single-molecule experiments (e.g. internal distances) and simulations (e.g. projections onto dominant principal modes in Principal Component Analysis) [37-39, 41-43, 68-71].

In these measurements (i) applies as soon as the latent degrees of freedom (DOF) (those being effectively integrated out) evolve on a time-scale similar to the monitored observable. In contrast, (i) does not apply when the latent DOF relax much faster than the observable, for example when neglecting inertia and integrating out solvent degrees of freedom of a colloidal particle in a low Reynolds number environment. Condition (ii) applies whenever the observable evolves from a non-stationary initial condition. This includes all experiments involving an instantaneous perturbation of the observable in equilibrium (e.g. magnetization or dielectric, rheological and mechanical response), and all experiments involving evolution from a quench, such as in temperature, pressure, or volume (which inter alia includes scattering experiments on supercooled liquids). Condition (ii) also holds in situations where the observable is neither perturbed nor quenched but is initially under-sampled from equilibrium, that is, when it is sampled from equilibrium with a limited number of repetitions (say $1-10^{3}$ ) such as in single-molecule FRET, AFM or optical tweezers experiment, as well as particle-based computer simulations. This yields a distribution that does not converge to the invariant measure. In fact, as regards DTA we prove quenching and the under-sampling of equilibrium to be qualitatively equivalent. Whenever both conditions (i) and (ii) are fulfilled, DTA emerges irrespective of the details of the dynamics.

In the main text and in the examples we focus on systems whose dynamics obey detailed balance and, as a whole, are initially prepared at equilibrium. The monitored lower-dimensional observable is assumed to evolve from some non-equilibrium initial distribution (i.e. not the marginalized equilibrium distribution [72]). Generalizations to a non-equilibrium preparation of the full system (e.g. by a temperature quench) are discussed in detail the Appendix.

\section{THEORY}

We consider a mechanical system at least weakly coupled to a thermal reservoir, such that the full system's dynamics (i.e. all degrees of freedom; Fig. 1a, red trajectory) obeys a time-homogeneous Markovian stochastic equation of motion [73] (for details see Appendix), which generates ergodic dynamics in phase space. That is, starting from any initial condition the system is assumed to evolve to a unique stationary distribution in a finite, but potentially extremely long, time that may or may not be reached during an observation. This assumption is true for a vast majority of soft matter and biological systems of interest and also includes glassy materials. To impose only the mildest of assumptions we consider that the full system is prepared in an equilibrium state at $t=0$, i.e. the full system was created at a time $t=-\infty$ and the initiation of an experiment or phenomenon imposes a time origin at $t=0$, whereas the actual observation starts after some time $t_{a} \geq 0$ (see Fig. $1 \mathrm{~b}$ ), where $t_{a}$ is the so-called aging (or waiting) time and the measurement time-window is the time delay $\tau=t-t_{a}$. The more restrictive assumption of a non-stationary preparation (e.g. a temperature quench $[14,19]$ ) is treated in the Appendix B 2. In practice, a stationary preparation means that at $t=0$ the full system's configuration is distributed according to a stationary, invariant probability measure. This refers either to the initial statistical ensemble of configurations in a bulk system or to the repeated sampling of individual initial configurations (say in a single molecule experiment), which are drawn randomly from the invariant probability measure. We assume that only the projected observable is being monitored at all times $t \geq 0$. The assumptions stated above suffice to prove our claims (for details see Appendix).

For simplicity we use $\langle\cdot\rangle$ interchangeably to denote the average over an ensemble of trajectories at a given time and over time along a given trajectory, respectively, keeping in mind that they are identical only when the trajectory is much longer than the longest relaxation time $t_{\text {rel }}$. The state of the observable is denoted by $q(t) \in \Xi$ (Fig. 1a, black trajectory), which we assume, without loss of generality, to be one dimensional (for the general case 
a)

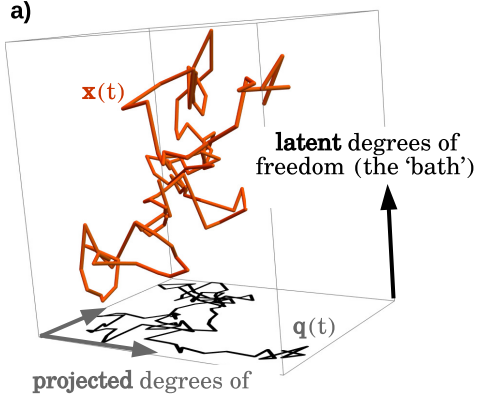

projected degrees of

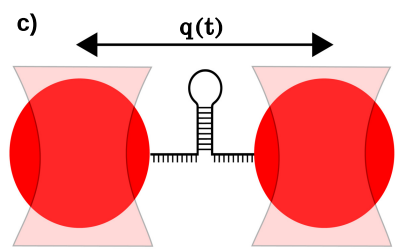

d) b)
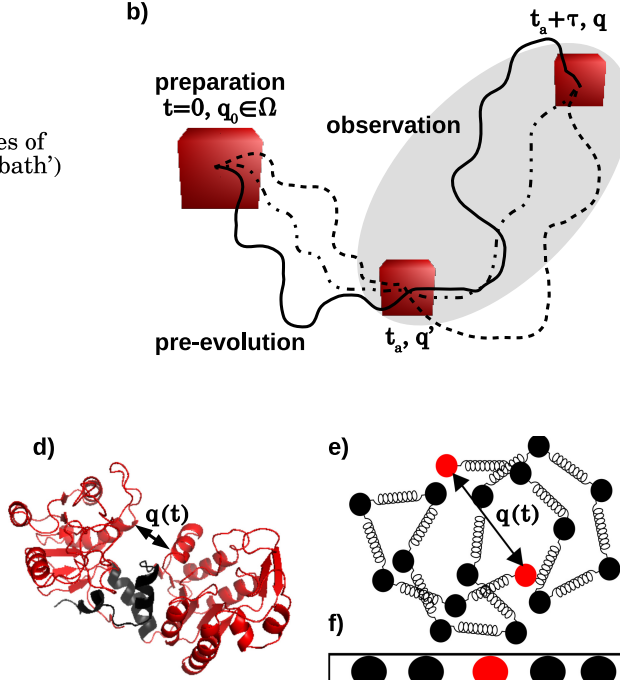

e)

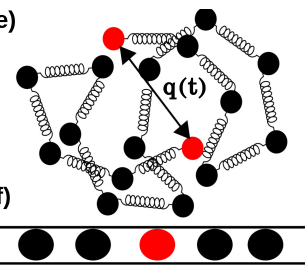

Figure 1. Schematics of projected observables, multi-point propagation and model systems. a) A physical observable corresponding to a simple lower-dimensional projection (shadow trajectory) of the full system's trajectory (red line), defining projected and latent (hidden) degrees of freedom. b) Trajectories of length $t$ evolving from preparation, through an aging (or pre-evolution) period of length $t_{a}$, followed by the observation of duration $\tau=t-t_{a}$. c) Optical tweezers experimental set-up probing DNA-hairpin dynamics; d) Structure of the yeast PGK protein with the reduced coordinate represented by the arrow. e) Rouse model of a polymer chain, comprising Hookean springs with a zero rest-length immersed in a heat bath. The reduced coordinate corresponds to the endto-end distance. f) Single file model with the tracer particle depicted in red. see the Appendix). Theoretically, each repetition of the experiment/process leads to an initial condition for $q(t)$ drawn randomly from the reduced stationary probability density $p_{\text {inv }}\left(q_{0}\right)$. In practice, however, this is not necessarily the case. For example, supercooled liquids [32-34] as well as polymeric [26, 27], spin [28, 29], and colloidal $[30,31]$ glasses are prepared by a quench in an external parameter (typically temperature) [26-31], such that the observable $q(t)$ nominally attains a non-stationary initial condition. A process may also start with the observable internally constrained to a subdomain of $p_{\text {inv }}\left(q_{0}\right)$, e.g. a chaperone stabilizing a particular configuration of a folded protein, with the biological process starting upon unbinding of the chaperone [74]. In another example single-molecule enzyme experiments may monitor the statistics of substrate turnover, where $q(t)$ reflects the geometry of the catalytic site of an enzyme that is reactive only for a specific sub-ensemble of configurations [4143]. Binding of a substrate molecule enforces an initial constraint on $q(t)$ thereby imposing non-stationary initial conditions on the chemical reaction. Alternatively, we may simply choose to initialize the experiment (i.e. reset our clock) a posteriori, such that $q(0)$ has a preset value, or we are dealing with a single, or a limited number of time-series [39] which do not sample $p_{\text {inv }}\left(q_{0}\right)$ sufficiently. In all these cases the observable is effectively not prepared in a stationary state, i.e. $p_{0}\left(q_{0}\right) \neq p_{\text {inv }}\left(q_{0}\right)$.

The dynamics in aging systems is conventionally analyzed via the normalized two-time correlation function $[15,28,45-48]$

$$
C_{t_{a}}(\tau)=\frac{\left\langle q\left(\tau+t_{a}\right) q\left(t_{a}\right)\right\rangle-\left\langle q\left(\tau+t_{a}\right)\right\rangle\left\langle q\left(t_{a}\right)\right\rangle}{\left\langle q\left(t_{a}\right)^{2}\right\rangle-\left\langle q\left(t_{a}\right)\right\rangle^{2}} .
$$

A system is often said to be aging if $C_{t_{a}}(\tau)$ strongly depends on $t_{a}$ in the sense that the relaxation of a system takes place on time-scales that grow with the age of the system $t_{a}$, and continue to do so beyond the largest times accessible within an experiment or simulation $[21,23$ $25,65]$.

However, the analysis and interpretation of time-series of physical observables that show DTA require a fundamentally different approach irrespective of whether equilibrium is attainable in an experiment or not. We prove below that $C_{t_{a}}(\tau)$ cannot conclusively indicate whether time-translation invariance is broken (see Appendix C, Lemma 2); in particular it cannot disentangle broken time-translation invariance from "trivial" correlations with a non-stationary initial condition (i.e. from "weak" or "second order" non-stationarity [75]). This is particularly problematic if one uses Eq. (1) as a "definition of DTA" to infer whether a complex experimental system, such as an individual protein molecule [38, 39], evolves with broken time-translation invariance or not. Eq. (1) is nevertheless reasonable, albeit sub-optimal, for quantifying DTA in materials that are known to posses a broken time-translation invariance.

Our aim is to conclusively and unambiguously infer whether relaxation evolves with a broken timetranslation invariance that is encoded in $G\left(q, t_{a}+\right.$ $\left.\tau \mid q^{\prime}, t_{a}, q_{0} \in \Omega_{0}\right)$, the probability density for the observable to be found in an infinitesimal volume element centered at $q$ at time $\tau+t_{a}$ given that it was at $q^{\prime}$ at time $t_{a}$ and started at $t=0$ somewhere in a subdomain $q_{0} \in \Omega_{0} \subset \Xi$ (Fig. 1b) with probability $p_{0}\left(q_{0}\right) . \Omega_{0}$ is strictly non-empty and may be a point, an interval or a union of intervals.

The dynamics of an observable $q(t)$ is generally said to be time-translation invariant (mathematically referred to as "strictly stationary" $[75,76]$ or "well-aged" [77]) if the underlying (effective) equations of motion that govern the evolution of $q(t)$ do not explicitly depend on time. That is, the probability of a path $\{q(t)\}$ for $t \in\left[t_{a}, t_{a}+\tau\right]$ does not depend on $t_{a}$. This is the case, e.g. in Newtonian dynamics or Langevin dynamics driven by Gaussian white noise [75] as well as generalized Langevin dynam- 
ics driven by stationary Gaussian colored noise [78-80]. Here $q(t)$ is said to be time-translation invariant if and only if (see also Definition 1 in Appendix C)

$$
G\left(q, t_{a}+\tau \mid q^{\prime}, t_{a}, q_{0} \in \Omega_{0}\right)=G\left(q, t^{\prime}+\tau \mid q^{\prime}, t^{\prime}, q_{0} \in \Omega_{0}\right),
$$

holds for any $\tau$ and $t^{\prime}$ [81]. Conversely, if time-translation invariance is broken we say that the system is dynamically time asymmetric. That is, time-translation invariance is broken if and only if the two-point conditioned Green's function $G\left(q, t_{a}+\tau \mid q^{\prime}, t_{a}, q_{0} \in \Omega_{0}\right)$ depends on $t_{a}$ (see also Definition 2 in Appendix C). The two-point conditioned Green's function is defined as

$$
G\left(q, t_{a}+\tau \mid q^{\prime}, t_{a}, q_{0} \in \Omega_{0}\right) \equiv \frac{P\left(q, t_{a}+\tau, q^{\prime}, t_{a}, q_{0} \in \Omega_{0}\right)}{P\left(q^{\prime}, t_{a}, q_{0} \in \Omega_{0}\right)},
$$

where $P\left(q, t_{a}+\tau, q^{\prime}, t_{a}, q_{0} \in \Omega_{0}\right)$ denotes the joint density of $q(t)$ to be found initially within $\Omega_{0}$ and to pass $q^{\prime}$ at time $t_{a}$ and to end up in $q$ at time $t_{a}+\tau$, and $P\left(q^{\prime}, t_{a}, q_{0} \in\right.$ $\left.\Omega_{0}\right)$ the joint density of $q(t)$ to be found initially within $\Omega_{0}$ and to pass $q^{\prime}$ at time $t_{a}$.

Note that there seems to be some relation between DTA and aging. A system is typically said to be aging if $C_{t_{a}}(\tau)$ in Eq. (1) depends on $t_{a}$ (i.e. that $q(t)$ is weakly non-stationary) but in a specific manner, e.g. the socalled "slow", non-stationary component of $C_{t_{a}}(\tau)$ must scale for all large $t_{a}$ as some power of $\tau / t_{a}[21,23,24]$ (for a rigorous discussion see [65]). However, this does not require that time-translation invariance (i.e. Eq. (2)) is broken [21, 23, 24]. So-called kinetically constrained models [25] and the spherical p-spin model [52, 53, 82], for example, have correlation functions Eq. (1) that show aging, but, when fully observed and not averaged over disorder (and only then), satisfy Eq. (2. Clearly, if timetranslation invariance is broken (see also Definition 1 in the Appendix C) then $C_{t_{a}}(\tau)$ automatically depends on $t_{a}$. If the dynamics is furthermore such that $C_{t_{a}}(\tau)$ depends on $t_{a}$ as some power of $\tau / t_{a}$ (see Eq. (8) below as well as Eqs. (C7) and (C10) as well as [45, 83, 84]) and, in addition, equilibrium cannot be attained during an observation then DTA also implies aging dynamics. However, the converse is not true.

To connect the aging correlation function in Eq. (1) with Eq. (2) we note that the numerator in Eq. (1) involves averages

$$
\begin{aligned}
& \langle q(t)\rangle \equiv \int_{\Xi} q G\left(q, t \mid q_{0} \in \Omega_{0}\right) d q \\
& \left\langle q\left(\tau+t_{a}\right) q\left(t_{a}\right)\right\rangle \equiv \int_{\Xi} \int_{\Xi} q q^{\prime} G\left(q, \tau+t_{a}, q^{\prime}, t_{a} \mid q_{0} \in \Omega_{0}\right) d q d q^{\prime}
\end{aligned}
$$

where the conditional density of the projected observable $G\left(q, t \mid q_{0} \in \Omega_{0}\right)$ is discussed in $[19,85]$ and in Appendix B 2 (see Eq. (B2)). The three-point conditional probability density $G\left(q, \tau+t_{a}, q^{\prime}, t_{a} \mid q_{0} \in \Omega_{0}\right)$ - the probability density for the observable to pass through an infinitesimal volume element centered at $q^{\prime}$ at time $t_{a}$ and end up in $q$ at time $\tau+t_{a}$ having started at $t=0$ in a subdomain $q_{0} \in \Omega_{0} \subset$
$\Xi$ with probability $p_{0}\left(q_{0}\right)$, is defined as (for details see Appendix B 2, Eq. (B20))

$$
G\left(q, t_{a}+\tau, q^{\prime}, t_{a} \mid q_{0} \in \Xi_{0}\right) \equiv \frac{P\left(q, t_{a}+\tau, q^{\prime}, t_{a}, q_{0} \in \Omega_{0}\right)}{P\left(q_{0} \in \Omega_{0}\right)} .
$$

Based on the mathematical properties of $G\left(q, t_{a}+\right.$ $\left.\tau \mid q^{\prime}, t_{a}, q_{0} \in \Omega_{0}\right)$ and $G\left(q, t_{a}+\tau, q^{\prime}, t_{a} \mid q_{0} \in \Xi_{0}\right)$ we prove in the Appendix C (see Theorem 1, Corollary 1.1 and, Lemma 2) that $C_{t_{a}}(\tau)$ in Eq. (1) can show a $t_{a^{-}}$ dependence even if Eq. (2) is satisfied, i.e. when the system is time-translation invariant. That is, if the system is dynamically time asymmetric then $C_{t_{a}}(\tau)$ depends on $t_{a}$, whereas the converse is not necessarily true. In turn this implies that one cannot determine on the basis of $C_{t_{a}}(\tau)$ derived from a time-series $q(t)$ whether time-translation invariance is broken, and a definitive and unambiguous indicator must be sought for.

We demonstrate this using the cleanest and most elementary example of a time-translation invariant system - a Brownian particle confined to a box of unit length (i.e. $L=1$ ) evolving from a a point $\Omega_{0}=x_{0}$ and from a uniform distribution within an interval $\Omega_{0}=[a, b]$ for some $0<a<b<1$. For this example the denominator in Eq. (3) is defined as $P\left(q^{\prime}, t, q_{0} \in \Omega_{0}\right) \equiv \int_{a}^{b} Q\left(q^{\prime}, t \mid q_{0}\right) d q_{0}$ and the numerator as $P\left(q, t_{a}+\tau, q^{\prime}, t_{a}, q_{0} \in \Omega_{0}\right) \equiv$ $Q\left(q, \tau+t_{a} \mid q^{\prime}\right) P\left(q^{\prime}, t_{a}, q_{0} \in \Omega_{0}\right)$, where $Q\left(x, t \mid x_{0}\right)$ denotes the propagator of the confined Brownian particle. Plugging into Eq. (3) confirms the validity of Eq. (2) and hence time-translation invariance. Nevertheless, the very same system exhibits a $t_{a}$-dependence of the aging autocorrelation function defined in Eq. (1) over more than two orders of magnitude in time measured in units of the relaxation time $t_{\text {rel }}=L^{2} / D \pi^{2}$ as depicted explicitly in Fig. 2. Note that by allowing the box to become macroscopic in size (i.e. $L \rightarrow \infty$ ) the relaxation time and thereby the extent of the $t_{a}$-dependence can become arbitrarily large when expressed in absolute units.

A general mathematical analysis (see Appendix B 2) therefore necessarily ties DTA to the three-point (nonMarkovian) conditional probability density, $G(q, \tau+$ $\left.t_{a}, q^{\prime}, t_{a} \mid q_{0} \in \Omega_{0}\right)$. If the projected dynamics is Markovian it is in turn fully described by two-point conditional densities $G_{\text {Markov }}\left(q, \tau+t_{a}, q^{\prime}, t_{a} \mid q_{0} \in \Omega_{0}\right)=$ $G\left(q, \tau \mid q^{\prime}, 0\right) G\left(q^{\prime}, t_{a} \mid q_{0} \in \Omega_{0}\right)$. If, on the other hand, the reduced dynamics is non-Markovian but the initial condition is sampled from the full (invariant) stationary density $p_{0}\left(q_{0}\right) \rightarrow p_{\text {inv }}\left(q_{0}\right)$ (or equivalently, $\Omega_{0}=\Xi$ ), we have $G\left(q, \tau+t_{a}, q^{\prime}, t_{a} \mid q_{0} \in \Xi\right)=G\left(q, \tau \mid q^{\prime}, 0\right) p_{\text {inv }}\left(q^{\prime}\right)$. In both cases there is no DTA (see Appendix C). To quantify broken time-translation invariance on the level of reduced phase space probability densities we therefore define the 

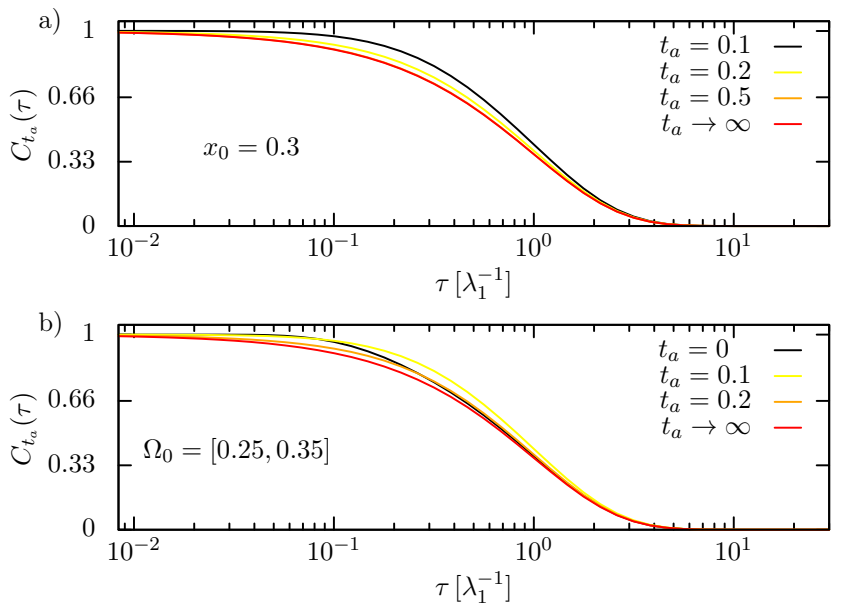

Figure 2. Aging correlation functions display fictitious dynamical time asymmetry in time-translation invariant systems. Analytical results for the aging correlation function $C_{t_{a}}(\tau)$ defined in Eq. (1) for a Brownian particle confined to a unit box evolving from a) the point $\Omega_{0}=0.3$ and b) the interval $\Omega_{0}=[0.25,0.35]$ for several values of the aging time $t_{a}$. Time $\tau$ is expressed in units of the relaxation time $\lambda_{1}^{-1}$.

\section{time asymmetry index as}

$$
\begin{aligned}
\Upsilon_{\Omega_{0}}\left(t_{a}, \tau\right) \equiv \int_{\Omega} d q & \int_{\Omega} d q^{\prime}\left[G\left(q, \tau+t_{a}, q^{\prime}, t_{a} \mid q_{0} \in \Omega_{0}\right) \times\right. \\
& \left.\ln \frac{G\left(q, \tau+t_{a}, q^{\prime}, t_{a} \mid q_{0} \in \Omega_{0}\right)}{G\left(q, \tau \mid q^{\prime}\right) G\left(q^{\prime}, t_{a} \mid q_{0} \in \Omega_{0}\right)}\right]
\end{aligned}
$$

where for notational convenience we henceforth drop the explicit dependence on $\Omega_{0}$, i.e. $\Upsilon_{\Omega_{0}}\left(t_{a}, \tau\right) \equiv \Upsilon\left(t_{a}, \tau\right)$. The time asymmetry index measures the relative entropy between the actual evolution of the observable and a corresponding "fictitious" dynamics that has the same probability density of the intermediate point $q$ at time $t_{a}$ but where at time $t_{a}$ the latent degrees of freedom are instantaneously quenched to equilibrium. Broken time translation invariance reflects that the effective equations of motion that govern the evolution of $q(t)$ change in time as a result of the relaxation of the hidden DOF the observable is coupled to. That is, if one were e.g. to derive an effective generalized Langevin equation for $q(t)$ the latter would contain a memory kernel and noise that depend explicitly on the time elapsed since the preparation of the system (see e.g. [86]).

A broken time-translation invariance is evidently a clear signature of non-equilibrium dynamics and therefore intimately related to entropy production. $\Upsilon$ may thus also be given a thermodynamic interpretation as an entropy associated with the breaking of time-translation invariance in analogy to the "instantaneous excess free energy" - the relative entropy between $G\left(q, t \mid q_{0} \in \Omega_{0}\right)$ and $p_{\text {inv }}(q)$ [19, 87-89]. Therefore it appears that the entropy of breaking time-translation invariance measures the instantaneous thermodynamic displacement of latent degrees of freedom at time $t_{a}$ from their stationary state. Note that $\Upsilon\left(t_{a}, \tau\right)>0$ also implies a violation of the fluctuation-dissipation theorem for non-Markovian system because it implies that the "bath" is non-stationary [90]. In general $\Upsilon$ is experimentally measurable simply by monitoring the time-series of the observable $q(t)$ (for details see Appendix D 4).

The relative entropy is a pseudo-metric and therefore the absolute value of the time asymmetry index (other than $\Upsilon\left(t_{a}, \tau\right)=0$ implying time-translation invariance and $\Upsilon\left(t_{a}, \tau\right)>0$ its violation) does not necessarily immediately allow for a quantitative comparison of DTA in different systems with disparate dimensionality. It is always meaningful when one considers a comparison of the same system and observable under different conditions (e.g. initial conditions, values of control parameters etc.). If one aims at comparing quantitatively DTA in different systems and/or observables one should instead consider a symmetrized version of the relative entropy (see e.g. [91]).

The time asymmetry index is constructed to detect and quantify conclusively broken time-translation invariance according to Eq. (2). It effectively measures the instantaneous relaxation of the latent degrees of freedom and is unaffected by spurious non-stationarity due to correlations between the value of the observable at time $t_{a}+\tau$ and the particular "initial" value at time $t_{a}$. These correlations are spurious because they exist for any $t_{a}$ and relax as a function of $\tau$ irrespective of whether a system is time-translation invariant or not.

By construction $\Upsilon\left(t_{a}, \tau\right) \geq 0$ and is identically zero for any $t_{a}$ and $\tau$ if and only if $q(t)$ is time-translation invariant. In turn, the observable $q(t)$ is time-translation invariant if and only if it is Markovian and/or $q(t=0)$ is sampled from a distribution converging in law to the invariant measure (the proof is presented in the Appendix C, Theorem 2 and Corollary 1.1). As a result $\Upsilon\left(t_{a}, \tau\right)$ is identically zero for all $\tau$ and $t_{a}$ for the timetranslation invariant dynamics of a confined Brownian particle evolving from a non-equilibrium initial condition (see, however, the fictitious DTA due to weak nonstationarity that is implied by the aging autocorrelation function in Fig. 2). Moreover, the extent of DTA is limited by the relaxation time $t_{\text {rel }}$ such that $\Upsilon\left(t_{a}, \tau\right) \rightarrow 0$ whenever $t_{a} \gg t_{\mathrm{rel}}$ or $\tau \gg t_{\mathrm{rel}}$. Obviously, if the full system is initially quenched into any non-stationary initial condition (see e.g. [19]), then $\Upsilon\left(t_{a}, \tau\right)>0$ as long as the projection renders the reduced dynamics non-Markovian. Therefore, as soon as $\Upsilon\left(t_{a}, \tau\right) \neq 0$ for some values $t_{a}$ and $\tau$ smaller than $t_{\text {rel }}$, the dynamics is time asymmetric, in specific cases with a self-similar scaling (see Appendix C, Propositions $1 \& 2$ ). In addition the following generic structure emerges:

$$
C_{t_{a}}(\tau)=(1-\varphi) g_{1}(\tau)+\varphi g_{2}\left(\tau, t_{a}\right),
$$

with $0<\varphi<1$ and $g_{1,2}$ depending on the details of the 
dynamics (see Appendix C, Theorem 3) in agreement with the properties of aging systems [15, 28, 45-49, 54]. These results are universal - they are independent of details of the dynamics, and, in particular, the underlying energy landscape.

Microscopically reversible dynamics in general allows for a spectral expansion of propagators and thus correlation and response functions (see e.g. Appendix B). Moreover, in specific cases the projection renders the observed dynamics self-similar with parameter $\alpha$, that is, a change of time-scale merely effects an $\alpha$-dependent renormalizion of the spectrum (for details see Definition 4 in the Appendix B 2). This arises, for example, when the observable corresponds to an internal distance within a single polymer molecule [92] (studied here in Figs. 3a and 4) or within individual protein molecules [93, 94], as well as in diffusion on fractal objects [95]. The aging correlation function in Eq. (1) then displays a power-law scaling for $\alpha>0$ (as in Fig. 3d and Eq. (C5) in the Appendix C) or, when $\alpha=0$ a logarithmic behavior (as observed in [38]; see also Eq. (C8) in the Appendix C). The latter is mathematically equivalent to the logarithmic relaxation found in [96]. For more details see Propositions 1 and 2 in the Appendix C, respectively. In particular for $\tau / t_{a} \gg 1$, in the glassy literature referred to as the "full aging" [20, 49, 96, 97] regime, we find (see Appendix C, Eqs. (C7) and (C10))

$$
C_{t_{a}}(\tau) \simeq A+ \begin{cases}B_{\alpha}\left(\frac{t_{a}}{\tau}\right)^{\alpha} & , \alpha>0, \\ B_{\alpha}\left(\frac{t_{a}}{\tau}\right) & , \alpha=0 .\end{cases}
$$

with constants $A$ and $B_{\alpha}$ that depend on the details of the dynamics. On a transient time-scale the asymptotic results in Eq. (8) agree with predictions of minimalistic "trap" models $[21,23,24]$ as well as fractional dynamics and random walks with diverging waiting times $[45,58,98]$ (for more details see also Remark 2.1 in the Appendix C). Fractional dynamics and random walks with long waiting times (that as well display DTA $[83,84,98])$ were in fact explicitly shown to arise as transients in projected dynamics when the latent degrees of freedom are orthogonal to $q(t)$ [85] and in the spatial coarse-graining of continuous dynamics on networks [99]. The phenomenology of systems displaying an algebraic scaling of $C_{t_{a}}(\tau)$ as in Eq. (8) is therefore by no means unique, and represents only a specific class of dynamical systems with a broken time-translation invariance. Dynamical time asymmetry is much more general.

\section{EXAMPLES}

It is not difficult to verify the above claims in practice as all corresponding quantities can readily be obtained from experimental or simulation-derived time-series. To that end we analyze DTA in four very different systems (see Fig. 1c-e): DNA hairpin dynamics measured by dual optical tweezers experiments, where $q(t)$ reflects the end- to-end distance (Fig. 1c and Appendix D 4 a) [68, 69], extensive MD simulations of internal motions of yeast PGK, where $q(t)$ corresponds to the inter-domain distance (Fig. 1d and Appendix D 4 b) [39], as well as two theoretical examples: the end-to-end distance fluctuations of a Rouse polymer chain [100] (Fig. 1e and Appendix D8) and tracer particle dynamics in a single file of impenetrable diffusing particles, where $q(t)$ reflects the position of the tracer particle [19, 85, 101] (Fig. If and Appendix D 3). The underlying energy landscapes of these four systems are fundamentally very different; the DNA-hairpin exhibits two well-defined metastable conformational states/ensembles [68, 69], the yeast PGK has a very rugged and apparently fractal energy landscape [39], that of the Rouse polymer is perfectly smooth and exactly parabolic, and that of the single file is flat with the tracer motion confined to a hyper-cone as a result of the non-crossing condition between particles. Yet, despite these striking differences, all systems display the same qualitative time asymmetric behavior, consistent with the proven universality of DTA.

The aging correlation functions $C_{t_{a}}(\tau)$ and time asymmetry indices $\Upsilon\left(t_{a}, \tau\right)$ are shown in Fig. 3. With the exception of the PGK protein, which does not equilibrate within the duration of the trajectory, in agreement with previous findings [39], DTA is manifested as a transient phenomenon. The precise form of $C_{t_{a}}(\tau)$ depends on the details of the dynamics, which naturally vary between the systems. Moreover, the dependence of $C_{t_{a}}(\tau)$ on $t_{a}$ is non-monotonic. The generic form of $\Upsilon\left(t_{a}, \tau\right)$ displays an initial increase towards a plateau, followed by a longtime decay to zero, which can be understood as follows. Irrespective of the details a finite time is required in order to allow for a build-up of memory, that is, of correlations between the instantaneous state of the projected observable and the initial condition of the latent variables. The memory at some point reaches a maximum. Afterwards, the memory of the preparation of the system is progressively lost as a result of the mixing of trajectories in full phase space during relaxation. Due to a relatively higher sampling frequency and sufficiently long sampling times that extend beyond the relaxation time all these effects are resolved in the experimental DNA-hairpin data but not in the case of the PGK simulation.

Moreover, a hallmark of aging is that at least part of the relaxation of a system takes place on time-scales that grow with the age of the system $t_{a}$, and continue to do so up to the largest times accessible within an experiment or simulation. Interestingly, Figs. 3 and 4 show that the relaxation time increases (at least transiently) with the aging time, i.e. $\Upsilon\left(t_{a}, \tau\right)$ decays with $t$ more slowly as $t_{a}$ grows at least up to a threshold time. If an experiment or simulation does not reach this threshold time the breaking of time-translation invariance would seemingly take place on timescales that grow indefinitely, somewhat similar to the aging phenomenon. Note that the threshold time may become arbitrarily large in large systems (e.g. the relaxation time and thus the threshold time in natu- 

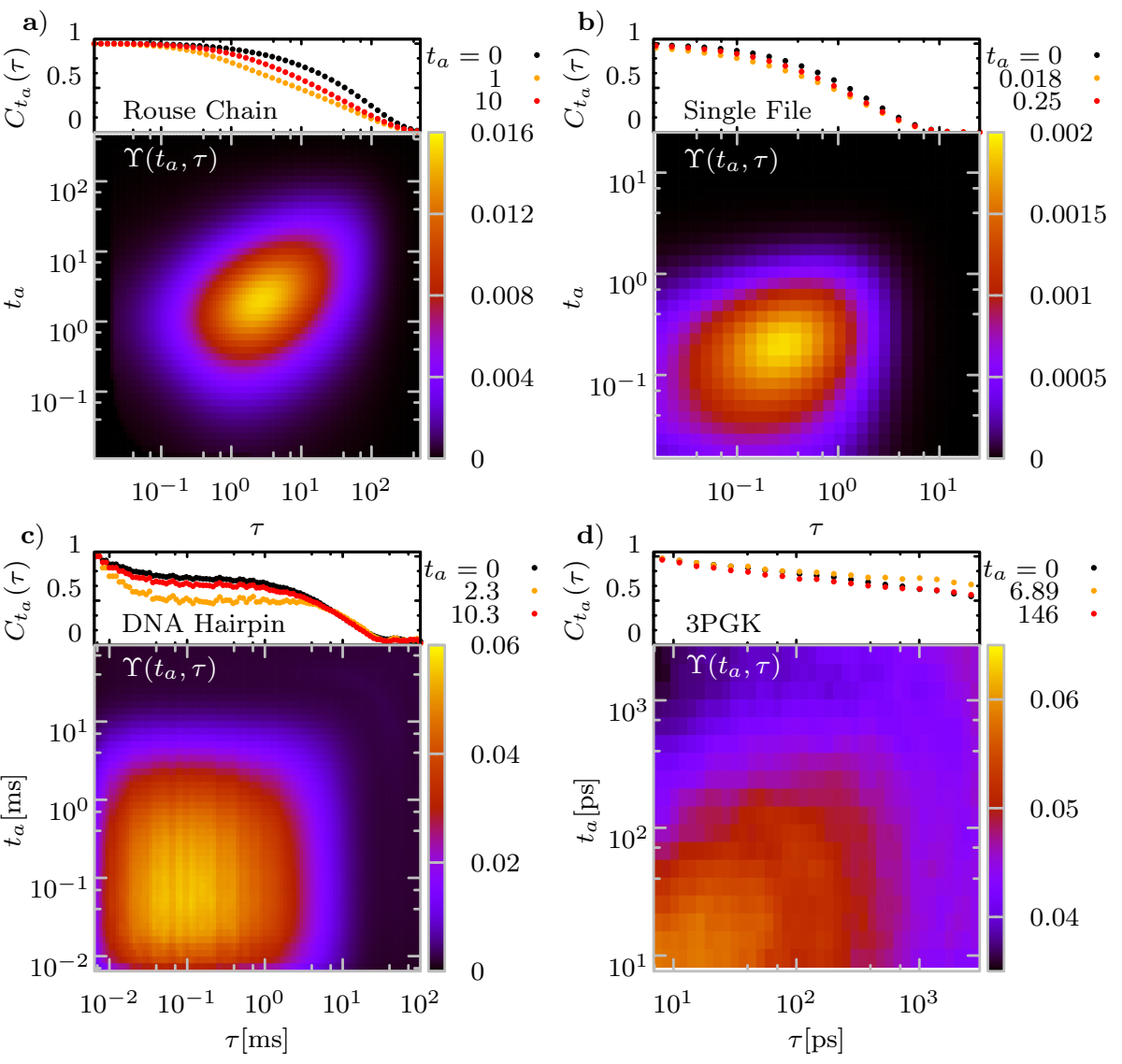

Figure 3. Aging two-point correlation function and time asymmetry index. $C_{t_{a}}(\tau)$ for different values of aging time $t_{a}$ and corresponding $\Upsilon\left(t_{a}, \tau\right)$ for: a) the Rouse polymer chain with 50 beads with initial end-to-end distance in dimensionless units equal to $q_{0}=9.85$, which corresponds to the most likely end-to-end distance (the dimensionless relaxation time here corresponds to $t_{\mathrm{rel}} \simeq 253.38$ ); b) tracer-particle dynamics in a single file with $N=5$ confined to a box of unit length, tagging the central particle particle with initial condition $q_{0}=0.5$ (the relaxation time measured in natural units of the "collision time" of is $t_{\mathrm{rel}} \simeq 2.5$ ), c) the DNA-hairpin extension determined from a trajectory of length of $2.75 \cdot 10^{4} \mathrm{~ms}$ sampled at $400 \mathrm{kHz}$. The initial condition was taken at the absolute maximum of equilibrium probability density $q_{0}=2.0 \pm 1 \mathrm{~nm}$, and $q$ refers to deviations from the mean distance $\langle d\rangle$, i.e. $q(t)=d(t)-\langle d\rangle$ (the relaxation time is $t_{\mathrm{rel}} \approx 15 \mathrm{~ms}$ ); The statistical error in determining $\Upsilon\left(t_{a}, \tau\right)$ from the hairpin data is less than 1\% (see Fig. D6 in the Appendix D $4 \mathrm{a}$ ); d) inter-domain motion between the centers of mass of the N-terminal (residues 1-185) and C-terminal domains (residues 200-389) in yeast PGK determined from a $200 \mathrm{~ns}$ atomistic MD simulation sampled every $150 \mathrm{ps}$. The initial condition was $q_{0}=0.01 \pm 0.2 \mathrm{~nm}$ relative to the average inter-domain distance $\langle d\rangle$, i.e. $q(t)=d(t)-\langle d\rangle$. c) was obtained from experimental data of Refs. [68, 69] and d) was determined from molecular dynamics simulations in Ref. [39]. Further details can be found in Appendix D. "Transient aging" in $C_{t_{a}}(\tau)$ arises whenever there is a region $\left(t_{a}, \tau\right)$ where $\Upsilon\left(t_{a}, \tau\right)>0$. In the case of PGK (panel d) $t_{\text {rel }}$ is not reached within the simulation time, which renders the system virtually eternally time asymmetric and "forever aging" [39, 102].

ral units for the Rouse polymer and single file grow with the number of particles as $\propto N^{2}$ (see e.g. Fig. D2 in the Appendix D8); for any duration of an observation one may find a $N$ that makes DTA appear as everlasting).

One appreciates that $\Upsilon\left(t_{a}, \tau\right)$ truly quantifies the degree of broken time-translation invariance and not correlations with the value of the observable at $t_{a}$. This is also the reason why $\Upsilon\left(t_{a}, \tau\right)$ decays to zero on a timescale shorter than $C_{t_{a}}(\tau)$. $C_{t_{a}}(\tau)$ starts at 1 and decays to zero as a result of "forgetting the initial condition". Because the probability density of being found at a given point always depends trivially on $t_{a} \neq 0$ (see Eq. (5)) irre- spective of whether time-translation invariance in Eq. (2) is broken or satisfied, $C_{t_{a}}(\tau)$ displays non-stationarity manifested in a $t_{a}$-dependence even for time-translation symmetric dynamics. Conversely, $\Upsilon\left(t_{a}, \tau\right)$ is constructed to not be affected by such spurious non-stationarity. Instead, it reflects how far the latent degrees of freedom are displaced from equilibrium at time $t_{a}$. In other words, $\Upsilon\left(t_{a}, \tau\right)$ compares the probability densities of the actual dynamics with those of fictitious dynamics that have the same probability density at time $t_{a}$ but in which at time $t_{a}$ the latent degrees of freedom are quenched to equilibrium (see Eq. (B25)). 

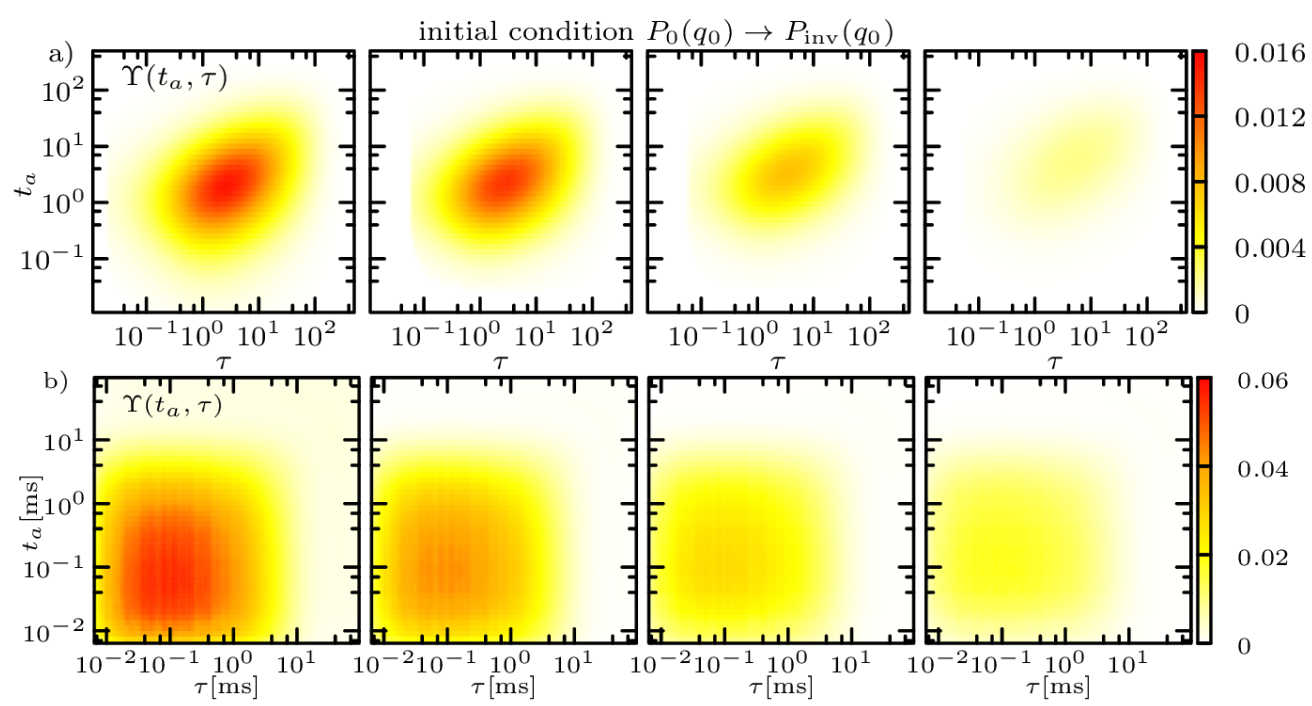

Figure 4. Attenuation and disappearance of dynamical time asymmetry upon approaching stationary initial conditions. Gradual vanishing of the time asymmetry index $\Upsilon\left(t_{a}, \tau\right)$ when the initial distribution of the projected coordinate $p_{0}\left(q_{0}\right)$ is sampled from a distribution being closer and closer to the density of the invariant measure, $p_{\text {inv }}\left(q_{0}\right)$ for: a) Rouse model of a polymer chain (the parameters are the same as in Fig. 3); The initial end-to-end distance is sampled from intervals (from left to right): $q_{0}=9.85, q_{0} \in[9-11], q_{0} \in[7-13]$ and $q_{0} \in[4-16]$, respectively. b) experimental data for the DNA-hairpin. The initial conditions (relative to the mean value $\langle d\rangle$, that is, $d(t)=q(t)+\langle d\rangle$ ) are sampled (from left to right) from the following intervals: $q_{0} \in[1,3] \mathrm{nm}, q_{0} \in[-3,7] \mathrm{nm}, q_{0} \in[-6,10]$ and $q_{0} \in[-8,12] \mathrm{nm}$, respectively. When the initial condition is sampled from a distribution closer to the invariant measure, DTA vanishes confirming the claims of our theory.

One can look at $\Upsilon\left(t_{a}, \tau\right)$ in two ways; as a function of $\tau$ at fixed $t_{a}$ and as a function of $t_{a}$ at fixed $\tau$. While the former intuitively reflects how the relaxation of the observable to equilibrium depends on the instantaneous ("initial") state of the latent degrees of freedom at time $t_{a}$, the latter measures how the correlation of the value of the observable at two times separated by $\tau$ changes due to the relaxation of the latent degrees of freedom to equilibrium. The time asymmetry index therefore provides access to the dynamics of hidden degrees of freedom coupled to the observable through an analysis of time-series derived from measurements on the observable.

A verification that a breaking of time-translation invariance occurs whenever the distribution of initial conditions sampled by the experiment has not converged to the equilibrium distribution follows from inspection of $\Upsilon\left(t_{a}, \tau\right)$ evolving from an ensemble of initial conditions being closer and closer to an equilibrium distribution, i.e. $\Omega_{0} \rightarrow \Xi$ (see Fig. 4 for the Rouse chain and DNAhairpin). Indeed, $\Upsilon\left(t_{a}, \tau\right)$ progressively vanishes when the initial condition becomes sampled from a distribution approaching the invariant measure, $p_{0}\left(q_{0}\right) \rightarrow p_{\text {inv }}\left(q_{0}\right)$. In the Appendix $\mathrm{C}$ we prove that this is a general effect (Theorem 1), independent of any details of the dynamics.

\section{DISCUSSION}

Non-stationary behavior of physical observables is traditionally considered as being important in systems with glassy, aging dynamics, such as polymer, spin or colloidal glasses, that attain glassy properties upon a quench in an external parameter [26-31]. During, for example, a temperature quench, the system (e.g. a supercooled liquid or a set of spins) at some point cannot keep pace with rapid changes in the bath, and is pushed out of equilibrium [14]. After the quench at $t=0$ the observable is thus (at least weakly) non-stationary - it is sampled from and averaged over a non-equilibrium ensemble, i.e. $p_{0}\left(q_{0}\right) \neq p_{\text {inv }}\left(q_{0}\right)$. The absence of such an obvious quench rendered the origin of non-stationary, apparent aging behavior in biological macromolecules somewhat mysterious [36, 37, 3943]. However, in biological systems the observable can become quenched implicitly, e.g. by the 'locking in' of a protein's configuration by a chaperone [74], the configurational requirements for enzymatic catalysis [41-43], or simply by the under-sampling of equilibrium such as in single-molecule experiments and particle-based computer simulations [39], such that $p_{0}\left(q_{0}\right) \neq p_{\text {inv }}\left(q_{0}\right)$. In an experiment one can check for non-stationarity of initial conditions, e.g. by inspecting whether histograms of the observable (also referred to as the "occupation time fraction" or "empirical density") at $t=0$ and at all later times coincide [103].

Here, we highlight a more general and wide-spread as- 
pect of out-of-equilibrium dynamics of physical observables - dynamical time asymmetry. The requirements for DTA to occur are much weaker than for aging, and it is manifested in a very broad variety of experimental situations, and in particular, one may also expect aging physical observables probed in many experiments to display DTA. Even measurements on polymer, spin and colloidal glasses have built-in underlying projections. For example, in tensile creep experiments in polymeric glasses the motion in a (cold) polymer is projected onto a local, effectively one-dimensional flow [26]. In supercooled liquids and colloidal glasses the dynamics is typically projected onto local particle displacements, pair correlation functions and structure factors $[30,31,33,34]$. In bulk experiments with spin glasses and supercooled liquids one measures quantities such as the average single-spin autocorrelation function $[21,104]$, magnetization, conductance or the dielectric constant, which correspond to projections of many-particle dynamics onto a scalar parameter $[29,32,49]$. In biological macromolecules the projection may correspond to [37, 39] or depend on [41-43] some internal distance within the macromolecule. These projections lead to non-Markovian observables evolving from non-stationary initial conditions which are in turn expected to show DTA. In fact we can appreciate that the physical origin of DTA in both, 'traditional' glassy systems [26-31] and biological matter [36, 37, 39-43], is qualitatively the same and simply results from nonstationary initial conditions of non-Markovian observables (see Observation 2 in the Appendix C). In most of these aforementioned systems the dynamics is also aging $[26-31,36,37,39]$.

It is important to realize that it is not possible to infer from a finite measurement whether the observed process is genuinely non-ergodic (i.e. a result of some true localization phenomenon in phase space) or whether the observation is made on an ergodic system but on a timescale shorter the relaxation time [85] (note that a comparison of the dynamics of PGK in Fig. 3d with a transient shorter than the relaxation time in any of the remaining examples in Fig. 3a-c shows no qualitative difference). A theoretical description of both scenarios on time-scales shorter than the relaxation time is in fact identical (for details see [85] as well as [24] in the context of glasses).

Although sporting characteristics commonly associated with aging, DTA and aging are not quite the same thing. DTA does not require the relaxation to take place on time-scales that grow indefinitely with the age of the system $t_{a}$ beyond the largest times accessible within an experiment or simulation, nor does it impose requirements on the precise form of the dependence on $t_{a}$. It is likely to be a ubiquitous phenomenon that is frequently observed in measurements of projected observables. In turn, aging does not imply a broken time-translation invariance according to Eq. (2).

Note, however, that many paradigmatic models of aging dynamics (e.g. continuous-time random walks with diverging mean waiting times and fractional diffusion
$[45,83,84])$ display a (strongly) broken time-translation invariance. Furthermore, most experimental observations of aging dynamics monitor projected observables, e.g. magnetization, single-spin auto-correlation functions averaged over the sample and potentially also over disorder $[26-31,36,37,39]$. The dynamics of these observables is thus almost surely non-Markovian [85] and expected to display DTA.

The observation of $\Upsilon\left(t_{a}, \tau\right)>0$ on a given scale of $t_{a}$ and $\tau$ implies that the dynamics of the observable $q(t)$ fundamentally changes in the course of time as a results of the relaxation of hidden DOF, and does not reflect correlations with the value of the observable at zero time $q(0)$. That is, the effective equations of motion for $q(t)$ truly change in time. In biological systems and in particular enzymes and other protein nanomachines non-stationary effects are thought to influence function, e.g. memory effects in catalysis [41-43]. This is particularly important because some larger proteins potentially never relax within their life-times, i.e. before they become degraded (note that relaxation corresponds to attaining the spontaneous unfolding-refolding equilibrium). This renders the dynamically time asymmetric regime virtually 'forever lasting' and implies that the system is aging [39]. As proteins are produced in the cell in an ensemble of folded configurations under the surveillance of chaperones [74], our theory implies that DTA during function [41-43] should arise naturally and generically due to the memory of a protein's preparation.

We expect DTA to be particularly pronounced in measurements on systems with entropy-dominated, temporally heterogeneous collective conformational dynamics involving (transient) local structure-formation where the background DOF evolve on the same time-scale as the observable [38], and we suggest the breaking of time-translation invariance to be closely related to the phenomenological notion of "dynamical disorder" in biomolecular dynamics [41-43, 71].

Our results have some intriguing implications. First, a quench in an external parameter and the mere undersampling of equilibrium distributions give rise to qualitatively equivalent manifestations (but potentially with a largely different magnitude and duration) of DTA as soon as the observable follows a non-Markovian evolution (see Appendix C, Observation 2). This has important practical consequences in fields such as single-molecule spectroscopy and computer simulations of soft and biological matter, which often suffer from sampling constraints. Second, broken time-translation invariance is 'in the eye of the beholder', insofar as its degree depends on the specific observable; there should exist a (potentially less) reduced coordinate, not necessarily accessible to experiment (e.g. when we follow all degrees of freedom), according to which the same system will exhibit virtually time-translation invariant dynamics. However, auto-correlation functions will show a $t_{a}$-dependence for essentially any non-stationary initial condition in any system. 
A broken time-translation invariance was shown to be linked to a form of entropy embodied in a time asymmetry index that is a measure of the instantaneous thermodynamic displacement of latent, hidden degrees of freedom from their stationary state. The time asymmetry index may therefore be used to probe systematically the timescale of dynamics of hidden, slowly relaxing degrees of freedom relative to the time-scale of the evolution of the observable. In particular, it may be useful as a practical tool to discriminate between situations where the hidden degrees of freedom evolve through a sequence of local equilibria that would yield small values of the time asymmetry index $\Upsilon$ from those cases where their evolution is transient and slow on the time-scale of the observable thus implying a significant $\Upsilon$. For example, $\Upsilon$ may potentially provide additional insight into the dominant folding mechanism of a protein from single-molecule force-spectroscopy data [105], in particular about the much debated heterogeneity of folding trajectories and its functional relevance $[106,107]$.

The present theory ties dynamical time asymmetry in a general setting to both the non-stationary preparation of an observable and its non-Markovian time evolution. Thereby it connects aspects of the better known phenomenology of aging of projected observables with the broken time-translation invariance observed in recent measurements on in soft and biological materials on a common footing. Moreover, dynamical time asymmetry is suggested to be a ubiquitous phenomenon in biological and materials systems.

\section{ACKNOWLEDGMENTS}

We thank Krishna Neupane and Michael T. Woodside for providing unlimited access to their DNA-hairpin data and Peter Sollich for clarifying discussion about physical aging and critical reading of the manuscript. The financial support from the Deutsche Forschungsgemeinschaft (DFG) through the Emmy Noether Program "GO 2762/1-1" (to AG) and from the Department of Eenergy through the grant DOE BER FWP ERKP752 (to JCS) are gratefully acknowledged. 


\begin{abstract}
APPENDIX
In this Appendix we present the main theorems needed for the article with the corresponding proofs. We treat the problem in a general setting, that is, not assuming that the full system is initially prepared in equilibrium. Further included are analytical results with details of calculations for the Rouse polymer and single file diffusion, all details of the numerical analyses of the DNA-hairpin and protein PGK data and further supporting results.
\end{abstract}

\title{
CONTENTS
}

A. Definitions, notation and preliminaries

B. Dynamics of the projected lower-dimensional observable 3

1. Spectral theory of projected dynamics 3

2. Three-point dynamics and breaking of time-translation invariance 5

C. Main theorems with proofs 6

D. Physical models, experimental and simulation data 10

1. Fictitious dynamical time asymmetry in a time-translation invariant system: the Brownian particle in a box10

2. Rouse polymer model 11

3. Single file diffusion 15

4. Analysis of experimental and simulation data 17

$\begin{array}{ll}\text { a. DNA-hairpin } & 18\end{array}$

b. Yeast 3-phosphoglycerate kinase (PGK) 20

References

\section{Appendix A: Definitions, notation and preliminaries}

We consider a stable conservative mechanical system in a continuous domain $\Omega \in \mathbb{R}^{d}$ that is at least weakly coupled to a thermal bath with Gaussian statistics with the longest correlation time $\tau_{b}$ being much shorter than that of the system, $\tau_{s}$ (i.e. $\tau_{b} \ll \tau_{s}$ ) such that the bath can be considered as representing stationary white noise on the time-scale of the system's dynamics [73]. The thermal bath is either external or the result of integrating out an additional subset of internal degrees of freedom that relaxes much faster than the system. At any time $t$ the state of the system is specified by a $d$-dimensional state (column) vector $\mathbf{x}_{t} \in \mathbb{R}^{d}$, whose entries are generalized coordinates $x_{t, i}$. Note that the dynamics in soft matter and biological systems is typically strongly overdamped which we also assume here. The extension to underdamped systems is conceptually straightforward (since we consider microscopically reversible dynamics) [108], but since a broken time-translation invariance in soft and biological matter is not tied to momenta, we omit these for convenience. We are strictly interested in the evolution of $\mathbf{x}_{t}$ for $t \gg \tau_{b}$. It is well known that under certain technical conditions imposed on the dynamics of the bath [73], which we will not further detail here but are strictly granted for the physical systems relevant to the discussion, $\mathbf{x}_{t}$ evolves according to the Itô equation

$$
d \mathbf{x}_{t}=\mathbf{F}\left(\mathbf{x}_{t}\right) d t+\boldsymbol{\sigma} d \mathbf{W}_{t}
$$

where $\mathbf{W}_{t}$ is a $d$-dimensional vector of independent Wiener processes whose increments have a Gaussian distribution with zero mean and variance $d t$, i.e. $\mathbb{E}\left[d W_{t, i} d W_{t^{\prime}, j}\right]=\delta_{i j} \delta\left(t-t^{\prime}\right) d t, \mathbb{E}[\cdot]$ denotes the expectation over the ensemble of Wiener increments and where $\boldsymbol{\sigma}$ is a $d \times d$ symmetric noise matrix. If momentum coordinates were included $\boldsymbol{\sigma}$ would be positive semi-definite with zeros in the sector of position variables and non-zero terms proportional to the friction constant $\gamma$ in the momentum sector, and is strictly positive definite with terms $\propto \gamma^{-1}$ for over-damped dynamics (i.e. for $\gamma \gg 1$ ) [108]). We focus on microscopically reversible dynamics, that is, we consider $d$-dimensional Markovian diffusion with a $d \times d$ symmetric positive-definite diffusion matrix $\mathbf{D}=\boldsymbol{\sigma} \boldsymbol{\sigma}^{T} / 2$ and mobility tensor $\mathbf{M}=\mathbf{D} / k_{\mathrm{B}} T$ (with $\beta^{-1} \equiv k_{\mathrm{B}} T$ being the thermal energy) in a drift field $\mathbf{F}(\mathbf{x})$, such that $\mathbf{M}^{-1} \mathbf{F}(\mathbf{x})=-\nabla \varphi(\mathbf{x})$ is a gradient flow. The drift field $\mathbf{F}(\mathbf{x}): \mathbb{R}^{d} \rightarrow \mathbb{R}^{d}$, is either nominally confining (in this case $\Omega$ is open) or is accompanied by corresponding reflecting boundary conditions at $\partial \Omega$ (in this case $\Omega$ is closed) thus guaranteeing the existence of an invariant measure and hence ergodicity [73, 108]. 
On the level of probability measures in phase space the dynamics is governed by the (forward) Fokker-Planck operator $\hat{\mathcal{L}}: V \rightarrow V$, where $V$ is a complete normed linear vector space with elements $f \in C^{2}\left(\mathbb{R}^{d}\right)$. In particular,

$$
\hat{\mathcal{L}}=\nabla \cdot \mathbf{D} \nabla-\nabla \cdot \mathbf{F}(\mathbf{x})
$$

$\mathbf{F}(\mathbf{x})$ is assumed to be sufficiently confining, i.e. $\lim _{\mathbf{x} \rightarrow \infty} P(\mathbf{x}, t)=0, \forall t$ sufficiently fast to assure that $\hat{\mathcal{L}}$ corresponds to a coercive and densely defined operator on $V$ with a pure point spectrum [109-111]. $\hat{\mathcal{L}}$ propagates probability measures $\mu_{t}(\mathbf{x})$ in time, which will throughout be assumed to possess well-behaved probability density functions $P(\mathbf{x}, t)$, i.e. $d \mu_{t}(\mathbf{x})=P(\mathbf{x}, t) d \mathbf{x}$. The nullspace of $\hat{\mathcal{L}}$ (i.e. the solution of $\hat{\mathcal{L}} P_{\text {eq }}(\mathbf{x})=0$ ) is the equilibrium (Maxwell)Boltzmann-Gibbs distribution, $P_{\text {eq }}(\mathbf{x})=Q^{-1} \mathrm{e}^{-\beta \varphi(\mathbf{x})}$, with partition function $Q=\int_{\Omega} d \mathbf{x e} \mathrm{e}^{-\beta \varphi(\mathbf{x})}$. We define the (forward) propagator $\hat{U}(t)=\mathrm{e}^{\hat{\mathcal{L}} t}$ that is the generator of a semi-group $\hat{U}\left(t+t^{\prime}\right)=\hat{U}(t) \hat{U}\left(t^{\prime}\right)$. The formal solution of the Fokker-Planck equation $\left(\partial_{t}-\hat{\mathcal{L}}\right) P(\mathbf{x}, t)=0$ is thereby given as $P(\mathbf{x}, t)=\hat{U}(t) P(\mathbf{x}, 0)$. The expectation over the ensemble of paths $\mathbf{x}_{t}$ will be denoted by $\langle\cdot\rangle$ and in the case of a physical observable $\mathcal{B}\left(\mathbf{x}_{t}\right)$ is given by

$$
\left\langle\mathcal{B}\left(\mathbf{x}_{t}\right)\right\rangle \equiv \int \mathcal{B}(\mathbf{x}) d \mu_{t}(\mathbf{x}) \equiv \int_{\Omega} \mathcal{B}(\mathbf{x}) P(\mathbf{x}, t) d \mathbf{x} \equiv \int_{\Omega} \mathcal{B}(\mathbf{x}) \hat{U}(t) P(\mathbf{x}, 0) d \mathbf{x}
$$

Part of the analysis will involve the use of spectral theory in Hilbert space, for which it is convenient to introduce the bra-ket notation; the 'ket' $|g\rangle$ represents a vector in $V$ written in position basis as $g(\mathbf{x}) \equiv\langle\mathbf{x} \mid g\rangle$, and the 'bra' $\langle h|$ as the integral $\int d \mathbf{x} h^{\dagger}(\mathbf{x})$. The scalar product is defined with the Lebesgue integral $\langle h \mid g\rangle=\int d \mathbf{x} h^{\dagger}(\mathbf{x}) g(\mathbf{x})$. In this notation we have the following evolution equation for the probability density function starting from an initial condition $\left|p_{0}\right\rangle:\left|p_{t}\right\rangle=\mathrm{e}^{\hat{\mathcal{L}} t}\left|p_{0}\right\rangle$. Since the process is ergodic we have $\lim _{t \rightarrow \infty} \mathrm{e}^{\hat{\mathcal{L}} t}\left|p_{0}\right\rangle=\mid$ eq $\rangle$, where $\langle\mathbf{x}|$ eq $\rangle=P_{\text {eq }}(\mathbf{x})$. We also define the (typically non-normalizable) 'flat' state $|-\rangle$, such that $\langle\mathbf{x} \mid-\rangle=1$ and $\left\langle-\mid p_{t}\right\rangle=1$. Hence, $\partial_{t}\left\langle-\mid p_{t}\right\rangle=0$ and $\langle-| \hat{\mathcal{L}}=0$.

Whereas $\hat{\mathcal{L}}$ by itself is not self-adjoint, it is orthogonally equivalent to a self-adjoint operator, i.e. the operator $\hat{\mathcal{L}}_{s}=\mathrm{e}^{\beta \varphi(\mathbf{x}) / 2} \hat{\mathcal{L}} \mathrm{e}^{-\beta \varphi(\mathbf{x}) / 2}$ is self-adjoint, and, moreover the operator $\mathrm{e}^{\beta \varphi(\mathbf{x})} \hat{\mathcal{L}}$ is self-adjoint (for a proof see $[108]$ ). Because any self-adjoint operator in Hilbert space is diagonalizable, $\hat{\mathcal{L}}$ is diagonalizable as well, but with a separate set of left and right bi-orthonormal eigenvectors $\left\langle\psi_{k}^{L}\right|$ and $\left|\psi_{k}^{R}\right\rangle$, respectively. That is, $\hat{\mathcal{L}}\left|\psi_{k}^{R}\right\rangle=-\lambda_{k}\left|\psi_{k}^{R}\right\rangle$ and $\left\langle\psi_{k}^{L}\right| \hat{\mathcal{L}}=$ $-\lambda_{k}\left\langle\psi_{k}^{L}\right|$ with real eigenvalues $\lambda_{k} \geq 0$ (assured by detailed balance) and where $\lambda_{0}=0,\left|\psi_{0}^{R}\right\rangle=\mid$ eq $\rangle,\left\langle\psi_{0}^{L}\right|=\langle-|$, and $\left\langle\psi_{k}^{L} \mid \psi_{l}^{R}\right\rangle=\delta_{k l}$. Moreover, since $\mathrm{e}^{\beta \varphi(\mathbf{x})} \hat{\mathcal{L}}$ is self-adjoint it follows that that $\left|\psi_{k}^{L}\right\rangle=\mathrm{e}^{\beta \varphi(\mathbf{x})}\left|\psi_{k}^{R}\right\rangle$. The resolution of identity is given by $\mathbf{1}=\sum_{k}\left|\psi_{k}^{R}\right\rangle\left\langle\psi_{k}^{L}\right|$ and the propagator by $\hat{U}(t)=\sum_{k}\left|\psi_{k}^{R}\right\rangle\left\langle\psi_{k}^{L}\right| \mathrm{e}^{-\lambda_{k} t}$.

The Markovian Green's function of the process $\mathbf{x}_{t}$ corresponds to the conditional probability density function for a localized initial condition $\left\langle\mathbf{x} \mid p_{0}\right\rangle=\delta\left(\mathbf{x}-\mathbf{x}_{0}\right)$ and is defined as $Q\left(\mathbf{x}, t \mid \mathbf{x}_{0}, 0\right)=\left\langle\mathbf{x}|\hat{U}(t)| \mathbf{x}_{0}\right\rangle$, such that the probability density starting from a general initial condition $\left|p_{0}\right\rangle$ becomes $P\left(\mathbf{x}, t, p_{0}\right)=\left\langle\mathbf{x}|\hat{U}(t)| p_{0}\right\rangle \equiv \int d \mathbf{x}_{0} p_{0}\left(\mathbf{x}_{0}\right) Q\left(\mathbf{x}, t \mid \mathbf{x}_{0}, 0\right)$. In the spectral representation the Green's function reads

$$
Q\left(\mathbf{x}, t \mid \mathbf{x}_{0}, 0\right)=\sum_{k} \psi_{k}^{R}(\mathbf{x}) \psi_{k}^{L}\left(\mathbf{x}_{0}\right) \mathrm{e}^{-\lambda_{k} t}
$$

where the semi-group property means that $Q\left(\mathbf{x}, \tau \mid \mathbf{x}_{0}, 0\right)=Q\left(\mathbf{x}, t+\tau \mid \mathbf{x}_{0}, t\right)$ is independent of $t$ as is easily verified via

$$
\int_{\Omega} d \mathbf{x}^{\prime} Q\left(\mathbf{x}, t \mid \mathbf{x}^{\prime}, t^{\prime}\right) Q\left(\mathbf{x}^{\prime}, t^{\prime} \mid \mathbf{x}_{0}, 0\right)=\sum_{k, l} \psi_{k}^{R}(\mathbf{x})\left\langle\psi_{k}^{L} \mid \psi_{l}^{R}\right\rangle \psi_{l}^{L}\left(\mathbf{x}_{0}\right) \mathrm{e}^{-\lambda_{k}\left(t-t^{\prime}\right)-\lambda_{l} t^{\prime}} \equiv Q\left(\mathbf{x}, t \mid \mathbf{x}_{0}, 0\right),
$$

where we have used that $\left\langle\psi_{k}^{L} \mid \psi_{l}^{R}\right\rangle=\delta_{k, l}$.

In the presence of a time-scale separation giving rise to local equilibrium the system's dynamics may be coarsegrained further into a discrete-state Markov jump master equation (see e.g. $[112,113]$ ). In this case the configuration space would be discrete and $d$-dimensional, $\hat{\mathcal{L}}$ would be replaced by a $d \times d$ symmetric stochastic matrix $\mathbf{M}$, and the Fokker-Planck equation by the master equation $\frac{d}{d t} Q=\mathbf{M} Q$. Since this situation corresponds to an approximate, lower-resolution dynamics of the system that is mathematically simpler and the mapping between the Fokker-Planck equation and Markov-state jump dynamics is well-known [103, 108, 112] and does not introduce any further conceptual changes (the complete spectral-theoretic approach in particular remains unchanged), we will without any loss of generality focus on the continuous scenario. 
Appendix B: Dynamics of the projected lower-dimensional observable

In order to describe the dynamics of the $r$-dimensional projected observable $\mathbf{q}=\boldsymbol{\Gamma}(\mathbf{x}): \mathbb{R}^{d} \rightarrow \mathbb{R}^{r}$ with $r<d$ and $\mathbf{q}$ lying in some orthogonal system in Euclidean space $\mathbf{q} \in \Xi\left(\mathbb{R}^{r}\right) \subset \Omega\left(\mathbb{R}^{d}\right)$, we define the operator $\hat{\mathcal{P}}_{\mathbf{x}}(\boldsymbol{\Gamma} ; \mathbf{q})$, such that, when applied to some function $Z(\mathbf{x}) \in V, \hat{\mathcal{P}}_{\mathbf{x}}(\boldsymbol{\Gamma} ; \mathbf{q})$ gives (see [85])

$$
\hat{\mathcal{P}}_{\mathbf{x}}(\boldsymbol{\Gamma} ; \mathbf{q}) Z(\mathbf{x}) \equiv \int_{\Omega} d \mathbf{x} \delta(\mathbf{\Gamma}(\mathbf{x})-\mathbf{q}) Z(\mathbf{x})
$$

where $\delta(\mathbf{y})$ is to be understood in the distributional sense. We can now define the (in general) non-Markovian twopoint conditional probability density of projected dynamics starting from $\mathbf{q}_{0} \in \Xi_{0}$, where the subdomain $\Xi_{0}$ is not necessarily simply connected, with the extended operator $\hat{\mathcal{P}}_{\mathbf{x}}\left(\boldsymbol{\Gamma} ; \mathbf{q} \in \Xi_{0}\right)=\int_{\Xi_{0}} d \mathbf{q} \hat{\mathcal{P}}_{\mathbf{x}}(\boldsymbol{\Gamma} ; \mathbf{q})$ in terms of the single-point and joint two-point density $P_{p_{0}}^{0}\left(\mathbf{q}_{0} \in \Xi_{0}\right)$ and $P_{p_{0}}\left(\mathbf{q}, t, \mathbf{q}_{0} \in \Xi_{0}\right)$, respectively, as

$$
G_{p_{0}}\left(\mathbf{q}, t \mid \mathbf{q}_{0} \in \Xi_{0}\right)=\frac{P_{p_{0}}\left(\mathbf{q}, t, \mathbf{q}_{0} \in \Xi_{0}\right)}{P_{p_{0}}^{0}\left(\mathbf{q}_{0} \in \Xi_{0}\right)} \equiv \frac{\hat{\mathcal{P}}_{\mathbf{x}}(\Gamma ; \mathbf{q}) \hat{\mathcal{P}}_{\mathbf{x}_{0}}\left(\boldsymbol{\Gamma} ; \mathbf{q}_{0} \in \Xi_{0}\right) Q\left(\mathbf{x}, t \mid \mathbf{x}_{0}, 0\right) p_{0}\left(\mathbf{x}_{0}\right)}{\hat{\mathcal{P}}_{\mathbf{x}_{0}}\left(\boldsymbol{\Gamma} ; \mathbf{q}_{0} \in \Xi_{0}\right) p_{0}\left(\mathbf{x}_{0}\right)}
$$

with the convention that $P_{p_{0}}\left(\mathbf{q}, t, \mathbf{q}_{0}\right)$ and $G_{p_{0}}\left(\mathbf{q}, t \mid \mathbf{q}_{0}\right)$ stand for $\Xi_{0}$ corresponding to a single point $\mathbf{q}_{0}$. The full system is said to have a stationary preparation if and only if $p_{0}\left(\mathbf{x}_{0}\right)=P_{\mathrm{eq}}(\mathbf{x})$, whereas the projected observable is said to have a stationary preparation if and only if $\Xi_{0}=\Xi$. Note that $\lim _{t \rightarrow \infty} P_{p_{0}}\left(\mathbf{q}, t, \mathbf{q}_{0} \in \Xi_{0}\right)=P_{\mathrm{eq}}(\mathbf{q}) \int_{\Xi_{0}} d \mathbf{q}_{0} P_{p_{0}}\left(\mathbf{q}_{0}\right)$, where we have defined $P_{\text {eq }}(\mathbf{q}) \equiv \hat{\mathcal{P}}_{\mathbf{x}}(\Gamma ; \mathbf{q}) P_{\text {eq }}(\mathbf{x})$ as well as $P_{p_{0}}\left(\mathbf{q}_{0}\right) \equiv \hat{\mathcal{P}}_{\mathbf{x}_{0}}\left(\boldsymbol{\Gamma} ; \mathbf{q}_{0}\right) p_{0}\left(\mathbf{x}_{0}\right)$. In turn it follows that $\lim _{t \rightarrow \infty} G_{p_{0}}\left(\mathbf{q}, t \mid \mathbf{q}_{0} \in \Xi_{0}\right)=P_{\text {eq }}(\mathbf{q})$. Eq. (B2) demonstrates that the entire time evolution of projected dynamics starting from a fixed condition $\mathbf{q}_{0}$ depends on the initial preparation of the full system $p_{0}\left(\mathbf{x}_{0}\right)$ as denoted by the subscript, which is the first signature of the non-stationary nature of projected dynamics. In addition, the dynamics described by Eq. (B2) is, except for quite exotic projections $\boldsymbol{\Gamma}(\mathbf{x})$, non-Markovian (see [85]).

We can now define averages and two-point correlation functions of $\mathbf{q}(t)$. The $n$-th moment of the position averaged over an ensemble of all projected non-Markovian evolutions prepared in the point $\mathbf{q}_{0}$ while the full system at $t=0$ is prepared in the state $p_{0}\left(\mathbf{x}_{0}\right)$ is given by

$$
\left\langle\mathbf{q}(t)^{n}\right\rangle_{p_{0}}^{\Xi_{0}} \equiv \int_{\Xi} d \mathbf{q} \mathbf{q}^{n} G_{p_{0}}\left(\mathbf{q}, t \mid \mathbf{q}_{0} \in \Xi_{0}\right), \quad\left\langle\mathbf{q}^{n}\right\rangle_{p_{0}}^{\Xi_{0}} \equiv \int_{\Xi_{0}} d \mathbf{q}_{0} \mathbf{q}_{0}^{n} P_{p_{0}}\left(\mathbf{q}_{0}\right)
$$

where we are here only interested in $n=1,2$, whereas the most general tensorial two-point $(0, t)$ (non-aging) correlation (i.e. covariance) matrix is defined as

$$
\begin{aligned}
\mathbf{C}_{\Xi_{0}}\left(t ; p_{0}\right) & \equiv\langle\mathbf{q}(t) \otimes \mathbf{q}(0)\rangle_{p_{0}}^{\Xi_{0}}-\langle\mathbf{q}(t)\rangle_{p_{0}}^{\Xi_{0}} \otimes\langle\mathbf{q}\rangle_{p_{0}}^{\Xi_{0}} \\
& =\int_{\Xi} d \mathbf{q} \int_{\Xi_{0}} d \mathbf{q}_{0}\left(\mathbf{q} \otimes \mathbf{q}_{0}\right) P_{p_{0}}\left(\mathbf{q}, t, \mathbf{q}_{0}\right)-\langle\mathbf{q}(t)\rangle_{p_{0}}^{\Xi_{0}} \otimes\langle\mathbf{q}\rangle_{p_{0}}^{\Xi_{0}},
\end{aligned}
$$

such that $\lim _{t \rightarrow \infty} \mathbf{C}\left(t ; p_{0}\right)=0, \forall p_{0}$, where from the scalar version is in turn obtained by taking the trace

$$
C_{\Xi_{0}}\left(t ; p_{0}\right) \equiv\langle\mathbf{q}(t) \cdot \mathbf{q}(0)\rangle_{p_{0}}^{\Xi_{0}}-\langle\mathbf{q}(t)\rangle_{p_{0}}^{\Xi_{0}} \cdot\langle\mathbf{q}\rangle_{p_{0}}^{\Xi_{0}}=\operatorname{Tr} \mathbf{C}_{\Xi_{0}}\left(t ; p_{0}\right)
$$

with the convention $\left.\mathbf{C}_{\Xi_{0}}\left(t ; P_{\text {eq }}\right)=\langle\mathbf{q}(t) \cdot \mathbf{q}(0)\rangle_{\text {eq }}^{\Xi_{0}}-\langle\mathbf{q}(t)\rangle\right\rangle_{\text {eq }}^{\Xi_{0}} \cdot\langle\mathbf{q}\rangle_{\text {eq }}^{\Xi_{0}} \equiv \mathbf{C}_{\Xi_{0}}(t)$. We can equivalently define the timedependent variance of $\mathbf{q}(t)$ with $\mathbf{q}(0)=\mathbf{q}_{0} \in \Xi_{0}$ as

$$
\sigma_{\Xi_{0}}^{2}\left(t ; p_{0}\right) \equiv\left\langle\mathbf{q}(t)^{2}\right\rangle_{p_{0}}^{\Xi_{0}}-\left(\langle\mathbf{q}(t)\rangle_{p_{0}}^{\Xi_{0}}\right)^{2}
$$

\section{Spectral theory of projected dynamics}

We now use spectral theory of the Markovian Green's function in Eq. (A4) to analyze the general properties of the non-Markovian time evolution of the projected lower-dimensional observable $\mathbf{q}(t)$. As the initial preparation of the full system $p_{0}\left(\mathbf{x}_{0}\right)$ was found to determine the point-to-point propagation of the probability density of $\mathbf{q}$, we begin by expanding the initial condition of the full system $p_{0}\left(\mathbf{x}_{0}\right)$ in the eigenbasis of $\hat{\mathcal{L}}$, i.e. $p_{0}\left(\mathbf{x}_{0}\right)=\sum_{l}\left|\psi_{l}^{R}\right\rangle\left\langle\psi_{l}^{L} \mid p_{0}\right\rangle$. The only assumptions made for $p_{0}\left(\mathbf{x}_{0}\right)$ are that it is normalized, Lebesgue integrable (such that $\left\langle\psi_{l}^{L} \mid p_{0}\right\rangle$ exists) and locally sufficiently compact to assure that the projection at time $t=0$ does not project onto an empty set of the observable 
$\mathbf{q}_{0}$. By further introducing the elements of the following infinite-dimensional matrices

$$
\Psi_{k l}(\mathbf{q})=\left\langle\psi_{k}^{L}|\delta(\boldsymbol{\Gamma}(\mathbf{x})-\mathbf{q})| \psi_{l}^{R}\right\rangle, \quad \Psi_{k l}\left(\Xi_{0}\right)=\int_{\Xi_{0}} d \mathbf{q}\left\langle\psi_{k}^{L}|\delta(\boldsymbol{\Gamma}(\mathbf{x})-\mathbf{q})| \psi_{l}^{R}\right\rangle
$$

where $\lim _{\Xi_{0} \rightarrow \mathbf{q}} \Psi_{k l}\left(\Xi_{0}\right)=\Psi_{k l}(\mathbf{q})$, we can express $P_{p_{0}}\left(\mathbf{q}, t \mid \mathbf{q}_{0} \in \Xi_{0}\right)$ in Eq. (B2) as

$$
P_{p_{0}}\left(\mathbf{q}, t, \mathbf{q}_{0} \in \Xi_{0}\right)=\sum_{k} \mathrm{e}^{-\lambda_{k} t} \Psi_{0 k}(\mathbf{q}) \sum_{l} \Psi_{k l}\left(\Xi_{0}\right)\left\langle\psi_{l}^{L} \mid p_{0}\right\rangle
$$

and since the preparation of the projected observable is $P_{p_{0}}\left(\mathbf{q}_{0} \in \Xi_{0}\right)=\sum_{l} \Psi_{0 l}\left(\Xi_{0}\right)\left\langle\psi_{l}^{L} \mid p_{0}\right\rangle$, the conditional nonMarkovian two-point density as

$$
G_{p_{0}}\left(\mathbf{q}, t \mid \mathbf{q}_{0} \in \Xi_{0}\right)=\frac{\sum_{k} \mathrm{e}^{-\lambda_{k} t} \Psi_{0 k}(\mathbf{q}) \sum_{l} \Psi_{k l}\left(\Xi_{0}\right)\left\langle\psi_{l}^{L} \mid p_{0}\right\rangle}{\sum_{l} \Psi_{0 l}\left(\Xi_{0}\right)\left\langle\psi_{l}^{L} \mid p_{0}\right\rangle} .
$$

For a stationary preparation of the full system, i.e. $p_{0}\left(\mathbf{x}_{0}\right)=P_{\text {eq }}\left(\mathbf{x}_{0}\right)$, we have that $\left\langle\psi_{l}^{L} \mid P_{\text {eq }}\right\rangle=\delta_{l, 0}$ and hence $P_{\text {eq }}\left(\mathbf{q} \in \Xi_{0}\right)=\Psi_{00}\left(\Xi_{0}\right)$ as well as

$$
G_{\mathrm{eq}}\left(\mathbf{q}, t \mid \mathbf{q}_{0} \in \Xi_{0}\right)=\frac{P_{\mathrm{eq}}\left(\mathbf{q}, t, \mathbf{q}_{0} \in \Xi_{0}\right)}{P_{\mathrm{eq}}\left(\mathbf{q}_{0} \in \Xi_{0}\right)}=\frac{\sum_{k} \Psi_{0 k}(\mathbf{q}) \Psi_{k 0}\left(\Xi_{0}\right) \mathrm{e}^{-\lambda_{k} t}}{\Psi_{00}\left(\Xi_{0}\right)}
$$

As a result

$$
\begin{aligned}
& \langle\mathbf{q}(t)\rangle_{p_{0}}^{\Xi_{0}}=\sum_{k} \mathrm{e}^{-\lambda_{k} t}\left(\int_{\Xi} d \mathbf{q q} \Psi_{0 k}(\mathbf{q})\right) \frac{\sum_{l} \Psi_{k l}\left(\Xi_{0}\right)\left\langle\psi_{l}^{L} \mid p_{0}\right\rangle}{\sum_{l} \Psi_{0 l}\left(\Xi_{0}\right)\left\langle\psi_{l}^{L} \mid p_{0}\right\rangle} \\
& \langle\mathbf{q}(t)\rangle_{\mathrm{eq}}^{\Xi_{0}}=\sum_{k} \mathrm{e}^{-\lambda_{k} t}\left(\int_{\Xi} d \mathbf{q q} \Psi_{0 k}(\mathbf{q})\right) \frac{\Psi_{k 0}\left(\Xi_{0}\right)}{\Psi_{00}\left(\Xi_{0}\right)} .
\end{aligned}
$$

Furthermore, we find that

$$
\lim _{\Xi_{0} \rightarrow \Xi} \Psi_{k l}\left(\Xi_{0}\right)=\int_{\Xi} d \mathbf{q}\left\langle\psi_{k}^{L}|\delta(\boldsymbol{\Gamma}(\mathbf{x})-\mathbf{q})| \psi_{l}^{R}\right\rangle=\left\langle\psi_{k}^{L}\left|\int_{\Xi} d \mathbf{q} \delta(\boldsymbol{\Gamma}(\mathbf{x})-\mathbf{q})\right| \psi_{l}^{R}\right\rangle=\left\langle\psi_{k}^{L} \mid \psi_{l}^{R}\right\rangle=\delta_{k, l}
$$

where the order of integration can be exchanged since the delta function in the distributional sense is smooth (i.e. the limit to a 'true' delta-function is taken after the integrals) and the domain of the $\mathbf{q}$ integration $\Xi$ by definition includes all mappings $\mathbf{q}=\boldsymbol{\Gamma}(\mathbf{x})$ such that $\int_{\Xi} d \mathbf{q} \delta(\boldsymbol{\Gamma}(\mathbf{x})-\mathbf{q})=1$. As a result $\lim _{\Xi_{0} \rightarrow \Xi} G_{\mathrm{eq}}\left(\mathbf{q},\left.t\right|_{\mathbf{q}_{0}} \in \Xi_{0}\right)=\Psi_{00}(\mathbf{q})=P_{\mathrm{eq}}(\mathbf{q}), \forall t$. Using these spectral-theoretic results it follows immediately that the elements of the general tensorial second moment matrix read

$$
\begin{aligned}
& \left\langle(\mathbf{q}(t) \otimes \mathbf{q}(0))_{i j}\right\rangle_{p_{0}}^{\Xi_{0}}=\sum_{k} \mathrm{e}^{-\lambda_{k} t}\left(\int_{\Xi_{i}} d q_{i} q_{i} \Psi_{0 k}\left(q_{i}\right)\right) \sum_{l}\left\langle\psi_{l}^{L} \mid p_{0}\right\rangle\left(\int_{\Xi_{0, j}} d q_{0, j} q_{0, j} \Psi_{k l}\left(q_{0, j}\right)\right) \\
& \left\langle(\mathbf{q}(t) \otimes \mathbf{q}(0))_{i j}\right\rangle_{\mathrm{eq}}^{\Xi_{0}}=\sum_{k} \mathrm{e}^{-\lambda_{k} t}\left(\int_{\Xi_{i}} d q_{i} q_{i} \Psi_{0 k}\left(q_{i}\right)\right)\left(\int_{\Xi_{j, 0}} d q_{0, j} q_{0, j} \Psi_{k 0}\left(q_{0, j}\right)\right),
\end{aligned}
$$

which, once plugged into Eq. (B4) together with Eq. (B11) and the right member of Eq. (B3), yield the tensorial correlation (or covariance) matrix $\mathbf{C}\left(t ; p_{0}\right)$. The case treated in the main text, that is, when the projected coordinate is one-dimensional and the full-system's preparation is stationary, follows trivially by appropriate simplification of Eq. (B1) and insertion into Eq. (B10), which leads to

$$
\begin{aligned}
C(t ; \mathrm{eq}) & \equiv\langle q(t) q(0)\rangle_{\mathrm{eq}}^{\Xi_{0}}-\langle q(t)\rangle_{\mathrm{eq}}^{\Xi_{0}}\langle q(0)\rangle_{\mathrm{eq}}^{\Xi_{0}} \\
& =\sum_{k} \mathrm{e}^{-\lambda_{k} t}\left(\int_{\Xi} d q q \Psi_{0 k}(q)\right)\left(\int_{\Xi_{0}} d q_{0} q_{0} \Psi_{k 0}\left(q_{0}\right)-\frac{\Psi_{k 0}\left(\Xi_{0}\right)}{\Psi_{00}\left(\Xi_{0}\right)} \int_{\Xi_{0}} d q_{0} q_{0} \Psi_{00}\left(q_{0}\right)\right) .
\end{aligned}
$$

As we now show in the following section dynamical time asymmetry (i.e. broken time-translation invariance) is inherently tied to non-Markovian three-point probability density functions of the projected observable. 


\section{Three-point dynamics and breaking of time-translation invariance}

In order to describe dynamical time asymmetry we introduce two times, the so-called "aging" (or "waiting") time, $t_{a}$, and the observation time window $\tau=t-t_{a}$. More precisely, we consider, as in the previous section, that the full system was prepared at $t=0$ in a general (not necessarily stationary) state $p_{0}\left(\mathbf{x}_{0}\right)$, whereby the choice of time origin is dictated by the initiation of an experiment or the onset of a phenomenon. The actual observation starts at some later (aging) time $t_{a} \geq 0$ and is carried out until a time $t$ and hence has a duration $\tau=t-t_{a}$. An example of a non-stationary preparation of a full system would be a temperature quench of a system equilibrated at some different temperature. We assume, as before, that only the lower-dimensional observable $\mathbf{q}(t)$ is observed for all times $t \geq 0$.

We now define time-delayed, "aging" observables. The normalized tensorial aging correlation matrix is defined as

$$
\hat{\mathbf{C}}_{t_{a}}^{\Xi_{0}}\left(\tau ; p_{0}\right) \equiv \frac{\mathbf{C}_{t_{a}}^{\Xi_{0}}\left(\tau ; p_{0}\right)}{\mathbf{C}_{t_{a}}^{\Xi_{0}}\left(0 ; p_{0}\right)}=\frac{\left\langle\mathbf{q}\left(\tau+t_{a}\right) \otimes \mathbf{q}\left(t_{a}\right)\right\rangle_{p_{0}}^{\Xi_{0}}-\left\langle\mathbf{q}\left(\tau+t_{a}\right)\right\rangle_{p_{0}}^{\Xi_{0}} \otimes\left\langle\mathbf{q}\left(t_{a}\right)\right\rangle_{p_{0}}^{\Xi_{0}}}{\left.\left.\left\langle\mathbf{q}\left(t_{a}\right) \otimes \mathbf{q}\left(t_{a}\right)\right\rangle\right\rangle_{p_{0}}^{\Xi_{0}}-\left\langle\mathbf{q}\left(t_{a}\right)\right\rangle\right\rangle_{p_{0}}^{\Xi_{0}} \otimes\left\langle\mathbf{q}\left(t_{a}\right)\right\rangle{\overline{p_{0}}}_{\Xi_{0}}^{\Xi_{0}}}
$$

such that $\hat{C}_{t_{a}}\left(\tau ; p_{0}\right) \equiv \operatorname{Tr} \hat{\mathbf{C}}_{t_{a}}\left(\tau ; p_{0}\right)$ and for the one-dimensional coordinate starting from a system prepared in a stationary state that is studied in the main paper

$$
\hat{C}_{t_{a}}^{\Xi_{0}}(\tau, \text { eq }) \equiv \hat{C}_{t_{a}}^{\Xi_{0}}(\tau) \equiv \frac{C_{t_{a}}^{\Xi_{0}}(\tau)}{C_{t_{a}}^{\Xi_{0}}(0)}=\frac{\left\langle q\left(\tau+t_{a}\right) q\left(t_{a}\right)\right\rangle^{\Xi_{0}}-\left\langle q\left(\tau+t_{a}\right)\right\rangle^{\Xi_{0}}\left\langle q\left(t_{a}\right)\right\rangle^{\Xi_{0}}}{\left\langle q\left(t_{a}\right)^{2}\right\rangle \Xi_{0}-\left(\left\langle q\left(t_{a}\right)\right\rangle^{\Xi_{0}}\right)^{2}}
$$

From the definitions of aging observables in Eqs. (B15-B16) it follows that these are inherently tied to three-point probability density functions at times $0, t_{a}$, and $t_{a}+\tau$. The full system's dynamics, corresponding to a Hamiltonian dynamics coupled to a Markovian heat bath, is Markovian and time-translation invariant. The three-point joint density therefore reads

$$
P_{\text {full }}^{p_{0}}\left(\mathbf{x}, t_{a}+\tau, \mathbf{x}^{\prime}, t_{a}, \mathbf{x}_{0}\right)=Q\left(\mathbf{x}, t_{a}+\tau \mid \mathbf{x}^{\prime}, t_{a}\right) Q\left(\mathbf{x}^{\prime}, t \mid \mathbf{x}_{0}, 0\right) p_{0}\left(\mathbf{x}_{0}\right)
$$

Using the definitions from the previous section and introducing the shorthand notation $\hat{\mathcal{P}}_{\mathbf{x}, \mathbf{x}^{\prime}, \mathbf{x}_{0}}\left(\boldsymbol{\Gamma} ; \mathbf{q}, \mathbf{q}^{\prime}, \mathbf{q}_{0} \in \Xi_{0}\right) \equiv$ $\hat{\mathcal{P}}_{\mathbf{x}}(\boldsymbol{\Gamma} ; \mathbf{q}) \hat{\mathcal{P}}_{\mathbf{x}^{\prime}}\left(\boldsymbol{\Gamma} ; \mathbf{q}^{\prime}\right) \hat{\mathcal{P}}_{\mathbf{x}_{0}}\left(\boldsymbol{\Gamma} ; \mathbf{q}_{0} \in \Xi_{0}\right)$ the three-point joint density is defined as

$$
\begin{aligned}
P_{p_{0}}\left(\mathbf{q}, t_{a}+\tau, \mathbf{q}^{\prime}, t_{a}, \mathbf{q}_{0} \in \Xi_{0}\right) & \equiv \hat{\mathcal{P}}_{\mathbf{x}, \mathbf{x}^{\prime}, \mathbf{x}_{0}}\left(\boldsymbol{\Gamma} ; \mathbf{q}, \mathbf{q}^{\prime}, \mathbf{q}_{0} \in \Xi_{0}\right) P_{\text {full }}^{p_{0}}\left(\mathbf{x}, t_{a}+\tau, \mathbf{x}^{\prime}, t_{a}, \mathbf{x}_{0}\right) \\
& =\sum_{k, l} \mathrm{e}^{-\lambda_{k} \tau-\lambda_{l} t_{a}} \Psi_{0 k}(\mathbf{q}) \Psi_{k l}\left(\mathbf{q}^{\prime}\right) \sum_{m} \Psi_{l m}\left(\Xi_{0}\right)\left\langle\psi_{m}^{L} \mid p_{0}\right\rangle .
\end{aligned}
$$

Under the milder (as far as the non-stationarity of $\mathbf{q}(t)$ is concerned) assumption that the full system at $t=0$ is in equilibrium, that is $p_{0}\left(\mathbf{x}_{0}\right)=P_{\mathrm{eq}}\left(\mathbf{x}_{0}\right)$ as we have assumed in the main text, $\left\langle\psi_{m}^{L} \mid P_{\mathrm{eq}}\right\rangle=\delta_{m, 0}$ and Eq. (B18) simplifies to

$$
\begin{aligned}
P_{\mathrm{eq}}\left(\mathbf{q}, t_{a}+\tau, \mathbf{q}^{\prime}, t_{a}, \mathbf{q}_{0} \in \Xi_{0}\right) & \equiv \hat{\mathcal{P}}_{\mathbf{x}, \mathbf{x}^{\prime}, \mathbf{x}_{0}}\left(\boldsymbol{\Gamma} ; \mathbf{q}, \mathbf{q}^{\prime}, \mathbf{q}_{0} \in \Xi_{0}\right) P_{\mathrm{full}}^{P_{\mathrm{eq}}}\left(\mathbf{x}, t_{a}+\tau, \mathbf{x}^{\prime}, t_{a}, \mathbf{x}_{0}\right) \\
& =\sum_{k, l} \mathrm{e}^{-\lambda_{k} \tau-\lambda_{l} t_{a}} \Psi_{0 k}(\mathbf{q}) \Psi_{k l}\left(\mathbf{q}^{\prime}\right) \Psi_{l 0}\left(\Xi_{0}\right)
\end{aligned}
$$

The corresponding three-point conditional probability densities are in turn defined by

$$
\begin{aligned}
G_{p_{0}}\left(\mathbf{q}, t_{a}+\tau, \mathbf{q}^{\prime}, t_{a} \mid \mathbf{q}_{0} \in \Xi_{0}\right) & \equiv \frac{P_{p_{0}}\left(\mathbf{q}, t_{a}+\tau, \mathbf{q}^{\prime}, t_{a}, \mathbf{q}_{0} \in \Xi_{0}\right)}{P_{p_{0}}\left(\mathbf{q}_{0} \in \Xi_{0}\right)} \\
& =\frac{\sum_{k, l} \mathrm{e}^{-\lambda_{k} \tau-\lambda_{l} t_{a}} \Psi_{0 k}(\mathbf{q}) \Psi_{k l}\left(\mathbf{q}^{\prime}\right) \sum_{m} \Psi_{l m}\left(\Xi_{0}\right)\left\langle\psi_{m}^{L} \mid p_{0}\right\rangle}{\sum_{l} \Psi_{0 l}\left(\Xi_{0}\right)\left\langle\psi_{l}^{L} \mid p_{0}\right\rangle}, \\
G_{\mathrm{eq}}\left(\mathbf{q}, t_{a}+\tau, \mathbf{q}^{\prime}, t_{a} \mid \mathbf{q}_{0} \in \Xi_{0}\right) & \equiv \frac{P_{\mathrm{eq}}\left(\mathbf{q}, t_{a}+\tau, \mathbf{q}^{\prime}, t_{a}, \mathbf{q}_{0} \in \Xi_{0}\right)}{P_{\mathrm{eq}}\left(\mathbf{q}_{0} \in \Xi_{0}\right)} \\
& =\frac{\sum_{k, l} \mathrm{e}^{-\lambda_{k} \tau-\lambda_{l} t_{a}} \Psi_{0 k}(\mathbf{q}) \Psi_{k l}\left(\mathbf{q}^{\prime}\right) \Psi_{l 0}\left(\Xi_{0}\right)}{\Psi_{00}\left(\Xi_{0}\right)} .
\end{aligned}
$$

A broken time-translation invariance is, however, most explicitly visible by means of what we will refer to as the 
two-point conditioned Green's function:

$$
\begin{aligned}
\tilde{G}_{p_{0}}\left(\mathbf{q}, t_{a}+\tau \mid \mathbf{q}^{\prime}, t_{a}, \mathbf{q}_{0} \in \Xi_{0}\right) & \equiv \frac{P_{p_{0}}\left(\mathbf{q}, t_{a}+\tau, \mathbf{q}^{\prime}, t_{a}, \mathbf{q}_{0} \in \Xi_{0}\right)}{P_{p_{0}}\left(\mathbf{q}, t, \mathbf{q}_{0} \in \Xi_{0}\right)} \\
& =\frac{\sum_{k, l} \mathrm{e}^{-\lambda_{k} \tau-\lambda_{l} t_{a}} \Psi_{0 k}(\mathbf{q}) \Psi_{k l}\left(\mathbf{q}^{\prime}\right) \sum_{m} \Psi_{l m}\left(\Xi_{0}\right)\left\langle\psi_{m}^{L} \mid p_{0}\right\rangle}{\sum_{k} \mathrm{e}^{-\lambda_{k} t_{a}} \Psi_{0 k}\left(\mathbf{q}^{\prime}\right) \sum_{l} \Psi_{k l}\left(\Xi_{0}\right)\left\langle\psi_{l}^{L} \mid p_{0}\right\rangle}, \\
\tilde{G}_{\mathrm{eq}}\left(\mathbf{q}, t_{a}+\tau \mid \mathbf{q}^{\prime}, t_{a}, \mathbf{q}_{0} \in \Xi_{0}\right) & \equiv \frac{P_{\mathrm{eq}}\left(\mathbf{q}, t_{a}+\tau, \mathbf{q}^{\prime}, t_{a}, \mathbf{q}_{0} \in \Xi_{0}\right)}{P_{\mathrm{eq}}\left(\mathbf{q}, t_{a}, \mathbf{q}_{0} \in \Xi_{0}\right)} \\
& =\frac{\sum_{k, l} \mathrm{e}^{-\lambda_{k} \tau-\lambda_{l} t_{a}} \Psi_{0 k}(\mathbf{q}) \Psi_{k l}\left(\mathbf{q}^{\prime}\right) \Psi_{l 0}\left(\Xi_{0}\right)}{\sum_{k} \mathrm{e}^{-\lambda_{k} t_{a}} \Psi_{0 k}\left(\mathbf{q}^{\prime}\right) \Psi_{k 0}\left(\Xi_{0}\right)} .
\end{aligned}
$$

By means of Eqs. (B20) and (B21) we can now determine aging expectation values entering Eq. (B15) and Eq. (B16), which, for a general matrix element $\left\langle q_{i}\left(\tau+t_{a}\right) q_{j}\left(t_{a}\right)\right\rangle$ read

$$
\begin{aligned}
\left\langle q_{i}\left(\tau+t_{a}\right) q_{j}\left(t_{a}\right)\right\rangle_{p_{0}}^{\Xi_{0}} & =\int_{\Xi_{j}} d q_{i} \int_{\Xi_{j}} d q_{j} q_{i} q_{j} G_{p_{0}}\left(q_{i}, t_{a}+\tau, q_{j}, t_{a} \mid \mathbf{q}_{0} \in \Xi_{0}\right) \\
\left\langle q_{i}\left(\tau+t_{a}\right) q_{j}\left(t_{a}\right)\right\rangle_{\mathrm{eq}}^{\Xi_{0}} & =\int_{\Xi_{i}} d q_{i} \int_{\Xi_{j}} d q_{j} q_{i} q_{j} G_{\mathrm{eq}}\left(q_{i}, t_{a}+\tau, q_{j}, t_{a} \mid \mathbf{q}_{0} \in \Xi_{0}\right)
\end{aligned}
$$

The dynamics of the projected observable $\mathbf{q}(t)$ is typically referred to as aging if correlation functions like $\hat{\mathbf{C}}_{t_{a}}\left(\tau ; p_{0}\right), \hat{C}_{t_{a}}\left(\tau ; p_{0}\right)$ and/or $C_{t_{a}}(\tau)$ defined in Eqs. (B4-B5) depend on $t_{a}$. However, the observables in Eq.(B24) only capture linear correlations in systems with broken time-translation invariance, and moreover display a $t_{a}$-dependence even in Markovian systems which are time-translation invariant but evolve from a non-stationary initial condition (see Lemma 2 below). These correlation functions are therefore by no means conclusive indicators of broken timetranslation invariance. We therefore propose the time asymmetry index, $\Upsilon$ - a new, conclusive (albeit not unique) indicator of broken time-translation invariance, which we define as

$$
\Upsilon_{\Xi_{0}}\left(t_{a}, \tau\right) \equiv \hat{\mathcal{D}}_{\mathbf{q}, \mathbf{q}^{\prime}}\left[G_{p_{0}}\left(\mathbf{q}, \tau+t_{a}, \mathbf{q}^{\prime}, t_{a} \mid \mathbf{q}_{0} \in \Xi_{0}\right)|| G_{p_{0}}\left(\mathbf{q}, \tau \mid \mathbf{q}^{\prime}\right) G_{p_{0}}\left(\mathbf{q}^{\prime}, t_{a} \mid \mathbf{q}_{0} \in \Xi_{0}\right)\right]
$$

where we have introduced the Kullback-Leibler divergence (or relative entropy)

$$
\hat{\mathcal{D}}_{\mathbf{y}_{1}, \mathbf{y}_{2}}[p \| q] \equiv \iint_{\operatorname{supp} p} d \mathbf{y}_{1} d \mathbf{y}_{2} p\left(\mathbf{y}_{1}, \mathbf{y}_{2}\right) \ln \frac{p\left(\mathbf{y}_{1}, \mathbf{y}_{2}\right)}{q\left(\mathbf{y}_{1}, \mathbf{y}_{2}\right)}
$$

which has the property $\hat{\mathcal{D}}_{\mathbf{y}_{1}, \mathbf{y}_{2}}[p \| q] \geq 0$ with the equality being true if and only if $p\left(\mathbf{y}_{1}, \mathbf{y}_{2}\right)$ is equal to $q\left(\mathbf{y}_{1}, \mathbf{y}_{2}\right)$ almost everywhere [114]. The rationale behind this choice is that it is defined to measure exactly the existence and degree of broken time-translation invariance and we will use this property in the following section to assert the necessary and sufficient conditions for the emergence of dynamical time asymmetry. We are now in a position to prove the central claims in the manuscript.

\section{Appendix C: Main theorems with proofs}

Definition 1. Time-translation invariance [76, 115]. The dynamics of the observable $\mathbf{q}(t)$ resulting from the projection defined in Eq. (B1) of the full Markovian dynamics $\mathbf{x}_{t}$ evolving according to Eq. (A1) is said to relax to equilibrium in a time-translation invariant manner (i.e. stationary) if and only if the two-point conditioned Green's function in Eqs. (B22-B23) does not depend on $t_{a}$, that is

$$
\tilde{G}_{p_{0}}\left(\mathbf{q}, t_{a}+\tau \mid \mathbf{q}^{\prime}, t_{a}, \mathbf{q}_{0} \in \Xi_{0}\right)=\tilde{G}_{p_{0}}\left(\mathbf{q}, t^{\prime}+\tau \mid \mathbf{q}^{\prime}, t^{\prime}, \mathbf{q}_{0} \in \Xi_{0}\right), \forall \tau, t^{\prime}>0 .
$$

Definition 2. Dynamical time asymmetry. The dynamics of the projected observable $\mathbf{q}(t)$ is said to be dynamically time asymmetric if its relaxation to equilibrium is not time-translation invariant.

Definition 3. Trivial non-stationarity. The dynamics of the projected observable $\mathbf{q}(t)$ is said to be trivially nonstationary if the relaxation is time-translation invariant but evolves from a non-equilibrium initial condition of the full 
system, $p_{0}\left(\mathbf{x}_{0}\right) \neq P_{\text {eq }}\left(\mathbf{x}_{0}\right)$.

Theorem 1. The dynamics of the observable $\mathbf{q}(t)$ resulting from the projection defined in Eq. (B1) of the full Markovian dynamics $\mathbf{x}_{t}$ evolving according to Eq. (A1) is time-translation invariant if and only if at least one of the following is true:

(1) the projected dynamics $\mathbf{q}(t)$ is Markovian

(2) the full system and projected observable are both prepared in and sampled from equilibrium, that is $p_{0}\left(\mathbf{x}_{0}\right)=P_{\mathrm{eq}}(\mathbf{x})$, $\Xi_{0} \rightarrow \Xi$ such that $\lim _{\Xi_{0} \rightarrow \Xi} P_{\mathrm{eq}}\left(\mathbf{q}_{0} \in \Xi_{0}\right) \rightarrow 1$.

If either of these two assumptions is true $\Upsilon_{\Xi_{0}}\left(t_{a}, \tau\right)=0, \forall t_{a}, \tau>0$.

Proof. We first prove sufficiency. If the projection $\hat{\mathcal{P}}$ is such that 1 . above holds then $G_{p_{0}}\left(\mathbf{q}, \tau+t_{a}, \mathbf{q}^{\prime}, t_{a} \mid \mathbf{q}_{0} \in \Xi_{0}\right)=$ $G_{p_{0}}\left(\mathbf{q}, \tau \mid \mathbf{q}^{\prime}\right) G_{p_{0}}\left(\mathbf{q}^{\prime}, t_{a} \mid \mathbf{q}_{0} \in \Xi_{0}\right)$ for any $\tau, t_{a}, \mathbf{q}, \mathbf{q}^{\prime}, \mathbf{q}_{0}, \Xi_{0}$ and $p_{0}\left(\mathbf{x}_{0}\right)$, such that the logarithmic term in Eq. (B26) is identically zero everywhere and hence $\Upsilon_{\Xi_{0}}\left(t_{a}, \tau\right)=0, \forall t_{a}, \tau$. Conversely, if 2. is true then due to Eq. (B12) we have $G_{\text {eq }}\left(\mathbf{q}, t \mid \mathbf{q}_{0} \in \Xi\right)=\Psi_{00}(\mathbf{q})=P_{\text {eq }}(\mathbf{q}), \forall t$ and according to Eq. (B20) also $G_{p_{0}}\left(\mathbf{q}, t_{a}+\tau, \mathbf{q}^{\prime}, t_{a} \mid \mathbf{q}_{0} \in \Xi\right)=G_{p_{0}}\left(\mathbf{q}, \tau \mid \mathbf{q}^{\prime}\right)$, such that the logarithmic term in Eq. (B26) is again identically zero everywhere and hence $\Upsilon_{\Xi_{0}}\left(t_{a}, \tau\right)=0, \forall t_{a}, \tau$. This proves sufficiency.

To prove necessity we first recall that $\hat{\mathcal{D}}_{\mathbf{y}_{1}, \mathbf{y}_{2}}[p \| q]=0$ if and only if $p\left(\mathbf{y}_{1}, \mathbf{y}_{2}\right)$ is equal to $q\left(\mathbf{y}_{1}, \mathbf{y}_{2}\right)$ almost everywhere [114]. In addition, as a result of Eq. (B7) and irrespective of the projection (as long as it does not project onto an empty set) the time evolution of $G_{p_{0}}\left(\mathbf{q}, \tau+t_{a}, \mathbf{q}^{\prime}, t_{a} \mid \mathbf{q}_{0} \in \Xi_{0}\right)$ in Eq. (B20) and Eq. (B21) as well as $G_{p_{0}}\left(\mathbf{q}, \tau \mid \mathbf{q}^{\prime}\right)$ and $G_{p_{0}}\left(\mathbf{q}^{\prime}, t_{a} \mid \mathbf{q}_{0} \in \Xi_{0}\right)$ in Eq. (B9) is smooth and continuous $\forall t_{a}>0, \tau>0$. Moreover, $\Psi_{0 k}(\mathbf{q}) \neq \Psi_{l k}(\mathbf{q})$ for $l \neq 0$ except for potentially on a set of $\mathbf{q}$ with zero measure because of Eq. (B7) and since $\left\langle\psi_{0}^{L}\right|$ and $\left\langle\psi_{l}^{L}\right|$ are linearly independent. Therefore, because $G_{p_{0}}\left(\mathbf{q}, \tau+t_{a}, \mathbf{q}^{\prime}, t_{a} \mid \mathbf{q}_{0} \in \Xi_{0}\right) \geq 0, \forall \tau, t_{a}, \mathbf{q}, \mathbf{q}^{\prime}, \mathbf{q}_{0}, \Xi_{0}$ and $p_{0}\left(\mathbf{x}_{0}\right)$ the Kullback-Leibler divergence in Eq. (B26) cannot not be zero almost everywhere except if either one or both of the statements 1. or 2. above are true. This completes the proof of necessity.

Corollary 1.1. The dynamics of the projected observable $\mathbf{q}(t)$ displays a dynamical time asymmetry as soon as the projection $\hat{\mathcal{P}}$ renders it non-Markovian and it is initially not prepared in, and averaged over, an equilibrium initial condition, i.e. $P_{p_{0}}\left(\mathbf{q}_{0} \in \Xi_{0}\right) \neq P_{\mathrm{eq}}\left(\mathbf{q}_{0} \in \Xi\right)=1$. If this is true then $\Upsilon_{\Xi_{0}}\left(t_{a}, \tau\right)>0$ at least on a dense set of $t_{a}$ and $\tau$ with non-zero measure.

Proof. The proof follows immediately from a straightforward extension of the proof of Theorem 1.

Lemma 2. Aging correlation functions like $\hat{\mathbf{C}}_{t_{a}}\left(\tau ; p_{0}\right), \hat{C}_{t_{a}}\left(\tau ; p_{0}\right)$ and/or $C_{t_{a}}(\tau)$ defined in Eqs. (B4-B5) are not conclusive indicators of the dynamical time asymmetry because they cannot discriminate between trivial non-stationarity and broken time-translation invariance, that is, they can display a dependence on $t_{a}$ even if the relaxation to equilibrium is time-translation invariant.

Proof. A simple example suffices to prove this claim. Consider that the observable $\mathbf{y}_{t}$ is evolving according to Markov dynamics (for conditions imposed on $\hat{\mathcal{P}}$ for this to occur please see [85]) with Green's function $Q\left(\mathbf{y}, t \mid \mathbf{y}_{0}\right)$. It is not difficult to show that the relaxation to equilibrium is time-translation invariant. Namely, consider, the probability density of $\mathbf{y}$ at a time $\tau+t_{a}$ given that at time $t_{a}$ the system was found in a point $\mathbf{q}^{\prime}$ whereby it evolved there from an initial probability density $p_{0}(\mathbf{y})$ :

$$
\begin{aligned}
\tilde{G}_{p_{0}}\left(\mathbf{y}, \tau+t_{a} \mid \mathbf{y}^{\prime}, t_{a}, \mathbf{y}_{0}\right) & \equiv \frac{\int_{\Xi_{0}} d \mathbf{y}_{0} Q\left(\mathbf{y}, \tau+t_{a} \mid \mathbf{y}^{\prime}, t_{a}\right) Q\left(\mathbf{y}^{\prime}, t_{a} \mid \mathbf{y}_{0}\right) p_{0}\left(\mathbf{y}_{0}\right)}{\int_{\Xi_{0}} d \mathbf{y}_{0} Q\left(\mathbf{y}^{\prime}, t_{a} \mid \mathbf{y}_{0}\right) p_{0}\left(\mathbf{y}_{0}\right)} \\
& =Q\left(\mathbf{y}, \tau+t_{a} \mid \mathbf{y}^{\prime}, t_{a}\right)=Q\left(\mathbf{y}, \tau \mid \mathbf{y}^{\prime}\right),
\end{aligned}
$$

where we allow (redundantly) and under-sampling of $p_{0}$ by setting $\Xi_{0} \neq \Xi$. Clearly, and expectedly, the relaxation process $\mathbf{y}\left(t_{a}\right) \rightarrow \mathbf{y}\left(t_{a}+\tau\right)$ is time-translation invariant - it depends only on $\mathbf{y}^{\prime}=\mathbf{y}\left(t_{a}\right)$ but does not depend on how this state was reached. Analogously to Eq. (B20) we also define the three-point joint probability density of $\mathbf{y}$ evolving from an initial probability density $p_{0}(\mathbf{y})$

$$
\begin{aligned}
G_{p_{0}}\left(\mathbf{y}, \tau+t_{a}, \mathbf{y}^{\prime}, t_{a} \mid \Xi_{0}\right) & \equiv \frac{\int_{\Xi_{0}} d \mathbf{y}_{0} Q\left(\mathbf{y}, \tau+t_{a} \mid \mathbf{y}^{\prime}, t_{a}\right) Q\left(\mathbf{y}^{\prime}, t_{a} \mid \mathbf{y}_{0}\right) p_{0}\left(\mathbf{y}_{0}\right)}{\int_{\Xi_{0}} d \mathbf{y}_{0} p_{0}\left(\mathbf{y}_{0}\right)} \\
& =Q\left(\mathbf{y}, \tau \mid \mathbf{y}^{\prime}\right) Q\left(\mathbf{y}^{\prime}, t_{a} \mid \Xi_{0}\right),
\end{aligned}
$$

where the propagation from $\mathbf{y}^{\prime}=\mathbf{q}\left(t_{a}\right) \rightarrow \mathbf{q}\left(t_{a}+\tau\right)$ only depends on $\mathbf{q}\left(t_{a}\right)$ but not on how this state was reached. It follows immediately that the time asymmetry index $\Upsilon$ for this process is identically zero (See Theorem 1 and Corollary 1.1). Nevertheless, because $G_{p_{0}}\left(\mathbf{y}, \tau+t_{a}, \mathbf{y}^{\prime}, t_{a} \mid \Xi_{0}\right)$ in Eq. (C2) depends on the probability that the system 
is found at time $t_{a}$ in $\mathbf{q}^{\prime}$ (but not on how it got there) the aging correlation function obtained from Eq. (C2) would display a dependence on $t_{a}$ as long as $p_{0}\left(\mathbf{y}_{0}\right) \neq P_{\mathrm{eq}}$ and $\Xi_{0} \neq \Xi$, which implies non-stationarity in a trivial sense (i.e. this would equally well be the case even simple Brownian diffusion of a particle in a box, which is manifestly time-translation invariant).

Conversely, it is also possible that the relaxation is indeed not translationally invariant according to Definition 1 but a dependence on $t_{a}$ only arises in the evolution of higher order (even or odd) correlation functions and is not visible in first order correlations in Eqs. (B15-B16).

Observation 1. Characteristic scaling of aging correlation functions. An interesting and very common observation in the existing literature on glassy, aging dynamics is an irreducible structure $C_{t_{a}}\left(\tau ; p_{0}\right)=f\left(\tau+t_{a}, t_{a}\right)$ (see e.g. $[46,47,116,117])$ frequently accompanied by a characteristic power-law scaling of aging autocorrelation functions [39, 46, 47, 117]. A particularly striking observation is the frequently observed so-called 'full aging' regime where the observation time window $\tau$ becomes much longer than the aging time $t_{a}, \tau \gg t_{a}$, and the following simple scaling emerges $C_{t_{a}}\left(\tau ; p_{0}\right) \propto t_{a} / \tau$ [20, 49, 96, 97]. Below we explain the emergence of irreducible structure as well as powerlaw-decaying aging autocorrelation functions incl. the full aging within the context of our spectral-theoretic approach.

Theorem 3. A representation result. Let $\varphi$ be a positive real number smaller than $1,0<\varphi<1$, and the functions $g_{1}(t): \mathbb{R}^{+} \rightarrow \mathbb{R}$ and $g_{2}(\tau, t): \mathbb{R}^{+} \times \mathbb{R}^{+} \rightarrow \mathbb{R}$ be smooth for $t>0$ and $t, \tau>0$, respectively. A matrix element of aging correlation functions, $C_{t_{a}, i, j}\left(\tau ; p_{0}\right)$ or $C_{t_{a}}\left(\tau ; p_{0}\right)$, defined in Eqs. (B15-B16) has the following irreducible structure in the form of a stationary contribution $g_{1}(t)$ and a non-stationary contribution $g_{2}(\tau, t)$ :

$$
C_{t_{a}, i, j}\left(\tau ; p_{0}\right)=(1-\varphi) g_{1}(\tau)+\varphi g_{2}\left(\tau, t_{a}\right)
$$

Proof. We recall the definition of aging expectation values in Eq. (B24) and simply split the double sum in Eqs. (B20) and (B21) into two parts $\sum_{k \geq 0} \sum_{l \geq 0} \rightarrow \sum_{k \geq 0 ; l=0}+\sum_{k \geq 0} \sum_{l \geq 1}$. The second term in the numerator of Eqs. (B15-B16) is nominally non-stationary (i.e. depends on both $\tau=\bar{t}-t_{a}$ and $t_{a}$ ). Collecting terms we obtain the representation stated in the theorem.

Definition 4. Self-similar dynamics. Let us write a general non-aging correlation function in Eq. (B4) as

$$
\mathbf{C}_{i j}\left(t ; p_{0}\right)=\sum_{k>0} w_{k}^{i j}\left(\Xi_{0} ; p_{0}\right) \mathrm{e}^{-\lambda_{k} t}
$$

The dynamics of the projected observable $\mathbf{q}(t)$ is said to be (transiently) self-similar on a time-scale $0<t \lesssim \lambda_{k_{\min }}^{-1}$ (see e.g. [118]) if a time-scale change $t \rightarrow t \delta$ does not change the relaxation beyond a renormalization of the weights, $w_{k}^{i j} \rightarrow \tilde{w}_{k}^{i j}(\delta)$. That is if $\lambda_{k}$ and $w_{k}$ are not independent, such that $\exists k_{\min } \in \mathbb{Z}^{+}$and constants $\left(\tau_{0}, \alpha, \delta, y\right) \in \mathbb{R}^{+}$with $0<\delta<1$ and $y<1$ such that for $k \in \mathbb{Z}^{+}>k_{\min }$ we have $\lambda_{k}=\left(\delta^{k} \tau_{0}\right)^{-1}$ and $w_{k}=\delta^{-\alpha k} y$. Then we have, on the time-scale $0<\tau_{0} \ll t \lesssim \lambda_{k_{\text {min }}}^{-1}$

$$
\begin{aligned}
\mathbf{C}_{i j}\left(t ; p_{0}\right) & =\sum_{0<k<k_{\min }} w_{k}^{i j}\left(\Xi_{0} ; p_{0}\right) \mathrm{e}^{-\lambda_{k} t}+\sum_{k \geq k_{\min }}^{\infty} w_{k}^{i j}\left(\Xi_{0} ; p_{0}\right) \mathrm{e}^{-\lambda_{k} t} \\
& \simeq \text { const }+y \int_{k_{\min }}^{\infty} d x \delta^{-\alpha x} \mathrm{e}^{-t \delta^{-x} / \tau_{0}} \\
& =\text { const }+\frac{y}{\ln \delta^{-1}}\left(\frac{t}{\tau_{0}}\right)^{-\alpha} \Gamma\left(\alpha, \lambda_{k_{\min }} t\right) \\
& =\text { const }+\frac{y}{\ln \delta^{-1}}\left[\Gamma(\alpha)\left(\frac{t}{\tau_{0}}\right)^{-\alpha}-\mathcal{O}\left(\frac{\delta^{-\alpha k_{\min }}}{\alpha}\right)\right]
\end{aligned}
$$

where $\Gamma(\alpha)$ and $\Gamma(\alpha, z)$ denote the complete and upper incomplete Gamma functions, respectively, and $\simeq$ stands for asymptotic equality, that is that the fraction of the left and the right hand side converges to 1 for $0<\tau_{0} \ll t \lesssim \lambda_{k_{\text {min }}}^{-1}$. $\mathbf{C}_{i j}\left(t ; p_{0}\right)$ thus transiently decays asymptotically according to a power-law with exponent $\alpha$.

Proposition 1. Self-similar time asymmetric dynamics. Let us write the non-normalized aging correlation function, i.e. the numerator in Eqs. (B15-B16), compactly as

$$
\mathbf{C}_{t_{a}, i j}\left(t ; p_{0}\right)=\sum_{k>0} \sum_{l>0}\left(w_{k l}^{i j}\left(\Xi_{0} ; p_{0}\right) \mathrm{e}^{-\lambda_{k}\left(t-t_{a}\right)-\lambda_{l} t_{a}}-\tilde{w}_{k l}^{i j}\left(\Xi_{0} ; p_{0}\right) \mathrm{e}^{-\lambda_{k} t-\lambda_{l} t_{a}}\right) .
$$


We now extend the idea of self-similar scaling in Definition 4 to aging correlations. Let $C_{1}, C_{2}, C_{4} \in \mathbb{R}^{+}, C_{3} \in \mathbb{R}$, $\lambda_{k}=\left(\delta_{1}^{k} \tau_{0}\right)^{-1}, w_{k l}=\delta_{1}^{-\alpha k} \delta_{2}^{-\alpha l} y_{1}, \tilde{w}_{k l}=\delta_{3}^{-\alpha k} \delta_{2}^{-\alpha l} y_{1}$ for suitably chosen $0<\delta_{1}, \delta_{2}, \delta_{3}<1, y_{1}<1, \tau=t-t_{a}$ and $\left(\tau_{0}, \alpha, k_{\min }\right)$ as in Definition 4. Then we have, for $0<\tau_{0} \ll t, \tau, t_{a} \lesssim \lambda_{k_{\min }}^{-1}$ asymptotically

$$
\begin{aligned}
\mathbf{C}_{t_{a}, i j}\left(t ; p_{0}\right) & \simeq C_{1}+y_{1} \Gamma(\alpha)\left[\frac{C_{2}}{\ln \delta_{1}^{-1}}\left(\frac{\tau}{\tau_{0}}\right)^{-\alpha}-\frac{C_{4}}{\ln \delta_{3}^{-1}}\left(\frac{t}{\tau_{0}}\right)^{-\alpha}\right] \\
& +\frac{y_{1} \Gamma(\alpha)}{\ln \delta_{2}^{-1}}\left(\frac{t_{a}}{\tau_{0}}\right)^{-\alpha}\left[C_{3}+\frac{\Gamma(\alpha)}{\ln \delta_{1}^{-1}}\left(\frac{\tau}{\tau_{0}}\right)^{-\alpha}-\frac{\Gamma(\alpha)}{\ln \delta_{3}^{-1}}\left(\frac{t}{\tau_{0}}\right)^{-\alpha}\right]+\mathcal{O}\left(\kappa_{\max }\right)
\end{aligned}
$$

where $\kappa_{\max } \equiv \alpha^{-1} \max \left(\frac{C_{2} \delta_{1}^{-\beta_{\min }}}{\ln \delta_{1}^{-1}}, \frac{C_{4} \delta_{3}^{-\beta_{\min }}}{\ln \delta_{3}^{-1}}, \frac{C_{3} \delta_{2}^{-\beta_{\min }}}{\ln \delta_{2}^{-1}}, \frac{\Gamma(\alpha)\left(\delta_{1} \delta_{2}\right)^{-\beta_{\min }}}{\ln \delta_{1} \ln \delta_{2}}, \frac{\Gamma(\alpha)\left(\delta_{3} \delta_{2}\right)^{-\beta_{\min }}}{\ln \delta_{3} \ln \delta_{2}}\right)$ and $\beta_{\min }=\alpha k_{\min }$. On a "good" scale of $t_{a}$, i.e. where $y_{1} \Gamma(\alpha) C_{3}\left(\tau_{0} / t_{a}\right)^{\alpha} / \ln \delta_{1}^{-1} \simeq$ const $\equiv B$ is effectively constant (that is, varies slowly) with respect to $\left(t_{a} / t\right)^{\alpha}$ then

$$
\mathbf{C}_{t_{a}, i j}\left(t ; p_{0}\right) \simeq C_{1}+B\left[1+\frac{C_{2} \ln \delta_{2}}{C_{3} \ln \delta_{1}}\left(\frac{t_{a}}{\tau}\right)^{\alpha}-\frac{C_{4} \ln \delta_{3}}{C_{3} \ln \delta_{1}}\left(\frac{t_{a}}{\tau+t_{a}}\right)^{\alpha}\right]+\mathcal{O}\left(\left[\frac{\tau_{0}^{2}}{t_{a} \tau}\right]^{\alpha}\right)
$$

Moreover, when $\tau \gg t_{a}$ we have the the "anomalous full aging" scaling

$$
\begin{aligned}
\mathbf{C}_{t_{a}, i j}\left(t ; p_{0}\right) & \simeq C_{1}+B\left[1+\frac{C_{2} \ln \delta_{2}-C_{4} \ln \delta_{3}}{C_{3} \ln \delta_{1}}\left(\frac{t_{a}}{\tau}\right)^{\alpha}+\alpha \frac{C_{4} \ln \delta_{3}}{C_{3} \ln \delta_{1}}\left(\frac{t_{a}}{\tau}\right)^{\alpha+1}\right]+\mathcal{O}\left(\left[\frac{t_{a}}{\tau}\right]^{\alpha+2}\right) \\
& =B_{1}+B_{2}\left(\frac{t_{a}}{\tau}\right)^{\nu}+\mathcal{O}\left(\left[\frac{t_{a}}{\tau}\right]^{\alpha+2}\right)
\end{aligned}
$$

where $\nu=\alpha$ if $C_{2} \ln \delta_{2} \neq C_{4} \ln \delta_{3}$ and $\nu=\alpha+1$ otherwise.

Proof. To prove the proposition we split each of the double sums as

$$
\sum_{k>0} \sum_{l>0}=\sum_{0<k<k_{\min }} \sum_{0<l<k_{\min }}+\sum_{k \geq k_{\min }} \sum_{0<l<k_{\min }}+\sum_{0<k<k_{\min }} \sum_{l \geq k_{\min }}+\sum_{k \geq k_{\min }} \sum_{l \geq k_{\min }}
$$

and note that $\int_{0}^{k_{\min }} d x \delta^{-\alpha x} \mathrm{e}^{-t /\left(\delta^{x} \tau_{0}\right)} \simeq$ const for $t \lesssim \lambda_{k_{\min }}^{-1}$. The rest follows directly from the computation in Definition 4 upon rearranging and collecting terms. To the the "anomalous full aging" scaling we expand $\left(t_{a}\left[\tau+t_{a}\right]\right)^{\alpha}=$ $\left(1+\tau / t_{a}\right)^{-\alpha}=\left(t_{a} / \tau\right)^{\alpha}\left(1-\alpha \tau / t_{a}+\mathcal{O}\left(\left(t_{a} / \tau\right)^{2}\right)\right.$ and collect terms. Upon identifying the constants $B_{1}$ and $B_{2}$ we arrive at Eq. (C7) which completes the proof.

Remark 1.1. Note that the scaling-from in Definition 4 arises, for example, when the observable corresponds to an internal distance within a single macromolecule (such e.g as the Rouse chain) [92] or within individual protein molecules [93, 94], as well as in diffusion on fractal objects [95].

Proposition 2. Logarithmic relaxation and 'full aging'. Let $\mathbf{C}_{t_{a}, i j}\left(t ; p_{0}\right)$ be written as in Eq. (C4) in Proposition 1 and let $\alpha=0, \delta_{1}=\delta_{3}$ and $C_{2}=C_{4}=C \in \mathbb{R}^{+}$(i.e. $1 / f$ self-similar scaling with logarithmic relaxation [20, 49, 96]; in fact a simple change of integration variable $x \rightarrow \delta^{-x}$ in Eqs. (C3)-(C5) with $\alpha=0$ shows that this case is mathematically equivalent to the analysis in [49, 96]). Then we have, for $0<\tau_{0} \ll t, \tau, t_{a} \lesssim \lambda_{k_{\min }}^{-1}$ asymptotically

$$
\mathbf{C}_{t_{a}, i j}\left(t ; p_{0}\right) \simeq C_{1}+\frac{C_{2} y_{1}}{\ln \delta_{1}^{-1}} \ln \left(\frac{\tau+t_{a}}{\tau}\right)+\frac{y_{1}}{\ln \delta_{2}^{-1}} \ln \left(\frac{1}{\lambda_{k_{\min }} t_{a}}\right)\left[C_{3}+\ln \left(\frac{\tau+t_{a}}{\tau}\right)\right]+\mathcal{O}(\kappa)
$$

where $\kappa=\max \left(\lambda_{k_{\min }} t, \lambda_{k_{\min }} \tau, \lambda_{k_{\min }} t_{a}\right)$. Moreover, in te limit $\tau \gg t_{a}$ we find

$$
\mathbf{C}_{t_{a}, i j}\left(t ; p_{0}\right) \simeq C_{1}+\frac{y_{1}}{\ln \delta_{1}^{-1}}\left(C_{2}+\frac{\ln \delta_{1}^{-1}}{\ln \delta_{2}^{-1}} \ln \left(\frac{1}{\lambda_{k_{\min }} t_{a}}\right)\right) \frac{t_{a}}{\tau}+\frac{C_{3} y_{1}}{\ln \delta_{2}^{-1}} \ln \left(\frac{1}{\lambda_{k_{\min }} t_{a}}\right)+\mathcal{O}(\kappa),
$$

such that when $\lambda_{k_{\min }} t_{a}=\mathcal{O}(1)$ we recover the so-called 'full aging' scaling [49, 96, 97]

$$
\mathbf{C}_{t_{a}, i j}\left(t ; p_{0}\right) \simeq C_{1}+C_{2} \frac{t_{a}}{\tau}
$$


Proof. The proof of the proposition is straightforward and follows from noticing that in the limit $x \ll 1$ we have $\Gamma(0, x)=-\ln (x)+\gamma+\mathcal{O}(x)$. Plugging into the expression in Proposition 1 we find, upon elementary manipulations, the result in Proposition 2. The 'full aging' scaling is further obtained by Taylor expanding the logarithm to first order.

Remark 2.1. The representation of $C_{t_{a}, i, j}\left(\tau ; p_{0}\right)$ given in Theorem 3 is indeed frequently observed in experiments on glassy systems [117], while self-similar aging dynamics with a power law scaling as in Proposition 1 has been observed both in glassy systems [47, 116, 117] as well as in individual protein molecules [39] and, in a similar form, emerges in the case of phenomenological so-called continuous time random walk models with diverging waiting times [119]. Notably, in the specific case of $1 / f$ self-similar dynamics our analysis also recovers the well-known, yet puzzling, "full aging" limiting scaling of $C_{t_{a}, i, j}\left(\tau ; p_{0}\right)$ (see Eq. (C10) [20, 49, 96, 97], and in particular the presence of the logarithmic correction in $t_{a}$ to the full aging scaling in Eq. (C9) may potentially explain the observation that a perfect $f\left(t_{a} / \tau\right)$ collapse is only observed for a specific "good" range values of the aging time $t_{a}$ [97]. Moreover, the power-law aging in Eq. (C7) for $t \gg t_{a}$ agrees with the "renewal aging" in fractional dynamics [45] (since $z^{\alpha}$ is the leading order term of the expansion of the incomplete Beta function, $B(z, \alpha, 1-\alpha)$, as $z \rightarrow 0)$. These specific results, as wall as others, therefore emerge as special cases of the framework presented in this work.

Observation 2. With respect to dynamical time asymmetry, the under-sampling of equilibrium is equivalent to a temperature quench. Let $P_{\mathrm{eq}}^{T}(\mathbf{x})$ be the equilibrium probability density function of the full system at a temperature $T$ prior to a quench in temperature, that is different from the ambient temperature $T_{0}$, i.e. $T>T_{0}$. Expanding $P_{\mathrm{eq}}^{T}(\mathbf{x})$ in the eigenbasis of $\hat{\mathcal{L}}$ (at the ambient temperature) we find $P_{\mathrm{eq}}^{T}(\mathbf{x})=\sum_{l}\left|\psi_{l}^{R}\right\rangle\left\langle\psi_{l}^{L} \mid P_{\mathrm{eq}}^{T}\right\rangle$, where $\lim _{T \rightarrow T_{0}}\left\langle\psi_{l}^{L} \mid P_{\mathrm{eq}}^{T}\right\rangle=\delta_{l 0}$. Let us further assume that the observable $\mathbf{q}$ is fully sampled from $P_{\mathrm{eq}}^{T}(\mathbf{x})$ (like in the case of a supercooled liquid), i.e. $\Xi_{0}=\Xi$, such that according to Eq. (B12) we have $\Psi_{k l}(\Xi)=\delta_{k l}$.

$$
\begin{gathered}
G_{P_{\mathrm{eq}}^{T}}\left(\mathbf{q}, t \mid \mathbf{q}_{0} \in \Xi\right)=\sum_{k} \mathrm{e}^{-\lambda_{k} t} \Psi_{0 k}(\mathbf{q})\left\langle\psi_{k}^{L} \mid P_{\mathrm{eq}}^{T}\right\rangle \\
G_{P_{\mathrm{eq}}^{T}}\left(\mathbf{q}, t_{a}+\tau, \mathbf{q}^{\prime}, t_{a} \mid \mathbf{q}_{0} \in \Xi\right)=\sum_{k, l} \mathrm{e}^{-\lambda_{k} \tau-\lambda_{l} t_{a}} \Psi_{0 k}(\mathbf{q}) \Psi_{k l}\left(\mathbf{q}^{\prime}\right)\left\langle\psi_{l}^{L} \mid P_{\mathrm{eq}}^{T}\right\rangle .
\end{gathered}
$$

since $\Psi_{k k}(\Xi)=\left\langle-\mid P_{\mathrm{eq}}^{T}\right\rangle=1$. According to Theorem 1 and Corollary 1.1 a temperature quench gives rise to broken time-translation invariance as longs as the projection renders the dynamics non-Markovian. Now consider an system prepared in equilibrium $p_{0}(\mathbf{x})=P_{\mathrm{eq}}(\mathbf{x})$ but with the projected observable undersampled from said equilibrium, i.e. for a domain $\mathbf{q}_{0} \in \Xi_{0} \subset \Xi$ such that $P_{\mathrm{eq}}\left(\mathbf{q}_{0} \in \Xi_{0}\right) \neq 1$. Then (see Eq. (B10) and Eq. (B21))

$$
\begin{gathered}
G_{\mathrm{eq}}\left(\mathbf{q}, t \mid \mathbf{q}_{0} \in \Xi_{0}\right)=\sum_{k} \mathrm{e}^{-\lambda_{k} t} \Psi_{0 k}(\mathbf{q}) \frac{\Psi_{k 0}\left(\Xi_{0}\right)}{\Psi_{00}\left(\Xi_{0}\right)} \\
G_{\mathrm{eq}}\left(\mathbf{q}, t_{a}+\tau, \mathbf{q}^{\prime}, t_{a} \mid \mathbf{q}_{0} \in \Xi_{0}\right)=\sum_{k, l} \mathrm{e}^{-\lambda_{k} \tau-\lambda_{l} t_{a}} \Psi_{0 k}(\mathbf{q}) \Psi_{k l}\left(\mathbf{q}^{\prime}\right) \frac{\Psi_{l 0}\left(\Xi_{0}\right)}{\Psi_{00}\left(\Xi_{0}\right)},
\end{gathered}
$$

which has a broken time-translation invariance as long as the projection renders the dynamics non-Markovian according to Theorem 1 and Corollary 1.1. Clearly, the only difference between the two non-Markovian time evolutions is in the factor $\psi_{l}^{L}\left|P_{\mathrm{eq}}^{T}\right\rangle$ versus $\Psi_{l 0}\left(\Xi_{0}\right) / \Psi_{00}\left(\Xi_{0}\right)$, which demonstrates that the effect of temperature quench and under-sampling of equilibrium are indeed (qualitatively) virtually indistinguishable as stated in the observation.

\section{Appendix D: Physical models, experimental and simulation data}

\section{Fictitious dynamical time asymmetry in a time-translation invariant system: the Brownian particle in a} box

Consider the propagator (i.e. the probability density) of a Browninan particle with diffusion coefficient $D 1$ confined in a box of unit length $L, G\left(x, t \mid x_{0}, 0\right)$ (without loss of generality we express length in units of $L$ and time in units of $L^{2} / D$ such that $x \rightarrow x / L$ and $\left.t \rightarrow t D / L^{2}\right)$ with $x \in[0,1]$, evolving according the Fokker-Planck equation

$$
\partial_{t} G\left(x, t \mid x_{0}, 0\right)=\partial_{x}^{2} G\left(x, t \mid x_{0}, 0\right),\left.\quad \partial_{x} G\right|_{x=0}=\left.\partial_{x} G\right|_{x=1}=0
$$


with initial condition $G\left(x, 0 \mid x_{0}\right)=\delta\left(x-x_{0}\right)$. The spectral expansion of the Green's function of the problem reads

$$
G\left(x, t \mid x_{0}, 0\right)=\sum_{k=0}^{\infty} \psi_{k}(x) \psi_{k}\left(x_{0}\right) \mathrm{e}^{-\lambda_{k} t}, \quad \psi_{k}(x)=\sqrt{2-\delta_{0, k}} \cos (k \pi x), \lambda_{k}=k^{2} \pi^{2},
$$

where we note that the problem is self-adjoint and hence $\psi_{k}^{R}(x)=\psi_{k}^{L}(x)=\psi_{k}(x)$. Let us define

$$
\begin{aligned}
I_{k} & \equiv 2^{-\delta_{k 0}}+\left(1-\delta_{k 0}\right) \sqrt{2}(\cos (k \pi)-1) / k^{2} \pi^{2} \\
J_{k, l} & \equiv 2^{-\delta_{k 0} \delta_{l 0}}+I_{k} \delta_{l 0}+I_{l} \delta_{k 0}+\delta_{k l} / 2 k \pi+2\left(1-\delta_{k l}\right)\left(k^{2}+l^{2}\right)[\cos (k \pi) \cos (l \pi)-1] /\left[\left(k^{2}-l^{2}\right) \pi\right]^{2} .
\end{aligned}
$$

Then we have

$$
\begin{aligned}
& \langle x(t)\rangle=\int_{0}^{1} d x G\left(x, t \mid x_{0}, 0\right) x=\sum_{k=0}^{\infty} I_{k} \psi_{k}\left(x_{0}\right) \mathrm{e}^{-\lambda_{k} t} \\
& \left\langle x\left(\tau+t_{a}\right) x\left(t_{a}\right)\right\rangle=\int_{0}^{1} d x \int_{0}^{1} d x_{1} G\left(x, \tau+t_{a} \mid x_{1}, t_{a}\right) G\left(x_{1}, t_{a} \mid x_{0}, 0\right) x x_{1}=\sum_{k=0}^{\infty} I_{k} \mathrm{e}^{-\lambda_{k}\left(\tau+t_{a}\right)} \sum_{l=0}^{\infty} J_{k, l} \psi_{l}\left(x_{0}\right) \mathrm{e}^{-\lambda_{l} t_{a}},
\end{aligned}
$$

which enter the definition of the aging correlation function

$$
C_{t_{a}, x_{0}}(\tau)=\frac{\left\langle x\left(\tau+t_{a}\right) x\left(t_{a}\right)\right\rangle-\left\langle x\left(\tau+t_{a}\right)\right\rangle\left\langle x\left(t_{a}\right)\right\rangle}{\left\langle x\left(t_{a}\right) x\left(t_{a}\right)\right\rangle-\left\langle x\left(t_{a}\right)\right\rangle^{2}} .
$$

When the initial distribution is not a point but is sampled from a flat distribution between $a$ and $b$ (as in the example in the main text), i.e. uniformly from a domain $\Omega_{0}=[a, b]$, we instead define

$$
L_{k}(b, a)=\delta_{k 0}+\left(1-\delta_{k 0}\right) \sqrt{2}[\sin (k \pi b)-\sin (k \pi a)] / k \pi(b-a)
$$

and then

$$
\begin{aligned}
\langle x(t)\rangle_{\Omega_{0}} & =\int_{0}^{1} d x \int_{\Omega_{0}} d x_{0} G\left(x, t \mid x_{0}, 0\right) P_{0}\left(x_{0}\right) x=\sum_{k=0}^{\infty} I_{k} L_{k}(b, a) \mathrm{e}^{-\lambda_{k} t} \\
\left\langle x\left(\tau+t_{a}\right) x\left(t_{a}\right)\right\rangle_{\Omega_{0}} & =\int_{0}^{1} d x \int_{0}^{1} d x_{1} \int_{\Omega_{0}} d x_{0} G\left(x, \tau+t_{a} \mid x_{1}, t_{a}\right) G\left(x_{1}, t_{a} \mid x_{0}, 0\right) P_{0}\left(x_{0}\right) x x_{1} \\
& =\sum_{k=0}^{\infty} I_{k} \mathrm{e}^{-\lambda_{k}\left(\tau+t_{a}\right)} \sum_{l=0}^{\infty} J_{k, l} L_{l}(b, a) \mathrm{e}^{-\lambda_{l} t_{a}} .
\end{aligned}
$$

Once inserted in Eq. (D5) Eq. (D7) deliver the aging autocorrelation function shown in Fig. 2 in the main text that displays fictitious dynamical time asymmetry (i.e. trivial dependence on $t_{a}$ ). In the meantime, the relaxation dynamics is time-translation invariant according to Definition 1 as a result of Theorem 1 (see also Eq. (2) in the main text), since it is Markovian and thus satisfies the Chapman-Kolmogorov semi-group property (see Lemma 2). The fictitious dynamical time asymmetry is thus a result of trivial non-stationarity in Definition 3.

\section{Rouse polymer model}

The Rouse polymer chain $[120,121]$ is a flexible macromolecule consisting of harmonic springs of zero rest-length. The potential energy of the macromolecule with $N+1$ point-like units (here referred to as 'beads') with a configuration $\mathbf{R} \in \mathbb{R}^{3(N+1)} \equiv\left\{\mathbf{R}_{i}\right\}$, where $\mathbf{R}_{i} \in \mathbb{R}^{3}$ is given by $U(\mathbf{R})=\sum_{i=1}^{N} \frac{3}{\beta b^{2}}\left|\mathbf{R}_{i+1}-\mathbf{R}_{i}\right|^{2}$, where $b$ is the so-called Kuhn length describing the size of a chain segment (i.e. the characteristic distance between two beads) and will for convenience (and without any loss of generality) here be set to $b=\sqrt{3}$. The dynamics is assumed to evolve according to overdamped diffusion (with all beads having a equal diffusion coefficient $D$ ) in a heat bath with zero mean Gaussian white noise, i.e. according to the system of coupled Itô equations

$$
d \mathbf{R}_{t}=-\beta D \underline{\mathbf{M}} \mathbf{R}_{t} d t+\sqrt{2 D} d \hat{\mathbf{W}}_{t},
$$


where $\hat{\mathbf{W}}_{t}$ denotes for a $3(N+1)$-dimensional vector of independent Wiener processes whose increments have a Gaussian distribution with zero mean and variance $d t: \mathbb{E}\left[d \hat{W}_{t, i} d \hat{W}_{t^{\prime}, j}\right]=\delta_{i j} \delta\left(t-t^{\prime}\right) d t$. $\mathbb{E}[\cdot]$ denotes the expectation over the ensemble of Wiener increments. The interaction matrix $\underline{\mathbf{M}}$ is the $3(N+1) \times 3(N+1)$ tridiagonal Rouse super-matrix whose elements are $\mathbf{M}_{i j} \mathbb{1}$ (where $\mathbb{1}$ denotes the $3 \times 3$ unit matrix) and the $(N+1) \times(N+1)$ matrix $\mathbf{M}$ has elements $\mathbf{M}_{i i}=\left(2-1^{\delta_{i 1}}-1^{\delta_{i N}}\right)$ and $\mathbf{M}_{i i+1}=\mathbf{M}_{i i-1}=-1$. On the level of a probability density function the Itô process Eq. (D8) corresponds to the $N$-body Fokker-Planck equation, which, introducing the operator $\nabla \equiv\left\{\nabla_{i}\right\}$ reads

$$
\partial_{t} P(\mathbf{R}, t)=D\left(\boldsymbol{\nabla}^{T} \boldsymbol{\nabla}+\beta \boldsymbol{\nabla}^{T} \underline{\mathbf{M}} \mathbf{R}\right) P(\mathbf{R}, t)
$$

which has the structure of Eq. (A2) and can be decoupled as follows. We first rotate the coordinate system to normal coordinates $\mathbf{Q} \in \mathbb{R}^{3(N+1)}=\left\{\mathbf{Q}_{i}\right\}, \forall i \in[0, N][122]$, i.e. $\mathbf{R}_{i}=\mathbf{S Q}_{i}\left(\mathbf{Q}_{i} \in \mathbb{R}^{3}\right)$ and $\nabla_{i}=\mathbf{S} \nabla_{\mathbf{Q}_{i}}$ with the $(N+1) \times(N+1)$ orthogonal matrix $\mathbf{S}, \mathbf{S}^{-1}=\mathbf{S}^{T}$, which diagonalizes the Rouse matrix, $\boldsymbol{\Lambda}=\mathbf{S}^{T} \mathbf{M S}$, where

$$
\boldsymbol{\Lambda}_{i k}=4 \sin ^{2}\left(\frac{k \pi}{2(N+1)}\right) \delta_{i k} \equiv \lambda_{k} \delta_{i k}, \quad \mathbf{S}_{i k}=\sqrt{\frac{2}{N+1}} \cos \left(\frac{(2 i-1) k \pi}{2(N+1)}\right), \forall k>0,
$$

and $\mathbf{S}_{i 0}=(N+1)^{-1 / 2}, \forall i$ (which is not required; see footnote). Introducing the $3(N+1) \times 3(N+1)$ super-matrix $\underline{\mathbf{S}}$, whose elements are $\mathbf{S}_{i k} \mathbb{1}$, the transformation to normal coordinates is found to decouple the Fokker-Planck equation Eq. (D9):

$$
\begin{aligned}
\partial_{t} P(\mathbf{Q}, t) & =D\left(\boldsymbol{\nabla}^{T} \underline{\mathbf{S}}^{T} \underline{\mathbf{S}} \boldsymbol{\nabla}+\beta \boldsymbol{\nabla}^{T} \underline{\mathbf{S}}^{T} \underline{\mathbf{M S}} \mathbf{Q}\right) P(\mathbf{Q}, t) \\
& =D \sum_{i=1}^{N}\left[\partial_{\mathbf{Q}_{i}}^{2}+\beta \lambda_{i} \partial_{\mathbf{Q}_{i}} \mathbf{Q}_{i}\right] P(\mathbf{Q}, t)
\end{aligned}
$$

whose structure implies that the solution factorizes $P(\mathbf{Q}, t)=\prod_{i=1}^{N} P\left(\mathbf{Q}_{i}, t\right)$ and $P\left(\mathbf{Q}_{i}, t\right)$ is simply the well-known solution of a 3-dimensional Ornstein-Uhlenbeck process. In particular, the density of the invariant measure and Green's function read

$$
\begin{aligned}
P_{\mathrm{eq}}(\mathbf{Q}) & =\prod_{i=1}^{N}\left(\frac{\lambda_{i}}{2 \pi}\right)^{3 / 2} \mathrm{e}^{-\lambda_{i} \mathbf{Q}_{i}^{2} / 2} \\
Q\left(\mathbf{Q}, t \mid \mathbf{Q}^{\prime}, t^{\prime}\right) & =\prod_{i=1}^{N}\left(\frac{\lambda_{i}}{2 \pi\left(1-\mathrm{e}^{-2 \lambda_{i}\left(t-t^{\prime}\right)}\right)}\right)^{3 / 2} \exp \left(-\frac{\lambda_{i}\left(\mathbf{Q}_{i}-\mathbf{Q}_{i}^{\prime} \mathrm{e}^{-\lambda_{i}\left(t-t^{\prime}\right)}\right)^{2}}{2\left(1-\mathrm{e}^{-2 \lambda_{i}\left(t-t^{\prime}\right)}\right)}\right) .
\end{aligned}
$$

The Gaussian structure of the solution will permit explicit results not requiring a spectral decomposition of the Fokker-Planck operator (which, however, is well-known [120]).

We are here interested in the dynamics of the end-to-end distance of the polymer, $q(t) \equiv|\mathbf{q}(t)|=\left|\mathbf{R}_{N+1}(t)-\mathbf{R}_{1}(t)\right|$, which would be typically probed in a single-molecule FRET or optical tweezers experiment. To make minimal assumptions we assume a stationary initial preparation of the full system, i.e. $P_{0}(\mathbf{R})=P_{\text {eq }}(\mathbf{R})$. Since $q(t)$ at any instance depends on all other degrees of freedom $\mathbf{R}_{k}(t), \forall k \in[2, N]$ its dynamics is strongly non-Markovian. In normal coordinates this corresponds to

$$
q(t)=\sum_{i=1}^{N}\left|\mathcal{A}_{i} \mathbf{Q}_{i}\right|=\sum_{i=1}^{N} \sqrt{\frac{2}{N+1}}\left|\left[\cos \left(\frac{(2 N-1) k \pi}{2(N+1)}\right)-\cos \left(\frac{k \pi}{2(N+1)}\right)\right] \mathbf{Q}_{i}\right|,
$$

having defined $\mathcal{A}_{i}$ in Eq. (D14), such that introducing $d \mathbf{Q} \equiv \prod_{i=1}^{N} d \mathbf{Q}_{i}$ the projection operator Eq. (B1) can be shown to correspond to

$$
\hat{\mathcal{P}}_{\mathbf{Q}}(\boldsymbol{\Gamma} ; q)=q^{2} \int_{0}^{2 \pi} d \varphi \int_{0}^{\pi} d \theta \int_{\Omega} d \mathbf{Q} \delta\left(\sum_{i=1}^{N} \mathcal{A}_{i} \mathbf{Q}_{i}-\mathbf{q}(q, \varphi, \theta)\right)
$$

and the non-Markovian conditional two-point probability density is calculated according to Eq. (B2) and leads, upon a lengthy but straightforward computation via a Fourier transform $\mathrm{FT}\{\mathbf{q} \rightarrow \mathbf{v}\}$, i.e. $\hat{\mathcal{P}}_{\mathbf{Q}}(\boldsymbol{\Gamma} ; q) f(\mathbf{Q}) \stackrel{\mathrm{FT}}{\longrightarrow} \tilde{f}(\mathbf{v}) \stackrel{\mathrm{FT}^{-1}}{\longrightarrow}$ 
$f(\mathbf{q}) \stackrel{\int d \varphi \int d \theta}{\longrightarrow} f(q)$, to

$$
G_{\mathrm{eq}}\left(q, t \mid q_{0}\right)=\frac{1}{\sqrt{\pi \gamma}} \frac{\phi(t)^{-1}}{\sqrt{1-\phi(t)^{2}}} \frac{q}{q_{0}} \exp \left(-\frac{q^{2}+q_{0}^{2}}{4 \gamma\left(1-\phi(t)^{2}\right)}+\frac{q_{0}^{2}}{4 \gamma}\right) \sinh \left(\frac{q q_{0} \phi(t)}{2 \gamma\left(1-\phi(t)^{2}\right)}\right),
$$

where we have defined

$$
\phi(t)=\sum_{i=1}^{N} \frac{\mathcal{A}_{i}^{2}}{2 \lambda_{i}} \mathrm{e}^{-\lambda_{i} t}, \quad \gamma=\phi(0) .
$$

The (non-aging) autocorrelation function in Eq. (B5), $C(t)=\langle q(t) q(0)\rangle-\langle q(t)\rangle\langle q(0)\rangle$, can in turn be shown to be given by

$$
C(t)=\frac{4 \gamma}{\pi}\left[3+\left(\frac{1}{\phi(t)}+2\right) \arctan \left(\frac{\phi(t)}{\sqrt{1-\phi(t)^{2}}}\right)\right]-\frac{16 \gamma}{\pi}
$$

which decays to zero as $t \rightarrow \infty$. We now address the three-point conditional probability density Eq. (B21) and aging autocorrelation function Eq. (B16), which are much more challenging. As such a complex calculation has, to the best of our knowledge, not been performed before for any stochastic system, we here present a more detailed derivation.

We start with Eq. (B17), plug in Eqs. (D12) and use Eq. (D15) to first calculate the three-point joint density of the vectorial counterpart, i.e. $P_{\text {eq }}\left(\mathbf{q}, t_{a}+\tau, \mathbf{q}^{\prime}, t_{a}, \mathbf{q}_{0}\right)$. We now perform a triple Fourier transform

$$
\tilde{P}_{\mathrm{eq}}\left(\mathbf{u}, t_{a}+\tau, \mathbf{v}, t_{a}, \mathbf{w}\right) \equiv \int d \mathbf{q} \mathrm{e}^{-i \mathbf{u} \cdot \mathbf{q}} \int d \mathbf{q}^{\prime} \mathrm{e}^{-i \mathbf{v} \cdot \mathbf{q}^{\prime}} \int d \mathbf{q}_{0} \mathrm{e}^{-i \mathbf{w} \cdot \mathbf{q}_{0}} P_{\mathrm{eq}}\left(\mathbf{q}, t_{a}+\tau, \mathbf{q}^{\prime}, t_{a}, \mathbf{q}_{0}\right)
$$

and carry out all integrations over $\mathbf{Q}, \mathbf{Q}^{\prime}$ and $\mathbf{Q}_{0}$ (a total of $3 N$ integrals each) and introduce the short-hand notation $S_{t}=\phi(t)$ to find

$$
\tilde{P}_{\mathrm{eq}}\left(\mathbf{u}, t_{a}+\tau, \mathbf{v}, t_{a}, \mathbf{w}\right)=\frac{1}{(2 \pi)^{9}} \exp \left(-\gamma\left(\mathbf{w}^{2}-\mathbf{v}^{2}-\mathbf{u}^{2}\right)-2 S_{t_{a}} \mathbf{w}^{T} \mathbf{v}-2 S_{t} \mathbf{u}^{T} \mathbf{w}-2 S_{\tau} \mathbf{u}^{T} \mathbf{v}\right)
$$

We now invert back all three Fourier transforms and introduce auxiliary functions $\mathcal{X}_{\tau, t_{a}} \equiv \gamma^{3}-\gamma\left(S_{t_{a}}^{2}-S_{t}^{2}-S_{\tau}^{2}\right)+$ $2 S_{t_{a}} S_{t} S_{\tau}$ as well as $\mathcal{Y}_{\tau, t_{a}} \equiv \gamma S_{t}-S_{t_{a}} S_{\tau}$ and $\mathcal{Z}_{\tau, t_{a}} \equiv \gamma S_{\tau}-S_{t} S_{t_{a}}$ (keeping in mind that $t=\tau+t_{a}$ ) to find

$$
\begin{aligned}
P_{\mathrm{eq}}\left(\mathbf{q}, t, \mathbf{q}^{\prime}, t_{a}, \mathbf{q}_{0}\right) & =\left(4^{3} \pi^{3} \mathcal{X}_{\tau, t_{a}}\right)^{-3 / 2} \\
& \times \exp \left(-\frac{\left(\gamma \mathcal{X}_{\tau, t_{a}}+\mathcal{Y}_{\tau, t_{a}}\right) \mathbf{q}_{0}^{2}+\left(\gamma \mathcal{X}_{\tau, t_{a}}+\mathcal{Z}_{\tau, t_{a}}\right) \mathbf{q}^{\prime 2}+\left(\gamma^{2}-S_{t_{a}}^{2}\right)^{2} \mathbf{q}^{2}}{4\left(\gamma^{2}-S_{t_{a}}^{2}\right) \mathcal{X}_{\tau, t_{a}}}\right) \\
& \times \exp \left(-\frac{\left(S_{t_{a}} \mathcal{X}_{\tau, t_{a}}+\mathcal{Y}_{\tau, t_{a}} \mathcal{Z}_{\tau, t_{a}}\right)}{2\left(\gamma^{2}-S_{t_{a}}^{2}\right) \mathcal{X}_{\tau, t_{a}}} \mathbf{q}^{\prime T} \mathbf{q}_{0}+\frac{\mathcal{Y}_{\tau, t_{a}}}{2 \mathcal{X}_{\tau, t_{a}}} \mathbf{q}^{T} \mathbf{q}_{0}+\frac{\mathcal{Z}_{\tau, t_{a}}}{2 \mathcal{X}_{\tau, t_{a}}} \mathbf{q}^{T} \mathbf{q}^{\prime}\right) .
\end{aligned}
$$

Before we perform the angular integrations, $\left(q q^{\prime} q_{0}\right)^{2} \int_{0}^{2 \pi} d \varphi \int_{0}^{\pi} d \theta \int_{0}^{2 \pi} d \varphi^{\prime} \int_{0}^{\pi} d \theta^{\prime} \int_{0}^{2 \pi} d \varphi_{0} \int_{0}^{\pi} d \theta_{0}$, we introduce the final set of auxiliary functions (i.e. the third in the hierarchy of our notation):

$$
\begin{gathered}
\Lambda_{1}^{\tau, t_{a}} \equiv \frac{S_{t_{a}} \mathcal{X}_{\tau, t_{a}}-\mathcal{Y}_{\tau, t_{a}} \mathcal{Z}_{\tau, t_{a}}}{2\left(\gamma^{2}-S_{t_{a}}^{2}\right) \mathcal{X}_{\tau, t_{a}}}, \quad \Lambda_{2}^{\tau, t_{a}} \equiv \frac{\mathcal{Y}_{\tau, t_{a}}}{2 \mathcal{X}_{\tau, t_{a}}}, \quad \Lambda_{3}^{\tau, t_{a}} \equiv \frac{\mathcal{Z}_{\tau, t_{a}}}{2 \mathcal{X}_{\tau, t_{a}}} \\
\Omega_{\Lambda_{\tau, t_{a}}}\left(\begin{array}{cc}
q & q^{\prime} \\
a & q_{0} \\
a & b
\end{array}\right) \equiv \operatorname{erfi}\left(\frac{a \Lambda_{1}^{\tau, t_{a}} \Lambda_{3}^{\tau, t_{a}} q^{\prime}+b \Lambda_{2}^{\tau, t_{a}}\left|\Lambda_{1}^{\tau, t_{a}} q_{0}+c \Lambda_{3}^{\tau, t_{a}} q\right|}{\sqrt{2 \Lambda_{1}^{\tau, t_{a}} \Lambda_{2}^{\tau, t_{a}} \Lambda_{3}^{\tau, t_{a}}}}\right),
\end{gathered}
$$


which, after a long and laborious computation leads to the exact result

$$
\begin{aligned}
& P_{\mathrm{eq}}\left(q, \tau+t_{a}, q^{\prime}, t_{a}, q_{0}\right)=\frac{q q^{\prime} q_{0}}{16 \pi}\left(\frac{\gamma^{2}-S_{t_{a}}^{2}}{\left[S_{t_{a}} \mathcal{X}_{\tau, t_{a}}-\mathcal{Y}_{\tau, t_{a}} \mathcal{Z}_{\tau, t_{a}}\right] \mathcal{Y}_{\tau, t_{a}} \mathcal{Z}_{\tau, t_{a}}}\right)^{1 / 2} \\
& \times \exp \left(-\frac{S_{t_{a}}\left(\gamma^{2}-S_{t_{a}}^{2}\right) q^{2}}{4\left(S_{t_{a}} \mathcal{X}_{\tau, t_{a}}-\mathcal{Y}_{\tau, t_{a}} \mathcal{Z}_{\tau, t_{a}}\right)}-\frac{\left(\gamma+S_{t_{a}} \frac{\mathcal{Z}_{\tau, t_{a}}}{\mathcal{Y}_{\tau, t_{a}}}\right) q^{\prime 2}+\left(\gamma+S_{t_{a}} \frac{\mathcal{Y}_{\tau, t_{a}}}{\mathcal{Z}_{\tau, t_{a}}}\right) q_{0}^{2}}{4\left(\gamma^{2}-S_{t_{a}}^{2}\right)}\right)
\end{aligned}
$$

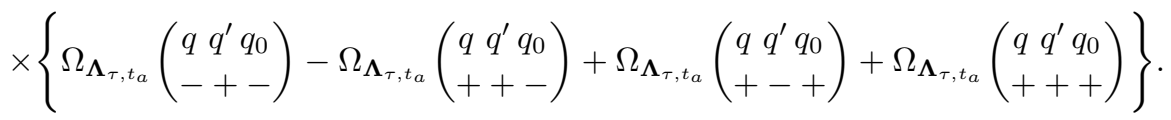

The conditional three-point density is in turn obtained from Eq. (D23) by

$$
G_{\mathrm{eq}}\left(q, \tau+t_{a}, q^{\prime}, t_{a} \mid q_{0}\right)=P_{\mathrm{eq}}\left(q, \tau+t_{a}, q^{\prime}, t_{a}, q_{0}\right) / P_{\mathrm{eq}}\left(q_{0}\right)
$$

Having obtained all quantities required for the computation of the aging correlation function and the time asymmetry index $\Upsilon$, the remaining integrals

$$
C_{t_{a}}(\tau)=\int_{0}^{\infty} d q \int_{0}^{\infty} d q^{\prime} q q^{\prime} G_{\mathrm{eq}}\left(q, \tau+t_{a}, q^{\prime}, t_{a} \mid q_{0}\right)-\int_{0}^{\infty} d q q G_{\mathrm{eq}}\left(q, \tau+t_{a} \mid q_{0}\right) \int_{0}^{\infty} d q q G_{\mathrm{eq}}\left(q, t_{a} \mid q_{0}\right)
$$

as well as

$$
\Upsilon_{q_{0}}\left(t_{a}, \tau\right)=\int_{0}^{\infty} d q \int_{0}^{\infty} d q^{\prime} G_{\mathrm{eq}}\left(q, \tau+t_{a}, q^{\prime}, t_{a} \mid q_{0}\right) \log \left(\frac{G_{\mathrm{eq}}\left(q, \tau+t_{a}, q^{\prime}, t_{a} \mid q_{0}\right)}{G_{\mathrm{eq}}\left(q, \tau \mid q^{\prime}\right) G_{\mathrm{eq}}\left(q^{\prime}, t_{a} \mid q_{0}\right)}\right)
$$

are performed using an adaptive Gauss-Kronrod routine [123]. The results for a Rouse chain with $N=50$ beads are presented in Fig. D1 and the corresponding time asymmetry index $\Upsilon_{q_{0}}\left(t_{a}, \tau\right)$ in Fig. 3a in the manuscript.
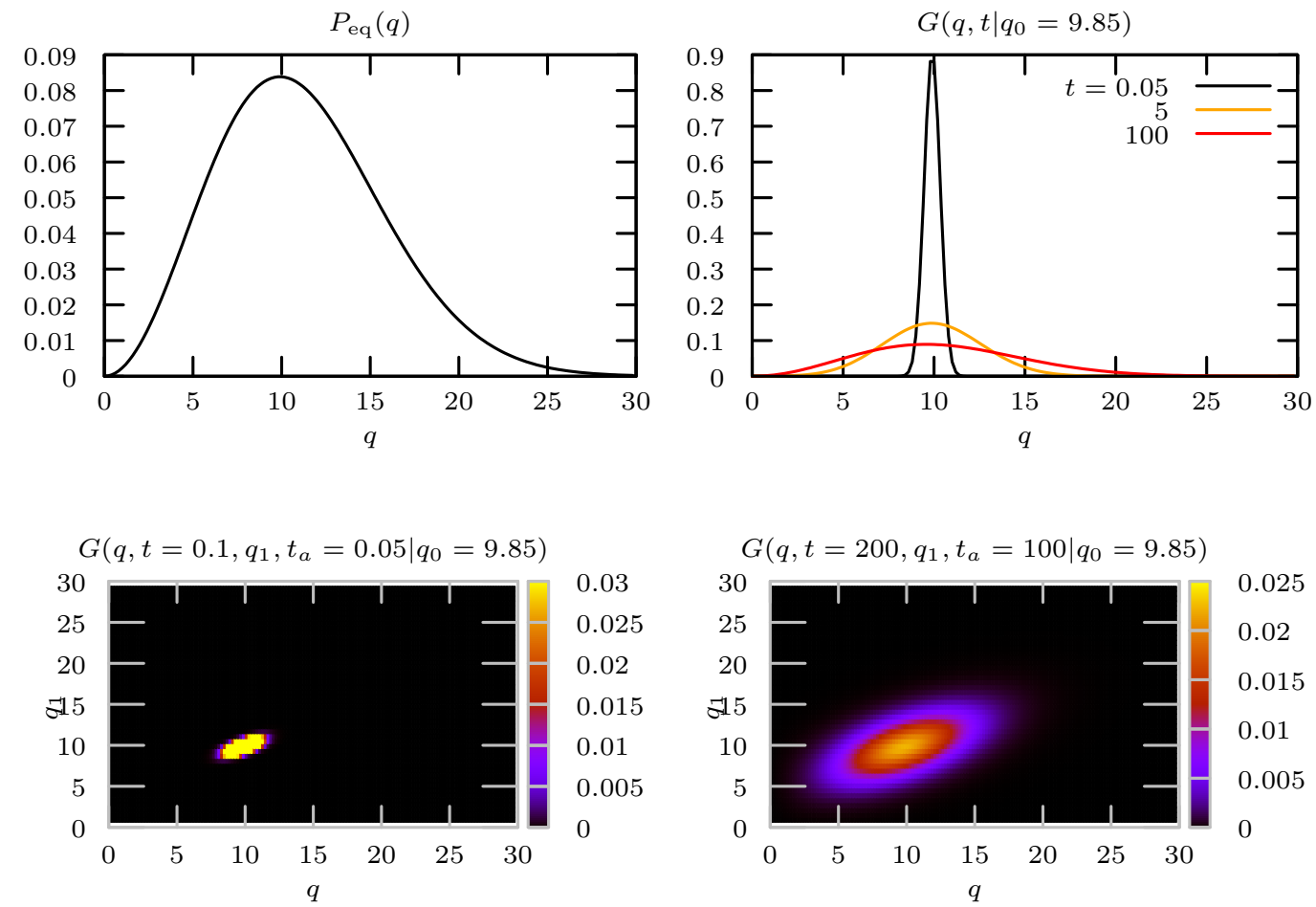

Figure D1. The top left panel shows the density of the invariant measure $P_{\text {eq }}(q)$, while the top right panel depicts the conditional two-point density $G_{\text {eq }}\left(q, t \mid q_{0}\right)$ in Eq. (D16) for $q_{0}=9.85$ and three different $t$. The bottom panels show the conditional threepoint probability density function $G_{\mathrm{eq}}\left(q, \tau+t_{a}, q^{\prime}, t_{a} \mid q_{0}\right)$ in Eq. (D24) for two combinations of $t=\tau+t_{a}$ and $t_{a}$. 
The density of the invariant measure of the end-to-end distance, $P_{\mathrm{eq}}(q)$ (Fig. D1, top left) is concentrated in the regime $0<q<30$ with a maximum at $q_{\text {peak }}=9.85 \approx 10$. The evolution of the conditional two-point conditional probability density for an ensemble of trajectories starting at the typical distance $q_{\text {peak }}, G_{\text {eq }}\left(q, t \mid q_{\text {peak }}\right)$ (Fig. D1, top right) evolves smoothly towards $P_{\mathrm{eq}}(q)$ with a relaxation time $t_{\mathrm{rel}}=\lambda_{1}^{-1} \approx 253.4$. Notably, the corresponding threepoint density $G_{\text {eq }}\left(q, t, q^{\prime}, t_{a} \mid q_{\text {peak }}\right)$ (Fig. D1, bottom) shows strong long-time correlations in the evolution of $q(t)$, e.g. even for aging times $t_{a}=100$ (which are already of the order of, but still smaller than, $t_{\text {rel }}$ ) the value of $q$ at time $t=2 t_{a}$ is strongly correlated with its value $q^{\prime}$ at $t_{a}$ (Fig. D1, bottom right). This long-lasting correlations, which are the result of the projection of the full $3(N+1)$-dimensional dynamics of the polymer onto a single distance coordinate $q(t)$, are responsible for the dynamical time asymmetry.

Note that the relaxation time scales quadratically with the length of the chain, in.e. $t_{\text {rel }} \propto N^{2}$ and therefore the dynamical time asymmetry extends, for long polymers $N \gtrsim 10^{4}$ over many orders in time. However, for such long chains the computation or $\Upsilon$ at short $t_{a}, \tau$ becomes numerically unstable. In Fig. D2 we demonstrate the quadratic growth of relaxation time-scales displaying dynamical time asymmetry with increasing $N$.
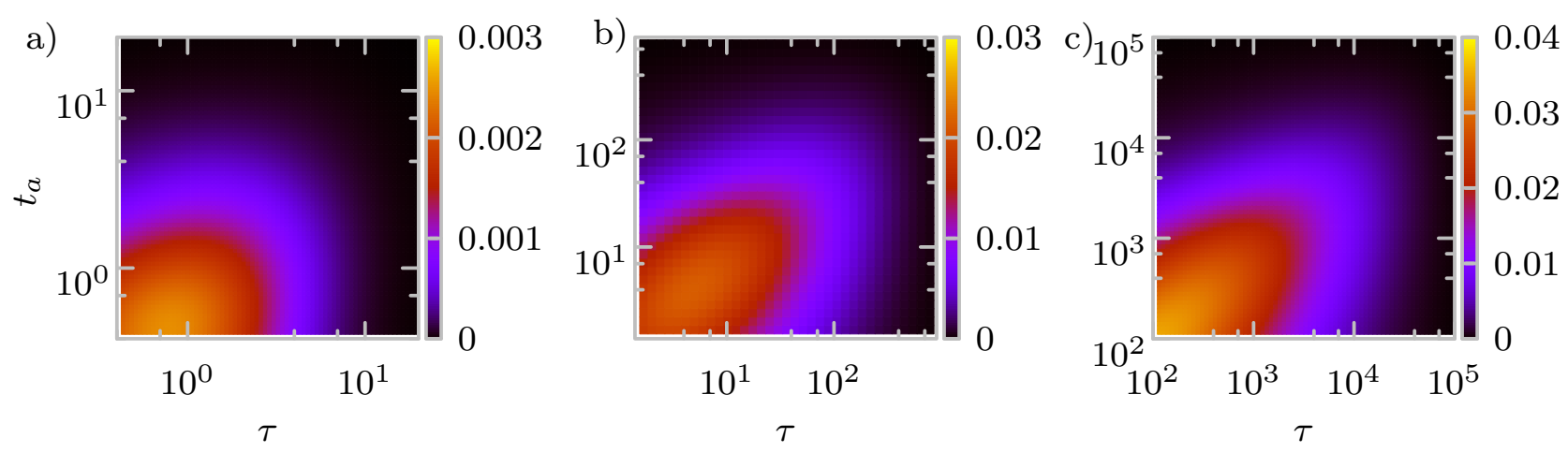

Figure D2. $\Upsilon\left(t_{a}, \tau\right)$ for a) $N=10$, b) $N=10^{2}$, and c) $N=10^{3}$ portraying a growing time-scale of dynamical time asymmetry.

\section{Single file diffusion}

The single file model refers to the overdamped Brownian motion of a system of $N$ particles with hard core exclusion interactions, which for simplicity (and because the finite-size scenario is obtained by a simple re-scaling of space) we assume to be point-like and confined to an interval of unit length $L=1[101,124,125]$. We express length in units of $L$ and time in units of $\tau=D / L^{2}$, where $D$ corresponds to the diffusion coefficient which is assumed to be equal for all particles. The state of the system is completely described with the vector of particle positions $\mathbf{x}=\left(x_{1}, \ldots, x_{N}\right)$. We are interested in tagged-particle dynamics and therefore our projected observable corresponds to the position of the $i$-th particle, $q(t)=x_{i}(t)$. The full system's dynamics is driven solely by entropic driving forces because the potential energy is strictly zero. In turn, the free energy landscape (i.e. the potential of the mean force acting on the tagged particle) corresponds to the entropic landscape, whereas the potential energy hypersurface is perfectly flat.

The Fokker-Planck equation for the Green's function with initial condition $Q\left(\mathbf{x}, t=0 \mid \mathbf{x}_{0}\right)$ describing the dynamics of $N$ reads

$$
\left(\partial_{t}-\sum_{i} \partial_{x_{i}}^{2}\right) Q\left(\mathbf{x}, t \mid \mathbf{x}_{0}\right)=0, \quad Q\left(\mathbf{x}, t=0 \mid \mathbf{x}_{0}\right)=\prod_{i=1}^{N} \delta\left(x_{i, 0}-x_{i}\right),
$$

which is solved under $N-1$ non-crossing boundary conditions

$$
\lim _{x_{i+1} \rightarrow x_{i}}\left(\partial_{x_{0, i+1}}-\partial_{x_{0, i}}\right) Q\left(\mathbf{x}, t \mid \mathbf{x}_{0}\right)=0, \quad \forall i
$$

The system is exactly solvable with the coordinate Bethe ansatz, which yields explicit results for the spectral expansion of $Q\left(\mathbf{x}, t \mid \mathbf{x}_{0}\right)[101,125]$, i.e. $Q\left(\mathbf{x}, t \mid \mathbf{x}_{0}\right)=\sum_{\mathbf{k}} \psi_{\mathbf{k}}^{R}(\mathbf{x}) \psi_{\mathbf{k}}^{L}\left(\mathbf{x}_{0}\right) \mathrm{e}^{-\lambda_{\mathbf{k}} t}$ according to Eq. (A4), where we introduced the $N$ tuple $\mathbf{k}=\left(k_{1}, \ldots, k_{N}\right), k_{i} \in \mathbb{N}, \forall i$. Expressions for the eigenfunctions $\psi_{\mathbf{k}}^{R}(\mathbf{x}), \psi_{\mathbf{k}}^{L}(\mathbf{x})$ are given in [101, 125] and the eignevalues corresponding to $\lambda_{\mathbf{k}}=\sum_{i=1}^{N} k_{i} \pi^{2}$. Note that the relaxation time $t_{\mathrm{rel}}=1 / \lambda_{\mathbf{1}}$ once re-scaled to natural 
units in terms of the collision time (i.e. $t_{\text {col }}=(L / N)^{2} / D$ scales as $\propto N^{2}$.

The projection operator is turn defined by Eq. (B1) with $\delta\left(x_{i}-q\right)$, which according to Eq. (B7) yields, upon some tedious algebra, $G_{\text {eq }}\left(q, t \mid q_{0} \in \Xi_{0}\right)$ in Eq. (B10) and $G_{\text {eq }}\left(q, t, q^{\prime}, t_{a} \mid q_{0} \in \Xi_{0}\right)$ in Eq. (B21) with matrix elements

$$
\Psi_{\mathbf{k l}}(x)=\frac{\mathbf{m}_{\mathbf{l}} !}{N_{L} ! N_{R} !} \sum_{\left\{n_{i}\right\}} T_{j}(x) \prod_{i=1}^{j-1} L_{i}(x) \prod_{i=j+1}^{N} R_{i}(x)
$$

where $\mathbf{m}_{\mathbf{l}} !=\prod_{i} m_{k_{i}}$ is the multiplicity of the eigenstate with $m_{k_{i}}$ corresponds to the number of times a particular value of $k_{1}$ appears in the tuple and $N_{L}, N_{R}$ are the number of particles to the left and right from the tagged particle, respectively. The sum $\sum_{\left\{n_{i}\right\}}$ is over all permutations of the elements of the $N$-tuple k. For the equilibrium density we find $P_{\text {eq }}(q)=\frac{N !}{N_{L} ! N_{R} !} q^{N_{L}}(1-q)^{N_{R}}$. In Eq. (D29) we have defined the auxiliary functions

$$
\begin{gathered}
T_{j}(x)=\left\{\begin{array}{l}
1 \quad \lambda_{k}=\lambda_{l}=0 \\
\sqrt{2} \cos \left(\lambda_{k} / l \pi x\right) \\
2 \cos \left(\lambda_{k} \pi x\right) \cos \left(\lambda_{l} \pi x\right) \quad \text { otherwise }
\end{array}\right. \\
L_{j}(x)=\left\{\begin{array}{ll}
x \quad \lambda_{k}=\lambda_{l}=0 \\
\sqrt{2} \frac{\sin \left(\lambda_{k / l} \pi x\right)}{\lambda_{k / l} \pi} \quad \lambda_{k}=0 \text { or } \lambda_{l}=0 \\
x+\frac{\sin \left(2 \lambda_{k} \pi x\right)}{2 \lambda_{k} \pi x} \quad \lambda_{k}=\lambda_{l} \\
2 \frac{\lambda_{k} \cos \left(\lambda_{l} \pi x\right) \sin \left(\lambda_{k} \pi x\right)-\lambda_{l} \cos \left(\lambda_{k} \pi x\right) \sin \left(\lambda_{l} \pi x\right)}{\pi\left(\lambda_{k}^{2}-\lambda_{l}^{2}\right)}
\end{array} \quad\right. \text { otherwise } \\
R_{j}(x)= \begin{cases}1-x \quad \lambda_{k}=\lambda_{l}=0 & \text { otherwise } \\
-\sqrt{2} \frac{\sin \left(\lambda_{k / l} \pi x\right)}{\lambda_{k / l} \pi} & \lambda_{k}=0 \text { or } \lambda_{l}=0 \\
1-x-\frac{\sin \left(2 \lambda_{k} \pi x\right)}{2 \lambda_{k} \pi x} & \lambda_{k}=\lambda_{l} \\
2 \frac{-\lambda_{k} \cos \left(\lambda_{l} \pi x\right) \sin \left(\lambda_{k} \pi x\right)+\lambda_{l} \cos \left(\lambda_{k} \pi x\right) \sin \left(\lambda_{l} \pi x\right)}{\pi\left(\lambda_{k}^{2}-\lambda_{l}^{2}\right)}\end{cases}
\end{gathered}
$$

The autocorrelation functions $C(t)$ and $C_{t_{a}}(\tau)$ can now be calculated using Eqs. (B14) and (B16), respectively, where trivially $\langle q(t)\rangle=\left(N_{l}+1\right) /(N+1)$ and $\left\langle q(0)^{2}\right\rangle=\left(N_{L}+2\right)\left(N_{L}+1\right) /[(N+2)(N+1)]$. As it was impossible to carry out this final step analytically, we carried out the integrals in Eqs. (B14) and (B16) depicted in Fig. 3b in the main text numerically according to the trapezoidal rule.

The computation of the time asymmetry index $\Upsilon$ in Eq. (B25), which was as well performed using the trapezoidal rule on a grid of 100 points, is extremely challenging even for moderate values of $N$. By repeating the integration using a smaller grid of 50 points we double-checked that the integration routine converged to a sufficient degree. The results for a single file of $N=5$ particles tagging the third particle are presented in Fig. $3 \mathrm{~b}$ in the main text and in Fig. D3.

The density of the invariant measure of the tagged central particle (Fig. D3, top left) peaks in the center of the unit box, $q_{\text {peak }}=0.5$, and decays towards the borders due to the entropic repulsion with the neighbors. The evolution of the conditional two-point conditional probability density for an ensemble of trajectories starting at the typical distance $q_{\text {peak }}, G_{\text {eq }}\left(q, t \mid q_{\text {peak }}\right)$ (Fig. D3, top right) evolves smoothly towards $P_{\text {eq }}(q)$ with a relaxation time $t_{\text {rel }}=\lambda_{1}^{-1} \approx 2.5$.

Similar to the end-to-end distance of the Rouse polymer the corresponding three-point density of the tagged particle, $G_{\text {eq }}\left(q, t, q^{\prime}, t_{a} \mid q_{\text {peak }}\right)$ (Fig. D3, bottom) shows strong long-time correlations in the evolution of $q(t)$, e.g. even for aging times $t_{a}=0.125$ (which are already of the order of, but still smaller than, $t_{\text {rel }}$ ) the value of $q$ at time $t=2 t_{a}$ is strongly correlated with its value $q^{\prime}$ at $t_{a}$ (Fig. D1, bottom right). This long-lasting correlation reflects prolonged entropic bottlenecks (i.e. 'traffic-jams'), which require collective rearrangements of many particles and thus decorrelate slowly, giving rise to strong memory effects and dynamical time asymmetry (see Fig. $3 \mathrm{~b}$ in the main text) as soon as $q_{0}$ is initially not sampled from $P_{\text {eq }}\left(q_{0}\right)$. 

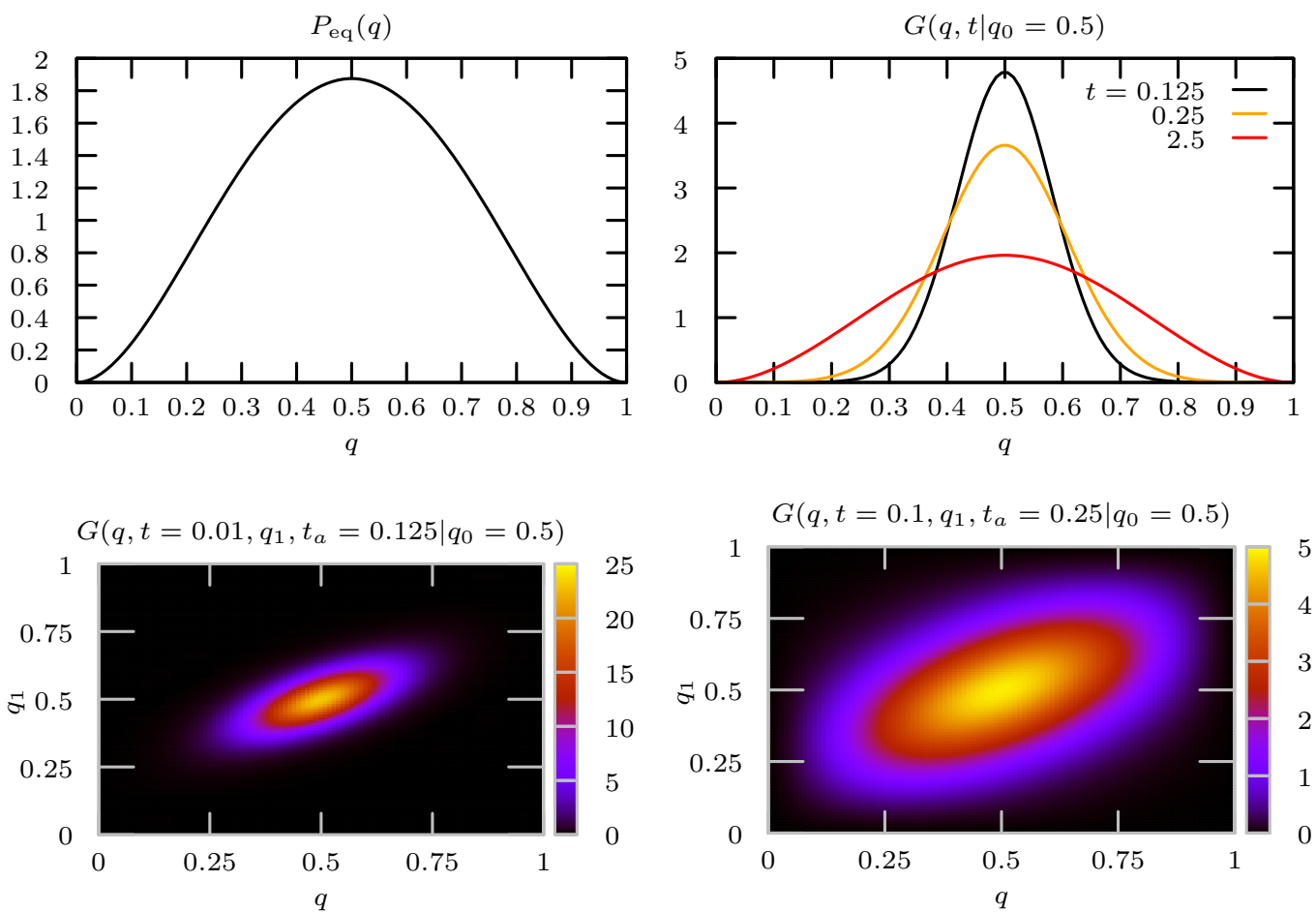

Figure D3. Single file. The top left panel depicts the density of the equilibrium measure $P_{\text {eq }}(q)$ of the third particle in a single file of five particles, $q(t)=x_{3}(t)$. The top right panel shows a two-point conditional probability density function $G\left(q, t \mid q_{0}\right)$ for different values of $t$, where $q_{0}=0.5$ (i.e. the maximum of the $P_{\text {eq }}(q)$ ). The bottom panels depict the three-point density $G\left(q, t, q^{\prime}, t_{a} \mid q_{0}\right)$ at different $\tau$ and $t_{a}$ evolving from the same initial condition. To produce $G\left(q, t, q^{\prime}, t_{a} \mid q_{0}\right)$ the spectral expansion Eq. (A4) was truncated at maximum Bethe eigenvalue $225 \pi^{2}$.

\section{Analysis of experimental and simulation data}

We now consider the time series of a low-dimensional projected observable sampled at discrete time steps that is frequently encountered in the analysis of experimental data. Therefore we first translate all definitions in Sec. B to discrete time series and explain in detail how to carry out the complete analysis of aging dynamics for such systems. To ease the application of these new concepts we also provide a $\mathrm{C}++$ routine TSymmetryFinder that will be made available on GitHub.

We consider a discrete time series of length $N$ - in our case a one-dimensional physical observable $q\left(t_{i}\right)$ - sampled at constant time intervals with spacing $t_{i+1}-t_{i}=\Delta t, \forall i$. We pre-process the data by evaluating the mean value over the time series $\bar{q}=N^{-1} \sum_{i=1}^{N} q\left(t_{i}\right)$ and then center the data by subtracting the mean value, $q\left(t_{i}\right) \rightarrow q\left(t_{i}\right)-\bar{q}$. In the first stage we determine the (non-aging) autocorrelation function

$$
C\left(\tau=n_{\tau} \Delta t\right)=\frac{1}{\left(N-n_{\tau}\right) \Delta t} \sum_{i=1}^{N-n_{\tau}} q\left(t_{i+n_{\tau}}\right) q\left(t_{i}\right) \Delta t, \quad n_{\tau} \ll N
$$

and determine the relaxation time as $t_{r}: \min _{t_{i}}\left[\mathcal{C}\left(t_{i}\right) / \mathcal{C}(0)<\epsilon\right]$, where we choose $\epsilon=0.05$. All data are henceforth analyzed such that $n_{\max } \equiv n_{\tau_{\max }} \approx n_{r}=t_{r} /(\Delta t)$ in order to assure sufficient sampling when evaluating sliding averages.

Next we determine the equilibrium probability density function and two-point conditional probability density (Eq. (B2)) as a histogram taken over the data. We introduce bins $\mathcal{B}_{i}$ centered at $q_{i}$ with a width $\delta q$ and define the characteristic function of a bin $\mathbb{1}_{\mathcal{B}_{i}}\left[q\left(t_{i}\right)\right]=1$ if $q_{i}-\delta q / 2 \leq q\left(t_{i}\right)<q_{i}+\delta q / 2$ and zero otherwise and let $\mathbb{1}_{\Xi_{0}}[q]$ be the indicator function of the initial condition. Let us further define $n_{l}\left(n_{\tau}\right)=\max _{i} q\left(t_{i}\right) \in \Xi_{0}: n_{l}+n_{\tau} \leq N$. The 
equilibrium probability density function is then determined as

$$
P_{\mathrm{eq}}\left(q_{i}\right)=(N \delta q)^{-1} \sum_{i=1}^{N} \mathbb{1}_{\mathcal{B}_{i}}\left[q\left(t_{i}\right)\right]
$$

and the 2-point conditional probability density as

$$
\begin{aligned}
G\left(q_{i}, n_{\tau} \mid q_{0} \in \Xi_{0}\right) & =\frac{\sum_{i=1}^{n_{l}\left(n_{\tau}\right)} \mathbb{1}_{\mathcal{B}_{i}}\left[q\left(t_{i+n_{\tau}}\right)\right] \mathbb{1}_{\Xi_{0}}\left[q\left(t_{i}\right)\right]}{\delta q \sum_{i=1}^{n_{l}\left(n_{\tau}\right)} \mathbb{1}_{\Xi_{0}}\left[q\left(t_{i}\right)\right]}, \\
G\left(q_{i}, n_{\tau} \mid q_{j}\right) & =\frac{\sum_{i=1}^{n_{l}\left(n_{\tau}\right)} \mathbb{1}_{\mathcal{B}_{i}}\left[q\left(t_{i+n_{\tau}}\right)\right] \mathbb{1}_{\mathcal{B}_{j}}\left[q\left(t_{i}\right)\right]}{\delta q \sum_{i=1}^{n_{l}\left(n_{\tau}\right)} \mathbb{1}_{\Xi_{0}}\left[q\left(t_{i}\right)\right]},
\end{aligned}
$$

The three-point conditional density Eq. (B21) is defined for $n_{\tau^{\prime}} \leq n_{\tau}$ analogously as

$$
G\left(q_{i}, n_{\tau}, q_{j}, n_{\tau^{\prime}} \mid q_{0} \in \Xi_{0}\right)=\frac{\sum_{i=1}^{n_{l}\left(n_{\tau}\right)} \mathbb{1}_{\mathcal{B}_{i}}\left[q\left(t_{i+n_{\tau}}\right)\right] \mathbb{1}_{\mathcal{B}_{j}}\left[q\left(t_{i+n_{\tau^{\prime}}}\right)\right] \mathbb{1}_{\Xi_{0}}\left[q\left(t_{i}\right)\right]}{\delta q^{2} \sum_{i=1}^{n_{l}\left(n_{\tau}\right)} \mathbb{1}_{\Xi_{0}}\left[q\left(t_{i}\right)\right]} .
$$

Introducing $\delta n=n_{\tau}-n_{a}$ the time-asymmetry index is in turn determined as a double sum

$$
\Upsilon_{\Xi_{0}}\left(t_{a}, \tau\right)=\delta q^{2} \sum_{i, j} G\left(q_{i}, \delta n+n_{a}, q_{j}, n_{a} \mid q_{0} \in \Xi_{0}\right) \log \frac{G\left(q_{i}, \delta n+n_{a}, q_{j}, n_{a} \mid q_{0} \in \Xi_{0}\right)}{G\left(q_{i}, \delta n+n_{a} \mid q_{j}\right) G\left(q_{i}, n_{a} \mid q_{0} \in \Xi_{0}\right)},
$$

whereas the normalized aging correlation function (Eq. (B16) is determined according to

$$
\begin{aligned}
\hat{C}_{t_{a}}(\tau) & =\frac{\left\langle q\left(t_{\delta n+n_{a}}\right) q\left(t_{n_{a}}\right)\right\rangle-\left\langle q\left(t_{\delta n+n_{a}}\right)\right\rangle\left\langle q\left(t_{n_{a}}\right)\right\rangle}{\left\langle q^{2}\left(t_{n_{a}}\right)\right\rangle-\left\langle q\left(t_{n_{a}}\right)\right\rangle^{2}}, \\
\left\langle q\left(t_{\delta n+n_{a}}\right) q\left(t_{n_{a}}\right)\right\rangle & \equiv \sum_{i, j} q_{i} q_{j} G\left(q_{i}, \delta n+n_{a}, q_{j}, n_{a} \mid q_{0} \in \Xi_{0}\right), \\
\left\langle q\left(t_{i}\right)\right\rangle & \equiv \sum_{i, j} q_{i} G\left(q_{i}, n_{i} \mid q_{0} \in \Xi_{0}\right),
\end{aligned}
$$

where $\tau=\delta n \Delta t$ and $t_{a}=n_{a} \Delta t$ and we note that by construction (i.e. due to the centering of data) $\left\langle q\left(t_{i}\right)\right\rangle=0, \forall i$. 100 bins in $q\left(t_{\delta n+n_{a}}\right)$ and 100 bins in $q\left(t_{n_{a}}\right)$ were used for each combination of $\tau$ and $t_{a}$ to determine $G\left(q_{i}, \delta n+\right.$ $\left.n_{a}, q_{j}, n_{a} \mid q_{0} \in \Xi_{0}\right), G\left(q_{i}, \delta n+n_{a} \mid q_{j}\right)$ and $G\left(q_{i}, n_{a} \mid q_{0} \in \Xi_{0}\right)$ and in turn $\Upsilon_{\Xi_{0}}\left(t_{a}, \tau\right)$.

\section{a. DNA-hairpin}

Dual optical tweezers data of the DNA hairpin were kindly provided by the Woodside group [69] in the form of a constant trap measurements of the DNA hairpin 30R50T4, sampled at $400 \mathrm{kHz}$, for trap stiffness $0.63 p N / n m$ in one optical trap and $1.1 \mathrm{pN} / \mathrm{nm}$ in the other. The time series was $2.75 \cdot 10^{4} \mathrm{~ms}$ long. The normalized aging correlation function $C_{t_{a}}(\tau)$ and dynamical time asymmetry index $\Upsilon$ are depicted in Figs. 3c and $4 \mathrm{~b}$ in the manuscript. Here, we additionally present in Fig. D4, for illustrative purposes and for the sake of completeness, the density of the invariant (equilibrium) measure $P_{\mathrm{eq}}(q)$ and exemplary two-point conditional probability $G\left(q, t \mid q_{0} \in \Xi_{0}\right)$ and the three-point conditional density $G\left(q, \tau+t_{a}, q^{\prime}, t_{a} \mid q_{0} \in \Xi_{0}\right)$, respectively, for various $t$. The histograms in the relative deviations $q\left(t_{i}\right)=q_{\text {raw }}\left(t_{i}\right)-\bar{q}$ were determined by binning the interval from $-25 \mathrm{~nm}$ to $+15 \mathrm{~nm}$ into 100 bins and $\bar{q}=3.47 \mathrm{~nm}$. These probability density functions are shown in Fig. D4.

The density of the invariant measure of the extension of the hairpin is bimodal, reflecting the existence of two long-lived conformational states (Fig. D5, top left). The evolution of the conditional two-point conditional probability density for an ensemble of trajectories starting at the typical distance $q_{\text {peak }}, G_{\text {eq }}\left(q, t \mid q_{\text {peak }}\right)$ (Fig. D5, top right) evolves smoothly towards $P_{\mathrm{eq}}(q)$ with a relaxation time $t_{\mathrm{rel}}=\lambda_{1}^{-1} \approx 15 \mathrm{~ms}$, and nicely depicts the onset of conformational transitions (see red line).

Another striking feature of hairpin dynamics is seen in the corresponding three-point density, $G_{\text {eq }}\left(q, t, q^{\prime}, t_{a} \mid q_{\text {peak }}\right)$ (Fig. D5, bottom), which depicts, alongside the linear correlations along and near the diagonal $q=q^{\prime}$ that were also present in the Rouse polymer and tagged-particle diffusion in a single-file, prominent non-linear correlations (see off-diagonal peaks). This readily reveals that temporal correlations in the motion persist beyond the time-scale of 

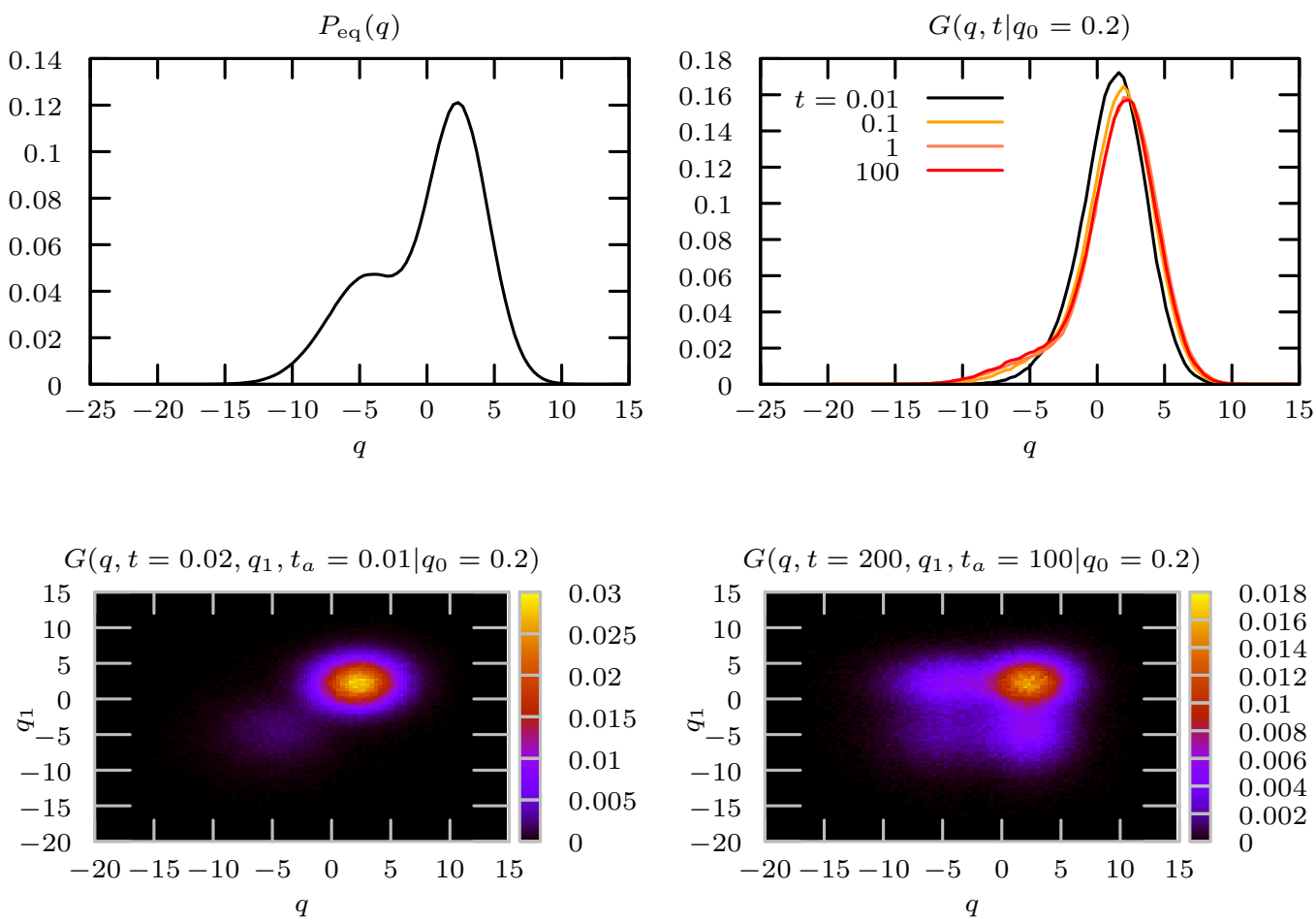

Figure D4. DNA Hairpin. In the top left panel depicts the density of the equilibrium measure $P_{\text {eq }}(q)$ of the centered time series $q\left(t_{i}\right)=q_{\text {raw }}\left(t_{i}\right)-\bar{q}$, while the top right shows a two-point conditional probability density function $G\left(q, t \mid q_{0} \in \Xi_{0}\right)$ for different values of $t$, where $\Xi_{0}=[0.2-0.2,0.2+0.2] \mathrm{nm}$. The bottom panels depict the three-point density $G\left(q, t, q^{\prime}, t_{a} \mid q_{0} \in \Xi_{0}\right)$ at different $\tau$ and $t_{a}$ evolving from the same initial condition.

$q_{0}=0.2 \pm 0.2 \mathrm{~nm}$

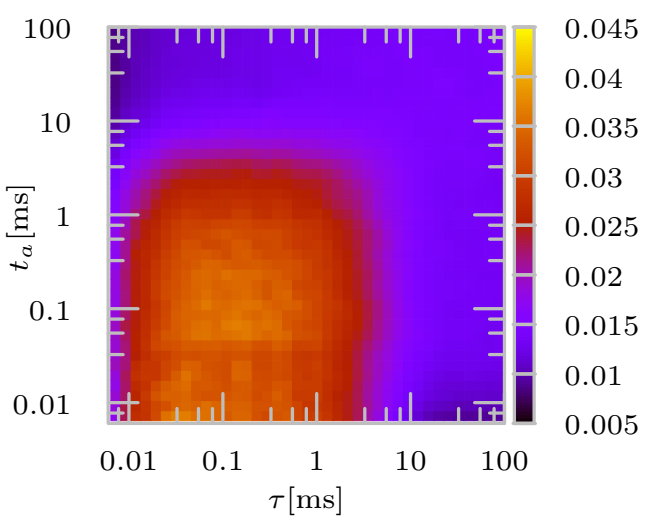

$q_{0}=-5.0 \pm 0.2 \mathrm{~nm}$

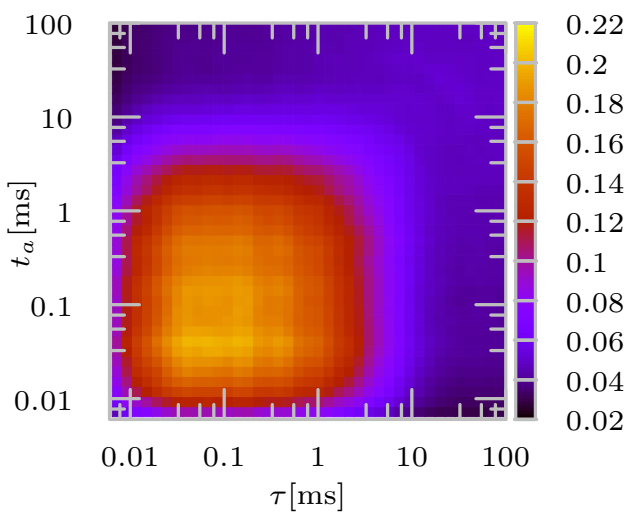

Figure D5. DNA Hairpin, second example. The time asymmetry index for the DNA hairpin data as in Fig. D4 but with the initial condition $\Xi_{0}=[-5-0.2,-5+0.2] \mathrm{nm}$.

conformational transitions, that is, the hairpin relaxation dynamics post transition remembers the configurations prior to the transition even on time-scales of $\gtrsim 200 \mathrm{~ms}$, which reflects a very long range of broken Markovianity. However, a comparison with the corresponding time asymmetry index in Fig. 3c in the main text shows that at aging times $t_{a}=100 \mathrm{~ms}$ the dynamics is already time-translation invariant. This is a nice and clear practical demonstration of the important conceptual difference between the notion of relaxation with a broken time-translation invariance and 
memory effects in time-translation invariant relaxation of a low-dimensional physical observable.

In order to demonstrate the robustness of these observations with respect to specific the initial condition $q_{0}=1(0)$ (as long as $p_{0}\left(q_{0}\right) \neq P_{\text {eq }}\left(q_{0}\right)$ that is) we also present in Fig. D5 the results for a different set of initial conditions. The results in Fig. D5 show qualitatively the same features and are fully consistent with the statements in the manuscript.

Finally, we asses the statistical uncertainty of determining $\Upsilon$ from the experimental time-series. We do so by performing the analysis on an ensemble of trajectories obtained by randomly removing 10 (from the total of 50 , i.e. $20 \%$ of the data) trajectories and averaging over 20 repetitions of a data-set created in this manner. We quantify the statistical error by determining the local standard deviation of $\Upsilon$, i.e. $\sigma_{\Upsilon}=\sqrt{N_{i}^{-1} \sum_{i=1}^{N_{i}} \Upsilon_{i}^{2}-\left(N_{i}^{-1} \sum_{i=1}^{N_{i}} \Upsilon_{i}\right)^{2}}$ where $N_{i}=20$. The results are shown in Fig. D6 and depict a local error that is smaller than $1 \%$.
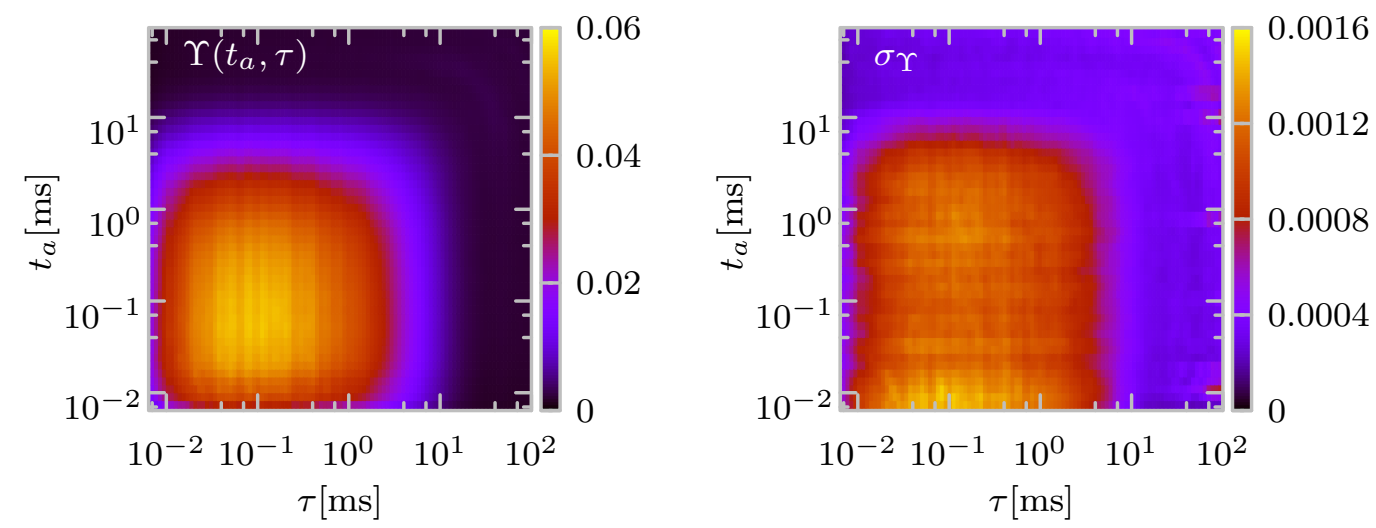

Figure D6. Statistical error in the DNA-hairpin analysis. a) average $\Upsilon\left(\tau, t_{a}\right)$ determined from an ensemble with forced under-sampling (i.e. by omiting $20 \%$ of the data); b) the standard deviation of the local $\Upsilon\left(\tau, t_{a}\right)$.

b. Yeast 3-phosphoglycerate kinase (PGK)

Atomistic Molecular Dynamics (MD) simulation of yeast PGK were carried out by $\mathrm{Hu}$ et al. [39], starting from the PDB structure 3PGK with a duration of $1.71 \cdot 10^{5} \mathrm{ps}$. The observable $q\left(t_{i}\right)$ refers here to the distance between the center of mass of the N-terminal domain (residues 1-185) and the center of mass of C-terminal domain (residues 200-389). In Fig. D7 we depict the density of the invariant (equilibrium) measure $P_{\text {eq }}(q)$ and exemplary two-point conditional probability $G\left(q, t \mid q_{0} \in \Xi_{0}\right)$ and the three-point conditional density $G\left(q, \tau+t_{a}, q^{\prime}, t_{a} \mid q_{0} \in \Xi_{0}\right)$, respectively, for various $t$. The histograms in the relative deviations $q\left(t_{i}\right)=q_{\mathrm{raw}}\left(t_{i}\right)-\bar{q}$ were determined by binning the interval from $-0.2 \mathrm{~nm}$ to $+0.3 \mathrm{~nm}$ into 100 bins and $\bar{q}=0.67 \mathrm{~nm}$.

The density of the invariant measure $P_{\text {eq }}(q)$ (Fig. D7, top left) is unimodal and effects of a poorer statistics are readily discernable through the roughness of the curve. The evolution of the conditional two-point conditional probability density for an ensemble of trajectories starting at the typical distance $q_{\text {peak }}, G_{\text {eq }}\left(q, t \mid q_{\text {peak }}\right)$ is shown in Fig. D7 (top right panel) and reveals that the the dynamics along $q$ is strongly localized (i.e. $G_{\text {eq }}\left(q, t \mid q_{\text {peak }}\right.$ barely changes between $t=100 \mathrm{ps}$ and $t=3000)$. Note that PGK did not relax within the duration of the trajectory. The corresponding three-point density, $G_{\text {eq }}\left(q, t, q^{\prime}, t_{a} \mid q_{\text {peak }}\right)$ (Fig. D7, bottom), shows that the observable almost does not relax at all within $6 \times 10^{4} \mathrm{ps}$ (compare left and right panel). As in the case of the Rouse polymer $G_{\text {eq }}\left(q, t, q^{\prime}, t_{a} \mid q_{\text {peak }}\right)$ shows strong and long-lasting correlations between positions, and comparison with the dynamical time asymmetry index in Fig. $3 \mathrm{~d}$ in the main text reveals that the dynamics has a strongly time-translation invariance. These findings corroborate the original analysis of $\mathrm{Hu}$ et al. [39] who observed aging effects.

Similar to the DNA hairpin we also present in Fig. D8 the results for the dynamical time asymmetry index for a different set of initial conditions and two different choices of the observable $q(t)$ for PGK, which demonstrate the robustness of the results.

PGK obviously does not relax within the duration of the trajectory and more generally it is conceivable that larger, complex proteins do not relax at all during their life-time [39], which makes them virtually 'forever aging' [126], which may have important consequences for their biological function. Such aging effects on function were observabed in single-enzyme turnover statistics [41-43] and have so-far been rationalized only with ad-hoc phenomenological models $[42,43]$. The present theoretical framework provides a unifying mechanistic understanding dynamics with 

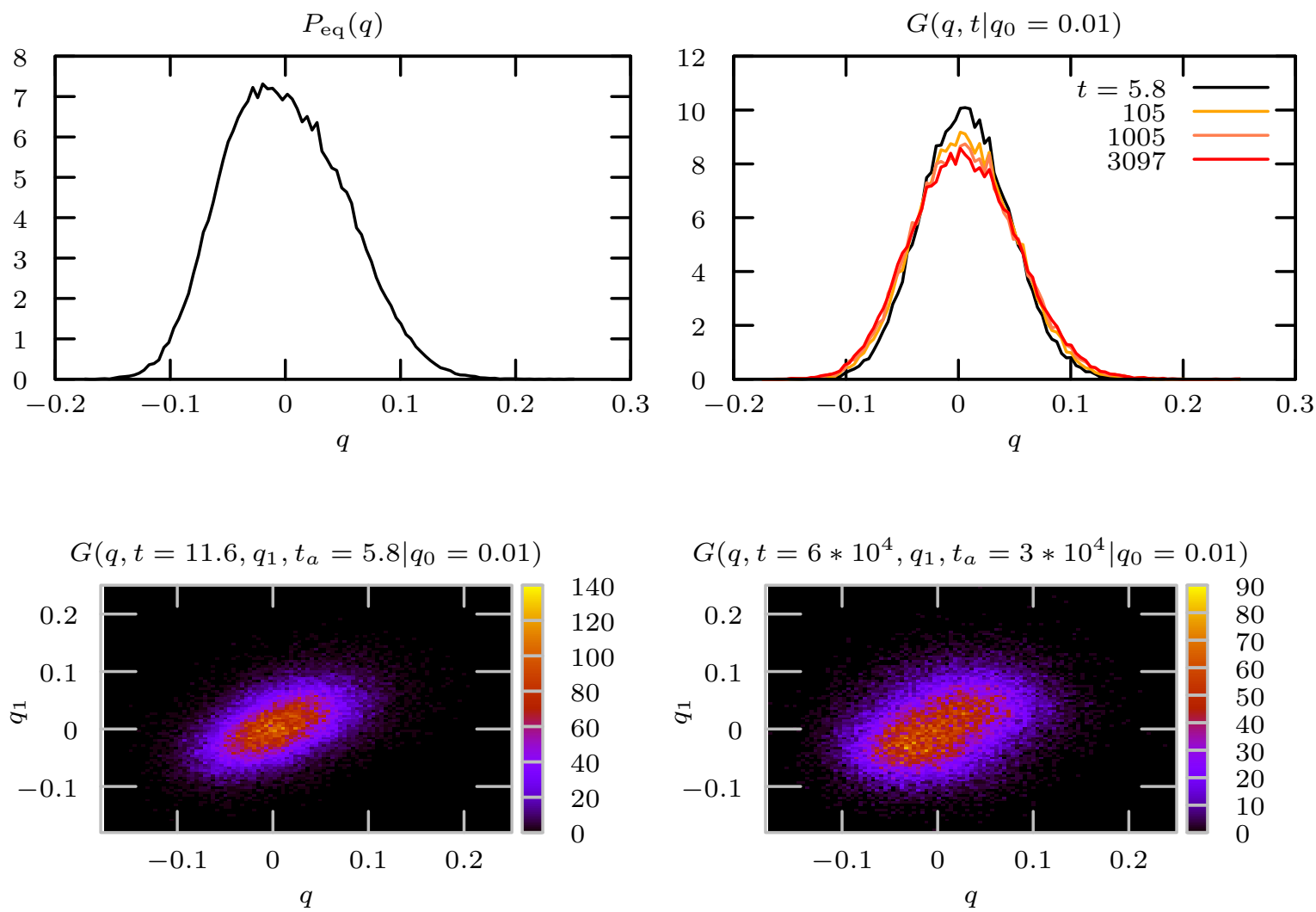

Figure D7. PGK. The top left panel depicts the density of the equilibrium measure $P_{\text {eq }}(q)$ of the centered time series $q\left(t_{i}\right)=q_{\mathrm{raw}}\left(t_{i}\right)-\bar{q}$, while the top right shows a two-point conditional probability density function $G\left(q, t \mid q_{0} \in \Xi_{0}\right)$ for different values of $t$, where $\Xi_{0}=[0.01-0.005,0.01+0.005] \mathrm{nm}$. The bottom panels depict the three-point density $G\left(q, t, q^{\prime}, t_{a} \mid q_{0} \in \Xi_{0}\right)$ at different $\tau$ and $t_{a}$ evolving from the same initial condition.

broken time-translation invariance in soft and biological matter and will pave the way for deeper and more systematic investigations of the potential biological relevance of memory and dynamical time asymmetry for enzymatic catalysis. 
$q_{0}=0.03 \pm 0.2 \mathrm{~nm}$, res : $C M-C M$
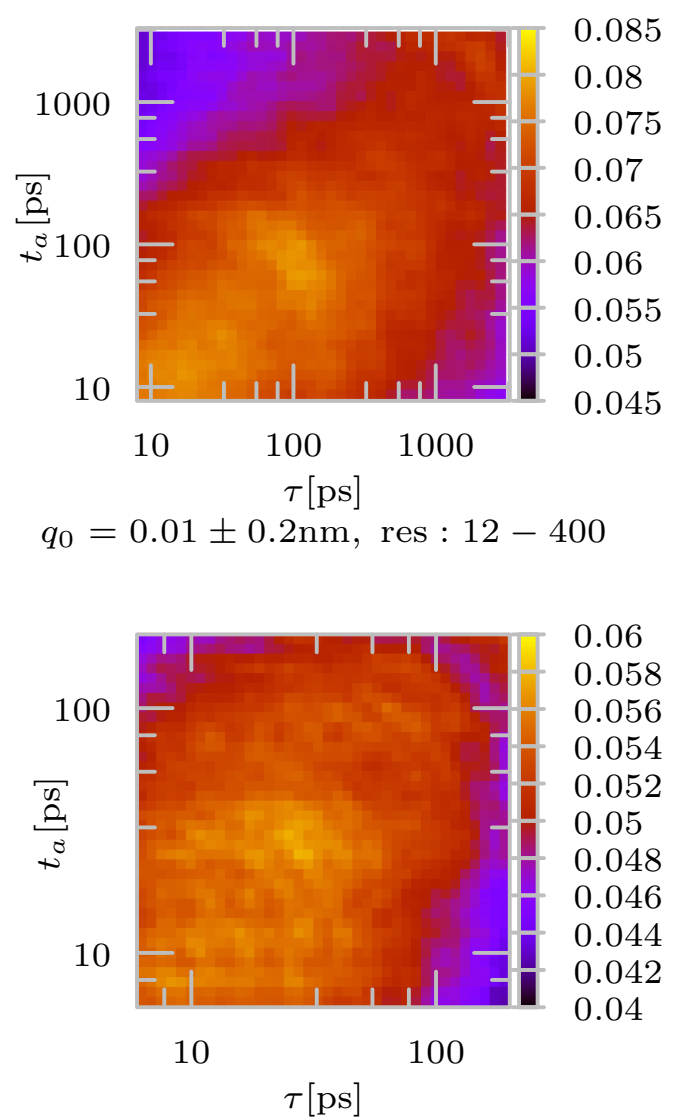

$q_{0}=-0.03 \pm 0.2 \mathrm{~nm}$, res : $C M-C M$
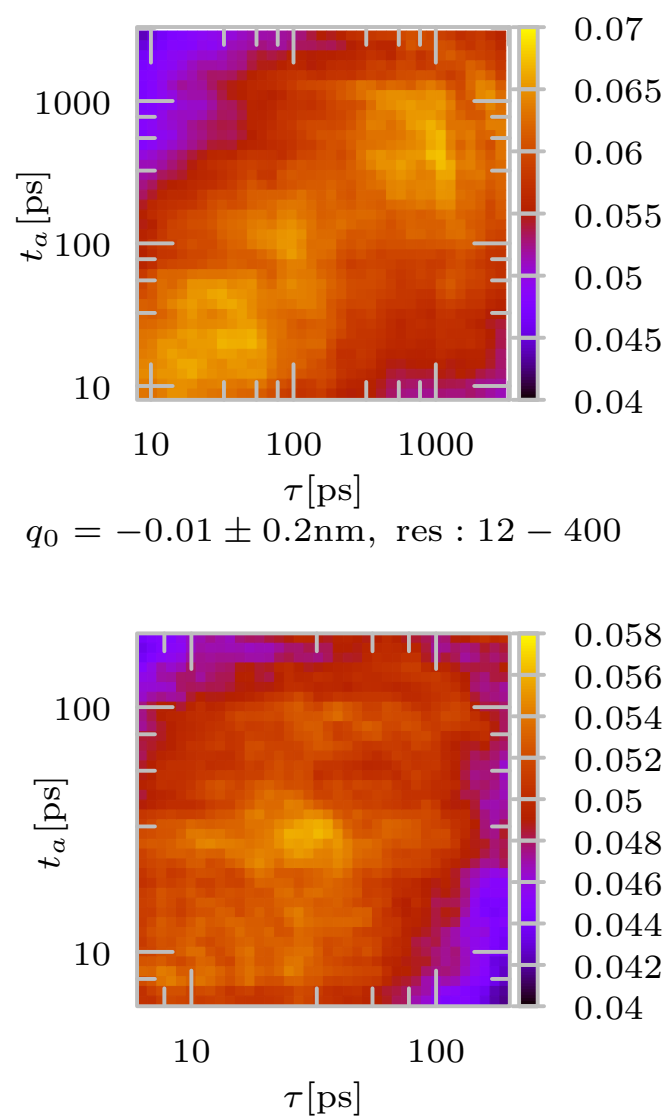

Figure D8. PGK, second example. Top: dynamical time asymmetry index for yeast PGK when $q(t)$ corresponds to distance between the center of masses of the N- and C-terminal domains (respectively residues 1-185 and 200-389) for a pair different initial conditions. Bottom: dynamical time asymmetry index for yeast PGK when $q(t)$ corresponds to the distance between two specific residues (the 12th and the 400th) for two different initial conditions. 
[1] A. Farhan, P. M. Derlet, A. Kleibert, A. Balan, R. V. Chopdekar, M. Wyss, J. Perron, A. Scholl, F. Nolting, and L. J. Heyderman, Direct Observation of Thermal Relaxation in Artificial Spin Ice, Phys. Rev. Lett. 111, 057204 (2013).

[2] S. Dattagupta, Relaxation phenomena in condensed matter physics (Elsevier, 2012).

[3] R. Kubo, M. Toda, and N. Hashitsume, Statistical Physics II (Springer Berlin Heidelberg, 1991).

[4] H. Chen, E. Rhoades, J. S. Butler, S. N. Loh, and W. W. Webb, Dynamics of equilibrium structural fluctuations of apomyoglobin measured by fluorescence correlation spectroscopy, Proc. Natl. Acad. Sci. USA 104, 10459-10464 (2007)

[5] L. Onsager, Reciprocal relations in irreversible processes. i., Phys. Rev. 37, 405 (1931).

[6] L. Onsager, Reciprocal relations in irreversible processes. ii., Phys. Rev. 38, 2265 (1931).

[7] R. Kubo, M. Yokota, and S. Nakajima, Statisticalmechanical theory of irreversible processes. ii. response to thermal disturbance, J. Phys. Soc. Jpn. 12, 1203-1211 (1957).

[8] R. Metzler, E. Barkai, and J. Klafter, Anomalous diffusion and relaxation close to thermal equilibrium: A fractional fokker-planck equation approach, Phys. Rev. Lett. 82, 3563 (1999).

[9] C. Maes, K. Netočný, and B. Wynants, Monotonic Return to Steady Nonequilibrium, Phys. Rev. Lett. 107, 010601 (2011).

[10] M. Baiesi and C. Maes, New J. Phys. 15, 013004 (2013).

[11] M. Polettini and M. Esposito, Nonconvexity of the relative entropy for Markov dynamics: A Fisher information approach, Phys. Rev. E 88, 012112 (2013).

[12] C. Maes, Phys. Rev. Lett. 119, 160601 (2017).

[13] C. Maes, Frenesy: Time-symmetric dynamical activity in nonequilibria, Phys. Rep. 850, 1 (2020).

[14] J. Kurchan, In and out of equilibrium, Nature 433, 222-225 (2005).

[15] L. F. Cugliandolo, D. S. Dean, and J. Kurchan, Fluctuation-dissipation theorems and entropy production in relaxational systems, Phys. Rev. Lett. 79, 2168 (1997).

[16] Z. Lu and O. Raz, Nonequilibrium thermodynamics of the Markovian Mpemba effect and its inverse, Proc. Natl. Acad. Sci. USA 114, 5083 (2017).

[17] I. Klich, O. Raz, O. Hirschberg, and M. Vucelja, Phys. Rev. X 9, 021060 (2019)

[18] N. Shiraishi and K. Saito, Information-Theoretical Bound of the Irreversibility in Thermal Relaxation Processes, Phys. Rev. Lett. 123, 110603 (2019).

[19] A. Lapolla and A. Godec, Faster uphill relaxation in thermodynamically equidistant temperature quenches, Phys. Rev. Lett. 125, 110602 (2020).

[20] J. P. Bouchaud, Weak ergodicity breaking and aging in disordered systems, J. de Physique I 2, 1705-1713 (1992).

[21] E. Vincent, J. Hammann, M. Ocio, J.-P. Bouchaud, and L. F. Cugliandolo, Slow dynamics and aging in spin glasses, Lecture Notes in Physics , 184-219.

[22] J.-P. Bouchaud, L. F. Cugliandolo, J. Kurchan, and M. Mézard, Out of equilibrium dynamics in spin-glasses and other glassy systems, in Spin Glasses and Random Fields (World Scientific, 1997) pp. 161-223.

[23] C. Monthus and J.-P. Bouchaud, Models of traps and glass phenomenology, J. Phys. A: Math. Gen. 29, 3847-3869 (1996).

[24] J.-P. Bouchaud and D.S. Dean, Aging on parisi's tree, J. Phys. I France 5, 265 (1995).

[25] F. Ritort and P. Sollich, Glassy dynamics of kinetically constrained models, Adv. Phys. 52, 219 (2003).

[26] I. M. Hodge, Physical aging in polymer glasses, Science 267, 1945-1947 (1995).

[27] H. Oukris and N. E. Israeloff, Nanoscale nonequilibrium dynamics and the fluctuation-dissipation relation in an ageing polymer glass, Nat. Phys. 6, 135-138 (2009).

[28] D. Hérisson and M. Ocio, Fluctuation-dissipation ratio of a spin glass in the aging regime, Phys. Rev. Lett. 88, 257202 (2002).

[29] G. G. Kenning, G. F. Rodriguez, and R. Orbach, End of aging in a complex system, Phys. Rev. Lett. 97, 057201 (2006).

[30] J. Mattsson, H. M. Wyss, A. Fernandez-Nieves, K. Miyazaki, Z. Hu, D. R. Reichman, and D. A. Weitz, Soft colloids make strong glasses, Nature 462, 83-86 (2009).

[31] L. Cipelletti, S. Manley, R. C. Ball, and D. A. Weitz, Universal aging features in the restructuring of fractal colloidal gels, Phys. Rev. Lett. 84, 2275 (2000).

[32] P. Lunkenheimer, R. Wehn, U. Schneider, and A. Loidl, Glassy aging dynamics, Phys. Rev. Lett. 95, 055702 (2005).

[33] V. Lubchenko and P. G. Wolynes, Theory of structural glasses and supercooled liquids, Annu. Rev. Phys. Chem. 58, 235 (2007).

[34] H. E. Castillo and A. Parsaeian, Local fluctuations in the ageing of a simple structural glass, Nat. Phys. 3, 26-28 (2006).

[35] M. Utz, P. G. Debenedetti, and F. H. Stillinger, Atomistic simulation of aging and rejuvenation in glasses, Phys. Rev. Lett. 84, 1471 (2000).

[36] H. Frauenfelder, S. Sligar, and P. Wolynes, The energy landscapes and motions of proteins, Science 254, 1598-1603 (1991).

[37] J. Brujić, R. I. Hermans Z., K. A. Walther, and J. M. Fernandez, Single-molecule force spectroscopy reveals signatures of glassy dynamics in the energy landscape of ubiquitin, Nat. Phys. 2, 282-286 (2006).

[38] I. L. Morgan, R. Avinery, G. Rahamim, R. Beck, and O. A. Saleh, Glassy dynamics and memory effects in an intrinsically disordered protein construct, Phys. Rev. Lett. 125, 058001 (2020).

[39] X. Hu, L. Hong, M. Dean Smith, T. Neusius, X. Cheng, and J. Smith, The dynamics of single protein molecules is non-equilibrium and self-similar over thirteen decades in time, Nat. Phys. 12, 171-174 (2015).

[40] Q. Xue and E. S. Yeung, Differences in the chemical reactivity of individual molecules of an enzyme, Nature 373, 681-683 (1995).

[41] H. P. Lu, L. Xun, and X. S. Xie, Single-molecule enzymatic dynamics, Science 282, 1877 (1998).

[42] A. M. van Oijen, P. C. Blainey, D. J. Crampton, 
C. C. Richardson, T. Ellenberger, and X. S. Xie, Singlemolecule kinetics of $\lambda$ exonuclease reveal base dependence and dynamic disorder, Science 301, 1235 (2003).

[43] B. P. English, W. Min, A. M. van Oijen, K. T. Lee, G. Luo, H. Sun, B. J. Cherayil, S. C. Kou, and X. S. Xie, Ever-fluctuating single enzyme molecules: Michaelismenten equation revisited, Nat. Chem. Biol. 2, 87-94 (2005).

[44] N. C. Keim, J. D. Paulsen, Z. Zeravcic, S. Sastry, and S. R. Nagel, Memory formation in matter, Rev. Mod. Phys. 91, 035002 (2019).

[45] S. Burov, R. Metzler, and E. Barkai, Aging and nonergodicity beyond the khinchin theorem, Proc. Natl. Acad. Sci. 107, 13228 (2010).

[46] S. Franz and J. Hertz, Glassy transition and aging in a model without disorder, Phys. Rev. Lett. 74, 2114 (1995).

[47] F. Ritort, Glassiness in a model without energy barriers, Phys. Rev. Lett. 75, 1190 (1995).

[48] L. F. Cugliandolo, J. Kurchan, and P. Le Doussal, Large time out-of-equilibrium dynamics of a manifold in a random potential, Phys. Rev. Lett. 76, 2390 (1996).

[49] A. Amir, Y. Oreg, and Y. Imry, On relaxations and aging of various glasses, Proc. Natl. Acad. Sci. 109, 1850 (2012).

[50] L. F. Cugliandolo and J. Kurchan, Analytical solution of the off-equilibrium dynamics of a long-range spin-glass model, Phys. Rev. Lett. 71, 173 (1993).

[51] L. F. Cugliandolo and J. Kurchan, On the out-ofequilibrium relaxation of the sherrington-kirkpatrick model, J. Phys. A: Math. Gen. 27, 5749 (1994).

[52] G. Folena, S. Franz, and F. Ricci-Tersenghi, Rethinking mean-field glassy dynamics and its relation with the energy landscape: The surprising case of the spherical mixed $p$-spin model, Phys. Rev. X 10, 031045 (2020).

[53] S. Franz, M. Mézard, G. Parisi, and L. Peliti, Measuring equilibrium properties in aging systems, Phys. Rev. Lett. 81, 1758 (1998).

[54] P. Mayer, S. Léonard, L. Berthier, J. P. Garrahan, and $\mathrm{P}$. Sollich, Activated aging dynamics and negative fluctuation-dissipation ratios, Phys. Rev. Lett. 96, 030602 (2006).

[55] A. Dechant, E. Lutz, D. A. Kessler, and E. Barkai, Scaling green-kubo relation and application to three aging systems, Phys. Rev. X 4, 011022 (2014).

[56] P. Charbonneau, J. Kurchan, G. Parisi, P. Urbani, and F. Zamponi, Fractal free energy landscapes in structural glasses, Nat. Commun. 5, 10.1038/ncomms4725 (2014).

[57] L. F. Cugliandolo, J. Kurchan, G. Parisi, and F. Ritort, Matrix models as solvable glass models, Phys. Rev. Lett. 74, 1012 (1995).

[58] E. Barkai, Aging in subdiffusion generated by a deterministic dynamical system, Phys. Rev. Lett. 90, 104101 (2003).

[59] D. Hartich and A. Godec, Duality between relaxation and first passage in reversible markov dynamics: rugged energy landscapes disentangled, New J. Phys. 20, 112002 (2018).

[60] D. Hartich and A. Godec, Interlacing relaxation and first-passage phenomena in reversible discrete and continuous space markovian dynamics, J. Stat. Mech. Theor. Exp. 2019, 024002 (2019).

[61] T. Speck and U. Seifert, Integral fluctuation theorem for the housekeeping heat, J. Phys. A: Math. Gen. 38,
L581 (2005).

[62] R. Chétrite, S. Gupta, I. Neri, and É. Roldán, Martingale theory for housekeeping heat, EPL 124, 60006 (2019).

[63] H.-M. Chun and J. D. Noh, Universal property of the housekeeping entropy production, Phys. Rev. E 99, 012136 (2019).

[64] K. Liu, Z. Gong, and M. Ueda, Thermodynamic uncertainty relation for arbitrary initial states, Phys. Rev. Lett. 125, 140602 (2020).

[65] A. Bovier and A. Faggionato, Spectral characterization of aging: The rem-like trap model, Ann. Appl. Probab. 15, 1997 (2005).

[66] S. M. Fielding, P. Sollich, and M. E. Cates, Aging and rheology in soft materials, J. Rheol. 44, 323 (2000).

[67] P. Sollich, F. m. c. Lequeux, P. Hébraud, and M. E. Cates, Rheology of soft glassy materials, Phys. Rev. Lett. 78, 2020 (1997).

[68] K. Neupane, F. Wang, and M. T. Woodside, Direct measurement of sequence-dependent transition path times and conformational diffusion in dna duplex formation, Proc. Natl. Acad. Sci. 114, 1329 (2017).

[69] K. Neupane, N. Q. Hoffer, and M. T. Woodside, Measuring the local velocity along transition paths during the folding of single biological molecules, Phys. Rev. Lett. 121, 018102 (2018).

[70] A. Solanki, K. Neupane, and M. T. Woodside, Singlemolecule force spectroscopy of rapidly fluctuating, marginally stable structures in the intrinsically disordered protein $\alpha$-synuclein, Phys. Rev. Lett. 112, 158103 (2014).

[71] C. Hyeon, M. Hinczewski, and D. Thirumalai, Evidence of disorder in biological molecules from single molecule pulling experiments, Phys. Rev. Lett. 112, 138101 (2014).

[72] From a thermodynamic point of view such systems are characterized by a transiently positive entropy production that vanishes upon reaching equilibrium.

[73] M. I. Freidlin and A. D. Wentzell, Random Perturbations of Dynamical Systems (Springer Berlin Heidelberg, 2012).

[74] F. U. Hartl, A. Bracher, and M. Hayer-Hartl, Molecular chaperones in protein folding and proteostasis, Nature 475, 324-332 (2011).

[75] G. A. Pavliotis, Stochastic Processes and Applications (Springer New York, 2014).

[76] L. E. Reichl, A Modern Course in Statistical Physics, 2nd Ed. (John Wiley \& Sons, 1998).

[77] J. Keizer, Statistical Thermodynamics of Nonequilibrium Processes (Springer New York, 1987).

[78] P. Hänggi, Path integral solutions for non-markovian processes, Z. Physik B Cond. Mat. 75, 275-281 (1989).

[79] P. Hänggi and P. Jung, Colored noise in dynamical systems, Adv. Chem. Phys. , 239-326 (2007).

[80] R. F. Fox, The generalized langevin equation with gaussian fluctuations, J. Math. Phys. 18, 2331 (1977), https://doi.org/10.1063/1.523242.

[81] Generally speaking "strict stationarity" and Definition 1 in Appendix C are equivalent only if $q(t)$ is a Gaussian process. If this is not the case Definition 1 imposes a milder condition on time-translation symmetry.

[82] G. B. Arous, A. Dembo, and A. Guionnet, Aging of spherical spin glasses, Probab. Theory Relat. Fields 
120, 1-67 (2001).

[83] E. Barkai, Aging in subdiffusion generated by a deterministic dynamical system, Phys. Rev. Lett. 90, 104101 (2003).

[84] E. Barkai and Y.-C. Cheng, Aging continuous time random walks, J. Chem. Phys. 118, 6167 (2003).

[85] A. Lapolla and A. Godec, Manifestations of projectioninduced memory: General theory and the tilted single file, Front. Phys. 7, 182 (2019).

[86] B. Robertson, Equations of motion in nonequilibrium statistical mechanics, Phys. Rev. 144, 151 (1966).

[87] J. L. Lebowitz and P. G. Bergmann, Ann. Phys. 1, 1 (1957).

[88] M. C. Mackey, The dynamic origin of increasing entropy, Rev. Mod. Phys. 61, 981-1015 (1989).

[89] H. Qian, A decomposition of irreversible diffusion processes without detailed balance, J. Math. Phys. 54, 053302 (2013).

[90] M. Medina-Noyola and J. Del Rio-Correa, The fluctuation-dissipation theorem for non-markov processes and their contractions: The role of the stationarity condition, Physica A 146, 483 (1987).

[91] F. Nielsen, On the jensen-shannon symmetrization of distances relying on abstract means, Entropy 21, $10.3390 / \mathrm{e} 21050485$ (2019).

[92] J. Tang and R. A. Marcus, Chain dynamics and powerlaw distance fluctuations of single-molecule systems, Phys. Rev. E 73, 022102 (2006).

[93] R. Granek and J. Klafter, Fractons in proteins: Can they lead to anomalously decaying time autocorrelations?, Phys. Rev. Lett. 95, 098106 (2005).

[94] W. Glöckle and T. Nonnenmacher, A fractional calculus approach to self-similar protein dynamics, Biophys. J. 68, 46-53 (1995).

[95] J. Kigami and M. L. Lapidus, Weyl's problem for the spectral distribution of laplacians on p.c.f. self-similar fractals, Commun. Math. Phys. 158, 93-125 (1993).

[96] A. Amir, Y. Oreg, and Y. Imry, Slow relaxations and aging in the electron glass, Phys. Rev. Lett. 103, 10.1103/physrevlett.103.126403 (2009).

[97] G. F. Rodriguez, G. G. Kenning, and R. Orbach, Full aging in spin glasses, Phys. Rev. Lett. 91, 10.1103/physrevlett.91.037203 (2003).

[98] J. H. P. Schulz, E. Barkai, and R. Metzler, Aging renewal theory and application to random walks, Phys. Rev. X 4, 011028 (2014).

[99] D. Hartich and A. Godec, Emergent memory and kinetic hysteresis in strongly driven networks (2020), arXiv:2011.04628 [cond-mat.stat-mech].

[100] C. W. Pyun and M. Fixman, Intrinsic viscosity of polymer chains, J. Chem. Phys. 42, 3838 (1965).

[101] A. Lapolla and A. Godec, Bethesf: Efficient computation of the exact tagged-particle propagator in single-file systems via the bethe eigenspectrum, Comput. Phys. Commun. 258, 107569 (2021).

[102] R. Metzler, Forever ageing, Nat. Phys. 12, 113 (2015).

[103] A. Lapolla, D. Hartich, and A. Godec, Spectral theory of fluctuations in time-average statistical mechanics of reversible and driven systems, Phys. Rev. Research 2, 043084 (2020).

[104] A. Buhot and J. P. Garrahan, Fluctuation-dissipation relations in the activated regime of simple strong-glass models, Phys. Rev. Lett. 88, 225702 (2002).

[105] M. T. Woodside and S. M. Block, Reconstructing folding energy landscapes by single-molecule force spectroscopy, Annu. Rev. Biophys. 43, 19 (2014), https://doi.org/10.1146/annurev-biophys-051013022754 .

[106] S. W. Englander and L. Mayne, The case for defined protein folding pathways, Proc. Natl. Acad. Sci. 114, 8253-8258 (2017).

[107] W. A. Eaton and P. G. Wolynes, Theory, simulations, and experiments show that proteins fold by multiple pathways, Proc. Natl. Acad. Sci. 114, E9759-E9760 (2017).

[108] H. Risken, The Fokker-Planck Equation (Springer Berlin Heidelberg, 1989).

[109] B. Helffer and F. Nier, Hypoelliptic Estimates and Spectral Theory for Fokker-Planck Operators and Witten Laplacians (Springer, Berlin, Heidelberg, Heidelberg, 2005).

[110] L. Chupin, Fokker-Planck equation in bounded domain, Ann. Inst. Fourier. 60, 217 (2010).

[111] M. Reed and B. Simon, Methods of Modern Mathematical Physics I: Functional Analysis (Academic Press, New York, 1972).

[112] N. V. KAMPEN, Chapter v - the master equation, in Stochastic Processes in Physics and Chemistry (Third Edition), North-Holland Personal Library, edited by N. van Kampen (Elsevier, Amsterdam, 2007) third edition ed., pp. $96-133$.

[113] M. F. Weber and E. Frey, Master equations and the theory of stochastic path integrals, Rep. Prog. Phys. 80, 046601 (2017).

[114] S. Kullback and R. Leibler, On information and sufficiency, Ann. Math. Statist 22, 79 (1951).

[115] E. Noether, Invariante variationsprobleme, Nachrichten von der Gesellschaft der Wissenschaften zu Göttingen, Mathematisch-Physikalische Klasse 1918, 235 (1918).

[116] D. Hérisson and M. Ocio, Fluctuation-dissipation ratio of a spin glass in the aging regime, Phys. Rev. Lett. 88, 10.1103/physrevlett.88.257202 (2002).

[117] M. Rubí and C. Pérez-Vicente, Complex Behaviour of Glassy Systems (Springer Berlin Heidelberg, 1997).

[118] E. W. Montroll and M. F. Shlesinger, On $1 / \mathrm{f}$ noise and other distributions with long tails, Proc. Natl. Acad. Sci. 79, 3380 (1982), https://www.pnas.org/content/79/10/3380.full.pdf.

[119] E. Barkai and I. M. Sokolov, Multi-point distribution function for the continuous time random walk, J. Stat. Mech. Theor. Exp. 2007, P08001 (2007).

[120] C. W. Pyun and M. Fixman, Intrinsic viscosity of polymer chains, J. Chem. Phys. 42, 3838 (1965), https://doi.org/10.1063/1.1695848.

[121] G. Wilemski and M. Fixman, Diffusion-controlled intrachain reactions of polymers. I Theory, J. Chem. Phys. 60, 866 (1974).

[122] Note that $\mathbf{Q}_{0}$ corresponds to the center of mass, which does not affect the dynamics of internal coordinates.

[123] https://www.boost.org/doc/libs/1_71_0/libs/ math/doc/html/quadrature.html (20/09/2019) (2019).

[124] L. Lizana and T. Ambjörnsson, Single-File Diffusion in a Box, Phys. Rev. Lett. 100, 200601 (2008).

[125] A. Lapolla and A. Godec, Unfolding tagged particle histories in single-file diffusion: exact single- and two-tag local times beyond large deviation theory, New J. Phys. 20, 113021 (2018).

[126] R. Metzler, Forever ageing, Nat. Phys. 12, 113 (2015). 


\section{Chapter 11}

\section{Time- and ensemble-average statistical mechanics of the Gaussian Network Model}

This article is currently under review [58. The pre-print is freely available from the ArXiv repository. The Gaussian Network Model describes an isotropic network of beads in a thermal bath connected by non-Hookean springs, i.e. the springs have a non-zero rest length at zero-temperature. This model has been largely used for the study of large amplitude motions in proteins, since it takes into account the internal rigidity of the system. In the publication we focus on the statistics of the intra-beads distance. We obtain analytical expressions for the propagator, the autocorrelation function and the local time fluctuations for the distance between two beads or between two center of masses of a subset of beads. The final results are computationally challenging to evaluate, therefore the publication is accompanied by a $\mathrm{C}++$ code implementing the analytical formulae. The analytical expression for the covariance matrix of this model, a common observable in Molecular Dynamics simulations, has been found as well. We apply our findings to protein structures and rigid frames. 


\title{
Time- and ensemble-average statistical mechanics of the Gaussian Network Model
}

\author{
Alessio Lapolla, Maximilian Vossel and Aljaž Godec \\ Mathematical bioPhysics group, Max Planck Institute for Biophysical Chemistry, Am \\ Fassberg 11, Göttingen 37077, Germany \\ E-mail: agodec@mpibpc.mpg.de
}

\begin{abstract}
We present analytical results (up to a numerical diagonalization of a real symmetric matrix) for a set of time- and ensemble-average physical observables in the non-Hookean Gaussian Network Model (GNM) - a generalization of the Rouse model to elastic networks with links with a certain degree of extensional and rotational stiffness. We focus on a set of coarse-grained observables that may be of interest in the analysis of GNM in the context of internal motions in proteins and mechanical frames in contact with a heat bath. A $\mathrm{C}++$ computer code is made available that implements all analytical results.
\end{abstract}

Submitted to: J. Phys. A: Math. Theor.

\section{Introduction}

Proteins utilize their unique dynamic character encoded in internal motions to execute a biological function [1]. These motions span fs to s time-scales and their study thus requires a multitude of experimental and/or computational methods [1]. The most detailed, atomically resolved information about these motions comes - with a grain of salt because of an underlying approximate, empirical potential energy function from Molecular Dynamics (MD) simulations [2, 3]. However, even if the state-of-the-art hardware and highly parallel algorithms allow to reach ms time-scales [4] a substantial time-scale gap remains. In addition, the sheer amount of detail in such tour de force simulations [4] often poses a challenge if one aims at extracting minimal, "leading order" physical principles underlying protein internal motions. Moreover, physical or even topological properties alone may accurately predict selected features of protein dynamics $[5,6]$.

To describe internal motions in proteins on an effective, coarse-grained level disregarding chemical details Tirion introduced the so-called Elastic Network Model (ENM) [7] akin to the seminal works of Rouse [8] and Flory [9] in polymer physics. The basic idea underlying ENMs is an elastic network connecting those residues, more 
precisely the respective $\mathrm{C} \alpha$ atoms, that lie within a cutoff distance typically chosen in the range $7-16 \AA$. Subsequent works considered various alternative models, e.g. socalled Gaussian Network Model (GNM) [10, 11] and the Anisotropic Network Model (ANM) [12, 13].

Up do date elastic network models in various forms have have been successfully applied (and extended) to refine NMR- [14] and X-ray crystallography-derived protein structures [15], derive NMR-structural order parameters [16], investigate structural correlations [17], function [18, 19, 20, 21], conformational transitions [22], and allosteric effects [23] in proteins, and to identify and decompose protein domains [24]. Further applications involve improving Molecular Dynamics simulations [25], the study of protein evolution [26], investigations of smart polymers [27, 28], viruses [29], membrane channels [30,31], and nucleic acids [32], as well as the prediction of rupture points in single-molecule pulling experiments [33],

Most of these works rely on "standard" Normal Mode Analysis (NMA) [34, 35], i.e. on spectral characteristics of the underlying mechanical vibration spectrum. In the particular context of proteins NMA has been used predominantly to identify the large-scale collective motions encoded in the eigenvector corresponding to the principal eigenvalue of the Hessian. Notably, the low-frequency modes are quite insensitive to the precise value of the cutoff distance [36].

Here we go beyond and present analytical results for time- and ensemble-average characteristics of internal "reaction coordinates" in GNM in contact with a heat bath at a finite temperature. More precisely, we consider the non-Markovian dynamics of internal distances at equilibrium. Our results may be relevant for interpreting singlemolecule spectroscopy data or Molecular Dynamics simulations.

\section{The Gaussian Network Model}

The Rouse model [8] is one of the earliest "elastic network" models of flexible linear polymers (later on extended to more general network structures [9]). It neglects excluded volume effects and hydrodynamic interactions. Within this theoretical framework beads are connected by ideal, Hookean springs with vanishing resting length (i.e. at $T=0$ the beads' positions would coincide). The strength of the springs is proportional to the temperature $T$ of the heat bath. The model does not accurately capture the features of molecules with a non-negligibly internal rigidity.

ENMs [7] extend these core ideas by including a non-zero resting length, i.e. at $T=0$ the residues are assumed to have distinct positions that are fixed in space. This idea is consistent with the results of NMR and X-ray crystallography that yield a set of positions $\mathbf{R}^{0}=\left\{\mathbf{r}_{i}^{0}\right\}$ of the $N+1$ residues to which we refer as "the structure" of a protein (NMR experiments in fact yield an ensemble of such structures).

In GNMs a pair of residues $i, j$ within a cutoff distance (i.e. $\left|\mathbf{r}_{i}^{0}-\mathbf{r}_{i}^{0}\right| \leq r_{c}$ ) are assumed to be connected by identical (for sake of simplicity) but non-Hookean springs with a constant $K$. The interaction energy as a function of the particles' positions 
$\mathbf{R}=\left\{\mathbf{r}_{i}\right\}$ is written as

$$
U_{\mathrm{GNM}}\left(\left\{\mathbf{r}_{i j}\right\}\right)=\frac{K}{2} \sum_{\langle i, j\rangle}\left(\mathbf{r}_{i j}-\mathbf{r}_{i j}^{0}\right)^{T}\left(\mathbf{r}_{i j}-\mathbf{r}_{i j}^{0}\right),
$$

where the sum spans all connected pairs. We now introduce for convenience the deviation from the (equilibrium) "structure", $\Delta \mathbf{R}=\left\{\Delta \mathbf{r}_{i} \equiv \mathbf{r}_{i}-\mathbf{r}_{i}^{0}\right\}$. The main simplifying hypothesis of the GNM is that $\Delta \mathbf{R}$ at tempertature $T$ corresponds to an isotropic Gaussian random super-vector, i.e.

$$
P(\Delta \mathbf{R})=\left[(2 \pi)^{N} \tilde{K} \operatorname{det} \Gamma^{-1}\right]^{-3 / 2} \exp \left(-\frac{\tilde{K}}{2} \Delta \mathbf{R}^{T} \boldsymbol{\Gamma} \Delta \mathbf{R}\right),
$$

where $\tilde{K} \equiv K / k_{\mathrm{B}} T$ is the dimensionless strength (in units of thermal energy $k_{\mathrm{B}} T$ ) and $\boldsymbol{\Gamma}$ is a $3(N+1) \times 3(N+1)$ block matrix in which each diagonal block is the connectivity (or Kirchhoff) matrix $\Gamma$ with elements

$$
\Gamma_{i j}= \begin{cases}-1, & \text { if } i \neq j \text { and }\left|\mathbf{r}_{i}^{0}-\mathbf{r}_{j}^{0}\right| \leq r_{c} \\ 0, & \text { if } i \neq j \text { and }\left|\mathbf{r}_{i}^{0}-\mathbf{r}_{j}^{0}\right|>r_{c} \\ -\sum_{j, j \neq i}^{N+1} \Gamma_{i j}, & \text { if } \mathrm{i}=\mathrm{j} .\end{cases}
$$

The dynamics of the beads' positions (i.e. deviations from the equilibrium "structure") is assumed to follow the Itô equation

$$
d \Delta \mathbf{R}(t)=-\xi K \mathbf{\Gamma} \Delta \mathbf{R}(t) d t+\sqrt{2 D} d \mathbf{W}(t),
$$

where $D$ is the diffusion coefficient and $\xi \equiv D / k_{\mathrm{B}} T$ the mobility both assumed to be equal for all beads, and $d \mathbf{W}(t)$ is the increment of the multi-dimensional Wiener process (i.e. Gaussian white noise) with zero mean and covariance $\left\langle d W_{i}(t) d W_{j}\left(t^{\prime}\right)\right\rangle=\delta_{i j} \delta\left(t-t^{\prime}\right)$. A discussion of ANM would require a different matrix $\Gamma$ to take into account for anisotropic interactions between beads. Moreover, the potential energy $U_{E_{N M}}$ would depend only on distances between the residues [35]. We do not treat this model here.

Henceforth we measure energy in units of thermal energy $k_{\mathrm{B}} T$ (i.e. $U \rightarrow U / k_{\mathrm{B}} T$ ), distances in units of the cutoff distance $r_{c}$ (i.e. $\Delta R_{i} \rightarrow \Delta R_{i} / r_{c}$ ) and time in units of the diffusion time, $t_{D} \equiv r_{c}^{2} / D$ - the time required for a bead with a diffusion coefficient $D$ to diffuse a distance $r_{c}$ (i.e. $t \rightarrow t / t_{D}$ ).

It is convenient to pass to normal super-coordinates $\mathbf{Q}=\left\{\mathbf{q}_{k}\right\}$ that diagonalize $\boldsymbol{\Gamma}$, i.e. $\mathbf{Q}^{T} \mathbf{\Gamma Q}=\operatorname{diag}(\boldsymbol{\mu})$ with $(\mathbf{Q})_{i j} \equiv Q_{i j} \mathbb{1}, \mathbb{1}$ being the $3 \times 3$ identity matrix and where the matrix $Q$ diagonalizes the Kirchoff matrix, i.e. $Q^{T} \Gamma Q=\operatorname{diag}\left(\mu_{i}\right)$, and therefore $\operatorname{diag}(\boldsymbol{\mu})_{i i}=\mu_{i} \mathbb{1}$. For convenience we let $k \in\{0, \cdots, N\}$ with $\mu_{0}=0$ and $Q_{i, 0}$ referring to the center of mass motion, while $i \in\{1, \cdots, N+1\}$ such that

$$
\Delta \mathbf{r}_{i}=\sum_{k=0}^{N} Q_{i k} \mathbf{q}_{k}, \forall i\{1, \cdots, N+1\} .
$$

In this notation the Itô equation corresponds to the Fokker-Planck equation describing $N$ independent isotropic three-dimensional Ornstein-Uhlenbeck processes. Neglecting 
the center of mass motion we obtain the following equation for the Green's function (i.e. transition probability density function)

$$
\partial_{t} G\left(\mathbf{Q}, t \mid \mathbf{Q}_{0}\right)=\sum_{k=1}^{N}\left[\partial_{\mathbf{q}_{k}}^{2}+\mu_{k} \partial_{\mathbf{q}_{k}} \mathbf{q}_{k}\right] G\left(\mathbf{Q}, t \mid \mathbf{Q}_{0}\right),
$$

with localized initial condition $G\left(\mathbf{Q}, t=0 \mid \mathbf{Q}_{0}\right)=\delta\left(\mathbf{Q}-\mathbf{Q}_{0}\right)$ and natural boundary conditions $\lim _{|\mathbf{Q}| \rightarrow \infty} G\left(\mathbf{Q}, t \mid \mathbf{Q}_{0}\right)=0$. We solve Eq. (6) by means of an eigendecomposition [37] yielding

$$
G\left(\mathbf{Q}, t \mid \mathbf{Q}_{0}\right)=\sum_{\mathbf{N}} \Psi_{\mathbf{N}}^{R}(\mathbf{Q}) \Psi_{\mathbf{N}}^{L}\left(\mathbf{Q}_{\mathbf{0}}\right) \mathrm{e}^{-\Lambda_{\mathbf{N}} t}
$$

where $\Lambda_{\mathbf{N}}$ denote eigenvalues, $\mathbf{N}$ being a multiset of integer-triples $\left\{\mathbf{n}_{1}, \cdots, \mathbf{n}_{N}\right\}$ with $\mathbf{n}_{i}=\left\{n_{i x}, n_{i y}, n_{i z}\right\}$ such that

$$
\Lambda_{\mathbf{N}}=\sum_{i=1}^{N}\left(n_{i x}+n_{i y}+n_{i z}\right) \mu_{i}
$$

and $\Psi_{\mathbf{N}}^{L}(\mathbf{Q})$ and $\Psi_{\mathbf{N}}^{R}(\mathbf{Q})$ are the corresponding left and right eigenfunction given by

$$
\Psi_{\mathbf{N}}^{L}(\mathbf{Q})=\prod_{i=1}^{N} \psi_{\mathbf{n}_{i}}\left(\mathbf{q}_{i}\right), \quad \Psi_{\mathbf{N}}^{R}(\mathbf{Q})=P_{\mathrm{eq}}(\mathbf{Q}) \prod_{i=1}^{N} \psi_{\mathbf{n}_{i}}\left(\mathbf{q}_{i}\right)
$$

where $P_{\mathrm{eq}}(\mathbf{Q}) \prod_{i=1}^{N}\left(\mu_{i} / 2 \pi\right)^{3 / 2} \mathrm{e}^{-\mu_{i} \mathbf{q}_{i}^{2} / 2}$ is the equilibrium probability density function of normal coordinates and

$$
\psi_{\mathbf{n}_{i}}\left(\mathbf{q}_{i}\right)=\frac{H_{n_{i x}}\left(\mu_{i} q_{i}^{x} / 2\right) H_{n_{i y}}\left(\mu_{i} q_{i}^{y} / 2\right) H_{n_{i z}}\left(\mu_{i} q_{i}^{z} / 2\right)}{\sqrt{2^{n_{i x}+n_{i y}+n_{i z}} n_{i x} ! n_{i y} ! n_{i z} !}},
$$

where $q^{x}, q^{y}$ and $q^{z}$ are the components of the vector $\mathbf{q}$, and $H_{n}(x)$ denotes the $n$th "physicist's" Hermite polynomial [38]. Using Mehler's formula [39]

$$
\sum_{n=0}^{\infty} \frac{(y / 2)^{n}}{n !} H_{n}(x) H_{n}(z)=\frac{1}{\sqrt{1-y^{2}}} \exp \left(-\frac{y^{2}[x-z]^{2}}{1-y^{2}}\right)
$$

and recalling that $\mu_{0}=0$ we can also write Eq. (7) in a closed form [40]

$$
G\left(\mathbf{Q}, t \mid \mathbf{Q}_{0}\right)=\prod_{i=1}^{N}\left(\frac{\mu_{i}}{2 \pi\left(1-\mathrm{e}^{-2 \mu_{i} t}\right)}\right)^{3 / 2} \exp \left(-\frac{\mu_{i}\left(\mathbf{q}_{i}-\mathbf{q}_{i 0} \mathrm{e}^{-\mu_{i} t}\right)^{2}}{2\left(1-\mathrm{e}^{-2 \mu_{i} t}\right)}\right),
$$

where the equilibrium probability density function corresponds

$$
P_{\text {eq }}(\mathbf{Q}) \equiv \lim _{t \rightarrow \infty} G\left(\mathbf{Q}, t \mid \mathbf{Q}_{0}\right)
$$

In what follows we will use both forms of the Green's function, i.e. Eq. (7) and Eq. (12). 


\section{Conformational dynamics}

Throughout we are interested in conformational motions encoded in the dynamics of some internal distance $d$, e.g. the distance between two beads $i$ and $j, l=\left|\mathbf{r}_{i}-\mathbf{r}_{j}\right|$ or the distance between the center of masses of two sets of beads $\Omega_{1}, \Omega_{2}$ with $\Omega_{1} \cap \Omega_{2}=\{0\}$, $l_{\Omega_{1}, \Omega_{2}}=\left|\sum_{i \in \Omega_{1}} \mathbf{r}_{i} / \operatorname{card}\left(\Omega_{1}\right)-\sum_{i \in \Omega_{2}} \mathbf{r}_{j} / \operatorname{card}\left(\Omega_{2}\right)\right|$ where $\operatorname{card}\left(\Omega_{i}\right)$ is the cardinality the set $\Omega_{i}$. Without loss of generality we may thus focus on the distance between two arbitrary beads. Note that in absence of any dynamics in an equilibrium at $T=0$ such a distance is constant and equal to $d_{0}$. Expressed in normal coordinates we in turn have

$$
\mathbf{l} \equiv \mathbf{r}_{i}-\mathbf{r}_{j}=\sum_{k=1}^{N}\left(Q_{i k}-Q_{j k}\right) \mathbf{q}_{k}+\mathbf{r}_{i}^{0}-\mathbf{r}_{j}^{0} \equiv \sum_{k=1}^{N} A_{k} \mathbf{q}_{k}+\mathbf{d}_{0},
$$

where in the second equality we have defined $A_{k}$ and $\mathbf{d}_{0}$ and omitted the labels $i, j$ to simplify the notation. Note, moreover, that $l \equiv|\mathbf{I}|$ and the generalization to $l_{\Omega_{1}, \Omega_{2}}$ follows by linear superposition.

We will focus on four types of observables. The first one is the (non-Markovian) conditional probability density of the time series of the coordinate, $l_{t}$, defined as

$$
\mathcal{G}_{d_{0}}\left(l, t \mid l_{0}\right) \equiv \mathbb{P}\left(l_{t} \in l \mathrm{~d} l \mid l_{t=0} \in l_{0} \mathrm{~d} l\right)=\frac{\left\langle\delta\left(l\left(\mathbf{Q}_{t}\right)-l\right) \delta\left(l\left(\mathbf{Q}_{0}\right)-l_{0}\right)\right\rangle_{\mathbf{Q}_{t}}}{\left\langle\delta\left(l\left(\mathbf{Q}_{0}\right)-l_{0}\right)\right\rangle_{\mathrm{eq}}}
$$

with $\lim _{t \rightarrow \infty} \mathcal{G}_{d_{0}}\left(l, t \mid l_{0}\right) \equiv \mathcal{P}_{d_{0}}^{\mathrm{eq}}(l)=\langle\delta(l(\mathbf{Q})-l)\rangle_{\mathrm{eq}}$, and where in the second equality we have used the law of conditional probability and introduced the expectation over all Markovian paths of the full system evolving from equilibrium $\langle\cdot\rangle_{\mathbf{Q}_{t}}$, i.e.

$$
\langle\mathcal{B}\rangle_{\mathbf{Q}_{t}} \equiv \int d \mathbf{Q} \int d \mathbf{Q}_{0} \mathcal{B}\left(\mathbf{Q}, \mathbf{Q}_{0}\right) G\left(\mathbf{Q}, t \mid \mathbf{Q}_{0}\right) P_{\mathrm{eq}}\left(\mathbf{Q}_{0}\right)
$$

and the expectation of any observable $\mathcal{B}(\mathbf{Q})$ over the equilibrium measure $\langle\cdot\rangle_{\text {eq }}$ is $\langle\mathcal{B}\rangle_{\mathrm{eq}} \equiv \int d \mathbf{Q B}(\mathbf{Q}) P_{\mathrm{eq}}(\mathbf{Q})$. The second observable is the normalized equilibrium autocorrelation function

$$
\mathcal{C}_{d_{0}}(t) \equiv \frac{\langle l(t) l(0)\rangle-\langle l(t)\rangle\langle l(0)\rangle}{\left\langle l^{2}\right\rangle_{\mathrm{eq}}-\langle l\rangle_{\mathrm{eq}}^{2}}
$$

where we have introduced th expectations

$$
\begin{aligned}
& \langle l(t) l(0)\rangle \equiv\left\langle l\left(\mathbf{Q}_{t}\right) l\left(\mathbf{Q}_{0}\right)\right\rangle_{\mathbf{Q}_{t}}=\int_{0}^{\infty} d l \int_{0}^{\infty} d l_{0} l l_{0} \mathcal{G}_{d_{0}}\left(l, t \mid l_{0}\right) \mathcal{P}_{d_{0}}^{\mathrm{eq}}\left(l_{0}\right) \\
& \langle l(t)\rangle \equiv \frac{\left\langle l\left(\mathbf{Q}_{t}\right) \delta\left(l\left(\mathbf{Q}_{0}\right)-l_{0}\right)\right\rangle_{\mathbf{Q}_{t}}}{\left\langle\delta\left(l\left(\mathbf{Q}_{0}\right)-l_{0}\right)\right\rangle_{\mathrm{eq}}}=\int_{0}^{\infty} d l l \mathcal{G}_{d_{0}}\left(l, t \mid l_{0}\right) \\
& \left\langle l^{n}\right\rangle_{\text {eq }} \equiv\left\langle l^{n}(\mathbf{Q})\right\rangle_{\text {eq }}=\int_{0}^{\infty} d l l^{n} \mathcal{P}_{d_{0}}^{\mathrm{eq}}(l)
\end{aligned}
$$

The third observable is the $3(N+1) \times 3(N+1)$ position-covariance matrix [41] whose elements are defined as

$$
C_{\alpha \beta}^{i j}\left(t, t_{0}\right)=\left\langle\left(\mathbf{r}_{i, \alpha}\left(t+t_{0}\right)-\mathbf{r}_{i, \alpha}^{0}\right)\left(\mathbf{r}_{j, \beta}\left(t_{0}\right)-\mathbf{r}_{j, \beta}^{0}\right)\right\rangle_{\mathbf{Q}_{t}},
$$

where $\mathbf{r}_{i, \alpha}$ is the $\alpha=\{x, y, z\}$ component of the position vector of bead $i, \mathbf{r}_{i}$. 
The fourth, time-average observable is a functional of the projected path $l_{\tau}$ evolving from $l_{\tau=0}$ called the fraction of occupation time or "empirical density" [42]

$$
\theta_{d_{0}}(l ; t) \equiv t^{-1} \int_{0}^{t} \delta\left(l_{\tau}-l\right) d \tau .
$$

Note that all observables defined above are assumed to evolve from equilibrium. However, except for $C_{\alpha \beta}^{i j}\left(t, t_{0}\right)$, the initial distribution in fact corresponds to equilibrium constrained to a given value of the tagged distance $l_{0}$, i.e. from all those equilibrium configurations drawn from $P_{\mathrm{eq}}(\mathbf{Q})$ that are compatible with $l_{0}$. This introduces memory in the dynamics of $l_{t}[43]$.

\subsection{Projected propagator}

The non-Markovian projected propagator $\mathcal{G}_{d_{0}}\left(l, t \mid l_{0}\right)$ defined in Eq. (15) denotes the probability density that the distance between the two tagged beads is equal to $l$ at time $t$ given that it was initially equal to $l_{0}$. Introducing the auxiliary functions

$$
\eta_{t} \equiv \sum_{k=1}^{N} \frac{A_{k}^{2}}{2 \mu_{k}} \mathrm{e}^{-\mu_{k} t}, \quad \Xi_{t}\left(d_{0}, l, l^{\prime}\right) \equiv \operatorname{erfi}\left(\frac{d_{0}\left(\eta_{0}-\eta_{t}\right)+\eta_{t}\left(l+l^{\prime}\right)}{2 \sqrt{\eta_{t}\left(\eta_{0}^{2}-\eta_{t}^{2}\right)}}\right)
$$

we find (for details of the calculation see Appendix A)

$$
\begin{aligned}
\mathcal{P}_{d_{0}}^{\mathrm{eq}}\left(l_{0}\right) \mathcal{G}_{d_{0}}\left(l, t \mid l_{0}\right)= & \frac{l l_{0} \exp \left(-\frac{\left(l^{2}+l_{0}^{2}\right) \eta_{t}+\left(\eta_{0}-\eta_{t}\right) d_{0}^{2}}{4 \eta_{t}\left(\eta_{0}-\eta_{t}\right)}\right)}{8 \sqrt{\pi \eta_{t}} d_{0}\left(\eta_{0}-\eta_{t}\right)}\left[\Xi_{t}\left(d_{0},-l,-l_{0}\right)-\right. \\
& \left.\Xi_{t}\left(d_{0},-l, l_{0}\right)+\Xi_{t}\left(d_{0}, l, l_{0}\right)+\Xi_{t}\left(-d_{0},-l, l_{0}\right)\right]
\end{aligned}
$$

where erfi $(x)$ is the imaginary error function [38] and

$$
\mathcal{P}_{d_{0}}^{\mathrm{eq}}(l)=\frac{l}{d_{0}} \frac{\mathrm{e}^{-\left(l^{2}+d_{0}^{2}\right) / 4 \eta_{0}}}{\sqrt{\pi \eta_{0}}} \sinh \left(\frac{l d_{0}}{2 \eta_{0}}\right),
$$

We also derive the spectral expansion of $\mathcal{G}_{d_{0}}\left(l, t \mid l_{0}\right)$ that reads (see Appendix B)

$$
\mathcal{G}_{d_{0}}\left(l, t \mid l_{0}\right)=V_{\mathbf{0 0}}\left(l_{0} ; d_{0}\right)^{-1} \sum_{\mathbf{N}} V_{\mathbf{0 N}}\left(l ; d_{0}\right) V_{\mathbf{N} \mathbf{0}}\left(l_{0} ; d_{0}\right) \mathrm{e}^{-\Lambda_{N} t},
$$

where the overlap elements $V_{\mathbf{O N}}$ and $V_{\mathbf{N} \mathbf{0}}$ admit a closed-form expression that is, however, somewhat complicated and thus given in Appendix B. Note that "the ground state" element is simple and corresponds to $V_{\mathbf{0 0}}\left(l ; d_{0}\right)=\mathcal{P}_{d_{0}}^{\mathrm{eq}}(l)$.

\subsection{Equilibrium distance autocorrelation function}

The (normalized) autocorrelation function defined in Eq. (17) is made explicit by means of the following results

$$
\begin{aligned}
& \langle l\rangle_{\mathrm{eq}}=2 \sqrt{\frac{\eta_{0}}{\pi}} \mathrm{e}^{-d_{0}^{2} / 4 \eta_{0}}+\left(d_{0}+\frac{2 \eta_{0}}{d_{0}}\right) \operatorname{erf}\left(\frac{d_{0}}{2 \sqrt{\eta_{0}}}\right), \\
& \left\langle l^{2}\right\rangle_{\mathrm{eq}}=d_{0}^{2}+6 \eta_{0}
\end{aligned}
$$


where erf is the error function. Eqs. (26) follow from direct integration of the last line of Eq. (18) with the aid of Eq. (23). Conversely, an analytic computation of $\langle l(t) l(0)\rangle$ is possible only using the spectral expansion in Eq. (24) and yields,

$$
\begin{aligned}
& \langle l(t) l(0)\rangle=\sum_{\mathbf{N}} \mathcal{V}_{\mathbf{0 N}}^{d_{0}} \mathcal{V}_{\mathbf{N} \mathbf{0}}^{d_{0}} \mathrm{e}^{-\Lambda_{N} t}, \\
& \mathcal{V}_{\mathbf{O N}}^{d_{0}}=\int_{0}^{\infty} d l l V_{\mathbf{0 N}}\left(l ; d_{0}\right), \quad \mathcal{V}_{\mathbf{N} \mathbf{0}}^{d_{0}}=\int_{0}^{\infty} d l l V_{\mathbf{N} \mathbf{0}}\left(l ; d_{0}\right) .
\end{aligned}
$$

The analytic expression of the coefficients $\mathcal{V}_{\mathbf{0 N}}^{d_{0}}$ is lengthy and can be found in Appendix D. Plugging Eqs. (28) and Eq. (26) into Eq. (17) delivers an exact analytical result for the equilibrium distance autocorrelation function $\mathcal{C}_{d_{0}}(t)$. Alternatively one may also evaluate $\mathcal{C}_{d_{0}}(t)$ by numerical integration of the first line of Eq. (16) using Eq. (22), which may in fact be numerically more convenient than implementing the analytical solution.

\subsection{Position covariance matrix}

In the analysis of atomistic Molecular Dynamics (MD) simulations one often focuses on the position covariance matrix $C_{\alpha \beta}^{i j}\left(t, t_{0}\right)$ [41] and its eigendecomposition. The trajectory derived from an MD stimulation is then projected on the eigenvector (or principal component) corresponding the largest eigenvalue of the covariance matrix with the aim to identify the most important (potentially functionally relevant) motion in a protein [41]. To facilitate a comparison between the aforementioned analysis of MD simulation with GNM we compute $C_{\alpha \beta}^{i j}\left(t, t_{0}\right)$ analytically. Passing as before to normal coordinates we find

$$
C_{\alpha \beta}^{i j}\left(t, t_{0}\right)=\left\langle\sum_{k=1}^{N} Q_{i k} q_{k \alpha}\left(t+t_{0}\right) \sum_{l=1}^{N} Q_{j l} q_{l \beta}\left(t_{0}\right)\right\rangle,
$$

where the matrix elements $Q_{i j}$ do not depend on the spatial coordinate because the GNM is isotropic. Each process $\mathbf{q}_{k, \alpha}$ corresponds to an independent Ornstein-Uhlenbeck process, i.e. the solution of the Itô integral [44] (setting all constant to unity)

$$
q_{k \alpha}(t)=\sqrt{2} \int_{0}^{t} \mathrm{e}^{-\mu_{k}(t-s)} d W_{k \alpha}(s) .
$$

Since by construction (i.e. as a result of isotropy) only the elements of the same spatial coordinate for any given normal mode survive the averaging in Eq. (29), the elements of the covariance matrix read explicitly

$$
C_{\alpha \alpha}^{i j}\left(t, t_{0}\right)=\sum_{k=1}^{N} \frac{Q_{i k} Q_{j k}}{\mu_{k}} \mathrm{e}^{-\mu_{k}\left|t-t_{0}\right|} .
$$

Obviously $C_{\alpha \alpha}^{i j}\left(t, t_{0}\right)$ is stationary (i.e. depends only on the time difference, $C_{\alpha \alpha}^{i j}\left(t, t_{0}\right)=$ $\left.C_{\alpha \alpha}^{i j}\left(\left|t-t_{0}\right|\right)\right)$. 


\subsection{Fluctuations of occupation time}

Single molecule experiments typically probe time-averaged observables. For example, Förster resonance energy transfer (FRET) [45] and plasmon ruler experiments [46] have been used to extract information about conformational motions of macro-molecules. A fundamental quantity to that underlies this kind of observables is the fraction of occupation time, $\theta_{d_{0}}(l ; t)$, defined in Eq. (20) $[47,48,49,50,51,42]$ - the random fraction of time a time-series (in our case an internal distance between two beads or between two center of masses) of length $t$ attains a given value of $l$.

In previous publications we have shown how to obtain the mean and the variance of $\theta_{d_{0}}(l ; t)[51,42]$. Along these lines we here focus on the mean, $\left\langle\theta_{d_{0}}(l ; t)\right\rangle$, and the variance, $\sigma_{\theta ; d_{0}}^{2}(l ; t) \equiv\left\langle\theta_{d_{0}}^{2}(l ; t)\right\rangle-\left\langle\theta_{d_{0}}(l ; t)\right\rangle^{2}$, of the occupation time fraction at equilibrium that read, respectively (for a derivation see Appendix F)

$$
\begin{aligned}
& \left\langle\theta_{d_{0}}(l)\right\rangle=\mathcal{P}_{d_{0}}^{\mathrm{eq}}(l), \\
& \sigma_{\theta ; d_{0}}^{2}(l, t)=\frac{2}{t} \sum_{\mathbf{N} \neq \mathbf{0}} \frac{V_{\mathbf{0 N}}\left(d ; d_{0}\right) V_{\mathbf{N} \mathbf{0}}\left(d ; d_{0}\right)}{\Lambda_{\mathbf{N}}}\left(1-\frac{1-\mathrm{e}^{-\Lambda_{\mathbf{N}} t}}{\Lambda_{\mathbf{N}} t}\right) .
\end{aligned}
$$

Note that $\left\langle\theta_{d_{0}}(l ; t)\right\rangle$ corresponds to the equilibrium probability density for all times $t$ since we are considering an ergodic system evolving from equilibrium initial conditions. The variance of the occupation time fraction can equivalently be obtained from (see e.g. [52])

$$
\sigma_{\theta ; d_{0}}^{2}(l, t)=\frac{2}{t} \mathcal{P}_{d_{0}}^{\mathrm{eq}}(l)\left[\int_{0}^{t}(1-\tau / t) \mathcal{G}_{d_{0}}(l, \tau \mid l)-\mathcal{P}_{d_{0}}^{\mathrm{eq}}(l)\right] d \tau
$$

The integral in Eq. (34) does not admit an explicit solution. However, it can easily be computed via numerical quadrature. Moreover, it is possible to expand $\mathcal{G}_{d_{0}}(l, \tau \mid l)$ for short times (details are given in Appendix E) yielding the small deviation limit

$$
\mathcal{G}_{d_{0}}(l, t \mid l) \stackrel{t \rightarrow 0}{=} 2 \sqrt{\frac{1}{\pi}}\left(\frac{2}{\sqrt{\kappa t}}+\frac{\sqrt{\kappa t}}{l^{2}}\right)+\mathcal{O}\left(t^{3 / 2}\right)
$$

where we have introduced the shorthand notation $\kappa=\sum_{k=1}^{N} A_{k}^{2}$. Plugging Eq. (35) into Eq. (34) and performing the integral in turn yields

$$
\sigma_{\theta ; d_{0}}^{2}(d, t) \stackrel{t \rightarrow 0}{\simeq} 2 \mathcal{P}_{d_{0}}^{\mathrm{eq}}(l)\left(\frac{8}{3 \sqrt{\kappa \pi t}}+\frac{4}{15 l^{2}} \sqrt{\frac{\kappa t}{\pi}}-\mathcal{P}_{d_{0}}^{\mathrm{eq}}(l)\right)
$$

Since the dynamics of every stable system at equilibrium can be "linearized" for sufficiently small times $t$ the small deviation asymptotic in Eqs. (36) and (35) is in fact a general result for the (large) fluctuations of $\theta_{d_{0}}(l ; t)$ at sufficiently short times.

\section{Examples}

We now apply the result of the previous section to the analysis of a Gaussian Network Model of a protein called adenylate kinase and the analysis of toy-model mechanical frames. 
a)

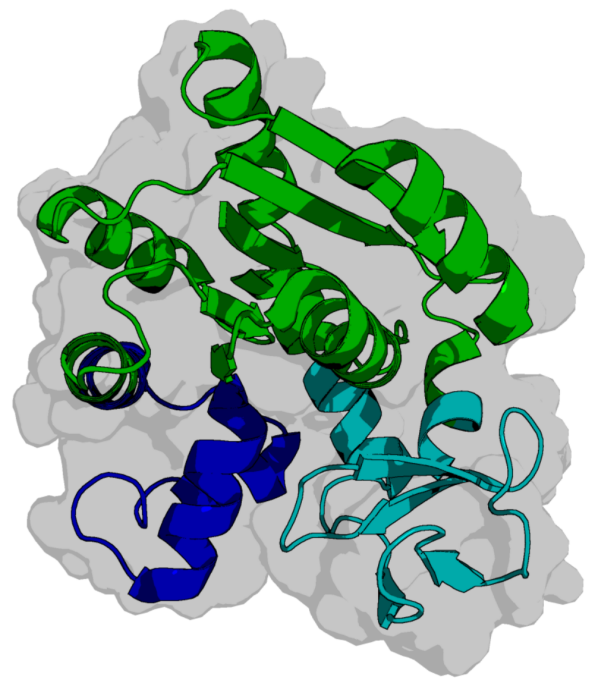

c)

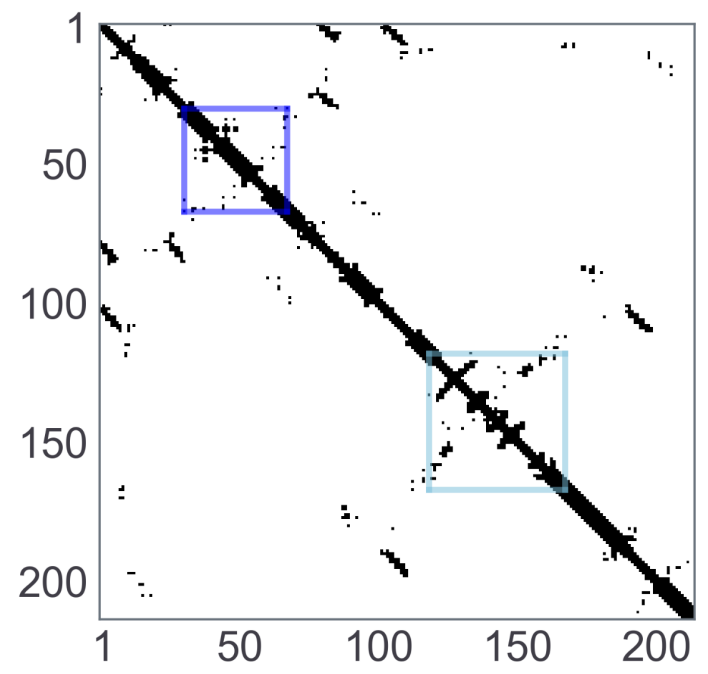

b)

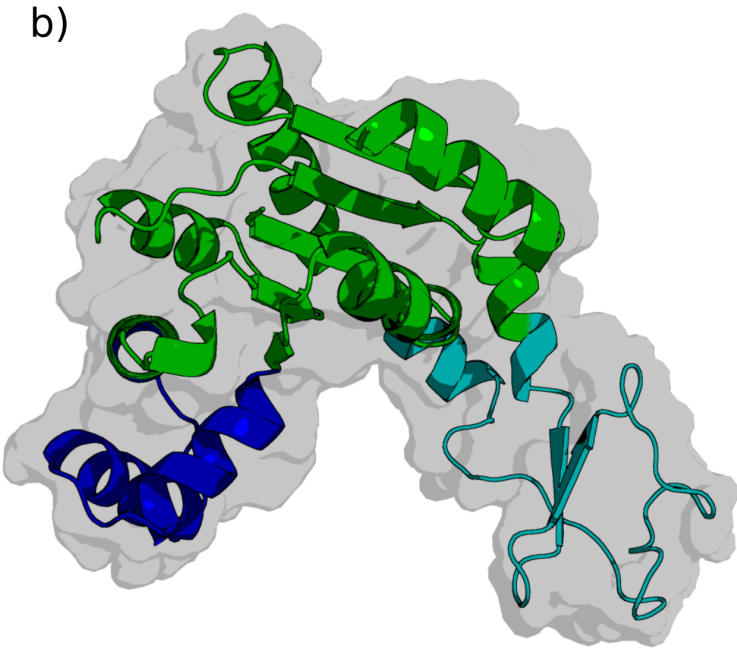

d)

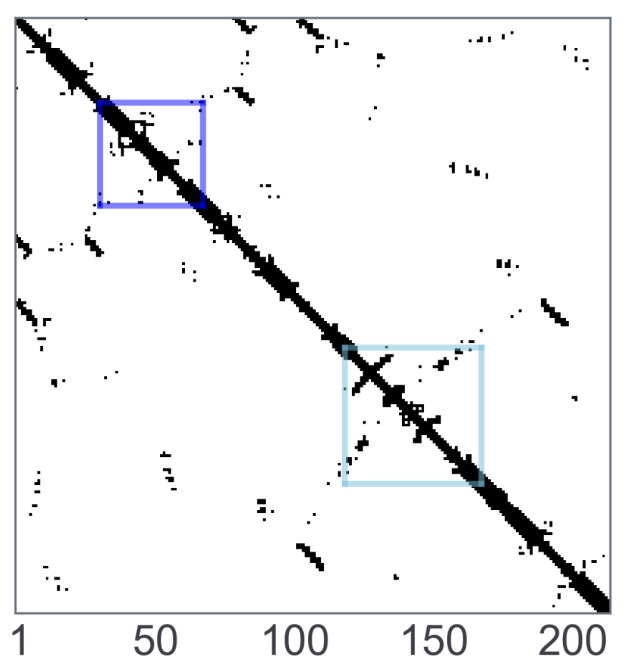

Figure 1. Panels (a) and (b) depict a cartoon and the molecular surface (gray) of the two protein structures, called (a) "the closed" configuration 1AKE and (b) "the open" configuration 4AKE. Panels (c) and (d) show the corresponding connectivity matrices for $1 \mathrm{AKE}$ and $4 \mathrm{AKE}$, respectively. The blue and cyan square enclose, respectively, the NMP and LID residues. The cutoff distance used to obtain these matrices was $8 \AA$.

\subsection{Gaussian Network Model of adenylate kinase}

Adenylate kinase (ADK) is an enzyme catalyzing the reversible phosphorylation reaction that transforms adenosine monophosphate (AMP) to adenosine triphosphate (ATP). The structure of ADK has been resolved using X-ray crystallography that uncovered two distinct conformations of the protein that are deposited in the Protein Data Bank (PDB ID: 1AKE [53] and PDB ID: 4AKE [54]) and shown in Fig. 1.

ADK consists of 214 residues divided in 3 macro-domains called CORE (residues $1-29,68-116$, and $160-214$ ), LID (residues 118-160), and NMP (residues 30-67). Distinct studies suggest the function to be coupled to open-closed transitions of both, 
Table 1. Distance between the center of masses of the three domains for both structures of ADK. All distances are expressed in units of the cutoff distance $r_{c}=8 \AA$.

\begin{tabular}{c|c|c}
$d_{0}\left[r_{c}\right]$ & $1 \mathrm{AKE}$ & $4 \mathrm{AKE}$ \\
\hline $\mathrm{CORE-LID}$ & 2.6 & 3.8 \\
CORE-NMP & 2.3 & 2.7 \\
LID-NMP & 2.6 & 4.5
\end{tabular}

LID and NMP domains with respect to the CORE domain $[53,54]$. These transitions have been observed even in absence of nucleotides [55, 56]. However, there is a lively debate in the biophysical community about the precise mechanism and rate-limiting steps in the catalytic function of ADK [57].

Here we analyze the autocorrelation functions of distances between the center of mass of LID, NMP, and CORE using the results described in the previous sections. Note that each GNM describes only a single stable structure and therefore cannot capture transitions between the two structures. Nevertheless, the comparison between the two respective GNMs may highlight some differences of the dynamics around the two distinct stable minima.

We obtain the connectivity matrices (shown in Fig. 1) of the two GNMs using the Prody package [58] with a cutoff distance $r_{c}=8 \AA$. The static (zero-temperature) distances between the center of masses of the three domains in both structures are given in Table 1.

Fig. 2 shows the equilibrium probability density function $\mathcal{P}_{d_{0}}^{\text {eq }}(l)$ (panels a and b) as well as the autocorrelation function $\mathcal{C}_{d_{0}}(t)$ (panels $\mathrm{c}$ and $\mathrm{d}$ ) for all considered distances of the two GNMs representing the two conformational states of ADK. The structure 1AKE is evidently more compact than $4 \mathrm{AKE}$ and its corresponding autocorrelation functions consistently decay faster. Moreover, the CORE-NMP distance autocorrelation function decays faster compared to the other two distances whose autocorrelation functions are almost identical (see Fig. 2d). This difference in relaxation is a result of differences in the respective projection, i.e. whereas the eigenvalues of the underlying generator are identical (see Eq. (28)) the numerical coefficients $\mathcal{V}_{\mathbf{0 N}}^{d_{0}}$ and $\mathcal{V}_{\mathbf{N} \mathbf{0}}^{d_{0}}$ depend strongly on the particular type of projection and thus modify the relaxation rate substantially [59].

The lines in Figs. 2c) and 2d) have been obtained by means of a numerical integration of the first line of Eq. (18) using the Gauss-Kronrod quadrature [60]. Unfortunately the evaluation of the integrand is challenging for very short-times because it is a function sharply peaked along the diagonal of the $l, l_{0}$-plane. This feature prohibits us to obtain reliably (that is, due to numerical imprecision) the autocorrelation function for very short times.

Next we inspect the covariance matrix in Eq. (31) to identify the dominant, potentially functional important, motions in ADK. In order to reduce the information content while retaining the most essential physics about the extent of local fluctuations and how much the motion of each bead correlated to the motion of other beads we 

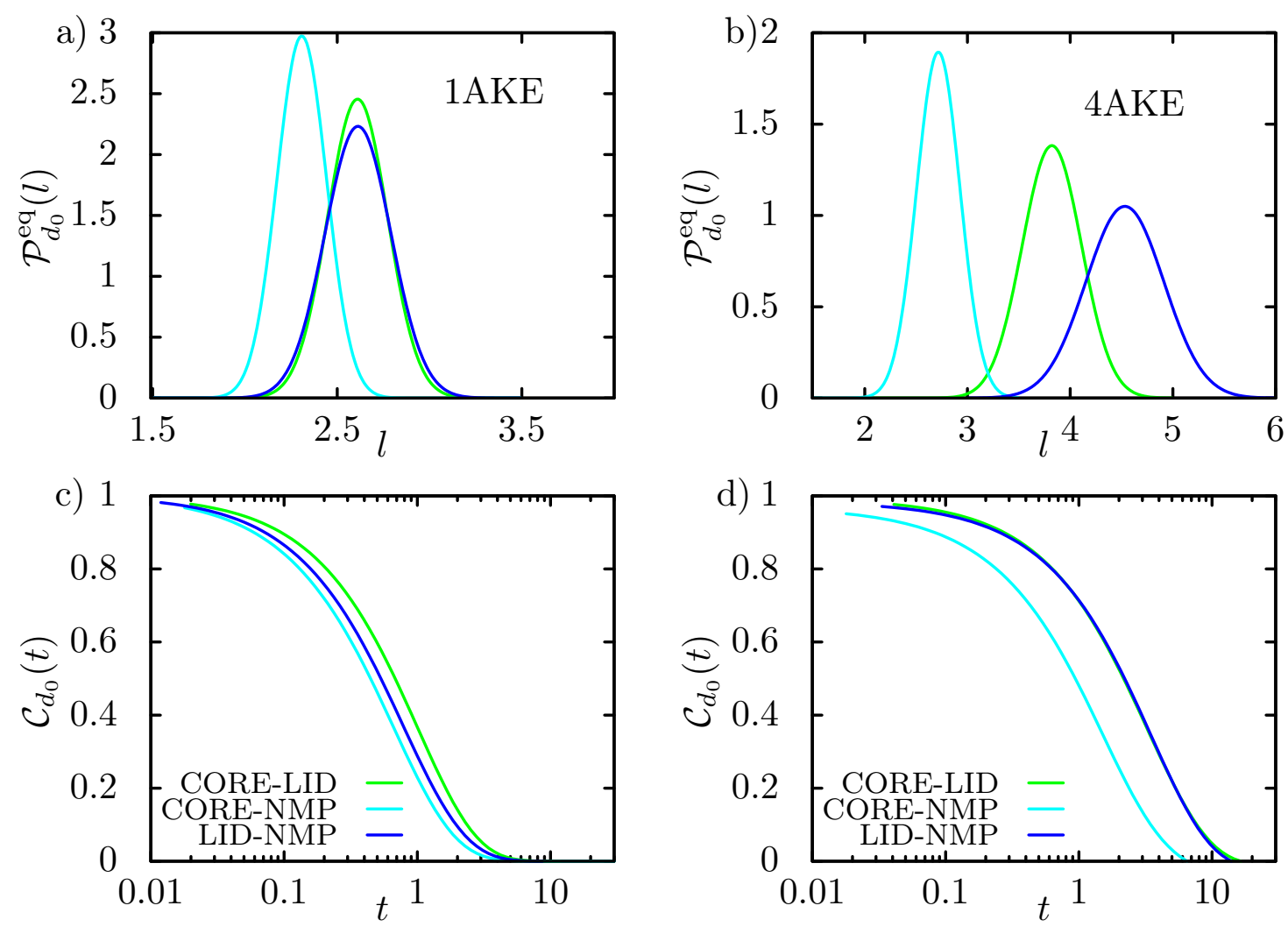

Figure 2. Panels (a) and (b) show the equilibrium probability density function for the three center-of-mass distances for both structures (see Table 1 for the numerical values of $\left.d_{0}\right)$. Panels (c) and (d) depict the respective distance autocorrelation functions. Note that the first structure is more compact and its $\mathcal{C}_{d_{0}}(t)$ decorrelates faster. Moreover, panel (d) reveals that the CORE-NMP distance decorrelates faster than the remaining two distances.

introduce the covariance-time defined as

$$
\tau_{i j \alpha}=\int_{0}^{\infty} C_{\alpha \alpha}^{i j}(t) d t
$$

which may be interpreted in a manner analogous to the correlation time $[61,62,63]$, i.e. as a measure of how much the motion between the beads $i$ and $j$ is correlated over time. To measure how much the motion of a single bead is correlated with the rest of the system we consider the total the total covariance-time $\tau_{i, \alpha}^{\text {tot }} \equiv \sum_{j \neq i}\left|\tau_{i j \alpha}\right|$. Conversely, the total variance-time is quantified directly by $\tau_{i i \alpha}$. Note that the model is isotropic and thus independent of $\alpha$. The results are shown in Fig. 3.

Notably, one can immediately observe that those residues that are involved in the large-scale open-closed motion (i.e. residues with a large $\tau_{i i \alpha}$ ) also participate in correlated motions denoted by large values $\tau_{i, \alpha}^{\text {tot }}$. For the open structure $4 \mathrm{AKE}$ (see Fig. $3 \mathrm{a}$ and $\mathrm{b}$ ) the two ends of the LID and NMP domains move in a particularly correlated fashion. These residues are in fact those that move towards the core region in the functional open-closed motion of the protein [55, 56]. A remnant of this collective 
a)

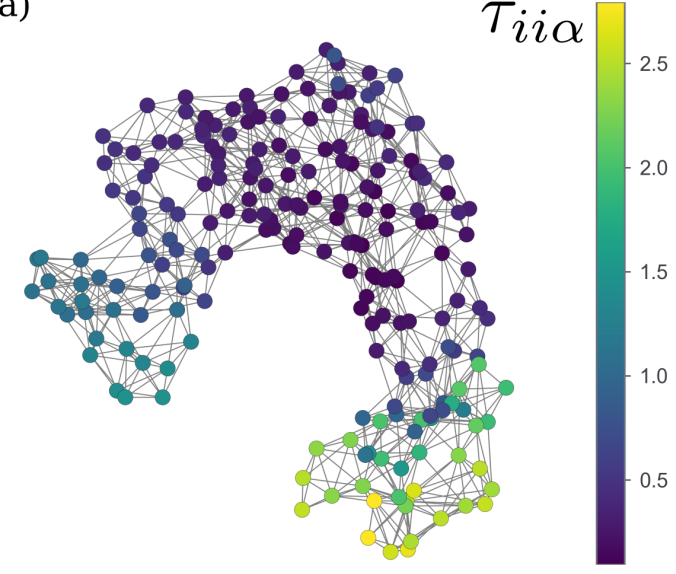

c)

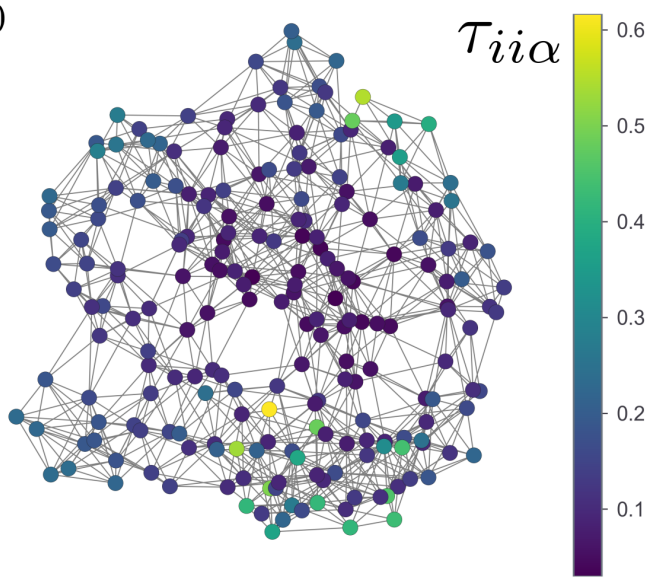

b)

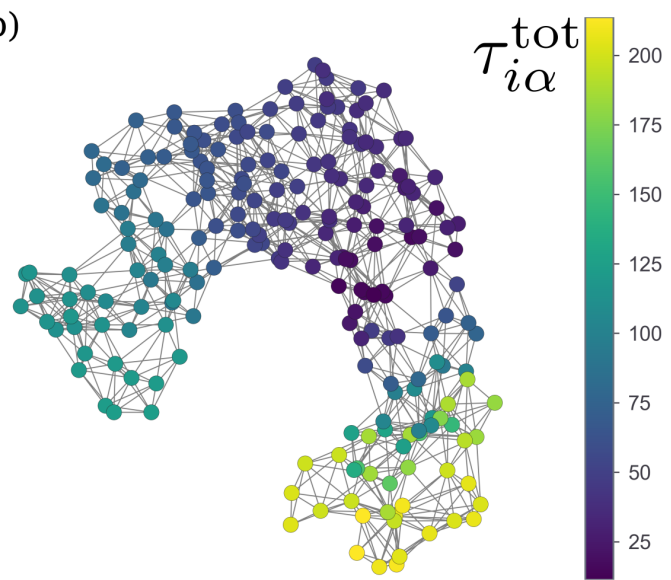

d)

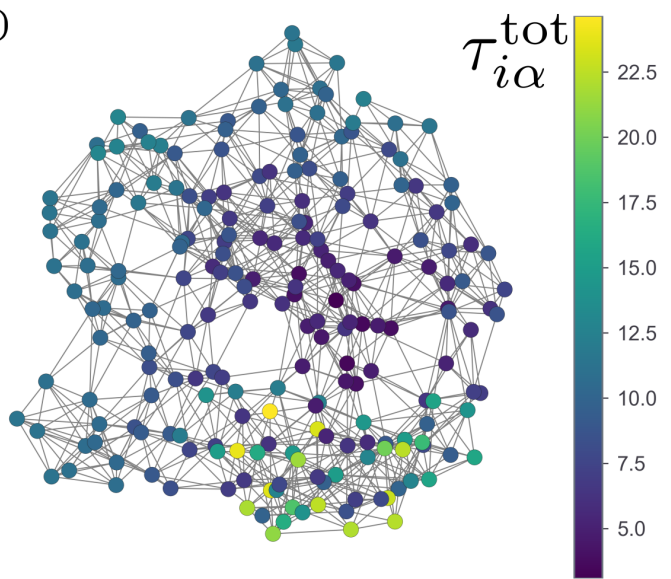

Figure 3. (a) and (c) depict $\tau_{i i \alpha}$ and (b) and (d) $\tau_{i, \alpha}^{\text {tot }}$ for each bead in the $4 \mathrm{AKE}$ and $1 \mathrm{AKE}$ structures, respectively. Notably, the beads in the LID and NMP domains in the 4AKE structure display a particularly large covariance-time.

motion can also be seen in the closed structure 1AKE (Fig. $3 \mathrm{c}$ and d), where the same beads as in $4 \mathrm{AKE}$ have a larger $\tau_{i, \alpha}^{\text {tot }}$. This is likely a result of a higher local connectivity.

\subsection{Simple mechanical frames}

Although GNMs were originally developed to describe proteins they can in fact be used to model any mechanical system in which some underlying network of links imposes constraints on the position of nodes while allowing small, Gaussian fluctuations driven by thermal noise. Examples may include nano-machines such as piezoelectric actuators that move probe-tips in atomic force microscopes $[64,65]$.

In the generic context of "mechanical frames" the theory of structural rigidity deals with the question of whether frames are rigid or not [66]. A frame is said to be rigid if one cannot change the distance between pairs of nodes without simultaneously altering the 

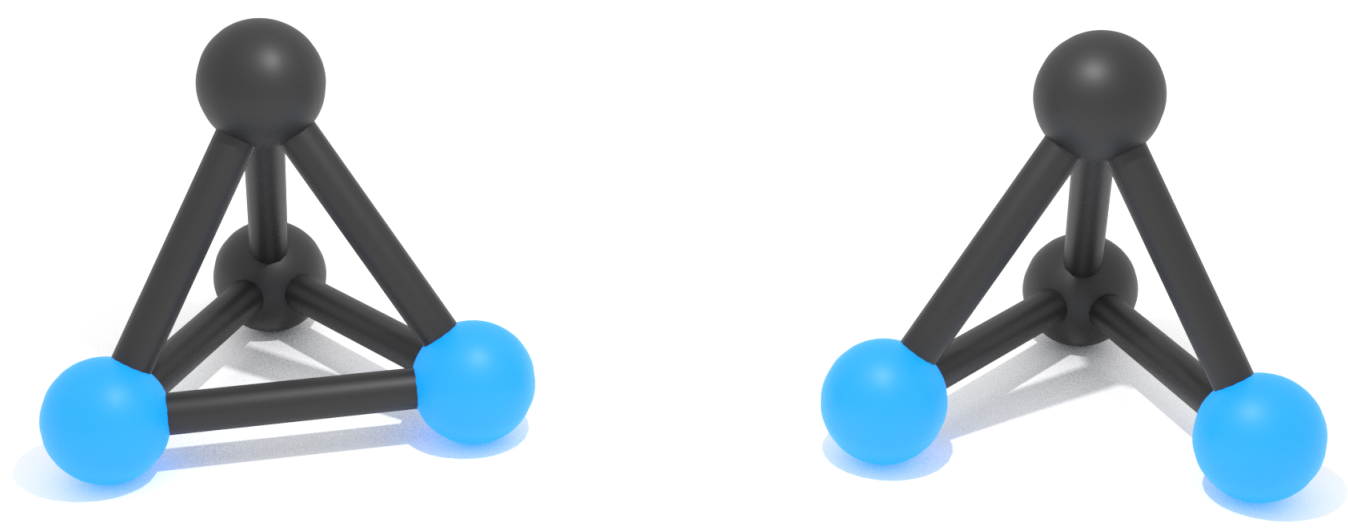

Figure 4. A schematic representation of a stable (left) and an unstable (right) frame we consider below.

length of at least one connection. A structure that is not rigid is in turn said to allow for inextensional mechanisms. These arise due to a too low number or a particular arrangement of links. In addition, in frames with redundant links there exist states of self-stress. Under given circumstances these states of self-stress impart stiffness to inextensional mechanisms [67].

As anticipated by Maxwell such a classification of mechanical frames is often nontrivial and may require more information than encoded in the topology of the network [68]. A complete analysis of the mechanisms of a given frame can be obtained by a "singular value decomposition" of the respective Equilibrium Matrix A [69] that relates forces $\mathbf{f}$ on the nodes with tensions $\mathbf{t}$ in the links

$$
\mathbf{A t}=\mathbf{f} .
$$

Singular value decomposition of $\mathbf{A}$ allows (amongst other things) to determine the rank $r$ of $\mathbf{A}$ and thereby the number of inextensional mechanisms $m$ and states of self-stress $s$ via $s=b-r$ and $m=3 j-6-r$, where $j$ is the number of joints and $b$ the number of links in the structure, and note that there are in general 6 rigid-body motions in 3 spatial dimensions. Maxwell's well-known formula $b=3 j-6$ is then extended to:

$$
b-3 j+6=s-m .
$$

To illustrate the concept we consider two toy-model frames depicted in Fig. 4. Both have $j=4$ nodes and $s=0$ states of self-stress. The rigid structure with $b=6$ links has no inextensional mechanism (i.e. $6-12-6=0-0$ ) while the structure with $b=5$ links has exactly $m=1$ mechanism (i.e. $5-12-6=0-1$ ).

To highlight the rôle of rigidity and to investigate the effect of a heat-bath we first analyze the autocorrelation function between the blue beads (see Fig. 4) as a function of the rest-length $d_{0}$. Notably, in a GNM such distance fluctuations do not depend on the equilibrium structure $\mathbf{R}^{0}$. Only the equilibrium distance between the tagged beads, $d_{0}=\left|\mathbf{r}_{i}^{0}-\mathbf{r}_{j}^{0}\right|$, is relevant. In turn there is a redundancy - many distinct equilibrium 

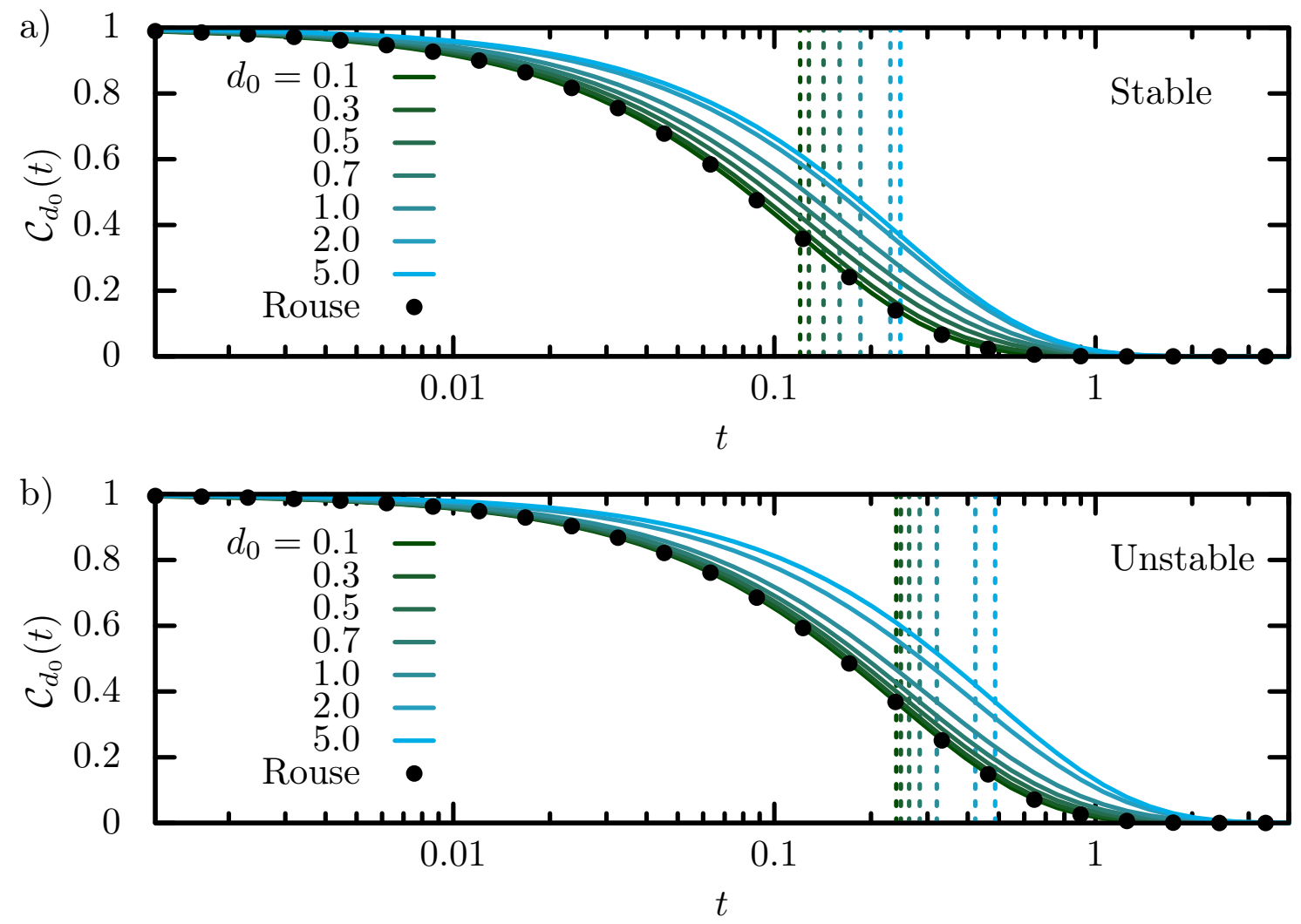

Figure 5. Distance autocorrelation function $\mathcal{C}_{d_{0}}(t)$ for various values of the rest length $d_{0}$ for the rigid (top panel) and non-rigid (bottom panel) frames depicted in Fig. 4. The black dots depict $\mathcal{C}_{d_{0}}(t)$ in the Rouse limit $d_{0}=0$ (see Appendix Appendix D for details). The vertical dashed lines corresponds to the time $t_{c}$ at which $\mathcal{C}_{d_{0}}\left(t_{c}\right)=\mathrm{e}^{-1}$. Note that the unstable structure relaxes slower.

structures $\mathbf{R}^{0}$ may yield the same result that depends only on the connectivity matrix $\Gamma$ and $d_{0}$.

The (normalized) distance autocorrelation function $\mathcal{C}_{d_{0}}(t)$ (see Eq. (17)) for the two frames is shown Fig. 5. For $d_{0} \lesssim 0.5$ (in dimensionless units) $\mathcal{C}_{d_{0}}(t)$ depends only very weakly on $d_{0}$. For larger values of $d_{0}$ the relaxation time (see dashed vertical lines in Fig. 5) increases. This observation may be explained by noticing that entropy dominates the motion for small $d_{0}$. That is, in the limit of small $d_{0}$ the rest length may be neglected and the "Rouse limit" suffices to explain the dynamics essentially quantitatively. Conversely, as $d_{0}$ increases a certain "stiffness" emerges in the frame and the (random) oscillations become localized around the equilibrium value $d_{0}$. Note that the entropic contribution to $\mathcal{C}_{d_{0}}(t)$ is more important for the non-rigid frame (see right panel in Fig. 5) as we increase the value of $d_{0}$ (see Fig. 5b)). Conversely, the departure from the Rouse limit towards the "large stiffness" case is faster in the stable frame (see Fig. 5a)). A larger $d_{0}$ leads to a slower decay of the autocorrelation function $\mathcal{C}_{d_{0}}(t)$.

Next we consider the fraction of occupation time $\theta_{d_{0}}(l ; t)[42]$. We assume that the 

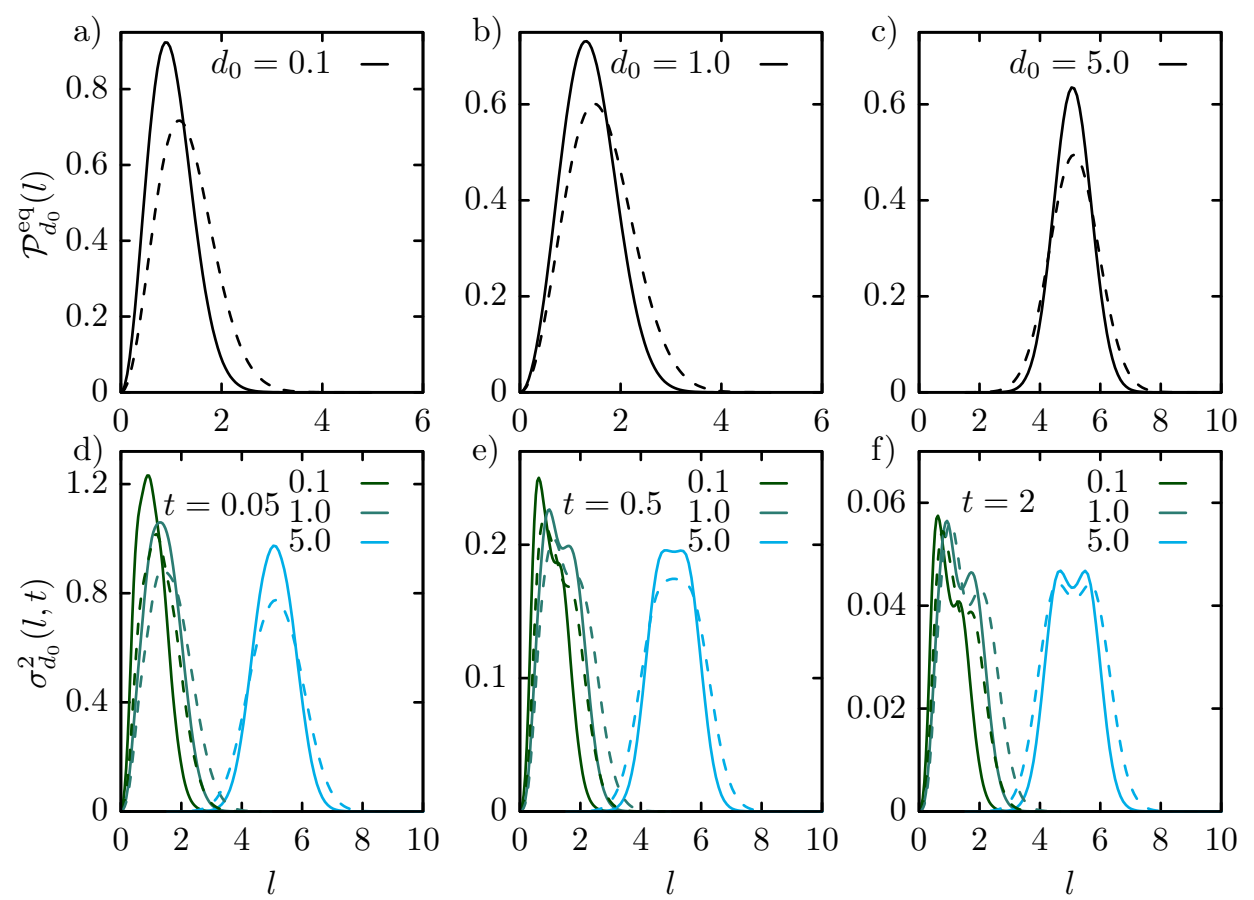

Figure 6. Panels a-c show the equilibrium probability density $\mathcal{P}_{d_{0}}^{\mathrm{eq}}(l)$ for the stable (full lines) and unstable (dashed lines) structure for several values of $d_{0}$. Panels ef depict the variance of the occupation time $\sigma_{\theta ; d_{0}}^{2}(l, t)$ for the stable (full lines) and unstable (dashed lines) structure, respectively, for different values of $d_{0}$. The length of the trajectory $t$ increases from $\mathrm{d}$ to $\mathrm{f}$.

initial condition evolves from equilibrium and therefore $\left\langle\theta_{d_{0}}(l ; t)\right\rangle=\mathcal{P}_{d_{0}}^{\mathrm{eq}}(l)$ whereas $\sigma_{\theta ; d_{0}}^{2}(l, t)$ depends on time (see Eq. (33) as well as $[51,42]$ ). The aforementioned dominance of the entropic (heat bath) contribution at small values of $d_{0}$ is also noticeable the the fluctuations of $\theta_{d_{0}}(l ; t)$ as depicted in Fig. 6. Notably, as $d_{0}$ increases the support of $\sigma_{\theta ; d_{0}}^{2}(l, t)$ progressively shifts towards larger $l$ and concentrates near $d_{0}$.

Notably, the variance of the occupation time fraction $\sigma_{\theta ; d_{0}}^{2}(l, t)$ changes shape from unimodal shape at short times $t$ to bimodal at long $t$. Such a behavior is characteristic for stochastic process in spatial confinement [42], i.e. fluctuations of $\theta_{d_{0}}(l ; t)$ are larger in the vicinity of confining boundaries (even if these boundaries are "soft").

Moreover as $d_{0}$ increases the shape of both, $\mathcal{P}_{d_{0}}^{\text {eq }}(l)$ as well as $\sigma_{\theta ; d_{0}}^{2}(l, t)$ becomes more symmetric. The reason seems to be that the effect of the confining boundary at $l=0$ becomes irrelevant as the support of $\sigma_{\theta ; d_{0}}^{2}(l, t)$ begins to concentrate near a substantial $d_{0}$. In other words although the projection of the dynamics of a link in 3-dimensional space onto a (1-dimensional) distance destroys the Gaussian behaviour, the latter becomes (partially) restored at large values of $d_{0}$. 


\section{Conclusions}

We presented analytical results (up to a numerical diagonalization of a symmetric matrix) for a selection of relevant time- and ensemble-average physical observables in the Gaussian Network Model (GNM). One may think of GNM as certain generalization of the Rouse model to networks with links with a certain degree of extensional and rotational stiffness. We determined a set of coarse-grained observables - internal distances - that may be of interest in the analysis of GNM in the context of internal motions in proteins or mechanical frames in contact with a heat bath. We hope that our results will enable and motivate a more systematic analysis of GNM derived from proteins [58]. To this end a $\mathrm{C}++$ computer code is provided in the Supplementary material that implements all result (for more details about the implementation see Appendix Appendix G).

\section{Acknowledgments}

The authors thank David Hartich and Lars Bock for the useful discussions. The financial support from the German Research Foundation (DFG) through the Emmy Noether Program GO 2762/1-1 to AG is gratefully acknowledged.

\section{Appendix A. Derivation of the equilibrium probability density}

The equilibrium probability density function of any link-vector $\mathbf{l}$ is defined by

$$
\mathcal{P}_{\mathbf{d}_{0}}^{\mathrm{eq}}(\mathbf{l})=V_{\mathbf{0 0}}\left(\mathbf{l} ; \mathbf{d}_{0}\right) \equiv \int d \mathbf{Q} \Psi_{\mathbf{0}}^{R}(\mathbf{Q}) \delta\left(\sum_{k=1}^{N} A_{k} \mathbf{q}_{k}+\mathbf{d}_{0}-\mathbf{l}\right) \Psi_{\mathbf{0}}^{L}(\mathbf{Q})
$$

Applying the Fourier transform $\tilde{f}(s)=\frac{1}{2 \pi} \int_{-\infty}^{\infty} \mathrm{d} x f(x) \mathrm{e}^{-i s x}$ component-wise to Eq. (A.1) (i.e. $\mathbf{l} \rightarrow$ s) yields

$$
\begin{aligned}
& \frac{1}{(2 \pi)^{3}} \int d \mathbf{Q} \prod_{k=1}^{N}\left(\frac{\mu_{k}}{2 \pi}\right)^{3 / 2} \exp \left(-\sum_{k=1}^{N} \frac{\mu_{k}}{2} \mathbf{q}_{k}^{2}+i\left(A_{k} \mathbf{q}_{k}+\mathbf{d}_{0}\right) \mathbf{s}\right)= \\
& \frac{1}{(2 \pi)^{3}} \mathrm{e}^{-s^{2} \sum_{k=1}^{N} A_{k}^{2} / 2 \mu_{k}+i \mathbf{d}_{0} \mathbf{s}} .
\end{aligned}
$$

Inverting the Fourier transform we obtain, defining $\eta_{0}=\sum_{k=1}^{N} A_{k}^{2} /\left(2 \mu_{k}\right)$,

$$
\mathcal{P}_{\mathbf{d}_{0}}^{\mathrm{eq}}(\mathbf{l})=V_{\mathbf{0 0}}\left(\mathbf{l} ; \mathbf{d}_{0}\right)=\frac{1}{(2 \pi)^{3}}\left(\frac{\pi}{\eta_{0}}\right)^{3 / 2} \mathrm{e}^{-\left(\mathbf{l}-\mathbf{d}_{0}\right)^{2} / 4 \eta_{0}} .
$$

Since we are only interested in the distance and not the direction we need to marginalize over angles, i.e.

$$
\int_{0}^{\infty} \mathrm{d} x x^{2} \int_{0}^{2 \pi} \mathrm{d} \phi \int_{-1}^{1} \mathrm{~d}(\cos \theta) V_{\mathbf{0 0}}(\mathbf{x}) \delta(|\mathbf{x}|-x)
$$


where $\phi$ is the polar angle, $\theta$ is the azimuthal angle and without loss of generality we choose a frame of reference such that the vector $\mathbf{d}_{0}$ is parallel to the $z$-axis. The solution of this integral finally gives Eq (23):

$$
\mathcal{P}_{d_{0}}^{\mathrm{eq}}(l)=V_{\mathbf{0 0}}\left(l ; d_{0}\right)=\frac{1}{\sqrt{\pi \eta_{0}}} \frac{l}{d_{0}} \mathrm{e}^{-\left(l^{2}+d_{0}^{2}\right) / 4 \eta_{0}} \sinh \left(\frac{l d_{0}}{2 \eta_{0}}\right) .
$$

\section{Appendix B. Spectral solution for $\mathcal{G}_{d_{0}}$}

In the spectral solution for the Green's function in Eq. (24) we have defined the elements $V_{\mathbf{0 N}}\left(l ; d_{0}\right), V_{\mathbf{N} \mathbf{0}}\left(l ; d_{0}\right)$ which are derived as follows. Let

$$
\begin{aligned}
& V_{\mathbf{0 N}}\left(\mathbf{l} ; \mathbf{d}_{0}\right)=\int d \mathbf{Q} \Psi_{\mathbf{N}}^{R}(\mathbf{Q}) \delta\left(\sum_{k=1}^{N} A_{k} \mathbf{q}_{k}+\mathbf{d}_{0}-\mathbf{l}\right) \Psi_{\mathbf{0}}^{L}(\mathbf{Q}), \\
& V_{\mathbf{N} \mathbf{0}}\left(\mathbf{l} ; \mathbf{d}_{0}\right)=\int d \mathbf{Q} \Psi_{\mathbf{0}}^{R}(\mathbf{Q}) \delta\left(\sum_{k=1}^{N} A_{k} \mathbf{q}_{k}+\mathbf{d}_{0}-\mathbf{l}\right) \Psi_{\mathbf{N}}^{L}(\mathbf{Q}) .
\end{aligned}
$$

Fortunately, the above elements $V_{\mathbf{0 N}}$ and $V_{\mathbf{N}}$ are identical (cf. Eq (9)). Therefore what we need to solve for is

$$
\begin{aligned}
& V_{\mathbf{N} \mathbf{0}}\left(\mathbf{l} ; \mathbf{d}_{\mathbf{0}}\right)=\prod_{k=1}^{N} \int d \mathbf{q}_{k}\left(\frac{\mu_{k}}{2 \pi}\right)^{\frac{3}{2}} \sqrt{\frac{1}{2^{n_{k x}+n_{k y}+n_{k z}} n_{k x} ! n_{k y} ! n_{k z} !}} \times \\
& H_{n_{k x}}\left(\sqrt{\frac{\mu_{k}}{2}} q_{k}^{x}\right) H_{n_{k y}}\left(\sqrt{\frac{\mu_{k}}{2}} q_{k}^{y}\right) H_{n_{k z}}\left(\sqrt{\frac{\mu_{k}}{2}} q_{k}^{z}\right) \times \\
& \mathrm{e}^{-\mu_{k} \mathbf{q}_{k}^{2} / 2} \delta\left(\sum_{k=1}^{N} A_{k} \mathbf{q}_{k}+\mathbf{d}_{0}-\mathbf{l}\right) .
\end{aligned}
$$

It is convenient to define the auxiliary variables $\left\{\mathbf{q}_{k}^{\prime}\right\} \equiv\left\{q_{k}^{x}-d_{0}^{x}, q_{k}^{y}-d_{0}^{y}, q_{k}^{z}-d_{0}^{z}\right\}$, and then perform the Fourier transform $\mathbf{l} \rightarrow \mathbf{s}$ to obtain

$$
\begin{aligned}
& \frac{1}{(2 \pi)^{3}} \prod_{k=1}^{N} \int d \mathbf{q}_{k}^{\prime}\left(\frac{\mu_{k}}{2 \pi}\right)^{\frac{3}{2}} \sqrt{\frac{1}{2^{n_{k x}+n_{k y}+n_{k z}} n_{k x} ! n_{k y} ! n_{k z} !}} \times \\
& H_{n_{k x}}\left(\sqrt{\frac{\mu_{k}}{2}} q_{k}^{\prime x}\right) H_{n_{k y}}\left(\sqrt{\frac{\mu_{k}}{2}} q_{k}^{\prime x}\right) H_{n_{k z}}\left(\sqrt{\frac{\mu_{k}}{2}} q_{k}^{\prime z}\right) \\
& \mathrm{e}^{-\mu_{k} \mathbf{q}_{k}^{\prime 2} / 2-i A_{k} \mathbf{s} \cdot \mathbf{q}_{k}^{\prime}} .
\end{aligned}
$$

Factorizing in the three spatial dimensions, completing the square in the exponential, and changing the variable to $t_{k}^{h}=\sqrt{\mu_{k}} q_{k}^{\prime h} / \sqrt{2}$ (where the subscript $h$ denotes the respective spatial coordinate) we find

$$
\begin{aligned}
& \frac{1}{(2 \pi)^{3}} \prod_{k=1}^{N}\left(\frac{1}{\pi}\right)^{\frac{3}{2}} \prod_{h=1}^{3} \sqrt{\frac{1}{2^{n_{k h}} n_{k h} !}} \mathrm{e}^{-s_{h}^{2}\left(A_{k}\right)^{2} / 2 \mu_{k}} \times \\
& \int_{-\infty}^{\infty} d t_{k}^{h} H_{n_{k h}}\left(t_{k}^{h}\right) \exp \left(-\left[t_{k}^{h}-\left(-\frac{i A_{k}}{\sqrt{2 \mu_{k}}} s_{h}\right)\right]^{2}\right)
\end{aligned}
$$


whose solution is [70]

$$
\frac{1}{(2 \pi)^{3}} \prod_{k=1}^{N} \prod_{h=1}^{3} \sqrt{\frac{2^{n_{k h}}}{n_{k h} !}}\left(-\frac{i A_{k}}{\sqrt{2 \mu_{k}}} s_{h}\right)^{n_{k h}} \mathrm{e}^{-s_{h}^{2}\left(A_{k}\right)^{2} / 2 \mu_{k}} .
$$

It turns out to be convenient to write Eq. (B.6) as

$$
\frac{1}{(2 \pi)^{3}}\left[\prod_{k=1}^{N} \prod_{h=1}^{3} \sqrt{\frac{2^{n_{k h}}}{n_{k h} !}}\left(-\frac{i A_{k}}{\sqrt{2 \mu_{k}}}\right)^{n_{k h}}\right] \prod_{h=1}^{3} s_{h}^{\sum_{k=1}^{N} n_{k h}} \mathrm{e}^{-s_{h}^{2} \sum_{k=1}^{N}\left(A_{k}\right)^{2} / 2 \mu_{k}},
$$

and to define $\ddagger$

$$
\begin{aligned}
& M \equiv \prod_{k=1}^{N} \prod_{h=1}^{3} \sqrt{\frac{2^{n_{k h}}}{n_{k h} !}}\left(-\frac{i A_{k}}{2 \mu_{k}}\right)^{n_{k h}}= \\
& \sqrt{\frac{2^{\sum_{k=1}^{N} \sum_{h=1}^{3} n_{k h}}}{\prod_{k=1}^{N} \prod_{h=1}^{3} n_{k h} !}}(-i)^{\sum_{k=1}^{N} \sum_{h=1}^{3} n_{k h}} \prod_{k=1}^{N}\left(\frac{A_{k}}{\sqrt{2 \mu_{k}}}\right)^{n_{k x}+n_{k y}+n_{k z}} .
\end{aligned}
$$

We now invert the Fourier transform

$$
\frac{M}{(2 \pi)^{3}} \prod_{h=1}^{3} \int_{-\infty}^{\infty} d s_{h} s_{h}^{\sum_{k=1}^{N} n_{k h}} \mathrm{e}^{-\eta_{0} s_{h}^{2}+i s_{h} d_{h}} .
$$

Completing the square in the exponential and defining $t_{h}=\sqrt{\eta_{0}} s_{h}$, we can write:

$$
\frac{M}{(2 \pi)^{3}} \prod_{h=1}^{3} \frac{\mathrm{e}^{-d_{h}^{2} / 4 \eta_{0}}}{{\sqrt{\eta_{0}}}^{\sum_{k=1}^{N} n_{k h}+1}} \int_{-\infty}^{\infty} d t_{h} t_{h}^{\sum_{k=1}^{N} n_{k h}} \mathrm{e}^{-\left(t_{h}-i d_{h} / 2 \sqrt{\eta_{0}}\right)^{2}},
$$

the integral in the previous equation can be solved analytically [70]

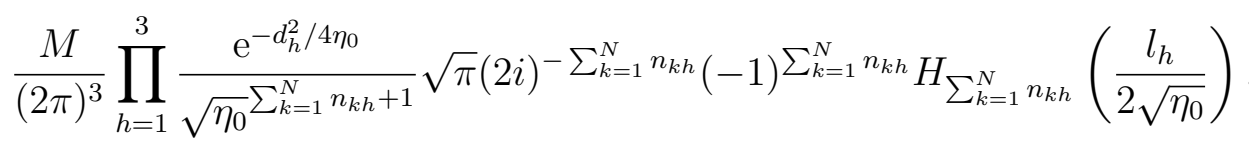

Using the definition of $M$ in Eq. (B.8), defining $N_{h}=\sum_{k=1}^{N} n_{k h}$ and $\mathcal{N}=N_{x}+N_{y}+N_{z}$, and going back to the original, non-shifted coordinates we arrive at the following form of Eq. (B.3)

$$
\begin{aligned}
& V_{\mathbf{0 N}}\left(\mathbf{l} ; \mathbf{d}_{0}\right)=\frac{1}{(2 \sqrt{\pi})^{3}} \sqrt{\frac{1}{2^{\mathcal{N}} \prod_{k=1}^{N} n_{k x} ! n_{k y} ! n_{k z} !}} \prod_{k=1}^{N}\left(\frac{A_{k}}{\sqrt{2 \mu_{k}}}\right)^{n_{k x}+n_{k y}+n_{k z}} \times \\
& \left.\frac{1}{{\sqrt{\eta_{0}}}^{\mathcal{N}+3}} H_{N_{x}}\left(\frac{l^{x}-d_{0}^{x}}{2 \sqrt{\eta_{0}}}\right) H_{N_{y}}\left(\frac{l^{y}-d_{0}^{y}}{2 \sqrt{\eta_{0}}}\right) H_{N_{z}}\left(\frac{l^{z}-d_{0}^{z}}{2 \sqrt{\eta_{0}}}\right) \mathrm{e}^{-\left(\mathbf{l}-\mathbf{d}_{0}\right)^{2} / 4 \eta_{0}} . \text { (B. } 12\right)
\end{aligned}
$$

To integrate over the angular part it is convenient to use the following expansion of the Hermite polynomials [38]:

$$
H_{n}(x)=n ! \sum_{m=0}^{\left\lfloor\frac{n}{2}\right\rfloor} \frac{(-1)^{m}}{m !(n-2 m) !}(2 x)^{n-2 m} .
$$

$\ddagger$ We use the convention $0^{0}=1$ for terms $\left(\frac{A_{k}}{\sqrt{2 \mu_{k}}}\right)^{n_{k x}+n_{k y}+n_{k z}}$. 
If we rotate our frame of reference such that $\hat{z} \| d_{0}^{z}$, (i.e.: $d_{0}^{x}=0, d_{0}^{y}=0$ and $d_{0}^{z}=d_{0}$ ) we find

$$
\begin{aligned}
& V_{\mathbf{0 N}}\left(\mathbf{l} ; \mathbf{d}_{0}\right)=\frac{1}{(2 \sqrt{\pi})^{3}} \sqrt{\frac{1}{2^{\mathcal{N}} \prod_{k=1}^{N} n_{k x} ! n_{k y} ! n_{k z}} !} \prod_{k=1}^{N}\left(\frac{A_{k}}{\sqrt{2 \mu_{k}}}\right)^{n_{k x}+n_{k y}+n_{k z}} \times \\
& \frac{1}{{\sqrt{\eta_{0}}}^{\mathcal{N}+3}} N_{x} ! N_{y} ! N_{z} ! \mathrm{e}^{-\frac{d^{2}+d_{0}^{2}}{4 \eta_{0}}} \times \\
& \sum_{a=0}^{\left\lfloor N_{x} / 2\right\rfloor} \sum_{b=0}^{\left\lfloor N_{y} / 2\right\rfloor} \sum_{c=0}^{\left\lfloor N_{z} / 2\right\rfloor} \frac{(-1)^{a+b+c}}{a ! b ! c !\left(N_{x}-2 a\right) !\left(N_{y}-2 b\right) !\left(N_{z}-2 c\right) !} \times \\
& \left(\frac{l^{x}}{\sqrt{\eta_{0}}}\right)^{N_{x}-2 a}\left(\frac{l^{y}}{\sqrt{\eta_{0}}}\right)^{N_{y}-2 b}\left(\frac{l^{z}-d_{0}}{\sqrt{\eta_{0}}}\right)^{N_{z}-2 c} \mathrm{e}^{\mathbf{l} \cdot \mathbf{d}_{0} / 2 \eta_{0}} .
\end{aligned}
$$

Using the binomial expansion of $\left.\left(l^{z}-d_{0}\right) / \sqrt{\eta_{0}}\right)^{N_{z}-2 c}$ we obtain

$$
\begin{aligned}
& V_{\mathbf{0 N}}\left(\mathbf{l} ; \mathbf{d}_{0}\right)=\frac{1}{(2 \sqrt{\pi})^{3}} \sqrt{\frac{1}{2^{\mathcal{N}} \prod_{k=1}^{N} n_{k x} ! n_{k y} ! n_{k z} !}} \prod_{k=1}^{N}\left(\frac{A_{k}}{\sqrt{2 \mu_{k}}}\right)^{n_{k x}+n_{k y}+n_{k z}} \times \\
& \frac{1}{{\sqrt{\eta_{0}}}^{\mathcal{N}+3}} N_{x} ! N_{y} ! N_{z} ! \mathrm{e}^{-\left(l^{2}+d_{0}^{2}\right) / 4 \eta_{0}} \times \\
& \sum_{a=0}^{\left\lfloor N_{x} / 2\right\rfloor} \sum_{b=0}^{\left\lfloor N_{y} / 2\right\rfloor} \sum_{c=0}^{\left\lfloor N_{z} / 2\right\rfloor} \frac{(-1)^{a+b+c}}{a ! b ! c !\left(N_{x}-2 a\right) !\left(N_{y}-2 b\right) !}\left(\frac{l^{x}}{\sqrt{\eta_{0}}}\right)^{N_{x}-2 a}\left(\frac{l^{y}}{\sqrt{\eta_{0}}}\right)^{N_{y}-2 b} \mathrm{e}^{\mathbf{l} \cdot \mathbf{d}_{0} / 2 \eta_{0}} \times \\
& \sum_{m=0}^{N_{z}-2 c} \frac{1}{m !\left(N_{z}-2 c-m\right) !}\left(\frac{l^{z}}{\sqrt{\eta_{0}}}\right)^{N_{z}-2 c-m}\left(-\frac{d_{0}}{\sqrt{\eta_{0}}}\right)^{m} .
\end{aligned}
$$

At this point we can integrate over the angles of $\mathbf{l}$ fixing the length, hence

$$
\begin{aligned}
& V_{\mathbf{0 N}}\left(l, d_{0}\right)=\frac{1}{(2 \sqrt{\pi})^{3}} \sqrt{\frac{1}{2^{\mathcal{N}} \prod_{k=1}^{N} n_{k x} ! n_{k y} ! n_{k z} !}} \prod_{k=1}^{N}\left(\frac{A_{k}}{2_{k}}\right)^{n_{k x}+n_{k y}+n_{k z}} \times \\
& \frac{1}{\eta_{0}^{\mathcal{N}+1}} N_{x} ! N_{y} ! N_{z} ! \mathrm{e}^{-\left(l^{2}+d_{0}^{2}\right) / 4 \eta_{0}^{2}} \times \\
& \sum_{a=0}^{\left\lfloor N_{x} / 2\right\rfloor} \sum_{b=0}^{\left\lfloor N_{y} / 2\right\rfloor} \sum_{c=0}^{\left\lfloor N_{z} / 2\right\rfloor} \frac{(-1)^{a+b+c}}{a ! b ! c !\left(N_{x}-2 a\right) !\left(N_{y}-2 b\right) !}\left(\frac{l}{\eta_{0}}\right)^{N_{x}+N_{y}-2(a+b)+2} \times \\
& \int_{0}^{2 \pi} d \phi(\sin \phi)^{N_{y}-2 b}(\cos \phi)^{N_{x}-2 a} \times \\
& \sum_{m=0}^{N_{z}-2 c} \frac{1}{m !\left(N_{z}-2 c-m\right) !}\left(\frac{l}{\eta_{0}}\right)^{N_{z}-2 c-m}\left(-\frac{d_{0}}{\eta_{0}}\right)^{m} \times \\
& \int_{0}^{\pi} d \theta(\sin \theta)^{N_{x}+N_{y}-2(a+b)+1}(\cos \theta)^{N_{z}-2 c-m} \mathrm{e}^{\cos \theta l d_{0} / 2 \eta_{0}^{2}} .
\end{aligned}
$$

The first integral is

$$
\int_{0}^{2 \pi} d \phi \cos ^{n} \phi \sin ^{m} \phi=\frac{\pi n ! m !}{2^{n+m-1}\left(\frac{n}{2}\right) !\left(\frac{m}{2}\right) !\left(\frac{n+m}{2}\right) !},
$$


and is non-zero only if $n$ and $m$ are even [71]. Therefore $N_{x}$ and $N_{x}$ must be even. While the second integral reads [71]

$$
\begin{aligned}
& \int_{0}^{\pi} d \theta(\sin \theta)^{n}(\cos \theta)^{m} \mathrm{e}^{k \cos \theta}= \\
& \frac{\sqrt{\pi}}{4} \gamma\left(\frac{1+n}{2}\right)\left[2\left(1+(-1)^{m}\right) \gamma\left(\frac{1+m}{2}\right){ }_{1} \tilde{F}_{2}\left(\frac{1+m}{2} ; \frac{1}{2}, \frac{2+m+n}{2} ; \frac{k^{2}}{4}\right)\right. \\
& \left.-\left(-1+(-1)^{m}\right) k \gamma\left(1+\frac{m}{2}\right){ }_{1} \tilde{F}_{2}\left(\frac{2+m}{2} ; \frac{3}{2}, \frac{3+m+n}{2} ; \frac{k^{2}}{4}\right)\right],
\end{aligned}
$$

where we have introduced the Euler's gamma function $\gamma(x)$ as well as the regularized hypergeometric function ${ }_{p} \tilde{F}_{q}\left(a_{1}, \cdots, a_{p} ; b_{1}, \cdots, b_{q} ; x\right)$ [38]. Putting all together we finally arrive at

$$
\begin{aligned}
& V_{\mathbf{0 N}}\left(l ; d_{0}\right)=\frac{1}{16} \sqrt{\frac{1}{2^{\mathcal{N}} \prod_{k=1}^{N} n_{k x} ! n_{k y} ! n_{k z} !} \prod_{k=1}^{N}\left(\frac{A_{k}}{\sqrt{2 \mu_{k}}}\right)^{n_{k x}+n_{k y}+n_{k z}} \times} \\
& \frac{1}{{\sqrt{\eta_{0}}}^{\mathcal{N}+1}} N_{x} ! N_{y} ! N_{z} ! \mathrm{e}^{-\left(d^{2}+d_{0}^{2}\right) / 4 \eta_{0}} \times \\
& \sum_{a=0}^{N_{x} / 2} \sum_{b=0}^{N_{y} / 2} \sum_{c=0}^{\left\lfloor N_{z} / 2\right\rfloor} \frac{(-1)^{a+b+c}}{a ! b ! c !\left(\frac{N_{x}-2 a}{2}\right) !\left(\frac{N_{y}-2 b}{2}\right) ! 2^{N_{x}+N_{y}-2(a+b)}} \times \\
& \sum_{m=0}^{N_{z}-2 c} \frac{1}{m !\left(N_{z}-2 c-m\right) !}\left(\frac{l}{\sqrt{\eta_{0}}}\right)^{\mathcal{N}-2(a+b+c)-m+2}\left(-\frac{d_{0}}{\sqrt{\eta_{0}}}\right)^{m} \times \\
& {\left[2\left(1+(-1)^{N_{z}-2 c-m}\right) \gamma\left(\frac{1+N_{z}-2 c-m}{2}\right) \times\right.} \\
& { }_{1} \tilde{F}_{2}\left(\frac{1+N_{z}-2 c-m}{2} ; \frac{1}{2}, \frac{3+\mathcal{N}-2(a+b+c)-m}{2} ; \frac{l^{2} d_{0}^{2}}{16 \eta_{0}^{2}}\right) \\
& -\left(-1+(-1)^{N_{z}-2 c-m}\right) \frac{l d_{0}}{2 \eta_{0}} \gamma\left(1+\frac{N_{z}-2 c-m}{2}\right) \times \\
& \left.{ }_{1} \tilde{F}_{2}\left(\frac{2+N_{z}-2 c-m}{2} ; \frac{3}{2}, \frac{4+\mathcal{N}-2(a+b+c)-m}{2} ; \frac{l^{2} d_{0}^{2}}{16 \eta_{0}^{2}}\right)\right]
\end{aligned}
$$

which finally allows us to write down the non-Markovian Green's function expressed as an infinite series in Eq. 24. In addition, the series expansion allows us the compute the cross conditioned Green's function

$$
\mathcal{G}_{d_{0}, d_{0}^{\prime}}\left(l, t \mid l^{\prime}\right)=V_{\mathbf{0 0}}\left(l^{\prime} ; d_{0}^{\prime}\right)^{-1} \sum_{\mathbf{N}} V_{\mathbf{0 N}}\left(l ; d_{0}\right) V_{\mathbf{N} \mathbf{0}}\left(l^{\prime} ; d_{0}^{\prime}\right) \mathrm{e}^{-\Lambda_{N} t}
$$

that is the probability that the distance between the beads $i$ and $j$ is equal to $l$ at time $t$ conditioned to the fact that the distance between the beads $k$ and $l$ at time 0 was equal to $l^{\prime}$, assuming that these two distances have rest lengths $d_{0}$ and $d_{0}^{\prime}$, respectively. In particular in order to evaluate $V_{\mathbf{N o}}\left(l^{\prime} ; d_{0}^{\prime}\right)$ we need to consider that the distance $\mathbf{l}^{\prime}$ is expressed via the normal coordinates as

$$
\mathbf{d}^{\prime}=\mathbf{r}_{k}-\mathbf{r}_{l}=\sum_{i=1}^{N} B_{i} \mathbf{q}_{i}
$$


and we in turn use these coefficients to define $\zeta_{t}=\sum_{k=1}^{N} B_{k}^{2} / 2 \mu_{k} \mathrm{e}^{-\mu_{k} t}$ and $\zeta_{0}=$ $\sum_{k=1}^{N} B_{k}^{2} / 2 \mu_{k}$ instead of $\eta_{t}$ and $\eta_{0}$.

\section{Appendix C. Closed form solution for $\mathcal{G}_{d_{0}}$}

In order to obtain the equivalent result in a closed form solution we should consider the following integral:

$$
\begin{aligned}
& \mathcal{J}_{\mathbf{d}_{0}}\left(\mathbf{l}, t ; \mathbf{l}_{1}\right)=\int \mathrm{d} \mathbf{Q} \int \mathrm{d} \mathbf{Q}_{1} G\left(\mathbf{Q}, t \mid \mathbf{Q}_{1}\right) P_{\text {eq }}\left(\mathbf{Q}_{1}\right) \times \\
& \delta\left(\sum_{k=1}^{N} A_{k} \mathbf{q}_{1 k}+\mathbf{d}_{0}-\mathbf{l}_{1}\right) \delta\left(\sum_{k=1}^{N} A_{k} \mathbf{q}_{k}+\mathbf{d}_{0}-\mathbf{l}\right) .
\end{aligned}
$$

Performing the first Fourier transform, between $\mathbf{l}_{1} \rightarrow \mathbf{u}$ the above integral becomes

$$
\begin{aligned}
& \int d \mathbf{Q} \delta\left(\sum_{k=1}^{N} A_{k} \mathbf{q}_{k}+\mathbf{d}_{0}-\mathbf{l}\right) \mathrm{e}^{-i \mathbf{d}_{\mathbf{0}} \cdot \mathbf{u}} \times \\
& \left(\frac{1}{2 \pi}\right)^{3} \int d \mathbf{q}_{1 k} \prod_{k=1}^{N}\left(\frac{\mu_{k}^{2}}{2 \pi}\right)^{3 / 2}\left(\frac{\mu_{k}}{2 \pi\left(1-\mathrm{e}^{-2 \mu_{k} t}\right)}\right)^{3 / 2} \times \\
& \exp \left[-\frac{\mu_{k}}{2\left(1-\mathrm{e}^{-2 \mu_{k}^{2} t}\right)}\left(\mathbf{q}_{k}^{2}+\mathbf{q}_{1 k}^{2} \mathrm{e}^{-2 \mu_{k} t}-2 \mathbf{q}_{k} \cdot \mathbf{q}_{1 k} \mathrm{e}^{-\mu_{k} t}\right)\right] \times \\
& \mathrm{e}^{-i A_{k} \mathbf{q}_{1 k} \cdot \mathbf{u}} \mathrm{e}^{-\mu_{k} \mathbf{q}_{1 k}^{2} / 2} ;
\end{aligned}
$$

and the integration yields

$$
\begin{aligned}
& \left(\frac{1}{2 \pi}\right)^{3} \int d \mathbf{Q} \delta\left(\sum_{k=1}^{N} A_{k} \mathbf{q}+\mathbf{d}_{0}-\mathbf{l}\right) \mathrm{e}^{-i \mathbf{d}_{\mathbf{0}} \cdot \mathbf{u}} \times \\
& \prod_{k=1}^{N}\left(\frac{\mu_{k}^{2}}{2 \pi}\right)^{3 / 2} \exp \left[-\frac{\mu_{k}}{2}\left(\mathbf{q}_{k}^{2}+2 i \frac{A_{k}}{\mu_{k}} \mathrm{e}^{-\mu_{k} t} \mathbf{u} \cdot \mathbf{q}_{k}+\frac{A_{k}^{2}}{\mu_{k}^{2}}\left(1-\mathrm{e}^{-2 \mu_{k} t}\right) \mathbf{u}^{2}\right)\right]
\end{aligned}
$$

Performing the second Fourier transform $\mathbf{l} \rightarrow \mathbf{v}$ we find

$$
\begin{aligned}
& \mathrm{e}^{-i \mathbf{d}_{0} \cdot(\mathbf{u}+\mathbf{v})}\left(\frac{1}{2 \pi}\right)^{6} \prod_{k=1}^{N} \int \mathrm{d} \mathbf{q}_{k}\left(\frac{\mu_{k}}{2 \pi}\right)^{3 / 2} \times \\
& \exp \left[-\frac{\mu_{k}}{2}\left(\mathbf{q}_{k}^{2}+2 i \frac{A_{k}}{\mu_{k}}\left(\mathrm{e}^{-\mu_{k} t} \mathbf{u}+\mathbf{v}\right) \cdot \mathbf{q}_{k}+\frac{A_{k}^{2}}{\mu_{k}^{2}}\left(1-\mathrm{e}^{-2 \mu_{k} t}\right) \mathbf{u}^{2}\right)\right],
\end{aligned}
$$

that reads

$$
\mathrm{e}^{-i \mathbf{d}_{0} \cdot(\mathbf{u}+\mathbf{v})}\left(\frac{1}{2 \pi}\right)^{6} \prod_{k=1}^{N} \exp \left[-\frac{A_{k}^{2}}{2 \mu_{k}}\left(\mathbf{u}^{2}+\mathbf{v}^{2}\right)+2 \frac{A_{k}^{2}}{2 \mu_{k}} \mathrm{e}^{-\mu_{k} t} \mathbf{u} \cdot \mathbf{v}\right] .
$$

It is convenient to define

$$
\sum_{k=1}^{N} \frac{A_{k}^{2}}{2 \mu_{k}} \mathrm{e}^{-\mu_{k} t}=\eta_{t} \rightarrow \sum_{k=1}^{N} \frac{A_{k}^{2}}{2 \mu_{k}}=\eta_{0}
$$


so the Fourier transform of the joint-density is:

$$
\tilde{\mathcal{J}}_{\mathbf{d}_{0}}(\mathbf{v}, t ; \mathbf{u})=\frac{1}{(2 \pi)^{6}} \exp \left(-\eta_{0} \mathbf{u}^{2}-\eta_{0} \mathbf{v}^{2}+2 \eta_{t} \mathbf{u} \cdot \mathbf{v}+i \mathbf{d}_{\mathbf{0}} \cdot(\mathbf{u}+\mathbf{v})\right)
$$

The inversion of the two Fourier transforms gives straightforwardly

$$
\begin{aligned}
& \mathcal{J}_{\mathbf{d}_{0}}\left(\mathbf{l}, t ; \mathbf{l}_{1}\right)=\frac{1}{2^{6} \pi^{3}}\left(\frac{1}{\eta_{0}^{2}-\eta_{t}^{2}}\right)^{3 / 2} \times \\
& \exp \left[-\frac{\eta_{0}\left(\mathbf{l}-\mathbf{d}_{\mathbf{0}}\right)^{2}+\eta_{0}\left(\mathbf{l}_{1}-\mathbf{d}_{\mathbf{0}}\right)^{2}-2 \eta_{t}\left(\mathbf{l}-\mathbf{d}_{\mathbf{0}}\right) \cdot\left(\mathbf{l}_{1}-\mathbf{d}_{0}\right)}{4\left(\eta_{0}^{2}-\eta_{t}^{2}\right)}\right],
\end{aligned}
$$

We now marginalize over the angles

$$
\mathcal{J}_{d_{0}}\left(l, t ; l_{1}\right) \equiv \int d \mathbf{d} \int d \mathbf{d}_{1} \int d \mathbf{d}_{0} \delta\left(\left|\mathbf{d}_{0}\right|-d_{0}\right) \delta\left(\left|\mathbf{l}_{1}\right|-l_{1}\right) \delta(|\mathbf{l}|-l) \mathcal{J}_{\mathbf{d}_{0}}\left(\mathbf{l}, t ; \mathbf{l}_{1}\right),
$$

by moving to a frame of reference where $\mathbf{d}_{0}$ is parallel to the the $z$ axis, and express all the vectors in spherical coordinates. This removes all delta-functions and $d_{0}$ in the new frame of reference is just a scalar. By doing so we obtain

$$
\begin{aligned}
& \mathcal{J}_{d_{0}}\left(l, t ; l_{1}\right)=\frac{1}{2^{6} \pi^{3}}\left(\frac{1}{\eta_{0}^{2}-\eta_{t}^{2}}\right)^{3 / 2} \exp \left(-\frac{\eta_{0} l^{2}+\eta_{0} l_{1}^{2}+2\left(\eta_{0}-\eta_{t}\right) d_{0}^{2}}{4\left(\eta_{0}^{2}-\eta_{t}^{2}\right)}\right) l^{2} l_{1}^{2} \times \\
& \int_{0}^{2 \pi} d \phi \int_{0}^{2 \pi} d \phi^{\prime} \int_{-1}^{1} d(\cos \theta) \int_{0}^{\pi} d\left(\cos \theta^{\prime}\right) \times \\
& \exp \left[\frac{\left(\eta_{0}-\eta_{t}\right) l d_{0}}{2\left(\eta_{0}^{2}-\eta_{t}^{2}\right)} \cos \theta+\frac{\left(\eta_{0}-\eta_{t}\right) l_{1} d_{0}}{2\left(\eta_{0}^{2}-\eta_{t}^{2}\right)} \cos \theta^{\prime}+\frac{\eta_{t} l l_{1}}{2\left(\eta_{0}^{2}-\eta_{t}^{2}\right)} \times\right. \\
& \left.\left(\cos \phi \cos \phi^{\prime} \sin \theta \sin \theta^{\prime}+\sin \phi \sin \phi^{\prime} \sin \theta \sin \theta^{\prime}+\cos \theta \cos \theta^{\prime}\right)\right] .
\end{aligned}
$$

The two integrals over $\phi$ and $\phi^{\prime}$ (keeping in mind that $\cos \left(\phi-\phi^{\prime}\right)=\cos \phi \cos \phi^{\prime}+$ $\sin \phi \sin \phi^{\prime}$ ) give us

$$
\begin{aligned}
& \frac{1}{16 \pi}\left(\frac{1}{\eta_{0}^{2}-\eta_{t}^{2}}\right)^{3 / 2} \exp \left(-\frac{\eta_{0} l^{2}+\eta_{0} l_{1}^{2}+2\left(\eta_{0}-\eta_{t}\right) d_{0}^{2}}{4\left(\eta_{0}^{2}-\eta_{t}^{2}\right)}\right) l^{2} l_{1}^{2} \times \\
& \int_{-1}^{1} d(\cos \theta) \int_{0}^{\pi} d\left(\cos \theta^{\prime}\right) \exp \left[\frac{\left(\eta_{0}-\eta_{t}\right) l d_{0}}{2\left(\eta_{0}^{2}-\eta_{t}^{2}\right)} \cos \theta+\frac{\left(\eta_{0}-\eta_{t}\right) l_{1} d_{0}}{2\left(\eta_{0}^{2}-\eta_{t}^{2}\right)} \cos \theta^{\prime}\right. \\
& \left.+\frac{\eta_{t} l l_{1}}{2\left(\eta_{0}^{2}-\eta_{t}^{2}\right)} \cos \theta \cos \theta^{\prime}\right] I_{0}\left(\frac{\eta_{t} l l_{1}}{2\left(\eta_{0}^{2}-\eta_{t}^{2}\right)} \sqrt{1-\cos ^{2} \theta} \sqrt{1-\cos ^{2} \theta^{\prime}}\right)
\end{aligned}
$$

where $I_{0}(x)$ is the modified Bessel function of the first kind. The first integral in $\cos \theta^{\prime}$ is solvable [70], and by changing the variable $\cos \theta \rightarrow x$ we are left with

$$
\begin{gathered}
\frac{1}{8 \pi}\left(\frac{1}{\eta_{0}^{2}-\eta_{t}^{2}}\right)^{3 / 2} \exp \left(-\frac{\eta_{0} l^{2}+\eta_{0} l_{1}^{2}+2\left(\eta_{0}-\eta_{t}\right) d_{0}^{2}}{4\left(\eta_{0}^{2}-\eta_{t}^{2}\right)}\right) l^{2} l_{1}^{2} \times \\
\int_{-1}^{1} d x \mathrm{e}^{\frac{\left(\eta_{0}-\eta_{t}\right) l d_{0}}{2\left(\eta_{0}^{2}-\eta_{t}^{2}\right)} x} \frac{\sinh \left(\sqrt{\frac{\left(\eta_{0}-\eta_{t}\right)^{2} l_{1}^{2} d_{0}^{2}+\eta_{t}^{2} l^{2} l_{1}^{2}+2 \eta_{t}\left(\eta_{0}-\eta_{t}\right) l l_{1}^{2} d_{0} x}{4\left(\eta_{0}^{2}-\eta_{t}^{2}\right)^{2}}}\right)}{\sqrt{\frac{\left(\eta_{0}-\eta_{t}\right)^{2} l_{1}^{2} d_{0}^{2}+\eta_{t}^{2} l^{2} l_{1}^{2}+2 \eta_{t}\left(\eta_{0}-\eta_{t}\right) l l_{1}^{2} d_{0} x}{4\left(\eta_{0}^{2}-\eta_{t}^{2}\right)^{2}}}} .
\end{gathered}
$$


And the final integral yields [71]

$$
\begin{aligned}
& \mathcal{J}_{d_{0}}\left(l, t ; l_{1}\right)=\frac{1}{16 \sqrt{\pi}}\left(\frac{1}{\eta_{0}^{2}-\eta_{t}^{2}}\right)^{3 / 2} \exp \left(-\frac{\eta_{0} l^{2}+\eta_{0} l_{1}^{2}+2\left(\eta_{0}-\eta_{t}\right) d_{0}^{2}}{4\left(\eta_{0}^{2}-\eta_{t}^{2}\right)}\right) \times \\
& l^{2} l_{1}^{2} \frac{\mathrm{e}^{-a b / c-c / 4 a}}{\sqrt{a c}}\left[\operatorname{erfi}\left(\frac{2 a \sqrt{b-c}-c}{2 \sqrt{a c}}\right)-\operatorname{erfi}\left(\frac{2 a \sqrt{b-c}+c}{2 \sqrt{a c}}\right)+\right. \\
& \left.\operatorname{erfi}\left(\frac{c-2 a \sqrt{b+c}}{2 \sqrt{a c}}\right)+\operatorname{erfi}\left(\frac{c+2 a \sqrt{b+c}}{2 \sqrt{a c}}\right)\right]
\end{aligned}
$$

having defined

$$
\begin{aligned}
& a=\frac{\left(\eta_{0}-\eta_{t}\right) l d_{0}}{2\left(\eta_{0}^{2}-\eta_{t}^{2}\right)} \\
& b=\frac{\left(\eta_{0}-\eta_{t}\right)^{2} l_{1}^{2} d_{0}^{2}+\eta_{t}^{2} l^{2} l_{1}^{2}}{4\left(\eta_{0}^{2}-\eta_{t}^{2}\right)^{2}} \\
& c=\frac{\eta_{t}\left(\eta_{0}-\eta_{t}\right) l l_{1}^{2} d_{0}}{2\left(\eta_{0}^{2}-\eta_{t}^{2}\right)^{2}}
\end{aligned}
$$

the direct substitution of these auxiliary variables gives, upon division by $\mathcal{P}_{d_{0}}^{\text {eq }}$ and some simplification, Eq. (22).

\section{Appendix D. Derivation of equilibrium autocorrelation function}

In order to compute the autocorrelation function in Eq. (28) the following integrals must be evaluated

$$
\mathcal{V}_{\mathbf{0 N}}^{d_{0}}=\int_{0}^{\infty} d x V_{\mathbf{0 N}}\left(x, d_{0}\right) x, \quad \mathcal{V}_{\mathbf{N} \mathbf{0}}^{d_{0}}=\int_{0}^{\infty} d x V_{\mathbf{N} \mathbf{0}}\left(x, d_{0}\right) x .
$$

These two integrals are identical and the integration yields [70]

$$
\begin{aligned}
& \mathcal{V}_{\mathbf{0 N}}^{d_{0}}=\frac{1}{16} \sqrt{\frac{1}{2^{\mathcal{N}} \prod_{k=1}^{N} n_{k x} ! n_{k y} ! n_{k z} !}} \prod_{k=1}^{N}\left(\frac{A_{k}}{\sqrt{2 \mu_{k}}}\right)^{n_{k x}+n_{k y}+n_{k z}} \frac{1}{{\sqrt{\eta_{0}}}^{\mathcal{N}+1}} N_{x} ! N_{y} ! N_{z} ! \mathrm{e}^{-d_{0}^{2} / 4 \eta_{0}} \times \\
& \sum_{a=0}^{N_{x} / 2} \sum_{b=0}^{N_{y} / 2} \sum_{c=0}^{\left.N_{z} / 2\right\rfloor} \frac{(-1)^{a+b+c}}{a ! b ! c !\left(\frac{N_{x}-2 a}{2}\right) !\left(\frac{N_{y}-2 b}{2}\right) ! 2^{N_{x}+N_{y}-2(a+b)}} \sum_{l=0}^{N_{z}-2 c} \frac{1}{l !\left(N_{z}-2 c-l\right) !}\left(-\frac{d_{0}}{\sqrt{\eta_{0}}}\right)^{l} \times \\
& {\left[\left(1+(-1)^{N_{z}-2 c-l}\right) \gamma\left(\frac{1+N_{z}-2 c-l}{2}\right) 2^{\mathcal{N}-2(a+b+c)-l+4} \eta_{0} \gamma\left(\frac{\mathcal{N}-2(a+b+c)-l+4}{2}\right) \times\right.} \\
& \left.\left(-1+(-1)^{N_{z}-2 c-l}\right) d_{0} \sqrt{\eta_{0}} \gamma\left(1+\frac{N_{z}-2 c-l}{2}\right) \times \frac{1}{2}, \frac{3+\mathcal{N}-2(a+b+c)-l}{2} ; \frac{d_{0}^{2}}{4 \eta_{0}}\right)- \\
& 2_{2}\left(\frac{1+N_{z}-2 c-l}{2}, \frac{\mathcal{N}-2(a+b+c)-l+4}{2}\right) \times \\
& \left.{ }_{2} \tilde{F}_{2}\left(\frac{2+N_{z}-2 c-l}{2}, \frac{\mathcal{N}-2(a+b+c)-l+5}{2} ; \frac{3}{2}, \frac{4+\mathcal{N}-2(a+b+c)-l}{2} ; \frac{d_{0}^{2}}{4 \eta_{0}}\right)\right]
\end{aligned}
$$


If we are instead interested in the cross-correlation the more general Eq (B.20) must be used and the two integrals differ in therms of some constants, i.e. they are obtained by changing the following variables $d_{0} \rightarrow d_{0}^{\prime},\left\{A_{k}\right\} \rightarrow\left\{B_{k}\right\}$ and $\eta_{t} \rightarrow \zeta_{t}$.

\section{Appendix D.1. Rouse-limit autocorrelation function}

In Fig. 5 we showed how the autocorrelation for a GNM compares to the autocorrelation in the Rouse limit (i.e. $d_{0} \rightarrow 0$ ). The latter can be obtained in a closed form [59]

$$
\begin{aligned}
& \mathcal{C}(t)=\frac{\langle l(t) l(0)\rangle-\langle l\rangle^{2}}{\left\langle l^{2}\right\rangle-\langle l\rangle^{2}} \\
& \langle l(t) l(0)\rangle=\frac{4\left[3 \eta_{t} \sqrt{\eta_{0}^{2}-\eta_{t}^{2}}+2\left(\eta_{0}^{2}+\eta_{t}^{2}\right) \arctan \left(\eta_{t} /\left(\eta_{0}^{2}-\eta_{t}^{2}\right)\right)\right]}{\pi \eta_{t}} \\
& \langle l\rangle=4 \sqrt{\eta_{0} / \pi}, \quad\left\langle l^{2}\right\rangle=6 \eta_{0} .
\end{aligned}
$$

\section{Appendix E. Short-time expansion of $\mathcal{G}_{d_{0}}$}

Introducing the auxiliary variable $\phi(t)=\eta_{t} / \eta_{0}$ in Eq. (22) we can write the return joint-density as and expanding to linear order in $t$ using

$$
\phi(t) \stackrel{t \rightarrow 0}{\simeq} 1-\frac{\sum_{k=1} A_{k}^{2} t}{2 \eta_{0}} \phi^{2}(t) \stackrel{t \rightarrow 0}{\simeq} 1-\sum_{k=1} A_{k}^{2} \frac{t}{\eta_{0}} ;
$$

we find the partial limits

$$
\begin{aligned}
& \exp \left(-\frac{2 d^{2} \phi(t)+(1-\phi(t)) d_{0}^{2}}{4 \eta_{0} \phi(t)(1-\phi(t))}\right) \stackrel{t \rightarrow 0}{\sim} \mathrm{e}^{-1 / t} \rightarrow 0, \\
& \operatorname{erfi}\left(\frac{ \pm 2 d \phi(t)+d_{0}(1-\phi(t))}{2 \sqrt{\eta_{0} \phi(t)\left(1-\phi(t)^{2}\right)}}\right) \stackrel{t \rightarrow 0}{\sim} \operatorname{erfi}\left( \pm t^{-1 / 2}\right) \rightarrow \pm \infty, \\
& \operatorname{erfi}\left(\frac{d_{0}(1-\phi(t))}{2 \sqrt{\eta_{0} \phi(t)\left(1-\phi(t)^{2}\right)}}\right) \stackrel{t \rightarrow 0}{\sim} \operatorname{erfi}(\sqrt{t}) \rightarrow 0 ;
\end{aligned}
$$

where all the convergences are of exponential order. Therefore, while we can neglect the second erfi, we need to retain the product between the exponential and the two diverging erfis and only then plug them into in Eq. (E.1). Thus considering the expansion for large and real arguments of erfi [38]

$$
\operatorname{erfi}(x) \stackrel{x \rightarrow \pm \infty, x \in \mathbb{R}}{\simeq} \mp i+\left(\frac{1}{x}+\frac{1}{2 x^{3}}+O\left(x^{-5}\right)\right) \frac{\mathrm{e}^{x^{2}}}{\sqrt{\pi}},
$$

and explicitly, multiplying by the remaining exponentials Eq. (22) becomes (note that $\left.\mathcal{P}_{d_{0}}^{\mathrm{eq}}(l) \mathcal{G}_{d_{0}}(l, t \mid l) \equiv \mathcal{J}_{d_{0}}(l, t ; l)\right)$

$\mathcal{J}_{d_{0}}(d, t ; d) \stackrel{t \rightarrow 0}{\simeq} \frac{d^{2}}{8 \pi d_{0}} \mathrm{e}^{-\left(l^{2}+d_{0}^{2}\right) / 2 \eta_{0}(1+\phi(t))} \times$ 


$$
\begin{aligned}
& \left\{\left[\frac{2 \sqrt{1+\phi(t)}}{\sqrt{1-\phi(t)} \eta_{0}\left(-2 d \phi(t)+d_{0}(1-\phi(t))\right)}+4 \frac{\phi(t) \sqrt{1-\phi(t)}(1+\phi(t))^{3 / 2}}{\left(-2 d \phi(t)+d_{0}(1-\phi(t))\right)^{3}}\right] \mathrm{e}^{-l d_{0} / \eta_{0}(1+\phi(t))}\right. \\
& \left.\left.+\left[\frac{2 \sqrt{1+\phi(t)}}{\sqrt{1-\phi(t)} \eta_{0}\left(2 d \phi(t)+d_{0}(1-\phi(t))\right)}+4 \frac{\phi(t) \sqrt{1-\phi(t)}(1+\phi(t))^{3 / 2}}{\left(2 d \phi(t)+d_{0}(1-\phi(t))\right)^{3}}\right] \mathrm{e}^{l d_{0} / \eta_{0}(1+\phi(t))}\right]\right\}
\end{aligned}
$$

Using Eq. (E.1) and expanding $t=0$ and introducing $\kappa=\sum_{k=1}^{N} A_{k}^{2}$ we finally arrive at Eq. (35).

\section{Appendix F. Evaluation of the variance of the occupation time fraction}

The direct implementation of Eq. (33) suffers from slow convergence issues. We suspect that this problem has his roots in the (well-known) slow convergence of series involving Hermite polynomials [72]. We therefore combine the analytical short-time asymptotics in Eq.(36) with the spectral solution. Defining a small cutoff time $t_{s} \ll 1$ and rewriting Eq. (34) (using the linearity of integration) as

$\sigma_{d_{0}}^{2}(l, t)=\frac{2 \mathcal{P}_{d_{0}}^{\mathrm{eq}}(l)}{t} \int_{0}^{t_{s}} d \tau(1-\tau / t) \mathcal{G}_{d_{0}}(l, \tau \mid l)+\frac{2 \mathcal{P}_{d_{0}}^{\mathrm{eq}}(l)}{t} \int_{t_{s}}^{t} d \tau(1-\tau / t)\left[\mathcal{G}_{d_{0}}(l, \tau \mid l)-\mathcal{P}_{d_{0}}^{\mathrm{eq}}(l)\right] .($

We can explicitly evaluate the first addend using Eq. (36) and evaluate the second term using the spectral expansion (24). Note that the first term in the series (with $\left.\Lambda_{0}=0\right)$ must be treated in a manner different thant the rest. Therefore $\sigma_{d_{0}}^{2}(l, t)$ can be conveniently written (and implemented) in the form

$$
\begin{aligned}
& \sigma_{d_{0}}^{2}(d, t)=2 \mathcal{P}_{d_{0}}^{\mathrm{eq}}(l)\left(\frac{8}{3 \sqrt{\kappa \pi t}}+\frac{4}{15 l^{2}} \sqrt{\frac{\kappa t}{\pi}}-\mathcal{P}_{d_{0}}^{\mathrm{eq}}(l)\right) \\
& +\frac{2}{t^{2}} \sum_{\mathbf{N} \neq \mathbf{0}} V_{\mathbf{N} \mathbf{0}}\left(l ; d_{0}\right) V_{\mathbf{0 N}}\left(l ; d_{0}\right)\left[\left(t-t_{s}\right) \frac{\mathrm{e}^{-\Lambda_{\mathbf{N}} t_{s}}}{\Lambda_{\mathbf{N}}}-\frac{\mathrm{e}^{-\Lambda_{\mathbf{N}} t_{s}}-\mathrm{e}^{-\Lambda_{\mathbf{N}} t}}{\Lambda_{\mathbf{N}}^{2}}\right] \\
& +\mathcal{P}_{d_{0}}^{\mathrm{eq}}(l)^{2}\left(\frac{t_{s}}{t}-2\right) \frac{t_{s}}{t} .
\end{aligned}
$$

\section{Appendix G. Notes on the numerical implementation of the results}

Accompanying this article there is a $\mathrm{C}++$ implementation of all analytical results. The code allows the computation the Green's function $\mathcal{G}_{d_{0}}$, the mean $\left\langle\theta_{t}\left(l, d_{0}\right)\right\rangle$ and variance $\sigma_{d_{0}}^{2}(d, t)$ of the occupation time fraction, as well as the autocorrelation function $\mathcal{C}_{d_{0}}(t)$ for a generic Gaussian Network. The connectivity matrix of the network $\Gamma$ must be provided as a plain text file and is diagonalized using the Armadillo libray [73, 74].

A closed-form expression of the joint density in Eq. (22) is implemented in the available $\mathrm{C}++$ code. However, for numerical stability and speed of computation it is convenient to implement Eq. (C.12) and perform the final integral numerically using a Gauss-Kronrod quadrature routine [60]. 
Our main results are based on the evaluation of both, Eq. (B.19) and Eq. (D.2). Both require the evaluation of the less common regularized hypergeometric functions ${ }_{p} \tilde{F}_{q}$. A notable exception is the Arblib library [75], that implements several "special" functions using arbitrary precision arithmetic. The reliable evaluation of such functions is challenging and often requires several different methods to cover the entire domain [76]. Unfortunately this higher reliability comes with a higher computational cost compared to machine precision arithmetic. However hypergeometric functions converge on the entire complex plane if $p \leq q[76]$. In addition, we only need to evaluate them when all the parameters are positive real numbers. Therefore we implemented the series definitions of these function directly since in our case these converge reasonably fast to a desired accuracy as long as the parameters are not too large.

Many of our results, in particular the autocorrelation function and the variance of the fraction of occupation time, can only be expressed analytically using the eigendecomposition of the Fokker-Plank operator. Unfortunately the computational effort required in the generation of all necessary terms to achieve convergence is huge. In addition, this number scales non-polynomially with the number of beads in the network. Therefore the attached program should be used with care as it does not generate reliable results when the size of the network becomes too large. 
[1] Katherine Henzler-Wildman and Dorothee Kern. Dynamic personalities of proteins. Nature, 450(7172):964-972, Dec 2007.

[2] Wilfred F. van Gunsteren and Herman J. C. Berendsen. Computer simulation of molecular dynamics: Methodology, applications, and perspectives in chemistry. Angew. Chem. Int. Ed., 29(9):992-1023, Sep 1990.

[3] John L Klepeis, Kresten Lindorff-Larsen, Ron O Dror, and David E Shaw. Long-timescale molecular dynamics simulations of protein structure and function. Curr. Opin. Struc. Biol., 19(2):120-127, Apr 2009.

[4] David E. Shaw, Paul Maragakis, Kresten Lindorff-Larsen, Stefano Piana, Ron O. Dror, Michael P. Eastwood, Joseph A. Bank, John M. Jumper, John K. Salmon, Yibing Shan, and Willy Wriggers. Atomic-level characterization of the structural dynamics of proteins. Science, 330(6002):341$346,2010$.

[5] Marco Baiesi, Enzo Orlandini, Flavio Seno, and Antonio Trovato. Exploring the correlation between the folding rates of proteins and the entanglement of their native states. J. Phys. A: Math. Theor., 50(50):504001, December 2017.

[6] Cristian Micheletti, Jayanth R. Banavar, Amos Maritan, and Flavio Seno. Protein structures and optimal folding from a geometrical variational principle. Phys. Rev. Lett., 82:3372-3375, Apr 1999.

[7] Monique M. Tirion. Large Amplitude Elastic Motions in Proteins from a Single-Parameter, Atomic Analysis. Phys. Rev. Lett., 77(9):1905-1908, August 1996.

[8] Prince E. Rouse. A Theory of the Linear Viscoelastic Properties of Dilute Solutions of Coiling Polymers. J. Chem. Phys., 21(7):1272-1280, July 1953.

[9] Flory, P. J. Statistical thermodynamics of random networks. Proc. R. Soc. Lond. A, 351(1666):351-380, November 1976.

[10] Ivet Bahar, Ali Rana Atilgan, and Burak Erman. Direct evaluation of thermal fluctuations in proteins using a single-parameter harmonic potential. Folding and Design, 2(3):173-181, June 1997.

[11] Turkan Haliloglu, Ivet Bahar, and Burak Erman. Gaussian Dynamics of Folded Proteins. Phys. Rev. Lett., 79(16):3090-3093, October 1997.

[12] Pemra Doruker, Ali Rana Atilgan, and Ivet Bahar. Dynamics of proteins predicted by molecular dynamics simulations and analytical approaches: Application to $\alpha$-amylase inhibitor. Proteins, 40(3):512-524, 2000.

[13] A.R. Atilgan, S.R. Durell, R.L. Jernigan, M.C. Demirel, O. Keskin, and I. Bahar. Anisotropy of Fluctuation Dynamics of Proteins with an Elastic Network Model. Biophys. J., 80(1):505-515, January 2001.

[14] M. Delarue and P. Dumas. On the use of low-frequency normal modes to enforce collective movements in refining macromolecular structural models. Proc. Natl. Acad. Sci. USA, 101(18):6957-6962, May 2004.

[15] Gunnar F. Schröder, Axel T. Brunger, and Michael Levitt. Combining Efficient Conformational Sampling with a Deformable Elastic Network Model Facilitates Structure Refinement at Low Resolution. Structure, 15(12):1630-1641, December 2007.

[16] Dengming Ming and Rafael Brüschweiler. Reorientational Contact-Weighted Elastic Network Model for the Prediction of Protein Dynamics: Comparison with NMR Relaxation. Biophys. J., 90(10):3382-3388, May 2006.

[17] Qian-Yuan Tang, Yang-Yang Zhang, Jun Wang, Wei Wang, and Dante R. Chialvo. Critical Fluctuations in the Native State of Proteins. Phys. Rev. Lett., 118(8):088102, February 2017.

[18] Kay Hamacher, Joanna Trylska, and J. Andrew McCammon. Dependency Map of Proteins in the Small Ribosomal Subunit. PLoS Comput Biol, 2(2):e10, February 2006.

[19] A. J. Rader, G. Anderson, B. Isin, H. G. Khorana, I. Bahar, and J. Klein-Seetharaman. Identification of core amino acids stabilizing rhodopsin. Proc Natl Acad Sci USA, 101(19):72467251, May 2004. 
[20] Ozlem Keskin, Stewart R. Durell, Ivet Bahar, Robert L. Jernigan, and David G. Covell. Relating Molecular Flexibility to Function: A Case Study of Tubulin. Biophysi. J., 83(2):663-680, August 2002.

[21] Jingwei Weng, Jianpeng Ma, Kangnian Fan, and Wenning Wang. The Conformational Coupling and Translocation Mechanism of Vitamin B12 ATP-Binding Cassette Transporter BtuCD. Biophys. J., 94(2):612-621, January 2008.

[22] Ines Putz and Oliver Brock. Elastic network model of learned maintained contacts to predict protein motion. PLoS ONE, 12(8):e0183889, August 2017.

[23] Wenjun Zheng and Bernard Brooks. Identification of Dynamical Correlations within the Myosin Motor Domain by the Normal Mode Analysis of an Elastic Network Model. J. Mol. Bio., 346(3):745-759, February 2005.

[24] Sibsankar Kundu, Dan C. Sorensen, and George N. Phillips. Automatic domain decomposition of proteins by a Gaussian Network Model. Proteins, 57(4):725-733, December 2004.

[25] Zhiyong Zhang, Yunyu Shi, and Haiyan Liu. Molecular Dynamics Simulations of Peptides and Proteins with Amplified Collective Motions. Biophys. J., 84(6):3583-3593, June 2003.

[26] K. Hamacher. Relating sequence evolution of HIV1-protease to its underlying molecular mechanics. Gene, 422(1-2):30-36, October 2008.

[27] Jinjun Zhang, Bonsung Koo, Yingtao Liu, Jin Zou, Aditi Chattopadhyay, and Lenore Dai. A novel statistical spring-bead based network model for self-sensing smart polymer materials. Smart Mater. Struct., 24(8):085022, August 2015.

[28] Jinjun Zhang, Bonsung Koo, Nithya Subramanian, Yingtao Liu, and Aditi Chattopadhyay. An optimized cross-linked network model to simulate the linear elastic material response of a smart polymer. J. INTEL. MAT. SYST. STR., 27(11):1461-1475, July 2016.

[29] Florence Tama and Charles L. Brooks. The Mechanism and Pathway of pH Induced Swelling in Cowpea Chlorotic Mottle Virus. J. Mol. Bio., 318(3):733-747, May 2002.

[30] Indira H. Shrivastava and Ivet Bahar. Common Mechanism of Pore Opening Shared by Five Different Potassium Channels. Biophysical Journal, 90(11):3929-3940, June 2006.

[31] Nicolas Bocquet, Hugues Nury, Marc Baaden, Chantal Le Poupon, Jean-Pierre Changeux, Marc Delarue, and Pierre-Jean Corringer. X-ray structure of a pentameric ligand-gated ion channel in an apparently open conformation. Nature, 457(7225):111-114, January 2009.

[32] Giovanni Pinamonti, Sandro Bottaro, Cristian Micheletti, and Giovanni Bussi. Elastic network models for RNA: a comparative assessment with molecular dynamics and SHAPE experiments. Nucleic Acids Res, 43(15):7260-7269, September 2015.

[33] Joanna I. Sułkowska, Andrzej Kloczkowski, Taner Z. Sen, Marek Cieplak, and Robert L. Jernigan. Predicting the order in which contacts are broken during single molecule protein stretching experiments. Proteins, 71(1):45-60, April 2008.

[34] Herbert Goldstein, Charles P. Poole, and John L. Safko. Classical mechanics. Addison Wesley, San Francisco, NJ, 3. ed edition, 2002. OCLC: 248389949.

[35] Ivet Bahar, Timothy R. Lezon, Ahmet Bakan, and Indira H. Shrivastava. Normal Mode Analysis of Biomolecular Structures: Functional Mechanisms of Membrane Proteins. Chem. Rev., 110(3):1463-1497, March 2010.

[36] S. Nicolay and Y.-H. Sanejouand. Functional Modes of Proteins Are among the Most Robust. Phys. Rev. Lett., 96(7):078104, February 2006. Publisher: American Physical Society.

[37] Gerald Wilemski and Marshall Fixman. Diffusion-controlled intrachain reactions of polymers. I Theory. J. Chem. Phys., 60(3):866-877, February 1974.

[38] Milton Abramowitz and Irene A. Stegun, editors. Handbook of mathematical functions: with formulas, graphs, and mathematical tables. Dover books on mathematics. Dover Publ, New York, NY, 9. dover print. edition, 2013. OCLC: 935935300.

[39] Dominique Foata. Some hermite polynomial identities and their combinatorics. Adv. Appl. Math., 2(3):250-259, 1981.

[40] Hannes Risken and Till Frank. The Fokker-Planck Equation: Methods of Solution and 
Applications. Springer Series in Synergetics. Springer-Verlag, Berlin Heidelberg, 2 edition, 1996.

[41] Andrea Amadei, Antonius B. M. Linssen, and Herman J. C. Berendsen. Essential dynamics of proteins. Proteins, 17(4):412-425, December 1993.

[42] Alessio Lapolla, David Hartich, and Aljaž Godec. Spectral theory of fluctuations in time-average statistical mechanics of reversible and driven systems. Phys. Rev. Research, 2(4):043084, October 2020.

[43] Alessio Lapolla and Aljaž Godec. Manifestations of Projection-Induced Memory: General Theory and the Tilted Single File. Front. Phys., 7, 2019.

[44] Gardiner, C.W. Handbook of Stochastic Methods for Physics, Chemistry and Natural Sciences. Springer-Verlag, second edition, 1985.

[45] Kevin Truong and Mitsuhiko Ikura. The use of FRET imaging microscopy to detect protein-protein interactions and protein conformational changes in vivo. CURR OPIN STRUC BIOL, 11(5):573-578, September 2001.

[46] Weixiang Ye, Markus Götz, Sirin Celiksoy, Laura Tüting, Christoph Ratzke, Janak Prasad, Julia Ricken, Seraphine V. Wegner, Rubén Ahijado-Guzmán, Thorsten Hugel, and Carsten Sönnichsen. Conformational Dynamics of a Single Protein Monitored for $24 \mathrm{~h}$ at Video Rate. Nano Lett., 18(10):6633-6637, October 2018.

[47] Kac, M. On distributions of Certain Weiner functionals. Trans. Amer. Math. Soc, 65:1-13, 1949.

[48] Ju-Yi Yen and Marc Yor. Local times and excursion theory for Brownian motion: a tale of Wiener and Itô measures. Number 2088 in Lecture notes in mathematics. Springer, Cham, 2013. OCLC: 862993594.

[49] Satya N. Majumdar and Alain Comtet. Local and Occupation Time of a Particle Diffusing in a Random Medium. Phys. Rev. Lett., 89(6):060601, July 2002.

[50] Satya N. Majumdar. Brownian Functionals in Physics and Computer Science. In The Legacy of Albert Einstein, pages 93-129. WORLD SCIENTIFIC, December 2006.

[51] Alessio Lapolla and Aljaž Godec. Unfolding tagged particle histories in single-file diffusion: exact single- and two-tag local times beyond large deviation theory. New J. Phys., 20(11):113021, November 2018.

[52] Irina V. Gopich and Attila Szabo. Single-Macromolecule Fluorescence Resonance Energy Transfer and Free-Energy Profiles. J. Phys. Chem. B, 107(21):5058-5063, May 2003.

[53] Christoph W. Müller and Georg E. Schulz. Structure of the complex between adenylate kinase from Escherichia coli and the inhibitor Ap5A refined at $1.9 \AA$ resolution. J. Mol. Bio., 224(1):159-177, March 1992.

[54] Cw Müller, Gj Schlauderer, J Reinstein, and Ge Schulz. Adenylate kinase motions during catalysis: an energetic counterweight balancing substrate binding. Structure, 4(2):147-156, February 1996.

[55] Katherine A. Henzler-Wildman, Vu Thai, Ming Lei, Maria Ott, Magnus Wolf-Watz, Tim Fenn, Ed Pozharski, Mark A. Wilson, Gregory A. Petsko, Martin Karplus, Christian G. Hübner, and Dorothee Kern. Intrinsic motions along an enzymatic reaction trajectory. Nature, 450(7171):838-844, December 2007.

[56] J. A. Hanson, K. Duderstadt, L. P. Watkins, S. Bhattacharyya, J. Brokaw, J.-W. Chu, and H. Yang. Illuminating the mechanistic roles of enzyme conformational dynamics. Proc Natl Acad Sci USA, 104(46):18055-18060, November 2007.

[57] Yuqing Zheng and Qiang Cui. Multiple Pathways and Time Scales for Conformational Transitions in apo-Adenylate Kinase. J. Chem. Theory Comput., 14(3):1716-1726, March 2018.

[58] A. Bakan, L. M. Meireles, and I. Bahar. ProDy: Protein Dynamics Inferred from Theory and Experiments. Bioinformatics, 27(11):1575-1577, June 2011.

[59] Alessio Lapolla and Aljaž Godec. A Toolbox for Quantifying Memory in Dynamics Along Reaction Coordinates. arXiv:2101.05237 [cond-mat, physics:physics], January 2021. arXiv: 2101.05237.

[60] https://www.boost.org/doc/libs/1_73_0/libs/math/doc/html/math_toolkit/gauss_kronrod.html, August 2020.

[61] Giovanni Lipari and Attila Szabo. Model-free approach to the interpretation of nuclear magnetic 
resonance relaxation in macromolecules. 1. Theory and range of validity. J. Am. Chem. Soc., 104(17):4546-4559, 1982.

[62] Angelo Perico, Roberto Pratolongo, Karl F. Freed, Richard W. Pastor, and Attila Szabo. Positional time correlation function for one-dimensional systems with barrier crossing: Memory function corrections to the optimized Rouse-Zimm approximation. J. Chem. Phys., 98(1):564$573,1993$.

[63] Alessio Lapolla and Aljaž Godec. Single-file diffusion in a bi-stable potential: Signatures of memory in the barrier-crossing of a tagged-particle. J. Chem. Phys., 153(19):194104, 2020.

[64] Y. Tian, B. Shirinzadeh, and D. Zhang. Design and dynamics of a 3-DOF flexure-based parallel mechanism for micro/nano manipulation. Microelectron Eng, 87(2):230-241, February 2010.

[65] Dannelle P. Sierra, Nathan A. Weir, and James Frank Jones. A review of research in the field of nanorobotics. Sandia National Laboratories, October 2005.

[66] Henry Crapo. Structural rigidity. Structural topology, 1979, núm. 1, 1979. Publisher: Université du Québec à Montréal.

[67] C.R. Calladine. Buckminster Fuller's "Tensegrity" structures and Clerk Maxwell's rules for the construction of stiff frames. Int J Solids Struct, 14(2):161-172, 1978.

[68] J. Clerk Maxwell. L. On the calculation of the equilibrium and stiffness of frames. Lond.Edinb.Dubl.Phil.Mag., 27(182):294-299, April 1864.

[69] S. Pellegrino and C.R. Calladine. Matrix analysis of statically and kinematically indeterminate frameworks. Int J Solids Struct, 22(4):409-428, 1986.

[70] Gradshteyn, I. S. and Ryzhik, I. M. Table of integrals, series, and products. Elsevier/Academic Press, Amsterdam, seventh edition, 2007.

[71] Wolfram Research, Inc. Mathematica, Version 12.0, 2019.

[72] John P. Boyd. The rate of convergence of Hermite function series. Math. Comp., 35(152):13091309, January 1980.

[73] Conrad Sanderson and Ryan Curtin. Armadillo: a template-based C++ library for linear algebra. J. Open Source Softw., 1(2):26, 2016. Publisher: The Open Journal.

[74] Conrad Sanderson and Ryan Curtin. A User-Friendly Hybrid Sparse Matrix Class in C++. In James H. Davenport, Manuel Kauers, George Labahn, and Josef Urban, editors, Mathematical Software - ICMS 2018, pages 422-430, Cham, 2018. Springer International Publishing.

[75] Fredrik Johansson. Arb: Efficient Arbitrary-Precision Midpoint-Radius Interval Arithmetic. IEEE T COMPUT, 66(8):1281-1292, August 2017.

[76] Fredrik Johansson. Computing Hypergeometric Functions Rigorously. ACM Trans. Math. Softw., 45(3), August 2019. Place: New York, NY, USA Publisher: Association for Computing Machinery. 


\section{Chapter 12}

\section{Summary and Conclusions}

This thesis focused on the generic differences and similarities of Markovian and non-Markovian processes arising from a projection of high-dimensional dynamics onto a low-dimensional physical observable. Although the presence or absence of memory is not one of the most advanced but a rather fundamental way to categorize stochastic processes, the investigation of memory effects begun in the last century and continues to be a promising and active field of research in this one.

The most important contribution of this thesis has been the elucidation of properties of non-Markovian processes arising in two distinct ways: via time-averaging and from a projection. The former, irrespective of whether the process is Makovian or not, considers a stochastic functional of the process that takes into account its entire history via a time-averaging procedure. The latter has roots in the extensive work done in the Physics community in the 1960s. However, instead of analyzing memory effects due to the integration over degrees of freedom in deterministic systems obeying the Liouville equation, we here projected out degrees of freedom in a Markovian stochastic system and studied the solution of the resulting, in general non-Markovian, proceses. In contrast to the majority of the literature dealing with projected dynamics which is devoted to deriving effective equations of motion in the presence of memory, we instead focused on the generic properties of the corresponding solutions.

In Chapter 3 we considered the dynamics of a paradigmatic many-body system: the single file in a box [59]. In particular, we considered the Green's function describing the motion of a tagged particle, a paradigm for anomalous diffusion. To this end we implemented a coordinate Bethe-Ansatz solution. Then we studied the local time fraction statistic of this observable. Notably, studying the covariance of the local time between two particles, we uncovered a "jamming transition" as the density of the single file increases. 
In Chapter 4 we studied how the projection becomes manifested in the evolution of lower dimensional observables. Considering a multi-dimensional Markovian stochastic process we projected the full dynamics onto a lower dimensional subspace. We proved that the resulting process is almost always non-Markovian, and that this does not happen only if the projection is orthogonal to the latent hidden variables at all times. In addition, we proved a sufficient condition under which the projected process is a renewal process, a result that again involves the orthogonality between the projected and latent degrees of freedom. We applied the result to the single file in a box subject to a constant external potential. We used this toy-model to study the attenuation of memory as the systems equilibrates over time.

The publication linked to Chapter 5 focused on the efficient implementation of the formally exact Bethe-Ansatz solution of the tagged particle in a single file in the presence a potential. The coordinate Bethe-Ansatz solution for the Green's function is challenging to evaluate since it involves a sum over all possible permutations over the eigenvalues of the many-body generator, henceforth requiring a non-polynomial algorithm to compute the conditional probability. However, exploiting the exchange symmetry of single file systems, it was possible to greatly reduce the computational effort. We implemented the algorithm and made it publicly available as an opensource, easily extendable, $\mathrm{C}++$ code. We also analyzed the computational complexity of our implementation.

In Chapter 6 we studied how systems, in which thermal fluctuations play a predominant role, relax towards equilibrium. In particular we considered "thermodinamically equidistant temperature quenches", that is: two identical systems prepared, respectively, below and above the equilibrium temperature such that these initial conditions are thermodinamically equidistant. We then asked which system reaches equilibrium faster. The main finding of the article is in contradiction with common experience in the macroscopic world: in small systems displaying a single well energetic potential, the system starting from the lower temperature equilibrates faster than its hotter counterpart. In the case of multi-well potentials the phenomenology is richer and we provide insight on this case as well. The uncovered asymmetry is due to the fact that the evolution of small noisy systems does not pass through local equilibrium configurations.

In Chapter 7 we extended the findings on the local time statistic developed in Chapter 3 to ergodic systems evolving from general initial conditions in presence and absence of detailed balance. We presented a detailed derivation of the first two moments of the local time using two methods: perturbation theory for non-Hermitian operators and the Dyson series approach. 
Subsequently we applied our results to several toy-models and showed via direct computation the emergence of the Central Limit Theorem for long times. In addition, a theorem connecting the variance of the local time and the return probability of a stochastic process has been proven and applied to the precision of biochemical signaling by chemical receptors.

In Chapter 8 we again directed our attention to relaxation towards equilibrium in systems with an energetic barrier in their potential landscape. In particular, by analyzing a single file in a box with a square barrier we studied how the relaxation towards equilibrium changes for different tracer-particles (i.e. different projected observables) and how the height of the barrier influences the relaxation. In addition, memory kernels of the autocorrelation functions have been computed numerically and rationalized.

In Chapter 9 we continued our investigations of memory in the dynamics of projected observables. We devised two practical methods to quantify the extent and presence of memory in the time-evolution of a process. One method compares the relaxation with that of a surrogate Markovian process with the same equilibrium properties as the original one, then the differences in the evolution of these two processes are analyzed. The other method checks if the semi-group property is satisfied using the Chapman-Kolmogorov equation. We tested our methods for systems relevant in polymer physics: a theoretical one, the Rouse chain, and an experimantal time-trace describing the end-to-end distance of a DNA-hairpin.

In Chapter 10 we showed how the mere, experimentally imposed, act of projecting out some degrees of freedom can break time-translational invariance. This breaking happens if and only if the projected dynamics in nonMarkovian and if the initial condition of the non-Markovian dynamics is not drawn from the stationary distribution. The effect is rather general and does not require any assumption beyond the assumption that the full dynamics is Markovian; notably it does not depend on the properties of the (free-)energy landscape governing the diffusive process nor the scaling of the relaxation time with respect to the size of the system. In fact, we provided practical examples by considering two analytically solvable models presenting a very regular potential and a quadratic scaling of the relaxation time as a function of the system size. In addition, using data obtained from an optical-tweezers experiment on a DNA hairpin [60] and a Molecular Dynamics simulation of a protein called phosphoglycerate kinase [61], we demonstrated the consistency of our findings in real systems with complex dynamics.

In Chapter 11 we addressed the so-called Gaussian Network Model. The model has been found to be useful in the analysis of internal dynamics of proteins. In particular we focused on a one-dimensional non-Markovian observable: the distance between two (sets) of beads in the network. The corresponding projection operator reduces a high-dimensional problem to a single coordinate. We obtained an analytical expression for time- and 
ensemble-averaged statistical quantities describing the fluctuations of such distances. We applied our findings to the study of a protein displaying a biologically relevant open-closed transition: the adenylate kinase. The most interesting finding of the article is the recovery of Gaussian, yet still nonMarkovian, behavior for very rigid structures.

From a purely mathematical point of view we were able to obtain exact formulae for the time-average a class of functional (Chapters 3 and 7 ). Nevertheless, the understanding of these results is far from being a completed task. In principle the perturbative approach used to obtain the first two moments of a time-averaged observable can be extended to higher order moments. Nonetheless the computational cost of this extension is prohibitive and, therefore, the ambitious goal of obtaining the probability density function for all times for these functionals cannot be obtained using the methods presented in this thesis (though they could be of some help). Moreover, even the properties of the results obtained in this thesis have still not been exhaustively explained yet. In particular, a partial list of open questions that we did not attempt to solve in the thesis on the mean and variance of timeaveraged functionals are:

- How do these observables depend on the dimensionality of the stochastic process (we only looked at one dimensional systems or one dimensional projections/reaction coordinates)?

- In our models the variance has at most one maximum as a function of time. Is this a universal feature? How is the position of this maximum related to the details of the process described?

- How is the long-time Gaussian statistics approached for generic initial conditions, i.e.: how fast the higher order moments decay to zero?

Though we did not answer to these questions, we believe that an answer can be found on the basis of our results. In addition we did not apply our results on time-averages to experimental time-series; nevertheless, the full history of the process could lead to a deeper understanding of the physical system probed experimentally.

Notably this possibility has a strong link to the results obtained in Chapters 4 and 10 for projected observables. Single-molecule experiments, usually, only probe a subset of the internal degrees of freedom of the system. Since we proved that this projected observable is non-Markovian with very high probability (at least for not very long times), and since experimental limitations may cause an undersampling of the equilibrium distribution of the reaction coordinate, we henceforth expect a breaking of timetranslational invariance. Therefore, as a consequence, memory effects must 
be taken into account in order to unfold important features of the system when analyzing these trajectories.

We partially investigated in Chapter 8 the non-trivial connection between memory and barriers in the (free-)energy landscapes. We believe that this is a promising field to apply our results that will be developed in the future. In Chapter 9 we bridged the gap between theory and experiments by developing methods to analyze experimental time-series. Nevertheless, an extensive explanation of the consequences of projections and how these are linked to the physical and also biological (in the case of some systems) properties of real experimental systems is still elusive and deserves a deeper study.

An exception to the content of this thesis is the work presented in Chapter 6, the idea of comparing how non-Markovian and Markovian observables of the same systems relax towards equilibrium lead us to the discovery of a completely unexpected asymmetry between the "cooling" and "heating" of a system.

In conclusion, using a pictorial metaphor, we looked at shadows of trajectories that remember the bodies that generated them, and analyzed the "shape and forms" they assume. As usual in Science, this thesis barely scratched the surface of memory effects and left the field with more questions than answers. 


\section{Bibliography}

[1] Robert Brown. A brief account of microscopical observations made in the months of June, July and August 1827, on the particles contained in the pollen of plants; and on the general existence of active molecules in organic and inorganic bodies. The Philosophical Magazine, 4(21):161173, September 1828.

[2] Albert Einstein. Über die von der molekularkinetischen Theorie der Wärme geforderte Bewegung von in ruhenden Flüssigkeiten suspendierten Teilchen. Annalen der physik, 4, 1905.

[3] William Sutherland. A dynamical theory of diffusion for nonelectrolytes and the molecular mass of albumin. The London, Edinburgh, and Dublin Philosophical Magazine and Journal of Science, 9(54):781-785, 1905. Publisher: Taylor \& Francis.

[4] Marian Von Smoluchowski. Zur kinetischen theorie der brownschen molekularbewegung und der suspensionen. Annalen der physik, 326(14):756-780, 1906. Publisher: Wiley Online Library.

[5] Jean Perrin. Brownian movement and molecular reality, translated from the Annales de Chimie et de Physique. Londres, 1910.

[6] Paul Langevin. Sur la théorie du mouvement brownien. Compt. Rendus, 146:530-533, 1908.

[7] Adriaan Daniël Fokker. Die mittlere Energie rotierender elektrischer Dipole im Strahlungsfeld. Annalen der Physik, 348(5):810-820, 1914. Publisher: WILEY-VCH Verlag Leipzig.

[8] VM Planck. Über einen Satz der statistischen Dynamik und seine Erweiterung in der Quantentheorie. Sitzungberichte der, 1917.

[9] George E Uhlenbeck and Leonard S Ornstein. On the theory of the Brownian motion. Physical review, 36(5):823, 1930. Publisher: APS.

[10] Oskar Klein. Zur statistischen Theorie der Suspensionen und Lösungen. PhD Thesis, Almqvist \& Wiksell, 1921. 
[11] Hendrik Anthony Kramers. Brownian motion in a field of force and the diffusion model of chemical reactions. Physica, 7(4):284-304, 1940. Publisher: Elsevier.

[12] Norbert Wiener. Differential-Space. Journal of Mathematics and Physics, 2(1-4):131-174, 1923. Publisher: Wiley Online Library.

[13] Norbert Wiener and others. Generalized harmonic analysis. Acta mathematica, 55:117-258, 1930. Publisher: Institut Mittag-Leffler.

[14] Andrei Kolmogoroff. Über die analytischen Methoden in der Wahrscheinlichkeitsrechnung. Mathematische Annalen, 104(1):415-458, 1931. Publisher: Springer.

[15] A Kolmogoroff. Zur Theorie der stetigen zufälligen Prozesse. Mathematische Annalen, 108(1):149-160, 1933. Publisher: Springer.

[16] William Feller. Zur Theorie der stochastischen Prozesse. In Selected Papers I, pages 293-340. Springer, 2015.

[17] Paul Lévy and Michel Loeve. Processus stochastiques et mouvement brownien. Gauthier-Villars Paris, 1965.

[18] Paul Lévy. Sur certains processus stochastiques homogènes. Compositio mathematica, 7:283-339, 1940.

[19] Rafael Benguria and Mark Kac. Quantum langevin equation. Physical review letters, 46(1):1, 1981. Publisher: APS.

[20] A.O. Caldeira and A.J. Leggett. Path integral approach to quantum Brownian motion. Physica A: Statistical Mechanics and its Applications, 121(3):587-616, September 1983.

[21] Benjamin Svetitsky. Diffusion of charmed quarks in the quark-gluon plasma. Physical Review D, 37(9):2484, 1988. Publisher: APS.

[22] Hendrik van Hees and Ralf Rapp. Thermalization of heavy quarks in the quark-gluon plasma. Physical Review C, 71(3):034907, 2005. Publisher: APS.

[23] Mohamed Abdel Aziz and Sean Gavin. Causal diffusion and the survival of charge fluctuations in nuclear collisions. Physical Review C, 70(3):034905, 2004. Publisher: APS.

[24] Edmund Bertschinger. Brownian motion of stars, dust, and invisible matter. In AIP Conference Proceedings, volume 861, pages 97-105. American Institute of Physics, 2006. Issue: 1. 
[25] Brandon Wolfe and Fulvio Melia. Covariant kinetic theory with an application to the coma cluster. The Astrophysical Journal, 638(1):125, 2006. Publisher: IOP Publishing.

[26] Jörn Dunkel and Peter Hänggi. Relativistic Brownian motion. Physics Reports, 471(1):1-73, February 2009.

[27] Linda JS Allen. An introduction to stochastic processes with applications to biology. CRC Press, 2010.

[28] Eric Lauga and Thomas R Powers. The hydrodynamics of swimming microorganisms. Rep. Prog. Phys., 72(9):096601, September 2009.

[29] Mark Davis. Mathematics of financial markets. In Mathematics unlimited-2001 and beyond, pages 361-380. Springer, 2001.

[30] Mark Potters. Theory of financial risks: from statistical physics to risk management. Cambridge university press, 2000.

[31] Majumdar, Satya N. Brownian functionals in Physics and Computer Science. Current Science, 89(12):2079-2092, December 2005.

[32] Claudio Castellano, Santo Fortunato, and Vittorio Loreto. Statistical physics of social dynamics. Rev. Mod. Phys., 81(2):591-646, May 2009.

[33] Gardiner, C.W. Handbook of Stochastic Methods for Physics, Chemistry and Natural Sciences. Springer-Verlag, second edition, 1985.

[34] N.G. van Kampen. Remarks on Non-Markov Processes. Braz. J. Phys., 28(2), June 1998.

[35] Robert Zwanzig. Nonequilibrium statistical mechanics. Oxford Univ. Press, Oxford [ u.a. ], nachdr. edition, 2010. OCLC: 838376705.

[36] Ulrich Krengel and Antoine Brunel. Ergodic Theorems. De Gruyter, January 1985.

[37] Robert Zwanzig. Memory Effects in Irreversible Thermodynamics. Phys. Rev., 124(4):983-992, November 1961.

[38] William Feller. An introduction to probability theory and its applications. Vol. 1: ... Wiley series in probability and mathematical statistics. Wiley, S.l., 3. ed., rev. print., [nachdr.] edition, 2009. OCLC: 837266385 .

[39] William Feller. An introduction to probability theory and its applications. Vol. 2: ... Wiley series in probability and mathematical statistics. Wiley, S.1.., 2. ed., [nachdr.] edition, 2009. OCLC: 608467098. 
[40] Jeffrey S. Rosenthal. A first look at rigorous probability theory. World Scientific, New Jersey, NJ, 2. ed., reprint edition, 2009. OCLC: 845712156 .

[41] Kiyosi Itô. Stochastic integral. Proc. Imp. Acad., 20(8):519-524, 1944.

[42] Kiyosi Ito. On stochastic differential equations. Memoirs of the AMS, 0(4):0-0, 1951.

[43] Bernt Øksendal. Stochastic Differential Equations. Universitext. Springer Berlin Heidelberg, Berlin, Heidelberg, 2003.

[44] Hannes Risken and Till Frank. The Fokker-Planck Equation: Methods of Solution and Applications. Springer Series in Synergetics. SpringerVerlag, Berlin Heidelberg, 2 edition, 1996.

[45] Sidney Redner. A guide to first-passage processes. Cambridge Univ. Press, Cambridge, digitally printed version (with corrections) 2007 edition, 2007. OCLC: 830642837.

[46] Laurie M Brown and Richard Phillips Feynman. Feynman's thesis: a new approach to quantum theory, 2005. ISBN: 9789812563668 9789812567635 Place: Hackensack, NJ [u.a. OCLC: 836979915.

[47] Kac, M. On distributions of Certain Weiner functionals. Trans. Amer. Math. Soc, 65:1-13, 1949.

[48] Ju-Yi Yen and Marc Yor. Local times and excursion theory for Brownian motion: a tale of Wiener and Itô measures. Number 2088 in Lecture notes in mathematics. Springer, Cham, 2013. OCLC: 862993594.

[49] Tomas Björk. The Pedestrian's Guide to Local Time. arXiv:1512.08912 [math], December 2015. arXiv: 1512.08912.

[50] Alessio Lapolla and Aljaž Godec. Unfolding tagged particle histories in single-file diffusion: exact single- and two-tag local times beyond large deviation theory. New J. Phys., 20(11):113021, November 2018.

[51] Alessio Lapolla and Aljaž Godec. Manifestations of Projection-Induced Memory: General Theory and the Tilted Single File. Front. Phys., 7, 2019.

[52] Alessio Lapolla and Aljaž Godec. BetheSF: Efficient computation of the exact tagged-particle propagator in single-file systems via the Bethe eigenspectrum. Comput. Phys. Commun, page 107569, August 2020.

[53] Alessio Lapolla and Aljaž Godec. Faster Uphill Relaxation in Thermodynamically Equidistant Temperature Quenches. Phys. Rev. Lett., 125(11):110602, September 2020. 
[54] Alessio Lapolla, David Hartich, and Aljaž Godec. Spectral theory of fluctuations in time-average statistical mechanics of reversible and driven systems. Phys. Rev. Research, 2(4):043084, October 2020.

[55] Alessio Lapolla and Aljaž Godec. Single-file diffusion in a bi-stable potential: Signatures of memory in the barrier-crossing of a taggedparticle. J. Chem. Phys., 153(19):194104, November 2020.

[56] Alessio Lapolla and Aljaž Godec. A Toolbox for Quantifying Memory in Dynamics Along Reaction Coordinates. arXiv:2101.05237 [cond-mat, physics:physics], January 2021. arXiv: 2101.05237.

[57] Alessio Lapolla, Jeremy C. Smith, and Aljaž Godec. Ubiquitous Dynamical Time Asymmetry in Measurements on Materials and Biological Systems. arXiv:2102.01666 [cond-mat, physics:math-ph, physics:physics], February 2021. arXiv: 2102.01666.

[58] Alessio Lapolla, Maximilian Vossel, and Aljaž Godec. Time- and ensemble-average statistical mechanics of the Gaussian Network Model. arXiv:2102.11847 [cond-mat, physics:physics], February 2021. arXiv: 2102.11847 .

[59] L. Lizana and T. Ambjörnsson. Single-File Diffusion in a Box. Phys. Rev. Lett., 100(20):200601, May 2008.

[60] Krishna Neupane, Ajay P. Manuel, John Lambert, and Michael T. Woodside. Transition-Path Probability as a Test of ReactionCoordinate Quality Reveals DNA Hairpin Folding Is a One-Dimensional Diffusive Process. J. Phys. Chem. Lett., 6(6):1005-1010, March 2015.

[61] Xiaohu Hu, Liang Hong, Micholas Dean Smith, Thomas Neusius, Xiaolin Cheng, and Jeremy C. Smith. The dynamics of single protein molecules is non-equilibrium and self-similar over thirteen decades in time. Nature Phys, 12(2):171-174, February 2016. 


\section{Alessio Lapolla \\ Curriculum Vitae}

\section{PERSONAL DETAILS}

$\begin{array}{ll}\text { Birth } & 13 \text { October } 1993 \\ \text { Nationality } & \text { Italian }\end{array}$

\section{EDUCATION}

Doctoral Studies

2017-2021

Mathematical bioPhysics Group, Computational BioPhysics Department,

Max Planck Institute for BioPhysical Chemisty, Germany

MSc. Theoretical Physics

$2015-2017$

University of Padua, Italy

BSc. Physics

$2012-2015$

University of Padua, Italy

\section{ADDITIONAL EDUCATIONAL EXPERIENCE}

Erasmus+ progamme

Stockholm University, Sweden 